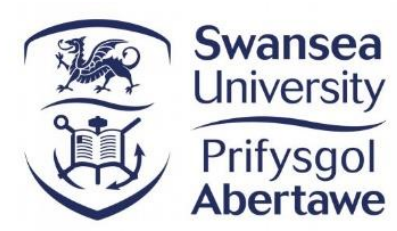

COLLEGE OF HUMAN AND HEALTH SCIENCES

Department of Nursing

\title{
Can 3D Camera Imaging Provide \\ Improved Information to Assess and \\ Manage Lymphoedema in Clinical \\ Practice?
}

Cheryl Elizabeth Pike BPhysT (Hons)

Submitted to Swansea University in fulfilment of the requirements for the Degree of Doctorate in Professional Practice 


\begin{abstract}

\section{Background}

Accurate diagnosis and measurement of limb volume in people with lymphoedema is important in order to provide best information for treatment, management and selfmanagement. Current assessment methods lack detail and accuracy. Three-dimensional camera imaging (3DCI) holds the potential to be cheap, accurate, and provide additional material about limb shape not provided by current methods. However, there is a need to ensure that this assessment method is valid and reliable.
\end{abstract}

\title{
Methodology
}

This prospective, observational, longitudinal study utilised a diagnostic test study framework to determine the validity, reliability and accuracy of 3DCI compared to circumferential tape measurement (CTM) and perometry and to explore whether shape is a feasible alternative to measure upper limb lymphoedema.

Twenty women with breast cancer-related lymphoedema were recruited. Phase one assessed criterion validity, intra-rater reliability, and accuracy of 3DCI by measuring limb volume of each participant with CTM, perometry and 3DCI four times over six months.

Phase two investigated the use of limb shape as a method of lymphoedema assessment using oedema maps and calculations of shape redundancy derived from the 3DCI images in phase one. These data sets were matched against limb volume to determine criterion validity, intra-rater reliability and accuracy.

\section{Results}

3DCI had high intra-rater correlation ( $\mathrm{ICC}=0.87 ; \mathrm{p}<0.00$ ). Concurrent validity ranged from 0.82 to 0.86 against perometry and CTM, with good sensitivity (91.7\% to $100 \%$ ) and moderate specificity (50\% to $66.7 \%)$.

Limb shape calculation (shape redundancy) had moderate intra-rater correlation ( $\mathrm{ICC}=0.71 ; \mathrm{p}=0.01$ ); but correlated poorly with limb volume ( $\mathrm{r}=0.19$ to 0.39 ). Coloured oedema maps were sensitive to change over time with colours clearly identifying problem areas and fluctuations within the affected limb.

\section{Conclusion}

Our study shows that 3DCI is a reliable, valid and accurate method of limb volume measurement, and that it could provide supportive information in clinical assessment. In addition, limb shape provides insight into localised areas of swelling, which other methods of lymphoedema measurement do not. However, shape redundancy requires further refinement. 


\section{Declarations and Statements}

This work has not previously been accepted in substance for any degree and is not being concurrently submitted in candidature for any degree.

Signed

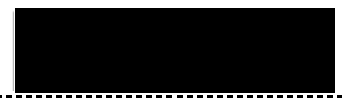
(candidate)

Date $\quad 26 / 05 / 2021$

\section{STATEMENT 1}

This thesis is the result of my own investigations, except where otherwise stated. Where correction services have been used, the extent and nature of the correction is clearly marked in a footnote(s).

Other sources are acknowledged by footnotes giving explicit references. A bibliography is appended.

Signed (candidate)

Date $. . .26 / 05 / 2021$

\section{STATEMENT 2}

I hereby give consent for my thesis, if accepted, to be available for photocopying and for inter-library loan, and for the title and summary to be made available to outside organisations.

Signed

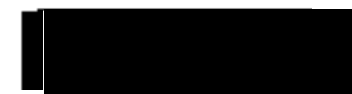
(Candidate)

Date $\quad 26 / 05 / 2021$ 


\section{Table of Contents}

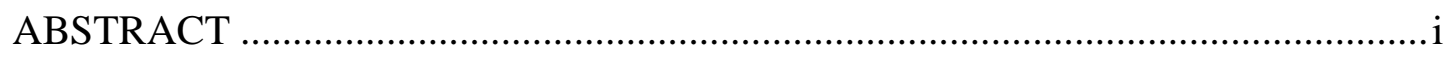

Declarations and Statements ............................................................................ ii

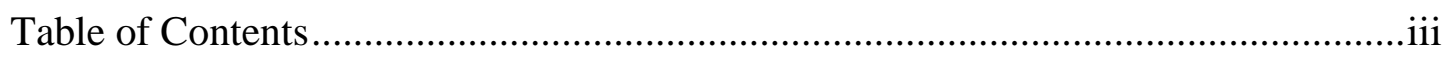

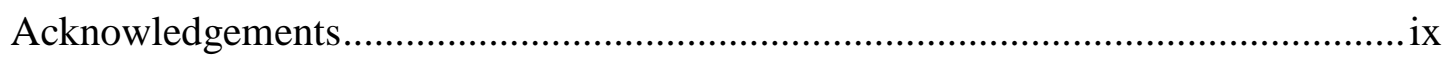

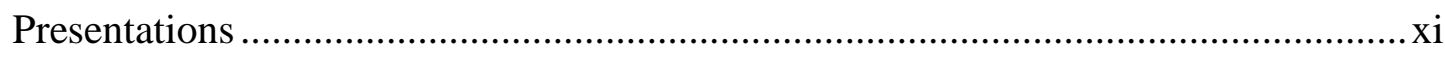

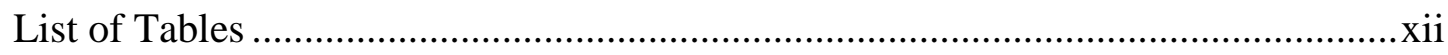

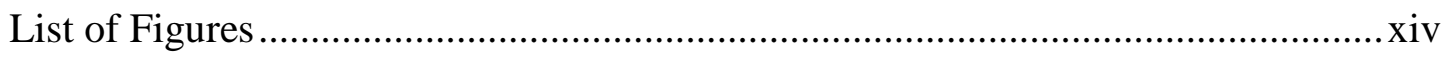

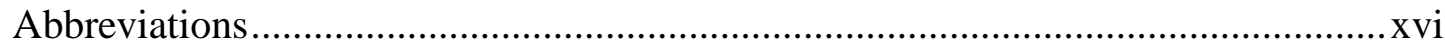

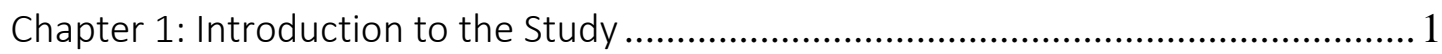

$1.1 \quad$ Background and Epidemiology of Lymphoedema ….................................... 3

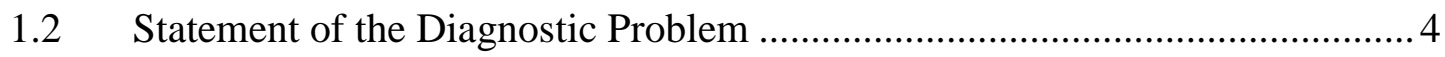

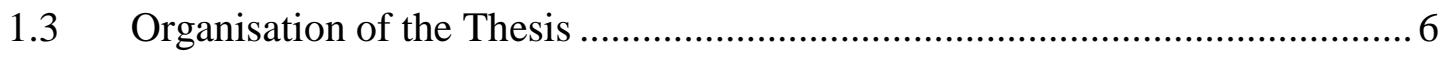

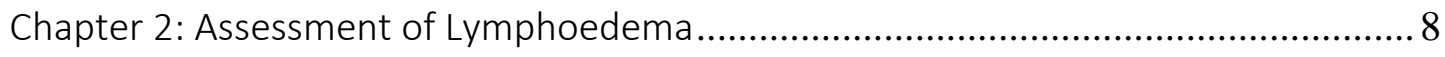

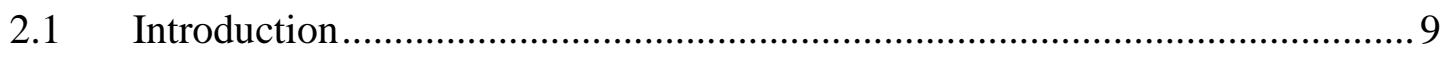

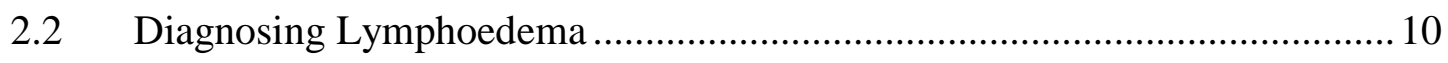

2.3 Staging of the Severity of Lymphoedema …............................................... 13

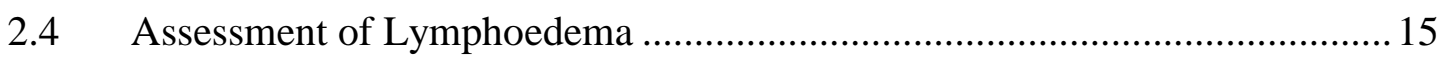

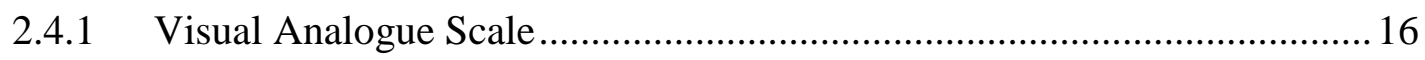

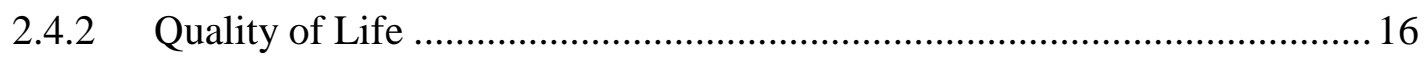

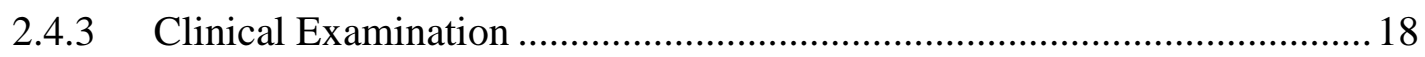

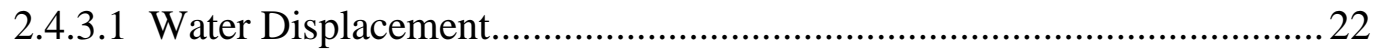

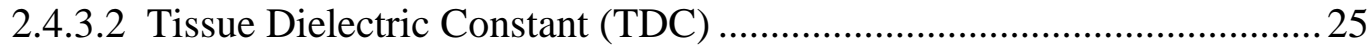

2.4.3.3 Bio-Impedance Spectroscopy (BIS) ............................................... 27

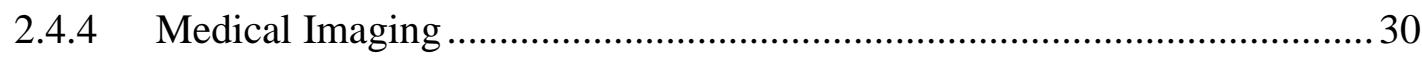

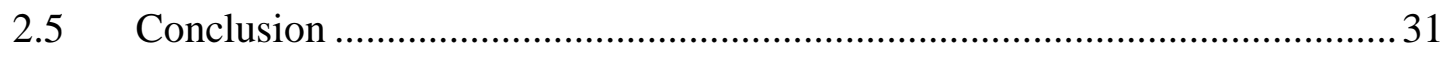




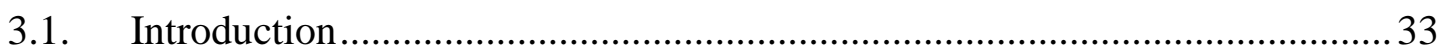

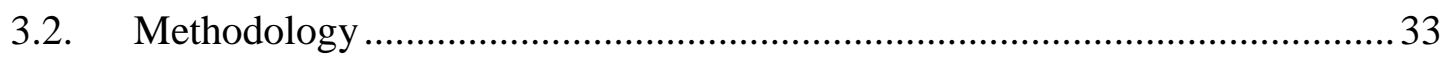

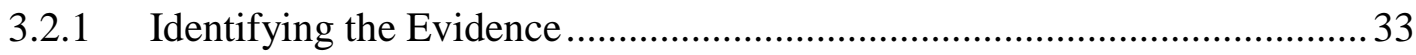

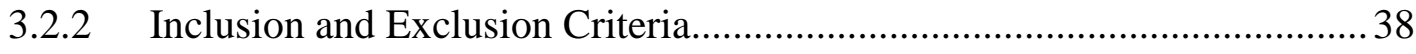

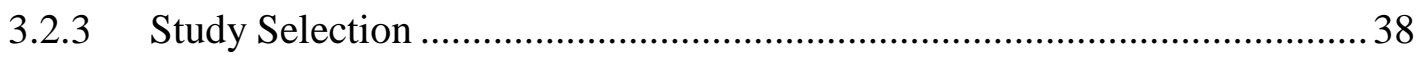

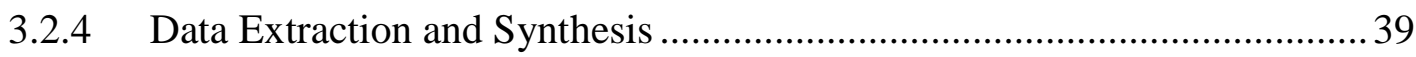

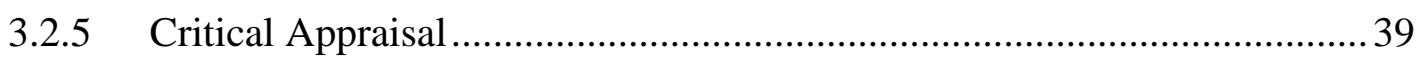

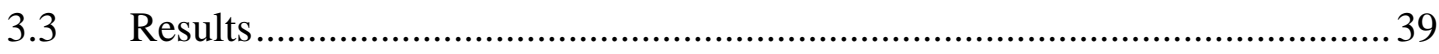

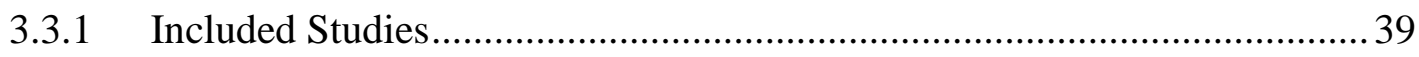

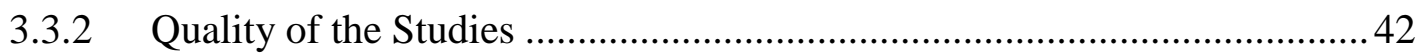

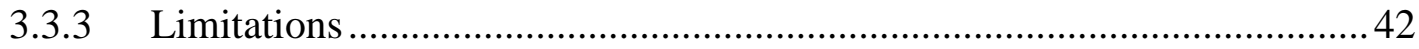

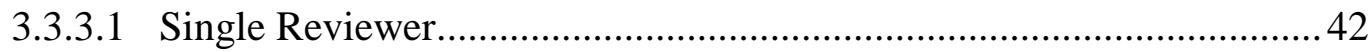

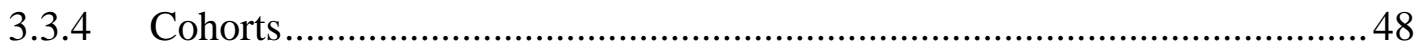

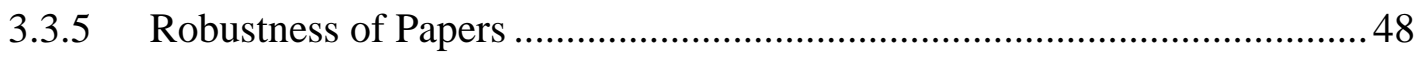

3.3.6 Circumferential Tape Measurement (CTM) ........................................ 48

3.3.6.1 Thresholds for Diagnosing Lymphoedema Using CTM 58

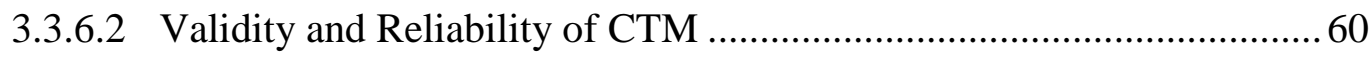

3.3.6.3 Benefits and Considerations of CTM ............................................. 61

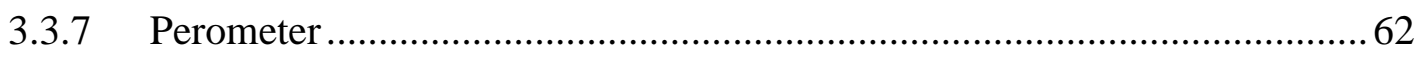

3.3.7.1 Thresholds for Diagnosing Lymphoedema Using Perometry ...............6 68

3.3.7.2 Validity and Reliability of Perometry .................................................. 69

3.3.7.3 Benefits and Considerations of Perometry ........................................ 70

3.3.8 Three-Dimensional Camera Imaging (3DCI) ..................................... 71

3.3.8.1 Thresholds for Diagnosing Lymphoedema Using 3DCI..................... 82

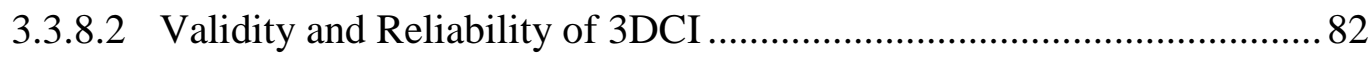

3.3.8.3 Benefits and Considerations of 3DCI................................................ 82 


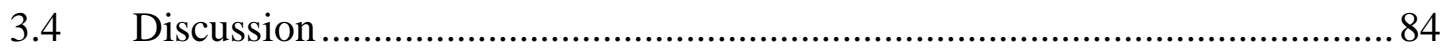

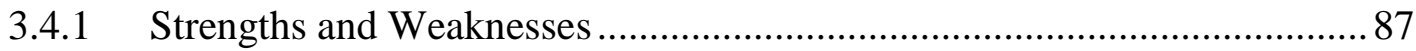

3.4.2 Implications for Daily Practice and Future Research ............................. 88

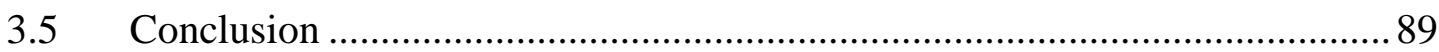

Chapter 4: Research Design and Methods ........................................................... 90

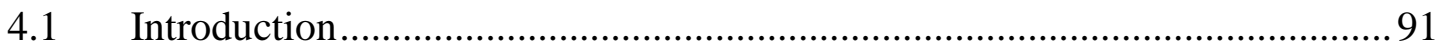

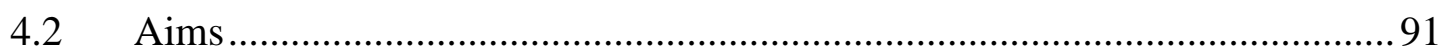

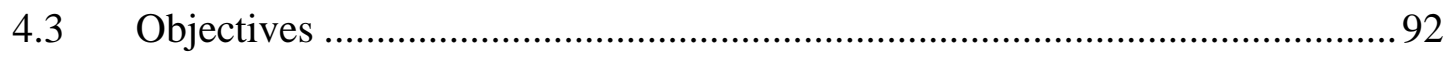

4.4 Research Methodology and Design ........................................................ 92

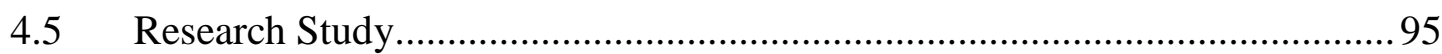

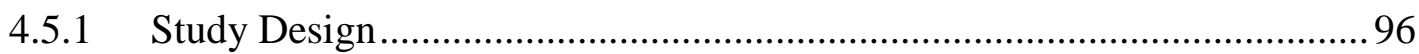

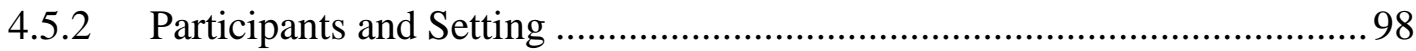

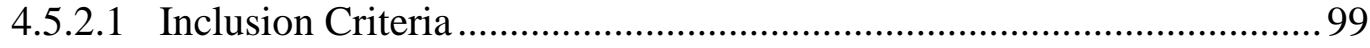

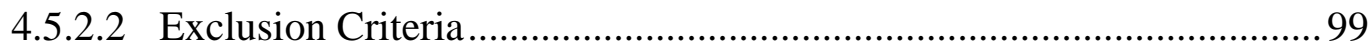

4.5.2.3 Criteria for Premature Withdrawal ..................................................... 99

4.5.3 Ethical Approval and Considerations ..................................................... 99

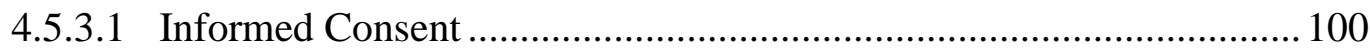

4.5.3.2 Participant Impact .................................................................. 101

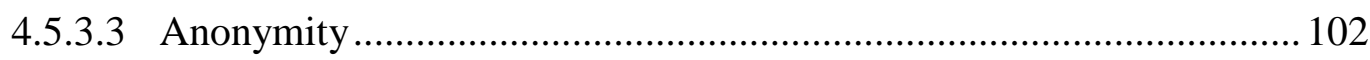

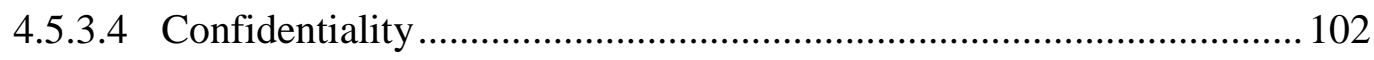

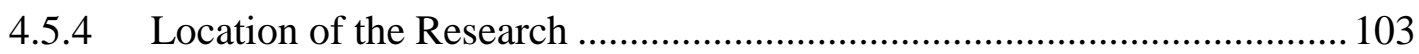

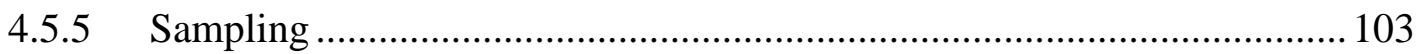

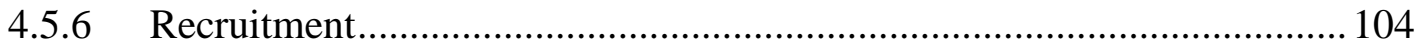

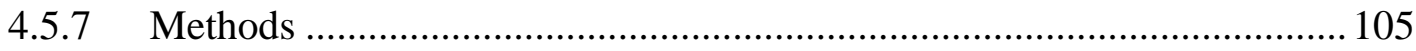

4.5.7.1 Demographic and Anthropometric Data ......................................... 105

4.5.7.2 Methods of Lymphoedema Measurements Used ............................... 107

4.5.8 Baseline Visit and Data Collection........................................................ 109 
4.6 Phase One: Diagnostic Test Study of Limb Volume ................................... 110

4.6.1 Phase One: Research Questions, Aim, and Objectives............................ 110

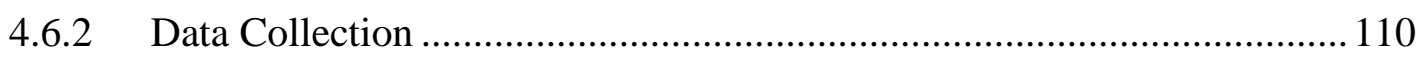

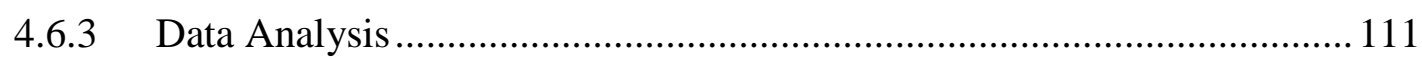

4.7 Phase Two: Diagnostic Test Study of Limb Shape .................................... 114

4.7.1 Phase Two Research Questions, Aim, and Objectives ............................ 114

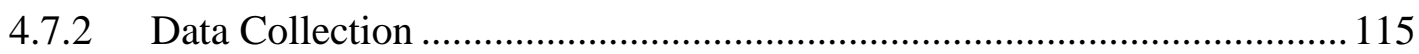

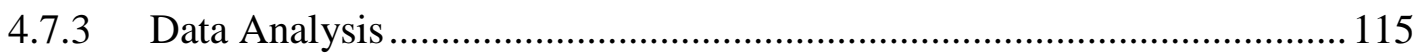

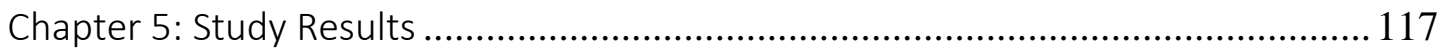

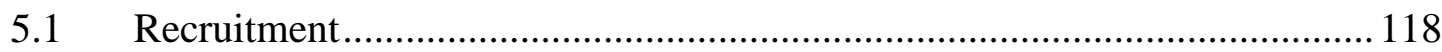

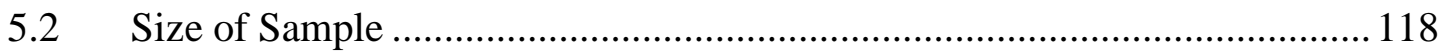

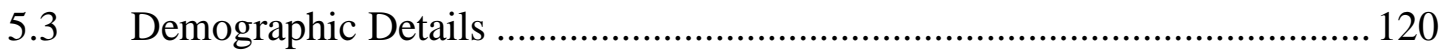

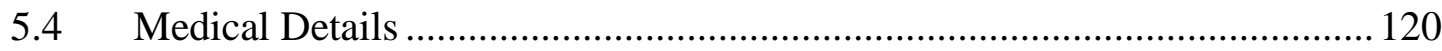

5.5 Phase One: Limb Volume Results and Analysis ...................................... 123

5.5.1 Objective 1.1: Determine the Reliability of 3DCI Compared to CTM and

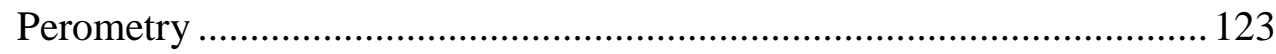

5.5.2 Objective 1.2: Assess the Validity of 3DCI Against Previously Validated Measures, i.e. CTM and Perometry ....................................................... 126

5.5.2.1 Relationship Between the Three Methods of Measurement Using Pearson's Correlation Coefficient ..................................................... 126

5.5.2.2 Agreement Between the Three Methods of Measurement Utilising BlandAltman Plots

5.5.3 Objective 1.3: Establish the Accuracy of 3DCI Compared to CTM and Perometry

5.6 Phase Two: Limb Shape Results and Analysis 130

5.6.1 Objective 2.1: Evaluate Limb Shape Derived from 3DCI as a New Method of Lymphoedema Measurement 
5.6.1.1 Objective 2.1a) Determine Trends Between Oedema Maps and Limb Volume

5.6.1.2 Reliability

5.6.1.3 Validity

5.6.1.4 Objective 2.1b) Use of Oedema Maps to Monitor Treatment Outcomes (Limb Volume Change)

5.6.1.5 Objective 2.1c) Consider Limb Shape as a Tool to Diagnose Lymphoedema

5.6.2 Objective 2.2: Ascertain 3DCI as a Sensitive Measure of Change in Lymphoedema

5.6.2.1 Objective 2.2a) Sensitivity to Change in Limb Volume Over time .... 137

5.6.2.2 Objective 2.2b) Sensitivity to Change in Limb Shape Over Time...... 137

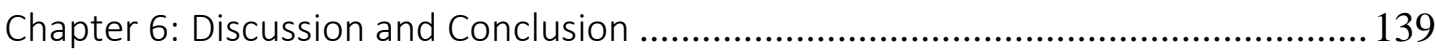

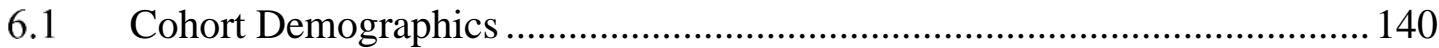

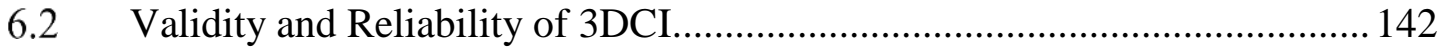

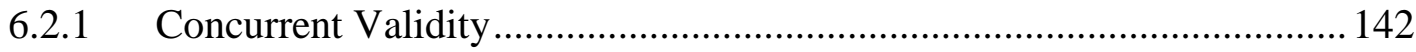

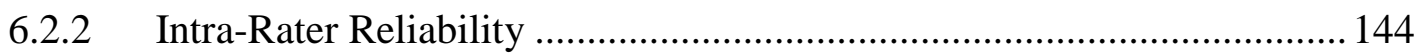

6.3 Establish the Accuracy of 3DCI Compared to CTM and Perometry............ 145

6.4 Ascertain Whether Limb Shape Derived from 3DCI is a Potential New

Method of Lymphoedema Measurement ................................................. 147

6.5 Potential Benefits of 3DCI in Clinical Practice .......................................... 148

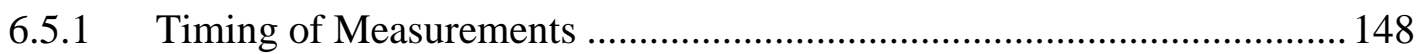

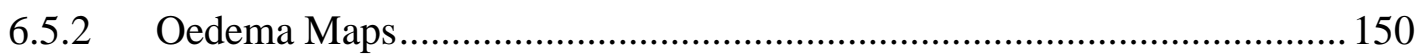

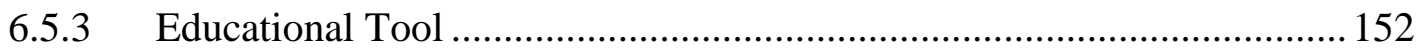

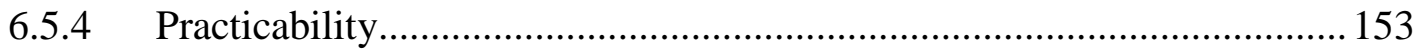

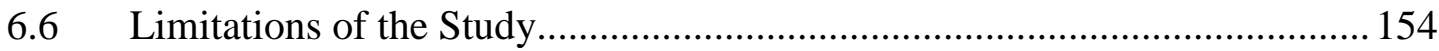

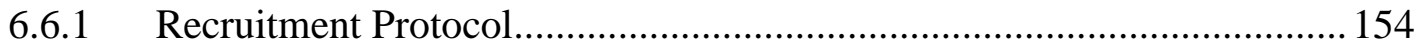

6.6.2 Unavailability of Oedema Maps ....................................................... 155 


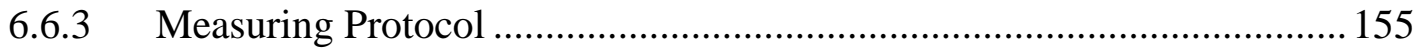

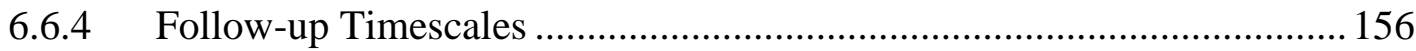

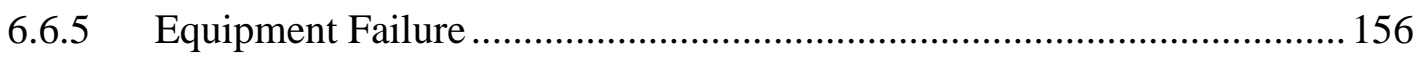

6.7 Recommendations for Further Research............................................... 156

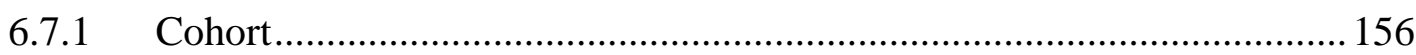

6.7.2 Extend to Lower Limb and Mid-Line Lymphoedema ............................ 157

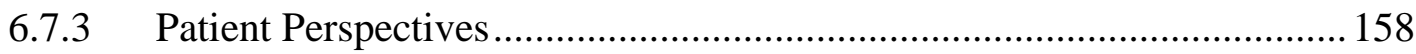

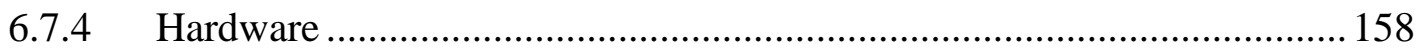

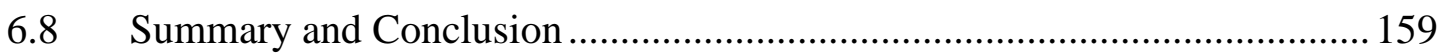

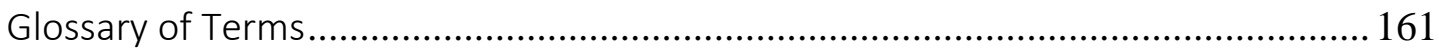

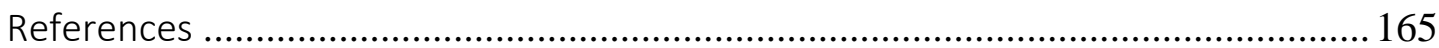

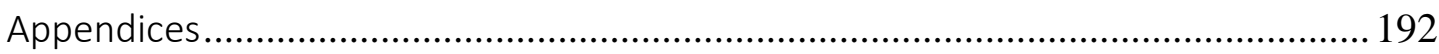

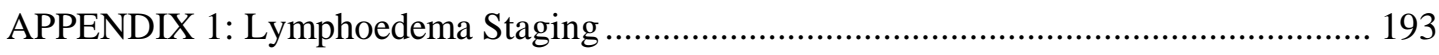

APPENDIX 2: Types of Lymphoedema Medical Imaging ................................................... 197

APPENDIX 3: Evidence Table of the Literature Reviewed on CTM, Perometry and 3DCI for

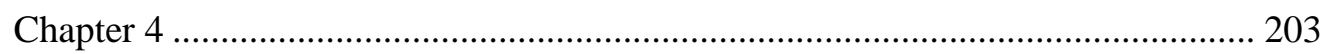

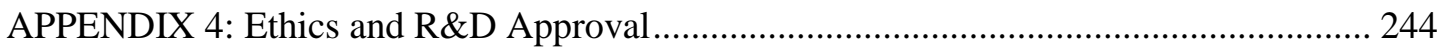

APPENDIX 5: Participant Information Sheet for Study ……............................................. 259

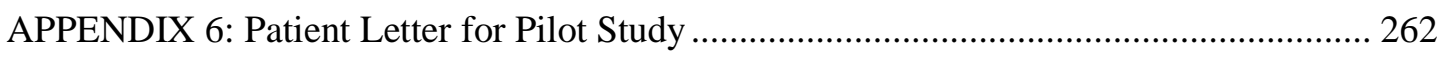

APPENDIX 7: Lymphoedema Network Wales Referral Form............................................ 263

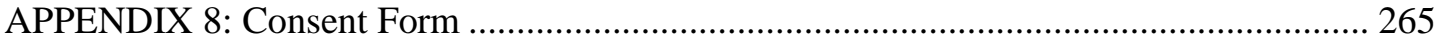

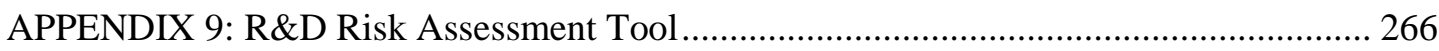

APPENDIX 10: 3D Study Assessment Form …….......................................................... 270

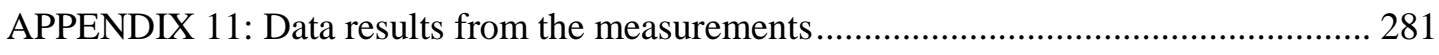

APPENDIX 12: The STARD 2015 List: An Updated List of Essential Items for Reporting

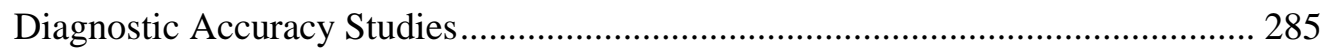

APPENDIX 13: Phase 1: Tests of Normality and Bland-Altman Plots .............................. 287

APPENDIX 14: Oedema Maps derived from 3DCI …………………............................. 295

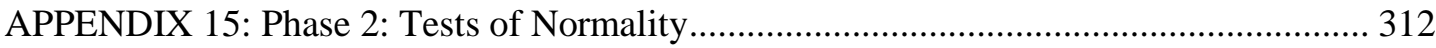




\section{Acknowledgements}

A huge thank you goes to all the women who participated in this research study. Your commitment to the time and travel required of you, and your patience during the data collection, was invaluable to the success of this study. You all demonstrated great enthusiasm and intrigue in the new methods of measuring your lymphoedema, which makes research studies like these possible.

My gratitude goes out to all my supervisors during my tenure at Swansea University. Dr Ruth Davies' interest and enthusiasm was the catalyst to my journey. Dr Raoul van Loon has been my crutch throughout my studies and is an exciting, inquisitive individual who encouraged me to push my boundaries in research. Dr Tessa Watts was instrumental in checking the processes for my research and literature review. Dr Darren Edwards gave me a greater appreciation of statistical analysis, and he is excellent for controversial and topical discussions! My thanks are extended to Prof. Sue Jordan, who helped me get back on track with my studies following a period of poor advancement for various reasons. Ioan Humphries helped me solve the riddle of health economics calculations - my thanks to you for your analytical knowledge. Finally, my heartfelt thanks and gratitude is extended to Prof. Debbie Fenlon, who was vital to the culmination of my thesis. Your time, guidance, research expertise and your endless patience and understanding were invaluable!

Without financial support, I would not have been in a position to undertake my studies. Enormous gratitude to Macmillan for sponsoring part of my studies annually. Thanks to the British Lymphology Society (BLS) for awarding me the Research Grant that enabled me to do the research study for this thesis.

Further thanks are extended to the various mathematical engineering students, including Kevork Karakashian, Lawrence Sheban and Daniel Watson, who helped design the programmes to extrapolate the data from the $3 \mathrm{D}$ images to create the oedema maps.

My friend, colleague and boss, Dr Melanie Thomas, has been responsible for this doctoral journey as she declared 'it will be good for you'! Thanks to you for your encouragement since the inception of my studies, to reading and proofreading my thesis 
at the end. Dr Rhian Noble-Jones for your wisdom, guidance and general sound mind thank you for all our chats and laughter over the last few years. Dr Marie Gabe-Walters for your thoughts and insight in the final draft of the thesis. To all my colleagues and friends in Lymphoedema Network Wales (LNW), thank you for your understanding, support, participation and patience over the years. I know it was challenging for all of us at times, but I hope the outcomes of this thesis shows you the potential of newer technologies that you and your patients may find useful.

To all my family and friends in the UK and South Africa, you have been so patient with me! You have also reminded me that there is more to life than studying, and I look forward to our visits and catch-ups in the future. A special thanks and cuddles to my dogs for their patience when their 'mommy' was typing away on her laptop instead of paying them attention or taking them for 'walkies'. I will miss my Sasha and Sandy who passed away in 2018 but hope to redeem myself with our old girl Tizzy, and our two youthful pups, Jack and Ripley.

Lastly, the biggest thanks go to my husband, soul mate and best friend, Graham. I could not have done this doctorate without your support, endless cups of tea (and snacks), and your big shoulders to lean on. I know the last few years have been challenging for both of us due to several reasons, but we can now look forward to a less stressful future together, and catch up on all those missed walks with our dogs. 


\section{Presentations}

Pike, C. E. (2017). Exploiting 3D scanning technology in lymphoedema for accurate and fast measurements of volume and shape. (Oral Presentation). Swansea University. Postgraduate Research (PGR) Conference, Swansea, Wales. September 2017

Pike, C. E. (2017). Exploiting 3D scanning technology in lymphoedema for accurate and fast measurements of volume and shape. (Oral Presentation). British Lymphology Society. National Conference, Stafford, England. October 2017

Pike, C. E. (2017). Assessment Using Evidence Based Techniques. (Oral Presentation). Lymphoedema Network Wales. Education Day for Qualified Therapists, Neath, Wales. December 2017

Pike, C. E., van Loon, R., \& Karakashian, K. (2018). Exploiting 3D scanning technology in lymphoedema for accurate and fast measurements of volume and shape. (Oral Presentation). International Lymphoedema Framework. International Conference, Rotterdam, Netherlands, May 2018

Pike, C. E. (2019). A pilot study exploiting 3D scanning technology in lymphoedema for accurate measurements of volume and shape. (Oral Presentation). International Lymphoedema Framework. International Conference, Chicago, USA, June 2018

Pike, C. E. (2020). Exploiting 3D scanning technology in lymphoedema for accurate and fast measurements of volume and shape. (Oral Presentation). British Lymphology Society. National Virtual Conference, Stafford, England. October 2020 


\section{List of Tables}

Table 1: Lymphoedema Treatment Outcomes ........................................................ 14

Table 2: Search strategies for Embase and Ovid Medline databases 2018 ................... 34

Table 3: Search strategies for Embase and Ovid Medline databases 2020 .................. 35

Table 4: Search strategy to identify papers reflecting 3D measurements for

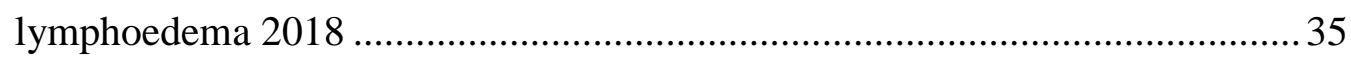

Table 5: Search strategy to identify papers reflecting 3D measurements for

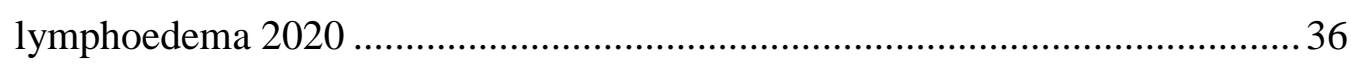

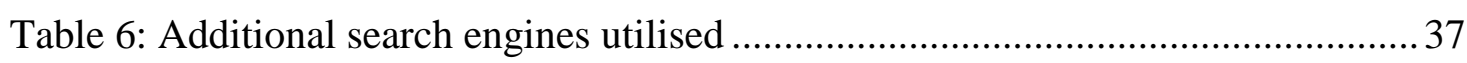

Table 7: Inclusion and exclusion criteria based on key words................................... 38

Table 8: Items of Critical Appraisal Skills Programme (CASP): Diagnostic Test Study specific for lymphoedema measurements ......................................................... 41

Table 9: Types of research methods from the systematic literature review ................. 42

Table 10: CASP outcomes of each research study paper.......................................... 43

Table 11: Methodology, cohort and limitations of each paper .....................................45

Table 12: Papers accessed on circumferential tape measurements (CTM)................. 49

Table 13: Variables for consideration when using CTM .........................................53

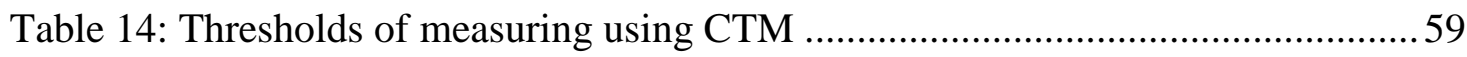

Table 15: Papers accessed on perometer measurements ......................................... 63

Table 16: Thresholds for diagnosing lymphoedema using perometry ........................ 69

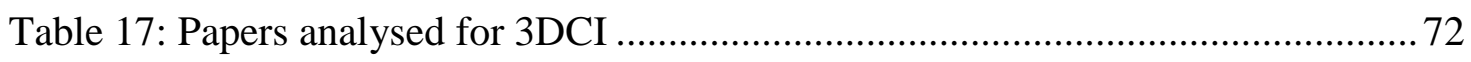

Table 18: Summary of the measurement properties of included studies ...................... 84

Table 19: Pooled data for ICC, SEM, and weighted mean SDC ................................ 85

Table 20: Research Objectives and Data Collection................................................. 98

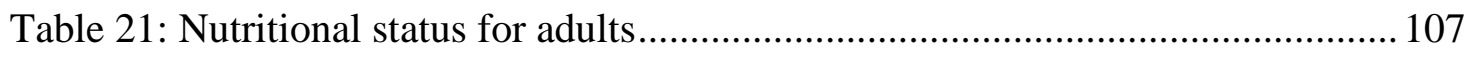

Table 22: Phase One objectives and methods of analysis ....................................... 110

Table 23: Phase Two objectives and methods of analysis ........................................ 114

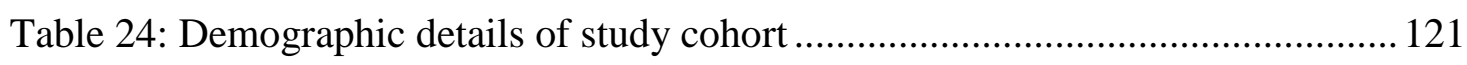

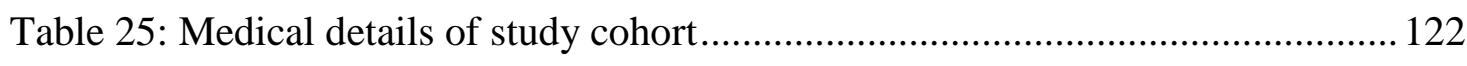

Table 26: Paired-samples correlations for CTM, perometry and 3DCI between sessions

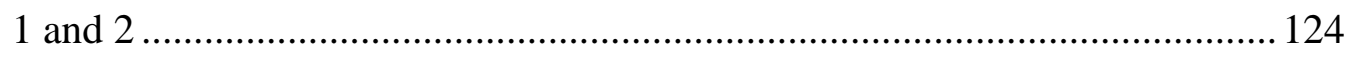

Table 27: Summary of all the data from CTM, perometry and 3DCI over the four

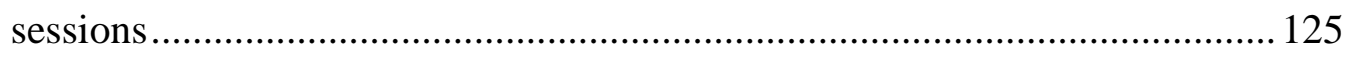


Table 28: Pearson's correlation coefficient between CTM, perometry and 3DCI's calculations of total volume difference

Table 29: Agreement between the methods of measurement through comparison of their volume differences 126

Table 30: $2 \times 2$ Crosstabulation of sensitivity and specificity between 3DCI, CTM and perometry

Table 31: Paired-samples correlations for Shape Redundancy (R-value) 132

Table 32: Summary of the data from R-value over the four sessions 132

Table 33: Pearson's correlation coefficient between R-value percentage difference (\%Diff) and 3DCI, CTM and perometry percentage volume difference (\%VD) 133

Table 34: Agreement between R-value percentage difference and 3DCI, CTM and perometry $\% \mathrm{VD}$ 134

Table 35: Agreement between the square root of R-value percentage difference and 3DCI, CTM and perometry \% VD. 134

Table 36: Oedema maps matching CTM, perometry and 3DCI \%VD over time 137

Table 37: 2x2 Crosstabulation of sensitivity and specificity between R-Value, 3DCI, CTM and perometry 138

Table 38: Timing of methods of measurement between Buffa et al. (2015), Landau et al. (2018), De Vrieze et al. (2019) and Thesis Study 150 


\section{List of Figures}

Figure 1: Photo of breast cancer-related lymphoedema.......................................... 2

Figure 2: Graphic illustration of the organisation of the thesis chapters ...................... 7

Figure 3: Treatment outcome using limb shape to aid categorisation of lymphoedema

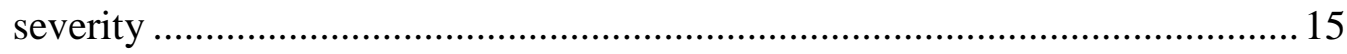

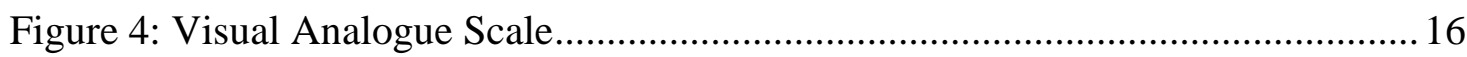

Figure 5: Positive Pitting Test and Positive Stemmer's Sign ..................................... 19

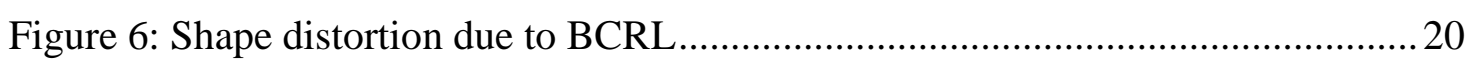

Figure 7: Measuring upper extremity limb volume by water displacement ................23

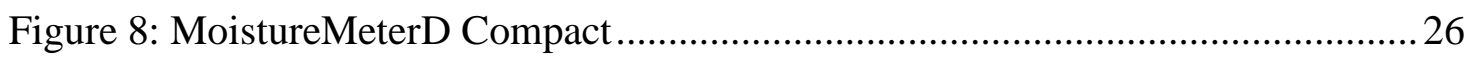

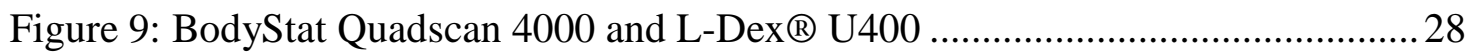

Figure 10: Preferred reporting items for systematic reviews and meta-analyses

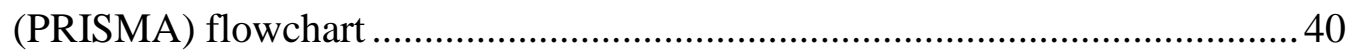

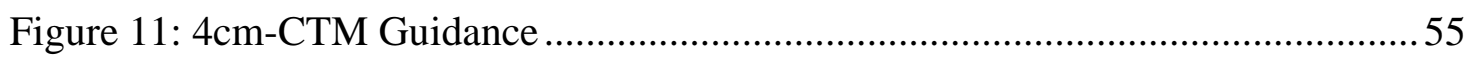

Figure 12: Geometric and mathematical representations of limb volume formulae: Frustum segment and Cylinder segment ................................................... 56

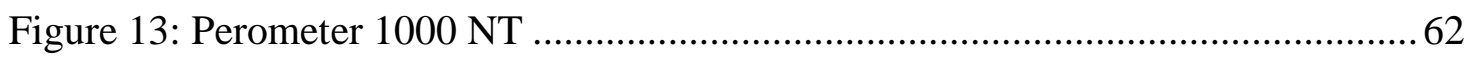

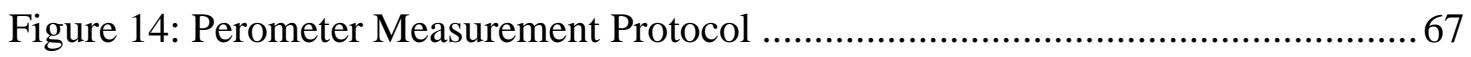

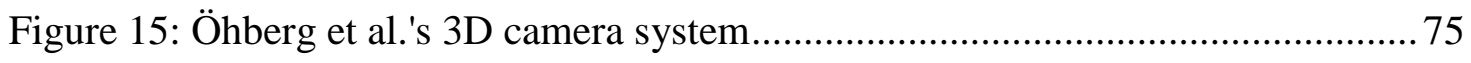

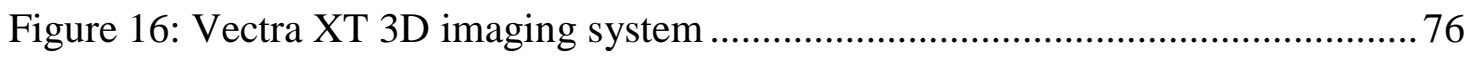

Figure 17: SkanLab system: Microsoft KinectTM camera attached to a rotating

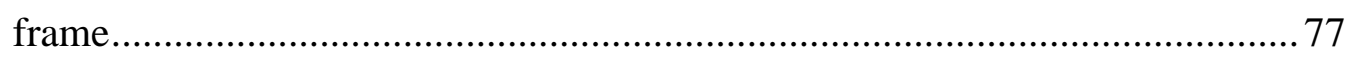

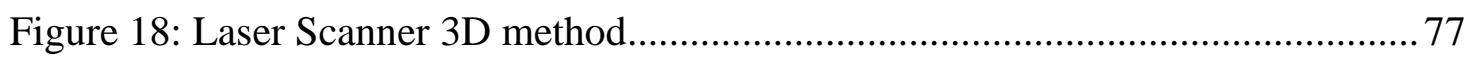

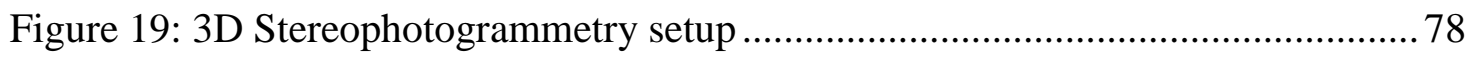

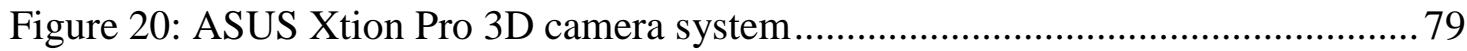

Figure 21: Preparation of 3D image to convert into limb volume data and radial

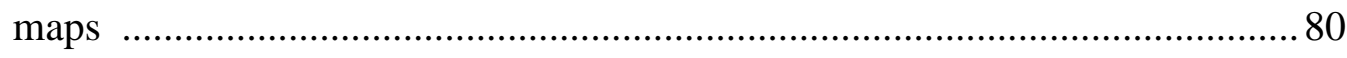

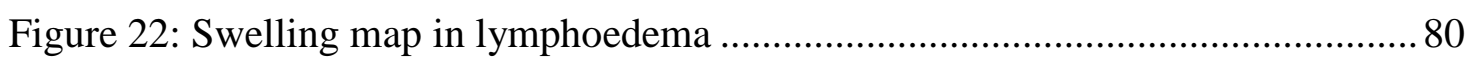

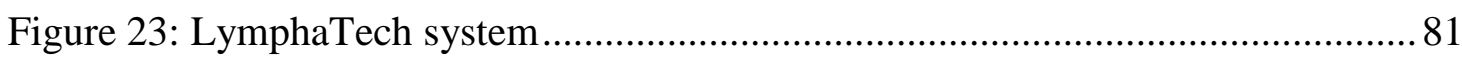

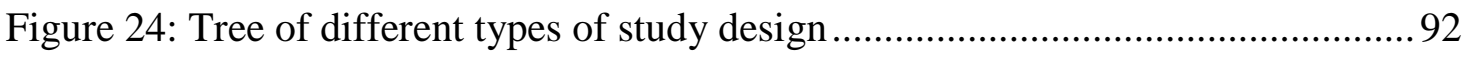

Figure 25: Diagram illustrating the two phases of the study ......................................96

Figure 26: Study Design Scheme giving an overview of the study protocol............... 97

Figure 27: Power of Analysis Results .............................................................. 104 
Figure 28: Schedule of Recruitment and Assessment - diagrammatic format of events from the time of receipt of referral to the first appointment 106

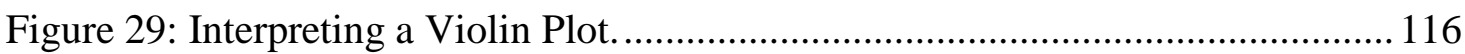

Figure 30: Flow diagram of recruitment study processes ....................................... 119

Figure 31: Bland-Altman Plot: a scatterplot of the mean of 3DCI and CTM methods plotted against the difference between the two methods.

Figure 32: Bland-Altman Plot: a scatterplot of the mean of 3DCI and perometry methods plotted against the difference between the two methods 128

Figure 33: Bland-Altman Plot: a scatterplot of the mean of the logarithm of CTM and perometry methods plotted against the difference between the two methods. 128

Figure 34: Oedema maps derived from 3DCI

Figure 35: Boxplot showing the mean values for the percentage differences of Shape Redundancy (R-value), 3DCI, CTM and perometry... 134

Figure 36: Violin Plots showing the probability of correlation between R-Value, 3DCI, CTM and perometry 135

Figure 37: NHS Wales COVID-19 Social Distancing Guidance 151 


\section{Abbreviations}

2SD

3D

3DCI

3SD

$\%$ AVD

$\% \mathrm{VD}$

ANOVA

AVD

BCRL

BIA

BIS

BLS

BMI

CASP

CEBM

$\mathrm{cm}$

CT (scan)

CTM

ICC

$\mathrm{ICC}_{\text {inter }}$

$\mathrm{ICC}_{\text {intra }}$

ISL

LAVI

LED

LNW

$\mathrm{MHz}$

Min

MRI

NHS

NICE

NIHR CRN

$\mathrm{Pa}$

PRISMA

$\mathrm{R} \& \mathrm{D}$

QoL

R-value

SD

SDC

SEM

TDC

UEL

VD
Two Standard Deviations

Three-Dimensional

Three-Dimensional Camera Imaging

Three Standard Deviations

Percentage Absolute Volume Difference

Percentage Volume Difference

Analysis of Variance

Absolute Volume Difference

Breast Cancer-Related Lymphoedema

Bioelectrical Impedance Analysis

Bio-Impedance Spectroscopy

British Lymphology Society

Body Mass Index

Critical Appraisal Skills Programme

Centre for Evidence-Based Medicine

Centimetre

Computed Tomography

Circumferential Tape Measurement

Intra-class (rater) Correlation Coefficient

Intra-class Correlation Coefficient for Interrater reliability

Intra-class Correlation Coefficient for Intrarater reliability

International Society of Lymphology

Localised Arm Volume Index

Light-Emitting Diode

Lymphoedema Network Wales

Megahertz

Minutes

Magnetic Resonance Imaging

National Health Service

National Institute for Clinical Excellence

National Institute for Health Research Clinical Research Network

Pascal

Preferred Reporting Items for Systematic Reviews and Meta-Analyses

Research and Development

Quality of Life

(Shape) Redundancy value

Standard Deviation

Smallest Detectable Change

Standard Error of Measurement

Tissue Dielectric Constant

Upper Extremity Lymphoedema Index

Volume Difference 


$$
\begin{aligned}
& \text { Chapter 1: } \\
& \text { Introduction to the } \\
& \text { Study }
\end{aligned}
$$


Lymphoedema is a dysfunction of the lymphatic system leading to chronic, progressive swelling. It is described as an excess accumulation of interstitial, protein-rich fluid in the subcutaneous periphery of the body that can also be a vital sign of ill health in clinical medicine (Mortimer \& Levick, 2004; Maclellan \& Greene, 2014). Lymphoedema is a morbid condition that can lead to painful limbs with a predisposition to infection, cutaneous changes, ulceration and in rare cases, malignant transformation (Grada \& Phillips, 2017). There are numerous causes for lymphoedema, both congenital and acquired, that can affect any part of the body, but occurs more commonly in the extremities (Thomas, 2017). Finnane, Janda, \& Hayes (2015), and Gebruers et al. (2017) report that lymphoedema is amongst the most feared consequences of cancer treatment because of its chronicity, and that it can result in significant physical, emotional, and psychological distress (due to altered appearance of the limb). Furthermore, there are social repercussions such as difficulty fitting into clothing, as well as loss of functional movement impacting activities (Maclellan \& Greene, 2014; Finnane, Janda, \& Hayes, 2015).

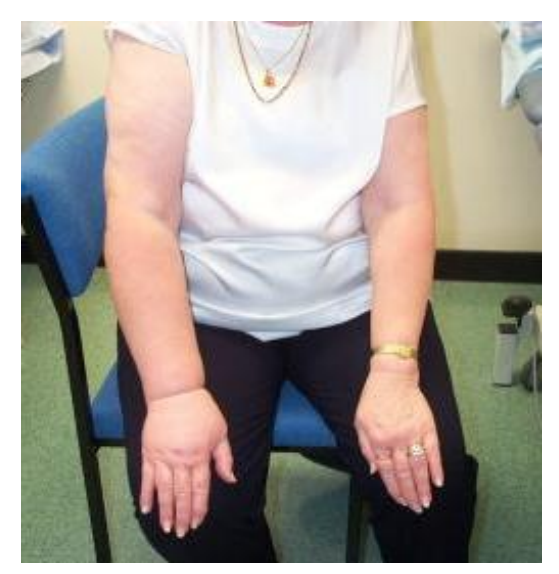

Figure 1: Photo of breast cancer-related lymphoedema

The most common cause of lymphoedema worldwide is filariasis (a parasitic worm), which is endemic in developing countries (Hadamitzky, Pabst, Gordon, \& Vogt, 2014). However, in developed countries, lymphoedema secondary to cancer treatment (Figure 1) is the more common cause of lymphoedema, with breast cancer-related lymphoedema (BCRL) making up the highest volume in lymphoedema clinic caseloads (Lu \& Dixon, 2019). The literature reports that $21 \%$ of people diagnosed with breast cancer develop lymphoedema due to their cancer treatment (DiSipio, Rye, Newman, \& Hayes, 2013), usually within a year of surgery and radiotherapy. Moffatt et al. (2003) 
raised awareness of the breadth of the impact lymphoedema has on the quality of life of people with BCRL. However Cornelissen et al. (2018) reported that BCRL was still considered to be one of the most distressing and underestimated side-effects of cancer treatment in their study cohort. Although this thesis will focus on the assessment of BCRL, it is important to consider the background and epidemiology of lymphoedema.

\subsection{Background and Epidemiology of Lymphoedema}

Lymphoedema is a chronic swelling (oedema) that persists for more than three months due to an overload of tissue fluid and is often used interchangeably with the term chronic oedema in the literature (National Lymphoedema Partnership, 2015). As such, chronic oedema can be classified as either:

- Lymphoedema - Primary or Secondary. Primary lymphoedema is caused by congenital disease or abnormality of the lymphatics; Secondary lymphoedema is due to damage of the lymphatic system through infection, trauma, cancer and its treatments including excision of lymph nodes and radiotherapy, and obstruction of the lymphatic system;

- Lymphovenous oedema - is due to the failure of the venous system such as deep vein thrombosis, valvular incompetence and varicose veins;

- Dependency oedema - loss of muscle pump causes poor venous return resulting in a build-up of tissue fluid;

- Lipoedema - is a swelling of fat deposits resulting in limb distortion but is sparing of the feet and hands.

(International Lymphoedema Framework, 2012; Maclellan \& Greene, 2014)

Obesity is both a cause and exacerbating factor of lymphoedema (Greene, Grant, Slavin, \& Maclellan, 2015). It can result in any of the first three types of chronic oedema, i.e. secondary lymphoedema due to obstruction as the fat cells compress the lymphatics; lymphovenous oedema due to increased pressure on the cardiovascular system resulting in venous disease; and dependency oedema due to reduced mobility and an inactive lifestyle.

The prevalence of lymphoedema has increased from 1.33 per 1000 (Moffatt et al., 2003) to 3.93 per 1000 , with the highest incidence for those aged 85 or above (28.75 per 1000) 
(Moffatt, Keeley, Franks, Rich, \& Pinnington, 2017). Following the inception of lymphoedema services in each of the Health Boards across Wales (Welsh Assembly Government, 2009), collected data captures the number of patients on each service caseload, including the number of new referrals and number of discharges each month. Since 2013, there has been a demonstrable increase in prevalence and incidence in Wales, with the prevalence increasing from 4.12 per 1000 to 6.83 per 1000 by the end of March 2020, and incidence increasing from 1.56 to 2.58 per 1000 (Lymphoedema Network Wales [LNW], 2020a). Cancer cases account for $30 \%$ of caseloads in Wales and of these, $85 \%$ are BCRL. Thus, the estimated prevalence of BCRL in Wales is 1.74 per 1000. With rising prevalence and limited capability, services have to ensure that health care provided is both valuable to the patient and the National Health Service (NHS).

Porter, Pabo, \& Lee (2013) express that the overarching strategy for health care should be to improve value for patients. They define value as patient outcomes achieved relative to the amount of money spent. The Allied Health Professions Outcome Measures UK Working Group (2019) developed a checklist for selecting outcomes measures. There is currently no standardised outcome measure for lymphoedema, and outcomes can vary from clinical decisions, such as volume reduction, a decrease in pain, reduced number of reported episodes of cellulitis, improved function and/or level of compression therapy required, to more practical outcomes for the patient such as impact on work, resuming hobbies, and wearing normal clothes. Thus, determining an assessment measurement tool that offers results that the therapist can easily interpret, and that the patient understands, is vital towards implementing Value-Based Healthcare.

\subsection{Statement of the Diagnostic Problem}

Diagnosing lymphoedema can be challenging due to a lack of national and international protocols and guidance regarding thresholds for its clinical diagnosis and the different methods of lymphoedema measurement used in clinical practice. Medical scanning and imaging of lymphoedema are costly and do not add value to the diagnosis or choice of conservative treatments. They are thus not routinely performed. Hence, clinicians need to rely on the person's history and symptom reports, physical assessment of the skin and soft tissues, and the measurement of the swollen limb (Maclellan \& Greene, 2014). 
Furthermore, observation of the limb shape comparing the affected to the unaffected side (see Figure 1) reveals areas of localised swelling of the affected arm that may require intensive intervention.

Physical measurements, such as circumferential tape measurement (CTM), water displacement, and perometry are used to calculate volume differences between the affected and unaffected sides to determine the extent of lymphoedema (Hidding et al., 2016). Newer technological devices are now available to determine tissue fluid content of a limb, and more recently, three-dimensional camera imaging (3DCI) has been used to capture limb volumes and limb volume differences (Levenhagen, Davies, Perdomo, Ryans, \& Gilchrist, 2017). Studies indicate that 3DCI could be used to assess limb shape, which may aid in the diagnosis of lymphoedema (Karakashian, Shaban, Pike, \& van Loon, 2018).

By utilising data from Three-Dimensional (3D) images, shape potentially offers a supplementary or alternative method of measuring lymphoedema. Using the images gained from 3DCI, areas of localised swelling can be objectively identified for the first time. 3DCI is non-invasive, fast to perform and relatively inexpensive compared to other technologies on the market. It provides data about the shape of the affected limb, something that current methods of measurement do not. However, what is not yet clear is whether this method is equivalent, or more accurate than current methods of volume measurement.

Clinical practice within the NHS is driven by the evidence-base, but with conflicting outcomes for measuring methods in the literature, and limited resources available to services, CTM is used most extensively since it requires minimal equipment, and can be used in most locations. However, no study to date has evaluated the economic value of each method of measurement; thus, no evidence exists to support that CTM may or may not be the most resource-effective method of assessing lymphoedema.

Due to ambiguity in the current methods of assessing lymphoedema, this thesis will present the investigations into the possibility of a new way of measuring for lymphoedema as opposed to current methods of volume and tissue water content. The overarching aims of this study were to determine the validity, reliability and accuracy 
of upper limb volume results obtained from 3DCI compared to CTM and perometry, and to analyse whether the shape of the limb is a feasible alternative to measure lymphoedema. Four objectives were identified to meet the aims of this study, which were to:

1. Determine whether 3DCI volume outcomes are comparable to CTM and perometry as a measure of intra-rater reliability

2. Assess the criterion validity of 3DCI against previously validated measures, i.e. CTM and perometry

3. Establish whether 3DCI is an accurate method of measurement for upper limb lymphoedema

4. Ascertain whether limb shape derived from 3DCI is a potential new method of lymphoedema measurement

\subsection{Organisation of the Thesis}

Figure 2 provides an overview of the layout of the thesis. The thesis comprises six chapters, with this first chapter as an introduction to the study undertaken. Chapter 2 explains how lymphoedema is assessed and classified. The systematic literature review, presented in Chapter 3, analyses the three measurements used in the study focussing on delivery, validity, reliability and the diagnostic thresholds.

Chapter 4 describes the study design that was used to address the research questions identified. The results of the study are presented in Chapter 5, with the discussion and conclusion of the findings conveyed in Chapter 6. This final chapter also identifies the limitations of the study and presents ideas for future research. 
Chapter 1:

Introduction

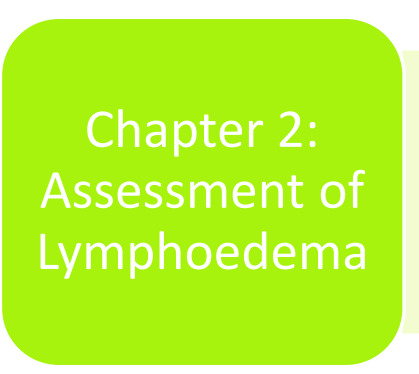

\section{Chapter 3:}

Systematic

Literature Review

- CTM, Perometer and 3DCI reviewed

- Analysis of the measurement procedure, reliability, validity and thresholds undertaken

- Clarifies the assessment process to diagnose lymphoedema

- Classification of Lymphoedema presented

- Elucidating the need for lymphoedema assessment

- Study design described

- Phase One: analysis of limb volume derived from $3 \mathrm{DCl}$ and compared to CTM and perometer

Chapter 4:

- Phase Two: investigates the use of limb shape as a

Methods method of lymphoedema assessment through the use of oedema maps and shape redundancy

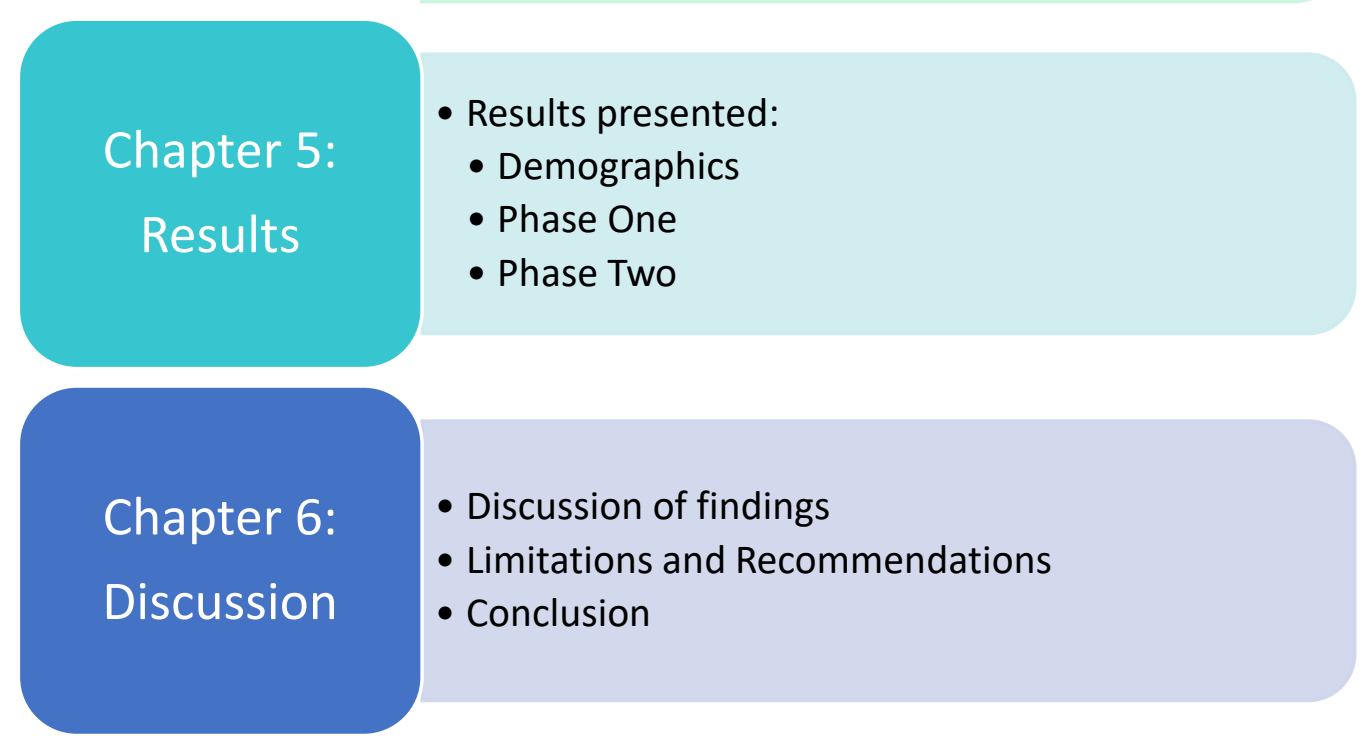

Figure 2: Graphic illustration of the organisation of the thesis chapters 
Chapter 2:
Assessment of
Lymphoedema 


\subsection{Introduction}

Unlike the triple test for diagnosing breast cancer, there is no protocol to help define a clinical diagnosis of upper limb lymphoedema. The lack of a clear protocol is compounded by the fact that there is no international consensus for the classification of lymphoedema, making a diagnosis even more challenging. There is also a lack of agreement when measuring to determine the extent of lymphoedema. Lu \& Dixon (2019) report that the most common approach to measure lymphoedema of the limb is using volumetric measurements. However, this method does not account for variations in muscle mass and adipose tissues, as the measurements include bone, fat, muscle and subcutaneous tissues and is unable to discern between these (Fu et al., 2013).

Following the principle of Archimedes, water displacement is considered the gold standard in measuring limb volume (Levenhagen et al., 2017) as the water displaced by an object equals the volume of that object. Clinically, the use of a tape measure to measure limb volume is the most popular method employed, while in research, perometry is highly valued due to its perceived accuracy in calculating the circumference of a limb through the use of infra-red and light-emitting diodes (LEDs) (Batista, Baiocchi, Campanholi, Bergmann, \& Duprat, 2018). However, measurement of actual tissue fluid would be more accurate than the volumetric value of a limb that includes bone, muscle, fat and skin. Thus technologies, such as tissue dielectric constant (TDC) (see section 2.4.3.2) and bio-impedance spectroscopy (BIS) (see section 2.4.3.3), which measure tissue fluid content, have been investigated for several years, but are yet to be used with confidence in clinical settings (Levenhagen et al., 2017).

3DCI has more recently been studied to determine limb volume; however, no research to date has evaluated the concept of determining the presence or extent of lymphoedema by objectively assessing the shape of the limb. The advantage of measuring the shape of a limb is that it can identify the amount of distortion in shape from the unaffected side, which would indicate the presence of localised lymphoedema, something that a volume measurement cannot. The importance of assessing for localised lymphoedema is that it informs the true severity of the lymphoedema and the need for specific compression therapy. Additionally, it provides a deeper understanding to support the patient's reported symptoms such as clothing not fitting. For example, real value for a 
patient is treatment that can enable them to button up a long sleeve shirt when their swelling is localised at the wrist.

Value-Based Healthcare aims to reduce variations in services to maximise the value of person-centred care (Gray, 2017). Research to establish what people desire in terms of monitoring their lymphoedema is lacking; thus, lymphoedema services are unable to evidence the value of their service to their stakeholders in these terms. Understanding whether patients comprehend their limb volume difference expressed as a percentage $(\%)$ or volume as millilitres $(\mathrm{ml})$ of the affected side's deviation from the unaffected is fundamental. Alternatively, therapists should determine whether it is easier to express this difference using a graph, or whether a picture could express the archetypical one thousand words. 3DCI has the potential of presenting swelling in a visual format, but this has not been researched previously. Thus, this thesis will present the research that proposes that limb shape, derived from 3DCI, could add a new way of assessing and monitoring upper limb lymphoedema. As the incidence of BCRL is 21\% (DiSipio et al., 2013) and predisposes a high caseload of patients, this thesis will study 3DCI limb volume and shape of the upper limb.

\subsection{Diagnosing Lymphoedema}

Diagnosis of lymphoedema can be established in $90 \%$ of cases by appraising a person's history, self-reported symptoms and undertaking a physical examination (Maclellan \& Greene, 2014; Perdomo, Davies, Levenhagen, \& Ryans, 2014). Not all upper limb lymphoedema is due to BCRL, thus indicative contributors to lymphoedema such as other cancers (melanoma), familial history (i.e. potential genetic link), trauma, number of infections (including cellulitis), surgery (including orthopaedic), obesity, and travel to countries endemic with filariasis need to be determined.

In the case of BCRL, a breast cancer diagnosis together with the type of surgery (wide local excision, mastectomy or primary reconstruction), the number of lymph nodes removed (sentinel lymph node biopsy and/or axillary node clearance), chemotherapy, radiotherapy, and/or hormone therapy will inform the risk and cause of the lymphoedema (Göker et al., 2013). In some cases, trauma or infection to the 'at risk' arm may be the trigger of the person's sudden onset of lymphoedema, as opposed to the 
slow progressive deterioration of lymphatic drainage of the 'at risk' arm due to either of the cancer treatments.

Patient self-reported symptoms may include swelling, heaviness, tightness, pain, discomfort, aching and numbness of the swollen area (Williams, Moffatt, \& Franks, 2004). Exploration of these symptoms includes identifying the location of the swelling, ascertaining when the person first noticed the swelling and what the behaviour of the symptoms are, i.e. is the swelling getting better, worse, stays the same or fluctuates. Personal experience of the lymphoedema must be considered, and these could include exacerbating factors such as heat, repetitive activities and static postures. Limitations in personal attire can also occur such as clothing too tight over a misshapen arm, rings no longer fitting on fingers; and functional difficulties like cooking, lifting pans or not being able to hang up washing due to the heaviness of the arm (Lam, Wallace, Burbidge, Franks, \& Moffatt, 2006). The level of treatment to be agreed takes into consideration the person's social circumstances in terms of their support structure, childcare arrangements, the responsibility of caring for elderly relatives, type of employment, as well as their social habits including smoking and drinking. Environmental factors such as the need to sleep in a chair (as this causes and exacerbates dependency lymphoedema), and mobility aids used, gives the therapist insight into the impact of lymphoedema and potential for improvement (Thomas \& Morgan, 2017).

Williams, Moffatt, \& Franks (2004) report that the impact of lymphoedema on a person can be considerable. Alcorso \& Sherman (2015) are more specific in stating that BCRL may impair quality of life, cause disturbance of body image and result in psychological distress. Their study indicates that an increase in lymphoedema symptoms is associated with psychological distress, including negatively affecting a person's self-identity and emotional disturbances such as fear, sadness and worry. Furthermore, Alcorso \& Sherman (2015) demonstrate that perceived treatment effectiveness, and the inability to self-regulate lymphoedema is associated with distress and body image disturbance, which can lead to psychological distress requiring appropriately targeted counselling and support.

Thomas \& Hamilton's (2014) qualitative study revealed that individuals who have BCRL report a loss of expression as they are unable to wear preferred clothing or 
jewellery due to the size of their affected arm. Their leisure activities are affected as they are no longer able to partake in previously enjoyed hobbies as their lymphoedema arm is too heavy. Consequently, this results in changes to self-perception and social isolation. They also opine a loss of independence in activities of daily living and selfcare. It is thus essential to identify psychosocial obstructions as part of the holistic lymphoedema assessment.

One of the most debilitating and frightening experiences as a direct complication of lymphoedema is cellulitis (skin infection / acute inflammatory episode) (Lam et al., 2006). Al-Niaimi \& Cox (2009) report that up to $25 \%$ of patients with lymphoedema, regardless of cause, may develop cellulitis. It can develop in the space of an hour with the person presenting with a red, swollen and hot to touch upper limb, and they feel generally unwell as if they have the flu. In some cases, cellulitis may grumble for weeks before a diagnosis is made. In more severe instances the person may have a fever and/or rigors, and in extreme situations, they may require hospitalisation as it could develop into sepsis (Al-Niaimi \& Cox, 2009; Cannon, Dyer, Carapetis, \& Manning, 2018; Moffatt, Doherty, Franks, \& Mortimer, 2018). Cellulitis damages the lymphatics, potentially further exacerbating lymphoedema. It is therefore vital to understand on assessment how many infections the person may have had in the previous 12 months as they may require prophylactic antibiotic management for at least a year in an attempt to prevent further damage to the lymphatics (Mason et al., 2014; BLS \& LSN, 2016). Enquiring whether they were hospitalised for cellulitis will give the therapist an indication of the level of damage to their lymphatic system. It is noteworthy that cellulitis can recur if not appropriately managed, or if lymphoedema is poorly controlled (Al-Niaimi \& Cox, 2009).

Once a lymphoedema diagnosis is suspected, further subjective and objective assessments need to be performed to confirm the type and severity of the lymphoedema, before a treatment plan is formulated. The next section explains how the presenting lymphoedema may be classified using a system of staging to identify the level of severity that can inform the response of the lymphoedema to treatment. 


\subsection{Staging of the Severity of Lymphoedema}

Defining and staging lymphoedema remains a subject of international debate. As such, there is no one system to aid clinicians in classifying lymphoedema, thus making it challenging to compare lymphoedema outcomes. Even though limb shape distortion is a recognised indicator of lymphoedema, currently no classification of lymphoedema includes limb shape distortion as a factor in the diagnosis, severity and staging of lymphoedema. Clinically, the greater the shape deviation from the normal, the more challenging and intensive the treatment will be (International Lymphoedema Framework, 2012). It is also unlikely for large limb shape deviations to have a positive outcome similar to that of a normal shaped limb following lymphoedema treatment. Thus, a value needs to be attributed to the amount of deviation of the limb from the normal shape to assist with the staging of the lymphoedema. Current staging focuses on limb volume and soft tissue changes in an attempt to capture the severity of the lymphoedema.

The International Society of Lymphology (ISL) has developed a lymphoedema staging (Appendix 1) based on the physical condition of the extremities (ISL, 2013). They acknowledge that this staging fails to capture the pathological and possible genetic defects of the condition. Thus, the ISL staging can be considered a crude descriptor of the presentation of lymphoedema. It is not sensitive to the different types of lymphoedema from the numerous causes (primary and secondary), nor does it allow for different presentations in one limb, for example, the hand may be soft and pitting (stage II), but the forearm may be firm with thickened skin (stage III).

The British Lymphology Society (BLS) have developed a classification and staging of lymphoedema based on the ISL staging of 2009 (British Lymphology Society, 2016). Although they appear similar, they do not directly correlate (Appendix 1). The BLS staging is also inclusive of the functional capabilities of the patient, making it more sensitive in terms of capturing the impact of the lymphoedema on the patient. The BLS also acknowledges that for people with advanced cancer, or for those who are at the end of life, they require a separate classification to address their unique needs (Appendix 1). 
In Wales, clinicians assign a 'Treatment Outcome' (Table 1) following each appointment to identify the severity of the lymphoedema and to determine the caseload within each clinic (LNW, 2020b). As a 'treatment outcome', it is not considered a classification, especially as it is not a recognised, nor validated tool. This is the only categorisation of lymphoedema severity in the world that refers to the shape of the limb as being 'normal' or 'distorted' (Figure 3) and relies on the ratio between the proximal (upper arm) to distal (forearm) segment of the limb being calculated using a programme called Lymcalc used for CTM.

Table 1: Lymphoedema Treatment Outcomes (LNW, 2020b)

\begin{tabular}{|c|l|}
\hline$\#$ & Description of Treatment Outcome \\
\hline 1 & $\begin{array}{l}\text { At Risk (no swelling) } \\
\text { No evidence of lymphoedema; has known risk factors } \\
\text { Limb shape normal: Proximal to Distal ratio of } \mathbf{0 . 0} / \mathbf{0 . 1}\end{array}$ \\
\hline 2 & $\begin{array}{l}\text { Mild (minimal swelling) } \\
5-10 \% \text { volume difference between limbs } \\
\text { Limb shape is normal: Proximal to Distal ratio of } \mathbf{0 . 0} / \mathbf{0 . 1}\end{array}$ \\
\hline 3 & $\begin{array}{l}\text { Moderate (moderate swelling) } \\
\text { Overall volume difference 11- 20\% between limbs. } \\
\text { Limb shape distortion: Proximal to Distal ratio 0.2 }\end{array}$ \\
\hline 4 & $\begin{array}{l}\text { Severe (severe swelling) } \\
21 \%-49 \% \text { total volume difference between limbs. } \\
\text { Limb shape distortion: Proximal to Distal ratio 0.3 }\end{array}$ \\
\hline 5 & $\begin{array}{l}\text { Complex } \\
50 \% \text { or more total volume difference between limbs. } \\
\text { Skin changes including Fibrosis, Peau d'orange, papillomatosis, hyperkeratosis, skin folds and cellulitis } \\
\text { Limb shape distortion: Proximal to Distal ratio 0.4 or above }\end{array}$ \\
\hline $5 \mathrm{~W}$ & $\begin{array}{l}\text { Complex with wound/leaking lymphorrhoea } \\
\text { Patient has lymphoedema and a wound/or 'wet legs' with leaking lymphorrhoea. The open wound can be } \\
\text { superficial or complex but requires dressings and compression. }\end{array}$ \\
\hline
\end{tabular}

With the introduction of fluorescent lymphography, Yamamoto et al. (2011a) have devised new classifications for lymphoedema, whereby the rate of 'arm dermal backflow' is staged (Appendix 1). They went on to devise a similar backflow staging for the lower limb, but Mihara et al. (2014) later advanced this staging based on the pumping function of the lymphatics to identify early-stage lymphedema and to evaluate the pathologic condition over a wider area, as opposed to being limited to the affected lower limb (Appendix 1). The limitations of fluorescent staging are that very few clinics have access to this equipment as the cost is prohibitive and staff training would have to be at a very high level to administer the Indocyanine Green dye. 

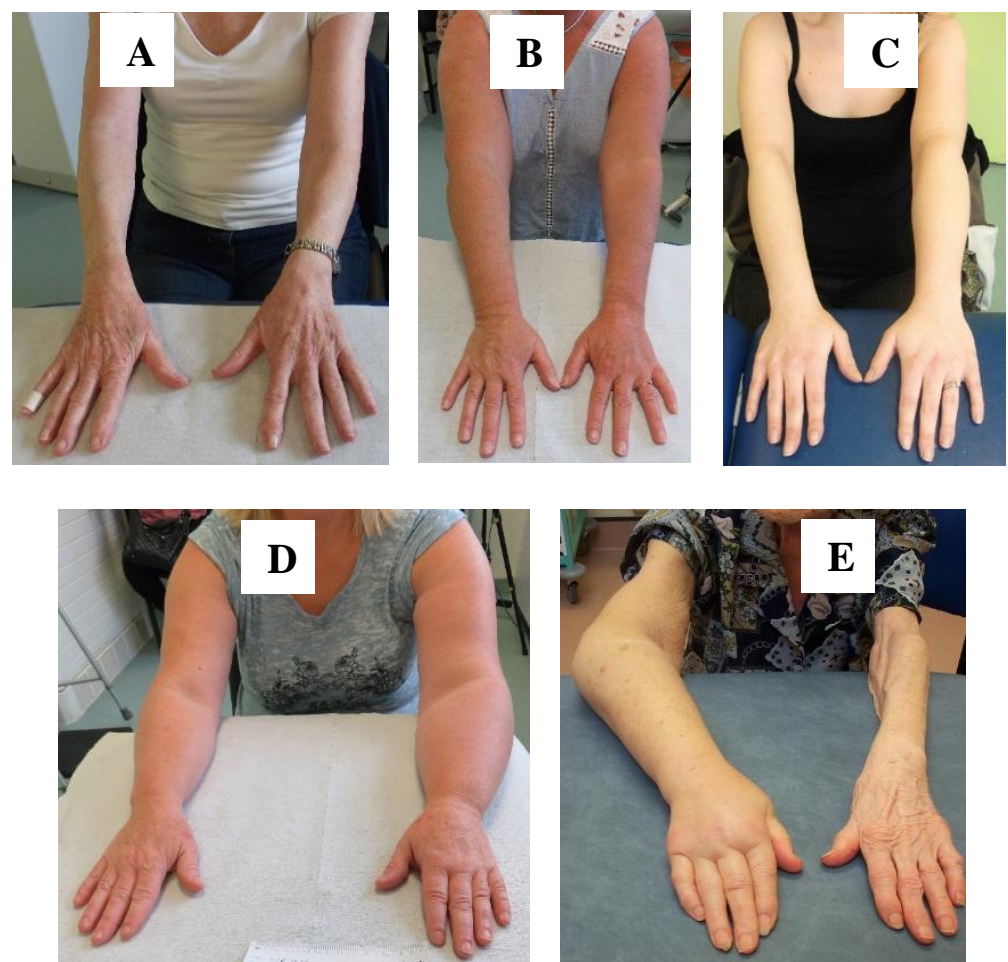

Figure 3: Treatment outcome using limb shape to aid categorisation of lymphoedema severity (photos with permission from LNW):

A= right affected, Code 1 (At Risk) with normal shape

$B=$ right affected, Code 2 (Mild) with normal shape

$\mathrm{C}=$ left affected, Code 3 (Moderate) with a distorted shape of the forearm

$\mathrm{D}=$ left affected, Code 4 (Severe) with shape distortion and large excess volume

$\mathrm{E}=$ right affected, Code 5 (Complex) with shape distortion and significant excess volume

\subsection{Assessment of Lymphoedema}

Understanding the development and behaviour of lymphoedema symptoms should facilitate confirmation of the diagnosis (Levenhagen et al., 2017) and commencement of treatment. Where the cause or phenotype of lymphoedema is unknown, a person may be referred for genetic testing, especially in cases of primary lymphoedema (Lymphoedema Framework, 2006; Lee et al., 2013).

The assessment process needs to capture both the patient's experience of their lymphoedema, together with the therapist's findings to ensure an accurate diagnosis and classification of the lymphoedema. The application of a simple visual analogue scale can capture the patient's experience of pain and heaviness related to their lymphoedema (section 2.4.1). Using validated quality of life questionnaires can also help the therapist understand the impact lymphoedema has on the patient and can be used to assess treatment outcomes (section 2.4.2). The clinical examination that follows needs to incorporate elements of skin and tissue observation and palpation, physical 
measurements of limb volume or tissue fluid (section 2.4.3), and the use of medical imaging such as lymphoscintigraphy (section 2.4.4).

\subsubsection{Visual Analogue Scale}

A Visual Analogue Scale (Figure 4) is a self-report measure that can be modified for swelling, heaviness or pain. It is a 10 centimetre $(\mathrm{cm})$ horizontal line with 'no (pain)' anchoring at the $0 \mathrm{~cm}$ end, and 'worst (pain)' at the $10 \mathrm{~cm}$ anchor. The distance of a mark made by the patient from $0 \mathrm{~cm}$ is measured with a ruler and can be recorded as 'how much pain, swelling, or heaviness' is present. The Visual Analogue Scale is a validated tool in the acute, chronic and oncology populations and has been used extensively in BCRL (Harrington, Gilchrist, \& Sander, 2014; Levenhagen et al., 2017).

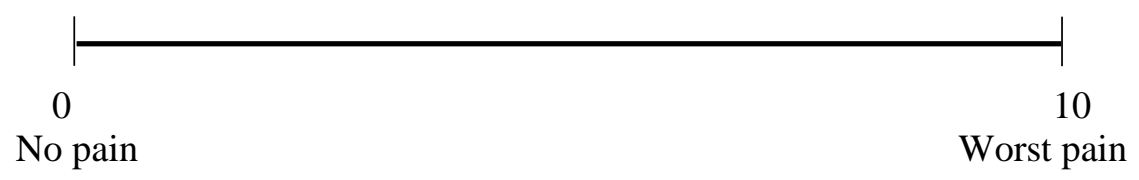

Figure 4: Visual Analogue Scale

Levenhagen, Davies, Perdomo, Ryans, \& Gilchrist's (2017) literature review found that self-report of swelling using a visual analogue scale demonstrated good inter-rater reliability (interclass correlation coefficient $[\mathrm{ICC}]=0.70$ ). Czerniec et al. (2010) used the Visual Analogue Scale in their study to assess how swollen the participant felt their arm was, with the $0 \mathrm{~cm}$ anchor indicating "not at all" and, the $10 \mathrm{~cm}$ anchor indicating "extremely so". They found the correlation between Visual Analogue Scale and upper limb volumes by CTM and perometry to be moderate ( $r=0.66$ and 0.65 respectively).

The main factors influencing the Visual Analogue Scale is subjectivity as it measures a person's experience of their lymphoedema, which is then compared to objective measurements. Subjective indicators can be influenced by emotions, perceptions and previous experiences, all of which may distort the person's account for their score.

\subsubsection{Quality of Life}

Lymphoedema of the arm secondary to breast cancer treatment is considered by some to be one of the most distressing and underestimated side effects (Cornelissen et al., 2018). Moffatt et al. (2003) highlighted the breadth of impact lymphoedema has on a 
person's Quality of Life (QoL), including physical, emotional, social functioning and physical functioning. They also found that there was a significant impact of cellulitis secondary to lymphoedema on people's lives; as well as restrictions in QoL in people's ability to function physically and socially, plus the potentially devastating emotional consequences.

Several tools exist to capture the impact that lymphoedema has on a person's life. Pusic et al. (2013) and Cornelissen et al. (2018) did systematic reviews of the literature to identify the optimal methods of assessing QoL for those people with Breast CancerRelated Lymphoedema (BCRL). They acknowledge that choosing the tool that would best inform treatment outcomes is often a challenge to clinicians who do not want to burden their patients with excessive paperwork, especially as many that have BCRL are often involved in clinical trials already requiring extensive questionnaires to be completed (Cornelissen et al., 2018).

Pusic et al.'s (2013) systematic review identified 39 studies with high-quality data on health-related QoL outcomes with 17 instruments used, with two specific to BCRL. They recommend the use of the Upper Limb Lymphoedema 27 (ULL-27) by Launois, Mègnigbêto, Pocquet, \& Alliot (2002) due to its strong psychometric properties. Cornelissen et al. (2018) also undertook a systematic review of which 49 studies met their inclusion criteria for QoL in BCRL, with 15 different questionnaires extracted. They concluded that the Lymphoedema Functioning, Disability, and Health (LymphICF) questionnaire by Devoogdt, Van Kampen, Geraerts, Coremans, \& Christiaens, (2011), and the Lymphoedema Quality of Life Inventory (LyQLI) questionnaire by Klernäs, Johnsson, Horstmann, Kristjanson, \& Johansson (2015) were the most complete and accurate questionnaires to assess QoL in patients with BCRL as these assess the largest number of QoL domains and specific arm symptoms. A key difference between these two measures is that the Lymph-ICF uses a Visual Analogue Scale, whereas the LyQLI uses categorical answers. Interestingly, even though the ULL-27 measure was included in Cornelissen et al.'s (2018) study, it was not critiqued against the other measures, possibly due to not fully meeting the criteria to assess global QoL and the different QoL domains, together with assessing all specific arm symptoms as required by this review. 
QoL measures in clinical practice can inform treatment and ensures that the assessment of lymphoedema focuses on the person rather than the condition. In research, the use of QoL measures is dependent on the research questions being asked, and the sensitivity required of the test. Thus, with the availability of several QoL tools ranging from the more generic and crude tools such as the EQ-5D-5L questionnaire (EuroQol, 2017) looking at function and well-being, to very subject-specific tools such as the Upper Limb Lymphoedema 27 (ULL-27) (Launois, Mègnigbêto, Pocquet, \& Alliot, 2002) and 'Quality of Life Measure for Limb Lymphoedema' (LYMQOL) (Keeley et al., 2010), clinicians and researchers need to understand the function of the tool before use.

Following on from the subjective information, an examination of the person's symptoms follows. Good clinical reasoning skills will pull all the subjective and objective data together to devise a treatment plan with the person to ensure they have ownership of the management of their lymphoedema.

\subsubsection{Clinical Examination}

The physical examination should include palpation of the soft tissues for fibrosis, pitting oedema, Stemmer's Sign test (Figure 5), and overall tissue quality (Levenhagen et al., 2017). This will include identification of skin changes such as dermal fibrosis, hyperkeratosis, papillomatosis, fungal infections, wounds (including ulcers), lymphangiectasia, lymphorrhoea, folliculitis, dermatitis and cellulitis (Fife et al., 2017). Observation and documentation of soft tissue changes, for example, skin folds, as well as skin colour and temperature, are essential in providing the therapist with evidence as to the severity of the person's symptoms. Limb shape distortion provides further evidence for the presence of lymphoedema. This is established by visually comparing the affected to the unaffected side to see if there is any deviation from the unaffected side. This deviation may be localised to the forearm, or there may be a noticeable difference in the size of the upper arm to the forearm. Currently, no measurement exists to capture limb shape distortion, although, as discussed in section 2.3 , a proximal to distal ratio can be calculated from CTM (LNW, 2017).

Additional clinical signs may include reduced visibility on the affected side of the subcutaneous veins of the anterior forearm and posterior hand. Furthermore, rounding 
or fullness of the elbow and distal upper arm regions are also clear signs of the presence of lymphoedema (Stanton et al., 2009).
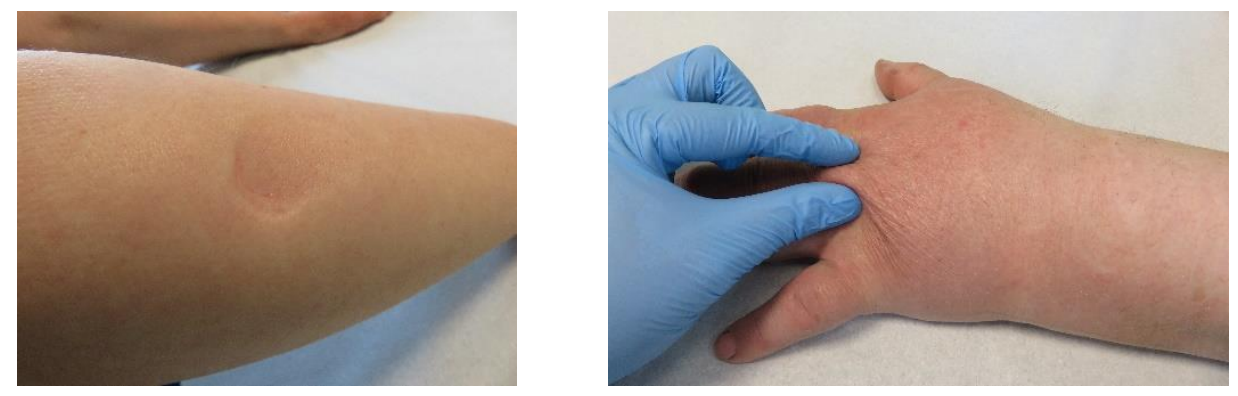

Figure 5: Positive Pitting Test (right) and Positive Stemmer's Sign (left)

A vascular examination is required to ensure the safety of the application of compression and to determine contributing factors to lymphoedema. This includes a visual and physical assessment of the arm to determine the presence of arterial insufficiency including hair loss, the colour of the nailbed, pain at night or rest, blanching on elevation and a capillary refill test (normal $\leq 3$ seconds). Should there be any concern following this examination, it is advised that a referral to a vascular clinic be made.

Assessment of upper limb functional movement is undertaken to determine restrictions in range of movement or muscle weakness as these may exacerbate lymphoedema. It is worth determining at this stage whether referral to a physiotherapist and/or occupational therapist is required as part of their rehabilitation package and lymphoedema management.

Measuring the arms by CTM, perometry, or water displacement determines volume differences between limbs that can help determine the severity of the lymphoedema (Levenhagen et al., 2017). Both arms are measured as the unaffected limb can be used as a reference or control when calculating excess volume in the presence of unilateral lymphoedema (Casley-Smith, 1994). Arm dominance must be known as it generally measures $1.6 \%$ to $4.7 \%$ bigger than the non-dominant limb (Smoot, Wong, \& Dodd, 2011). Mayrovitz, Macdonald, Davey, Olson, \& Washington (2007) state that assessing limb volume difference at each point of contact will determine effective treatment outcomes. Casual variation in a person's general body mass index (BMI) over time is negated by measuring both arms at each point of contact (Perdomo et al., 2014) as the 
volume of a limb includes bone, muscle, fat, interstitial fluid and the dermis (Fu et al., 2013).

Volume decrease or increase may be due to muscle atrophy or hypertrophy, and not necessarily an increase in the extracellular fluid (interstitial fluid) related to lymphoedema. It is therefore prudent that a therapist determines whether a person's activity may account for unilateral limb hypertrophy due to overuse, such as a change in profession requiring more muscle activity, or limb atrophy as a consequence of avoidance behaviour, for example, due to pain or fear of movement. A noticeable change in limb volume often follows a broken arm, when the muscles will be atrophied following immobilisation in a plaster cast. Measurement of the arm will not mean an improvement in the lymphoedema, as the muscle volume will be significantly reduced. The reduction can be disproportional due to changes in the shape of the arm as a result of the loss of muscle mass, and dependent on the location of the break.

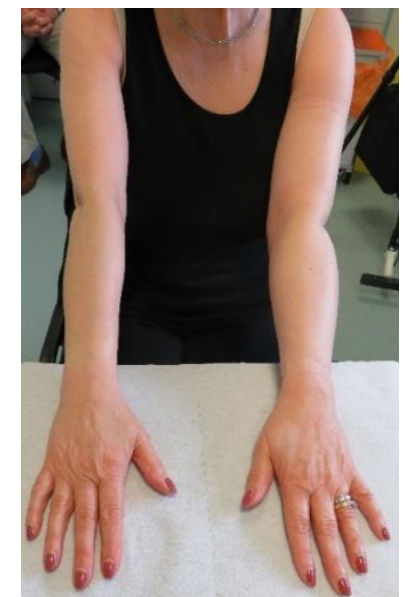

Figure 6: Shape distortion due to BCRL

Figure 6 portrays left arm lymphoedema secondary to breast cancer. The limb shape disproportion is evident when compared to the right arm. With mild to moderate lymphoedema, the calculated limb volumes may be much the same over time; however, the progression of the condition should also be monitored by assessing the shape of the limb and patient feedback. Often the patient will report that their clothes are tighter over their forearm, or their rings no longer fit their fingers, which are indicative of a change in shape, and not necessarily a volume change. This could be due to the presence of fibrosis, where the skin and tissues become thicker, distorting those tissues, without necessarily changing limb volume as such. Both shape and limb volume are important, 
but often clinicians only take limb volume into account in the treatment plan of mild to moderate lymphoedema, when a change in shape can indicate a change in the lymphoedema behaviour that may require more intensive treatment to stop the progression of the condition.

Measuring for lymphoedema is a challenging issue as a consensus for defining lymphoedema based on limb volume is non-existent (Tewari, Gill, Bochner, \& Kollias, 2008; Blaney et al., 2015). Various thresholds exist for diagnosing lymphoedema. The threshold measures compare the affected to the unaffected arm. The types of thresholds described in the literature include $\geq 2 \mathrm{~cm}$ circumference difference at any point; $\geq 5 \%$ increase in circumference at any site; $\geq 10 \%$ increase in circumference at any point; $5 \%$ difference in the sum of arm circumferences; $>10 \%$ difference in total limb volume; and $\geq 200 \mathrm{ml}$ volume difference between limbs, or a combination of these (Asim et al., 2012; Levenhagen et al., 2017). The threshold of a 10\% increase in inter-limb differences is frequently used; however, although it has good specificity (the test identifies the absence of a condition), it has poor sensitivity (the test identifies the presence of a condition) for detecting mild lymphoedema (Wang et al., 2017).

Some studies support limb measurements before breast cancer surgery as it helps take into consideration pre-existing asymmetry, is a baseline against which a diagnosis of early or mild lymphoedema can be determined within a screening programme, and are strongly encouraged (Blaney et al., 2015; Levenhagen et al., 2017; Bundred et al., 2020). Smoot et al. (2014) encourage pre-operative measurements to capture normal variability associated with limb dominance. Blaney et al. (2015) opine that patients are interested in, and value BCRL screening programmes based on the high recruitment $(85.7 \%)$ and retention rates $(83.8 \%)$ within their BCRL screening study. However, clinically, resources in terms of funding, staffing and time remain a challenge within the National Health Service (NHS). Thus screening programmes are not standardised, i.e. some services measure limbs pre-operatively as part of a one-year screening programme, while other services may not offer any form of screening for lymphoedema following breast cancer surgery (Bundred et al., 2020). For some patients awaiting surgery, preoperative measurements can be an inconvenience as it may be another appointment for them to attend, or it may interfere with their surgical preparation (Bundred et al., 2020). 
In some instances, it can lead to distress if people feel that their potential risk of lymphoedema is high due to their pending cancer surgery.

Technologies that can measure lymphoedema include perometry (Stanton, Northfield, Holroyd, Mortimer, \& Levick, 1997), bio-impedance spectroscopy (Cornish, Ward, Thomas, \& Bunce, 1998; Levenhagen et al., 2017), and dielectric constant devices (Nuutinen, Ikäheimo, \& Lahtinen, 2004; Levenhagen et al., 2017). Three-dimensional camera imaging (3DCI) has explored limb volume; however, this technology is underutilised, and prognostication of the image data is yet to be fully realised (Öhberg, Zachrisson, \& Holmner-Rocklöv, 2014). 3DCI has the potential to measure geometric parameters enabling an alternative method of measurement using limb shape (Cau et al., 2016). Karakashian, Shaban, Pike, \& van Loon (2018) developed swelling maps where colour is used to visualise areas of deviance from the non-lymphoedema arm. This could make diagnosing lymphoedema easier for a clinician, and it may enable a person with lymphoedema to understand the severity of their condition better by visualising the colours that show the areas and size of localised swelling.

The systematic literature review in Chapter 3 will critique three methods of measurement, namely CTM, as it is the most commonly used method of measurement in the clinical setting, Perometry, as it is widely used in lymphoedema research and 3DCI, the new method of lympheodema measurement. The systematic literature review will highlight the need for further research into the use of 3D technology, including the use of shape as a means of determining the severity of lymphoedema and the effectiveness of treatment outcomes.

The other methods of measurements used in clinical practice include water displacement, tissue dielectric constant (TDC) and bio-impedance spectroscopy (BIS). These are discussed next:

\subsubsection{Water Displacement}

Within lymphoedema research, water displacement is still considered the gold standard, or reference standard, of volume measurement (Devoogdt et al., 2010; Lee, Boland, Czerniec, \& Kilbreath, 2011; Adriaenssens, Buyl, Lievens, Fontaine, \& Lamote, 2013; Öhberg et al., 2014; Buffa et al., 2015; Mori, Lustman, \& Katz-Leurer, 2015; Hidding 
et al., 2016; Levenhagen et al., 2017), however, there is growing disagreement to this statement (Adriaenssens et al., 2013; Lu, Han, DeSouza, Armer, \& Shyu, 2014). Water displacement is easy to perform, painless, able to measure objects with an irregular shape and is highly sensitive, thus able to detect small volume changes (Chen, Tsai, Hung, \& Tsauo, 2008; Tewari et al., 2008; Perdomo et al., 2014). Water displacement (Figure 7) measures limb volumes most accurately and is based on Archimedes' Principle physical law of buoyancy (Öhberg et al., 2014). It states that the volume of an object immersed in a fluid is equal to the volume of the displaced fluid (Brorson \& Hoijer, 2012; Erends, van der Aa, van der Hulst, \& de Grzymala, 2014; Lu et al., 2014; Öhberg et al., 2014).

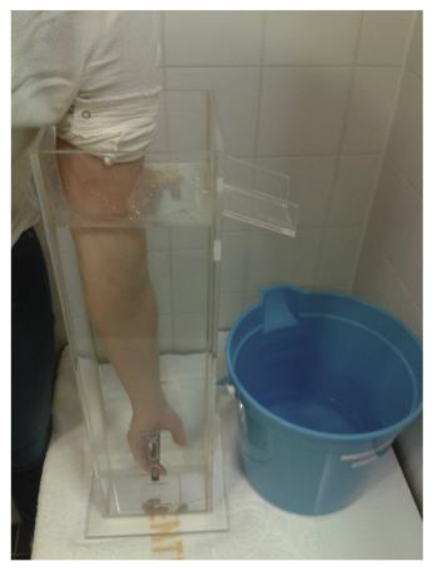

Figure 7: Measuring upper extremity limb volume by water displacement (Hameeteman, Verhulst, Vreeken, Maal, \& Ulrich, 2016) ${ }^{l}$

Consideration for the type of volumeter, methodology, water temperature, patient positioning and method of water displacement is essential for research and clinical use (Lee et al., 2011; Erends et al., 2014; Perdomo et al., 2014; Levenhagen et al., 2017) to ensure repeatability and standardisation of the technique. Water displacement has excellent reliability (inter- and intra-rater) and validity with ICC between 0.97 and 0.99 (Chen et al., 2008; Öhberg et al., 2014; Perdomo et al., 2014; Hidding et al., 2016; Levenhagen et al., 2017). Water displacement is reportedly reproducible with an error rate of $<1 \%$ (Barrio, Eaton, \& Frazier, 2015; Lee et al., 2011) and has proven concurrent and construct validity compared to computed tomography (CT scan) and magnetic resonance imaging (MRI) in the presence of lymphoedema (Sagen, Kårensen, Skaane, \& Risberg, 2009). There are however difficulties in defining and implementing the

\footnotetext{
1 Reprinted from Journal of Plastic, Reconstructive and Aesthetic Surgery, volume 69, issue 2, Hameeteman et al., 3D stereophotogrammetry in upper-extremity lymphedema: An accurate diagnostic method, pages 241-247., Copyright (2016), with permission from Elsevier
} 
upper level for immersion of limbs due to an inability to submerge the entire limb, thus there exist variations in limb lengths when measuring using water displacement (Tewari et al., 2008; Erends et al., 2014; Öhberg et al., 2014).

There is an ongoing discussion within the literature about how volume thresholds should define the presence of lymphoedema, i.e., the volume difference between the affected and unaffected arms that confirms the presence of lymphoedema. Seminal research performed by Stanton, Badger, \& Sitzia (2000), concluded that a total volume difference between arms of $>200 \mathrm{ml}$ as measured by water displacement is a sensitive indicator of lymphoedema. However, Taylor, Jayasinghe, Koelmeyer, Ung, \& Boyages (2006) report that a change of $>150 \mathrm{ml}$ is unlikely to be attributable to a chance variation or measurement error, thus this lower volume should be considered as a measure of the presence of lymphoedema in the upper limb. Sagen, Kårensen, Skaane, \& Risberg (2009) report that an increase of $10 \%$ volume between the affected and unaffected arm should be the threshold value for the diagnosis of lymphoedema. To date, there is no consensus as to which of these three thresholds should be used in clinical practice for the diagnosis of lymphoedema, and a study comparing these three thresholds to decide on the ideal value would benefit research and clinical practice.

Water displacement records volume as a single value, making it challenging to identify the areas of change in limb size (Rincon, Shah, Ramella-Roman, \& Bhansali, 2016); unlike CTM or perometry which are taken at fixed points making it easier to see where changes in limb size occur, thus informing the clinician about the impact of treatment (Belgrado et al., 2010). There is a close correlation between the methods of measuring volume in that all are reliable and provide similar estimates. However, due to limb length differences and variances in techniques, some studies report CTM underestimating limb volume compared to water displacement (Devoogdt et al., 2010; Brorson \& Hoijer, 2012; Erends et al., 2014; Öhberg et al., 2014), while others report CTM to overestimate volume (Tewari et al., 2008; Adriaenssens et al., 2013). Perometry was found to overestimate limb volume compared to water displacement in two studies (Lee et al., 2011; Adriaenssens et al., 2013); thus, the volumetric methods cannot be interchangeable due to the differences in outcomes (Devoogdt et al., 2010; Lee et al., 2011; Brorson \& Hoijer, 2012; Adriaenssens et al., 2013; Öhberg et al., 2014; Landau, Kim, Gould, \& Patel, 2018). 
The disadvantages of water displacement as a method are that it is inconvenient for routine clinical use as it is time-consuming, costly, cumbersome, impractical as a portable test, and can be unsanitary (Tewari et al., 2008; Devoogdt et al., 2010; Lee et al., 2011; Brorson \& Hoijer, 2012; Öhberg et al., 2014; Buffa et al., 2015; Hameeteman, Verhulst, Vreeken, Maal, \& Ulrich, 2016; Landau et al., 2018). Adriaenssens, Buyl, Lievens, Fontaine, \& Lamote (2013) state that water displacement is challenging to use in a clinical setting due to water spillage; and it is negated for certain medical conditions such as ulcers, open wounds, abrasions, and some skin conditions (Tewari et al., 2008; Lee et al., 2011; Erends et al., 2014; Buffa et al., 2015; Landau et al., 2018). Furthermore, water displacement is unable to provide information on the shape of the limb.

\subsubsection{Tissue Dielectric Constant (TDC)}

Tissue Dielectric Constant (TDC) measurements evaluate local tissue water in the skin and subcutaneous tissues via a non-invasive device (Mayrovitz, Carson, \& Luis, 2010; Mayrovitz, Corbitt, Grammenos, Abello, \& Mammino, 2017a; Mayrovitz, Fasen, Spagna, \& Wong, 2018a) and are performed at 300-megahertz (MHz) frequency using a concentric transmission line measurement technique. This, in turn, reflects electromagnetic energy dependant on the dielectric constant of the tissue, which is dependent on the amount of free and bound water in the tissue volume. As fat has lower water content, TDC values decrease with an increase in depth testing (Mayrovitz, 2010; Mayrovitz et al., 2010; Mayrovitz, Weingrad, \& Lopez, 2015; Mayrovitz, Grammenos, Corbitt, \& Bartos, 2017b).

There are two devices available to determine Local Tissue Water in the measurement of lymphoedema namely the 'MoistureMeterD' and the 'MoistureMeterD Compact' (Figure 8), manufactured by Delfin Technologies Ltd, Kuopio, Finland. The main differences are that the former unit is a multi-probe system consisting of various sized probes that allow measurement of TDC at different depths. In contrast, the latter device is a single depth, portable model of the MoistureMeterD with an effective measurement depth between 1.5 and 2.5mm (Mayrovitz, Weingrad, \& Lopez, 2016; Mayrovitz et al., 2017a; Bakar, Tuğral, \& Üyetürk, 2018; Mayrovitz, Arzanova, Somarriba, \& Eisa, 2018b). 


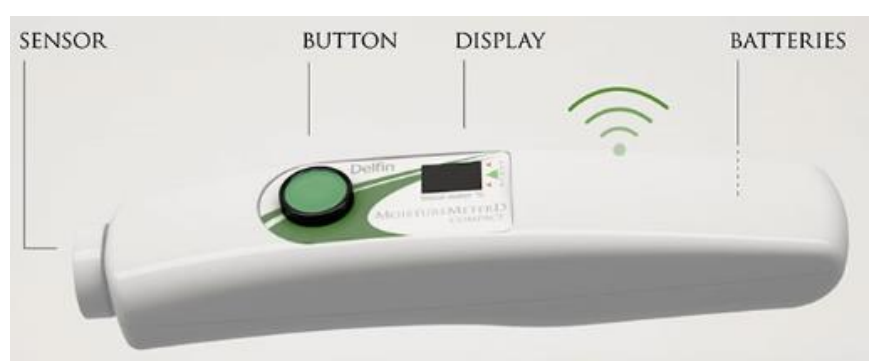

Figure 8: MoistureMeterD Compact (Delfin Technologies, 2017)

The MoistureMeterD Compact has a built-in pressure sensor to enhance repeatability and user independence and is programmed to convert the TDC into water percentage on a scale of 0 to $100 \%$. The water percentage value is calculated using the formulae:

$$
\text { Water } \%=\left[(\varepsilon-1) /\left(\varepsilon_{\mathrm{w}}-1\right)\right]^{*} 100 \%
$$

where $\varepsilon$ is the measured dielectric constant, and $\varepsilon_{\mathrm{w}}$ is the dielectric constant of water (Delfin Technologies Ltd, 2014).

TDC is a quick measurement and takes about 10 seconds for a reading, which is taken at multiple sites, measuring locally, thus making it a clinically efficient technique (Mayrovitz, Davey, \& Shapiro, 2008; Mayrovitz, Davey, \& Shapiro, 2009a; Mayrovitz, 2010; Mayrovitz et al., 2010; Czerniec, Ward, \& Kilbreath, 2015; Lahtinen, Seppala, Viren, \& Johansson, 2015; Mayrovitz et al., 2015; Mayrovitz et al., 2017b; Mayrovitz et al., 2018a; De Vrieze et al., 2020). Single measurements are as effective as triplicate measurements on the forearm and upper arm, thus less time consuming to measure within a clinical setting (Mayrovitz et al., 2009a; Mayrovitz et al., 2016; De Vrieze et al., 2020). TDC values are converted to a ratio to determine the presence of lymphoedema, thus both sides must be measured at congruent points (Mayrovitz et al., 2018b). Mayrovitz, Weingrad, \& Davey (2009b) concluded that a TDC ratio exceeding 1.26 was indicative of early lymphoedema in the 'at-risk' person. Application of the device needs to ensure repeatability, and thus pre-determined landmarks are required. All published research agrees that the midline of the anterior aspect of the arm should be used.

Original work by Nuutinen, Ikäheimo, \& Lahtinen (2004) validated the dielectric device as they found a statistically highly significant correlation between TDC and CTM of the 
$\operatorname{arm}(r=0.97, p<0.05)$. Levenhagen et al.'s (2017) literature review confirmed moderate to good correlations between arm TDC ratios and arm volume $(\mathrm{r}=0.69)$, as well as segmental volume measures (r=0.77). According to Bakar, Tuğral, \& Üyetürk (2018), TDC sensitivity and specificity was $65 \%$ and $94 \%$ respectively.

Levenhagen et al. (2017) recommend against the use of TDC as a diagnostic tool for lymphoedema due to its limited clinical utility and lack of diagnostic evidence in the literature. It does not, however, mean that it should not be used as an additional measurement tool for the assessment of lymphoedema, as it can contribute to the other examinations to decide on a lymphoedema diagnosis.

\subsubsection{Bio-Impedance Spectroscopy (BIS)}

Bio-Impedance Spectroscopy (BIS) is a non-invasive procedure whereby an alternating current (200-800 mA) is applied to a body segment via a set of cutaneous electrodes (Barrio et al., 2015; Svensson, Ward, Dylke, \& Kilbreath, 2017). In 1969, Bioelectrical Impedance Analysis (BIA) was used to measure total body water (Ward et al., 1992). The seminal paper by Ward et al. (1992) was able to demonstrate segmental analysis of extracellular fluid volume being accurately and reliably predicted by BIA over a range of frequencies ( $4 \mathrm{kHz}$ to $1 \mathrm{MHz}$ ).

In general, lower frequency currents pass through the extracellular fluid, whereas higher frequency currents cross both extracellular and intracellular fluid spaces as the reactance of the cell membranes decrease (York et al., 2009; Kim, Jeon, Sung, Jeong, \& Do, 2011; Fu et al., 2013; Rincon et al., 2016; Bundred et al., 2020). Impedance to current flow is inversely proportional to fluid accumulation, thus reduced impedance values in a measured extremity is considered indicative of the presence of lymphoedema (Ward, 2009; Ward, Czerniec, \& Kilbreath, 2009a; Czerniec et al., 2010; Jain, Danoff, \& Paul, 2010; Ward, Dylke, Czerniec, Isenring, \& Kilbreath, 2011; Svensson et al., 2017; Jung et al., 2018). BIS is ultimately the measure of resistance, or impedance, the current experiences while travelling through the cutaneous and subcutaneous matrixes. It is recognised as one of the few techniques that attempts to directly measure lymph fluid volume in unilateral lymphoedema (Bilir, DeKoven, \& Munakata, 2012; Dylke et al., 2016; Jung et al., 2018). 
Lymph fluid in lymphoedema is additional to the normal extracellular fluid compartment, thus results from BIS are considered as accurate measures of both lymph fluid impedance and total fluid impedance (Buendia et al., 2018). Extrapolation of properties unique to lymphatic fluid in measuring the flow of current is based on the principle that tissues such as fat and bone act as insulators, while electrolytic fluids conduct electricity (Seward et al., 2016). Buendia et al. (2018) later confirmed that BIS is not only useful for detecting lymph accumulation but is also able to estimate an increase in adipose tissue in BCRL.

There are two manufacturers providing devices for determining BIS for lymphoedema in the UK. The Bodystat Quadscan 4000 (Figure 9) from Bodystat Ltd, Cronkbourne, Isle of Man (Bodystat, 2017), provides accurate and immediate results of body composition, hydration status and fluid shifts in the extracellular and intracellular spaces. The L-Dex ${ }^{\circledR}$ U400 BIS (Figure 9) from ImpediMed Ltd, Brisbane, Australia (ImpediMed Ltd, 2017), is a multi-frequency BIA device (Levenhagen et al., 2017) that quantifies changes in the extracellular fluid by the allocation of an L-Dex score, to enable clinicians to easily monitor fluid content in people with, or at risk of, lymphoedema. An advantage of using the Bodystat Quadscan 4000 is that measurements can be taken segmentally or globally, whereas the L-Dex U400 only measures global fluid distribution.
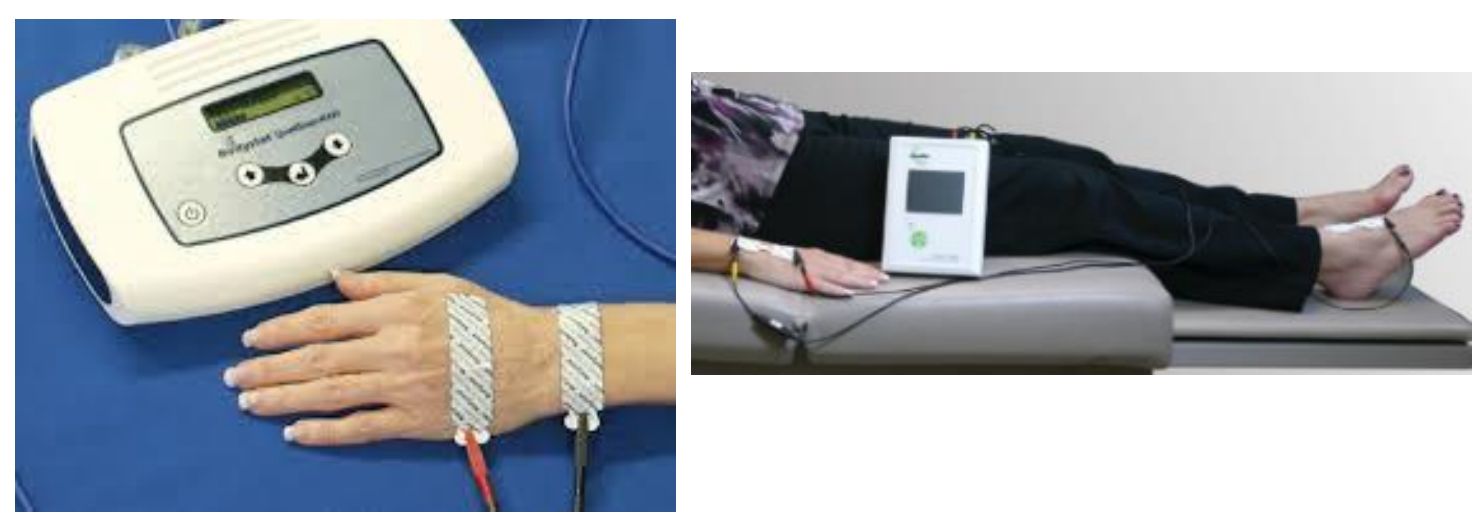

Figure 9: BodyStat Quadscan 4000 (left) and L-Dex® U400 (right)

Calculation of the BIS ratio involves normalising the affected side to that of the unaffected side and then comparing it to normative values (Ward et al., 2011; Bundred et al., 2020). BIS values differ between dominant and non-dominant sides due to the dominant limb containing more muscle and water as a result of common use in daily life 
(Dylke, Yee, Ward, Foroughi, \& Kilbreath, 2012; Smoot et al., 2014; Arinaga, Piller, \& Sato, 2016). Normative L-Dex scores lie between -10 and +10 , which is the equivalent of an impedance range of 0.935 to 1.139 for at-risk dominant arms, and 0.862 to 1.006 for at-risk non-dominant arms (Jain et al., 2010; Czerniec et al., 2011; Smoot et al., 2011; Fu et al., 2013; Blaney et al., 2015; Iyigun et al., 2015; Bundred et al., 2020). With an increase in extracellular fluid, the L-Dex value will increase, and early signs of lymphoedema are suggested at a reading above the normal range, or an increase of 10 L-Dex units from the baseline (Shah, Vicini, \& Arthur, 2016; National Institute for Clinical Excellence (NICE), 2017).

The normative population mean limb index ratio for BIS is 1.037 for the dominant side, and 0.964 for the non-dominant side (Ridner, Dietrich, Deng, Bonner, \& Kidd, 2009). Based on the standard deviation (SD) of 0.034 , thresholds have been set at three standard deviations (3SDs) above the normative mean at a ratio of 1.139 for the dominant side, or 1.066 for the non-dominant side (Dylke et al., 2016; Seward et al., 2016; Shah et al., 2016; Svensson et al., 2017).

The devices are small and portable, with built-in display screens; it utilises standardised anatomical landmarks and requires minimal training (Ridner et al., 2009; Ward, Czerniec, \& Kilbreath, 2009b; Ridner et al., 2014; NICE, 2017). It can be used on bedbound patients (Jain et al., 2010) and in private homes and offices (Ridner et al., 2009). BIS is quick and easy to perform (Ward et al., 2009a; Jung et al., 2018), taking less than one minute (min) (Ridner et al., 2009) but up to $5 \mathrm{~min}$ (Vicini, Shah, Lyden, \& Whitworth, 2012) on average. It is non-invasive (Ward et al., 2009a; Ward et al., 2009b; Berlit et al., 2012; Berlit et al., 2013; Barrio et al., 2015; Czerniec et al., 2015; Svensson, Dylke, Ward, \& Kilbreath, 2015; Jung et al., 2018) and relatively inexpensive compared to perometry (Ridner et al., 2009; Ward et al., 2009a; Buendia et al., 2018). A further advantage of BIS is that it can be used to measure bilateral upper limb lymphoedema by comparing it to a non-lymphoedematous leg (Ward, 2009; Ng \& Munnoch, 2010).

Disadvantages of BIS are the recurring cost of electrodes and the cost of the device itself (Perdomo et al., 2014; Hidding et al., 2016; Levenhagen et al., 2017; NICE, 2017). People with electrical devices such as pacemakers and metal implants should be excluded due to electrical interference (Ridner et al., 2009; Vicini et al., 2012; Hidding 
et al., 2016). BIS is limited to measuring the arm from the ulnar styloid process, thus excludes the hand in its results (Perdomo et al., 2014; Svensson et al., 2015). BIS is unable to capture tissue changes such as fibrosis or adipose infiltration; thus, it is unsuitable for later-stage lymphoedema assessment (Levenhagen et al., 2017). Some clinicians find it challenging to work with an index or ratio as opposed to the normative percentage volume differences, and it is not advised to convert either measure due to the questionable validity of calculations, especially as methods are not interchangeable (Ward et al., 2009a; Ward et al., 2009b). Clinical decisions need to be made with caution due to differences in conditions (environmental and physiological) affecting results (Timmer, Bosman, Geertzen, \& Dijkstra, 2020). As BIS captures full arm values and compares one side to the other, it cannot detect limb shape distortion utilising a distal to proximal ratio like CTM.

Tonometry is another means of assessing lymphoedema; however, the literature is weak and is not discussed as a valid measure of lymphoedema within this thesis.

In most instances, the subjective information and objective measures are sufficient to commence a programme of care and self-management for a person with lymphoedema. However, in cases where there is a lack of clarity as to the cause of the lymphoedema, medical imaging techniques can further assist the diagnosis, staging and treatment options available (Pappalardo \& Cheng, 2020).

\subsubsection{Medical Imaging}

A range of imaging techniques is available to aid in the diagnosis and understanding of lymphoedema. Direct imaging includes lymphography and lymphangiography. Indirect imaging, such as lymphoscintigraphy and fluorescent lymphography, is more commonly practised, and a variety of techniques exist to look at different aspects of the lymphatic system, and the impact lymphoedema has on the cutaneous and subcutaneous structures. The value of performing medical imaging is to gain a better insight as to what part of the lymphatic system is at fault, how the fluid flows and then backflows, and what lymphatic pathways exist that could be manipulated through intensive treatments to get a better reduction in volume and normalisation of the limb shape. Imaging can also inform the type of surgical intervention that could be offered to a patient with 
lymphoedema. Appendix 2 provides more detail into the type of medical imaging available to lymphoedema clinicians and patients.

\subsection{Conclusion}

Self-reported symptoms, psychosocial and quality of life factors form the subjective information required to understand the complexities of a person's experience of their lymphoedema. A physical examination, including measurement of the lymphoedema, together with the subjective assessment, is required to make a diagnosis of lymphoedema. Chapter 3 reports on a systematic literature review to provide an indepth analysis of three assessment methods used in this study, i.e. CTM, perometry and 3DCI. Medical imaging is used in limited cases to help with the diagnosis of lymphoedema, or as a means to determine surgical suitability.

Current classifications or staging of lymphoedema are limited in their description of the presenting swelling. The shape of the limb needs to be considered as a small volume difference between two arms may be low in terms of percentage, but if the forearm is disproportionately bigger than the upper arm or the opposite arm, then the staging would be inaccurate. However, the oversight of limb shape could be in part that there is no clinical measurement in use that can measure both limb volume and shape. Thus, a measurement technique that offers not only volume as a measurement outcome, but shape as an additional outcome, need to be considered as it would make diagnosing of lymphoedema easier for a clinician, treatment more specific and targeted, and it would assist the patient in understanding the severity of their condition. 
Chapter 3:

Systematic

Literature Review 


\subsection{Introduction}

Methods of lymphoedema measurement differ substantially in terms of application and thresholds used for diagnosing or categorising lymphoedema severity. This chapter will explore the empirical evidence available in the literature about the performance, validity and reliability of three methods of lymphoedema measurement, namely CTM, perometry and 3DCI.

This systematic literature review will also appraise how each method correlates to the other, to appreciate the relationships they may have in terms of measuring upper limb volume. The recommended thresholds for diagnosing lymphoedema for each method will also be reviewed to determine any commonality between these methods. Exploration of additional outcomes from 3DCI data will be investigated to determine the potential benefits of using limb shape as a means of assessing lymphoedema.

Thus, the research question for this systematic literature review is 'how effective is 3DCI compared to CTM and perometry?' Sub-objectives for this review include:

- explore the thresholds for diagnosing lymphoedema for each method of measurement

- analyse whether 3DCI is a valid measure of upper limb volume compared to CTM and perometry

- determining whether 3DCI is as reliable as CTM and perometry, and

- investigate whether 3DCI is capable of measuring limb shape.

\subsection{Methodology}

\subsubsection{Identifying the Evidence}

Consultation with a university hospital librarian ensured correct and comprehensive Medical Subject Heading (MeSH) terms to identify the various assessment methods used in diagnosing and assessing lymphoedema. The literature search was conducted from January 1, 2008, through to December 31, 2018; and a repeat search was conducted July 1, 2020, to check for missed articles collected from monthly alerts received from the NHS library services. 
Ovid Medline and Embase are considered comprehensive databases for medical and health care research papers as they contain the listings of more than 4,600 journals reporting on biomedical research, and together they contain a considerable number of search terms, subheadings and synonyms. An initial search strategy of Ovid Medline and Embase (Table 2) was performed, with search terms including 'lymphoedema' or 'lymphedema'; 'breast cancer' or 'breast neoplasm'; 'circumferen' (for circumferential measurements), 'limb volume'; 'anthropometry'; 'imaging, three-dimensional'; 'perometry' or 'perometer'; and a further limit of 'English language' was included. Due to research in lymphoedema measurement having advanced in the last ten years from the initial literature search in 2018, a 10-year limit was placed; thus abstracts published from '2008 to current' were included. However, earlier studies were retrieved if they were pertinent to this systematic literature review. This reduced the number of abstracts from 941 to 754 prior to deduplication, which then resulted in 537 abstracts for review.

Table 2: Search strategies for Embase and Ovid Medline databases 2018

\begin{tabular}{|l|l|l|}
\hline$\#$ & Searches & Results \\
\hline 1 & exp lymphoedema/ & 18557 \\
\hline 2 & (lymphoedema or lymphedema).mp. [mp=ti, ab, hw, tn, ot, dm, mf, dv, kw, fx, dq, nm, kf, ox, px, rx, an, ui, sy] & 27628 \\
\hline 3 & 1 or 2 & 30920 \\
\hline 4 & breast cancer.mp. [mp=ti, ab, hw, tn, ot, dm, mf, dv, kw, fx, dq, nm, kf, ox, px, rx, an, ui, sy] & 671509 \\
\hline 5 & breast neoplasm.mp. [mp=ti, ab, hw, tn, ot, dm, mf, dv, kw, fx, dq, nm, kf, ox, px, rx, an, ui, sy] & 2186 \\
\hline 6 & 4 or 5 & 672100 \\
\hline 7 & 3 and 6 & 5806 \\
\hline 8 & circumferen*.mp. [mp=ti, ab, hw, tn, ot, dm, mf, dv, kw, fx, dq, nm, kf, ox, px, rx, an, ui, sy] & 183205 \\
\hline 9 & limb volume.mp. [mp=ti, ab, hw, tn, ot, dm, mf, dv, kw, fx, dq, nm, kf, ox, px, rx, an, ui, sy] & 1060 \\
\hline 10 & anthropometry.mp. [mp=ti, ab, hw, tn, ot, dm, mf, dv, kw, fx, dq, nm, kf, ox, px, rx, an, ui, sy] & 93122 \\
\hline 11 & imaging, three-dimensional.mp. [mp=ti, ab, hw, tn, ot, dm, mf, dv, kw, fx, dq, nm, kf, ox, px, rx, an, ui, sy] & 65153 \\
\hline 12 & (perometry or perometer).mp. [mp=ti, ab, hw, tn, ot, dm, mf, dv, kw, fx, dq, nm, kf, ox, px, rx, an, ui, sy] & 274 \\
\hline 13 & 8 or 9 or 10 or 11 or 12 & 322936 \\
\hline 14 & 7 and 13 & 991 \\
\hline 15 & limit 14 to english language & 941 \\
\hline 16 & limit 15 to yr="2008 -Current" & 754 \\
\hline 17 & remove duplicates from 16 & 537 \\
\hline
\end{tabular}

The above search was repeated in 2020 and produced an additional 146 abstracts for review (Table 3). 
Table 3: Search strategies for Embase and Ovid Medline databases 2020

\begin{tabular}{|l|l|l|}
\hline$\#$ & Searches & Results \\
\hline 1 & exp lymphoedema/ & 18778 \\
\hline 2 & (lymphoedema or lymphedema).mp. [mp=ti, ab, hw, tn, ot, dm, mf, dv, kw, fx, dq, nm, kf, ox, px, rx, an, ui, sy] & 30752 \\
\hline 3 & 1 or 2 & 34038 \\
\hline 4 & breast cancer.mp. [mp=ti, ab, hw, tn, ot, dm, mf, dv, kw, fx, dq, nm, kf, ox, px, rx, an, ui, sy] & 782333 \\
\hline 5 & breast neoplasm.mp. [mp=ti, ab, hw, tn, ot, dm, mf, dv, kw, fx, dq, nm, kf, ox, px, rx, an, ui, sy] & 2839 \\
\hline 6 & 4 or 5 & 7803038 \\
\hline 7 & 3 and 6 & 7379 \\
\hline 8 & circumferen*.mp. [mp=ti, ab, hw, tn, ot, dm, mf, dv, kw, fx, dq, nm, kf, ox, px, rx, an, ui, sy] & 219712 \\
\hline 9 & limb volume.mp. [mp=ti, ab, hw, tn, ot, dm, mf, dv, kw, fx, dq, nm, kf, ox, px, rx, an, ui, sy] & 1300 \\
\hline 10 & anthropometry.mp. [mp=ti, ab, hw, tn, ot, dm, mf, dv, kw, fx, dq, nm, kf, ox, px, rx, an, ui, sy] & 99109 \\
\hline 11 & imaging, three-dimensional.mp. [mp=ti, ab, hw, tn, ot, dm, mf, dv, kw, fx, dq, nm, kf, ox, px, rx, an, ui, sy] & 75986 \\
\hline 12 & (perometry or perometer).mp. [mp=ti, ab, hw, tn, ot, dm, mf, dv, kw, fx, dq, nm, kf, ox, px, rx, an, ui, sy] & 340 \\
\hline 13 & 8 or 9 or 10 or 11 or 12 & 373623 \\
\hline 14 & 7 and 13 & 1342 \\
\hline 15 & limit 14 to english language & 1291 \\
\hline 16 & limit 15 to yr= $2019-2020$ & 231 \\
\hline 17 & remove duplicates from 16 & 146 \\
\hline
\end{tabular}

It was noted that few papers from the initial search identified 3DCI as a method of lymphoedema measurement method; thus a second, more specific search strategy of Ovid Medline and Embase was performed (Table 4) with search terms including 'lymphoedema' or 'lymphedema'; 'anthropometry'; '3d imaging' or '3d camera'; and 'English language' limitation was added. The same time scale was introduced (2008 to current), reducing the number of abstracts from 149 to 71, prior to deduplication which further reduced the abstracts to 54 for review. This search was repeated in 2020 and produced an additional 19 abstracts for appraisal (Table 5).

Table 4: Search strategy to identify papers reflecting 3D measurements for lymphoedema 2018

\begin{tabular}{|l|l|l|}
\hline$\#$ & Searches & Results \\
\hline 1 & exp lymphoedema/ & 18586 \\
\hline 2 & (lymphoedema or lymphedema).mp. [mp=ti, ab, hw, tn, ot, dm, mf, dv, kw, fx, dq, nm, kf, ox, px, rx, an, ui, sy] & 27667 \\
\hline 3 & 1 or 2 & 30963 \\
\hline 4 & anthropometry.mp. [mp=ti, ab, hw, tn, ot, dm, mf, dv, kw, fx, dq, nm, kf, ox, px, rx, an, ui, sy] & 93176 \\
\hline 5 & 3d imaging.mp. [mp=ti, ab, hw, tn, ot, dm, mf, dv, kw, fx, dq, nm, kf, ox, px, rx, an, ui, sy] & 7262 \\
\hline 6 & 3d camera.mp. [mp=ti, ab, hw, tn, ot, dm, mf, dv, kw, fx, dq, nm, kf, ox, px, rx, an, ui, sy] & 226 \\
\hline 7 & 4 or 5 or 6 & 100575 \\
\hline 8 & 3 and 7 & 165 \\
\hline 9 & limit 8 to english language & 149 \\
\hline 10 & limit 9 to yr="2008 -Current" & 71 \\
\hline 11 & remove duplicates from 10 & 54 \\
\hline
\end{tabular}


Table 5: Search strategy to identify papers reflecting 3D measurements for lymphoedema 2020

\begin{tabular}{|c|c|c|}
\hline \# & Searches & Results \\
\hline 1 & exp lymphoedema/ & 18778 \\
\hline 2 & (lymphoedema or lymphedema).mp. [mp=ti, ab, hw, tn, ot, dm, mf, dv, kw, fx, dq, nm, kf, ox, px, rx, an, ui, sy] & 30752 \\
\hline 3 & 1 or 2 & 34038 \\
\hline 4 & anthropometry.mp. [mp=ti, ab, hw, tn, ot, dm, mf, dv, kw, fx, dq, nm, kf, ox, px, rx, an, ui, sy] & 99109 \\
\hline 5 & 3d imaging.mp. [mp=ti, ab, hw, tn, ot, dm, mf, dv, kw, fx, dq, nm, kf, ox, px, rx, an, ui, sy] & 10760 \\
\hline 6 & 3d camera.mp. [mp=ti, ab, hw, tn, ot, dm, mf, dv, kw, fx, dq, nm, kf, ox, px, rx, an, ui, sy] & 417 \\
\hline 7 & 4 or 5 or 6 & 110150 \\
\hline 8 & 3 and 7 & 195 \\
\hline 9 & limit 14 to english language & 181 \\
\hline 10 & limit 15 to $y r=" 2019-2020^{\prime \prime}$ & 28 \\
\hline 11 & remove duplicates from 16 & 19 \\
\hline
\end{tabular}

To ensure the maximum number of papers were included within this systematic literature review, further databases were searched including Pubmed, ProQuest Dialog, Cochrane, and Google Scholar; and journal alerts were reviewed (see Table 6). The search term "upper limb" was used when searching PubMed and Google Scholar as the breast cancer terms produced too large a volume of irrelevant abstracts. Following deduplication, ten abstracts were identified for inclusion for review. Wiley Online Library, BMJ, Oxford Academic and Springer search engines were also utilised but produced no new abstracts for review. Monthly alerts were also created with the library for the above MeSH-terms, and intermittent literature searches were performed to ensure no relevant papers were omitted. Grey literature, including tweets and conference abstracts, were considered and followed up through contacting the authors to find out whether their research or clinical findings had been published. Citation tracking was also performed to determine the impact the articles may have had on other research.

As described, a comprehensive search of the various databases was performed. Articles were cross-referenced using their reference lists to ensure quality and validity and to locate papers that may not have been identified in the online literature search. 
Table 6: Additional search engines utilised

\begin{tabular}{|c|c|c|c|c|}
\hline $\begin{array}{l}\text { Search } \\
\text { Engine }\end{array}$ & Boolean Search Terms & $\begin{array}{l}\text { Abstracts } \\
\text { Identified }\end{array}$ & $\begin{array}{l}\text { Abstracts } \\
\text { Reviewed }\end{array}$ & $\begin{array}{c}\text { New } \\
\text { Abstracts }\end{array}$ \\
\hline \multirow{4}{*}{ PubMed } & $\begin{array}{l}\text { Lymphoedema/Lymphedema + 3D } \\
\text { Camera }\end{array}$ & 3 & 3 & 0 \\
\hline & $\begin{array}{l}\text { Lymphoedema/Lymphedema + 3D } \\
\text { Imaging + Upper Limb }\end{array}$ & 23 & 10 & 2 \\
\hline & $\begin{array}{l}\text { Lymphoedema/Lymphedema + } \\
\text { Perometer + Upper Limb }\end{array}$ & 28 & 11 & 1 \\
\hline & $\begin{array}{l}\text { Lymphoedema/Lymphedema + } \\
\text { Assessment + Upper Limb }\end{array}$ & 178 & 9 & 2 \\
\hline & Total & 232 & 33 & 5 \\
\hline \multirow{8}{*}{$\begin{array}{l}\text { ProQuest } \\
\text { Dialog }\end{array}$} & $\begin{array}{l}\text { Lymphoedema/Lymphedema + Breast } \\
\text { Cancer/Breast Neoplasm }+ \\
\text { circumference }+ \text { limb volume }\end{array}$ & 28 & 5 & 2 \\
\hline & $\begin{array}{l}\text { Lymphoedema/Lymphedema + Breast } \\
\text { Cancer/Breast Neoplasm + } \\
\text { anthropometric + perometer/perometry }\end{array}$ & 847 & & \\
\hline & $\begin{array}{l}\text { Lymphoedema/Lymphedema + Breast } \\
\text { Cancer/Breast Neoplasm + } \\
\text { perometer/perometry }\end{array}$ & 872 & & \\
\hline & $\begin{array}{l}\text { Lymphoedema/Lymphedema + Breast } \\
\text { Cancer/Breast Neoplasm + limb } \\
\text { volume + perometer/perometry }\end{array}$ & 913 & & \\
\hline & $\begin{array}{l}\text { Lymphoedema/Lymphedema + Breast } \\
\text { Cancer/Breast Neoplasm }+ \text { limb } \\
\text { volume }+ \text { perometer/perometry }+ \\
\text { circumference }\end{array}$ & 29 & 5 & 0 \\
\hline & $\begin{array}{l}\text { Lymphoedema/Lymphedema + Breast } \\
\text { Cancer/Breast Neoplasm + limb } \\
\text { volume + 3D imaging }\end{array}$ & 913 & & \\
\hline & $\begin{array}{l}\text { Lymphoedema/Lymphedema + Breast } \\
\text { Cancer/Breast Neoplasm + limb } \\
\text { volume }+ \text { perometer/perometry }+ \\
\text { circumference }+3 \text { D imaging }\end{array}$ & 29 & 5 & 0 \\
\hline & Total & 3631 & 15 & 2 \\
\hline \multirow[t]{2}{*}{ Cochrane } & Lymphoedema/Lymphedema & 19 & 0 & \\
\hline & Total & 19 & $\mathbf{0}$ & $\mathbf{0}$ \\
\hline \multirow[t]{2}{*}{$\begin{array}{l}\text { Google } \\
\text { Scholar }\end{array}$} & $\begin{array}{l}\text { Lymphoedema + Breast Cancer + limb } \\
\text { volume + circumference + } \\
\text { anthropometry after } 2008\end{array}$ & 226 & 14 & 0 \\
\hline & Total & 226 & 14 & $\mathbf{0}$ \\
\hline \multirow[t]{3}{*}{$\begin{array}{l}\text { Journal } \\
\text { Alerts }\end{array}$} & $\begin{array}{l}\text { Lymphoedema/Lymphedema + Breast } \\
\text { Cancer/Breast Neoplasm + limb } \\
\text { volume + perometer/perometry }+ \\
\text { circumference }+3 \mathrm{D} \text { imaging }\end{array}$ & 10 & 6 & 3 \\
\hline & Total & 10 & 6 & 3 \\
\hline & Grand Total & 4118 & 68 & 10 \\
\hline
\end{tabular}




\subsubsection{Inclusion and Exclusion Criteria}

There is more evidence in the literature for upper limb lymphoedema secondary to breast cancer compared to any other type or region of lymphoedema. It was therefore decided to focus on this group exclusively. Table 7 summarises the final inclusion and exclusion criteria for the systematic literature review based on the key words used. No filters were applied, as there were no clinical trials or randomised control trials available. The majority of the papers were diagnostic test studies.

Table 7: Inclusion and exclusion criteria based on key words

\begin{tabular}{|c|c|c|}
\hline \multirow{5}{*}{$\begin{array}{l}\text { Inclusion } \\
\text { Criteria }\end{array}$} & Population & $\begin{array}{l}\text { Adults (aged }>18 \text { years) with upper limb lymphoedema } \\
\text { secondary to breast cancer }\end{array}$ \\
\hline & Assessment & Upper Limb Lymphoedema Measurements \\
\hline & $\begin{array}{l}\text { Types of } \\
\text { Measurement }\end{array}$ & $\begin{array}{l}\text { At least one of the measures were required: } \\
\text { - } \quad \text { Circumferential Tape Measure (CTM) } \\
\text { - } \quad \text { Perometry } \\
\text { - } \quad \text { Three-Dimensional Camera Imaging (3DCI) }\end{array}$ \\
\hline & Limits & $\begin{array}{ll}\text { - } & \text { English language only } \\
\text { - } & \text { Date limit of } 2008 \\
\end{array}$ \\
\hline & $\begin{array}{l}\text { Types of evidence } \\
\text { included }\end{array}$ & $\begin{array}{l}\text { No filters applied to this topic as there were no clinical } \\
\text { trials or randomised control trials available }\end{array}$ \\
\hline \multirow{5}{*}{$\begin{array}{l}\text { Exclusion } \\
\text { Criteria }\end{array}$} & Assessment & $\begin{array}{l}\text { - } \text { Risk factors of lymphoedema } \\
\text { - } \quad \text { Early diagnosis criteria } \\
\text { - Treatment outcomes } \\
\text { - } \quad \text { Subjective reporting } \\
\text { - Incidences of lymphoedema } \\
\text { - } \quad \text { Measures truncal or lower limb oedema } \\
\end{array}$ \\
\hline & $\begin{array}{l}\text { Types of } \\
\text { Measurement }\end{array}$ & $\begin{array}{ll}\text { - } & \text { Use of advanced medical imaging } \\
\text { - } & \text { Other methods of measurements (Water } \\
& \text { Displacement, TDC, BIS) } \\
\text { - } & \text { Anatomical imaging } \\
\text { - } & \text { Simulation modelling } \\
\end{array}$ \\
\hline & Type of literature & $\begin{array}{ll}\text { - } & \text { Letters or editorials } \\
\text { - } & \text { Conference abstracts } \\
\text { - } & \text { Systematic, Literature or Methodological reviews }\end{array}$ \\
\hline & Irrelevant & $\begin{array}{l}\text { - Head \& neck, or genital oedema } \\
\text { - Animal trials }\end{array}$ \\
\hline & Other & - Non-English language \\
\hline
\end{tabular}

\subsubsection{Study Selection}

One person, the researcher, for appropriateness, reviewed retrieved article titles and abstracts with duplicates removed. Studies excluded during analysis of the full text were captured with the reasons for exclusion. 


\subsubsection{Data Extraction and Synthesis}

Data from each of the critiqued papers were extracted into an evidence table (Appendix 3) to document the analysis performed for each article. The subject headings used included the purpose of the study, research design, sample, validity of the study, the study results and the clinical benefit of the study. The findings of the evidence are summarised in a concise narrative below.

\subsubsection{Critical Appraisal}

Each paper was critiqued using the Critical Appraisal Skills Programme (CASP) (Critical Appraisal Skills Programme, 2017). As each study reported was related to diagnostics, the CASP for Diagnostic Test Studies was used. This 12-item checklist evaluates the validity, results and impact of each study. Table 8 provides an overview of the CASP Diagnostic Test Study criteria used for the lymphoedema methods of measurement studies. Papers scored '2' if answered yes, ' 1 ' if unsure, and ' 0 ' if no. Scores were totalled at the end of each row to a maximum value of 24 .

\subsection{Results}

\subsubsection{Included Studies}

The Preferred Reporting Items for Systematic Reviews and Meta-Analyses (PRISMA) flowchart (Shamseer et al., 2015; PRISMA, 2018) was used as a guide for the collation of the data (Figure 10). Articles identified from Ovid MEDLINE and Embase totalled 756. A further 68 abstracts were identified from the other search engines utilised (refer to Table 6). The total number of articles identified for the full search was 824 . This was reduced to 650 abstracts on removal of duplicates $(n=174)$. By implementing the exclusion criteria, the number of papers identified for full review was 44 . Of the 44 papers for the full review, one was excluded as irrelevant as the 3D data was extrapolated for simulation models. 


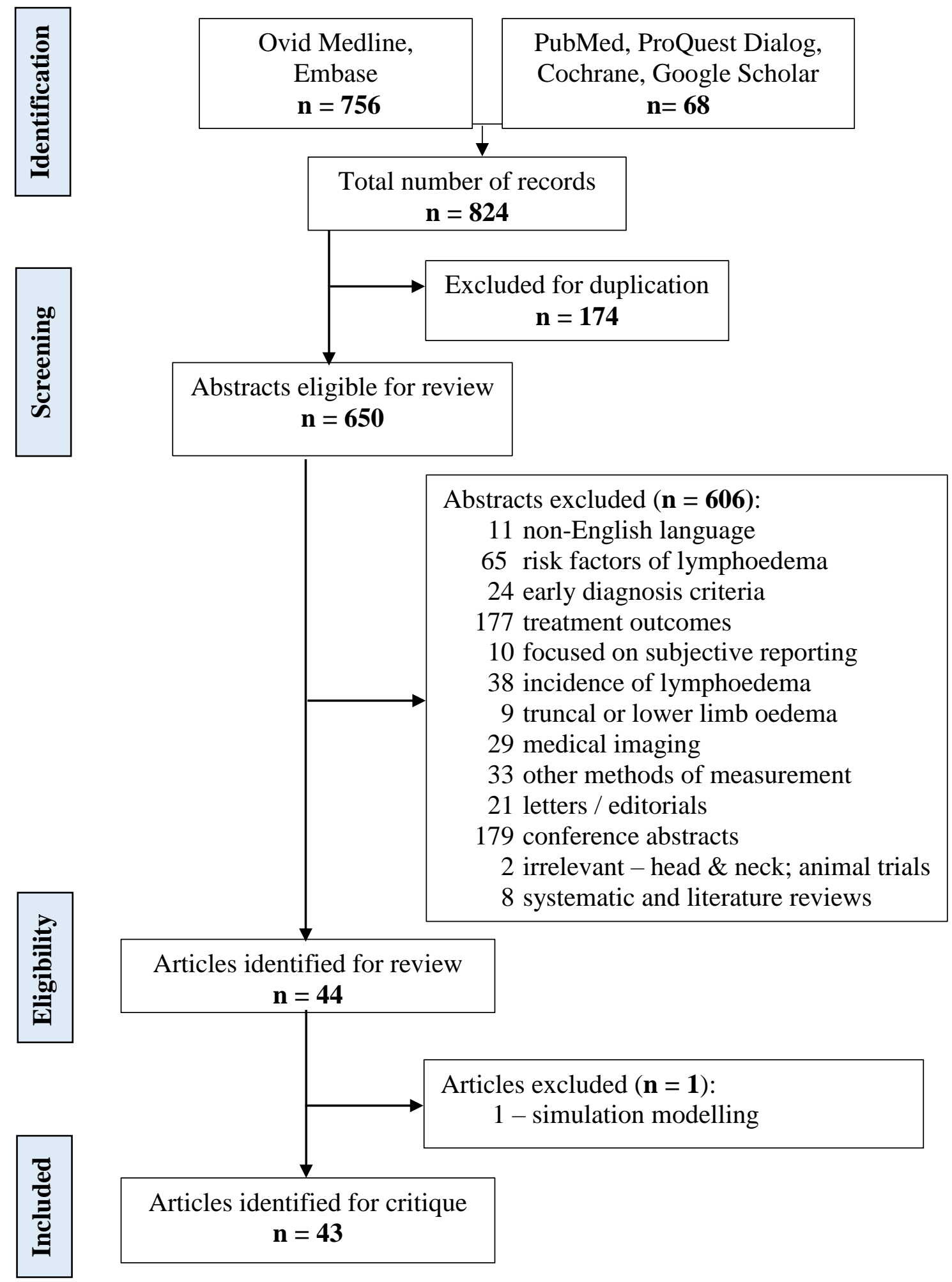

Figure 10: Preferred reporting items for systematic reviews and meta-analyses (PRISMA) flowchart 
Table 8: Items of Critical Appraisal Skills Programme (CASP) (Critical Appraisal Skills Programme, 2017): Diagnostic Test Study specific for lymphoedema measurements

\begin{tabular}{|c|c|c|c|c|}
\hline \multicolumn{2}{|c|}{ Item to Score } & Yes (2) & Unclear (1) & No (0) \\
\hline \multicolumn{5}{|c|}{ Validity } \\
\hline 1 & $\begin{array}{l}\text { Research } \\
\text { Question }\end{array}$ & $\begin{array}{l}\text { Clearly identifies the } \\
\text { population, test, setting and } \\
\text { outcomes }\end{array}$ & $\begin{array}{l}\text { Lacks clarity about } \\
\text { population, test, setting and } \\
\text { outcomes }\end{array}$ & $\begin{array}{l}\text { Fails to identify population, } \\
\text { test, setting and outcomes }\end{array}$ \\
\hline 2 & $\begin{array}{l}\text { Comparator } \\
\text { Measurement }\end{array}$ & $\begin{array}{l}\text { Appropriate reference } \\
\text { standard used, both sides } \\
\text { measured }\end{array}$ & $\begin{array}{l}\text { Incomplete reference } \\
\text { standard described, one or } \\
\text { both sides measured }\end{array}$ & $\begin{array}{l}\text { Inappropriate reference } \\
\text { standard used, one side } \\
\text { measured }\end{array}$ \\
\hline 3 & $\begin{array}{l}\text { Measurements } \\
\text { Performed }\end{array}$ & $\begin{array}{l}\text { All patients were measured } \\
\text { with all measurement } \\
\text { instruments on both sides }\end{array}$ & $\begin{array}{l}\text { One measurement } \\
\text { instrument was used on a } \\
\text { proportion of patients on } \\
\text { one or both sides }\end{array}$ & $\begin{array}{l}\text { All patients were measured } \\
\text { with one or more } \\
\text { measurement but only on } \\
\text { one side }\end{array}$ \\
\hline 4 & Blinding & $\begin{array}{l}\text { Results blinded, tester } \\
\text { unaware of outcome of } \\
\text { other test }\end{array}$ & $\begin{array}{l}\text { Unclear or }<30 \text { min between } \\
\text { measurements }\end{array}$ & Results not blinded \\
\hline 5 & $\begin{array}{l}\text { Included } \\
\text { participants }\end{array}$ & $\begin{array}{l}\text { Diagnosis of upper limb } \\
\text { lymphoedema, or at risk } \\
\text { clearly described }\end{array}$ & $\begin{array}{l}\text { Lymphoedema not clearly } \\
\text { defined, other oedema; age } \\
\text { unclear, inappropriate } \\
\text { exclusions }\end{array}$ & $\begin{array}{l}\text { No match on sexes/age } \\
\text { (>10-year difference; not } \\
\text { mentioned) }\end{array}$ \\
\hline 6 & $\begin{array}{l}\text { Measurement } \\
\text { Protocol }\end{array}$ & $\begin{array}{l}\text { Appropriate protocol } \\
\text { described for research } \\
\text { question, both sides } \\
\text { measured }\end{array}$ & $\begin{array}{l}\text { Incomplete protocol } \\
\text { described for research } \\
\text { question, one or both sides } \\
\text { measured }\end{array}$ & $\begin{array}{l}\text { Protocol not appropriate for } \\
\text { research question, one side } \\
\text { measured }\end{array}$ \\
\hline \multicolumn{5}{|c|}{ Results } \\
\hline 7 & Results & $\begin{array}{l}\text { Sensitivity and specificity } \\
\text { and/or likelihood ratios } \\
\text { clearly presented }\end{array}$ & $\begin{array}{l}\text { Sensitivity and specificity } \\
\text { and/or likelihood ratios } \\
\text { unclear }\end{array}$ & $\begin{array}{l}\text { Sensitivity and specificity } \\
\text { and/or likelihood ratios not } \\
\text { presented }\end{array}$ \\
\hline 8 & $\begin{array}{l}\text { Confidence in } \\
\text { Results }\end{array}$ & $\begin{array}{l}\text { Results intentional; clear } \\
\text { confidence limits identified }\end{array}$ & $\begin{array}{l}\text { Results intention clear; } \\
\text { confidence limits vague }\end{array}$ & $\begin{array}{l}\text { Results occurred by chance; } \\
\text { no confidence limits } \\
\text { identified }\end{array}$ \\
\hline \multicolumn{5}{|c|}{ Impact } \\
\hline 9 & $\begin{array}{l}\text { Results } \\
\text { transferable to } \\
\text { own population }\end{array}$ & $\begin{array}{l}\text { Results can be applied to } \\
\text { own population }\end{array}$ & $\begin{array}{l}\text { Some aspects of the results } \\
\text { can be applied to own } \\
\text { population such as age, sex, } \\
\text { ethnicity }\end{array}$ & $\begin{array}{l}\text { Results cannot be applied to } \\
\text { own population }\end{array}$ \\
\hline 10 & $\begin{array}{l}\text { Test applicative } \\
\text { to own } \\
\text { population }\end{array}$ & $\begin{array}{l}\text { Resources in terms of both } \\
\text { expertise and services } \\
\text { available to apply the test to } \\
\text { own patients }\end{array}$ & $\begin{array}{l}\text { Some resources in terms of } \\
\text { expertise and/or services } \\
\text { available to apply the test to } \\
\text { own patients }\end{array}$ & $\begin{array}{l}\text { Resources in terms of } \\
\text { expertise and services } \\
\text { unavailable to apply the test } \\
\text { to own patients }\end{array}$ \\
\hline 11 & $\begin{array}{l}\text { All outcomes } \\
\text { considered }\end{array}$ & $\begin{array}{l}\text { Outcomes will result in } \\
\text { improved patient well-being } \\
\text { and management }\end{array}$ & $\begin{array}{l}\text { Outcomes will result in } \\
\text { improved patient well-being } \\
\text { or management }\end{array}$ & $\begin{array}{l}\text { Outcomes will not result in } \\
\text { improved patient well-being } \\
\text { and management }\end{array}$ \\
\hline 12 & $\begin{array}{l}\text { Impact of using } \\
\text { test on } \\
\text { population }\end{array}$ & $\begin{array}{l}\text { Positive change to current } \\
\text { practice }\end{array}$ & $\begin{array}{l}\text { Modification to current } \\
\text { practice }\end{array}$ & $\begin{array}{l}\text { No change to current } \\
\text { practice }\end{array}$ \\
\hline
\end{tabular}




\subsubsection{Quality of the Studies}

The pivot table created from the database of articles analysed (Table 9) identified that 'diagnostic test study' ( $\mathrm{n}=36)$ was the most commonly used research method. The lack of standardisation in the measurement procedures, and the variations in thresholds used to diagnose lymphoedema, created confusion.

Table 9: Types of research methods from the systematic literature review
\begin{tabular}{lcc} 
Research Design & Count \\
\hline Blinded Repeat Measures Observational Study & 1 \\
Case-control study & 1 \\
Cross-sectional Study & 4 \\
Diagnostic Test Study & 36 \\
Prospective Cohort Study & 1 \\
Grand Total & $\mathbf{4 3}$
\end{tabular}

All studies were critiqued against the CASP criteria with the results shown in Table 10. Following a critique of the articles, 19 (44.2\%) studies scored 22 or more out of 24, with only two papers scoring the maximum of 24; these were De Vrieze et al. (2019) and Spinelli et al. (2019). The lowest scored papers at 17 out of 24, were Verhulst et al. (2017) and Yamamoto, Yamamoto, \& Yoshimatsu (2017).

\subsubsection{Limitations}

There are several limitations with the systematic literature review, most of which are attributed to the quality of the studies comparing or validating methods of measurement for lymphoedema. The limitations are identified in Table 11. Twenty-four of the reviewed studies did not identify any limitations, yet 19 of these were found to have limitations identified by the researcher. Blinding could not be identified in 25 papers, and blinding was unclear in eleven studies, bringing the quality and validity of these studies into question where tools that are prone to user error could be manipulated for favourable outcomes.

\subsubsection{Single Reviewer}

The researcher (Cheryl Pike) produced this systematic literature review. It has not been piloted in clinical practice, and it does not include perspectives of patients, peers or other health professionals in its inception and conduct. This limitation has been addressed through the review of two supervisors overseeing the construct of this thesis. 
Table 10: CASP outcomes of each research study paper

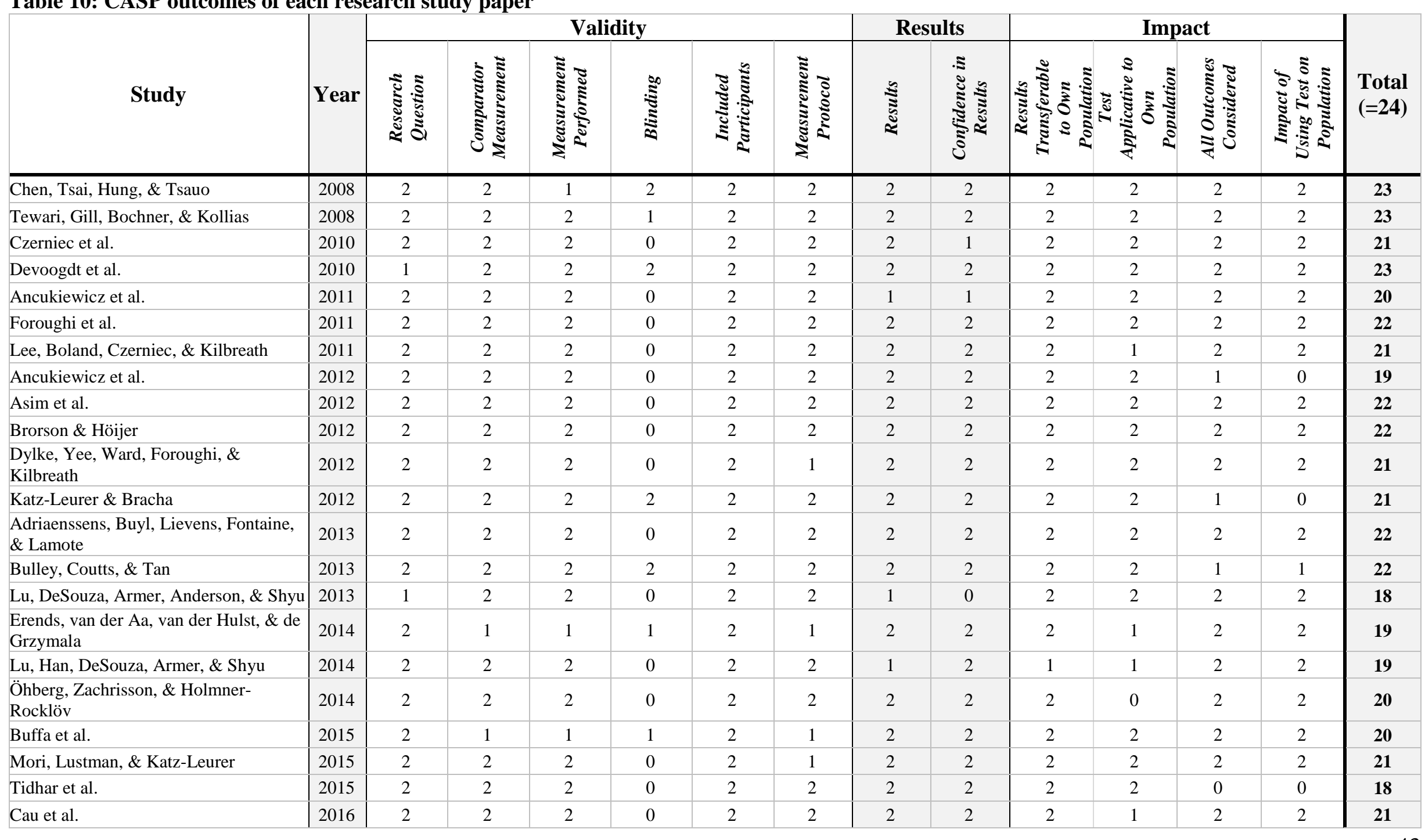




\begin{tabular}{|c|c|c|c|c|c|c|c|c|c|c|c|c|c|c|}
\hline \multirow[b]{2}{*}{ Study } & \multirow[b]{2}{*}{ Year } & \multicolumn{6}{|c|}{ Validity } & \multicolumn{2}{|c|}{ Results } & \multicolumn{4}{|c|}{ Impact } & \multirow[b]{2}{*}{$\begin{array}{l}\text { Total } \\
(=24)\end{array}$} \\
\hline & & 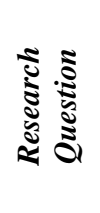 & 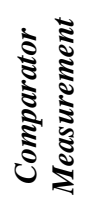 & 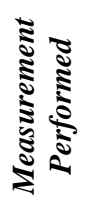 & 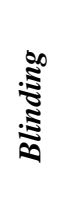 & 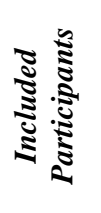 & 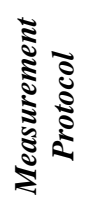 & 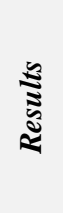 & 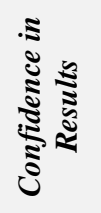 & 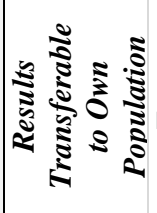 & 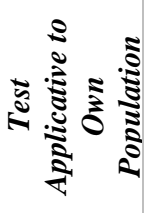 & 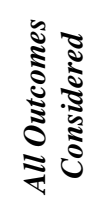 & 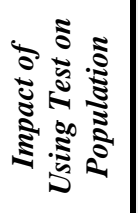 & \\
\hline $\begin{array}{l}\text { Hameeteman, Verhulst, Vreeken, Maal, } \\
\text { \& Ulrich }\end{array}$ & 2016 & 2 & 2 & 2 & 1 & 2 & 2 & 2 & 2 & 2 & 1 & 2 & 2 & 22 \\
\hline Hoevenaren et al. & 2016 & 2 & 2 & 2 & 0 & 2 & 2 & 2 & 2 & 2 & 1 & 2 & 2 & 21 \\
\hline Yamamoto et al. & 2016 & 2 & 2 & 2 & 0 & 2 & 2 & 2 & 2 & 2 & 2 & 2 & 2 & 22 \\
\hline Peleg \& Katz-Leurer & 2017 & 2 & 2 & 2 & 0 & 2 & 2 & 2 & 2 & 2 & 2 & 2 & 2 & 22 \\
\hline Verhulst et al. & 2017 & 2 & 1 & 1 & 1 & 2 & 1 & 0 & 2 & 2 & 1 & 2 & 2 & 17 \\
\hline Wang et al. & 2017 & 2 & 2 & 2 & 0 & 2 & 2 & 2 & 2 & 2 & 2 & 0 & 0 & 18 \\
\hline Yamamoto, Yamamoto, \& Yoshimatsu & 2017 & 2 & 2 & 2 & 0 & 2 & 2 & 2 & 1 & 2 & 2 & 0 & 0 & 17 \\
\hline $\begin{array}{l}\text { Batista, Baiocchi, Campanholi, } \\
\text { Bergmann, \& Duprat }\end{array}$ & 2018 & 2 & 2 & 2 & 1 & 2 & 2 & 2 & 2 & 2 & 2 & 2 & 0 & 21 \\
\hline Cau et al. & 2018 & 2 & 2 & 2 & 0 & 2 & 2 & 2 & 2 & 2 & 0 & 2 & 2 & 20 \\
\hline Karakashian, Shaban, Pike, \& van Loon & 2018 & 2 & 1 & 2 & 1 & 2 & 2 & 2 & 2 & 2 & 2 & 2 & 2 & 22 \\
\hline Landau, Kim, Gould, \& Patel & 2018 & 2 & 2 & 2 & 1 & 2 & 2 & 2 & 2 & 2 & 1 & 2 & 2 & 22 \\
\hline $\begin{array}{l}\text { Preuß, Killaars, de Grzymala, } \\
\text { Binnebösel \& Neumann }\end{array}$ & 2018 & 2 & 2 & 2 & 1 & 2 & 2 & 2 & 2 & 2 & 1 & 2 & 2 & 22 \\
\hline Sharkey et al. & 2018 & 1 & 2 & 2 & 0 & 0 & 2 & 2 & 1 & 2 & 2 & 2 & 2 & 18 \\
\hline Sun et al. & 2018 & 2 & 2 & 2 & 0 & 2 & 2 & 2 & 2 & 2 & 2 & 2 & 2 & 22 \\
\hline DeSnyder et al. & 2019 & 2 & 2 & 2 & 1 & 2 & 2 & 2 & 1 & 2 & 1 & 2 & 2 & 21 \\
\hline De Vrieze et al. & 2019 & 2 & 2 & 2 & 2 & 2 & 2 & 2 & 2 & 2 & 2 & 2 & 2 & 24 \\
\hline Karakashian, Pike, \& van Loon & 2019 & 2 & 1 & 1 & 0 & 2 & 2 & 2 & 2 & 2 & 2 & 2 & 2 & 20 \\
\hline Lu \& Dixon & 2019 & 2 & 2 & 2 & 0 & 2 & 2 & 2 & 2 & 2 & 2 & 2 & 2 & 22 \\
\hline $\begin{array}{l}\text { Rafn, McNeely, Camp, Midtgaard, \& } \\
\text { Campbell }\end{array}$ & 2019 & 2 & 2 & 2 & 1 & 2 & 2 & 2 & 2 & 2 & 2 & 2 & 2 & 23 \\
\hline Spinelli et al. & 2019 & 2 & 2 & 2 & 2 & 2 & 2 & 2 & 2 & 2 & 2 & 2 & 2 & 24 \\
\hline Binkley et al. & 2020 & 2 & 2 & 2 & 2 & 2 & 2 & 2 & 2 & 2 & 1 & 2 & 2 & 23 \\
\hline
\end{tabular}


Table 11: Methodology, cohort and limitations of each paper

\begin{tabular}{|c|c|c|c|c|}
\hline Author & Year & Cohort & Methodology & Limitations \\
\hline Chen et al. & 2008 & 14 BCRL & Diagnostic Test Study & $\begin{array}{l}\text { No ethics approval or consent gained reported. Small cohort may lower variability among } \\
\text { participants thus affecting the authenticity of the results }\end{array}$ \\
\hline Tewari et al. & 2008 & 87 mixed & Diagnostic Test Study & No consent reported. Hand volume not included. Blinding is unclear \\
\hline Czerniec et al. & 2010 & 51 mixed & Diagnostic Test Study & None identified by authors. No blinding identified \\
\hline Devoogdt et al. & 2010 & $112 \mathrm{BCRL}$ & Diagnostic Test Study & $\begin{array}{l}\text { No consent reported. Reliability measurements were performed on healthy arms of the BCRL } \\
\text { cohort. Surgery was performed during the two measurement sessions. }\end{array}$ \\
\hline Ancukiewicz et al. & 2011 & 677 BCRL & Diagnostic Test Study & None identified by authors. No consent reported. No blinding identified \\
\hline Foroughi et al. & 2011 & 57 healthy & Cross-sectional study & Only written instructions for self-measurement were provided \\
\hline Lee et al. & 2011 & 40 mixed & Diagnostic Test Study & None identified by authors. No blinding identified \\
\hline Ancukiewicz et al. & 2012 & 677 BCRL & Diagnostic Test Study & None identified by authors. No consent reported. No blinding identified \\
\hline Asim et al. & 2012 & 193 BCRL & Diagnostic Test Study & No ethics approval reported. No pre-surgery volume available for comparison over time. \\
\hline Brorson \& Hoijer & 2012 & $10 \mathrm{BCRL}$ & Diagnostic Test Study & $\begin{array}{l}\text { None identified by authors. No ethics approval reported. Very small cohort and no blinding } \\
\text { identified }\end{array}$ \\
\hline Dylke et al. & 2012 & 204 healthy & Diagnostic Test Study & $\begin{array}{l}\text { Only perometer circumferences were used and not the more commonly used tool of tape measure. } \\
\text { Hand dominance was determined by asking the participant, using clarification of which hand they } \\
\text { wrote with if needed. The validated method of the Edinburgh Handedness Inventory may be more } \\
\text { accurate; however, in clinics, the self-determined method is usually used. Equations for frustum } \\
\text { and cylinder methods provided, but not for perometer }\end{array}$ \\
\hline Katz-Leurer \& Bracha & 2012 & $16 \mathrm{BCRL}$ & Diagnostic Test Study & Lack of blinding for the second evaluation \\
\hline Adriaenssens et al. & 2013 & 80 mixed & Diagnostic Test Study & None identified by authors. No blinding identified \\
\hline Bulley et al. & 2013 & 30 healthy & Diagnostic Test Study & $\begin{array}{l}\text { Study would benefit from larger and more varied participants. Numbers of participants were not } \\
\text { consistent for the different pilot studies. }\end{array}$ \\
\hline Lu et al. & 2013 & 6 healthy & Diagnostic Test Study & $\begin{array}{l}\text { None identified by authors. No ethics approval or consent gained reported. Cohort small and only } \\
\text { healthy participants included }\end{array}$ \\
\hline Erends et al. & 2014 & 33 healthy & Diagnostic Test Study & None identified by authors. No ethics approval or consent gained reported \\
\hline Lu et al. & 2014 & 13 healthy & Diagnostic Test Study & $\begin{array}{l}\text { None identified by authors. No ethics approval or consent gained reported. Cohort small and only } \\
\text { healthy participants included }\end{array}$ \\
\hline Öhberg et al. & 2014 & $25 \mathrm{BCRL}$ & Diagnostic Test Study & None identified by authors. Relatively small cohort and no blinding identified \\
\hline Buffa et al. & 2015 & 30 healthy & Diagnostic Test Study & None identified by authors. Only healthy participants included \\
\hline Mori et al. & 2015 & 17 BCRL & Diagnostic Test Study & None identified by authors. Small cohort and no blinding identified \\
\hline
\end{tabular}




\begin{tabular}{|c|c|c|c|c|}
\hline Author & Year & Cohort & Methodology & Limitations \\
\hline Tidhar et al. & 2015 & 5 BCRL & Diagnostic Test Study & $\begin{array}{l}\text { Study design - study conducted as part of a conference day, thus not all therapists could measure } \\
\text { all the patients. Results of the inter-rater reliability of the arm measurements raised another } \\
\text { limitation as only } 56 \% \text { of the therapists measured within the limit of } \pm 5 \% \text { from the true mean. }\end{array}$ \\
\hline Cau et al. & 2016 & 12 healthy & Diagnostic Test Study & Small cohort. No blinding identified \\
\hline Hameeteman et al. & 2016 & $11 \mathrm{BCRL}$ & Diagnostic Test Study & $\begin{array}{l}\text { No ethics approval or consent gained reported. Lack of blinding causing potential diagnostic } \\
\text { review bias }\end{array}$ \\
\hline Hoevenaren et al. & 2016 & 27 mixed & Diagnostic Test Study & $\begin{array}{l}\text { None identified by authors. No ethics approval or consent gained reported. Relatively small cohort } \\
\text { and no blinding identified }\end{array}$ \\
\hline Yamamoto et al. & 2016 & 35 healthy & Diagnostic Test Study & $\begin{array}{l}\text { Relatively small cohort. All participants were Japanese females. Tape measure method, as opposed } \\
\text { to water displacement, was used for volumetry }\end{array}$ \\
\hline Peleg \& Katz-Leurer & 2017 & 30 mixed & Case-control study & $\begin{array}{l}\text { No ethics approval or consent gained reported. Limitations are related to selection bias, external } \\
\text { validity, the measurement tool and research manipulation }\end{array}$ \\
\hline Verhulst et al. & 2017 & 10 healthy & Diagnostic Test Study & None identified by authors. Small cohort and only healthy participants included \\
\hline Wang et al. & 2017 & 484 healthy & Diagnostic Test Study & $\begin{array}{l}\text { Normative 'absolute' thresholds do not identify all patients with lymphoedema in a Chinese } \\
\text { population. Limitations for absolute diagnostic thresholds for diagnosing lymphoedema was } \\
\text { recognised in the development of criteria for bio-impedance spectroscopy. }\end{array}$ \\
\hline Yamamoto et al. & 2017 & 54 healthy & Diagnostic Test Study & $\begin{array}{l}\text { No consent reported. Volumetry was determined by tape measurement and not the gold standard of } \\
\text { water volumetry. Only Japanese women were included in the cohort; thus results may differ for } \\
\text { men and other ethnic populations }\end{array}$ \\
\hline Batista et al. & 2018 & 91 mixed & Diagnostic Test Study & $\begin{array}{l}\text { Measurements were taken at } 10 \mathrm{~cm} \text { intervals, and measurements made only once on each subject. } \\
\text { This would justify the large random variability observed between methods. Cohort mainly people } \\
\text { without lymphoedema, which may result in a higher disagreement between the methods }\end{array}$ \\
\hline Cau et al. & 2018 & $200 \mathrm{BCRL}$ & $\begin{array}{l}\text { Prospective Cohort } \\
\text { Study }\end{array}$ & None identified by authors. No blinding identified \\
\hline Karakashian et al. & 2018 & 31 mixed & Diagnostic Test Study & $\begin{array}{l}\text { None identified by authors. Relatively small cohort; service evaluation, not a research study. No } \\
\text { protocol given for CTM as the comparator }\end{array}$ \\
\hline Landau et al. & 2018 & $11 \mathrm{BCRL}$ & Diagnostic Test Study & None identified by authors. No ethics approval reported. Very small cohort \\
\hline Preuß et al. & 2018 & 37 BCRL & Diagnostic Test Study & None identified by authors \\
\hline Sharkey et al. & 2018 & 35 healthy & Diagnostic Test Study & $\begin{array}{l}\text { None identified by authors. No consent reported. No standardised protocol for measurements for } \\
\text { experts and students. Researchers did not time the methods of measurements but instead estimated } \\
\text { the time taken to perform the tests }\end{array}$ \\
\hline Sun et al. & 2018 & $287 \mathrm{BCRL}$ & Diagnostic Test Study & $\begin{array}{l}\text { Simulated circumferential measurements do not account for test-retest reliability and human error } \\
\text { of tape measurement. Exclusion of the hand is a fundamental limitation of current perometry } \\
\text { methods }\end{array}$ \\
\hline DeSnyder et al. & 2019 & $30 \mathrm{BCRL}$ & Diagnostic Test Study & $\begin{array}{l}\text { None identified by authors. No consent reported. Reporting of repeatability and reproducibility in } \\
\text { terms of percentage makes it difficult for comparison to other papers }\end{array}$ \\
\hline
\end{tabular}




\begin{tabular}{|c|c|c|c|c|}
\hline Author & Year & Cohort & Methodology & Limitations \\
\hline De Vrieze et al. & 2019 & $30 \mathrm{BCRL}$ & Cross-sectional Study & $\begin{array}{l}\text { Relatively small cohort. A lower limb optoelectric device was utilised; this was remedied through } \\
\text { a strict positioning protocol }\end{array}$ \\
\hline Karakashian et al. & 2019 & $23 \mathrm{BCRL}$ & Diagnostic Test Study & Garment properties and geometries not included in this work \\
\hline Lu \& Dixon & 2019 & 73 mixed & Diagnostic Test Study & None identified by authors. No ethics approval reported. No blinding identified \\
\hline Rafn et al. & 2019 & 41 mixed & Cross-sectional Study & $\begin{array}{l}\text { No ethics approval reported. Disproportion of educational accomplishments compared to general } \\
\text { population; thus, there is a suspected higher engagement in research by the cohort. Study was } \\
\text { performed in a metropolitan area of high socioeconomic status. All participants had access to } \\
\text { internet thus performance of the written instructions alone was not tested }\end{array}$ \\
\hline Spinelli et al. & 2019 & $71 \mathrm{BCRL}$ & $\begin{array}{l}\text { Blinded Repeat } \\
\text { Measures } \\
\text { Observational Study }\end{array}$ & $\begin{array}{l}\text { Expert therapists performed the measurements. Intra-rater estimates were based on one rater. } \\
\text { Unable to recruit } 24 \text { patients in each stage category. Single scores to capture reduction of } \\
\text { lymphoedema severity raises concerns as to its value for clinical or research purposes }\end{array}$ \\
\hline Binkley et al. & 2020 & 66 mixed & $\begin{array}{l}\text { Cross-sectional } \\
\text { Reliability and } \\
\text { Validity Study }\end{array}$ & $\begin{array}{l}\text { Sample size not based on a formal sample size calculation. LymphaTech included both inter- and } \\
\text { intra-rater variance; perometer only measured intra-rater variance. }\end{array}$ \\
\hline
\end{tabular}

Key:

BCRL $=$ Breast Cancer-Related Lymphoedema 


\subsubsection{Cohorts}

Large-scale studies are a challenge where the epidemiology of upper limb lymphoedema is dependent upon one condition, i.e. the consequences of breast cancer treatment. Based on the Central Limit Theorem, at least 30 participants should be recruited for a study (Field, 2013). The cohort or sample size for the studies varied immensely from six to 677, some with healthy participants only, and others with a good mix of healthy participants and BCRL. The majority of studies had good-sized cohorts of 30 or more participants, while 14 studies recruited less than the recommended 30. Most studies focused on BCRL participants $(n=19)$, while 13 studies utilised healthy participants only, and 12 studies had a mixture of BCRL and healthy participants.

\subsubsection{Robustness of Papers}

Nineteen studies did not state whether consent or ethical approval was gained before embarking on the research (Table 11). Seven papers reported their results in a scientific format, but the abstract was a narrative (Dylke et al., 2012; Lu et al., 2013; Lu et al., 2014; Cau et al., 2016; Hoevenaren et al., 2016; Wang et al., 2017; Cau et al., 2018).

The following three sections will describe the procedure and analyse the outcomes of the three chosen methods of measurement, namely CTM, perometry and 3DCI.

\subsubsection{Circumferential Tape Measurement (CTM)}

Of the 43 papers reviewed, 23 studies were identified for CTM. Of the papers critiqued using CASP, five papers scored 23 or 24 (out of 24), these were De Vrieze et al. (2019), Rafn, McNeely, Camp, Midtgaard, \& Campbell (2019), Devoogdt et al. (2010), Chen, Tsai, Hung, \& Tsauo (2008) and Tewari, Gill, Bochner, \& Kollias (2008). Table 12 provides a summary of the findings from each of the critiqued papers relating to CTM.

Within the clinical setting, the most common technique for determining limb volume is CTM. CTM is a method of limb volume measurement whereby the circumference of the limb is measured at set intervals along the arm from the wrist to the axilla (armpit). The two more commonly used methods are 4cm-CTM and 5-point CTM (Table 13) where $4 \mathrm{~cm}-\mathrm{CTM}$ measures the arm at $4 \mathrm{~cm}$ intervals from the wrist to the axilla. The 5 point CTM method measures five set points up the arm, i.e. the wrist, widest part of the forearm, the elbow, widest part of the upper arm and the point nearest the axilla. 
Table 12: Papers accessed on circumferential tape measurements (CTM)

\begin{tabular}{|c|c|c|c|c|c|}
\hline $\begin{array}{l}\text { Author } \\
\text { (CASP score) }\end{array}$ & Year & Cohort & $\begin{array}{l}\text { Research } \\
\text { Design }\end{array}$ & Method & Primary Results \\
\hline $\begin{array}{l}\text { De Vrieze et } \\
\text { al. } \\
(24)\end{array}$ & 2019 & $\begin{array}{l}30 \text { women } \\
\text { with BCRL }\end{array}$ & $\begin{array}{l}\text { Cross-sectional } \\
\text { Study }\end{array}$ & $\begin{array}{l}\text { Compared WD to 5-point CTM and perometry } \\
\text { to establish which was the more reliable, time- } \\
\text { efficient and clinically feasible method of } \\
\text { measurement to use }\end{array}$ & $\begin{array}{l}\text { 5-point CTM was the best measurement method in terms of reliability (intra- and } \\
\text { inter-rater reliability was } 0.987 \text { and } 0.984 \text { respectively), low error rate, low cost, } \\
\text { few limitations and time spent ( } 264.13 \text { seconds) }\end{array}$ \\
\hline $\begin{array}{l}\text { Rafn et al. } \\
\text { (23) }\end{array}$ & 2019 & $\begin{array}{l}20 \text { with and } \\
21 \text { without } \\
\text { BCRL }\end{array}$ & $\begin{array}{l}\text { Cross-sectional } \\
\text { Reliability and } \\
\text { Validity Study }\end{array}$ & $\begin{array}{l}\text { Self-measurement using a tape measure by a } \\
\text { participant at home and in a laboratory is } \\
\text { compared to } 10 \mathrm{~cm}-\mathrm{CTM} \text { by a therapist and } \\
\text { perometry }\end{array}$ & $\begin{array}{l}\text { High to excellent intra-rater reliability between non-BCRL group and therapis } \\
10 \mathrm{~cm}-\mathrm{CTM} \text { (ICC } \geq 0.88 \text { ), and excellent for BCRL group }(\mathrm{ICC}>0.91) \text {. A near- } \\
\text { perfect correlation between BCRL group and perometry }(r=0.98, p<0.001) \text {, and } \\
\text { those without BCRL }(r=0.95, p<0.001)\end{array}$ \\
\hline $\begin{array}{l}\text { Batista et al. } \\
(21)\end{array}$ & 2018 & $\begin{array}{l}46 \text { at risk } \\
45 \text { healthy }\end{array}$ & $\begin{array}{l}\text { Diagnostic Test } \\
\text { Study }\end{array}$ & $\begin{array}{l}10 \mathrm{~cm}-\mathrm{CTM} \text { was compared to perometry to } \\
\text { determine the LOA }\end{array}$ & $\begin{array}{l}\text { Pearson's correlation coefficient for volume, VD and \%VD were } 0.99,0.88 \text { and } \\
0.86 \text {, respectively. The LOA for volume ranged from }-202 \text { to } 181 \mathrm{ml} \text {, for VD it } \\
\text { ranged from }-101 \text { to } 141 \mathrm{ml} \text { and } \% \text { VD ranged from }-5 \text { to } 6.8 \%\end{array}$ \\
\hline $\begin{array}{l}\text { Cau et al. } \\
(20)\end{array}$ & 2018 & $\begin{array}{l}200 \text { women } \\
\text { with BCRL }\end{array}$ & $\begin{array}{l}\text { Prospective } \\
\text { Cohort Study }\end{array}$ & $\begin{array}{l}\text { Circumferential and volume parameters were } \\
\text { defined to compare CTM to 3DCI. } \\
\text { Camera = Laser Scanner 3D system (LS3D) }\end{array}$ & $\begin{array}{l}\text { Good correlation between CTM and 3DCI on affected }\left(\mathrm{R}^{2}=0.738 ; \mathrm{p}<0.05\right) \text { and } \\
\text { unaffected side }\left(\mathrm{R}^{2}=0.762 ; \mathrm{p}<0.05\right) \text {. Statistical difference between } \mathrm{CTM} \text { and } 3 \mathrm{DCl} \\
\text { for circumference and volume on the affected side, and for circumference on the } \\
\text { unaffected side; interestingly, total volumes were identical on the unaffected side }\end{array}$ \\
\hline $\begin{array}{l}\text { Landau et al. } \\
\text { (22) }\end{array}$ & 2018 & $\begin{array}{l}11 \text { women } \\
\text { with BCRL }\end{array}$ & $\begin{array}{l}\text { Diagnostic Test } \\
\text { Study }\end{array}$ & $\begin{array}{l}\text { Arm 3D image captured with volumes analysed } \\
\text { by Mirror software. } 4 \mathrm{~cm}-\mathrm{CTM} \text { measured from } \\
\text { ulnar styloid to upper arm mark, and WD } \\
\text { measured to upper arm mark. All methods were } \\
\text { timed. Camera = Vectra 3D imaging system }\end{array}$ & $\begin{array}{l}\text { 3DCI correlated well with } 4 \mathrm{~cm}-\mathrm{CTM}\left(\mathrm{R}^{2}=0.991\right) \text { and had good agreement with } \\
\text { mean \% VD of } 2.8 \%(\mathrm{SD} 2.0) \text {. 3DCI and WD correlated well }\left(\mathrm{R}^{2}=0.987\right) \text { with good } \\
\text { agreement of } \% \mathrm{VD} \text { at } 2.0 \%(\mathrm{SD} 2.1) \text {. } \\
\text { Time efficiency indicated 3DCI needed } 9.8 \text { min less than } 4 \mathrm{~cm}-\mathrm{CTM} \text { to perform, } \\
\text { and } 19.0 \text { min less than WD. }\end{array}$ \\
\hline $\begin{array}{l}\text { Sharkey et al. } \\
\text { (18) }\end{array}$ & 2018 & $\begin{array}{c}35 \\
\text { volunteers }\end{array}$ & $\begin{array}{l}\text { Diagnostic Test } \\
\text { Study }\end{array}$ & $\begin{array}{l}\text { Assessing intra-and inter-rater reliability of } \\
4 \mathrm{~cm}-\mathrm{CTM} \text { and perometry to determine the } \\
\text { accuracy of CTM compared to perometry when } \\
\text { measured by experts versus students }\end{array}$ & $\begin{array}{l}\text { Experts had lower SEM than students }(25 \mathrm{ml} \text { vs } 29.55 \mathrm{ml}) \text { for } 4 \mathrm{~cm}-\mathrm{CTM} \text {. Moderate } \\
\text { intra-rater reliability with students (Cronbach's alpha }=0.6755) \text { but excellent for } \\
\text { expert group (Cronbach's alpha }=0.9947)\end{array}$ \\
\hline $\begin{array}{l}\text { Peleg \& } \\
\text { Katz-Leurer } \\
(22)\end{array}$ & 2017 & $\begin{array}{l}14 \text { BCRL } \\
16 \text { healthy }\end{array}$ & $\begin{array}{l}\text { Case-control } \\
\text { study }\end{array}$ & $\begin{array}{l}\text { CTM measurements are compared with the arm } \\
\text { in horizontal versus vertical position }\end{array}$ & $\begin{array}{l}\text { Intra-rater reliability for both groups was excellent }(\mathrm{ICC} \geq 0.997) \text {. A non-significan } \\
\text { interaction effect of arm size, position (horizontal vs vertical) and group was noted } \\
\left(\mathrm{F}_{1,28}=3.30, \mathrm{p}=0.08\right) \text {. A significant interaction was noted for arm volume between } \\
\text { positions and groups }\left(\mathrm{F}_{1,28}=4.42, \mathrm{p}=0.04\right)\end{array}$ \\
\hline $\begin{array}{l}\text { Wang et al. } \\
\text { (18) }\end{array}$ & 2017 & $\begin{array}{l}484 \text { healthy } \\
\text { women }\end{array}$ & $\begin{array}{l}\text { Diagnostic Test } \\
\quad \text { Study }\end{array}$ & $\begin{array}{l}\text { To determine whether the normative-based } \\
\text { thresholds by } 10 \mathrm{~cm}-\mathrm{CTM} \text { for the detection of } \\
\text { lymphoedema differed for a Chinese population }\end{array}$ & $\begin{array}{l}\text { Dominant arm circumferences were significantly larger than the non-dominan } \\
\text { arm }\left(\mathrm{F}_{1,4796}=9.2, \mathrm{p}=0.002\right) \text {. Dominant limb volume larger than non-dominan } \\
\left(\mathrm{F}_{1,3840}=6.6, \mathrm{p}=0.01\right) \text { and increased along length of limb }\left(\mathrm{F}_{3,3820}=2598.2, \mathrm{p}<0.001\right) \\
\text { no interaction identified. Age, body weight and BMI did not affect circumference } \\
\text { or volume. Cut-off thresholds were determined at Two Standard Deviations (2SD) }\end{array}$ \\
\hline $\begin{array}{l}\text { Yamamoto et } \\
\text { al. } \\
\text { (17) }\end{array}$ & 2017 & $\begin{array}{l}53 \text { healthy; } \\
1 \text { bilateral } \\
\text { BCRL }\end{array}$ & $\begin{array}{l}\text { Diagnostic Test } \\
\text { Study }\end{array}$ & $\begin{array}{l}\text { Development of a new mathematical model for } \\
\text { determining Localised Arm Volume Index by } \\
\text { using segmental CTM volumes }\end{array}$ & $\begin{array}{l}\text { Intra- and inter-rater reliability were all very high for all segments }(r>0.98) \\
\text { Differences were observed for different BMI groups with higher BMI having } \\
\text { statistically significantly higher volumes }(\mathrm{p}<0.001)\end{array}$ \\
\hline
\end{tabular}




\begin{tabular}{|c|c|c|c|c|c|}
\hline $\begin{array}{l}\text { Author } \\
\text { (CASP score) }\end{array}$ & Year & Cohort & $\begin{array}{l}\text { Research } \\
\text { Design }\end{array}$ & Method & Primary Results \\
\hline $\begin{array}{l}\text { Cau et al. } \\
(21)\end{array}$ & 2016 & $\begin{array}{l}12 \text { healthy } \\
\text { adults }\end{array}$ & $\begin{array}{l}\text { Diagnostic Test } \\
\text { Study }\end{array}$ & $\begin{array}{l}\text { Comparison of reliability and correlation } \\
\text { between CTM and 3DCI. One arm was } \\
\text { measured twice by each of the two raters for } \\
\text { both } 4 \mathrm{~cm}-\mathrm{CTM} \text { and 3DCI. }\end{array}$ & $\begin{array}{l}\text { CTM has no significant difference in terms of intra-reliability with no significant } \\
\text { differences for calculated volume. Note no ICC values given. Pearson's correlation } \\
\text { coefficient between CTM and 3DCI was very high }\left(\mathrm{R}_{2}=0.923, \mathrm{p}<0.05\right) \text {; however, } \\
\text { circumference and volume differences were statistically significant between } \\
\text { measures with a difference of }-0.05 \mathrm{dm}^{3} \text { (no p-values provided) }\end{array}$ \\
\hline $\begin{array}{l}\text { Yamamoto et } \\
\text { al. } \\
(22)\end{array}$ & 2016 & $\begin{array}{l}35 \text { healthy } \\
\text { adults }\end{array}$ & $\begin{array}{l}\text { Diagnostic Test } \\
\text { Study }\end{array}$ & $\begin{array}{l}\text { Comparing arm volumetry by CTM with four } \\
\text { truncated cone volumes to a new Upper } \\
\text { Extremity LO (UEL) index according to BMI } \\
\text { groups }\end{array}$ & $\begin{array}{l}\text { There was a statistically significant difference in arm volume between BMI groups } \\
(\mathrm{p}<0.001) \text {; however there was no statistically significant difference in UEL index } \\
\text { between BMI groups }(\mathrm{P}>0.667)\end{array}$ \\
\hline $\begin{array}{l}\text { Mori et al. } \\
\text { (21) }\end{array}$ & 2015 & $\begin{array}{l}17 \mathrm{BCRL} \\
\text { women }\end{array}$ & $\begin{array}{l}\text { Diagnostic Test } \\
\text { Study }\end{array}$ & $\begin{array}{l}\text { Self-administered CTM was compared to WD } \\
\text { and 5-point CTM to determine its reliability } \\
\text { and validity }\end{array}$ & $\begin{array}{l}\text { Self-measure had excellent reliability with ICC of affected side ranging } 0.97 \text { to } \\
0.99 \text {, and for the unaffected side ranging } 0.96 \text { to } 0.99 \text {. Validity: Pearson's } \\
\text { correlation coefficient between self-measure and WD was } 0.59-0.68(\mathrm{p}<0.01) \text {, and } \\
\text { with 5-point CTM was } 0.88-0.95(\mathrm{p}<0.01) \text {. }\end{array}$ \\
\hline $\begin{array}{l}\text { Tidhar et al. } \\
\text { (18) }\end{array}$ & 2015 & $\begin{array}{l}41 \text { physio- } \\
\text { therapists; } \\
5 \text { patients }\end{array}$ & $\begin{array}{l}\text { Diagnostic Test } \\
\text { Study }\end{array}$ & $\begin{array}{l}\text { Experienced physiotherapists measured both } \\
\text { arms of one patient three times using } 5 \text {-point } \\
\text { CTM to determine the SEM and the reliability } \\
\text { of measurements between therapists }\end{array}$ & $\begin{array}{l}\text { SEM was } 27.5 \mathrm{ml}(\mathrm{CI} 20.5-34.4 \mathrm{ml}) \text { with scaled SEM at } 0.82 \% .83 \% \text { of therapists } \\
\text { achieved SEM of }<1 \%(\mathrm{p}=0.847) \text {. No statistically significant difference seen } \\
\text { between affected and unaffected side measurements }(\mathrm{p}=0.945)\end{array}$ \\
\hline $\begin{array}{l}\text { Öhberg et al. } \\
\text { (20) }\end{array}$ & 2014 & $\begin{array}{l}25 \text { people } \\
\text { with LO }\end{array}$ & $\begin{array}{l}\text { Diagnostic Test } \\
\quad \text { Study }\end{array}$ & $\begin{array}{l}\text { Circumference and volume of both arms was } \\
\text { measured by WD, } 4 \mathrm{~cm}-\mathrm{CTM} \text { and } 3 \mathrm{DCI} \text { by one } \\
\text { of two physiotherapists to evaluate the } \\
\text { performance of 3DCI against commonly used } \\
4 \mathrm{~cm}-\mathrm{CTM}\end{array}$ & $\begin{array}{l}4 \mathrm{~cm}-\mathrm{CTM} \text { underestimates volume compared to WD but was not statistically } \\
\text { significant }(\mathrm{CI}=-99.78-51.22, \mathrm{p}=0.512) \text {. No statistically significant difference was } \\
\text { found between the methods }(\mathrm{p}>0.05) \text {. A systematic bias between therapists was } \\
\text { observed }(\mathrm{p}=0.019)\end{array}$ \\
\hline $\begin{array}{l}\text { Adriaenssens } \\
\text { et al. } \\
\text { (22) }\end{array}$ & 2013 & $\begin{array}{l}80 \text { people: } \\
31 \text { healthy; } \\
49 \mathrm{BC}\end{array}$ & $\begin{array}{l}\text { Diagnostic Test } \\
\text { Study }\end{array}$ & $\begin{array}{l}\text { Both arms were measured three times by } \\
\text { perometer and compared to the commonly used } \\
\text { methods of WD and CTM }\end{array}$ & $\begin{array}{l}\text { CTM produced highest volume and WD lowest volume, with perometer volumes } \\
\text { in between. Volume of dominant arm was } 2.2 \%>\text { non-dominant arm }\end{array}$ \\
\hline $\begin{array}{l}\text { Asim et al. } \\
(22)\end{array}$ & 2012 & $\begin{array}{l}193 \mathrm{BC} \\
\text { women }\end{array}$ & $\begin{array}{l}\text { Diagnostic Test } \\
\text { Study }\end{array}$ & $\begin{array}{l}\text { Used CTM to determine the prevalence of LO } \\
\text { in the cohort. Different methods of CTM were } \\
\text { used ( } 3 \text {-point vs } 10 \mathrm{~cm} \text { ), and different thresholds } \\
\text { were considered }\end{array}$ & $\begin{array}{l}\text { LO was defined as } \geq 7.5 \% \text { increase in any circumference in at-risk arm - produced } \\
23.3 \% \text { prevalence, with sensitivity of } 83 \% \text { and specificity of } 81 \% \text {. For } \geq 10 \mathrm{~cm} \\
\text { increases, prevalence was } 12.9 \% \text {, with sensitivity of } 66 \% \text { and specificity of } 89 \% \text {. } \\
\text { For }>2 \mathrm{~cm} \text { increase, prevalence was } 25.3 \% \text {, sensitivity } 66 \% \text { and specificity } 80 \%\end{array}$ \\
\hline $\begin{array}{l}\text { Brorson \& } \\
\text { Hoijer } \\
(22)\end{array}$ & 2012 & $\begin{array}{l}10 \text { women } \\
\text { with } \\
\text { unilateral } \\
\text { BCRL }\end{array}$ & $\begin{array}{l}\text { Diagnostic Test } \\
\text { Study }\end{array}$ & $\begin{array}{l}\text { Compared } 4 \mathrm{~cm} \text { - and } 5 \text {-point CTM to WD to } \\
\text { determine their correlation }\end{array}$ & $\begin{array}{l}\text { Coefficient of variance for } 4 \mathrm{~cm} \mathrm{CTM}=0.628 \%, 5 \text {-point } \mathrm{CTM}=0.632 \% \text { and WD } \\
=0.609 \% \text {. Difference between } 4 \mathrm{~cm} \text { and } 5 \text {-point CTM volumes was not } \\
\text { significant. All methods had high correlation coefficients }(\mathrm{r}=0.813-0.915) \text { and high } \\
\text { regression coefficients }(\beta=0.863-1.089) \text {. Excess volume had no statistical } \\
\text { significance between CTM methods but was statistically significant with WD } \\
(\mathrm{p}<0.05)\end{array}$ \\
\hline
\end{tabular}




\begin{tabular}{|c|c|c|c|c|c|}
\hline $\begin{array}{l}\text { Author } \\
\text { (CASP score) }\end{array}$ & Year & Cohort & $\begin{array}{l}\text { Research } \\
\text { Design }\end{array}$ & Method & Primary Results \\
\hline $\begin{array}{l}\text { Katz-Leurer } \\
\text { \& Bracha } \\
(21)\end{array}$ & 2012 & $\begin{array}{l}16 \text { women } \\
\text { with arm } \\
\text { LO }\end{array}$ & $\begin{array}{c}\text { Diagnostic Test } \\
\text { Study }\end{array}$ & $\begin{array}{l}\text { 5-point CTM was measured twice, one week } \\
\text { apart. Compared test-retest reliability of } \\
\text { segmental volume to total limb volume }\end{array}$ & $\begin{array}{l}\text { Total VD of LO arm was } 7 \%>\text { unaffected arm }(\mathrm{p}<0.01) \text {; and for segmented VD } \\
\text { this was } 7-8 \%>\text { unaffected }(\mathrm{p}<0.01) \text {. Circumferential test-retest values were } \\
\text { ICC }=0.96-0.99 \text { in LO arm and ICC }=0.98-1.00 \text { for unaffected arm. Test-retest } \\
\text { reliability for volume was excellent for both unaffected (ICC }=0.99) \text { and LO arms } \\
\text { (ICC }>0.98) \text {. SEM increased in unaffected arm when using segmental limb volume } \\
\text { (47.1 to } 66.6 \mathrm{ml}) \text {, and decreased on LO arm }(78.8 \text { to } 45.0 \mathrm{ml})\end{array}$ \\
\hline $\begin{array}{l}\text { Foroughi et } \\
\text { al. } \\
(22)\end{array}$ & 2011 & $\begin{array}{l}57 \\
\text { community- } \\
\text { dwelling } \\
\text { adults aged } \\
>40 \text { years }\end{array}$ & $\begin{array}{l}\text { Cross-sectional } \\
\text { study }\end{array}$ & $\begin{array}{l}\text { Inter-rater reliability testing of a home-CTM } \\
\text { protocol versus therapist CTM and perometry } \\
\text { within two weeks }\end{array}$ & $\begin{array}{l}\text { Moderate to high concordance between home-CTM and therapist-CTM }\left(\mathrm{r}_{\mathrm{c}}=0.68-\right. \\
0.93) \text {, and high concordance between therapist-CTM and perometry }\left(\mathrm{r}_{\mathrm{c}}=0.83-\right. \\
0.94) \text {. LOA varied dependent on the measurement location with a bias range of - } \\
5.5 \% \text { to } 1.5 \% \text { therapist-CTM vs perometer, and }-2.4 \% \text { to } 4.0 \% \text { therapist-CTM vs } \\
\text { home-CTM }\end{array}$ \\
\hline $\begin{array}{l}\text { Czerniec et } \\
\text { al. } \\
(21)\end{array}$ & 2010 & $\begin{array}{l}51 \text { people: } \\
33 \text { with } \mathrm{LO} \\
18 \text { non-LO }\end{array}$ & $\begin{array}{c}\text { Diagnostic Test } \\
\text { Study }\end{array}$ & $\begin{array}{l}\text { Participants were assessed twice over four } \\
\text { weeks in which both arms were measured by } \\
10 \mathrm{~cm}-\mathrm{CTM} \text {, perometer and BIS to determine } \\
\text { the relationship between each method and the } \\
\text { SEM }\end{array}$ & $\begin{array}{l}\text { Strongest relationship was for limb VD between } 10 \mathrm{~cm}-C T M \text { and perometer } \\
\left(\mathrm{r}_{\mathrm{c}}=0.99\right) \text {, and weakest between BIS and } 10 \mathrm{~cm}-\mathrm{CTM}\left(\mathrm{r}_{\mathrm{c}}=0.89\right) \text {. LOA showed } 10 \\
\mathrm{~cm}-\mathrm{CTM} \text { to underestimate limb volume compared to perometer. All } 3 \text { methods } \\
\text { had excellent intra-rater reliability (ICC CTM }=0.96 \text {; perometer }=0.99 \text {; BIS }=0.96) \text {. } \\
\text { SEM for } 10 \mathrm{~cm}-\mathrm{CTM} \text { was } 85 \mathrm{ml} \text {; perometer } 43 \mathrm{ml} \text {; and BIS ratio } 0.05\end{array}$ \\
\hline $\begin{array}{l}\text { Devoogdt et } \\
\text { al. } \\
(23)\end{array}$ & 2010 & $\begin{array}{l}112 \mathrm{BC} \\
\text { patients }\end{array}$ & $\begin{array}{c}\text { Diagnostic Test } \\
\text { Study }\end{array}$ & $\begin{array}{l}\text { The unaffected arm of patients was measured } \\
\text { twice over nine days by } 3 \text { therapists who were } \\
\text { blinded to each other's results. A new } 4 \mathrm{~cm} \text { - } \\
\text { CTM device was compared to WD to determine } \\
\text { its reliability and validity }\end{array}$ & $\begin{array}{l}\text { Circumference measurements demonstrated intra-rater reliability ICC ranging } \\
\text { from } 0.977-0.996 \text { and inter-rater reliability ICC ranging from } 0.942-0.994 \text {. Arm } \\
\text { volume intra-rater ICC was } 0.997 \text {, and inter-rater ICC was 0.994. Circumference } \\
\text { measurement and calculated volume intra-and inter-rater SEMs were low (range } \\
0.8 \%-2.0 \% \text { ). High correlation between CTM and WD ( } \mathrm{r}=0.973 \text { ). SRD noted at an } \\
\text { increase of } \geq 1 \mathrm{~cm} \text { in circumference, and } \geq 55 \mathrm{ml} \text { of arm volume was clinically } \\
\text { significant }\end{array}$ \\
\hline $\begin{array}{l}\text { Chen et al. } \\
(23)\end{array}$ & 2008 & $\begin{array}{c}14 \text { people } \\
\text { with BCRL }\end{array}$ & $\begin{array}{c}\text { Diagnostic Test } \\
\text { Study }\end{array}$ & $\begin{array}{l}\text { Investigated and compared the reliability of } \\
\text { WD and 3-point CTM by two blinded therapists } \\
\text { by taking two measurements } 10 \text { min apart }\end{array}$ & $\begin{array}{l}\text { Excellent intra-and inter-rater reliability observed between methods (ICCs for both } \\
\text { were }>0.99, \mathrm{p}<0.05 \text { ). SEMs for WD was } 27.3 \text {, and SEMs for CTM was low, } \\
\text { ranging from } 0.13-0.37 \text {. SRD for WD was } 75.6 \text {, and for CTM it ranged from } 0.37 \\
\text { to } 1.02\end{array}$ \\
\hline $\begin{array}{l}\text { Tewari et al. } \\
\text { (23) }\end{array}$ & 2008 & 87 women & $\begin{array}{l}\text { Diagnostic Test } \\
\text { Study }\end{array}$ & $\begin{array}{l}\text { Compared } 10 \mathrm{~cm}-\mathrm{CTM} \text {, using a narrow and } \\
\text { broad tape measure, to WD to assess for } \\
\text { accuracy }\end{array}$ & $\begin{array}{l}\text { Significant correlation in volume estimations between narrow and wide tape CTM } \\
(\mathrm{r}=0.95, \mathrm{p}<0.0001) \text {. Narrow tape CTM vs WD had Pearson's correlation } \\
\text { coefficient of } 0.92(\mathrm{p}<0.0001) \text {; and wide tape CTM vs WD was } 0.88(\mathrm{p}<0.0001)\end{array}$ \\
\hline
\end{tabular}

Key:

3DCI $=$ Three-Dimensional Camera Imaging BCRL $=$ Breast Cancer-Related LO

CTM = Circumferential Tape Measurement $\mathrm{SD}=$ Standard Deviation

UEL = Upper Extremity Lymphoedema
$\% \mathrm{VD}=$ Percentage Volume Difference BIS = Bio-impedance Spectroscopy ICC $=$ Intra-class Correlation Coefficient SDC $=$ Smallest Detectable Change TDC $=$ Tissue Dielectric Constant
AUC $=$ Area Under Curve

$\mathrm{BMI}=$ Body Mass Index

LO = Lymphoedema

SEM = Standard Error of Measurement

$\mathrm{VD}=$ Volume Difference
$\mathrm{BC}=$ Breast Cancer

$\mathrm{CI}=$ Confidence Interval

LOA = Limits of Agreement

$\mathrm{SRD}=$ Smallest Real Difference

$\mathrm{WD}=$ Water Displacement 
CTM is poorly standardised in both research and clinical practice, with taught methods, access to volumetric calculation programmes, and personal choice influencing this continued practice (Chen et al., 2008; Peleg \& Katz-Leurer, 2017). The lack of standardisation presents challenges in comparing findings and reduces the repeatability of the outcomes for continued monitoring of a patient if different methods of CTM are used between appointments. Variables upon which CTM should be performed and interpreted include 1) patient position [the position of the patient's arm in relation to their body]; 2) measurement points [first mark and points along the arm being measured]; 3) tape measure [type and method of application]; and 4) the mathematical formula used for volume calculation.

Patient positioning was mentioned in eight studies, with six of them using a seated position (Table 13). Eleven studies report positioning the arm in a horizontal position, with one study (Peleg \& Katz-Leurer, 2017) comparing the vertical (arm down by the side) position to the horizontal. This study concluded that the vertical position in healthy participants significantly increased limb volume compared to the horizontal position in the healthy cohort; however, this was not replicated in the lymphoedema cohort (Peleg $\&$ Katz-Leurer, 2017). The positioning of the forearm was documented in seven of these studies with five pronating (palm down) the forearm (Czerniec et al., 2010; Katz-Leurer \& Bracha, 2012; Peleg \& Katz-Leurer, 2017; Wang et al., 2017; Batista et al., 2018), while the other two studies had the participants supinating (palm up) their forearm (Cau et al., 2016; Cau et al., 2018). Incidentally, these last two studies were performed by the same researchers; hence there is insufficient evidence to recommend supination of the forearm. Thus, based on the evidence from the eight studies, the most commonly used posture for standardisation would be for both arms held in the horizontal position and forearms pronated.

The 'first mark' on the limb determines the reference point from which arm volume measurements commence. The first mark was identified in 16 studies with the majority opting for the 'wrist' as their identifying landmark from which the arm measurements were made to ensure repeatability on the opposite arm and between sessions (Table 13). The wrist was defined as the crease created when the hand is extended (lifted off the table), thus reducing variation between users. The second most common first mark used was the Ulnar Styloid process, mentioned in five studies (Table 13). Research 
Table 13: Variables for consideration when using CTM

\begin{tabular}{|c|c|c|c|c|c|c|c|c|c|c|c|c|c|c|c|c|c|c|c|c|c|c|}
\hline \multirow[b]{2}{*}{ Study Reference } & \multirow[b]{2}{*}{ Year } & \multicolumn{2}{|c|}{$\begin{array}{c}\text { Body } \\
\text { Position } \\
\end{array}$} & \multicolumn{2}{|c|}{ Arm Position } & \multicolumn{2}{|c|}{\begin{tabular}{l|} 
Forearm \\
Position \\
\end{tabular}} & \multicolumn{2}{|c|}{$\begin{array}{c}\text { Tool Used for } \\
\text { Marking }\end{array}$} & \multicolumn{5}{|c|}{ First Mark } & \multicolumn{6}{|c|}{ Points Measured on Arm } & \multicolumn{2}{|c|}{ Formulae Used } \\
\hline & & 兽 & 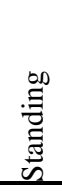 & 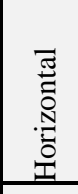 & 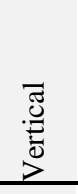 & 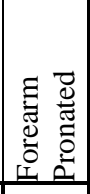 & 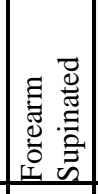 & 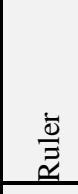 & 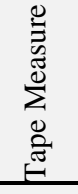 & ஜ̈ & $\frac{\vec{n}}{3}$ & 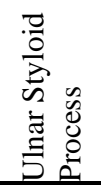 & 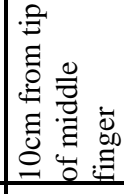 & 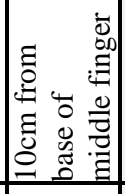 & 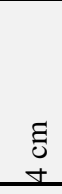 & $\begin{array}{l}\tilde{0} \\
\stackrel{0}{0} \\
\end{array}$ & 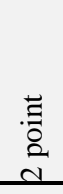 & 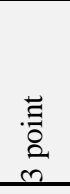 & 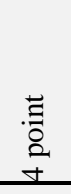 & 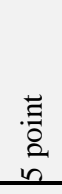 & 吾曾 & 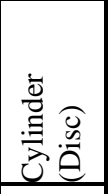 \\
\hline De Vrieze et al. & 2019 & & & & & & & & & & & & & & & & & & & $\sqrt{ }$ & $\sqrt{ }$ & \\
\hline Rafn et al. & 2019 & $\sqrt{ }$ & & & & & & & & & $\sqrt{ }$ & & & & & $\sqrt{ }$ & & & & & $\sqrt{ }$ & $\sqrt{ }$ \\
\hline Batista et al. & 2018 & & & & & $\sqrt{ }$ & & & & & $\sqrt{ }$ & & & & & $\sqrt{ }$ & & & & & & $\sqrt{ }$ \\
\hline Cau et al. & 2018 & & $\sqrt{ }$ & $\sqrt{ }$ & & & $\sqrt{ }$ & & & & & $\sqrt{ }$ & & & $\sqrt{ }$ & & & & & $\sqrt{ }$ & $\sqrt{ }$ & \\
\hline Landau et al. & 2018 & & & & & & & & & & & $\sqrt{ }$ & & & $\sqrt{ }$ & & & & & & $\sqrt{ }$ & \\
\hline Sharkey et al. & 2018 & & & $\sqrt{ }$ & & & & & & & & & & & $\sqrt{ }$ & & & & & & $\sqrt{ }$ & \\
\hline \begin{tabular}{|l|} 
Peleg \& Katz- \\
Leurer \\
\end{tabular} & 2017 & & & $\sqrt{ }$ & $\sqrt{ }$ & $\sqrt{ }$ & & & $\sqrt{ }$ & & $\sqrt{ }$ & & & & & & & & & & $\sqrt{ }$ & \\
\hline Wang et al. & 2017 & $\sqrt{ }$ & & $\sqrt{ }$ & & $\sqrt{ }$ & & & $\sqrt{ }$ & & & $\sqrt{ }$ & & & & $\sqrt{ }$ & & & & & & $\sqrt{ }$ \\
\hline Yamamoto et al. & 2017 & & & & & & & & & & & & & & & & & & $\sqrt{ }$ & $\sqrt{ }$ & $\sqrt{ }$ & \\
\hline Cau et al. & 2016 & & $\sqrt{ }$ & $\sqrt{ }$ & & & $\sqrt{ }$ & & & & $\sqrt{ }$ & & & & $\sqrt{ }$ & & & & & $\sqrt{ }$ & $\sqrt{ }$ & \\
\hline Yamamoto et al. & 2016 & & & & & & & & & & & & & & & & & & $\sqrt{ }$ & & $\sqrt{ }$ & \\
\hline Mori et al. & 2015 & & & & & & & & & & & & & & & & & & & & $\sqrt{ }$ & \\
\hline Tidhar et al. & 2015 & & & & & & & & & & & & & & & & & & & $\sqrt{ }$ & $\sqrt{ }$ & \\
\hline Öhberg et al. & 2014 & $\sqrt{ }$ & & $\sqrt{ }$ & & & & $\sqrt{ }$ & & & $\sqrt{ }$ & & & & $\sqrt{ }$ & & & & & & $\sqrt{ }$ & $\sqrt{ }$ \\
\hline Adriaenssens et al. & 2013 & $\sqrt{ }$ & & $\sqrt{ }$ & & & & & $\sqrt{ }$ & & & $\sqrt{ }$ & & & $\sqrt{ }$ & & & & & $\sqrt{ }$ & $\sqrt{ }$ & \\
\hline
\end{tabular}




\begin{tabular}{|c|c|c|c|c|c|c|c|c|c|c|c|c|c|c|c|c|c|c|c|c|c|c|}
\hline \multirow[b]{2}{*}{ Study Reference } & \multirow[b]{2}{*}{ Year } & \multicolumn{2}{|c|}{$\begin{array}{c}\text { Body } \\
\text { Position }\end{array}$} & \multicolumn{2}{|c|}{ Arm Position } & \multicolumn{2}{|c|}{$\begin{array}{l}\text { Forearm } \\
\text { Position }\end{array}$} & \multicolumn{2}{|c|}{$\begin{array}{l}\text { Tool Used for } \\
\text { Marking }\end{array}$} & \multicolumn{5}{|c|}{ First Mark } & \multicolumn{6}{|c|}{ Points Measured on Arm } & \multicolumn{2}{|c|}{ Formulae Used } \\
\hline & & 营 & 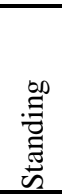 & 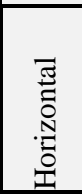 & $\begin{array}{l}\widetilde{0} \\
\stackrel{0}{0} \\
\end{array}$ & 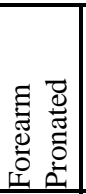 & 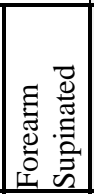 & $\frac{\bar{v}}{\overline{\underline{\theta}}}$ & 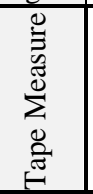 & $\stackrel{0}{z}$ & 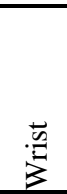 & 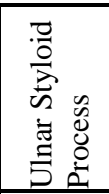 & 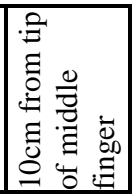 & 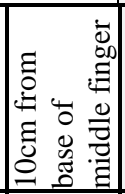 & $\begin{array}{l}\varepsilon \\
\vdots \\
+ \\
\end{array}$ & $\begin{array}{l}\tilde{J} \\
\varrho \\
\end{array}$ & $\begin{array}{l}\overrightarrow{.} \\
\text { 蒿 } \\
\text { c. }\end{array}$ & 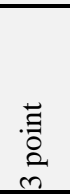 & 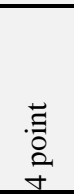 & 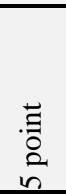 & 吾总 & 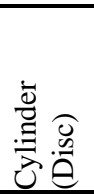 \\
\hline Asim et al. & 2012 & & & & & & & & & & & & & $\sqrt{ }$ & & $\sqrt{ }$ & & $\sqrt{ }$ & & & & \\
\hline Brorson \& Höijer & 2012 & & & & & & & & & & $\sqrt{ }$ & & & & $\sqrt{ }$ & & & & & & & \\
\hline $\begin{array}{l}\text { Katz-Leurer \& } \\
\text { Bracha }\end{array}$ & 2012 & $\sqrt{ }$ & & $\sqrt{ }$ & & $\sqrt{ }$ & & & & & $\sqrt{ }$ & & & & & & & & & & $\sqrt{ }$ & \\
\hline Foroughi et al. & 2011 & $\sqrt{ }$ & & $\sqrt{ }$ & & & & & & & $\sqrt{ }$ & & & & & & & & & & $\sqrt{ }$ & \\
\hline Czerniec et al. & 2010 & & & $\sqrt{ }$ & & $\sqrt{ }$ & & & & & & $\sqrt{ }$ & & & & $\sqrt{ }$ & & & & & $\sqrt{ }$ & \\
\hline Chen et al. & 2008 & & & $\sqrt{ }$ & & & & & & $\sqrt{ }$ & & & & & & & & $\sqrt{ }$ & & & $\sqrt{ }$ & \\
\hline Tewari et al. & 2008 & & & & & & & & $\sqrt{ }$ & & & & $\sqrt{ }$ & & & $\sqrt{ }$ & & & & & $\sqrt{ }$ & \\
\hline
\end{tabular}


demonstrates that all first marks are highly valid and reliable (Tewari et al., 2008) and that they should be used for the repeatability of CTM measures between sessions.

Brorson \& Hoijer's (2012) study comparing 4cm-CTM versus the 5-point method found that there was no statistically significant difference between these two methods of CTM. The advantage of the 4cm-CTM method is that it is more sensitive in detecting limb volume change and distortion in limb shape as it measures between 10 and 12 points along the arm compared to the 5-point method. Table 13 identifies six different points of measurement used to measure the arms using CTM.

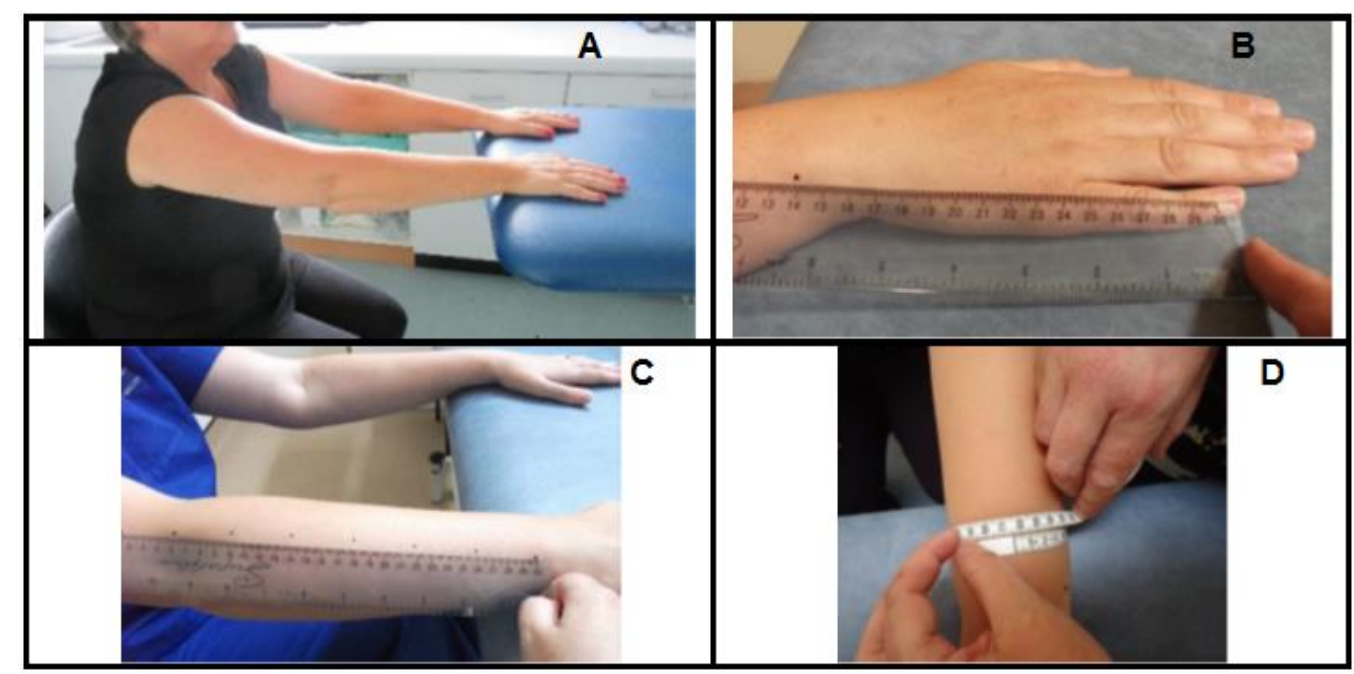

Figure 11: 4cm-CTM Guidance (LNW, 2017). Participant rests hands palm down on a table, arms straight at shoulder height (A). The 'first mark' is measured from the base of the little finger to above the ulnar styloid process (B). Measurements are taken every 4 $\mathrm{cm}$ along the outer arm to about $2 \mathrm{~cm}$ below the axilla crease (C). The tape measure is placed snugly around the limb to overlap the mark in the 'middle' (D).

Five studies mention the tool used to mark the participant's arms for taking circumferential measurements (Table 13). Öhberg, Zachrisson, \& Holmner-Rocklöv (2014) used a ruler to mark their participant's arms as they suggested that a tape measure would adhere to the irregular shape of the lymphoedematous arm thus distorting the actual distances between points (Figure 11). All other studies utilised a tape measure at tension to negate the distortion along the arm. When performing CTM, the type of tape measure used does not influence the reliability and validity of CTM (Tewari et al., 2008). Placement of the tape measure needs care to reduce errors such as applying the tape too tight, too loose, or by compressing tissue (Chen et al., 2008; Tewari et al., 2008; Asim et al., 2012). Research recommends placing the tape perpendicular to the limb with 
consistent tautness at each fixed point (Öhberg et al., 2014), or by placing the tape snugly around the arm without tightening the tape (Brorson \& Hoijer, 2012; Öhberg et al., 2014).

A further identifier of the lack of standardisation existent in CTM is that there are two types of calculation for limb volume, each based on the concept of the limb being defined as either a cylinder or truncated cone. As such, there are different methods for calculating limb volumes from the multiple circumferential measurements of the limb. 'Frustum' (also referred to as truncated cone) and 'Cylinder' (also referred to as disc method) volume methods are the more conventional geometric formulae used to calculate limb volume from circumferential measurements. From Table 13, it is evident that the most commonly used calculation in the literature is the frustum method, with 18 studies utilising this equation. Essentially, the total volume of geometry is subdivided into $n$ segments, and calculated by adding the volumes of each segment $i$, as shown in Figure 12. A benefit of this method of measurement is that the limb can be calculated in segments or as a whole (Katz-Leurer \& Bracha, 2012).

$C_{2}$

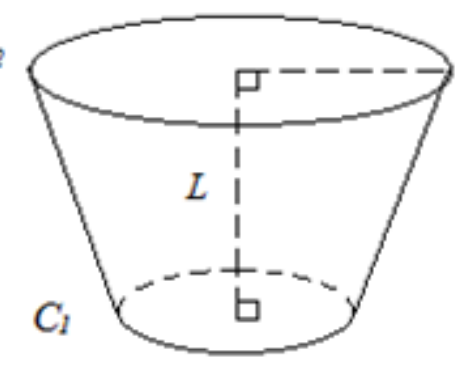

$V_{\text {frustrum }}=\frac{1}{12 \pi} \sum_{i=1}^{n} L\left(C_{1}^{2}+C_{1} C_{2}+C_{2}^{2}\right)$

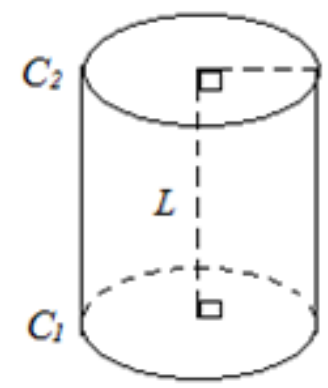

$V_{\text {cylinder }}=\frac{1}{4 \pi} L C_{1}^{2}$

Figure 12: Geometric and mathematical representations of limb volume formulae: Frustum segment (left) and Cylinder segment (right). C1 and C2 are the lower and upper circumferences of each segment, while $L$ is the length of each segment.

Influential work from Sitzia (1995), and later studies by Devoogdt et al. (2010), Foroughi et al. (2011) and Adriaenssens et al. (2013), demonstrates that the Cylinder method is inaccurate as it can overestimate limb volume by up to 5\%. Thus the frustum method is recommended as it assumes that the limb resembles a cone shape as opposed to a cylindrical shape. 
Arm dominance needs to be considered as the dominant limb generally measures $1.6 \%$ to $4.7 \%$ bigger than the non-dominant limb (Smoot et al., 2011; Wang et al., 2017). For this reason, it is ideal that pre-operative measurements are taken for those people having axillary node excision, as they will be at risk of developing lymphoedema. With this pre-operative measurement, the limb volume difference will be the baseline, and normal arm dominance will be accounted for (Smoot et al., 2014). However, as discussed in section 2.4 .3 (page 21), pre-operative measurements are rarely achieved in practice due to resource limitations on lymphoedema services.

Formative work by Casley-Smith (1994) demonstrated that patients (and therapists) are more likely to understand limb volume differences in terms of 'percentage of oedema' as opposed to 'altered percentage of normal' or 'altered percentage of the initial volume'. Within this paper, the recommended equation for presenting volume difference in unilateral limb lymphoedema is 'Difference in Oedema/Normal'; and for bilateral limb oedema, the equation is 'Difference in Volume/Initial Volume. The former method of calculation has been used in many research studies (Czerniec et al., 2010; Adriaenssens et al., 2013; Tidhar et al., 2015; Peleg \& Katz-Leurer, 2017; Wang et al., 2017) as it presents the least errors in calculation and appears to be the most meaningful way of expressing an alteration in the amount of lymphoedema (Casley-Smith, 1994). Absolute volume difference has also been used in research. It is calculated by subtracting the initial limb volume from the current limb volume of the same arm where a negative value indicates a reduction in limb volume (Casley-Smith, 1994). In clinical practice, patients have a better understanding of limb volume difference expressed as both a volume (cups or pints) and percentage of the lymphoedema arm compared to their 'normal' (unaffected) arm, thus supporting Casley-Smith's (1994) findings.

Newer models for determining the extent of lymphoedema and treatment outcomes are the 'Upper Extremity Lymphoedema Index' [UEL] and the 'Localised Arm Volume Index' [LAVI] (Wang et al., 2017; Sharkey et al., 2018). UEL is calculated by dividing the person's body mass index (BMI) into the summated squared circumferences, while LAVI is the percentage proportion of one localised UEL compared to the standard localised UEL. Both methods were found to be highly reproducible and were less affected by body physique compared with the frustum method, however, both studies were performed on healthy participants with no history of arm lymphoedema or breast 
cancer. The authors express that these methods would be transferable to a pathological cohort, i.e. BCRL, but to date, no study exists to confirm this. The calculations are not complex, but the points of measurement used are questionable as they measure the hand, wrist, elbow and $5 \mathrm{~cm}$ above and below the elbow. Therefore, a potential for error exists in the presentation of lymphoedema where localised swelling may be distal, or proximal, to the points measured.

De Vrieze et al. (2019) developed a list of 12 limitations regarding clinical feasibility which were scored for each method. Water displacement and CTM showed statistically significant differences in means against perometry $(\mathrm{p}<0.05)$. For time efficiency, perometry was the fastest in terms of set up time and execution of bilateral limb measurements with a mean time of 1:43 min. However, for clinical feasibility, perometry was rated second to CTM, resulting in an overall ranking of CTM as the highest, indicating that CTM is the most appropriate method of measurement for use in clinical practice.

\subsubsection{Thresholds for Diagnosing Lymphoedema Using CTM}

There exists a lack of consensus over diagnostic thresholds for the diagnosis of lymphoedema. Ten different thresholds were identified from the five studies that utilised volume measurement differences between arms in diagnosing lymphoedema (Table 14). The most commonly referenced methods were $\geq 10 \%$ volume difference in total upper limb volume, $\geq 200 \mathrm{ml}$ difference between arms, with the third commonly used threshold being $\geq 2 \mathrm{~cm}$ circumference difference at any point along the arm.

Asim et al. (2012) used CTM to determine the prevalence of lymphoedema in their breast cancer cohort $(n=193)$. Different methods of CTM were used (3-point vs $10 \mathrm{~cm}$ CTM), and different thresholds were considered. Interestingly, for $10 \mathrm{~cm}-\mathrm{CTM}$, thresholds chosen were $20 \%, 15 \%$ and $10 \%$, whilst for 3-point CTM the thresholds were $7.5 \%, 10 \%$ and $2 \mathrm{~cm}$ difference. The only discernible reason is that these thresholds were chosen to compare their study outcomes with the Sentinel Node Biopsy versus Axillary Clearance (SNAC) trial. Their results for 3-point CTM thresholds had the highest sensitivity for $\geq 7.5 \%$ increase in any circumference in the 'at-risk' arm at $83 \%$ (specificity was $81 \%$ ), and the highest specificity was $89 \%$ when using an increase of $\geq 10 \%$ threshold (sensitivity was $66 \%$ ). No sensitivity or specificity data was provided 
for the 10cm-CTM thresholds. No conclusion was given as to which method of CTM was more accurate, as they state that the protocol chose 3-point CTM from the International Breast Cancer Study Group Trial (IBCSG 10-93). They do however conclude that CTM is a simple 'office method' of screening for lymphoedema and that patient history and $\geq 10 \%$ increase in any circumference is optimal for determining lymphoedema as it has the highest specificity of the thresholds tested. However, with moderate sensitivity, this conclusion is debatable, as several false negatives will be missed, causing delays in treatment and risking complications such as fibrosis and the risk of cellulitis.

Table 14: Thresholds of measuring using CTM

\begin{tabular}{|c|c|c|c|c|c|c|c|c|c|c|c|}
\hline Author & Year & $\begin{array}{l}\text { Research } \\
\text { Method }\end{array}$ & 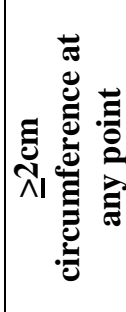 & 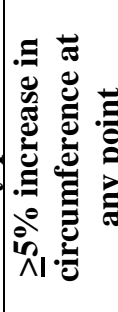 & 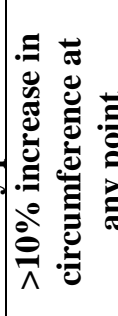 & 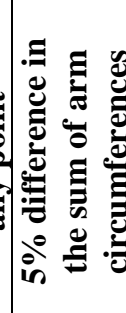 & 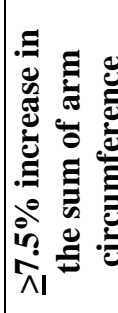 & 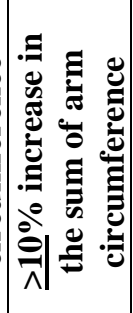 & 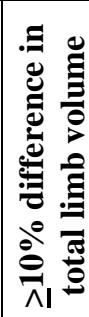 & 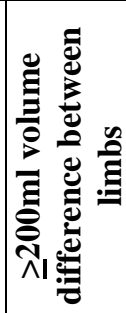 & 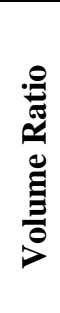 \\
\hline $\begin{array}{l}\text { Wang et } \\
\text { al. }\end{array}$ & 2017 & $\begin{array}{l}\text { Diagnostic } \\
\text { Test Study }\end{array}$ & $\sqrt{ }$ & & & & & & $\sqrt{ }$ & $\sqrt{ }$ & $\sqrt{ }$ \\
\hline $\begin{array}{l}\text { Mori et } \\
\text { al. }\end{array}$ & 2015 & $\begin{array}{l}\text { Diagnostic } \\
\text { Test Study }\end{array}$ & & & & & & & $\sqrt{ }$ & $\sqrt{ }$ & \\
\hline Fu et al. & 2013 & $\begin{array}{c}\text { Cross-sectional } \\
\text { Diagnostic } \\
\text { Study }\end{array}$ & $\sqrt{ }$ & & & & & & $\sqrt{ }$ & $\sqrt{ }$ & \\
\hline $\begin{array}{l}\text { Asim et } \\
\text { al. }\end{array}$ & 2012 & $\begin{array}{l}\text { Diagnostic } \\
\text { Test Study }\end{array}$ & $\sqrt{ }$ & $\sqrt{ }$ & $\sqrt{ }$ & $\sqrt{ }$ & $\sqrt{ }$ & $\sqrt{ }$ & & & \\
\hline $\begin{array}{l}\text { Czerniec } \\
\text { et al. }\end{array}$ & 2010 & $\begin{array}{l}\text { Diagnostic } \\
\text { Test Study }\end{array}$ & & & & & & & $\sqrt{ }$ & $\sqrt{ }$ & $\sqrt{ }$ \\
\hline
\end{tabular}

Wang et al. (2017) used the 10cm-CTM method and calculated cut-off thresholds at two standard deviations (2SD) ranging from 0.6 to $0.7 \mathrm{~cm}$ at the wrist, and 1.6 to $2.3 \mathrm{~cm}$ at $40 \mathrm{~cm}$ up the arm for the non-dominant and dominant sides respectively. This is a more labour intensive method to use in clinical practice in terms of needing to remember the differences in circumference required for diagnosing lymphoedema at each point up the arm.

In clinical practice, it is best to consider sensitivity testing for lymphoedema, especially for those at risk of lymphoedema, as early detection and treatment are known to minimise complications (Blaney et al., 2015). Thus, when considering Asim et al.'s (2012) results, the threshold of $\geq 7.5 \%$ is the more sensitive for detecting lymphoedema 
and should be considered for standardisation of diagnosing lymphoedema going forward.

\subsubsection{Validity and Reliability of CTM}

\section{Concurrent Validity:}

This looks at the similarity between a new measure against existing or comparable measures (Glen, 2015a). Eight papers report on concurrent validity, with correlations between CTM and water displacement, perometry or 3DCI of 0.91 (range $0.80-0.99$ ) (Tewari et al., 2008; Czerniec et al., 2010; Foroughi et al., 2011; Brorson \& Hoijer, 2012; Mori et al., 2015; Cau et al., 2016; Batista et al., 2018; Rafn, McNeely, Camp, Midtgaard, \& Campbell, 2019).

\section{Convergent Validity:}

Determines the extent to which measures that are related, correlate (Trochim, 2020). Three studies reported on this subcategory of construct validity. The correlation between different CTM intervals, 4cm-CTM to 5-point CTM, was 0.87 (range 0.810.92) (Brorson \& Hoijer, 2012). Tewari et al. (2008) report that the correlation between different widths of tape measure, $8 \mathrm{~mm}$ and $15 \mathrm{~mm}$, was 0.95 , whilst Mori, Lustman, \& Katz-Leurer, (2015) found the correlation between paper and plastic tape measures to be 0.98 .

\section{Reliability:}

To help distinguish between the various reported reliability tests, an explanation of each follows. Intra-class Correlation Coefficient (ICC) is a reliability index in test-retest, intra-rater $\left(\mathrm{ICC}_{\text {intra }}\right)$ and inter-rater ( $\left.\mathrm{ICC}_{\text {inter }}\right)$ reliability analysis (Koo \& $\left.\mathrm{Li}, 2016\right)$, with values closer to 1.00 indicating excellence. Standard Error of Measurement (SEM) quantifies the amount of variability in a test and is measured in the same units as the original measurement with values closer to 0 indicating stronger reliability (Huang \& Leong, 2016). For purposes of comparison, the SEM for each study was converted to percentages. Furthermore, the Smallest Detectable Change (SDC) has been calculated which can be defined as the smallest change that can be detected by a device beyond measurement error (de Vet \& Terwee, 2010). The SEM was used to calculate the SDC of each method of measurement using the formula: SDC $=$ SEM X 1.96 X $\sqrt{2}$ (Dontje et al., 2018). 
Eight papers reported excellent ICC values for CTM. Pooled ICC data showed an $\mathrm{ICC}_{\text {intra }}$ value of 0.97 (95\% CI=0.88, 1.00) (Chen et al., 2008; Czerniec et al., 2010; Devoogdt et al., 2010; Katz-Leurer \& Bracha, 2012; Mori et al., 2015; De Vrieze et al., 2019; Rafn et al., 2019) and an $\mathrm{ICC}_{\text {inter value } 0} 0.95$ (95\% CI=0.88, 0.99) (Devoogdt et al., 2010; De Vrieze et al., 2019; Rafn et al., 2019). The weighted mean SEM was 2.0\% $(\sigma=2.8 \%)$ and the weighted mean SDC was 5.5\% $(\sigma=8.3 \%) \quad$ (Czerniec et al., 2010; Devoogdt et al., 2010; Foroughi et al., 2011; Brorson \& Hoijer, 2012; Adriaenssens et al., 2013; Tidhar et al., 2015; Cau et al., 2016; Batista et al., 2018; Sharkey et al., 2018).

Three studies looked at self-measurement using CTM. Intra-rater reliability varied from 0.88 to 0.99 , with the lower values attributed to non-BCRL groups (Mori et al., 2015; Rafn et al., 2019). Foroughi et al. (2011) performed an inter-rater reliability test of a home-CTM protocol versus therapist-CTM and perometry within two weeks. Moderate to high concordance was observed between home-CTM and therapist-CTM $\left(r_{c}=0.68-0.93\right)$. From these three studies, self-administered CTM appears to be highly reliable with the use of a protocol.

\subsubsection{Benefits and Considerations of CTM}

The advantages of CTM are that it is easy to perform (Chen et al., 2008; Asim et al., 2012; Rafn et al., 2019), allows for analysis of segments individually or the total limb (Devoogdt et al., 2010; Brorson \& Hoijer, 2012), and is not reliant on physique (Blaney et al., 2015). It is portable to most environments and is low cost (Asim et al., 2012; Tidhar et al., 2015; Cau et al., 2016; Cau et al., 2018; Rafn et al., 2019). The added benefit of CTM to the therapist is that it aids clinical decisions such as choosing a garment size suitable for the person, deciding on the more appropriate material of the compression garment and determining when a change in treatment may be required (Tidhar et al., 2015). These latter decisions also take into account the person's subjective feedback about their lymphoedema management.

Conversely, CTM is user-dependent and can be prone to technical errors such as inconsistent pressure in tape application, inaccurately marked points on the limb, and deviation of the angle of the tape relative to the long axis of the limb (Chen et al., 2008; Tewari et al., 2008; Batista et al., 2018). It can be a time-consuming procedure (Devoogdt et al., 2010; Landau et al., 2018) with Landau, Kim, Gould, \& Patel (2018) 
reporting an average time of 15:42 min to perform the setup, execution and calculation of the results. It is also worth remembering that CTM is a single measure and is thus unable to differentiate between changes in muscle, bone, fat and fluid of the limb (Tewari et al., 2008; Czerniec et al., 2010; Peleg \& Katz-Leurer, 2017).

\subsubsection{Perometer}

Seventeen studies were identified and reviewed for perometry. Two of the papers analysed using CASP scored 24 (out of 24); these were De Vrieze et al. (2019) and Spinelli et al. (2019). Table 15 provides a summary of these critiqued papers.

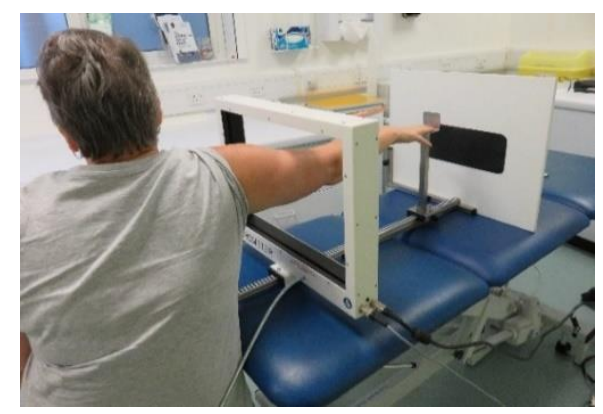

Figure 13: Perometer 1000 NT (portable model). The hand rests on the support while a frame is moved along the stand, capturing the circumference of the limb.

The perometer (Figure 13) is a technology-based circumferential measure. Specifically, it is an infrared optoelectric imaging system used for the indirect measurement of arm or leg volumes. It offers information on circumferences, contours and provides schematic representations of the cross-sectional area of the limb (Lee et al., 2011; Adriaenssens et al., 2013; Bulley, Coutts, \& Tan, 2013). The estimated time taken to perform the perometer measurement is on average $2 \mathrm{~min}$ per limb, as opposed to CTM which takes more than 10 min for both limbs (Sharkey et al., 2018); and is reportedly faster to perform than water displacement (Lee et al., 2011).

Pero-System Messgeraete GmbH, Wuppertal, Germany (Pero-System Messgeraete $\mathrm{GmbH}, 2017$ ), are the only manufacturers of the perometer. There are currently four perometer models, each comprising a square measuring frame that is moved manually back and forth at $90^{\circ}$ to the base plate (Czerniec et al., 2010; Foroughi et al., 2011). The frame contains parallel-acting light curtains made of photosensors and infrared lightemitting diodes (LEDs) which illuminate and scan the limb (Czerniec et al., 2010; Ancukiewicz et al., 2011; Foroughi et al., 2011; Dylke et al., 2012). The measurements 
Table 15: Papers accessed on perometer measurements

\begin{tabular}{|c|c|c|c|c|c|}
\hline $\begin{array}{c}\text { Author } \\
(\text { CASP Score })\end{array}$ & Year & Cohort & $\begin{array}{l}\text { Research } \\
\text { Design }\end{array}$ & Method & Primary Results \\
\hline $\begin{array}{l}\text { Binkley et al. } \\
\text { (23) }\end{array}$ & 2020 & $\begin{array}{l}66 \text { women } \\
\text { with BC }\end{array}$ & $\begin{array}{l}\text { Cross-sectional } \\
\text { Reliability and } \\
\text { Validity Study }\end{array}$ & $\begin{array}{l}\text { Compared new 3DCI device (LymphaTech) } \\
\text { to perometry to determine its reliability and } \\
\text { validity }\end{array}$ & $\begin{array}{l}\text { Both methods displayed ICCs of } \geq 0.99 \text {. SEM for both methods nearly identical, } \\
\text { ranging from } 28.3 \text { to } 32.8 \mathrm{ml} \text {. The convergent validity analysis demonstrated no } \\
\text { systematic difference between methods. The Bland Altman plot identifies a mean } \\
\text { volume difference between methods for the affected side of }-7.8 \mathrm{ml} \text {, and }-4.8 \mathrm{ml} \text { for } \\
\text { the unaffected side. It also shows volumes }>3000 \mathrm{ml} \text { has slightly greater variability } \\
\text { between methods. }\end{array}$ \\
\hline $\begin{array}{l}\text { DeSnyder et } \\
\text { al. } \\
\text { (21) }\end{array}$ & 2019 & $\begin{array}{l}50 \text { patients } \\
5 \text { volunteers } \\
23 \text { therapists }\end{array}$ & $\begin{array}{l}\text { Diagnostic Test } \\
\text { Study }\end{array}$ & $\begin{array}{l}\text { Both arms were measured by each therapist } \\
\text { three times, by one or two raters, or in groups } \\
\text { of } 5 \text { to } 6 \text { raters, using a perometer in two } \\
\text { positions to determine repeatability, } \\
\text { reproducibility and precision/tolerance }(\mathrm{P} / \mathrm{T}) \\
\text { ratios }\end{array}$ & $\begin{array}{l}\text { Baseline P/T ratio was } 19.9 \% \text {, repeatability was } 65.0 \% \text { and reproducibility } 19.0 \% \text {. } \\
\text { BMI, height and weight were all significant }(\mathrm{p} \leq 0.003) \text {. Difference from old to new } \\
\text { patient position reduced variations in limb volume by } 28 \%(\mathrm{p}=0.02) \text {. P/T ratio for } \\
\text { the new position was } 6.5 \% \text {, repeatability } 2.4 \% \text { and reproducibility } 6.1 \% \text {. Over- } \\
\text { diagnosis and under-diagnosis both reduced to } 0 \% \text { from } 3.5 \% \text { and } 2.3 \% \\
\text { respectively for threshold of } 10 \% \text { volume difference }\end{array}$ \\
\hline $\begin{array}{l}\text { De Vrieze et } \\
\text { al. } \\
(24)\end{array}$ & 2019 & $\begin{array}{l}30 \text { women } \\
\text { with BCRL }\end{array}$ & $\begin{array}{l}\text { Cross-sectional } \\
\text { Study }\end{array}$ & $\begin{array}{l}\text { Compared } 3 \text { types of WD to CTM and } \\
\text { perometry to establish which was the more } \\
\text { reliable, time-efficient and clinically feasible } \\
\text { method of measurement to use }\end{array}$ & $\begin{array}{l}\text { Intra- and inter-rater reliability for volume ranged from } 0.897 \text { to } 0.975 \text { with an } \\
\text { SEM of } 92.01 \mathrm{ml}(95 \% \mathrm{CI}) \text {. Perometry was fastest in terms of set up time and } \\
\text { execution of bilateral limb measurements with a mean time of } 1 \mathrm{~min} 43 \mathrm{sec} \text {. All } \\
\text { other measures showed statistically significant differences in the means against } \\
\text { perometry }(\mathrm{p}<0.05) \text {. For clinical feasibility, perometry was rated second to CTM }\end{array}$ \\
\hline $\begin{array}{l}\text { Spinelli et al. } \\
(24)\end{array}$ & 2019 & $\begin{array}{l}71 \text { women } \\
\text { with BC }\end{array}$ & $\begin{array}{l}\text { Blinded Repeat } \\
\text { Measures } \\
\text { Observational } \\
\text { Study }\end{array}$ & $\begin{array}{l}\text { Compared new assessment tool (Cancer- } \\
\text { related LO of the Upper Extremity [CLUE]) } \\
\text { to perometry by measuring twice by two } \\
\text { therapists over } 7-21 \text { days }\end{array}$ & $\begin{array}{l}\text { ICC for intra-rater reliability was } 0.93(95 \% \mathrm{CI}) \text {, and for inter-rater reliability it } \\
\text { was } 0.98(95 \% \mathrm{CI}) \text {. Pearson's correlation between the measurement tools was } 0.79\end{array}$ \\
\hline $\begin{array}{l}\text { Batista et al. } \\
(21)\end{array}$ & 2018 & $\begin{array}{l}91 \text { patients: } \\
46 \text { at risk } \\
45 \text { not at } \\
\text { risk }\end{array}$ & $\begin{array}{l}\text { Diagnostic Test } \\
\text { Study }\end{array}$ & $\begin{array}{l}\text { CTM was compared to perometry to } \\
\text { determine the LOA }\end{array}$ & $\begin{array}{l}\text { Very strong correlation for volume, VD and \%VD were observed with Pearson's } \\
\text { correlation coefficients of } \mathrm{r}=0.99,0.88 \text { and } 0.86 \text {, respectively. The LOA for } \\
\text { volume ranged from }-202 \text { to } 181 \mathrm{ml} \text {, for VD it ranged from }-101 \text { to } 141 \mathrm{ml} \text { and } \\
\% \text { VD ranged from }-5 \text { to } 6.8 \%\end{array}$ \\
\hline $\begin{array}{l}\text { Sharkey et al. } \\
\text { (18) }\end{array}$ & 2018 & $\begin{array}{l}35 \text { arm and } \\
26 \text { leg } \\
\text { volunteers }\end{array}$ & $\begin{array}{l}\text { Diagnostic Test } \\
\text { Study }\end{array}$ & $\begin{array}{l}\text { Assessing intra-and inter-rater reliability of } \\
\text { CTM and perometry to determine the } \\
\text { accuracy of CTM compared to perometry } \\
\text { when measured by experts versus students }\end{array}$ & $\begin{array}{l}\text { Perometer had lower SEMs for students compared to CTM ( } 24.88 \text { vs } 114.1) \text {; } \\
\text { however, SEM experts perometer was higher than CTM ( } 38.99 \text { vs } 25) \text {. There was } \\
\text { moderate intra-rater reliability with students (Cronbach's alpha=0.764) but } \\
\text { excellent ICC for the expert groups (Cronbach's alpha }=0.99)\end{array}$ \\
\hline $\begin{array}{l}\text { Sun et al. } \\
(22)\end{array}$ & 2018 & $\begin{array}{l}287 \text { women } \\
\text { with BC }\end{array}$ & $\begin{array}{l}\text { Diagnostic Test } \\
\text { Study }\end{array}$ & $\begin{array}{l}\text { Women with BC were screened for LO using } \\
\text { perometry and compared total limb VD to } \\
\text { simulated VD at } 4 \mathrm{~cm} \text { intervals, landmark (3- } \\
\text { point), and landmark-midpoint (5-point) } \\
\text { methods }\end{array}$ & $\begin{array}{l}\text { Correlation coefficient was consistently }>0.98 \text { for all simulated methods compared } \\
\text { to total limb perometry. Both landmark methods significantly underestimated } \\
\text { upper arm volume but overestimated forearm volume. Volume change for LO was } \\
\text { detected most commonly in the upper arm }(63.4 \%) \text {, forearm }(11.1 \%) \text { and equally } \\
\text { in both segments }(25 \%) \text {. Both landmark methods had greater sensitivity compared } \\
\text { to } 4 \mathrm{~cm} \text { and total limb }(93.1 \text { and } 90.3 \% \text { vs } 81.9 \text { and } 77.8 \%) \text { and greater specificity } \\
(93.5 \% \text { vs } 68.4 \text { and } 92.6 \%)\end{array}$ \\
\hline
\end{tabular}




\begin{tabular}{|c|c|c|c|c|c|}
\hline $\begin{array}{c}\text { Author } \\
(C A S P \text { Score })\end{array}$ & Year & Cohort & $\begin{array}{l}\text { Research } \\
\text { Design }\end{array}$ & Method & Primary Results \\
\hline $\begin{array}{l}\text { Adriaenssens } \\
\text { et al. } \\
(22)\end{array}$ & 2013 & $\begin{array}{l}80 \text { people: } \\
31 \text { healthy; } \\
49 \mathrm{BC}\end{array}$ & $\begin{array}{l}\text { Diagnostic Test } \\
\text { Study }\end{array}$ & $\begin{array}{l}\text { Both arms were measured three times by } \\
\text { perometer and compared to one measurement } \\
\text { of commonly used methods of WD and CTM }\end{array}$ & $\begin{array}{l}\text { Intra-class correlation for perometer was excellent (ICC >0.997 for healthy group, } \\
\text { and }>0.998 \text { for BC group). CTM produced highest volume and WD lowest volume, } \\
\text { with perometer volumes in between. Volume of dominant arm was } 2.2 \%>\text { non- } \\
\text { dominant arm. }\end{array}$ \\
\hline $\begin{array}{l}\text { Bulley et al. } \\
\text { (22) }\end{array}$ & 2013 & $\begin{array}{l}30 \text { healthy } \\
\text { volunteers }\end{array}$ & $\begin{array}{l}\text { Diagnostic Test } \\
\text { Study }\end{array}$ & $\begin{array}{l}\text { Develop a standardised protocol for the } \\
\text { upright perometer and test its reliability on } \\
\text { two separate occasions } 24-48 \mathrm{hrs} \text { apart by two } \\
\text { raters }\end{array}$ & $\begin{array}{l}\text { A series of pilot studies were undertaken to develop the final standardised protocol. } \\
\text { High intra-and inter-rater reliability observed (ICC=0.953-0.989); however, the } \\
\text { LOA results were less positive, ranging from }-941 \text { to } 915 \mathrm{ml} \text {. }\end{array}$ \\
\hline $\begin{array}{l}\text { Lu et al. } \\
\text { (18) }\end{array}$ & 2013 & $\begin{array}{l}6 \text { people: } \\
5 \text { healthy; } \\
1 \text { BCRL }\end{array}$ & $\begin{array}{l}\text { Diagnostic Test } \\
\text { Study }\end{array}$ & $\begin{array}{l}\text { Compared 3DCI to perometer in first } \\
\text { experiment; then determined accuracy and } \\
\text { ability of 3DCI to detect localised swells }\end{array}$ & $\begin{array}{l}\text { To note: authors state that 'perometer cannot be considered as ground truth, since } \\
\text { its application in LO is predicted upon its ability to provide repeatability, but not } \\
\text { necessarily accuracy in the readings'. Perometry underestimates volume compared } \\
\text { to 3DCI }(4.7-12.8 \%) \text {. }\end{array}$ \\
\hline $\begin{array}{l}\text { Ancukiewicz } \\
\text { et al. } \\
\text { (19) }\end{array}$ & 2012 & $\begin{array}{l}677 \text { people } \\
\text { with BC }\end{array}$ & $\begin{array}{l}\text { Diagnostic Test } \\
\text { Study }\end{array}$ & $\begin{array}{l}\text { Both arms were measured pre-and post- } \\
\text { operatively over } 3 \text { years together with BMI. } \\
\text { The purpose was to compare absolute change } \\
\text { in arm size }(2 \mathrm{~cm}) \text { to relative arm volume } \\
\text { change }(200 \mathrm{ml}) \text { to quantify BCRL }\end{array}$ & $\begin{array}{l}\text { Paper reports 'strong' correlation of unaffected arm volume with bodyweight } \\
\text { (Kendall's } \tau=0.65, \mathrm{p}<0.0001) \text {, and BMI (Kendall's } \tau=0.55, \mathrm{p}<0.0001) \text {, but values } \\
\text { are moderate. The median relative arm volume change was } 7.7 \% \text { resulting from a } \\
200 \mathrm{ml} \text { change in arm volume. For subset group }(\mathrm{n}=45 \text { ), local change of } 2 \mathrm{~cm} \text { in } \\
\text { circumference corresponded to a relative arm volume change of } 7.5 \% \text {. For subset } \\
\text { group }(\mathrm{n}=124) \text {, relative arm volume change in affected arm did not correlate to } \\
\text { weight or BMI }(\mathrm{P}>0.05)\end{array}$ \\
\hline $\begin{array}{l}\text { Dylke et al. } \\
\text { (21) }\end{array}$ & 2012 & $\begin{array}{l}204 \text { healthy } \\
\text { women }\end{array}$ & $\begin{array}{l}\text { Diagnostic Test } \\
\text { Study }\end{array}$ & $\begin{array}{l}\text { A single assessment was performed to } \\
\text { determine the normative values for inter-arm } \\
\text { differences by obtaining both circumferential } \\
\text { (10cm method) and volume measurements by } \\
\text { perometer. Volume calculations compared } \\
\text { included perometer, truncated cone and } \\
\text { cylinder (calculated) methods }\end{array}$ & $\begin{array}{l}\text { Volumes versus simulations had high correlation coefficients ranging from } 0.851 \\
\text { to } 0.988 \text {. Concordance between calculated methods was very high }(0.999-1.000) \text {. } \\
\text { Mean bias for total arm volume was } 2.9 \% \text { with bias largest in dominant arm. The } \\
\text { LOA (2SD) between methods was largest between perometry and truncated cone } \\
\text { method for dominant arm (LOA }=-1-10 \%) \text {. Paired t-tests between dominant and } \\
\text { non-dominant arm revealed significant differences at all but } 30 \mathrm{~cm} \text { point }(95 \% \mathrm{CI}) \text {. } \\
\text { All perometer segments were significantly different between sides ( }<0.03-0.001) \text {. } \\
\text { Diagnostic cut-offs for LO were determined at } 3 \mathrm{SD} \text { above the mean. Regression } \\
\text { analysis found age negatively related to inter-limb difference. }\end{array}$ \\
\hline $\begin{array}{l}\text { Ancukiewicz } \\
\text { et al. } \\
(20)\end{array}$ & 2011 & $\begin{array}{l}677 \text { women } \\
\text { with BC }\end{array}$ & $\begin{array}{l}\text { Diagnostic Test } \\
\text { Study }\end{array}$ & $\begin{array}{l}\text { Both arms were measured pre-and post- } \\
\text { operatively up to three times at a session. } \\
\text { The measurement protocol was revised } \\
\text { throughout the study to increase accuracy of } \\
\text { the measurements, and a formula for the } \\
\text { quantitative assessment of developing LO } \\
\text { was constructed }\end{array}$ & $\begin{array}{l}\text { At baseline measure, there was no statistically significant difference between sides } \\
(p=0.42) \text {. Statistical analysis identified volume ratios as most appropriate for } \\
\text { quantification of both asymmetry and temporal changes. Formula for } R V C \text { was } \\
\text { developed }=\left(A_{2} U_{1}\right) /\left(U_{2} A_{1}\right)-1 \text {, where } A_{1}, A_{2} \text { are arm volumes on affected side at } \\
\text { different time points, and } U_{1}, U_{2} \text { are volumes on the unaffected side. RVC is not } \\
\text { significantly associated with hand dominance, age or time since diagnosis of BC. } \\
\text { Baseline weight correlates ( }=0.0074) \text { with higher } R V C \text {; however, BMI or weight } \\
\text { changes over time do not. Protocol in sitting was developed for the perometer. }\end{array}$ \\
\hline
\end{tabular}




\begin{tabular}{|c|c|c|c|c|c|}
\hline $\begin{array}{c}\text { Author } \\
\text { (CASP Score) }\end{array}$ & Year & Cohort & $\begin{array}{l}\text { Research } \\
\text { Design }\end{array}$ & Method & Primary Results \\
\hline $\begin{array}{l}\text { Foroughi et } \\
\text { al. } \\
\text { (22) }\end{array}$ & 2011 & $\begin{array}{l}57 \\
\text { community- } \\
\text { dwelling } \\
\text { adults aged } \\
>40 \text { years }\end{array}$ & $\begin{array}{l}\text { Cross-sectional } \\
\text { study }\end{array}$ & $\begin{array}{l}\text { Inter-rater reliability testing of a home-CTM } \\
\text { protocol versus therapist CTM and perometry } \\
\text { within two weeks }\end{array}$ & $\begin{array}{l}\text { Moderate to high concordance between home-CTM and therapist CTM }\left(\mathrm{r}_{\mathrm{c}}=0.68-\right. \\
0.93) \text {, and high concordance between therapist-CTM and perometry }\left(\mathrm{r}_{\mathrm{c}}=0.83-0.94\right) \text {. } \\
\text { LOA varied dependent on the measurement location with a bias range of }-5.5 \% \text { to } \\
1.5 \% \text { therapist-CTM vs perometer, and }-2.4 \% \text { to } 4.0 \% \text { therapist-CTM vs home- } \\
\text { CTM }\end{array}$ \\
\hline $\begin{array}{l}\text { Lee et al. } \\
(21)\end{array}$ & 2011 & $\begin{array}{l}40 \text { women: } \\
20 \text { with LO; } \\
20 \text { non-LO }\end{array}$ & $\begin{array}{l}\text { Diagnostic Test } \\
\text { Study }\end{array}$ & $\begin{array}{l}\text { Only the LO side or dominant side (for non- } \\
\text { LO group) were measured in a single session. } \\
\text { Perometer measurement was done twice by } \\
\text { one rater, and once by a second rater, and one } \\
\text { measurement done by WD }\end{array}$ & $\begin{array}{l}\text { Perometer demonstrated excellent inter- }\left(\mathrm{ICC}_{2,1}=0.993\right) \text { and intra-rater reliability } \\
\left(\mathrm{ICC}_{2,1}=0.989\right) \text {. Paired } \mathrm{t} \text {-test showed no significant difference in hand volume } \\
\text { between raters. Strong concordance between perometry and WD }\left(\mathrm{r}_{\mathrm{c}}=0.88\right) \text {. LOA } \\
\text { showed perometry to overestimate volume compared to WD }\end{array}$ \\
\hline $\begin{array}{l}\text { Czerniec et } \\
\text { al. } \\
(21)\end{array}$ & 2010 & $\begin{array}{l}51 \text { people: } \\
33 \text { with } \mathrm{LO} \\
18 \text { non-LO }\end{array}$ & $\begin{array}{l}\text { Diagnostic Test } \\
\text { Study }\end{array}$ & $\begin{array}{l}\text { Participants were assessed twice over a } 4- \\
\text { week period in which both arms were } \\
\text { measured by } 10 \mathrm{~cm}-\mathrm{CTM} \text {, perometer and BIS } \\
\text { to determine the relationship between each } \\
\text { method and the SEM }\end{array}$ & $\begin{array}{l}\text { Strongest relationship was for limb VD between } 10 \mathrm{~cm}-C T M \text { and perometer } \\
\left(\mathrm{r}_{\mathrm{c}}=0.99\right) \text {, and weakest between BIS and } 10 \mathrm{~cm}-\mathrm{CTM}\left(\mathrm{r}_{\mathrm{c}}=0.89\right) \text {. LOA showed } \\
10 \mathrm{~cm}-\mathrm{CTM} \text { to underestimate limb volume compared to perometer. All } 3 \text { methods } \\
\text { had excellent intra-rater reliability (ICC CTM=0.96; perometer }=0.99 ; \mathrm{BIS}=0.96) \text {. } \\
\text { SEM for } 10 \mathrm{~cm}-\mathrm{CTM} \text { was } 85 \mathrm{ml} \text {; perometer } 43 \mathrm{mll} \text { and BIS ratio } 0.05 \text {. }\end{array}$ \\
\hline
\end{tabular}

Key:

2SD $=$ Two Standard Deviation

$\mathrm{BC}=$ Breast Cancer

$\mathrm{CI}=$ Confidence Interval

LOA $=$ Limits of Agreement

SEM $=$ Standard Error of Measurement
3DCI $=$ Three-Dimensional Camera Imaging $\mathrm{BCRL}=$ Breast Cancer-Related Lymphoedema $\mathrm{CTM}=$ Circumferential Tape Measurement

$\mathrm{P} / \mathrm{T}=$ Precision/Tolerance ratio

TDC $=$ Tissue Dielectric Constant
$3 \mathrm{SD}=$ Three Standard Deviation

BIS = Bio-impedance Spectroscopy ICC = Intra-class Correlation Coefficient RVC $=$ Relative Volume Change

$\mathrm{VD}=$ Volume Difference
$\% \mathrm{VD}=$ Percentage Volume Difference

$\mathrm{BMI}=$ Body Mass Index

LO = Lymphoedema

$\mathrm{SD}=$ Standard Deviation

$\mathrm{WD}=$ Water Displacement 
taken by the perometer are calculated to produce an overall limb volume for each arm, which are then compared to one another to provide the volume difference.

According to Lu, DeSouza, Armer, Anderson, \& Shyu (2013) and Sun et al. (2018), perometry is sensitive to limb volume change and is a specific method for assessing lymphoedema. The first paper published about perometry by Stanton, Northfield, Holroyd, Mortimer, \& Levick (1997) validated the perometer by comparing it to $4 \mathrm{~cm}$ CTM. They found the perometer to be highly reproducible, accurate and reliable.

From the literature, two studies recommended protocols for positioning when using the perometer. Ancukiewicz et al. (2011) designed a 'Perometer Measurement Protocol' (Figure 14) recommended for clinical and research use, and two years later Bulley, Coutts, \& Tan (2013) also developed a protocol using a different model of perometer. In essence, the limb is in the centre of the frame that is moved along the length of the arm from the wrist towards the top of the arm, and then back to the starting point. One study compared two positions for their perometer (DeSnyder et al., 2019). They determined that a baseline perometer measurement had repeatability of $65.0 \%$ and reproducibility of $19.0 \%$, and when re-measured using a new patient positioning, the repeatability reduced to $2.4 \%$, and reproducibility to $6.1 \%$. This change saw both overand under-diagnosis rates reducing to $0 \%$ from $3.5 \%$ and $2.3 \%$ respectively for a threshold of $10 \%$ volume difference between arms. There are, however, six studies that recommend a protocol on patient positioning for the type of perometer used to ensure standardisation, allowing for less error between sessions and different therapists (Ancukiewicz et al., 2011; Foroughi et al., 2011; Lee et al., 2011; Dylke et al., 2012; Bulley et al., 2013; Sun et al., 2018).

The LED lights of the perometer frame cast limb shadows in two planes, capturing limb diameters every $3.1 \mathrm{~mm}$ (Czerniec et al., 2010) or $4.7 \mathrm{~mm}$ (Foroughi et al., 2011; Bulley et al., 2013; Lu, DeSouza, Armer, Anderson, \& Shyu, 2013; Sharkey et al., 2018), dependent on the model and software of the device used. The perometer software uses these captured diameters to build an image of the limb and calculate its volume (Czerniec et al., 2010; Dylke et al., 2012). Stanton et al.'s (1997) work explain that volume calculations are based on the assumption of a circular or elliptical cross-section; therefore, hands cannot be reliably measured as they deviate markedly from the circle 


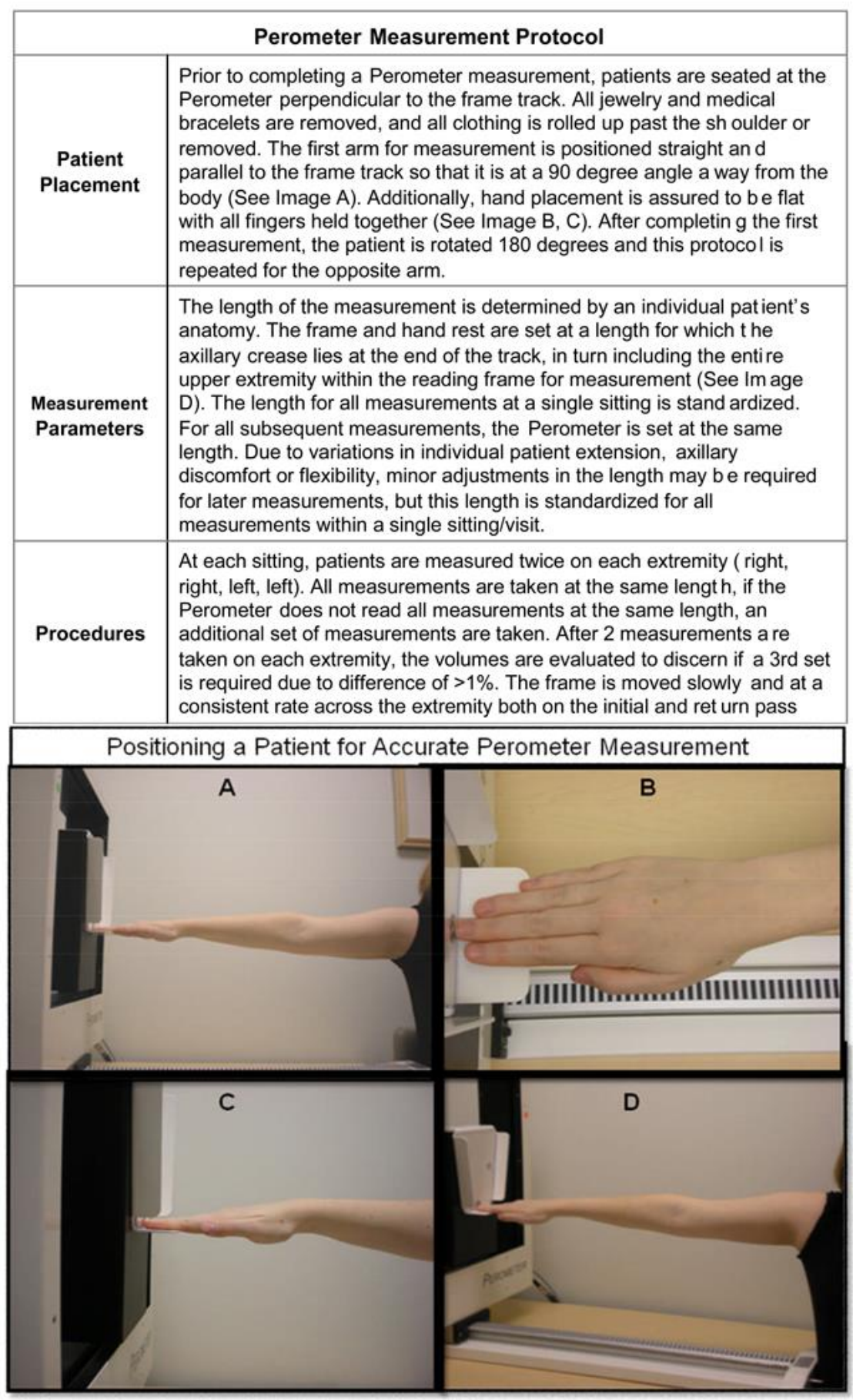

Figure 14: Perometer Measurement Protocol (Ancukiewicz et al., 2011)²

or ellipse shapes. Foroughi et al. (2011) measured each limb by perometer once, as their literature review showed it to have excellent reliability and reproducibility with repeated measures. Concurrently, Ancukiewicz et al. (2011) warn that caution should be taken when interpreting perometer results when a single measurement of a limb is made due

${ }^{2}$ Reprinted from International Journal of Radiation Oncology Biology Physics, volume 79, issue 5, Ancukiewicz et al., Standardised Method for Quantification of Developing Lymphedema in Patients Treated for Breast Cancer, pages 1436-1443., Copyright (2011), with permission from Elsevier 
to potential discrepancies in limb volumes of $3 \%$ for $17 \%$ of patients and more than $5 \%$ for $5.8 \%$ of patients.

The unaffected limb is used as the control for comparison in unilateral lymphoedema, using either a limb volume ratio (affected/unaffected), or limb volume difference (affected-unaffected) to gauge the severity of lymphoedema or treatment outcomes (Czerniec et al., 2010; Ancukiewicz et al., 2011; Dylke et al., 2012; Adriaenssens et al., 2013; Lu et al., 2013; Sun et al., 2018). Interestingly, Dylke, Yee, Ward, Foroughi, \& Kilbreath (2012) found that the frustum and cylinder formulae calculated from the perometer circumferences could be used interchangeably. However, these were not interchangeable with the perometer calculation even though the computer software sums the numerous elliptical discs to calculate limb volume (Bulley et al., 2013). This was due to significant differences in total volume differences between the equations used. For this reason, perometry cannot be used interchangeably with CTM or water displacement (Czerniec et al., 2010; Lee et al., 2011; Dylke et al., 2012; Adriaenssens et al., 2013; Sun et al., 2018) as these measurement techniques are inequivalent, producing different and incompatible total limb volumes.

\subsubsection{Thresholds for Diagnosing Lymphoedema Using Perometry}

As with CTM, there exists a lack of consensus over diagnostic thresholds in lymphoedema measurement using perometry. From the papers reviewed for perometry, five studies report a threshold used to diagnose lymphoedema, identifying four different thresholds, as summarised in Table 16. The most commonly used threshold methods reported were '10\% relative percentage difference' (Czerniec et al., 2010; Dylke et al., 2012; Adriaenssens et al., 2013; Bundred et al., 2020) and 'absolute interlimb volume difference of 200ml' (Czerniec et al., 2010; Ancukiewicz et al., 2012; Dylke et al., 2012; Bundred et al., 2020). The third commonly mentioned threshold was ' $1.5 \mathrm{~cm}$ to $2 \mathrm{~cm}$ interlimb circumference difference' (Ancukiewicz et al., 2012; Dylke et al., 2012; Bundred et al., 2020). To note, none of these studies take into account arm dominance.

Ancukiewicz et al. (2012) measured breast cancer patients' arms pre-and postoperatively over 3 years together with BMI. The purpose was to compare absolute change in arm size $(2 \mathrm{~cm})$ to relative arm volume change $(200 \mathrm{ml})$ to quantify BCRL. The median relative arm volume change was $7.7 \%$ resulting from a $200 \mathrm{ml}$ change in 
arm volume. A subset group of 45 participants had a localised change of $2 \mathrm{~cm}$ in circumference, which corresponded to a relative arm volume change of $7.5 \%$. For another subset group of 124 participants, the relative arm volume change in the affected arm did not correlate to weight or BMI ( $>0.05$ ). Thus the $2 \mathrm{~cm}$ and $200 \mathrm{ml}$ thresholds correlate at $7.5 \%-7.7 \%$ in diagnosing lymphoedema.

Table 16: Thresholds for diagnosing lymphoedema using perometry

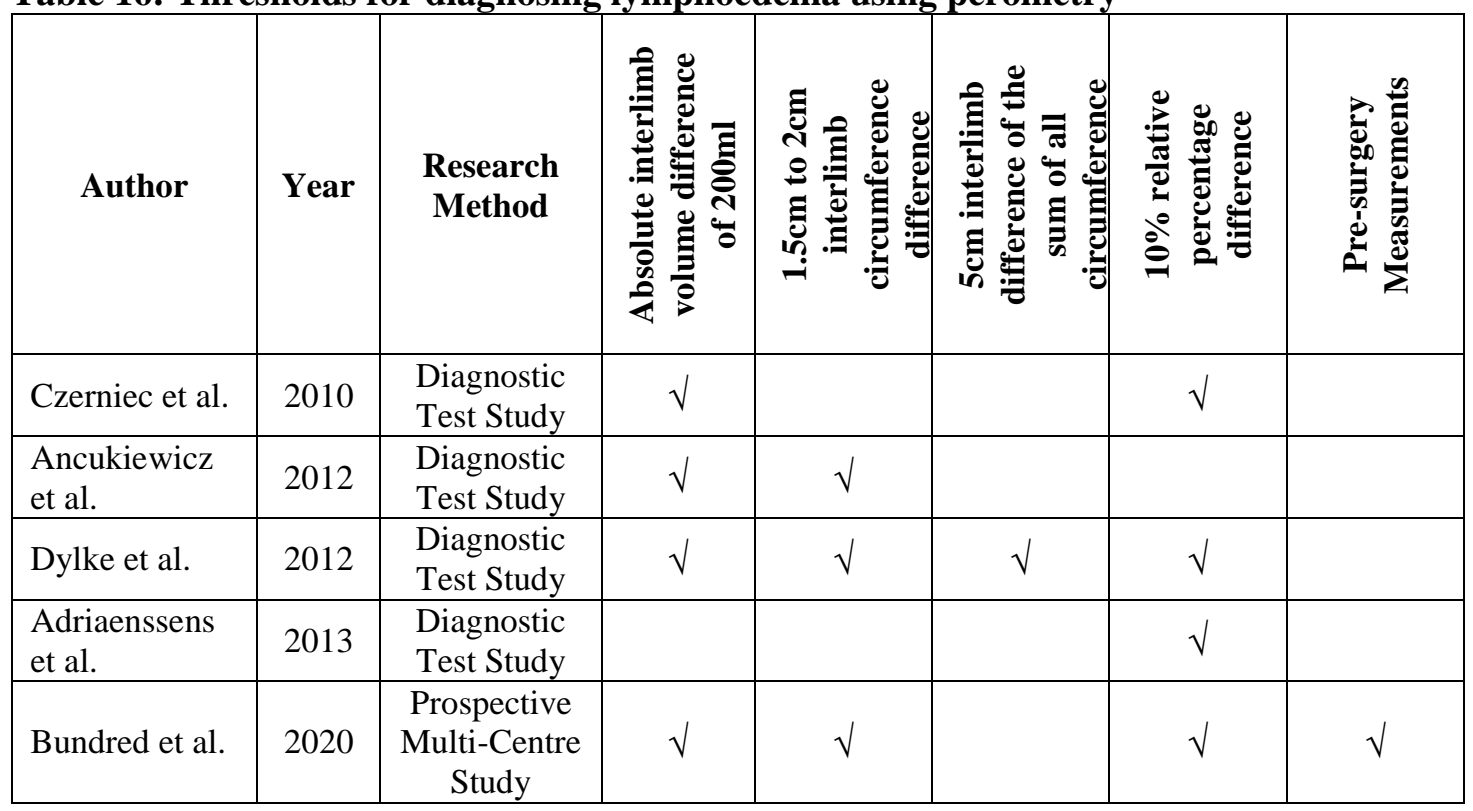

Dylke et al. (2012) found small, but significant differences where the mean bias for total arm volume was $2.9 \%$, and the largest bias in the dominant arm. They proposed a diagnostic cut-off of $3 \mathrm{SD}$ and determined cut-offs of $1.3 \mathrm{~cm}$ at the wrist to $3.1 \mathrm{~cm}$ at the axilla on the dominant side, and $1.2 \mathrm{~cm}$ to $2.7 \mathrm{~cm}$ for the non-dominant arm at the same landmarks. For volume cut-offs, they found that the perometer had higher thresholds than CTM for both the dominant and non-dominant arms, with all values above the recommended $200 \mathrm{ml}$ threshold. The higher volume thresholds are accounted for by the proposed 3SD as opposed to the more commonly used 2SD due to $8 \%$ of their cohort that would be incorrectly diagnosed with lymphoedema.

\subsubsection{Validity and Reliability of Perometry}

\section{Concurrent Validity:}

Four studies report on this, with correlations between perometry and CTM or water displacement of 0.92 (range 0.84-0.99) (Czerniec et al., 2010; Foroughi et al., 2011; Lee et al., 2011; Batista et al., 2018). 


\section{Convergent Validity:}

Two studies reported on this subcategory of construct validity using simulated models from the perometry data. The correlation between total limb perometry and various simulated CTM methods was 0.99 (range 0.98-1.00) (Dylke et al., 2012; Sun et al., 2018).

\section{Reliability:}

Nine papers reported good to excellent ICC values for perometry. Pooled ICC data showed an $\mathrm{ICC}_{\text {intra }}$ value of 0.97 (95\% CI=0.85, 1.00) (Czerniec et al., 2010; Lee et al., 2011; Dylke et al., 2012; Adriaenssens et al., 2013; Bulley et al., 2013; Sharkey et al., 2018; De Vrieze et al., 2019; Spinelli et al., 2019; Binkley et al., 2020) and an ICC $_{\text {inter }}$ value of 0.98 (95\% CI=0.90, 1.00) (Lee et al., 2011; Bulley et al., 2013; De Vrieze et al., 2019; Spinelli et al., 2019). The weighted mean SEM was 3.4\% $(\sigma=4.6 \%)$ and the weighted mean SDC was 9.4\% ( $\sigma=12.8 \%)$ (Czerniec et al., 2010; Foroughi et al., 2011; Dylke et al., 2012; Adriaenssens et al., 2013; Bulley et al., 2013; Lu et al., 2013; Batista et al., 2018; Sharkey et al., 2018; Binkley et al., 2020).

\subsubsection{Benefits and Considerations of Perometry}

The benefits of the perometer are that it requires little time to operate (Ancukiewicz et al., 2011; Lee et al., 2011; Adriaenssens et al., 2013; Lu et al., 2013), is safe to use in the presence of open wounds or infection as it does not require direct contact with the limb, and requires minimal technical training for staff (Lee et al., 2011). There is only one type of portable perometer available which offers more flexibility in terms of cost and use (Adriaenssens et al., 2013). The perometer software can build an image of the limb from the captured data (Czerniec et al., 2010; Dylke et al., 2012), which gives the therapist a 2-D view of the difference in shape between the limbs.

Disadvantages of perometry are that it is cost-prohibitive (Adriaenssens et al., 2013; Lu et al., 2013; Sharkey et al., 2018; Binkley et al., 2020), bulky in size requiring space for setting up (Binkley et al., 2020), calculates total limb volume thus is unable to discriminate between fat, fluid, muscle and bone (Czerniec et al., 2010), does not easily capture the extremity and root of the limb (Czerniec et al., 2010; Sun et al., 2018), and most devices are not portable (Lu et al., 2013). The images built from the data are not quantifiable and other than confirming the observed visual differences in shape, the 
images provide no further information to the therapist. Lastly, there are reports of software failure when using the perometer (Dylke et al., 2016).

\subsubsection{Three-Dimensional Camera Imaging (3DCI)}

$3 D C I$ has not been used in a lymphoedema clinical setting to date, but studies show its potential as a fast, accurate, and cost-effective method of measuring for lymphoedema. This newer method of determining limb volume using 3D modelling is derived from a variety of methods. From the 16 studies for 3DCI, seven types of 3D devices were identified, including Asus Xtion Pro 3D, Kinect Kinect ${ }^{\mathrm{TM}}$, LymphaTech system, Laser Scanner 3D system (LS3D), Vectra 3D imaging system, 5-pod stereophotogrammetry system and static 3D Microsoft Kinect ${ }^{\mathrm{TM}}$ system. The maximum score the papers in this group achieved on the CASP table was 23 by Binkley et al. (2020), while five papers scored 22, namely Hameeteman, Verhulst, Vreeken, Maal, \& Ulrich (2016), Karakashian et al. (2018), Landau et al. (2018), Preuß, Killaars, de Grzymala, Binnebösel, \& Neumann (2018) and Lu \& Dixon (2019). Table 17 provides a summary of the findings from each of the critiqued papers relating to 3DCI.

3DCI has been used in various clinical settings including respiratory monitoring, falls risk assessment, assessment of spatiotemporal gait variables (Öhberg et al., 2014), dermatology, rehabilitation, assisted living (Dylke et al., 2016), and for determining patient size for radiation dose monitoring during CT scanning (Cook, Couch, Couch, Kim, \& Boonn, 2013).

Initial studies utilising 3D technology focused on facial oedema (Aldridge, Boyadjiev, Capone, DeLeon, \& Richtsmeier, 2005; Plooij et al., 2009; Maal et al., 2010; van der Meer, Dijkstra, Visser, Vissinl, \& Ren, 2014). These studies have been expanded to include breast assessment (Henseler et al., 2012; Henseler, Kuznetsova, Vogt, \& Rosenhahn, 2014) and hand oedema (Vicini et al., 2012; Hoevenaren et al., 2015). 
Table 17: Papers analysed for 3DCI

\begin{tabular}{|c|c|c|c|c|c|c|}
\hline \multicolumn{7}{|c|}{ 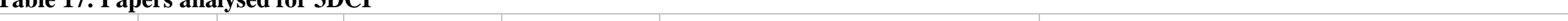 } \\
\hline $\begin{array}{c}\text { Author } \\
(\text { CASP Score })\end{array}$ & Year & Cohort & $\begin{array}{l}\text { Research } \\
\text { Design }\end{array}$ & 3DCI System & Method & Primary Results \\
\hline $\begin{array}{l}\text { Binkley et al. } \\
(20)\end{array}$ & 2020 & $\begin{array}{l}66 \text { women } \\
\text { with BC }\end{array}$ & $\begin{array}{l}\text { Cross-sectional } \\
\text { Reliability and } \\
\text { Validity Study }\end{array}$ & LymphaTech & $\begin{array}{l}\text { Compared new 3DCI device (LymphaTech) } \\
\text { to perometry to determine its reliability and } \\
\text { validity }\end{array}$ & $\begin{array}{l}\text { ICCs of } \geq 0.99 \text {. SEM ranged from } 28.3 \mathrm{ml} \text { to } 32.8 \mathrm{ml} \text {. The } \\
\text { convergent validity analysis demonstrated no systematic } \\
\text { difference between methods. The Bland Altman plot identifies } \\
\text { a mean volume difference between methods for the affected side } \\
\text { of }-7.8 \mathrm{ml} \text { and }-4.8 \mathrm{ml} \text { for the unaffected side. It shows volumes } \\
>3000 \mathrm{ml} \text { have slightly greater variability between methods. }\end{array}$ \\
\hline $\begin{array}{l}\text { Karakashian } \\
\text { et al. } \\
\text { (20) }\end{array}$ & 2019 & $\begin{array}{l}23 \text { women } \\
\text { with BCRL }\end{array}$ & $\begin{array}{l}\text { Diagnostic Test } \\
\text { Study }\end{array}$ & $\begin{array}{l}\text { Asus Xtion Pro } \\
\text { 3D }\end{array}$ & $\begin{array}{l}\text { The framework was built generating } \\
\text { computational models from 3DCI in which } \\
\text { Finite Element Analysis was performed to } \\
\text { determine the sub-garment pressures based } \\
\text { on the local curvature of each subject's arms. } \\
\text { Images were post-processed using } \\
\text { MATLAB. }\end{array}$ & $\begin{array}{l}\text { Average maximum sub-garment pressure was } 5100 \mathrm{~Pa} \\
(38.25 \mathrm{mmHg}) \text { as opposed to the intended } 2500 \mathrm{~Pa} \\
(18.75 \mathrm{mmHg}) \text {. Subcutaneous results show that pressure exerted } \\
\text { by garments exist more on adipose tissue than intended firmer } \\
\text { LO skin and tissues. A novel finding was that a negative sub- } \\
\text { garment gradient pressure exists from the wrist to the elbow }\end{array}$ \\
\hline $\begin{array}{l}\text { Lu \& Dixon } \\
(22)\end{array}$ & 2019 & $\begin{array}{c}73 \text { women: } \\
30 \text { BCRL } \\
43 \text { non- } \\
\text { BCRL }\end{array}$ & $\begin{array}{l}\text { Diagnostic Test } \\
\text { Study }\end{array}$ & $\begin{array}{l}\text { Kinect IR } \\
\text { sensor }\end{array}$ & $\begin{array}{l}\text { Both arms measured in single session once } \\
\text { by 3DCI and twice by Perometer } \\
\text { (consecutively). 3DCI scanned front and } \\
\text { back of patient for } 30 \text { seconds each side and } \\
\text { then post-processed using MATLAB. }\end{array}$ & $\begin{array}{l}\text { Validity had correlation of } 0.88 \text {. Absolute volume agreement } \\
\text { analysis resulted in a difference of }-117.6 \mathrm{ml} \text { (SD 228.6). Bland- } \\
\text { Altman analysis resulted in a \%VD of }-0.47 \% \text { (SD 6.01) with a } \\
\text { bias of } 6.02 \mathrm{ml} \text {. Comparing BCRL to non-BCRL, there is no } \\
\text { significant difference in \%VD ( }=0.28) \text {, but significantly greater } \\
\text { VD ( } \mathrm{p}=0.03 \text { ) between arms in BCRL women. }\end{array}$ \\
\hline $\begin{array}{l}\text { Cau et al. } \\
(20)\end{array}$ & 2018 & $\begin{array}{l}200 \text { women } \\
\text { with BCRL }\end{array}$ & $\begin{array}{l}\text { Prospective } \\
\text { Cohort Study }\end{array}$ & $\begin{array}{l}\text { Laser Scanning } \\
\text { 3D method }\end{array}$ & $\begin{array}{l}\text { Circumferential and volume parameters } \\
\text { were defined to compare CTM to 3DCI. }\end{array}$ & $\begin{array}{l}\text { Good correlation between } C T M \text { and } 3 D C I \text { on the affected } \\
\left(\mathrm{R}^{2}=0.74 ; \mathrm{p}<0.05\right) \text { and unaffected side }\left(\mathrm{R}^{2}=0.76 ; \mathrm{p}<0.05\right) \text {. There } \\
\text { was a statistical difference between CTM and 3DCI for } \\
\text { circumference and volume on the affected side and } \\
\text { circumference on the unaffected side. Interestingly, total } \\
\text { volumes were identical on the unaffected side. }\end{array}$ \\
\hline $\begin{array}{l}\text { Karakashian } \\
\text { et al. } \\
\text { (22) }\end{array}$ & 2018 & $\begin{array}{l}24 \text { women } \\
\text { with BCRL; } \\
7 \text { healthy } \\
\text { adults }\end{array}$ & $\begin{array}{l}\text { Diagnostic Test } \\
\text { Study }\end{array}$ & $\begin{array}{l}\text { Asus Xtion Pro } \\
\text { 3D }\end{array}$ & $\begin{array}{l}\text { Healthy group: one arm measured with } \\
\text { 3DCI to determine repeatability and } \\
\text { reliability } \\
\text { BCRL group: Both arms were measured } \\
\text { once by 3DCI and } 4 \mathrm{~cm}-\mathrm{CTM} \text {. Volume and } \\
\text { shape were analysed. Meshlab was used for } \\
\text { analysing 3DCI data. }\end{array}$ & $\begin{array}{l}\text { Healthy group: Repeatability mean volume was } 2.52 \text { litres (SD } \\
0.03 \text { ) with a coefficient of variation } 0.99 \% \text {. Speed of camera did } \\
\text { not influence the outcome. Reliability had ICC }(3,1) \text { of } 0.95 \text {. 3DCI } \\
\text { integral formula underestimated volume compared to CTM. } \\
\text { Shape related metrics such as circumference and circularity were } \\
\text { used to distinguish between LO and unaffected arms ( } \mathrm{p} \leq 0.05 \text { ). } \\
\text { Radial and oedema maps were developed using colour coding to } \\
\text { identify deviation of LO shape compared to unaffected side }\end{array}$ \\
\hline
\end{tabular}




\begin{tabular}{|c|c|c|c|c|c|c|}
\hline $\begin{array}{c}\text { Author } \\
(\text { CASP Score })\end{array}$ & Year & Cohort & $\begin{array}{l}\text { Research } \\
\text { Design }\end{array}$ & 3DCI System & Method & Primary Results \\
\hline $\begin{array}{l}\text { Landau et al. } \\
\text { (22) }\end{array}$ & 2018 & $\begin{array}{l}11 \text { women } \\
\text { with BCRL }\end{array}$ & $\begin{array}{l}\text { Diagnostic Test } \\
\text { Study }\end{array}$ & Vectra 3D & $\begin{array}{l}\text { Full arm 3D image captured with volumes } \\
\text { analysed by Mirror software. } 4 \mathrm{~cm}-\mathrm{CTM} \\
\text { measured from ulnar styloid to upper arm } \\
\text { mark, and WD measured to upper arm mark. } \\
\text { All methods were timed. }\end{array}$ & $\begin{array}{l}\text { 3DCI correlated well with CTM }\left(\mathrm{R}^{2}=0.99\right) \text { and had good } \\
\text { agreement with mean \% VD of } 2.8 \%(\mathrm{SD} 2.0) \text {. 3DCI and WD } \\
\text { correlated well }\left(\mathrm{R}^{2}=0.99\right) \text { with good agreement of } \% \mathrm{VD} \text { at } 2.0 \% \\
\text { (SD 2.1). Time efficiency indicated 3DCI needed } 9.8 \text { min less } \\
\text { than CTM to perform, and } 19.0 \text { min less than WD. }\end{array}$ \\
\hline $\begin{array}{l}\text { Preuß et al. } \\
(22)\end{array}$ & 2018 & $\begin{array}{l}37 \text { women } \\
\text { with BCRL }\end{array}$ & $\begin{array}{l}\text { Diagnostic Test } \\
\text { Study }\end{array}$ & Vectra 3D & $\begin{array}{l}\text { Both arms were measured twice using } 3 \mathrm{DCI} \\
\text { and WD over two sessions. Volumes were } \\
\text { analysed using Mirror software }\end{array}$ & $\begin{array}{l}\text { Validity: both healthy and LO arm correlations were } 1.00 \text {. WD } \\
\text { measured larger volumes than 3DCI. Paired-samples } t \text {-test } \\
\text { shows VD not significant for healthy arms ( }=0.06 \text { ), but } \\
\text { significant for LO arms }(\mathrm{p}<0.001) \text {. } \\
\text { Reliability: Intra-rater ICC was } 1.00 \text { for both arms. Paired- } \\
\text { samples } t \text {-test showed no significant VD between sessions. }\end{array}$ \\
\hline $\begin{array}{l}\text { Verhulst et } \\
\text { al. } \\
\text { (17) }\end{array}$ & 2017 & $\begin{array}{l}10 \text { healthy } \\
\text { adults }\end{array}$ & $\begin{array}{l}\text { Diagnostic Test } \\
\text { Study }\end{array}$ & $\begin{array}{l}\text { 3D } \\
\text { stereophoto- } \\
\text { grammetry }\end{array}$ & $\begin{array}{l}\text { Landmarks placed using 3D-printed device } \\
\text { to identify the wrist. The hand and forearm } \\
\text { of each subject were measured twice by first } \\
\text { rater and once by a second rater. Images } \\
\text { were analysed using } 3 \mathrm{ds} \text { Max } 2015 \text { software. }\end{array}$ & $\begin{array}{l}\text { Reproducibility mean difference was } 0.6 \mathrm{ml}(\mathrm{SD} 7.6) \text { and paired- } \\
\text { samples } t \text {-test had no significant difference between } \\
\text { measurements }(\mathrm{p}=0.75) \text {. Hand volume difference for intra-rater } \\
\text { was } 1 \mathrm{ml}(\mathrm{SD} 10.2) \text {, and between raters was } 1.4 \mathrm{ml} \text { (SD 9.2). } \\
\text { Paired-samples } t \text {-test showed no significance for intra-rater } \\
(\mathrm{p}=0.50) \text { measurements. ICC for both intra- and inter-rater } \\
\text { reliability was excellent (ICC }=0.99) \text {. }\end{array}$ \\
\hline $\begin{array}{l}\text { Cau et al. } \\
(21)\end{array}$ & 2016 & $\begin{array}{l}12 \text { healthy } \\
\text { adults }\end{array}$ & $\begin{array}{l}\text { Diagnostic Test } \\
\text { Study }\end{array}$ & $\begin{array}{l}\text { Laser Scanning } \\
\text { 3D method }\end{array}$ & $\begin{array}{l}\text { Comparison of reliability and correlation } \\
\text { between } 4 \mathrm{~cm}-\mathrm{CTM} \text { and } 3 \text { DCI. One arm } \\
\text { measured twice by each of two raters for } \\
\text { both } 4 \mathrm{~cm}-\mathrm{CTM} \text { and 3DCI. Data were } \\
\text { processed using Rodin4D software. }\end{array}$ & $\begin{array}{l}\text { Poor statistical data. Reliability (intra and inter) reported as } \\
\text { excellent, but no ICC given. Reproducibility very high with a } \\
\text { difference of } 0.02 \mathrm{dm}^{3} \text { between raters. Validity: correlation was } \\
0.92(\mathrm{p}<0.05) \text {. Bland-Altman plot demonstrates consistency } \\
\text { between measures with a mean difference of } \pm 1.96 \text {. }\end{array}$ \\
\hline $\begin{array}{l}\text { Hameeteman } \\
\text { et al. } \\
(22)\end{array}$ & 2016 & $11 \mathrm{BCRL}$ & $\begin{array}{l}\text { Diagnostic Test } \\
\text { Study }\end{array}$ & $\begin{array}{l}\text { 3D stereophoto- } \\
\text { grammetry }\end{array}$ & $\begin{array}{l}\text { Both arms measured twice by WD and 3DCI } \\
\text { to determine use of 3DCI in volume } \\
\text { measurements for arms. Volume } \\
\text { determined using Autodesk 3ds Max } 2012 \\
\text { software. }\end{array}$ & $\begin{array}{l}\text { Mean VD was } 13 \mathrm{ml} \text { (SD 15.6). Paired-sample t-test showed no } \\
\text { significant difference ( } \mathrm{p}=0.33 \text { ). Pearson's correlation coefficient } \\
\text { was 0.99 ( } \mathrm{p}=0.01) \text {. Bland-Altman plot indicates strong } \\
\text { correlation between methods but shows higher LOA between } \\
\text { 3DCI than WD measurements. 3DCI overestimates volume by } \\
11 \mathrm{ml} \text { (SD 77). Intra-class correlation coefficient was high: } 1.00\end{array}$ \\
\hline $\begin{array}{l}\text { Hoevenaren } \\
\text { et al. } \\
(21)\end{array}$ & 2016 & $\begin{array}{l}18 \text { women } \\
\text { with BCRL; } \\
9 \text { healthy } \\
\text { control } \\
\text { group }\end{array}$ & $\begin{array}{l}\text { Diagnostic Test } \\
\text { Study }\end{array}$ & $\begin{array}{l}\text { 3D } \\
\text { stereophoto- } \\
\text { grammetry }\end{array}$ & $\begin{array}{l}\text { 3DCI of both hands were captured using } \\
\text { study protocol to investigate the use of } 3 \text { DCI } \\
\text { as a reproducible and useful tool for } \\
\text { measuring LO of the hand. Data were } \\
\text { analysed using } 3 \text { ds Max } 2015 \text { software. }\end{array}$ & $\begin{array}{l}\text { Mean VD between hands was } 73 \mathrm{ml} \text { (SD 64). Healthy control } \\
\text { group had mean difference of } 8 \mathrm{ml} \text { with dominant hand bigger. } \\
\text { Reproducibility showed average difference of } 5.2 \mathrm{ml} \text { (SD 5.2) } \\
\text { with statistically significant difference in hand volume between } \\
\text { groups (chi-square }=18.9 ; \mathrm{p}<0.00 \text { ). }\end{array}$ \\
\hline $\begin{array}{l}\text { Buffa et al. } \\
(20)\end{array}$ & 2015 & $\begin{array}{l}30 \text { healthy } \\
\text { adults }\end{array}$ & $\begin{array}{l}\text { Diagnostic Test } \\
\text { Study }\end{array}$ & $\begin{array}{l}\text { Microsoft } \\
\text { Kinect }^{\mathrm{TM}} \\
\text { (SkanLab) }^{\text {Ska }}\end{array}$ & $\begin{array}{l}\text { Two raters measured one arm twice with } \\
\text { both WD and 3DCI. Plastic cylinders were } \\
\text { also measured. Study done to determine }\end{array}$ & $\begin{array}{l}\text { Both raters had similar results with bias range of } 21.7 \mathrm{ml} \text { and } \\
22.2 \mathrm{ml} \text {. 3DCI slightly underestimates volume compared to WD. } \\
\text { Linear regression shows no significant relationship between }\end{array}$ \\
\hline
\end{tabular}




\begin{tabular}{|c|c|c|c|c|c|c|}
\hline $\begin{array}{c}\text { Author } \\
\text { (CASP Score })\end{array}$ & Year & Cohort & $\begin{array}{l}\text { Research } \\
\text { Design }\end{array}$ & 3DCI System & Method & Primary Results \\
\hline & & & & & $\begin{array}{l}\text { accuracy and reliability of 3DCI for both } \\
\text { human arms and inanimate objects. All } \\
\text { measurements were timed. Data were } \\
\text { analysed using MeshLab software. }\end{array}$ & $\begin{array}{l}\text { volume and accuracy; however, highly significant relationship } \\
\text { between BMI and accuracy was found }(\mathrm{p}=0.004) \text {. Intra- and } \\
\text { inter-rater reliability of } 1.00 \text {. SEM between methods was } \\
\text { similar. Duration of tests was higher for WD }(2 \mathrm{~min} 3 \mathrm{sec}) \text { than } \\
\text { 3DCI }(42 \mathrm{sec})(\mathrm{p}<0.00) \text {. }\end{array}$ \\
\hline $\begin{array}{l}\text { Erends et al. } \\
\text { (19) }\end{array}$ & 2014 & $\begin{array}{l}33 \text { healthy } \\
\text { adults: } \\
20 \text { female } \\
13 \text { men }\end{array}$ & $\begin{array}{l}\text { Diagnostic Test } \\
\text { Study }\end{array}$ & Vectra XT & $\begin{array}{l}\text { Two raters measured the right arm of each } \\
\text { subject twice with both WD and 3DCI. The } \\
\text { 3DCI images were viewed and analysed } \\
\text { using Mirror software allowed for merging } \\
\text { of the anterior and posterior views taken } \\
\text { with 3DCI. The study was done to determine } \\
\text { the validity and reliability of 3DCI volume } \\
\text { measurements compared to WD. }\end{array}$ & $\begin{array}{l}\text { Validity: Correlation was } 0.98 \text {. 3DCI volumes were higher than } \\
\text { WD with mean VD of }-13.8 \mathrm{ml} \text { (SD 59.3). Paired-sample } t \text {-tes } \\
\text { showed no significant difference between methods (p=0.19). } \\
\text { Reliability: Intra-rater reliability was } 0.99 \text {. Paired-sample } t \text {-tes } \\
\text { showed no significant difference between two 3DCl } \\
\text { measurements }(0.67) \text {, with a mean volume difference of }-1.39 \mathrm{~m} \\
\text { (SD 18.8). Strong agreement between measures on Bland- } \\
\text { Altman plot: differences ranging from }-30.0 \text { to } 30.0 \mathrm{ml} \text {. }\end{array}$ \\
\hline $\begin{array}{l}\text { Lu et al. } \\
\text { (19) }\end{array}$ & 2014 & $\begin{array}{l}6 \\
\text { participants } \\
\quad \text { for } \\
\text { perometer; } \\
7 \text { for WD }\end{array}$ & $\begin{array}{l}\text { Diagnostic Test } \\
\text { Study }\end{array}$ & $\begin{array}{l}\text { Microsoft } \\
\text { Kinect }^{\mathrm{TM}}\end{array}$ & $\begin{array}{l}\text { System validated using a PVC pipe. Both } \\
\text { arms measured twice by perometer and } \\
\text { 3DCI. Localised swelling determined by } \\
\text { taping a pen to the forearm of one subject. } \\
\text { Both arms measured by 3DCI and WD twice } \\
\text { in sets of three with a 2-hour time-lapse. }\end{array}$ & $\begin{array}{l}\text { 3DCI overestimated volume compared to perometry with a bias } \\
\text { error ranging from }-0.4 \text { to } 7.8 \% \text {. 3DCI is sensitive to pick up } \\
\text { small change in volume of less than } 1 \% \text {. 3DCI overestimates } \\
\text { volume compared to WD with a bias error ranging from }-7.51 \text { to } \\
8.83 \% \text {; however, the mean volume difference between the two } \\
\text { methods was } 8.35 \mathrm{ml} \text {. Correlation was } 0.98 \text {. }\end{array}$ \\
\hline $\begin{array}{l}\text { Öhberg et al. } \\
(20)\end{array}$ & 2014 & $\begin{array}{l}25 \text { people } \\
\text { with LO }\end{array}$ & $\begin{array}{l}\text { Diagnostic Test } \\
\text { Study }\end{array}$ & $\begin{array}{l}\text { Static 3D } \\
\text { Microsoft } \\
\text { Kinect }^{\mathrm{TM}} \\
\text { system }\end{array}$ & $\begin{array}{l}\text { Circumference and volume of both arm } \\
\text { measured by WD, CTM and 3DCI by one of } \\
\text { two physiotherapists to evaluate the } \\
\text { performance of 3DCI against commonly } \\
\text { used CTM. MATLAB was used to analyse } \\
\text { the image data. }\end{array}$ & $\begin{array}{l}\text { No statistically significant difference between the methods } \\
(\mathrm{p}>0.05) \text {. A systematic bias between therapists was observed } \\
(\mathrm{p}=0.02) \text {. For circumference, 3DCI systematically differed to } \\
\text { CTM }(\mathrm{p}=0.002) \text { with increased disagreement the higher up the } \\
\text { limb measurements were taken. Inter- }(0.98, \mathrm{p}<0.001) \text { and intra- } \\
\text { rater }(0.99, \mathrm{p}<0.001) \text { reliability was excellent }\end{array}$ \\
\hline $\begin{array}{l}\text { Lu et al. } \\
\text { (18) }\end{array}$ & 2013 & $\begin{array}{l}6 \text { people: } \\
5 \text { healthy; } \\
1 \text { BCRL }\end{array}$ & $\begin{array}{l}\text { Diagnostic Test } \\
\text { Study }\end{array}$ & $\begin{array}{l}\text { Microsoft } \\
\text { Kinect }^{\mathrm{TM}}\end{array}$ & $\begin{array}{l}\text { Compared 3DCI to perometer in first } \\
\text { experiment; then determined accuracy and } \\
\text { ability of 3DCI to detect localised swells. } \\
\text { Localised swelling was tested by taping a } \\
\text { pen to the inner forearm of a subject }\end{array}$ & $\begin{array}{l}\text { 3DCI overestimates volume compared to perometry with a } \\
\text { percentage discrepancy ranging from } 4.7 \text { to } 12.8 \% \text {. 3DCI was } \\
\text { able to detect a change in volume as small as } 0.5 \% \text { of the arm } \\
\text { volume. }\end{array}$ \\
\hline
\end{tabular}

Key:

3D $=$ Three Dimensional

$\mathrm{BCRL}=$ Breast Cancer-Related Lymphoedema $\mathrm{LO}=$ Lymphoedema

$\mathrm{VD}=$ Volume Difference
3DCI $=$ Three-Dimensional Camera Imaging

$\mathrm{CI}=$ Confidence Interval

LOA = Limits of Agreemen

WD $=$ Water Displacement
$\% \mathrm{VD}=$ Percentage Volume Difference

$\mathrm{CTM}=$ Circumferential Tape Measurement $\mathrm{SD}=$ Standard Deviation
$\mathrm{BC}=$ Breast Cancer

ICC = Intra-class Correlation Coefficient $\mathrm{SEM}=$ Standard Error of Measurement 
Öhberg et al. (2014) compared a 3DCI system to water displacement and CTM to calculate upper limb volume. The 3DCI set up involved three fixed cameras within a predefined frame with a positional laser mounted to mark the distal end of the measurement space (Figure 15). They found that 3DCI volumes were overestimated compared to water displacement and that CTM volumes were underestimated compared to water displacement. Öhberg et al. (2014) confirmed recommendations of measures not being interchangeable, even though they correlated well. They concluded that 3DCI offered a modern alternative to CTM.

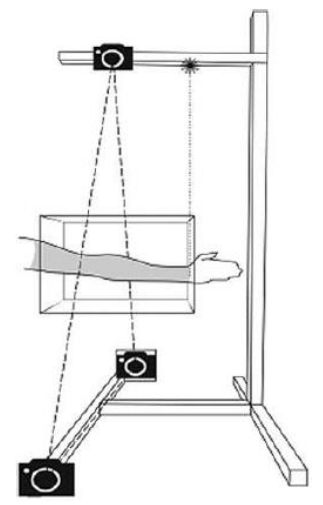

Figure 15: Öhberg et al.'s 3D camera system (2014). Utilises three Microsoft KinectTM cameras ( 0 ) with the 'box' indicating the measurement subspace aligned by a laser'

Öhberg et al. (2014) acknowledged that using 3DCI within a clinical setting was uncertain but that it could be an invaluable educative tool for patients as it would provide a graphical representation of how the shape of the limb changes over time. These visual changes could potentially motivate patients to be more concordant with their lymphoedema self-management, as they would be able to understand visually where the lymphoedema was improving or changing. They noted that at the time of their paper going to print that a newer model of 3D camera was to be launched later that year with a system using only a single handheld camera to perform volumetric measurements as opposed to their static three camera set up.

The Microsoft Kinect ${ }^{\mathrm{TM}}$ is a single unit combination device consisting of an RGB camera (a camera that delivers red, green and blue components) with an infrared depth sensor. This camera is used in the computer gaming industry, most notably XBox.

\footnotetext{
${ }^{3}$ Reprinted from Lymphatic Research and Biology, volume 12, issue 4, Öhberg et al., Three-Dimensional Camera System for Measuring Arm Volume in Women with Lymphoedema Following Breast Cancer Treatment, pages 267-274., Copyright (2014), with permission from LiebertPub
} 
Cook, Couch, Couch, Kim, \& Boonn (2013) did a proof of concept and initial validation of the Kinect camera to determine radiation dose monitoring by more accurately estimating patient volume thus enabling better dose prescription for CT scanning. Henseler, Kuznetsova, Vogt, \& Rosenhahn (2014) validated the Microsoft Kinect ${ }^{\mathrm{TM}}$ device for $3 \mathrm{D}$ breast assessment with the potential for better surgical outcomes for breast reconstruction surgery.

Lu et al. (2014) compared water displacement and perometry to a new algorithm for their 3DCI utilising the Microsoft Kinect ${ }^{\mathrm{TM}}$. Their algorithm, the Iterative Clustered Closest Points, required the 3DCI to be held $80 \mathrm{~cm}$ from the target limb to capture the image most accurately. They mobilised the 3DCI around the limb by attaching a gyroscope and accelerometer to the camera. The optimal position of the model's arm was at $90^{\circ}$ abduction. They report that the Kinect device is 'biased' by $5.16 \%$ and thus they 'corrected' their outputs accordingly.

Concurrently, Erends, van der Aa, van der Hulst, \& de Grzymala (2014), did a validity and reliability study for measuring upper limb volume on healthy participants. They compared their Vectra XT 3D imaging system (Canfield Imaging Systems, Fairfield, NJ) to water displacement. The Vectra XT 3D imaging system comprised of six digital single-lens reflex cameras divided over three stations with four flashes mounted within the Vectra unit (Figure 16). This system captures images in $180^{\circ}$; thus, the anterior and posterior sides have to be captured separately and then 'stitched' together. Within a clinical setting, this would be a time-consuming procedure marking up the person's arm on both sides and then ensuring the images align when they are amalgamated and analysed.

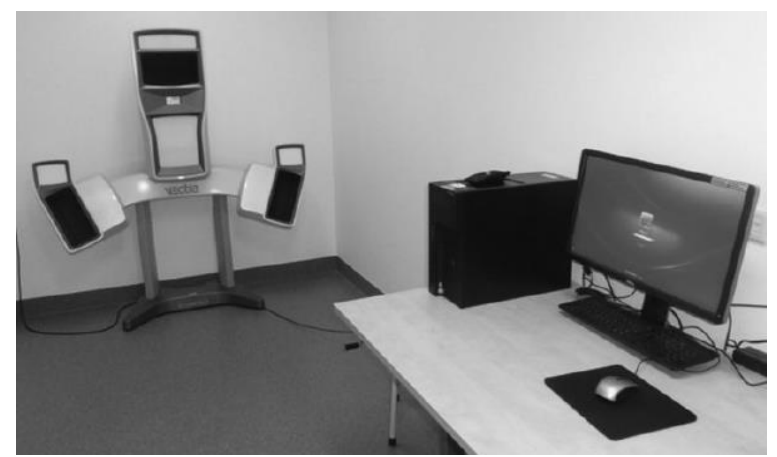

Figure 16: Vectra XT 3D imaging system (Erends et al., 2014) 


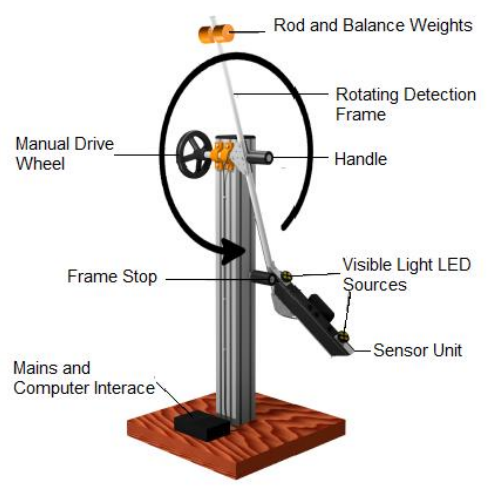

Figure 17: SkanLab system: Microsoft KinectTM camera attached to a rotating frame (Buffa et al., 2015)

Buffa et al. (2015) developed a SkanLab system utilising the Microsoft Kinect ${ }^{\mathrm{TM}}$ camera on a rotating stand (Figure 17) and using Skanect (Occipital, San Francisco, USA) software together with MeshLab (Visual Computing Lab, Pisa, Italy) to interpret the images. Time taken to perform each measure showed that 3DCI was slightly faster than water displacement $(\mathrm{p} \approx 0.00)$ at about $2.5 \mathrm{~min}$. They conclude that 3DCI may be useful for monitoring latent lymphoedema and early intervention due to its high accuracy in terms of bias $(0.6 \%)$ and limits of agreement $(-2.6 \%$ to $1.4 \%)$.

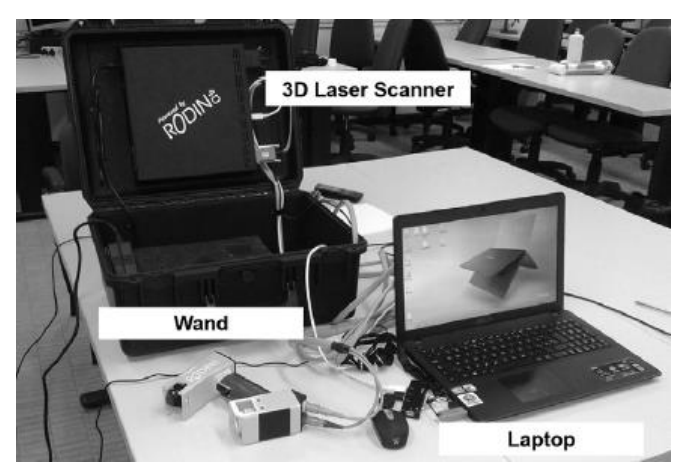

Figure 18: Laser Scanner 3D method (Cau et al., 2016). The system consists of a laser scanner (wand probe), a transmitter and a signal processing unit ${ }^{4}$

Cau et al. (2016) compared CTM to the laser scanner 3D method (O\&P Scan Rodin4D, Pessac, France). This particular device (Figure 18) is cited as being used in orthopaedics, on the design of orthoses, and other health and well-being applications as it has demonstrated accuracy and reproducibility compared to water displacement. The wand is kept at a fixed distance of one meter on a stand where the hand is supported and

\footnotetext{
${ }^{4}$ Reprinted from Journal of Vascular Surgery: Venous and Lymphatic Disorders, volume 4, issue 1, Cau et al., Comparative study between circumferential method and laser scanner 3D method for the evaluation of arm volume in healthy subjects, pages 64-72., Copyright (2016), with permission from Elsevier
} 
moved back and forth along a single scanning plane. Cau et al. (2016) report that 3DCI has the potential to measure geometric parameters and shape information of scanned limbs.

A 3D stereophotogrammetry system (3dMD Cranial, Atlanta, USA - Figure 19) was studied by Hoevenaren et al. (2016) initially looking at hand volume between healthy and lymphoedema hands. They found a statistically significant difference in hand volume between the groups $(\mathrm{p}<0.00)$, with an average volume difference of $73 \mathrm{ml}$ (22.5\%). Hand dominance may need to be accounted for as there was an $8 \mathrm{ml}(2.7 \%)$ difference in hand volumes in the healthy group. They concluded that 3DCI could be used for quantifying hand volumes as it is accurate and reproducible. Hameeteman et al. (2016) compared the 3D stereophotogrammetry (3dMD Cranial, Atlanta, USA Figure 20) to water displacement. They performed two landmark-based cut-off images of the forearm and upper arm from which volumes were calculated and added together for total arm volume. They conclude that 3DCI is an accurate and reliable method for measuring upper limb volume and can be used for early detection of a change in limb volume, and for monitoring conservative and surgical treatment outcomes.

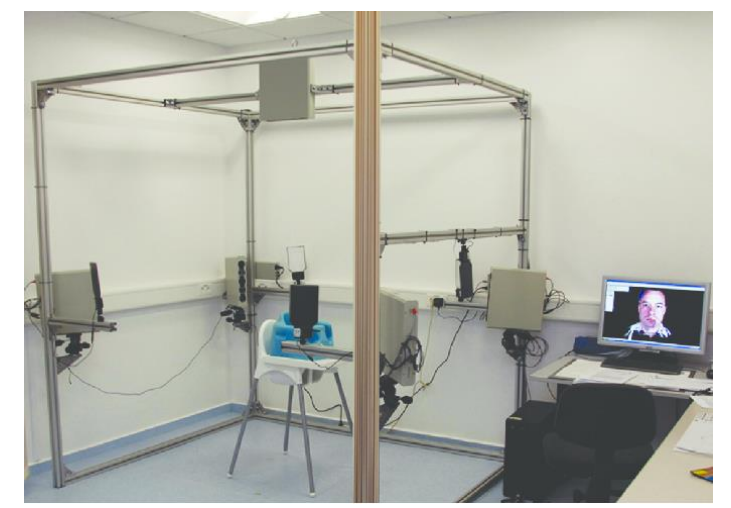

Figure 19: 3D Stereophotogrammetry setup: $3 \mathrm{dMD} 囚$ cranial system is a 5-pod system with 15 cameras to capture 3D images (Schaaf, 2009)

Karakashian et al. (2018) used a commercially available ASUS Xtion Pro 3D (A.X.Pro3D-ASUS, Taiwan) depth-sensing camera in conjunction with software from RecFusion (ImFusion, Munich, Germany). This camera is a single unit that is mounted on a camera tripod stand with a ball joint and an attached selfie stick with a 3D printed mount, and with the participant seated with their arm abducted, palm down, to shoulder height 
(Figure 20). Volume measurements were compared to $4 \mathrm{~cm}-\mathrm{CTM}$ as used in a clinical setting.

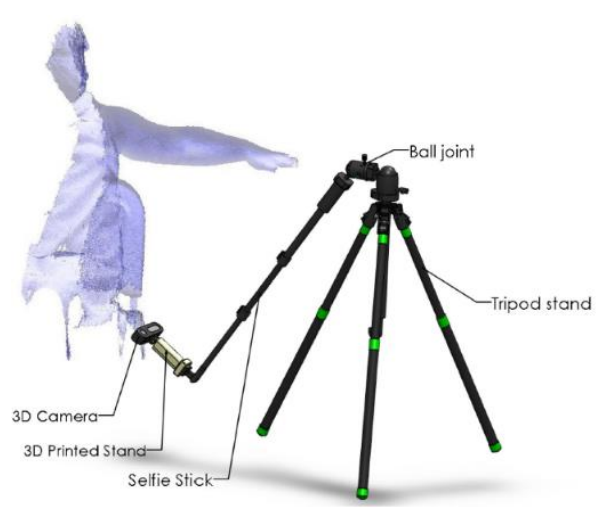

Figure 20: ASUS Xtion Pro 3D camera system (Karakashian et al., 2018). The 3D camera is attached to a selfie stick via a 3D printed mount and rotated using a tripod stand with a ball joint. The subject sits with the arm abducted, palm down, to shoulder height, with fingers in line with the ball joint of the tripod stand.

Data from the 3D images were selected, 'cleaned' from the surrounding clinical environment and cropped at the wrist and upper arm to isolate the area to be measured (Figure 21) (Karakashian et al., 2018). Volume calculations for this 3DCI method utilised the 'integral' formulae where triangle $j$ is one of the triangles describing the triangulated arm surface as

$$
V_{\text {integral }} \approx \sum_{j=1}^{N_{f}} \sum_{i=1}^{N_{d}} F_{j, i} n_{j, i} A_{j}
$$

where $N_{\mathrm{f}}$ is the number of vertices and $N_{\mathrm{d}}$ is the number of dimensions (Karakashian et al., 2018). $F$ is a vector field, $n$ is the surface normal and $\mathrm{A}_{\mathrm{j}}$ is the area of triangulation. The integral equation is considered more accurate than both the frustum and cylinder methods in the calculation of limb volume as the latter make simplified assumptions on the geometry, whilst the former takes into consideration the true limb shape (Karakashian et al., 2018).

The data was further extrapolated to produce radial maps where the affected and unaffected maps were subtracted (after mirroring one of the two) to produce an 'oedema distribution' map, i.e. swelling map (Figure 22). These maps are colour coded so that red demonstrates areas of greater deviance from the 'normal' thus indicating the location of the lymphoedema, cyan represents the correlation of size between the affected and 
unaffected limbs, and dark blue indicates areas smaller than the unaffected side. The maps clearly show that the lymphoedema is not homogeneously distributed along the arm, which is verified by the circumferential measurement data and cross-sectional area distributions from the 3D images. This study is the first of its kind and can be linked to the suggestions made by Öhberg et al. (2014) and Cau et al. (2016) to investigate limb shape as a tool for determining lymphoedema distribution. It has the potential to create new thresholds for the diagnosis of lymphoedema using topology and shape as opposed to volume.

A

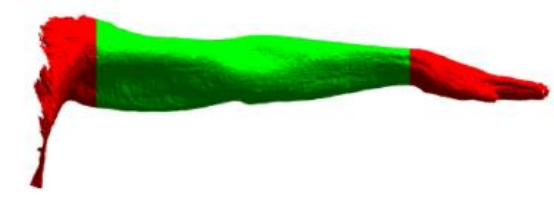

B

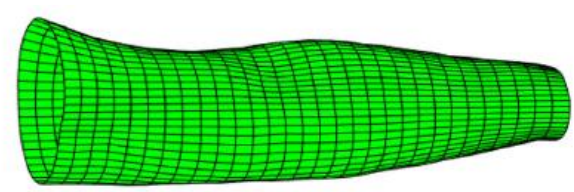

Figure 21: Preparation of 3D image to convert into limb volume data and radial maps (Karakashian et al., 2018). (A) Area selected (green) for analysis using an STL file to triangulate the surface. (B) The cropped arm mapped on a regular grid after being 'cleaned' from the surrounding clinical environment and unwanted body parts.
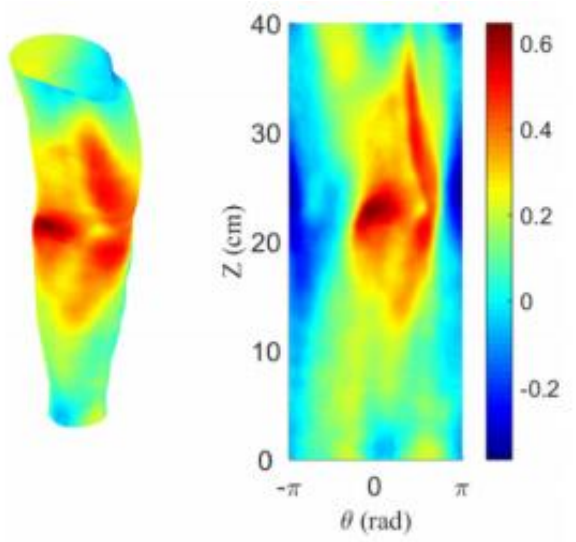

Figure 22: Swelling map in lymphoedema (Karakashian et al., 2018). The relative radial changes between the two arms are projected as a 'new arm' (left) and mapped as a $\theta$ - z graph (right). The colour scale changes from red (positive for swelling) to blue (negative difference) enabling the identification of localised oedema.

Karakashian, Pike, \& van Loon (2019) consequently utilised the 3D images and computational modelling to elucidate the effect that limb shape may have on subbandage /sub-garment and subcutaneous pressures, stresses and strains. This study demonstrated a large variation in pressures across each arm under the compression 
garment, with a maximum sub-garment pressure of 5,100 Pascal (Pa), exceeding the intended sub-garment pressure of 2,500 $\mathrm{Pa}$. Furthermore, a positive pressure gradient was found in the forearm, which is counterproductive to the required negative pressure gradient for lymph drainage. This study demonstrated how technology could be utilised in garment design and treatment strategies.

Of interest, Gonzalez-Jorgea, Riveirob, Vazquez-Fernandezc, Martinez-Sáncheza, \& Ariasa (2013) confirmed that the Microsoft Kinect ${ }^{\mathrm{TM}}$ and ASUS Xtion Pro 3D are very similar camera systems suitable for close-range imaging. However, an article published by Good on $25^{\text {th }}$ October 2017 has alerted the public to the demise of the Kinect camera. According to Good (2017), Microsoft has confirmed that they are no longer manufacturing the Kinect camera due to the evolution of the Xbox consoles and the rumoured ability of the camera to spy on individuals. Thus researchers who had hoped to expand the use of this camera system into a clinical setting will have to source a new $3 \mathrm{D}$ camera system and validate its use.

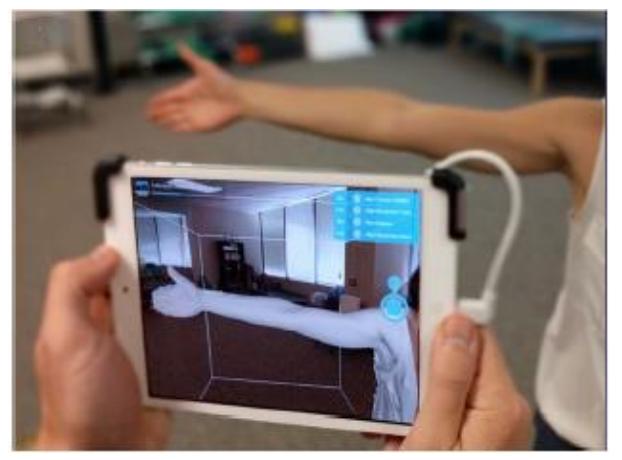

Figure 23: LymphaTech system: compatible depth sensor camera is attached to an iPad tablet via a custom bracket and integrates with the built-in camera of the device via the custom bracket to overlay the depth and colour imaging (Binkley et al., 2020) ${ }^{5}$

The newest method of 3DCI technology is the LymphaTech system (Binkley et al., 2020) which consists of a commercially available depth camera (Structure Sensor; Occipital, Inc, Boulder, CO, USA) interfaced with a smartphone or tablet computer (Figure 24). Currently, compatibility is limited to iOS devices, but there are plans to expand it to Android and Windows devices. Two software programmes were developed supporting the implementation of the hardware, to provide application and visual cues

5 Binkley et al., Assessing Arm Volume in People During an After Treatment for Breast Cancer: Reliability and Convergent Validity of the LymphaTech System, Physical Therapy, 2020, volume 100, issue 3, pages 1-11, by permission of Oxford University Press 
to capture the image initially, and secondly to carry out the image processing and analysis to quantify and track patient metrics. The area can be scanned at a distance of 0.5 to $1.5 \mathrm{~m}$. This device was compared to perometry by comparing limb volume and limb volume differences. Binkley et al. (2020) concluded that their 3DCI provided similar limb volumes to perometry and found that the results supported the reliability and validity of their LymphaTech system.

\subsubsection{Thresholds for Diagnosing Lymphoedema Using 3DCI}

Threshold testing was not considered in any of the studies, with only one study referencing a $10 \%$ volume difference or a $200 \mathrm{ml}$ difference between arms as diagnostic criteria for lymphoedema through water displacement as their comparator (Hammeteman et al. 2017).

\subsubsection{Validity and Reliability of 3DCI}

\section{Concurrent Validity:}

Eight studies report on concurrent validity with correlations between 3DCI and CTM, perometer, or water displacement of 0.94 (range 0.74-0.99) (Erends et al., 2014; Lu et al., 2014; Cau et al., 2016; Hameeteman et al., 2016; Cau et al., 2018; Landau et al., 2018; Preu $\beta$, Killaars, de Grzymala, Binnebösel, \& Neumann, 2018; Lu \& Dixon, 2019).

\section{Reliability:}

Eight papers reported excellent ICC values for 3DCI. Pooled ICC data showed an ICC $_{\text {intra value of }} 0.99$ (95\% CI=0.95, 1.00) (Erends et al., 2014; Öhberg et al., 2014; Buffa et al., 2015; Hameeteman et al., 2016; Verhulst et al., 2017; Karakashian et al., 2018; Preuß et al., 2018; Binkley et al., 2020) and an $\mathrm{ICC}_{\text {inter }}$ value of $1.00(95 \%$ $\mathrm{CI}=0.98,0.99$ ) (Buffa et al., 2015; Verhulst et al., 2017). The weighted mean SEM was $1.7 \%(\sigma=2.5 \%)$ and the weighted mean SDC was 4.7\% ( $\sigma=6.9 \%)$ (Lu et al., 2013; Buffa et al., 2015; Landau et al., 2018; Preuß et al., 2018; Lu \& Dixon, 2019; Binkley et al., 2020).

\subsubsection{Benefits and Considerations of 3DCI}

Advantages of using 3DCI are that it is a simple, fast, accurate, easy to use and safe means of providing limb volumetric measurements (Öhberg et al., 2014; Buffa et al., 2015; Hoevenaren et al., 2015; Cau et al., 2016; Cau et al., 2018; Landau et al., 2018; 
Binkley et al., 2020). It requires minimal training to operate (Lu et al., 2014; Öhberg et al., 2014; Verhulst et al., 2017), and the single-camera 3DCI devices are portable and relatively inexpensive, especially compared to the perometer ( $\mathrm{Lu}$ et al., 2014; Buffa et al., 2015; Cau et al., 2016; Verhulst et al., 2017; Cau et al., 2018; Binkley et al., 2020). As 3DCI is a non-invasive, contactless technique, there are no known contra-indications for its use; thus, it can be safely used in the presence of lesions, wounds and cellulitis (Erends et al., 2014; Cau et al., 2016; Hameeteman et al., 2016; Cau et al., 2018; Landau et al., 2018). 3DCI could also be used in clinical practice to inform garment design (Karakashian, Pike, \& van Loon, 2019).

3DCI measures less than $1 \mathrm{ml}$ variations in volume (Lu et al., 2013; Lu et al., 2014; Cau et al., 2016; Karakashian et al., 2018) and is recommended for use in the early detection of lymphoedema as it is an accurate measure which can also be used for self-monitoring of limb volume (Lu et al., 2014). 3DCI can capture the shape of the limb and quantify the differences in shape between the arms (Karakashian et al., 2018). Thus a further benefit of 3DCI is that it can be used as an educative tool for patients and therapists as they can visualise the effects of treatment and may become a motivational tool for treatment concordance (Öhberg et al., 2014; Karakashian et al., 2018).

A disadvantage of 3DCI is that not every clinic has the availability to a 3DCI system and trained staff to perform the measurement using the more complex 3DCI systems such as Vectra 3D Camera systems and 3D stereophotogrammetry (Hameeteman et al., 2016; Hoevenaren et al., 2016; Verhulst et al., 2017). Furthermore, people with limited shoulder range of movement may not be able to position their arm to accommodate this measurement.

As 3DCI is a form of photography, a therapist would need to gain written consent from the patient before their limbs are measured. This is not considered a disadvantage but a safeguard, to ensure the patient would be willing for their photographic data to be stored electronically. All NHS Health Boards have photographic consent form templates available from their Medical Photography Departments to facilitate this. 


\subsection{Discussion}

Table 18 provides a summary of the measurement properties analysed for this systematic review. The findings show clear evidence for excellent validity and reliability of CTM, perometry and 3DCI in measuring upper limb volume. Pooled results for the reliability of the measurements are summarised in Table 19. 3DCI had the lowest variance, SEM and SDC in the measurement of the upper limb, but these differences were small.

Table 18: Summary of the measurement properties of included studies

\begin{tabular}{|c|c|c|c|c|c|c|}
\hline \multirow[b]{2}{*}{ Study } & \multicolumn{4}{|c|}{ Reliability } & \multicolumn{2}{|c|}{ Validity } \\
\hline & ICC $_{\text {intra }}$ & ICC inter & SEM & SDC & $\begin{array}{c}\text { Concurrent } \\
\text { Validity } \\
\text { (Comparator } \\
\text { Instrument) }\end{array}$ & $\begin{array}{c}\text { Convergent } \\
\text { Validity }\end{array}$ \\
\hline & \multicolumn{6}{|c|}{ CTM } \\
\hline $\begin{array}{l}\text { De Vrieze et al. } \\
\text { (2019) }\end{array}$ & 0.99 & 0.98 & & & & \\
\hline Rafn et al. (2019) & 0.86 & 0.88 & $2.2 \%$ & $6.1 \%$ & $0.95(\mathrm{P})$ & \\
\hline Batista et al. (2018) & & & $-5 \%-6.8 \%$ & $\begin{array}{l}-13.9 \%- \\
18.8 \% \\
\end{array}$ & $0.86-0.99(\mathrm{P})$ & \\
\hline Sharkey et al. (2018) & 0.96 & & $1.2 \%$ & $3.3 \%$ & & \\
\hline Tewari et al. (2018) & & & & & $0.92(\mathrm{WD})$ & $\begin{array}{l}\text { Compare narrow } \\
\text { to wide tape: } 0.95\end{array}$ \\
\hline Cau et al. (2016) & & & $2.8 \%$ & $7.8 \%$ & 0.92 (3DCI) & \\
\hline Mori et al. (2015) & 0.98 & & & & $0.80(\mathrm{WD})$ & $\begin{array}{c}\text { Compare plastic } \\
\text { with paper tape: } \\
0.98\end{array}$ \\
\hline Tidhar et al. (2015) & & & $0.8 \%$ & $2.2 \%$ & & \\
\hline $\begin{array}{l}\text { Adriaenssens et al. } \\
\text { (2013) }\end{array}$ & 1.00 & & $2.2 \%$ & $6.1 \%$ & & \\
\hline $\begin{array}{l}\text { Brorson \& Hoijer } \\
\text { (2012) }\end{array}$ & & & $2 \%-3 \%$ & $5.5 \%-8.3 \%$ & $0.87(\mathrm{WD})$ & $\begin{array}{c}\text { Compare } 4 \mathrm{~cm} \text { vs } \\
\text { 5-point: } 0.87\end{array}$ \\
\hline $\begin{array}{l}\text { Katz-Leurer \& } \\
\text { Bracha (2012) }\end{array}$ & 0.99 & & $\begin{array}{c}45 \mathrm{ml}-66.6 \\
\mathrm{ml}\end{array}$ & $\begin{array}{c}124.7 \mathrm{ml}- \\
184.6 \mathrm{ml} \\
\end{array}$ & & \\
\hline Foroughi et al. (2011) & & & $0.8 \%-2 \%$ & $2.2 \%-5.5 \%$ & $0.84-0.94(\mathrm{P})$ & \\
\hline Czerniec et al. (2010) & 0.96 & & $7 \%$ & $19.4 \%$ & $0.99(\mathrm{P})$ & \\
\hline $\begin{array}{l}\text { Devoogdt et al. } \\
\text { (2010) }\end{array}$ & 1.00 & 0.99 & $1.3 \%-1.5 \%$ & $3.6 \%-4.2 \%$ & & \\
\hline \multirow[t]{2}{*}{ Chen et al. (2008) } & 0.99 & & & & & \\
\hline & \multicolumn{6}{|c|}{ Perometry $(\mathrm{P})$} \\
\hline Binkley et al. (2020) & 0.99 & & $1.3 \%-1.5 \%$ & $3.6 \%-4.2 \%$ & & \\
\hline $\begin{array}{l}\text { De Vrieze et al. } \\
(2019)\end{array}$ & 0.99 & 0.98 & & & & \\
\hline Spinelli et al. (2019) & 0.93 & 0.98 & & & & \\
\hline Sharkey et al. (2018) & 0.96 & & $0.8 \%$ & $2.2 \%$ & & \\
\hline Sun et al. (2018) & & & & & & $\begin{array}{c}\text { Full arm versus } \\
\text { simulated } 4 \mathrm{~cm} \text { and } \\
\text { 5-point: } 0.98\end{array}$ \\
\hline $\begin{array}{l}\text { Adriaenssens et al. } \\
\text { (2013) }\end{array}$ & 1.00 & & $2.2 \%$ & $6.1 \%$ & & \\
\hline Batista et al. (2013) & & & $0.9 \%$ & $2.5 \%$ & $\begin{array}{c}0.86-0.99 \\
(\mathrm{CTM})\end{array}$ & \\
\hline Bulley et al. (2013) & 0.98 & 0.98 & $6.9 \%-8.7 \%$ & $\begin{array}{l}19.1 \%- \\
24.1 \%\end{array}$ & & \\
\hline
\end{tabular}




\begin{tabular}{|c|c|c|c|c|c|c|}
\hline \multirow[b]{2}{*}{ Study } & \multicolumn{4}{|c|}{ Reliability } & \multicolumn{2}{|c|}{ Validity } \\
\hline & ICC $_{\text {intra }}$ & ICC inter & SEM & SDC & 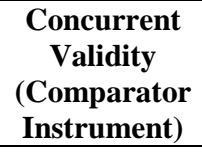 & $\begin{array}{c}\text { Convergent } \\
\text { Validity } \\
\end{array}$ \\
\hline Lu et al. (2013) & & & $9.8 \%$ & $27.2 \%$ & & \\
\hline Dylke et al. (2012) & 0.93 & & $-1 \%-10 \%$ & $\begin{array}{l}-2.7 \%- \\
39.2 \%\end{array}$ & & $\begin{array}{c}\text { Full arm versus } \\
\text { simulated } 10 \mathrm{~cm} \\
0.99 \\
\end{array}$ \\
\hline Foroughi et al. (2011) & & & $-5 \%-1.5 \%$ & $\begin{array}{c}-13.9 \%- \\
4.2 \% \\
\end{array}$ & $\begin{array}{c}0.84-0.94 \\
(\mathrm{CTM})\end{array}$ & \\
\hline Lee et al. (2011) & 1.00 & 0.99 & & & $0.88(\mathrm{WD})$ & \\
\hline \multirow[t]{2}{*}{ Czerniec et al. (2010) } & 0.99 & & $7 \%$ & $19.4 \%$ & $0.99(\mathrm{CTM})$ & \\
\hline & \multicolumn{6}{|c|}{ 3DCI } \\
\hline Binkley et al. (2020) & 0.99 & & $1.4 \%-1.6 \%$ & $3.9 \%-4.4 \%$ & & \\
\hline Lu \& Dixon (2019) & & & $-0.5 \%-6 \%$ & $\begin{array}{l}-1.4 \%- \\
16.6 \%\end{array}$ & $0.88(\mathrm{P})$ & \\
\hline Cau et al. (2018) & & & & & $0.74(\mathrm{CTM})$ & \\
\hline $\begin{array}{l}\text { Karakashian et al. } \\
(2018)\end{array}$ & 0.95 & & & & & \\
\hline Landau et al. (2018) & & & $0.5 \%-0.7 \%$ & $1.4 \%-1.9 \%$ & $0.99(\mathrm{CTM})$ & \\
\hline Preu $\beta$ et al. (2018) & 1.00 & & $0.2 \%-0.3 \%$ & $0.6 \%-0.8 \%$ & $1.00(\mathrm{WD})$ & \\
\hline Verhulst et al. (2017) & 0.99 & 0.99 & & & & \\
\hline Cau et al. (2016) & & & & & 0.92 (CTM) & \\
\hline $\begin{array}{l}\text { Hameeteman et al. } \\
(2016)\end{array}$ & 1.00 & & & & $0.99(\mathrm{WD})$ & \\
\hline Buffa et al. (2015) & 1.00 & 1.00 & $0.6 \%$ & & & \\
\hline Erends et al. (2014) & 0.99 & & & & $0.98(\mathrm{WD})$ & \\
\hline Lu et al. (2014) & & & & & $0.98(\mathrm{WD})$ & \\
\hline Öhberg et al. (2014) & 0.98 & & & & & \\
\hline Lu et al. (2013) & & & $6.5 \%$ & $18.0 \%$ & & \\
\hline
\end{tabular}

Table 19: Pooled data for ICC, SEM, and weighted mean SDC

\begin{tabular}{|c|c|c|c|c|c|c|c|}
\hline 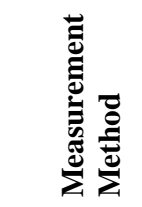 & 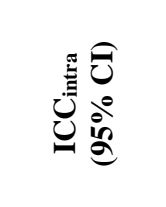 & : & 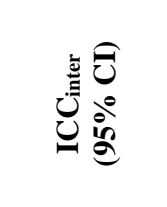 & : & 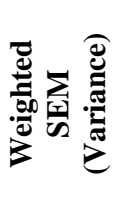 & 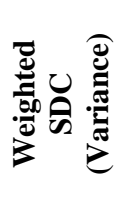 & 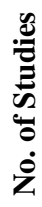 \\
\hline CTM & $\begin{array}{c}0.97 \\
(0.86,1.00)\end{array}$ & 0.04 & $\begin{array}{c}0.95 \\
(0.88,0.99)\end{array}$ & 0.01 & $\begin{array}{l}2.0 \% \\
(2.8 \%)\end{array}$ & $\begin{array}{l}5.5 \% \\
(8.3 \%)\end{array}$ & 8 \\
\hline Perometer & $\begin{array}{c}0.97 \\
(0.85,1.00)\end{array}$ & 0.03 & $\begin{array}{c}0.98 \\
(0.90,1.00)\end{array}$ & 0.03 & $\begin{array}{l}3.4 \% \\
(4.6 \%)\end{array}$ & $\begin{array}{c}9.4 \% \\
(12.8 \%)\end{array}$ & 9 \\
\hline 3DCI & $\begin{array}{c}0.98 \\
(0.95,1.00)\end{array}$ & 0.02 & $\begin{array}{c}0.99 \\
(0.98,0.99)\end{array}$ & 0.01 & $\begin{array}{l}1.7 \% \\
(2.5 \%)\end{array}$ & $\begin{array}{l}4.7 \% \\
(6.9 \%)\end{array}$ & 8 \\
\hline
\end{tabular}

Key for Tables 16 and 17:

3DCI $=$ Three-Dimensional Camera Imaging $\quad \mathrm{CI}=$ Confidence Interval

$\mathrm{CTM}=$ Circumferential Tape Measurement

nterrater reliability

ICC $_{\text {inter }}=$ Intra-class Correlation Coefficient for interrater reliability

$\mathrm{ICC}_{\text {intra }}=$ Intra-class Correlation Coefficient for intrarater reliability

SEM = Standard Error of Measurement $\quad$ SDC $=$ Smallest Detectable Change

$\mathrm{WD}=$ Water Displacement 
Based on the analysis of the studies, CTM, perometry and 3DCI can be recommended for clinical practice. However, none of the measurement methods is interchangeable, thus therapists need to keep this in mind (Ward et al., 2009a; Ward et al., 2009b; Devoogdt et al., 2010; Lee et al., 2011; Brorson \& Hoijer, 2012; Adriaenssens et al., 2013; Bulley et al., 2013; Öhberg et al., 2014; Landau et al., 2018). In addition to considering validity and reliability, the choice of measurement method will also depend on access, feasibility and costs. The perometer is an expensive device compared to CTM (Adriaenssens et al., 2013; Lu et al., 2013; Sharkey et al., 2018; Binkley et al., 2020); and dependent on the type of 3DCI utilised, this can also be a costly means of measuring limb volume.

Space is premium within any clinic, thus large devices such as the static perometers and some of the larger 3DCI devices, e.g. Vectra 3D, 3dD Stereophotogrammetry and static 3D Microsoft Kinect ${ }^{\mathrm{TM}}$ system, may not be feasible. In small clinical areas, CTM, mobile perometer and 3D devices would be more practical. As many lymphoedema services are community based, rarely having access to dedicated facilities, the portability of the device has to be a factor when measuring lymphoedema. Furthermore, to further promote self-management, patients could be encouraged to measure themselves at home following a protocol, and CTM has demonstrated to be an effective method of selfmeasurement (Foroughi et al., 2011; Mori et al., 2015; Rafn et al., 2019), whilst Lu, Han, DeSouza, Armer, \& Shyu (2014) suggest that 3DCI could also be used as a selfmonitoring tool.

None of the studies reported on the sensitivity and specificity of the methods used. Ideally, a measurement method should have a high sensitivity to detect all cases of lymphoedema, and high specificity to exclude false-positive outcomes. Further research is needed to analyse the different methods of volume measurement and to preferably compare it to a gold standard method of volume measurement, which according to some papers is water displacement (Devoogdt et al., 2010; Lee et al., 2011; Adriaenssens et al., 2013; Öhberg et al., 2014; Buffa et al., 2015; Mori et al., 2015).

Moreover, there is a general lack of evidence and consensus as to the recommended threshold for diagnosing lymphoedema based on volume measurement. However, two studies, one for CTM (Asim et al., 2012) and the other for perometry (Ancukiewicz et 
al., 2012) indicate that a 7.5\% volume difference between arms could be considered as a threshold for diagnosing lymphoedema. Hidding et al. (2016) explain that although SEM is the smallest amount of change that can reliably be measured, the SEM values are generally smaller than the normal differences between arms. They argue for the use of SDC as a better clinical reference point for commencing treatment for lymphoedema as there is no recognised threshold for lymphoedema diagnosis. This review presented the weighted mean SDC values of $4.7 \%$ for 3DCI, $5.5 \%$ for CTM, and $9.4 \%$ for perometry (refer to Table 19). Hidding et al. (2016) suggest that treatment before these cut-off points could lead to overtreatment, which should be avoided. Further research into the use of SDC versus threshold should also be investigated, as well as research looking at the sensitivity and specificity of the different thresholds for CTM and perometry, and to introduce this for 3DCI as well.

The time needed to perform a measurement was reported in three studies. Buffa et al. (2015) measured only the time taken to perform the measurement whilst Landau et al. (2018) timed the set-up, execution and calculation of limb volumes in their study. De Vrieze et al. (2019) timed the set-up and execution of the limb volume measurements between CTM, perometry and various methods of water displacement. They were also the only study to look at the burden of the different measurement methods and found CTM to be the better method of limb volume measurement. However, there are more factors to consider than the timing of the method, and they need to include the cost of the device (including depreciation and servicing), the cost of the therapist's time, therapist preference, but also patient choice based on their understanding of the results from the method of measurement. This would make a more economical and ValueBased argument for choosing one method over the other in clinical practice.

\subsubsection{Strengths and Weaknesses}

Through this systematic literature review, a meta-analysis for the reliability of CTM, perometry and 3DCI on measuring BCRL was performed. Based on the analysis, the use of CTM, perometry and 3DCI for measuring upper limb lymphoedema can be recommended, as many studies were included in the meta-analysis.

Limitations have also been identified. As there is no validated gold standard that could be used as a comparator test, all methods of measurement were reviewed independently. 
In the research literature, lymphoedema of the upper limb is predominantly described in women with breast cancer. Medical treatment received for breast cancer results in a higher risk for developing lymphoedema than other types of upper limb lymphoedema causes, except for melanoma (Rockson, 2018); however, melanoma numbers remain very low compared to BCRL, making recruitment difficult, hence, no study currently exists for this group either. As BCRL is largely a female condition, research studies exist in women more than men. Other causes of upper limb lymphoedema can include vascular insufficiency, for example, deep vein thrombus, dependency, example due to a stroke, filariasis and even primary lymphoedema. These cases are rare, thus poorly represented in the literature.

The quality of the studies varied immensely with numerous limitations identified, including cohort size, and lack of description of a clear methodology, including ethics gained, recruitment selection and measurement protocol. The risk of bias raised concerns, firstly about the diversity of the cohort selection, which was not always described, consecutive participants were included in some studies, and random selection via advertisement was mentioned. Secondly, only a few studies reported on the severity of the lymphoedema being measured. Thirdly, measurement protocols of the comparator test were poorly described, especially in studies on perometry and 3DCI. Lastly, the blinding of the investigators was described clearly in only seven studies. Therefore, bias cannot be excluded in this review.

\subsubsection{Implications for Daily Practice and Future Research}

All the methods of measurement have evidence for good reliability and validity. Based on the measurement properties and feasibility, it is recommended CTM be used as best practice in measuring lymphoedema in the upper limbs. Positioning for CTM should be standardised with the patient sitting down, hands resting palm down on a flat surface, and the starting point to be used is the crease of the wrist. Both arms must be measured, measurements calculated to volume, and the volume then needs to be calculated to percentage limb volume difference.

In future research, measurement protocols and results should be reported clearly and in a uniform way to facilitate the future synthesis of the literature. It is strongly 
recommended that attention be paid to sensitivity, specificity and diagnostic thresholds for the different types of lymphoedema measurement methods in studies.

\subsection{Conclusion}

From this systematic literature review, it is evident at this time that there is no study concurrently comparing CTM, perometry and 3DCI to determine validity, reliability and differences in the outcomes reported. CTM is a commonly used method of measuring limb volume within clinical practice, the perometer is considered by some to be a gold standard method of limb volume measurement and 3DCI, while being able to measure for limb volume, also has the potential benefit of interpreting shape as an additional diagnostic measure for lymphoedema. Currently, neither CTM nor perometry can identify areas of localised swelling. Thus swelling maps have the potential to identify localised oedema that can inform treatment.

3DCI is shown to be both a reliable and valid method of limb volume measurement compared to both CTM and perometry; however, the evidence is limited due to the varying devices used. Also, the systematic literature review has revealed that lymphoedema could be assessed through limb shape by utilisation of swelling maps from the data extrapolated from 3DCI. It has the potential for clinical use if utilising one of the more portable devices, i.e. LymphaTech, Microsoft Kinect ${ }^{\mathrm{TM}}$, Asus Xtion or Laser Scanner 3D systems. However, no studies to date have looked at this method within the clinical setting. Only one study exists looking at the use of limb shape as a means of assessing lymphoedema (Karakashian et al., 2018); thus further studies are needed to provide evidence of this new technological benefit. A longitudinal study would determine repeatability and sensitivity to change over time, including limb shape, and could be considered as part of a clinical programme of care.

Thus from the systematic literature review, and the research gaps identified, it would be worth comparing 3DCI to CTM and perometry concurrently to establish its reliability, validity and accuracy. Further analysis of swelling maps would add to the limited evidence to ascertain their value in the diagnosis of lymphoedema, and consideration for the use of 3DCI in clinical practice is also needed. 
Chapter 4:
Research Design
and Methods 


\subsection{Introduction}

The systematic literature review presented in Chapter 3 demonstrated that CTM, perometry and 3DCI have excellent validity and reliability, however, no study exists comparing these three methods within one study. Karakashian et al. (2018) to date presents the only study where images taken by 3D camera are converted into oedema maps, thus offering a different means of assessing lymphoedema through limb shape deviation. However, their study was part of a service evaluation and lacked clear protocols for the comparator measurements. A gap in knowledge proves the need for this study.

This chapter describes the research design and research methods used for this study. The data collected from the Asus Xtion Pro 3D camera was converted into limb volume, and volume difference was calculated and expressed in terms of millilitres ( $\mathrm{ml}$ ) and as a percentage (\%), and then compared to both CTM and perometry. Furthermore, the data was used to create oedema maps, which were compared to the volume measurements to determine any correlations between these different methods.

\subsection{Aims}

The study aimed to determine:

1) the validity, reliability and accuracy of upper limb volume results obtained from 3DCI compared to CTM and perometry; and

2) whether the shape of the limb is a feasible alternative to measure lymphoedema.

As there are two aims for this study, two hypotheses and null hypotheses are required. A hypothesis is a statement specifying the relationship between variables, with the null hypothesis being presumed to be initially true (Parahoo, 2014). The first hypothesis is that 3DCI is a valid, reliable and accurate method of measurement for lymphoedema; thus, the null hypothesis is '3DCI is not a valid, reliable nor accurate method of measurement for lymphoedema'. The second hypothesis is that shape entropy derived from 3DCI is an alternative method that correlates to arm volume measurement. The resultant null hypothesis is 'shape entropy derived from 3DCI is not an alternative method that correlates to arm volume measurement'. 


\subsection{Objectives}

The objectives drawn from the research questions posed in the introduction are:

1. Determine whether 3DCI volume outcomes are comparable to CTM and perometry as a measure of intra-rater reliability

2. Assess the criterion validity of 3DCI against previously validated measures, i.e. CTM and perometry

3. Establish whether 3DCI is an accurate method of measurement for upper limb lymphoedema

4. Ascertain whether limb shape derived from 3DCI is a potential new method of lymphoedema measurement

\subsection{Research Methodology and Design}

Antwi \& Hamza (2015) explains that research methodology is informed by the paradigm (a school of thought with a set of beliefs [Parahoo, 2014]) guiding a chosen research project. Due to the empirical nature of lymphoedema assessment and the research questions being asked, the quantitative research paradigm (also referred to as the deductive or scientific research paradigm), was the research methodology of choice (Atieno, 2009; Parahoo, 2014; Antwi \& Hamza, 2015). Atieno (2009) posits that this paradigm ensures validity by the process of rigorous clarification, definition, and use of pilot experiments, while Antwi \& Hamza (2015) explain that it is an approach to thinking about and doing research, thus testing hypotheses.

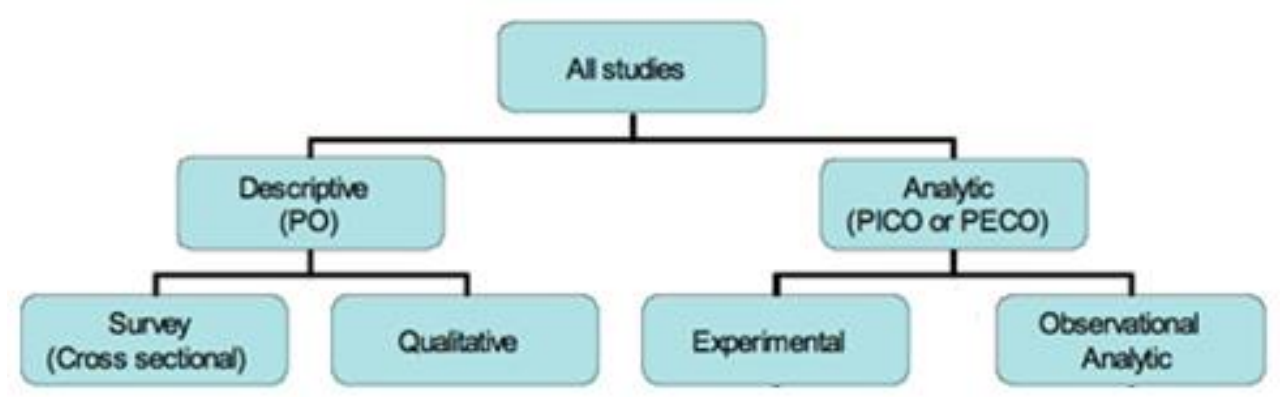

Figure 24: Tree of different types of study design (CEBM, 2014)

The Centre for Evidence-Based Medicine (CEBM) (2014) developed a 'tree of possible study designs' (Figure 24) whereby basic study designs are subdivided into descriptive or analytic studies, and analytic studies are further divided into experimental or 
observational studies. The analytic studies are analogous to the quantitative research paradigm as they are all empirical.

To determine whether 3DCI is comparable to CTM and perometry, the optimal methodological approach considered was an analytic, observational longitudinal study utilising a diagnostic test study framework. The CEBM (2014) explains that observational studies investigate and record exposures (such as interventions or risk factors) and observe outcomes (such as disease) as they occur. Howick (2013) states that observational studies dominate the literature and can be subdivided into exploratory, descriptive and analytical studies. Caruana, Roman, Hernández-Sánchez, \& Solli (2015) explain that longitudinal studies are a form of observational analytic study that employs continuous or repeated measures to follow particular individuals over some time. Data collected for individuals from a predefined group can be statistically analysed to determine change over time for the group, or particular individuals. A diagnostic test study evaluates how well a new test is able to predict or diagnose a disease (Bland, 2010). Bland (2010) clarifies that the basic design is to compare test results on people with the disease with test results on people without the disease by comparing the new test to a gold standard, or reference standard.

Consideration should be given to the experience of the key stakeholders, i.e. the participant (patient) and the researcher (clinician) involved in the study, to understand their thoughts on the interventions (measurements) received. Qualitative data utilising methods such as semi-structured interviews, focus groups or feedback (verbal or written), could determine what the stakeholders thought of the interventions (Hammerberg, Kirkman, \& de Lacey, 2016). However, qualitative data was not collected for this study as the first step was to identify some of the other parameters, such as, 'is 3DCI a valid method of measurement', 'is it accurate, reliable and repeatable', 'does it offer more data for lymphoedema assessment', and 'how else may the data from the images be manipulated for clinical use'? Subsequent qualitative studies may be required in the future once the feasibility of 3DCI has been established.

Daya (1996) posits that diagnostic tests are based on the principle that individuals with disease are different from those without disease, and that the test can distinguish between these two groups. Di Ruffano, Hyde, McCaffery, Bossuyt, \& Deeks (2012) advise that 
the priority for diagnostic test studies is to establish the benefit of the new test. Thus new tests should only be introduced into clinical practice when the evidence, through proper analysis, indicates that they have a better chance of improving patient health care than existing tests. Hence, diagnostic test studies aim to determine whether a test or method of measurement is useful in clinical practice.

For a test to be clinically meaningful, determination of how the test results will affect clinical decisions is integral, while also taking into consideration the costs, risks, and the acceptability of the test (Glasser, 2008). Ideally, a new test should be tested against a 'gold standard' or 'reference standard' (Daya, 1996; Rutjes, Reitsma, Coomarasamy, Khan, \& Bossuyt, 2007). However, from Chapter 2, it has been determined that there is currently dispute over the claimed gold standard of lymphoedema measurement, i.e. water displacement, especially as it is not used internationally.

Rutjes, Reitsma, Coomarasamy, Khan, \& Bossuyt (2007) identify four situations where the lack of a gold standard does not negate the value of a diagnostic test study. These include 1) ascribe or adjust for missing data on reference standard, 2) correct imperfect reference standard, 3) construct reference standard, and 4) validate index test results. The results are combined to construct a reference standard by 1) deterministic predefined rules (composite reference standard), 2) consensus procedure among experts and 3) a statistical model based on actual data (Knottnerus \& Muris, 2003; Rutjes et al., 2007). As previously reported, the most commonly used method of assessing for lymphoedema is CTM which can meet all the criteria as a construct reference standard for use in this methodology.

Furthermore, diagnostic tests require an evaluation of the reproducibility, accuracy, and variation among those with and without disease. In evaluating and applying the results from a diagnostic test study, the validity of the study requires an independent, blind comparison with a reference standard, the methodology must be fully explained, and the results should show sensitivity and specificity (Daya, 1996). Parahoo (2014) explains that when studying current phenomena by pursuing information from the future, a prospective design is advised. 
The diagnostic test study employed 3D scanning technology to determine its accuracy and speed of application compared to CTM and perometry, with the data collected fitting the remit of an observational analytic study. It was a prospective, longitudinal study taking place over six months, utilising sequential sampling with a diagnostic test study framework. Two phases were designed for the analysis of the results based on the distinct objectives focusing on limb volume and limb shape. Phase One analysed the validity, reliability and accuracy of limb volume between the three methods of measurements identified. Phase Two explored utilising 3DCI data as a new innovative method of assessing lymphoedema through limb shape.

The next sections will elucidate the study design, criteria, ethical considerations, and the data collection process. After that, the two phases will identify which of the research questions and objectives they will be analysing, along with the methods employed to do this.

\subsection{Research Study}

The title of the study submitted for ethical approval was 'exploiting 3D scanning technology in lymphoedema for accurate and fast measurements of volume and shape' (Appendix 4). The aims and objectives identified in sections 4.2 and 4.3, were utilised in the construct of this study. However, as the thesis developed, this title was changed to be more reflective of the gaps identified in the systematic literature review, together with the more focused aims and objectives of this thesis. Demographic and anthropometric data of the participants were collected and analysed. Due to the two distinct aims for the study, two phases of analysis were undertaken. Phase One focused on limb volume measurements from 3DCI, CTM and perometry, and Phase Two concentrated on limb shape derived from the 3DCI data as a measurement tool for lymphoedema. Figure 25 illustrates the two phases of the study. 


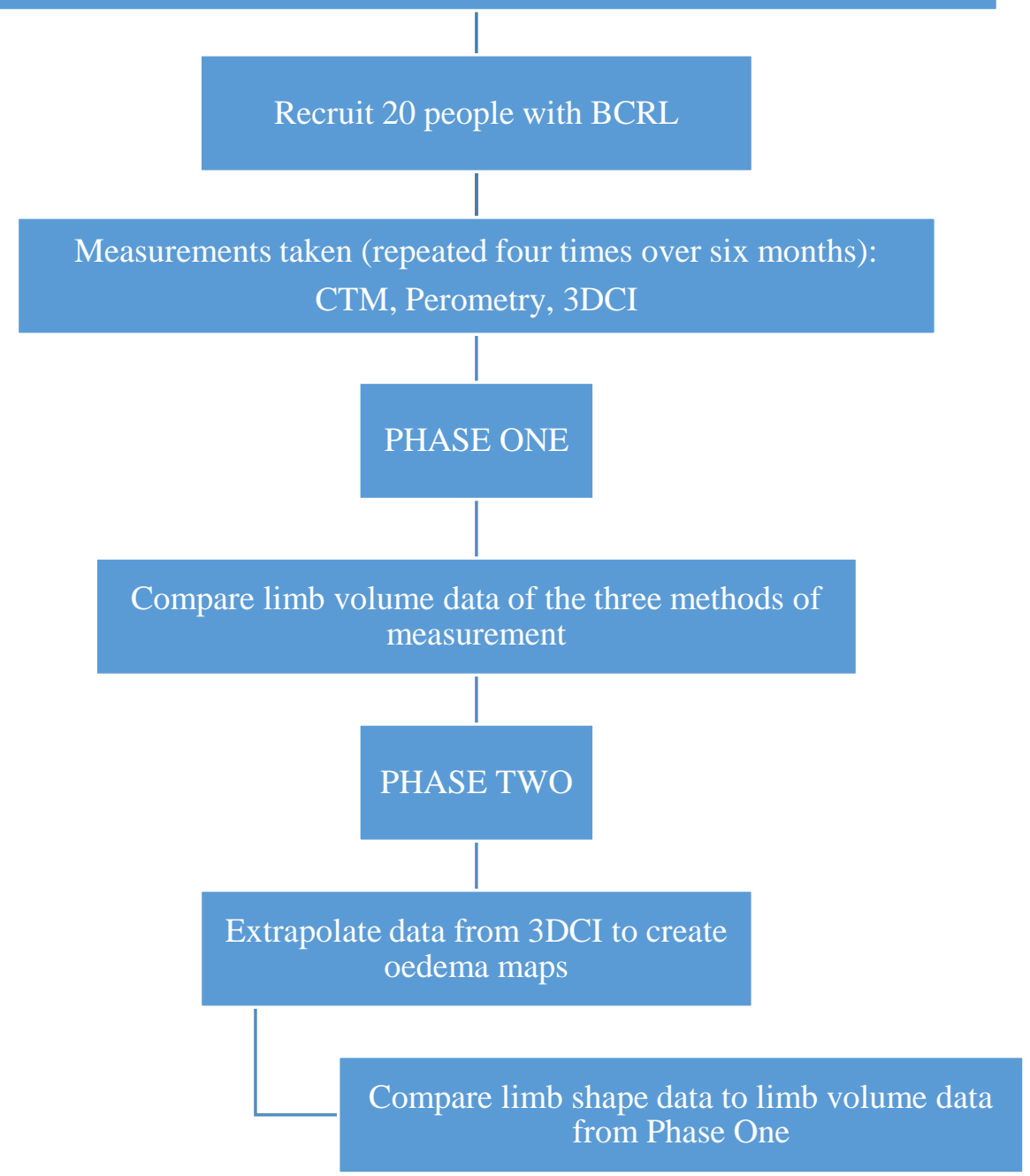

Figure 25: Diagram illustrating the two phases of the study

\subsubsection{Study Design}

The number of participants for recruitment was based on clinic referrals to the local NHS lymphoedema service within one year. A post-hoc sample size calculation was completed (see section 4.5.4) as result. Twenty participants referred to the local NHS lymphoedema service were recruited based on them meeting the eligibility criteria as described in section 4.5.2. After gaining consent, participants were assessed every two months for six months, comparing 3DCI to CTM and Perometry. The 'study scheme diagram' (Figure 26) clarifies and breaks down the study design 
Upon receipt of referral (Appendix 7) for person with unilateral upper limb lymphoedema, a trained therapist identified potential participants against study criteria

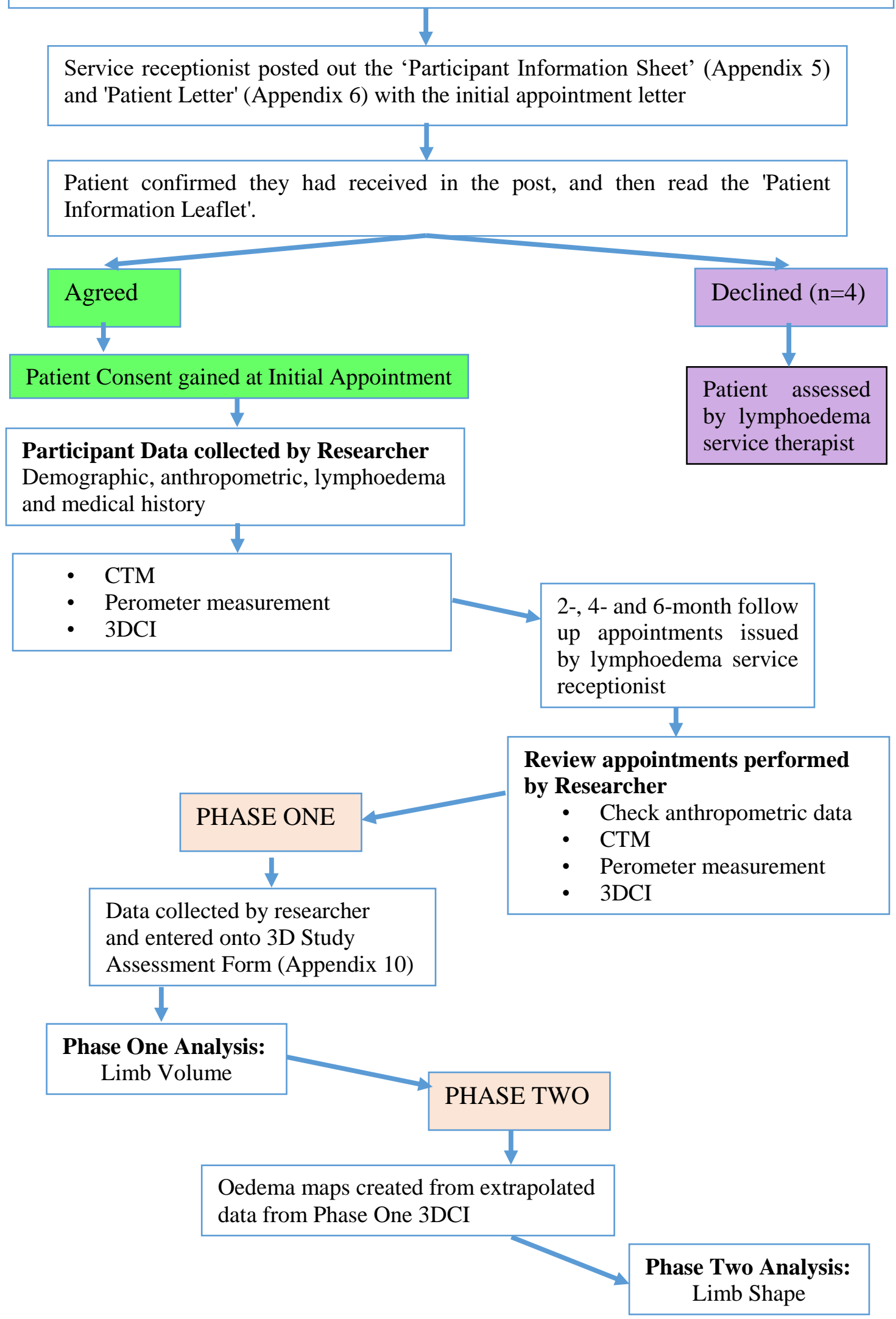

Figure 26: Study Design Scheme giving an overview of the study protocol 
Table 20 lists the methods of data collection for each objective which is discussed in more detail in sections 4.6.2 and 4.7.2 for each of the study phases. Objectives one to three were measured through the calculation of limb volume and used as a comparator amongst the three methods of lymphoedema measurement. Objective four explored limb shape, utilising the $3 \mathrm{D}$ data, compared to the results from objectives one to three.

Table 20: Research Objectives and Data Collection

\begin{tabular}{|c|c|}
\hline Objective & Method of Data Collection \\
\hline $\begin{array}{l}\text { 1. Determine whether 3DCI volume } \\
\text { outcomes are comparable to CTM and } \\
\text { perometry (intra-rater reliability) }\end{array}$ & $\begin{array}{l}\text { - Researcher captured measurements } \\
\text { manually and written on 3D Study } \\
\text { Assessment Form (Appendix 10). } \\
\text { - Data transferred by researcher onto } \\
\text { electronic databases (Excel \& SPSS) }\end{array}$ \\
\hline $\begin{array}{l}\text { 2. Assess the criterion validity of 3DCI } \\
\text { against previously validated measures, } \\
\text { i.e. CTM and perometry }\end{array}$ & $\begin{array}{l}\text { - Data captured by researcher on 3D Study } \\
\text { Assessment Form }\end{array}$ \\
\hline $\begin{array}{l}\text { 3. Establish whether } 3 \mathrm{DCI} \text { is an accurate } \\
\text { method of measurement for } \\
\text { lymphoedema }\end{array}$ & $\begin{array}{l}\text { - Data captured by researcher on 3D Study } \\
\text { Assessment Form }\end{array}$ \\
\hline $\begin{array}{l}\text { 4. Ascertain whether limb shape derived } \\
\text { from 3DCI is a potential new method of } \\
\text { lymphoedema measurement }\end{array}$ & $\begin{array}{l}\text { - Data from oedema maps and calculated } \\
\text { limb volumes as entered on 3D Study } \\
\text { Assessment Form by researcher }\end{array}$ \\
\hline
\end{tabular}

\subsubsection{Participants and Setting}

Twenty participants referred to their local NHS lymphoedema service in one NHS Wales Health Board were recruited into this study. Referrals to the service were done either by the General Practitioner, the Breast Care Nurse, or as a self-referral if the patient had attended a Lymphoedema Risk Reduction session. The lymphoedema service receptionist and a lymphoedema therapist were trained and familiarised with the study protocol. This enabled the lymphoedema therapist to identify potential participants from the referrals based on the study inclusion and exclusion criteria.

The lymphoedema receptionist sent the patient their initial assessment appointment, together with the 'Participant Information Sheet' (Appendix 5) and 'Patient Letter' (Appendix 6). It ensured that the potential participant had more than 24 hours to read the information about the study. 


\subsubsection{Inclusion Criteria}

Participants selected had unilateral (one-sided) lymphoedema of their upper limb secondary to breast cancer. Participants had to be adults (aged 18 or over), and both male and female genders were included.

\subsubsection{Exclusion Criteria}

Participants were excluded if they:

- were unable to consent due to poor mental health or dementia

- were unable to lift their arm to shoulder height

- were unable to commit to the scheduled appointments

- had active disease (cancer) or were under palliative care

- presented with a chronic or non-chronic disease, other than lymphoedema that may affect the swelling of their arm

- were women who were pregnant.

\subsubsection{Criteria for Premature Withdrawal}

Participants could withdraw from the study if they withdrew their consent, no longer wanted to partake in the study, or were unable to attend the scheduled appointments. Once recruited, the data was retained unless the participant requested that their data be excluded from the study.

\subsubsection{Ethical Approval and Considerations}

This study was conducted with adherence to the Health \& Care Professions Council Standards of Conduct, Performance and Ethics (2016), the Chartered Society of Physiotherapy's Code of Member's Professional Values and Behaviour (2011), and the guidance as set out by the Good Clinical Practice Reference Guide (National Institute for Health Research Clinical Research Network [NIHR CRN], 2016). The ethical principles as advocated by Beauchamp \& Childress (2012) and Parahoo (2014) including non-maleficence, beneficence, fidelity, veracity, confidentiality and justice were considered throughout the study to protect participants from harm. Thus, the rights, well-being, and safety of participants took precedence over the research objectives. 
Ethical approval was granted for the study in March 2016 from the College of Human Health Sciences and College of Medicine Research Ethics Committee, Swansea University. Subsequent application via the Integrated Research Application System (known as IRAS) received approval in June 2016 from the West of Scotland Research Ethics Service (REC Reference 16/WS/0127). The local NHS Research and Development (R\&D) approval was later granted in August 2016. Appendix 4 provides evidence of the ethics and R\&D approvals for this study.

The study protocol together with the aims and objectives were discussed in a meeting with the local lymphoedema service based in an NHS Wales Health Board. The benefits of the study to the service, and to the lymphoedema community were conveyed. The benefits included a comprehensive assessment of the patient's reported lymphoedema and the opportunity for a nurse or physiotherapist to partake in research (which meets one of their governance criteria for registration). Furthermore, the 3DCI equipment utilised would be handed over to the service for ongoing use, and the study would offer an exciting opportunity for the service to be part of a new and original lymphoedema assessment whereby the shape of the limb could be measured.

The lymphoedema receptionist and a lymphoedema therapist were trained for one hour to assist with the research recruitment and protocols. The inclusion and exclusion criteria were explained in detail to them, to ensure new referrals received to the service were scrutinised against these criteria to identify potential participants. The tasks given were for the lymphoedema therapist to triage the referrals against the criteria, and for the lymphoedema receptionist to send out the appointment letter together with the study literature described in section 4.5.2.

\subsubsection{Informed Consent}

Lymphoedema referral forms (Appendix 7) are standardised across Wales and require details on the site and severity of the lymphoedema, as well as the potential cause. All $(n=68)$ referrals for upper limb lymphoedema to the local NHS lymphoedema service from September 2016 to April 2018 were screened against the study inclusion and exclusion criteria. Information as described in section 4.5.2 was posted out to those meeting the criteria. As per standard operating procedures, this was within 48 hours of receipt of the referral. Both the researcher and lymphoedema therapist's details were 
included should a patient wish to contact them to gain more information about the study. Appointments were made for between two and four weeks from the date the referral was received on the allocated research days provided by the researcher. At the patient's initial appointment at the lymphoedema clinic, they were asked if they had received the research information, and whether they had read the information posted to them. They were given time to ask questions regarding the research and were then asked if they would like to participate in the study.

Efforts were made to ensure that patients were not coerced into partaking in the study by ensuring the patient information leaflet was provided to them before their appointment so that they had the opportunity to read it before their appointment. It was explained that their participation would be voluntary and that their decision would not affect how they were assessed and treated at the lymphoedema clinic. It was also explained that they could withdraw from the study at any time without giving a reason and that it would not impact their lymphoedema care from their lymphoedema clinic (Parahoo, 2014). Once a patient confirmed that they were willing to participate in the research study, they were asked to sign a Consent Form (Appendix 8) of which they received a copy, a copy was retained for the research portfolio, and the original was placed within the patient's clinic notes.

\subsubsection{Participant Impact}

The risks to harm for this study were considered to be low for the participants due to the study procedures being non-invasive (Appendix 9). At the initial assessment, some participants could experience mild discomfort when elevating their arm to shoulder height for the measurements to be taken. This was addressed by providing stretching exercises and physiotherapy to relieve the discomfort for future measurements.

Both Swansea University and the R\&D department at the NHS Wales Health Board considered the appointments to be part of a participant's usual lymphoedema reviews, thus no reimbursement for travel or out of pocket expenses could be offered. This was made clear in the Participant Information Sheet and was discussed before the consent was taken. 


\subsubsection{Anonymity}

Participants were allocated unique research numbers to anonymise their acquired data. This unique number was allocated in sequential order of recruited participants on the screening log. No identifiable patient information was collected. Only the lymphoedema service held the participant's details; the researcher accessed specified demographic, medical information and the required clinical assessments with nonpatient identifiable data on the 3D Study Assessment Form (Appendix 10), thus only allocated anonymous research numbers were visible. Participants were issued an appointment date before leaving the session for their review appointment.

For those participants who failed to attend their review appointment, the lymphoedema service tried to make contact via telephone and mail to enquire the reason for not attending. If the participant requested to withdraw from the study, their telephone number was provided to the researcher to enquire the reason for withdrawing to understand whether the information provide could be improved for future projects. Furthermore, the enquiry could determine what the participant's views and tolerance for the study were, and to ensure that the data collected previously could be used for the study $(n=0)$. Where participants failed to respond to contact attempts $(n=3)$, it was assumed that they wished to withdraw from the study and that their data could be used for statistical analysis. These were documented accordingly.

All data collected for the study were kept in a research file that was locked in a filing cabinet in the researcher's office. Only the researcher had access to the research files. Participants were assured of their anonymity throughout the thesis and in any publications by use of their allocated research numbers. This is in keeping with Good Clinical Practice guidelines (NIHR CRN, 2016).

\subsubsection{Confidentiality}

The study protocols followed Data Protection regulations (Data Protection Act, 2018) and Research Ethics guidance (NIHR CRN, 2016) ensuring participant confidentiality, safe record keeping, and qualified research staff with up to date Good Clinical Practice certification. Allocation of a unique research number to each participant ensured the anonymity of the database being analysed. Individuals were able to be tracked via the screening log which was kept in a file and locked in a cabinet in the researcher's office 
to which only the researcher had access. Data were entered into a password-protected spreadsheet, and the hard data copies were filed and locked in a filing cabinet in the researcher's office as per the Health Board Policy and Data Protection Act (2018).

Participant clinical and research notes are to be retained for seven years on completion of the study as per guidance from R\&D. Disposal of these notes will be performed per this guidance, which is currently via the secure data disposal services within the Health Board. The researcher is the data custodian.

\subsubsection{Location of the Research}

The study took place in one of the seven Health Boards in Wales. The population for this health board is more than 500,000, and they employ over 13,000 staff. Patients diagnosed with breast cancer attend a central hospital for their surgery, and since 2004, these patients have routinely been invited by the lymphoedema service to attend a lymphoedema risk reduction programme. Once patients have attended this programme, they can self-refer to the lymphoedema service at any point in the future.

The service currently has a caseload in excess of 3,000 patients, of which $30 \%$ have lymphoedema, or are at risk of lymphoedema, due to breast cancer treatment. There are currently 16 members of staff, both registered and unregistered professionals, who are strategically managed by Lymphoedema Network Wales. Potential and recruited participants were seen at one of two hospital sites within the Health Board. Participants could choose which location they preferred to be seen for the duration of the study, as is usual practice. The participant was seen by the researcher at each point of contact regardless of the venue they chose to be seen. Allocated treatment rooms at each site were utilised for the study.

\subsubsection{Sampling}

Twenty eligible people newly referred to the lymphoedema service were recruited for the study in the time available. Post-hoc calculations were performed to determine whether this sample size would be sufficient. When discussing statistical power, there are four interrelated concepts to consider, namely power, effect size, sample size and alpha (UCLA Institute for Digital Research and Education, 2019). For this study, post hoc analysis using the Analysis of Variance (ANOVA) repeated measures, within 
factors f-test, was used to determine the power of the sample size with an effect size of 0.25 . This small effect size was to ensure that any variance would be too small to detect other than statistically and is the lower limit of what is clinically relevant, which will help reduce the effect of a Type I error (UCLA Institute for Digital Research and Education, 2019).

In this study, the number of measurements was four (participants measured at their initial assessment, 2-, 4- and 6-month reviews), with the dependent variable being limb measurement (Laerd Statistics, 2018a). The Power of Analysis, using a software programme called G*Power (version 3.1.9.4), produced an Output Power of 0.73, with a standard margin of error of 0.05 (see Figure 27). It means the study sample will give a high effect to disprove the null hypothesis for the thesis.

$\begin{array}{lll}\text { F tests - ANOVA: Repeated measures, within factors } \\ \text { Analysis: } & \text { Post hoc: Compute achieved power } & \\ \text { Input: } & \text { Effect size f } & =0.25 \\ & \alpha \text { err prob } & =0.05 \\ & \text { Total sample size } & =20 \\ & \text { Number of groups } & =2 \\ & \text { Number of measurements } & =4 \\ & \text { Corr among rep measures } & =0.5 \\ & \text { Nonsphericity correction } \epsilon & =1 \\ \text { Output: } & \text { Noncentrality parameter } \lambda & =10.0000000 \\ & \text { Critical F } & =2.7757624 \\ & \text { Numerator df } & =3.0000000 \\ & \text { Denominator df } & =54.0000000 \\ & \text { Power }(1-\beta \text { err prob) } & =0.7270272\end{array}$

Figure 27: Power of Analysis Results (UCLA Institute for Digital Research and Education, 2019)

Missing data due to failure to attend sessions, equipment failure, or data processing, is considered as 'missing completely at random', and although Power may be lost in the study design, the analysis will remain unbiased (Kang, 2013). Statistics Solutions (2021) advise the use of a $t$-test following either pair-wise or list-wise deletion of missing data to check whether any difference in the samples between the data sets exists.

\subsubsection{Recruitment}

Recruitment commenced October 1, 2016, and finished April 30, 2018. Potential participants received the relevant letters and documents as described in section 4.5.2, and at their first appointment, the Participant Information Sheet (Appendix 5) was discussed, and any queries answered. At this stage, the patient could decide whether they would like to participate in the study. The patient was checked against the criteria, 
including ensuring they could elevate their arm to the required shoulder height level. If the patient was eligible, consent was gained and data collected. If the patient was ineligible, the reason for exclusion was documented on the screening log, and they were assessed under the standard lymphoedema service protocol. Figure 28 provides a diagrammatic representation of the recruitment process.

\subsubsection{Methods}

A means of controlling bias was done in the form of blinding, where the data collection sheets for each session were independent of all previous sessions. However, continuity in CTM was assured by transferring the value for the first mark, as well as the required number of marks to be measured, between sessions. The participant's height was transferred between sessions, as this was unlikely to change over six months; however, weight was re-measured at each point of contact, and BMI calculated using Lymcalc software.

\subsubsection{Demographic and Anthropometric Data}

Previous studies (Hidding et al., 2016; Levenhagen et al., 2017) indicate that certain patient demographics and anthropometric data may influence lymphoedema measurements. The participant's age, gender, handedness and affected side were documented. Some papers report (Wang et al., 2017) that ethnicity may influence measurements; thus, this was also collected.

The impact of a person's medical history and cancer treatment cannot be dismissed; consequently, a full medical history and the most commonly reported medications were collated, together with the type of cancer treatment the participant had had such as surgery, type of lymph node excision, chemotherapy, radiotherapy and hormone therapy. 


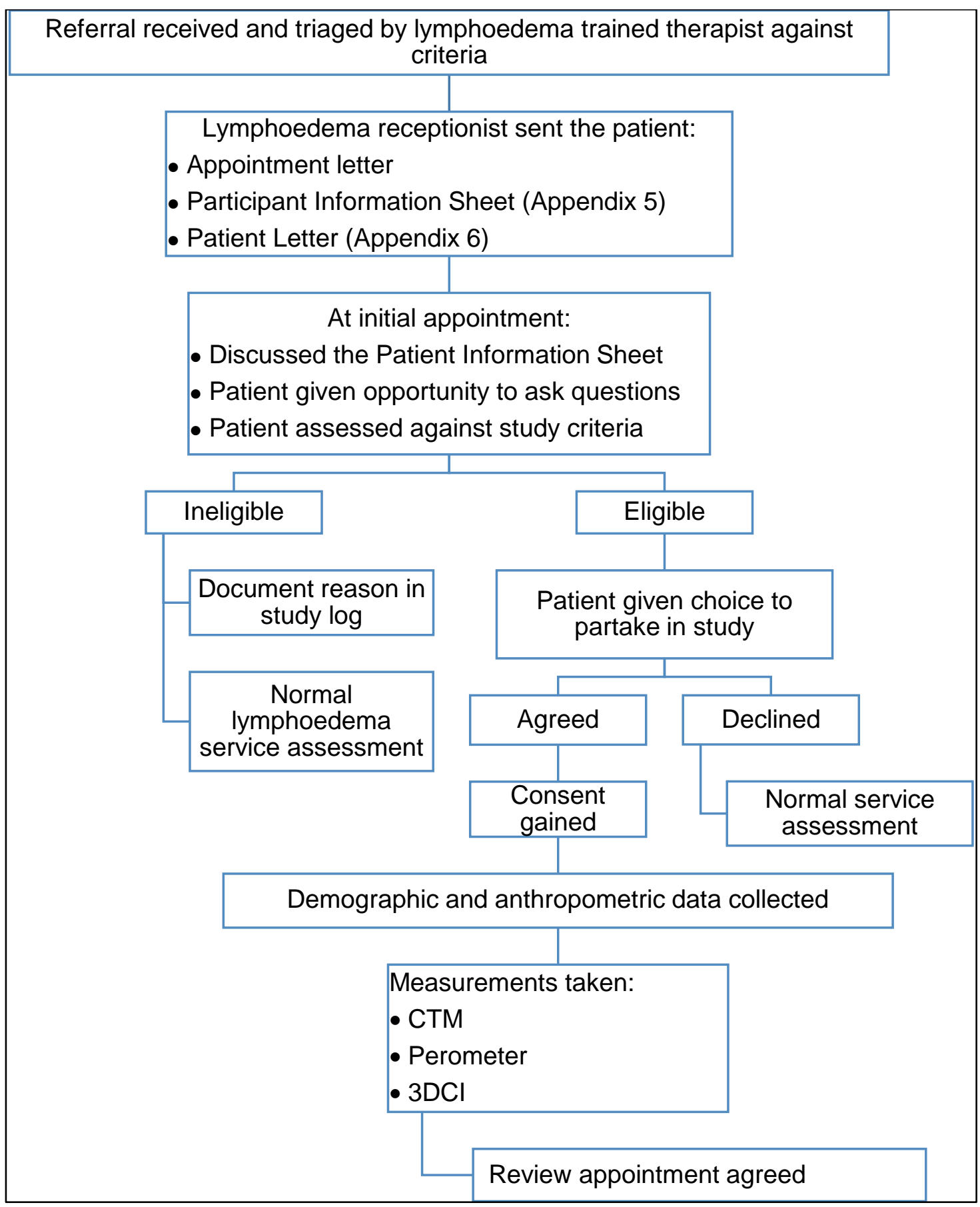

Figure 28: Schedule of Recruitment and Assessment - diagrammatic format of events from the time of receipt of referral to the first appointment

Cellulitis adversely affects lymphoedema (Lam et al., 2006), and this data was gathered to determine its effect on lymphoedema through the measurement methods. Time since surgery and time from surgery to onset of lymphoedema was collected to understand whether this would have an impact on the severity of the lymphoedema as measured by CTM, perometry and 3DCI. The staging of the participant's lymphoedema was determined following the measurements using both the ISL staging and BLS grouping, 
as discussed in section 2.3 and defined in Appendix 1. It helped classify the severity of the participant's lymphoedema.

Employment status was captured to determine how many of the participants were employed, and to explore if this had any relevance on the lymphoedema measurements. Obesity adversely affects lymphoedema (Mehrara \& Greene, 2014). Each participant had their height measured at the initial assessment, and their weight measured at each session to determine their BMI. The BMI range was determined using the World Health Organization's (2021) Nutritional Status for adults (Table 21).

Table 21: Nutritional status for adults (World Health Organization, 2021)

\begin{tabular}{|l|l|}
\hline BMI & Nutritional status \\
\hline Below 18.5 & Underweight \\
$25.0-24.9$ & Normal weight \\
\hline $30.0-34.9$ & Pre-obesity \\
$35.0-39.9$ & Obesity class I \\
\hline Above 40 & Obesity class II \\
\hline
\end{tabular}

Smoking is known to cause cardiovascular disease, including thickening and narrowing of the blood vessels and blood clots (Yanbaeva, Dentener, Creutzberg, Wesseling, \& Wouters, 2007), which will have a direct impact on the lymphatic system. However, no study exists to determine whether smoking has a direct impact on the lymphatics and resultant lymphoedema. Excessive alcohol consumption is known to cause liver disease and weakening of the immune system (Centers for Disease Control and Prevention, 2019), both of which will impact the lymphatic system. Therefore, exploring whether smoking and excessive alcohol consumption affect lymphoedema measurement could offer new information for clinical practice.

\subsubsection{Methods of Lymphoedema Measurements Used}

Chapter 3 provided a detailed description of the methods of measurement used in this study, including positioning and execution of each test. For clarity, CTM was performed per the 'All Wales Lymphoedema Guidance: Measuring Volumes to Determine Lymphoedema Outcome' (LNW, 2017). The protocol devised by Ancukiewicz et al. 
(2011) was used when executing the perometer measurements, and the protocol described by Karakashian et al. (2018) was used when performing 3DCI measurements.

CTM, perometry and 3DCI were executed at each session by the researcher. As discussed in section 4.5.7, bias was controlled as all data from previous sessions were blinded for both the researcher and the participant. Timing of each method was executed at the initial assessment only and included set up and execution of the test. Results were calculated using the various formulas for each method of measurement which was the cylinder method (Equation 1) for CTM, disc method (same as for cylinder) for perometer, and the more accurate integral formula (Equation 2) for 3DCI.

Equation 1

$$
V_{\text {cylinder } / \text { disc }}=\frac{1}{4 \pi} L C_{1}^{2}
$$

Equation 2

$$
V_{\text {integral }} \approx \sum_{j=1}^{N_{f}} \sum_{i=1}^{N_{d}} F_{j, i} n_{j, i} A_{j}
$$

The percentage volume difference $(\% V D)$ between the arms of a given participant was calculated by dividing the volume difference $(V D)$ by the unaffected arm volume $(L V u)$ and multiplying it by 100 (Equation 3).

Equation 3

$$
\% V D=\left(\frac{V D}{L V u}\right) * 100
$$

Absolute volume difference ( $A V D$ ) was calculated whereby the volumes of the same arm were compared over time to the initial assessment volume ( $\left.V_{I A}\right)$ (Equation 4). Percentage AVD (\%AVD) was calculated by dividing $A V D$ by $V_{I A}$ and multiplying by 100 (Equation 5).

Equation 4

$$
A V D=V-V_{I A}
$$

Equation 5

$$
\% A V D=\frac{A V D}{V_{I A}} * 100
$$


$\% V D$ and $\% A V D$ were calculated for each method of measurement and compared in the statistical analysis (Appendix 11).

Extrapolation of the 3DCI data produced lymphoedema maps as described by Karakashian et al. (2018). Furthermore, a new metric was introduced by Watson, Karakashian, Pike, \& van Loon (Submitted), which is a single value that describes the shape of the arm (the concept finds its origins in computer graphics) whereby the swollen arm is considered to be more 'round' and 'stretched', thus lacking in definition. This new metric is proposed to capture this change in shape without considering the actual volume of the arm (which is normalised to 1 for each participant). This metric is calculated for each arm, used for further analysis, and is known as redundancy (R), with values closer to 1.00 indicating no shape distortion.

\subsubsection{Baseline Visit and Data Collection}

As described in section 4.5.3.1, consent was gained before the commencement of the study assessment. A study log was kept of all persons screened and indicated those that met the criteria, and the reasons others were ineligible for the study. Participants' demographic and anthropometric data were collected, followed by the three investigative measures, i.e. CTM, perometry and 3DCI.

\subsubsection{Treatment of Participants}

Following the assessment, a treatment plan together with advice and compression garments was formulated and documented by the lymphoedema therapist. These outcomes were captured on the 3D Study Assessment Form (Appendix 10) for future research to explore how active treatment may affect limb volume and shape. Participants were reviewed at two, four and six months, where the researcher took repeat CTM, perometry and 3DCI measurements. The participant's lymphoedema treatment plan was reviewed and amended according to the measurement outcomes by the lymphoedema therapist. The study ended when the last of the 20 recruited participants attended their six-month review. 


\subsection{Phase One: Diagnostic Test Study of Limb Volume}

\subsubsection{Phase One: Research Questions, Aim, and Objectives}

The aim of Phase One was to determine the validity, reliability and accuracy of limb volume results obtained from 3DCI compared to CTM and perometry.

The objectives of Phase One were to:

1.1 Determine whether 3DCI volume outcomes are comparable to CTM and perometry (intra-rater reliability)

1.2 Assess the criterion validity of 3DCI against previously validated measures, i.e. CTM and perometry

1.3 Establish whether 3DCI is an accurate method of measurement for lymphoedema

Table 22 provides a breakdown of Phase One's objectives and the planned methods of analysis that were used to measure these objectives.

Table 22: Phase One objectives and methods of analysis

\begin{tabular}{|c|c|c|}
\hline Objective & Data Collection Method & Methods of Analysis \\
\hline $\begin{array}{l}\text { Determine the intra-rater } \\
\text { reliability of 3DCI compared } \\
\text { to CTM and perometry }\end{array}$ & $\begin{array}{l}\text { Researcher captured } \\
\text { measurements manually and } \\
\text { written on 3D Study Assessment } \\
\text { Form (Appendix 10). } \\
\text { - Data transferred by researcher } \\
\text { onto electronic databases (Excel } \\
\text { \& SPSS) }\end{array}$ & $\begin{array}{l}\text { - Intra-rater Correlation } \\
\text { Coefficient (ICC) with a } \\
\text { confidence interval of } 95 \% \\
\text { - Standard error of measurement } \\
\text { - } \quad \text { Minimum detectable change }\end{array}$ \\
\hline $\begin{array}{l}\text { 1.2 Assess the criterion validity of } \\
\text { 3DCI against previously } \\
\text { validated measures, i.e. CTM } \\
\text { and perometry }\end{array}$ & $\begin{array}{l}\text { - Data captured by researcher on } \\
\text { 3D Study Assessment Form }\end{array}$ & $\begin{array}{l}\text { - Pearson's Correlation } \\
\text { Coefficient } \\
\text { - Bland-Altman plots }\end{array}$ \\
\hline $\begin{array}{l}\text { 1.3 } \begin{array}{l}\text { Establish whether 3DCI is an } \\
\text { accurate method of } \\
\text { measurement for lymphoedema }\end{array} \\
\end{array}$ & $\begin{array}{l}\text { - Data captured by researcher on } \\
\text { 3D Study Assessment Form }\end{array}$ & $\begin{array}{l}\text { - Sensitivity and Specificity } \\
\text { using } 2 \times 2 \text { tables }\end{array}$ \\
\hline
\end{tabular}

\subsubsection{Data Collection}

To meet the three objectives, data were gathered on the demographic, lymphoedema and medical history, and anthropometric details of the participant at the initial assessment. Furthermore, data were collected on the timing and outcomes of the methods, which measured limb volume and $V D$ in millilitres $(\mathrm{ml})$, and $\% V D$ and $\% A V D$ in percentile. Measures were chosen based on clinical use (CTM), validated imaging comparator (perometry) and study method (3DCI). 
An assessment form (Appendix 10) was devised for this study to ensure the required data was collected and recorded.

\subsubsection{Data Analysis}

Guidance was sought from a senior statistician at Swansea University. Data handling and analysis were performed using IBM $^{\circledR}$ SPSS ${ }^{\circledR}$ Statistics, version 26 (IBM Corporation). Bossuyt et al. (2015) explain the development of 'The Standards for the Reporting of Diagnostic Accuracy Studies' statement which aims to improve the quality of reporting of diagnostic test studies and consists of a list of 30 essential items (Appendix 12) to ensure comprehensiveness and transparency in reporting of diagnostic test studies. These were reflected on to ensure concordance with this guidance during the reporting of the study.

The validity and reliability of measures are vital indicators of the quality of measuring instruments. Validity is often defined as the extent to which an instrument has measured what they purport to measure (Kumar, 2005; Kimberlin \& Winterstein, 2008; Salkind, 2011; Field, 2013). The concept of validity ensures that answers to a research question are gained through the correct procedures and can be applied to any aspect of the research process (Kumar, 2005). Thus, it looks at whether the instrument measures what it was designed to measure (Field, 2013).

In research, there are three types of validity, namely content validity, criterion validity (can be concurrent or predictive) and construct validity (Kumar, 2005; Salkind, 2011; Field, 2013). The first type, content validity, is relatively easy to apply as a logical link must exist between the objectives and the research questions (Kumar, 2005; Salkind, 2011). Criterion validity is where a new measure is compared to an existing or comparable measure to determine the same or similar outcomes. Concurrent validity, a sub-group of criterion validity, occurs when data are collected simultaneously using the new instrument and existing criteria, and predictive validity is when data from the new instrument are used to predict observations at a future point in time (Kumar, 2005; Salkind, 2011; Field, 2013). Construct validity, is a more sophisticated technique where the underlying construct or idea behind an instrument is validated by statistical procedures (Kumar, 2005; Salkind, 2011). 
Reliability is defined as the consistency of measurements (Mehta et al., 2018) and refers to the quality of a measurement process that provides accuracy and repeatability, i.e. an instrument should be interpreted consistently across different situations (Kumar, 2005; Salkind, 2011; Field, 2013). Different types of reliability exist. Repeatability, or testretest reliability, evaluates the stability of the measure administered over a set amount of time. Parallel forms of reliability are when the similarity between two different forms of the same test are examined. Inter-rater reliability is where different researchers apply the same test, and intra-rater reliability is where the same researcher performs the repeated tests. Internal consistency is the correlation of two halves of data from the same test construct (Kumar, 2005; Kimberlin \& Winterstein, 2008; Leddy, Crowner, \& Earhart, 2011; Salkind, 2011).

The development and validation of an instrument can be ascertained by reducing error, and the reporting of findings in an unbiased and objective way (Kumar, 2005; Kimberlin \& Winterstein, 2008). The ability of a measure to detect change over time is expressed as responsiveness (Kimberlin \& Winterstein, 2008). Reliability is a crucial component of responsiveness and must be appropriate to the people being studied. Sensitivity and specificity look at the probability of correctly identifying a condition and evaluating the probability of correctly identifying the absence of a condition, respectively (SAGE, 2019).

The goal of diagnostic test studies is to determine whether a test is useful in clinical practice. To accomplish this, the test needs to be reproducible, accurate, and be able to affect clinical decisions (Daya, 1996; Glasser, 2008). Kimberlin \& Winterstein (2008) explain that reliability evaluates the stability of measures administered at different times to the same individuals or using the same standard (test-retest or intra-rater reliability). Reliability coefficients range from 0.00 to 1.00 , with higher coefficients indicating higher levels of reliability (Kimberlin \& Winterstein, 2008).

Objective 1.1 compared the volumetric data from the three measurement methods to ensure that each method was repeatable, reproducible and thus reliable by calculating the ICC with a confidence interval of 95\%, standard deviation, standard error of measurement, and smallest detectable change. As only one rater was used per session, intra-rater reliability was tested. Intervals for test-retest studies should be over short 
periods of time, however with the sessions allocated as per the clinic standard operating procedures, and with known minimal change in clinical presentation over a period of four to six months with lymphoedema, only sessions one and two were compared (Ochalek, Gradalski, \& Szygula, 2015). All data for the six months were utilised to determine the mean values of limb volume for the affected and unaffected (control) arms, the volume difference (VD) between the affected and control arm, and the percentage volume difference (\%VD) at each point of assessment. The SD, SEM, and P-Values for each of the data were also analysed.

Leddy, Crowner, \& Earhart (2011) explain that a new assessment can be validated by comparing it to previously validated measures. For this study, concurrent validity ( $a$ subset of criterion validity) was assessed as a new method of measurement was being compared to existing measures to determine whether they produce the same or similar outcomes. Objective 1.2 required the calculation of correlation between the methods that were analysed through the application of Pearson's Correlation Coefficient (r). Agreement of the measures is possible through Bland-Altman plots. The Bland-Altman analysis determined the bias, or mean difference, between 3DCI and the two reference techniques (CTM and perometry), as a measure of validity and accuracy (Montenij, Buhre, Jansen, Kruitwagen, \& de Waal, 2016). However, before undertaking this analysis, the data requires a test of Normality to determine whether it followed a normal distribution (Glen, 2017). The Shapiro-Wilk and Kolmogorov-Smirnov tests of Normality, as well as Q-Q plots, were used as described by Field (2013). Where data deviated from the normal, normalisation of data was done using the Box-Cox power transformation (Currell, 2015; Glen, 2015b).

A test result is all that is known in clinical practice; thus confidence is required in knowing how good the test is at predicting abnormality, i.e. what proportion of patients with abnormal test results are genuinely abnormal? According to Glasser (2008), there are at least six ways to determine a test's accuracy, and these are all interrelated. These include sensitivity and specificity, $2 \times 2$ tables, predictive value, Bayes formula of conditional probability, likelihood ratio and Receiver Operator Characteristic curves. Determining which test to use is based on the question(s) being asked and personal preference. To note, Glasser (2008) reports that reproducibility does not require a gold (reference) standard, while accuracy does. Altman \& Bland (1994) and Daya (1996) 
describe sensitivity as the proportion of true-positives that are correctly identified by a test, and specificity as the proportion of true-negatives that are correctly identified by a test. Akobeng (2007) explains that sensitivity and specificity are inversely related according to the choice of cut-off values used, and Receiver Operator Characteristic curves can be used to identify these. The threshold used for diagnosing lymphoedema in this study was $\geq 5 \%$ difference in limb volume, as this threshold is used in clinical practice in Wales. For purposes of this study, calculations of sensitivity and specificity utilising $2 \times 2$ tables were used to address Objective 1.3 .

\subsection{Phase Two: Diagnostic Test Study of Limb Shape}

\subsubsection{Phase Two Research Questions, Aim, and Objectives}

The aim of Phase Two was to determine whether limb shape is a feasible alternative to measure lymphoedema.

The objectives for Phase Two were:

2.1 Evaluate limb shape derived from 3DCI as a new method of lymphoedema measurement

a) Determine trends between oedema maps and limb volume

b) Use of oedema maps to monitor treatment outcomes (limb volume change)

c) Consider limb shape as a tool to diagnose lymphoedema

2.2 Ascertain 3DCI as a sensitive measure of changes in lymphoedema

a) Sensitivity to change in limb volume over time

b) Sensitivity to change in limb shape over time

Table 23: Phase Two objectives and methods of analysis

\begin{tabular}{|c|c|}
\hline Objective & Method of Analysis \\
\hline $\begin{array}{l}\text { 2.1 Evaluate limb shape derived from 3DCI as a new } \\
\text { method of lymphoedema measurement: } \\
\text { a) Determine trends between oedema maps and } \\
\text { limb volume } \\
\text { b) Use of oedema maps to monitor treatment } \\
\text { outcomes (limb volume change) } \\
\text { c) Consider limb shape as a tool to diagnose } \\
\text { lymphoedema }\end{array}$ & $\begin{array}{l}\text { - } \quad \text { Compare shape redundancy (R-value) to } \\
\text { volumetric data from Phase One } \\
\text { - } \quad \text { Pearson's Correlation Coefficient } \\
\text { - } \quad \text { Bland-Altman plot } \\
\text { - } \quad \text { Violin plots }\end{array}$ \\
\hline $\begin{array}{l}\text { 2.2 Ascertain 3DCI as a sensitive measure of changes in } \\
\text { lymphoedema } \\
\text { a) Sensitivity to change in limb volume over time } \\
\text { b) Sensitivity to change in limb shape over time }\end{array}$ & - $\quad 2 \times 2$ tables \\
\hline
\end{tabular}


Table 23 provides a breakdown of Phase Two's objectives and the planned methods of analysis that were used to measure these objectives. The chosen methods used and the data collected for this phase of the study are discussed in the next section.

\subsubsection{Data Collection}

Data collected for the volume outcomes for Phase One were utilised to meet the two objectives of Phase Two. These include data from that used in clinics (CTM), the validated imaging comparator (perometry) and the study method (3DCI). The devised assessment form (Appendix 10) collected and recorded the data. As the 3D conversion software was still being refined at the time of the study, the 3DCI images were shared with the College of Engineering, Swansea University, who converted the images into oedema maps (swelling maps), and corresponding shape redundancy values. It is anticipated that this software will form a package that will automatically convert the 3DCI image into oedema maps at the point of measurement in the future. As described in section 4.5.7.2, calculation of shape redundancy provides an $R$-value, which was compared to the limb volume differences for analysis of correlation and agreement between the various methods of measurements to determine the value of shape as a new method of lymphoedema measurement. In addition, descriptive analysis of each oedema map is compared to the \%VDs reported for each method from Phase One.

\subsubsection{Data Analysis}

Working in collaboration with the Engineering Department of Swansea University, the 3DCI data were converted into oedema maps and shape redundancy (R-value) calculated (refer to section 4.5.7.2). These were compared to the volumetric data from Phase One, which enabled an interpretation of change in shape over time, potentially informing treatment outcomes.

Objective 2.1 determined any correlations between volumetric measurements and derived oedema maps from the 3DCI data. This was aided by the mathematical calculation of the limb shape through shape redundancy. The limb Shape Redundancy Value (R-value) was compared to the various limb \%VD through the use of Boxplots and Violin plots. A boxplot visually shows the distribution of numerical data and skewness through displaying data quartiles (or percentiles) and averages, including the minimum score, lower quartile, median, upper quartile, and maximum score (McLeod, 
2019). Violin plots (Figure 29) are similar to box plots as it shows the peaks in the data pertaining to the median and interquartile ranges, but uniquely the bulge of the shape demonstrates higher probability, while the thinner sections represent a lower probability of the measure (Carron, 2016). These plots determined the trends between limb shape and limb volume outcomes. As in Phase One, Pearson's Correlation Coefficients and Bland-Altman plots were utilised to ascertain the criterion validity of the oedema maps compared to limb volume outcomes.

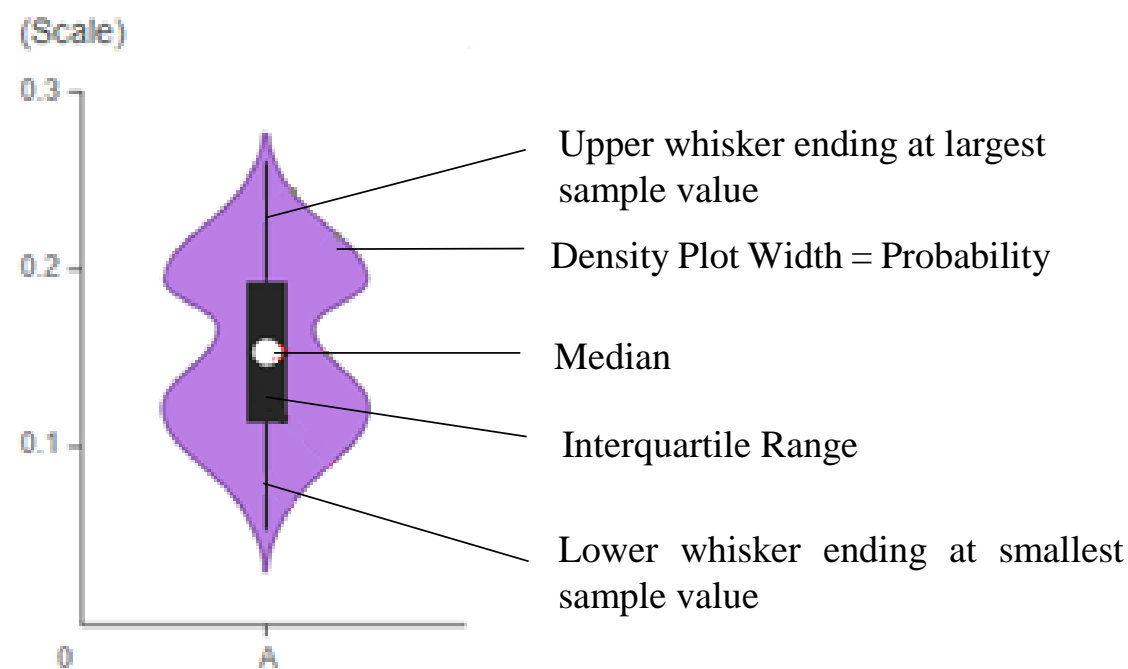

Figure 29: Interpreting a Violin Plot (Carron, 2016; Cohen, 2018). The white dot, thick black bar and thin black line represent the median (mid-point of the data), interquartile range (middle 50\% of scores for the group) and the rest of the distribution akin to a Box Plot. The wider the kernel (purple body) of the Violin Plot, the higher the probability of the measure.

As discussed in section 4.6.3, there are several methods to determine the sensitivity of a new method of measurement. For Objective 2.2, 2x2 tables were utilised by comparing $\mathrm{R}$-value to limb volume data as calculated by CTM, perometry and 3DCI, to determine the sensitivity and specificity of limb shape using 3DCI. 


$$
\begin{aligned}
& \text { Chapter 5: } \\
& \text { Study Results }
\end{aligned}
$$




\subsection{Recruitment}

The recruitment process for this study, together with the number recruited, is illustrated in Figure 30. Although this study was open to men and women, only women were recruited as no men had been referred to the lymphoedema service with upper limb lymphoedema during the 19-month duration of the study. Sixty-eight referrals to the NHS Wales Lymphoedema Service were screened from September 2016 to March 2018 against the study inclusion and exclusion criteria as described in sections 4.5.2.1 and 4.5.2.2.

Twenty women were recruited between October 2016 and April 2018. At the screening, participants were excluded if only hand $(n=2)$ or breast oedema $(n=1)$ was identified, presence of bilateral upper limb lymphoedema $(n=1)$, where no lymphoedema was observed or palpated $(n=29)$, or if the referral was inappropriate $(n=1)$. The inappropriate referral was for a patient with a musculoskeletal injury. Those participants who were unable to consent $(n=1)$ due to dementia, had active or palliative disease $(n=3)$, or insufficient shoulder range of movement $(n=6)$, were excluded. Thus $35.3 \%(n=24)$ of screened new referrals were eligible for the study. Four people who met the eligibility criteria declined to partake in the study due to time commitment $(\mathrm{n}=2)$, and travelling required for the sessions within the study $(n=2)$.

Recruitment was more challenging than initially envisioned possibly due to a successful Lymphoedema Risk Reduction programme at the lymphoedema service, which resulted in a smaller volume of referrals to the department than anticipated (refer to section 4.5.2).

\subsection{Size of Sample}

From the original 20 participants recruited, there was an attrition rate of $15 \%(n=3)$ in which one participant did not return following the initial assessment, and two failed to attend their final session. One of the last two participants missed their second session due to undergoing minor surgery at the time of their appointment. Contact could not be made with any of the three participants who did not return in an attempt to understand their reason for not attending their appointment. 
Every effort was made to retain participants, but attempts at contacting them were made no more than three times on separate days. None of these participants responded to mail correspondence sent by the lymphoedema service either. As none of these participants requested their data be excluded from the study, all measurements taken have been included for analysis, as agreed during the consenting process.

Missing data was managed as 'missing completely at random'. These data were deleted list-wise, and a $t$-test performed to check whether any difference in the sample sets existed (see section 4.5.5).

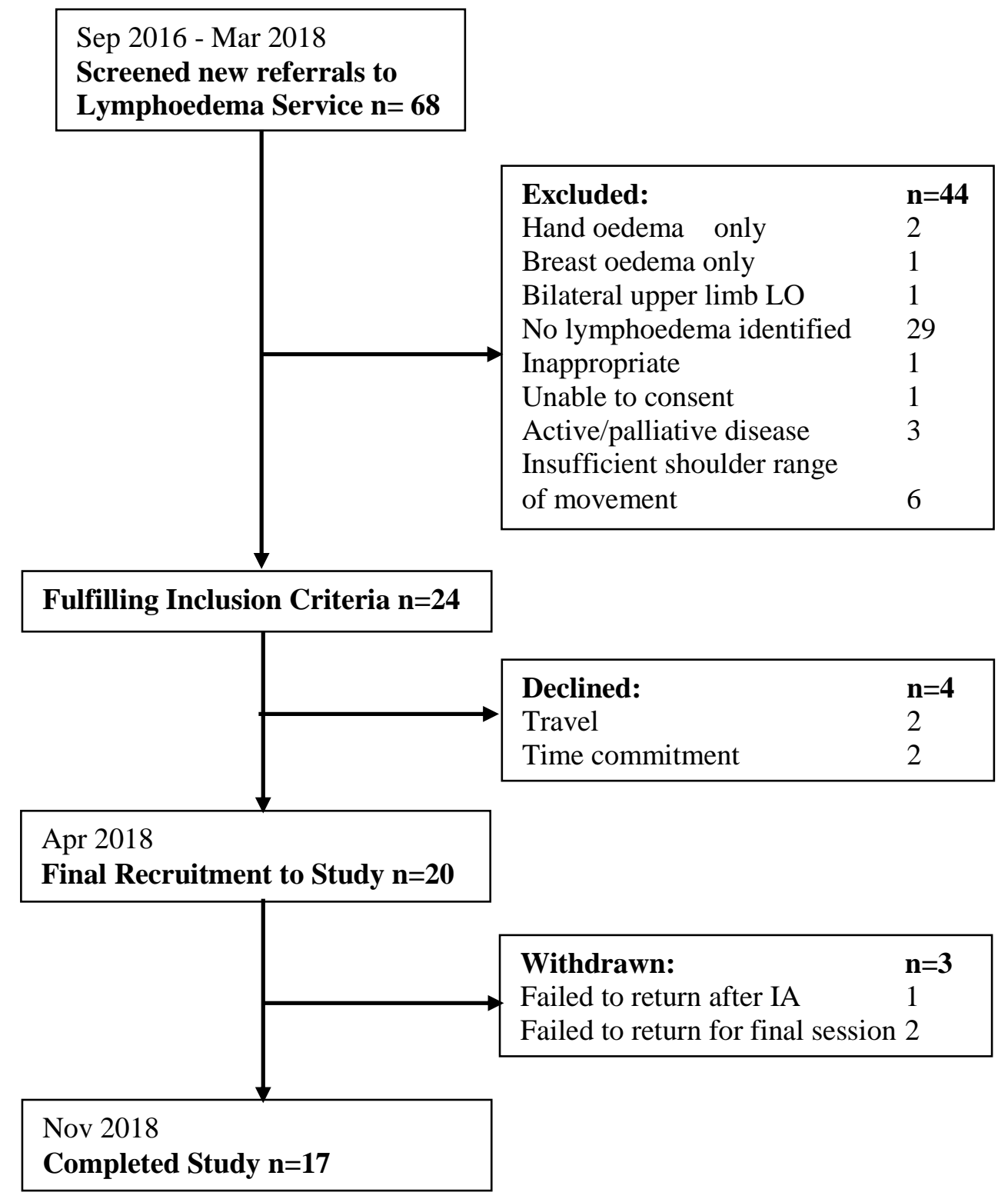

Figure 30: Flow diagram of recruitment study processes 


\subsection{Demographic Details}

The anthropometric data were collated with a summary of the descriptive demographic details in Table 24. The mean age was 58.9 years (range 43 to 77 years). The cohort ethnicity was 95\% Caucasian $(n=19)$, one of Asian origin. Most participants were still employed $(70 \%, \mathrm{n}=14), 85 \%(\mathrm{n}=17)$ did not smoke, and 35\% $(\mathrm{n}=7)$ drank occasionally. The mean weight was $82.7 \mathrm{~kg}$ (SD 17.0), and the mean BMI was $32.2 \mathrm{~kg} / \mathrm{m}^{2}$ (SD 7.0). From Table 24, it is evident that most of the participants $(90 \%, \mathrm{n}=18)$ were overweight or obese, with only two (10\%) participants in the normal weight category.

All participants were female with lymphoedema secondary to breast cancer treatment with the average time since surgery being three years (SD 3.70). The time of onset of lymphoedema since surgery was 1.89 years (SD 2.66 years); however, one participant was receiving neoadjuvant chemotherapy (i.e. chemotherapy before surgery) at the time of the assessment. Handedness was analysed in which 18 (90\%) participants were right hand dominant, with $50 \%(n=9)$ of these being the affected arm. There was good representation between those with affected dominant (55\%) versus affected nondominant $(45 \%)$ sides.

\subsection{Medical Details}

Table 25 gives a summary of the medical details about the cohort. Of the 19 participants who had had surgery, $12(60 \%)$ had a mastectomy and $16(80 \%)$ had axillary node clearance (ANC), with five of the participants having had sentinel lymph node biopsy prior to the ANC. Nine (45\%) participants had all four breast cancer treatments available i.e. surgery, radiotherapy, chemotherapy and hormone therapy. Of those who had hormone therapy, one had stopped following 10 years of treatment.

Lymphoedema ISL Staging (Appendix 1) shows most participants were in Stage I reversible lymphoedema $(70 \%, n=14)$, with the BLS Grouping (Appendix 1) mostly in Group 1 early lymphoedema $(65 \%, n=13)$. Three participants $(15 \%)$ had one or more previous episodes of cellulitis, but none were on prophylactic antibiotics. The most common chronic comorbidity was arthritis $(n=7)$, with cardiac/respiratory and skin conditions, each accounting for five participants. 
Table 24: Demographic details of study cohort

\begin{tabular}{|c|c|c|c|}
\hline Variable & Category & $\mathbf{n}$ & $(\%)$ \\
\hline \multirow{5}{*}{ Age Group } & $40-49$ & 3 & (15) \\
\hline & $50-59$ & 7 & $(35)$ \\
\hline & $60-69$ & 8 & $(40)$ \\
\hline & $70+$ & 2 & $(10)$ \\
\hline & TOTAL & 20 & (100) \\
\hline \multirow{4}{*}{ Ethnic Origin } & Caucasian & 19 & $(95)$ \\
\hline & Asian & 1 & $(5)$ \\
\hline & Other & 0 & $(0)$ \\
\hline & TOTAL & 20 & (100) \\
\hline \multirow{4}{*}{ Employment } & Employed & 14 & $(70)$ \\
\hline & Unemployed & 2 & (10) \\
\hline & Retired & 4 & (20) \\
\hline & TOTAL & 20 & (100) \\
\hline \multirow{9}{*}{ Lifestyle Choices } & Do not Smoke & 17 & $(85)$ \\
\hline & Smoke & 3 & $(15)$ \\
\hline & TOTAL & 20 & (100) \\
\hline & Do not drink alcohol & 5 & $(25)$ \\
\hline & Drink alcohol occasionally & 7 & $(35)$ \\
\hline & $1-5$ units per week & 5 & $(25)$ \\
\hline & $6-9$ units per week & 2 & (10) \\
\hline & 10 or more units per week & 1 & $(5)$ \\
\hline & TOTAL & 20 & (100) \\
\hline \multirow{6}{*}{ BMI Groups } & Normal weight (18.5-24.9) & 2 & (10) \\
\hline & Overweight $(25-29.9)$ & 7 & (35) \\
\hline & Obese Class 1 (30-34.9) & 4 & (20) \\
\hline & Obese Class 2 (35-39.9) & 5 & $(25)$ \\
\hline & Obese Class 3 (40 or more) & 2 & $(10)$ \\
\hline & TOTAL & 20 & (100) \\
\hline \multirow{5}{*}{ Time Since Surgery } & Less than 2 years & 8 & $(40)$ \\
\hline & $2-5$ years & 8 & $(40)$ \\
\hline & More than 5 years & 3 & $(15)$ \\
\hline & Not had surgery & 1 & $(5)$ \\
\hline & TOTAL & 20 & (100) \\
\hline \multirow{5}{*}{$\begin{array}{l}\text { Time from Surgery to } \\
\text { BCRL Onset }\end{array}$} & Less than 2 years & 10 & $(50)$ \\
\hline & $2-5$ years & 8 & (40) \\
\hline & More than 5 years & 1 & $(5)$ \\
\hline & Not had surgery & 1 & $(5)$ \\
\hline & TOTAL & 20 & (100) \\
\hline \multirow{3}{*}{ Handedness } & Right Dominant & 18 & (90) \\
\hline & Left Dominant & 2 & (10) \\
\hline & TOTAL & 20 & (100) \\
\hline \multirow{3}{*}{ Affected Side } & Right & 9 & $(45)$ \\
\hline & Left & 11 & (55) \\
\hline & TOTAL & 20 & (100) \\
\hline
\end{tabular}


Table 25: Medical details of study cohort

\begin{tabular}{|c|c|c|c|}
\hline Variable & Category & $\mathbf{n}$ & $(\%)$ \\
\hline \multirow{9}{*}{ Surgery Type } & Wide Local Excision & 7 & (35) \\
\hline & Mastectomy & 12 & $(60)$ \\
\hline & No Surgery & 1 & (5) \\
\hline & TOTAL & 20 & (100) \\
\hline & Sentinel Node Biopsy & 2 & (10) \\
\hline & Axillary Node Sampling & 1 & $(5)$ \\
\hline & Axillary Node Clearance & 16 & $(80)$ \\
\hline & No surgery & 1 & (5) \\
\hline & TOTAL & 20 & (100) \\
\hline \multirow{3}{*}{ Chemotherapy } & Yes & 15 & (75) \\
\hline & No & 5 & $(25)$ \\
\hline & TOTAL & 20 & (100) \\
\hline \multirow{3}{*}{ Radiotherapy } & Yes & 15 & $(75)$ \\
\hline & No & 5 & $(15)$ \\
\hline & TOTAL & 20 & (100) \\
\hline \multirow{6}{*}{$\begin{array}{l}\text { Hormone Therapy } \\
\text { - Type }\end{array}$} & Yes & 14 & $(70)$ \\
\hline & - $\quad$ Aromatase Inhibitor & 7 & $(35)$ \\
\hline & - Tamoxifen & 4 & $(20)$ \\
\hline & - Herceptin & 2 & $(10)$ \\
\hline & No & 6 & $(30)$ \\
\hline & TOTAL & 20 & (100) \\
\hline \multirow{3}{*}{ Multiple Treatments } & Surgery, Radiotherapy, Chemotherapy & 13 & $(65)$ \\
\hline & Surgery, Radiotherapy, Hormone Therapy & 11 & (55) \\
\hline & Surgery, Chemotherapy, Hormone Therapy & 10 & $(50)$ \\
\hline \multirow{8}{*}{$\begin{array}{l}\text { Lymphoedema } \\
\text { Staging }\end{array}$} & ISL Stage I - Reversible & 14 & (70) \\
\hline & ISL Stage IIa - Pitting & 4 & (20) \\
\hline & ISL Stage IIb - Non-pitting & 2 & $(10)$ \\
\hline & TOTAL & 20 & (100) \\
\hline & BLS Group 1 - Early & 13 & $(65)$ \\
\hline & BLS Group 2 - Uncomplicated & 3 & $(15)$ \\
\hline & BLS Group 3a-Complex One Limb & 4 & $(20)$ \\
\hline & TOTAL & 20 & (100) \\
\hline \multirow{3}{*}{ Cellulitis } & Yes & 3 & (15) \\
\hline & No & 17 & $(85)$ \\
\hline & TOTAL & 20 & $(\mathbf{1 0 0})$ \\
\hline \multirow{9}{*}{ Medical Conditions } & Arthritis & 7 & (35) \\
\hline & Cardiac/Respiratory Condition & 5 & $(25)$ \\
\hline & Skin Conditions & 5 & $(25)$ \\
\hline & Hypertension & 4 & $\frac{(20)}{(20)}$ \\
\hline & Stroke / Mini-stroke & 3 & $(15)$ \\
\hline & Hypercholesterolaemia & 3 & (15) \\
\hline & Diabetes & 2 & (10) \\
\hline & Hyperthyroidism & 2 & $(10)$ \\
\hline & Vascular Disease & 2 & (10) \\
\hline \multirow{3}{*}{ Allergies } & Elastoplast/Micropore & 4 & (20) \\
\hline & Penicillin & 3 & (15) \\
\hline & Erythromycin & 2 & (10) \\
\hline
\end{tabular}




\subsection{Phase One: Limb Volume Results and Analysis}

5.5.1 Objective 1.1: Determine the Reliability of 3DCI Compared to CTM and Perometry

The two-tailed paired-samples tests between sessions one and two, i.e. assessment and first follow up at 2-months, (Table 26) shows all measures to be significant $(\mathrm{p} \leq 0.00)$ with the intra-rater reliability of CTM being the highest at $0.92(95 \% \mathrm{CI}=0.89,0.94)$. Perometer had an $\mathrm{ICC}_{\text {intra }}$ value of $0.89(95 \% \mathrm{CI}=0.85,0.93)$, and $3 \mathrm{DCI} \mathrm{ICC}_{\text {intra }}$ was 0.87 (95\% CI $=0.80,0.93)$. The SEM for CTM, perometry and 3DCI was $0.95 \%, 1.33 \%$ and $1.72 \%$ respectively, with the SDC values at $2.63 \%, 3.69 \%$ and $4.77 \%$ respectively.

Table 27 summarises all the data from CTM, perometry and 3DCI over the four sessions in terms of mean values of limb volume for the affected and unaffected (control) arms, the $V D$ between the affected and control arm, and the \%VD at each point of assessment. Also presented are the SD, SEM, and P-Values for each of the data analysed. Overall, 3DCI showed the lowest mean limb volume for both the affected and unaffected arms, the smallest $V D$ between arms, as well as the smallest SD and SEM values with all data significant at $\mathrm{p}=0.00$. Incidentally, the lowest $\% V D$ was with $\mathrm{CTM}$, even though the limb volume difference was the highest of the three measurements. When comparing limb volumes of the affected and unaffected arms, 3DCI was found to underestimate limb volume by $278 \mathrm{ml}$ compared to CTM, and by $354 \mathrm{ml}$ compared to perometry.

3DCI was the fastest measurement to perform with an average time of 1:50 min (SD 0:17 min). To note, even though two participant's data could not be used due to corruption of the images, the measurements were still performed and timed. CTM took the longest at 5:54 min, but this can be explained by the need for repeated measuring of a limb, where the marking on the arm may have been incorrect due to either the participant's arm position or the researcher's error in the $4 \mathrm{~cm}$ spacing. Additionally, errors were made due to the patient engaging in conversation during the measuring process that distracted the researcher while recording the data. Perometry and 3DCI are automated methods of measuring, thus does not require the same level of concentration to perform as CTM. 
Table 26: Paired-samples correlations for CTM, perometry and 3DCI between sessions 1 and 2

\begin{tabular}{|c|c|c|c|c|c|c|c|}
\hline & \multirow{2}{*}{$\mathrm{ICC}_{\text {intra }}$} & \multirow{2}{*}{ Sig. } & \multirow{2}{*}{ Mean (SD) } & \multirow{2}{*}{ SEM } & \multirow{2}{*}{ SDC } & \multicolumn{2}{|c|}{$95 \% \mathrm{CI}$ of the Difference } \\
\hline & & & & & & Lower & Upper \\
\hline \multicolumn{8}{|l|}{ CTM (n=18) } \\
\hline Affected Arm (ml) & 0.92 & 0.00 & $225.67(550.51)$ & 129.76 & 359.68 & -48.09 & 499.43 \\
\hline Control Arm (ml) & 0.89 & 0.00 & $-159.56(553.07)$ & 125.65 & 300.31 & -424.64 & 105.53 \\
\hline Volume Difference (ml) & 0.94 & 0.00 & $67.67(137.49)$ & 32.41 & 89.84 & -0.70 & 136.04 \\
\hline Percentage Volume Difference (\%) & 0.92 & 0.00 & $2.11(4.04)$ & 0.95 & 2.63 & 0.10 & 4.12 \\
\hline \multicolumn{8}{|l|}{ Perometer $(n=16)$} \\
\hline Affected Arm (ml) & 0.90 & 0.00 & $28.88(277.29)$ & 69.32 & 165.68 & -118.88 & 176.63 \\
\hline Control Arm (ml) & 0.93 & 0.00 & $-16.06(255.77)$ & 63.94 & 177.23 & -152.35 & 120.23 \\
\hline Volume Difference (ml) & 0.85 & 0.00 & $44.94(148.63)$ & 37.16 & 103.00 & -34.26 & 124.14 \\
\hline Percentage Volume Difference (\%) & 0.86 & 0.00 & $2.06(5.32)$ & 1.33 & 3.69 & -0.77 & 4.90 \\
\hline \multicolumn{8}{|l|}{ 3DCI $(n=16)$} \\
\hline Affected Arm (ml) & 0.93 & 0.00 & $-1.66(209.03)$ & 52.26 & 144.86 & -113.04 & 109.73 \\
\hline Control Arm (ml) & 0.89 & 0.00 & $32.72(207.24)$ & 51.81 & 143.6 & -77.71 & 143.15 \\
\hline Volume Difference (ml) & 0.87 & 0.00 & $-34.31(147.45)$ & 36.86 & 102.17 & -112.87 & 44.26 \\
\hline Percentage Volume Difference (\%) & 0.80 & 0.00 & $-1.44(6.88)$ & 1.72 & 4.77 & -5.10 & 2.23 \\
\hline
\end{tabular}

Key:

3DCI $=$ Three-dimesional Camera Imaging $\quad \mathrm{CI}=$ Confidence Interval

$\mathrm{ICC}_{\text {intra }}=$ Intraclass Correlation Coefficient for intrarater reliability

CTM $=$ Circumferential Tape Measure

SDC $=$ Smallest Detectable Change

SEM $=$ Standard Error of Measure

$\mathrm{SD}=$ Standard Deviation

Sig. $=$ Significance 
Table 27: Summary of all the data from CTM, perometry and 3DCI over the four sessions

\begin{tabular}{|c|c|c|c|c|c|c|c|c|c|c|c|c|c|}
\hline & $\begin{array}{c}\text { Mean Volume } \\
\text { Affected Arm } \\
(\mathrm{ml})(\mathrm{SD}) \\
\end{array}$ & SEM & $\begin{array}{c}\mathrm{P}- \\
\text { Value }\end{array}$ & $\begin{array}{l}\text { Mean Volume } \\
\text { Control Arm } \\
\text { (ml) (SD) }\end{array}$ & SEM & $\begin{array}{c}\text { P- } \\
\text { Value }\end{array}$ & $\begin{array}{c}\text { Volume } \\
\text { Difference (ml) } \\
\text { (SD) }\end{array}$ & SEM & $\begin{array}{c}\text { P- } \\
\text { Value }\end{array}$ & $\begin{array}{c}\text { Percentage } \\
\text { Volume Difference } \\
(\%)(\mathrm{SD}) \\
\end{array}$ & SEM & $\begin{array}{c}\mathrm{P}- \\
\text { Value }\end{array}$ & $\begin{array}{l}\text { Mean Time } \\
\text { in minutes } \\
(\mathrm{SD})(\mathrm{n}=20)\end{array}$ \\
\hline \multicolumn{14}{|c|}{ CTM } \\
\hline $\begin{array}{c}\text { Session } 1 \\
(\mathrm{n}=20)\end{array}$ & $3090.8(1103.0)$ & 246.6 & 0.00 & $2829.3(787.0)$ & 176.0 & 0.00 & $317.2(395.4)$ & 88.4 & 0.00 & $10.2(9.7)$ & 2.2 & 0.00 & \multirow{5}{*}{$\begin{array}{c}05: 54 \\
(01: 17)\end{array}$} \\
\hline $\begin{array}{c}\text { Session } 2 \\
(\mathrm{n}=18)\end{array}$ & 2980.9 (108.9) & 261.4 & 0.00 & $2850.4(753.0)$ & 177.5 & 0.00 & $249.0(374.7)$ & 88.3 & 0.01 & $7.8(10.2)$ & 2.4 & 0.00 & \\
\hline $\begin{array}{c}\text { Session } 3 \\
(\mathrm{n}=19)\end{array}$ & $2881.4(1106.2)$ & 253.8 & 0.00 & $2781.3(758.0)$ & 173.9 & 0.00 & $206.3(387.7)$ & 88.9 & 0.03 & $6.3(9.9)$ & 2.3 & 0.01 & \\
\hline $\begin{array}{c}\text { Session } 4 \\
(\mathrm{n}=17)\end{array}$ & $3058.4(1128.1)$ & 273.6 & 0.00 & $2868.4(763.7)$ & 185.2 & 0.00 & $255.4(437.3)$ & 106.1 & 0.03 & $8.0(10.7)$ & 2.6 & 0.01 & \\
\hline $\begin{array}{c}\text { TOTAL } \\
(\mathrm{n}=74)\end{array}$ & $3002.9(1091.1)$ & 126.8 & 0.00 & $2831.1(750.9)$ & 87.3 & 0.00 & $257.9(392.5)$ & 45.6 & 0.00 & $8.1(10.0)$ & 1.2 & $\mathbf{0 . 0 0}$ & \\
\hline \multicolumn{14}{|c|}{ Perometer } \\
\hline $\begin{array}{c}\text { Session } 1 \\
(\mathrm{n}=20)\end{array}$ & $3168.5(983.0)$ & 219.8 & 0.00 & $2885.0(742.5)$ & 166.0 & 0.00 & $270.0(454.0)$ & 101.5 & 0.02 & $9.7(12.0)$ & 2.7 & 0.00 & \multirow{5}{*}{$\begin{array}{c}01: 56 \\
(00: 35)\end{array}$} \\
\hline $\begin{array}{c}\text { Session } 2 \\
(\mathrm{n}=16)\end{array}$ & $3043.4(565.4)$ & 141.4 & 0.00 & $2883.3(498.9)$ & 124.7 & 0.00 & $160.1(276.3)$ & 69.1 & 0.04 & $5.8(9.7)$ & 2.4 & 0.03 & \\
\hline $\begin{array}{c}\text { Session } 3 \\
(\mathrm{n}=19)\end{array}$ & $3129.2(861.5)$ & 197.6 & 0.00 & $2879.7(614.1)$ & 140.9 & 0.00 & $260.5(462.9)$ & 106.2 & 0.02 & $8.4(12.0)$ & 2.8 & 0.01 & \\
\hline $\begin{array}{c}\text { Session } 4 \\
(\mathrm{n}=17)\end{array}$ & $3152.0(1004.0)$ & 243.5 & 0.00 & $2866.4(703.6)$ & 170.6 & 0.00 & $274.8(494.5)$ & 119.9 & 0.04 & $8.8(14.0)$ & 3.4 & 0.02 & \\
\hline $\begin{array}{c}\text { TOTAL } \\
(n=72)\end{array}$ & $3126.4(862.3)$ & 101.6 & 0.00 & $2878.8(638.2)$ & 75.2 & 0.00 & $244.2(427.6)$ & 50.4 & 0.00 & $8.3(11.9)$ & 1.4 & 0.00 & \\
\hline \multicolumn{14}{|c|}{ 3DCI } \\
\hline $\begin{array}{c}\text { Session } 1 \\
(\mathrm{n}=18)\end{array}$ & $2762.2(533.8)$ & 125.8 & 0.00 & $2540.5(432.2)$ & 101.9 & 0.00 & $221.7(278.2)$ & 65.6 & 0.00 & $8.8(10.4)$ & 2.5 & 0.00 & \multirow{5}{*}{$\begin{array}{c}01: 50 \\
(00: 17)\end{array}$} \\
\hline $\begin{array}{c}\text { Session } 2 \\
(\mathrm{n}=16)\end{array}$ & $2726.6(566.5)$ & 141.6 & 0.00 & $2498.1(436.4)$ & 109.1 & 0.00 & $228.4(297.7)$ & 74.4 & 0.01 & $9.2(11.4)$ & 2.9 & 0.01 & \\
\hline $\begin{array}{c}\text { Session } 3 \\
(\mathrm{n}=17)\end{array}$ & $2743.4(541.9)$ & 131.4 & 0.00 & $2566.2(398.9)$ & 96.7 & 0.00 & $177.2(259.8)$ & 63.0 & 0.01 & $6.6(9.4)$ & 2.3 & 0.01 & \\
\hline $\begin{array}{c}\text { Session } 4 \\
(\mathrm{n}=15)\end{array}$ & $2745.4(527.8)$ & 136.3 & 0.00 & $2516.3(497.8)$ & 128.5 & 0.00 & $229.1(284.3)$ & 73.4 & 0.01 & $9.9(13.5)$ & 3.5 & 0.01 & \\
\hline $\begin{array}{c}\text { TOTAL } \\
(n=66)\end{array}$ & $2744.9(530.1)$ & 65.3 & 0.00 & $2531.3(431.3)$ & 53.1 & 0.00 & $213.6(274.2)$ & 33.8 & 0.00 & $8.6(11.0)$ & 1.4 & 0.00 & \\
\hline
\end{tabular}




\subsubsection{Objective 1.2: Assess the Validity of 3DCI Against Previously Validated Measures, i.e. CTM and Perometry}

\subsubsection{Relationship Between the Three Methods of Measurement Using Pearson's Correlation Coefficient}

The criterion validity of 3DCI at all time points through the application of Pearson's Correlation Coefficient demonstrated high correlations between methods, with marginally higher correlation with $\mathrm{CTM}(\mathrm{r}=0.86)$ than perometry $(\mathrm{r}=0.82)$ (refer to Table 28). The highest correlation was between CTM and perometry $(r=0.96)$. All correlations were significant with $\mathrm{p}<0.01$.

Table 28: Pearson's correlation coefficient between CTM, perometry and 3DCI's calculations of total volume difference

\begin{tabular}{|l|l|c|c|}
\hline \multicolumn{2}{|c|}{} & CTM Total VD & Perometer Total VD \\
\hline \multirow{2}{*}{ 3DCI Total VD } & Pearson Correlation & $.86^{* *}$ & $.82^{* *}$ \\
\cline { 2 - 4 } & Sig. (2-tailed) & .00 & .00 \\
\hline \multirow{2}{*}{ CTM Total VD } & Pearson Correlation & 1 & $.96^{* *}$ \\
\cline { 2 - 4 } & Sig. (2-tailed) & .00 \\
\hline \multirow{2}{*}{$* *$ Correlation is significant at the 0.01 level (2-tailed). } \\
\hline
\end{tabular}

\subsubsection{Agreement Between the Three Methods of Measurement Utilising Bland-Altman Plots}

Prior to normalisation of the total VD data for each method of measurement, Table 29 illustrates that there was some agreement between the tests as all p-values were $>0.05$.

Table 29: Agreement between the methods of measurement through comparison of their volume differences

\begin{tabular}{lc|c|c} 
& $\mathrm{t}$ & $\mathrm{df}$ & Sig. (2-tailed) \\
\hline Difference 3DCI-CTM & 1.100 & 65 & .275 \\
\hline Difference 3DCI-Pero & 1.819 & 64 & .074 \\
\hline Difference CTM-Pero & .432 & 71 & .667 \\
\hline
\end{tabular}

3DCI initially had a statistically non-significant Kolmogorov-Smirnov test $(p=0.68)$ that could be used to indicate a normal distribution. However, for CTM total VD, data were normalised using the logarithm of the data. Furthermore, due to an extreme deviation caused by data entry number 68 for perometry, this data was removed to allow for the normalisation of the data through the application of the logarithm to the adjusted total $V D$ data for perometry (Currell, 2015) (Appendix 13). 
For the Bland-Altman plot between 3DCI and CTM (Figure 31), the raw VD data for each method was used as it showed a strong distribution within the upper and lower limits of agreement (calculated at $1.96 \times \mathrm{SD}$ ), with two outliers above the upper limit of agreement. Using the raw $V D$ data for 3DCI and CTM, the mean calculated difference was $19.09 \mathrm{ml}$ (SD $140.95 \mathrm{ml})$. This large SD is due to the existent SD noted for both 3DCI and CTM as indicated in Table 27 which were higher than each of the VDs for each of the methods. The upper limit of agreement was $295.35 \mathrm{ml}$, and the lower limit of agreement $-257.17 \mathrm{ml}$. The associated coefficient chart (Appendix 13), had a B-value for the mean of 0.09 (close to zero), with a non-significant p-value of 0.21 , indicating no proportional bias; therefore, there was a level of agreement between 3DCI and CTM.

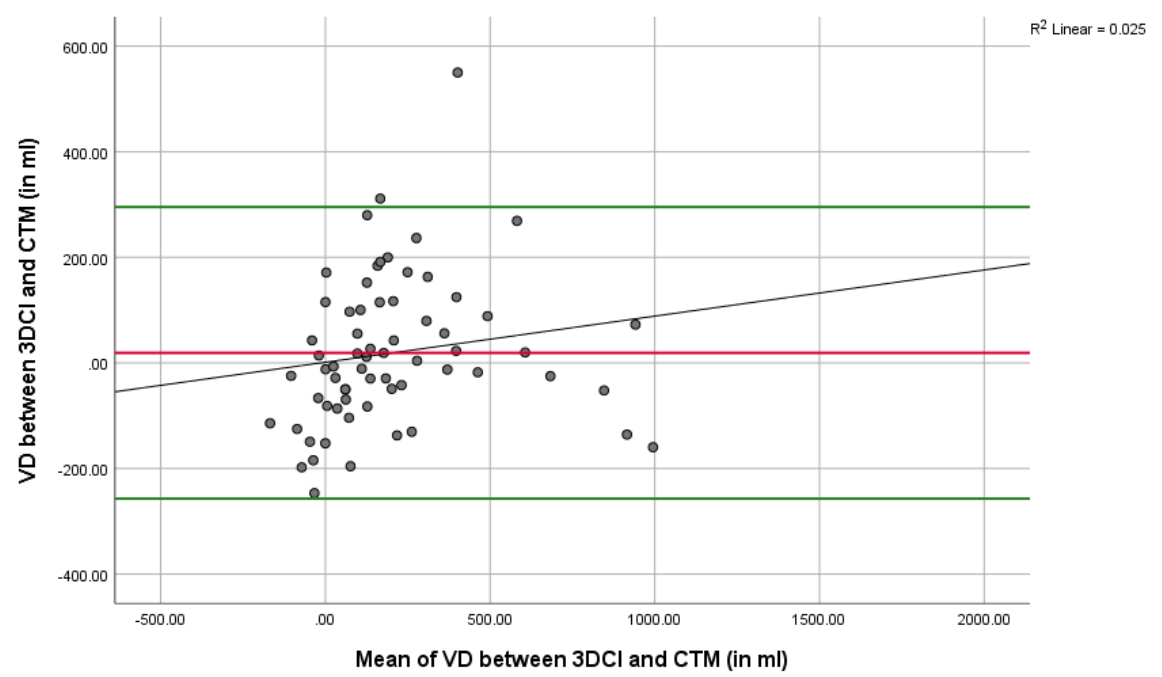

Figure 31: Bland-Altman Plot: a scatterplot of the mean of 3DCI and CTM methods plotted against the difference between the two methods

For the Bland-Altman plot between 3DCI and perometry (Figure 32), the raw VD data for each method was used as it showed a strong distribution within the upper and lower limits of agreement, with two outliers above the upper limit of agreement. Using the raw $V D$ data for 3DCI and perometry, the mean calculated difference was $36.72 \mathrm{ml}$ (SD $162.77 \mathrm{ml})$. Again, the large SD was due to the existent SD noted for both 3DCI and perometry as indicated in Table 27. The upper limit of agreement was $355.75 \mathrm{ml}$, and the lower limit of agreement $-282.32 \mathrm{ml}$. The associated coefficient chart (Appendix 13), had a B-value for the mean of 0.05 , with a non-significant $p$-value of 0.21 , indicating no proportional bias; therefore, there was a level of agreement between 3DCI and perometry. 


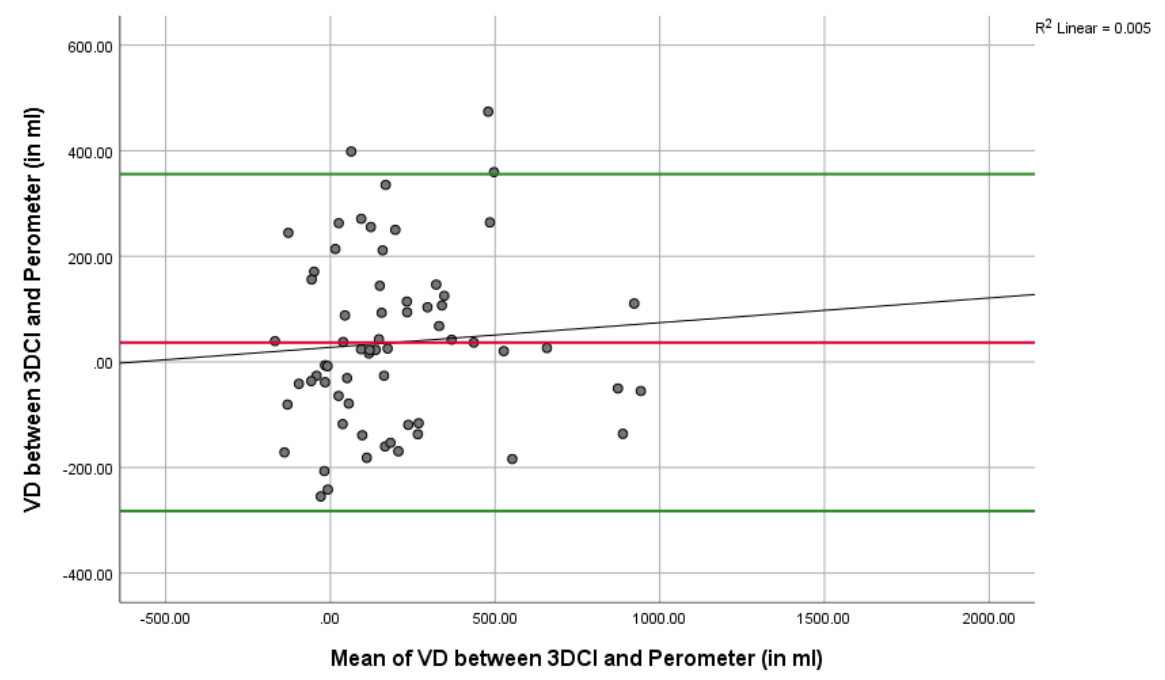

Figure 32: Bland-Altman Plot: a scatterplot of the mean of 3DCI and perometry methods plotted against the difference between the two methods

The Bland-Altman plot between CTM and perometry (Figure 33), required the normalised data for both methods using the logarithm of the $V D$ data (Appendix 13) as the raw data showed no proportional bias between the methods. Using this data, there was one outlier above the upper limit of agreement, and two below the lower limit of agreement. Using the normalised data for CTM and perometry, the mean calculated difference was $-0.08 \mathrm{ml}$ (SD $0.78 \mathrm{ml}$ ). The upper limit of agreement was $1.45 \mathrm{ml}$, and the lower limit of agreement $-1.61 \mathrm{ml}$. The associated coefficient chart (Appendix 13), had a B-value for the mean of 0.10 , with a non-significant $\mathrm{p}$-value of 0.75 , indicating no proportional bias; therefore, there was a level of agreement between CTM and perometry using normalised data.

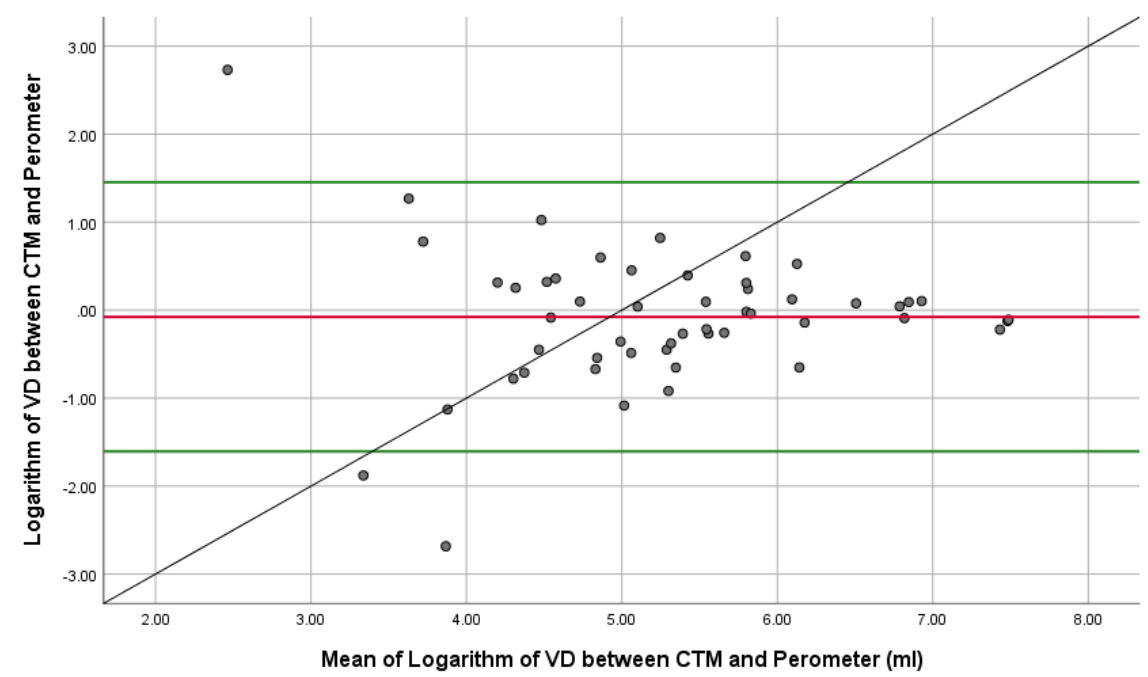

Figure 33: Bland-Altman Plot: a scatterplot of the mean of the logarithm of CTM and perometry methods plotted against the difference between the two methods 


\subsubsection{Objective 1.3: Establish the Accuracy of 3DCI Compared to CTM and Perometry}

As in clinical practice, lymphoedema was diagnosed at a $\geq 5 \%$ volume difference between the affected and unaffected arms. The results from the $2 \times 2$ crosstabulations (Table 30) indicate excellent sensitivity between 3DCI and CTM (91.7\%), and perometry $(100 \%)$, meaning that 3DCI has a very high probability of detecting lymphoedema compared to CTM and perometry. It also had a very low false-negative rate ranging from $0 \%$ to $8.3 \%$ compared to perometry and CTM respectively.

The specificity (the ability to detect no lymphoedema) of 3DCI was moderate compared to CTM $(66.7 \%)$ and perometry $(50.0 \%)$, with false positives ranging from $33.3 \%$ to $50 \%$ compared to CTM and perometry respectively.

Of note, the values between 3DCI and perometry are very similar to that between CTM and perometry, where the sensitivity was $100 \%$, and specificity slightly higher at $60 \%$.

Table 30: $2 \times 2$ Crosstabulation of sensitivity and specificity between 3DCI, CTM and perometry

\begin{tabular}{|c|c|c|c|c|c|}
\hline \multicolumn{2}{|c|}{} & \multicolumn{2}{c|}{ CTM } & \multicolumn{2}{c|}{ Perometer } \\
\cline { 3 - 6 } \multicolumn{2}{|c|}{} & Negative & Positive & Negative & Positive \\
\hline \multirow{3}{*}{$3 \mathrm{DCl}$} & Negative & $66.7 \%$ & $8.3 \%$ & $50.0 \%$ & $0.0 \%$ \\
\cline { 2 - 6 } & Positive & $33.3 \%$ & $91.7 \%$ & $50.0 \%$ & $100.0 \%$ \\
\hline \multirow{2}{*}{ CTM } & Negative & & $60.0 \%$ & $0.0 \%$ \\
\cline { 2 - 4 } & Positive & & $40.0 \%$ & $100.0 \%$ \\
\hline
\end{tabular}

Key:

Green highlight $=$ Sensitivity (true positive)

Purple highlight $=$ Specificity (true negative) 


\subsection{Phase Two: Limb Shape Results and Analysis}

5.6.1 Objective 2.1: Evaluate Limb Shape Derived from 3DCI as a New Method of Lymphoedema Measurement

There are three sub-objectives from this primary objective, which is to evaluate limb shape as a new method of lymphoedema measurement.

Figure 34 provides an example of the oedema maps created, with all oedema maps available for each participant for each session attended in Appendix 14. Each map has the relevant $\% V D$ for CTM, perometry and 3DCI, as well as the percentage difference between the calculated R-value. These will be referred to for the duration of phase two of the results.

\subsubsection{Objective 2.1a) Determine Trends Between Oedema Maps and} Limb Volume

Similar expressions of analysis were used to determine trends between oedema maps and limb volume. As for limb volume analysis in Phase 1, the intra-rater reliability and criterion validity of limb shape using the R-value were compared to the limb volume data from Phase One.

\subsubsection{Reliability}

The paired-samples tests between sessions one and two (Table 31) show the R-value for the lymphoedema arm to have a moderate correlation as the $\mathrm{ICC}_{\text {intra }}$ was 0.71 and statistically significant $(\mathrm{p}=0.01)$. The correlation for the control arm was moderate with an $\mathrm{ICC}_{\text {intra }}$ value of 0.50 but was not statistically significant as $\mathrm{p}>0.05$. The correlation for both the redundancy difference and redundancy percentage difference between the arms have negative poor $\mathrm{ICC}_{\text {intra }}$ of 0.24 and 0.22 respectively with no statistical significance $(\mathrm{p}>0.05)$.

Table 32 summarises all the data from R-value over the four sessions in terms of mean $R$-value of the affected and unaffected (control) arms, the difference between the affected and control arm, and the percentage difference at each session. Also presented are the SD, SEM, and P-Values for each of the data analysed. 


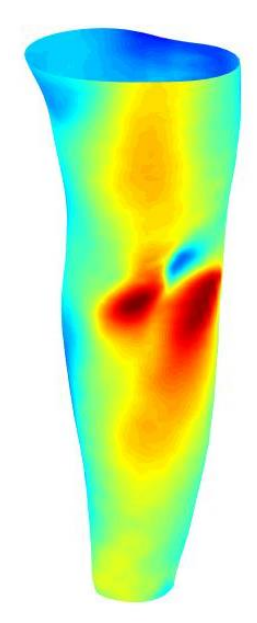

Initial Assessment

$\mathrm{CTM}=13 \%$

Perometer $=9.5 \%$

$3 \mathrm{DCI}=7 \%$

$\mathrm{R}$-value $=-1 \%$

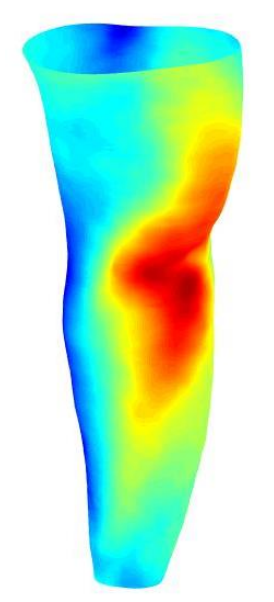

Follow up 2

$\mathrm{CTM}=11 \%$

Perometer $=7.5 \%$

$3 \mathrm{DCI}=12.8 \%$

$\mathrm{R}$-value $=-1 \%$
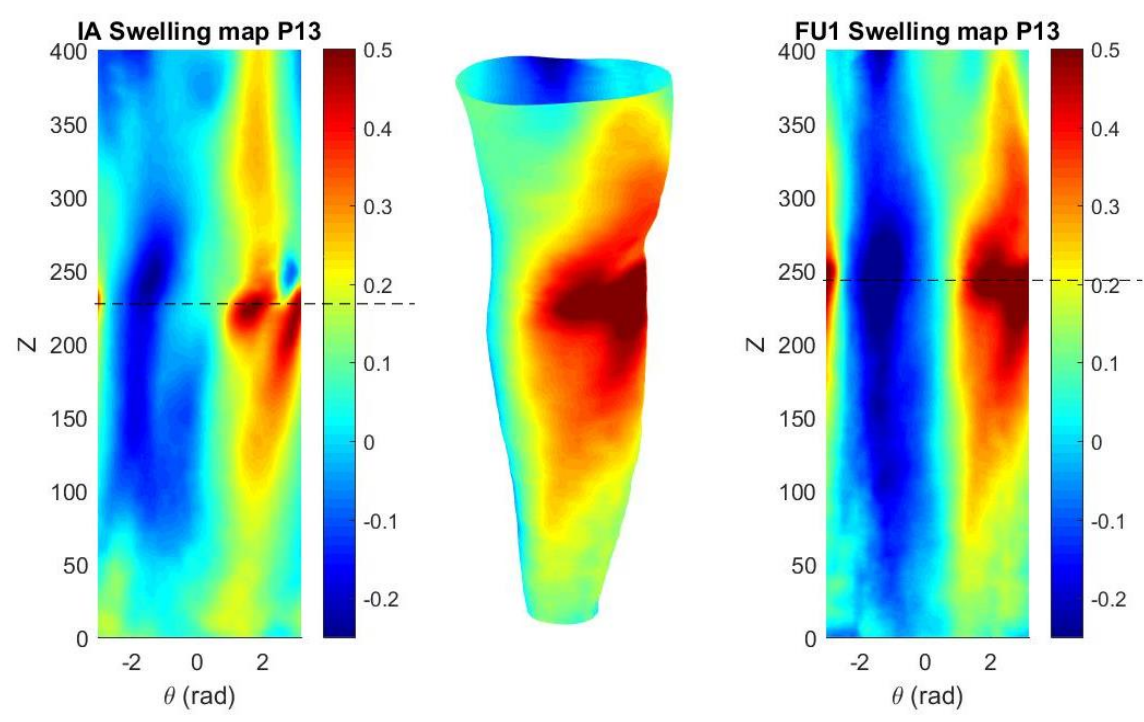

Follow up 1

$\mathrm{CTM}=9 \%$

Perometer $=8.8 \%$

$3 \mathrm{DCI}=15 \%$

$\mathrm{R}$-value $=4 \%$

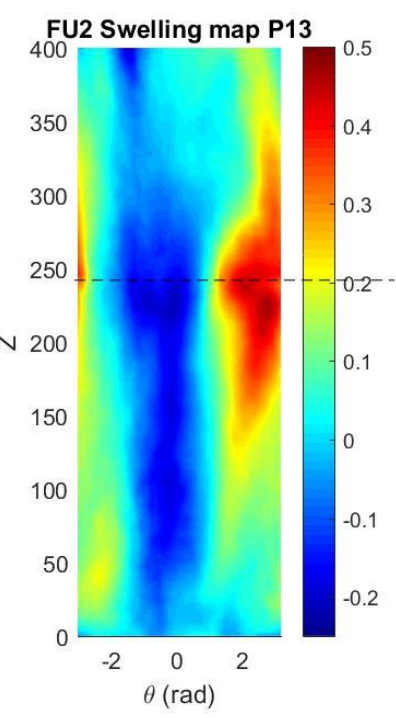

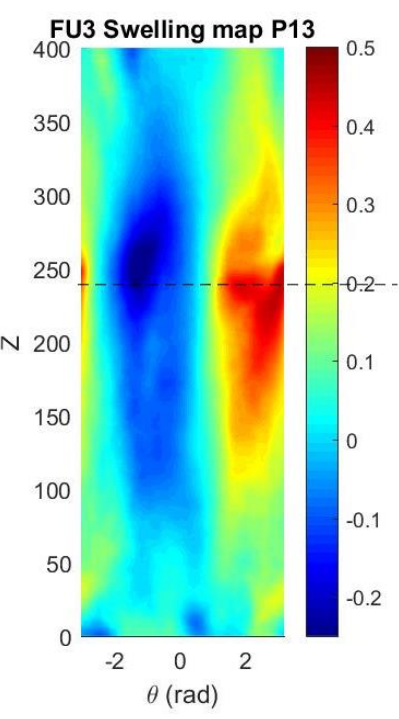

Follow up 3

$\mathrm{CTM}=13 \%$

Perometer $=10.7 \%$

$3 \mathrm{DCI}=15.1 \%$

$\mathrm{R}$-value $=0 \%$

Figure 34: Oedema maps derived from 3DCI: cyan = no difference between arms; yellow to red = larger than unaffected side; blue = smaller than unaffected side 
Table 31: Paired-samples correlations for Shape Redundancy (R-value)

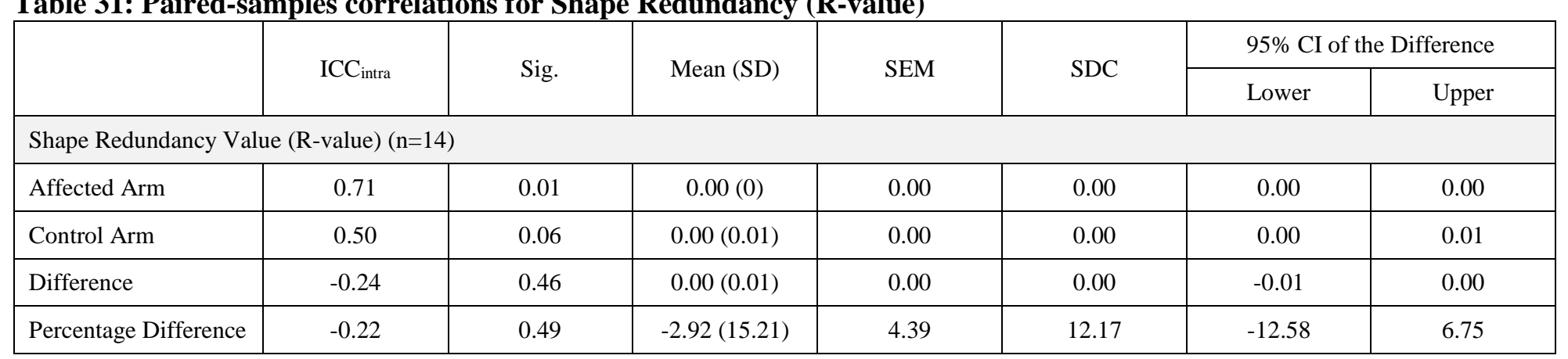

Table 32: Summary of the data from R-value over the four sessions

\begin{tabular}{|c|c|c|c|c|c|c|c|c|c|c|c|c|}
\hline & $\begin{array}{l}\text { R-value Affected } \\
\text { Arm (SD) }\end{array}$ & SEM & $\begin{array}{c}\text { P- } \\
\text { Value }\end{array}$ & $\begin{array}{l}\text { R-value Control } \\
\text { Arm (SD) }\end{array}$ & SEM & $\begin{array}{c}\mathrm{P}- \\
\text { Value }\end{array}$ & $\begin{array}{c}\text { R-value } \\
\text { Difference (SD) }\end{array}$ & SEM & $\begin{array}{c}\text { P- } \\
\text { Value }\end{array}$ & $\begin{array}{c}\text { R-value } \\
\text { Percentage } \\
\text { Difference (\%) } \\
\text { (SD) }\end{array}$ & SEM & $\begin{array}{c}\text { P- } \\
\text { Value }\end{array}$ \\
\hline \multicolumn{13}{|c|}{ СТМ } \\
\hline $\begin{array}{c}\text { Session } 1 \\
(\mathrm{n}=16)\end{array}$ & $0.07(0.01)$ & 0.00 & 0.00 & $0.07(0.01)$ & 0.00 & 0.00 & $0.00(0.01)$ & 0.00 & 0.53 & $2.00(8.67)$ & 2.17 & 0.37 \\
\hline $\begin{array}{c}\text { Session } 2 \\
(\mathrm{n}=13)\end{array}$ & $0.07(0.01)$ & 0.00 & 0.00 & $0.07(0.00)$ & 0.00 & 0.00 & $0.00(0.01)$ & 0.00 & 0.25 & 3.54 (10.23) & 2.84 & 0.24 \\
\hline $\begin{array}{c}\text { Session } 3 \\
(\mathrm{n}=14)\end{array}$ & $0.07(0.00)$ & 0.00 & 0.00 & $0.07(0.01)$ & 0.00 & 0.00 & $0.00(0.01)$ & 0.00 & 0.12 & $3.79(8.03)$ & 2.15 & 0.10 \\
\hline $\begin{array}{c}\text { Session } 4 \\
(\mathrm{n}=14)\end{array}$ & $0.07(0.01)$ & 0.00 & 0.00 & $0.07(0.01)$ & 0.00 & 0.00 & $0.00(0.01)$ & 0.00 & 0.05 & $4.50(8.17)$ & 2.18 & 0.06 \\
\hline $\begin{array}{c}\text { TOTAL } \\
(\mathbf{n}=57)\end{array}$ & $0.07(0.01)$ & 0.00 & 0.00 & $0.07(0.01)$ & 0.00 & 0.00 & $0.00(0.01)$ & 0.00 & 0.24 & $3.46(8.78)$ & 2.34 & 0.19 \\
\hline
\end{tabular}




\subsubsection{Validity}

Three methods of testing the criterion validity of limb shape through R-values were used, namely Pearson's Correlation, Bland-Altman plots and Box- and Violin Plots.

\section{Relationship Between Methods Using Pearson's Correlation Coefficient}

From Table 33 it can be seen that the percentage difference calculated for limb shape, i.e. R-value, has a poor Pearson's correlation with the $\% V D$ from all three methods of measurements (3DCI r=0.39; CTM r=0.23; perometer $r=0.19$ ). However, 3DCI volume measurements were statistically significant with the $R$-value $(p=0.002)$, even though there was a poor correlation. Thus, even though there was a poor correlation with the volume methods, there was a statistical significance with 3DCI volume measurements, indicating some agreement, although poor, between the volume and R-values calculated from 3DCI. Furthermore, as the R-value is a different construct to volume and $\% V D$, and based on these poor correlations, it is recommended not to compare these two constructs to each other.

Table 33: Pearson's correlation coefficient between R-value percentage difference (\% Diff) and 3DCI, CTM and perometry percentage volume difference (\%VD)

\begin{tabular}{|c|c|c|c|c|}
\hline \multicolumn{5}{|c|}{ Correlations } \\
\hline & & 3DCI \%VD & CTM \%VD & Perometer \%VD \\
\hline \multirow[t]{2}{*}{ R-value \% Diff } & Pearson Correlation & $.392^{* *}$ & .227 & .190 \\
\hline & Sig. (2-tailed) & .002 & .081 & .146 \\
\hline \multirow[t]{2}{*}{ 3DCI \% VD } & Pearson Correlation & 1 & $.787^{* * *}$ & $.766^{* * *}$ \\
\hline & Sig. (2-tailed) & & .000 & .000 \\
\hline \multirow[t]{2}{*}{ CTM \%VD } & Pearson Correlation & & 1 & $.932^{* * *}$ \\
\hline & Sig. (2-tailed) & & & .000 \\
\hline
\end{tabular}

\section{Agreement Between Shape Redundancy and the Three Methods of Measurement Utilising Bland-Altman Plots}

Table 34 illustrates that there was no agreement between the R-value and the volume measurement methods as all $\mathrm{p}$-values were $<0.05$.

Tests of normality show that the R-value had significance values of $<0.05$, with a Kolmogorov-Smirnov test p-value of 0.03, and Shapiro-Wilk of 0.00. The normalisation of data was done by transforming the data to the square root of the values (Appendix 15). This produced a statistically significant Kolmogorov-Smirnov test p-value of 0.20 and Shapiro-Wilk of 0.06. The differences between the normalised R-value data and 
3DCI, CTM and Perometry \%VD were calculated to determine agreement, however, all tests remained statistically significant $(\mathrm{p}<0.05)$, thus no level of agreement exists between R-value and the volume-based measurements (Table 35), confirming that these data should not be compared to each due to their different constructs.

Table 34: Agreement between R-value percentage difference and 3DCI, CTM and perometry \% VD

\begin{tabular}{lc|c|c|} 
& $\mathrm{t}$ & $\mathrm{df}$ & Sig. (2-tailed) \\
\hline Difference R-value-3DCI & -3.917 & 59 & .000 \\
\hline Difference R-value-CTM & -2.864 & 59 & .006 \\
\hline Difference R-value-Perometer & -2.683 & 59 & .009 \\
\hline
\end{tabular}

Table 35: Agreement between the square root of R-value percentage difference and 3DCI, CTM and perometry \% VD

\begin{tabular}{|l|r|r|r|} 
& $\mathrm{t}$ & $\mathrm{df}$ & \multicolumn{1}{|c|}{ Sig. (2-tailed) } \\
\hline Difference square root R-value-3DCI & -5.347 & 36 & .000 \\
\hline Difference square root R-value-CTM & -4.005 & 36 & .000 \\
\hline Difference square root R-value-Perometer & -3.561 & 36 & .001 \\
\hline
\end{tabular}

\section{Correlations Between Methods Utilising Box- and Violin Plots}

The Boxplot (Figure 35) shows that the mean values for the R-value are lower than for limb volume, with the interquartile range and adjacent values being much lower as well.

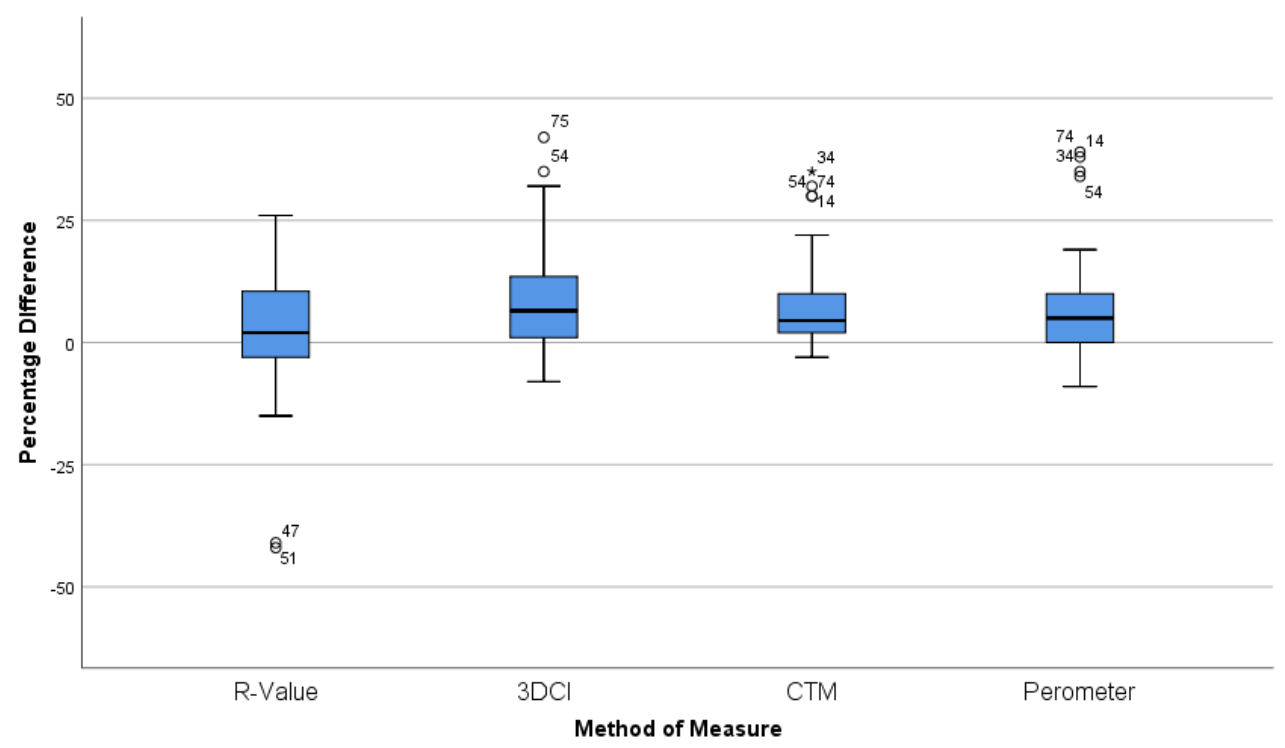

Figure 35: Boxplot showing the mean values for the percentage differences of Shape Redundancy (R-value), 3DCI, CTM and perometry. The $O$ and $\star$ indicate the case numbers as outliers and the overlap of cases respectively.

The Violin Plots (Figure 36) show a lower probability of correlation with the R-value compared to any of the three volumetric methods of measurement. From the Box Plots 
and Violin Plots, it is evident that there is a poor correlation between shape redundancy and limb volume calculations.
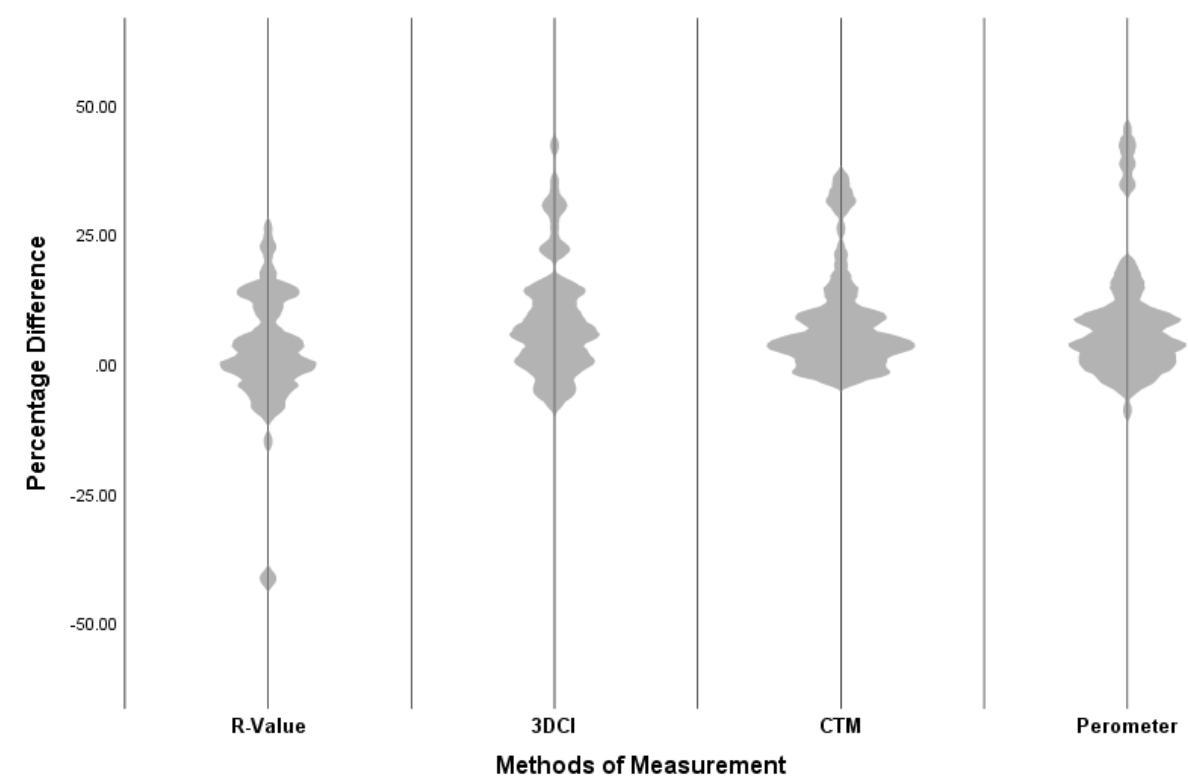

Figure 36: Violin Plots showing the probability of correlation between R-Value, 3DCI, CTM and perometry

\subsubsection{Objective 2.1b) Use of Oedema Maps to Monitor Treatment Outcomes (Limb Volume Change)}

From the oedema maps in Appendix 14, the range of colours from blue to red portrayed in the reconstructed arm highlight the deviation of the lymphoedema arm from the 'normal'. Cyan is when the two arms match in shape and girth, however the redder the image, the bigger the deviation, indicating the presence of lymphoedema. The concentration of the colours also indicates where the worst of the lymphoedema is, i.e. localised lymphoedema.

Shape deviation was primarily detected around the elbow with nine highlighting swelling around the back or outer elbow; three had swelling along the inner aspect, and two along the anterior (front) of the elbow. Swelling generally extended down the forearm and up into the upper arm, but only six had swelling along the length of their arm. 
The radial maps proved most useful in nine cases as the reconstructed arm was cyan or blue, which could be interpreted as no lymphoedema, however, looking at the radial maps highlighted significant shape distortion of the back of the arm.

In two cases (participant 2 and 11) changes in shape distortion were not identified by perometer, and in one case (participant 16), the perometer showed a significant increase in volume which neither the oedema map nor CTM or 3DCI identified.

Some problems were identified with 3D imaging in that it was not possible to identify the arms for participant 6 as they could not be distinguished from the trunk. The participant had a BMI of $49.2 \mathrm{~kg} / \mathrm{m}^{2}$ resulting in her upper arm resting on her trunk, even with her arm held out to her side at shoulder height. In two case (participant 9 and 10), overstretching of one arm to the other caused mismatching of the images, resulting in either false positive readings from the oedema map, or no oedema map to be constructed. There was also a case (participant 20) where images were taken as per protocol for all four sessions, however, the data were corrupted somehow, thus no 3DCI data was available for calculation or construction of the oedema maps.

Further analysis of the oedema maps and the $\% V D$ results of the three methods are presented in Table 36. This table provides a summary of whether the oedema maps match the \%VD data from CTM, perometry and 3DCI over time. From this, $14(82 \%)$ of CTM \%VD match the oedema maps, $11(65 \%)$ compared to perometry \%VD and 16 (94\%) against the 3DCI \%VD. This indicates a good trend of oedema maps corresponding with calculated $\% V D$ to $\mathrm{CTM}$ and $3 \mathrm{DCI}$, and moderate compared to perometry. However, the R-values do not match very well $(n=8 ; 47 \%)$, with five participant's R-values either changing drastically over time, missing or was a 50:50 split in matching or not; thus, caution is advised when using shape redundancy to diagnose lymphoedema.

\subsubsection{Objective 2.1c) Consider Limb Shape as a Tool to Diagnose Lymphoedema}

The current $R$-value does not seem a valid method of determining the presence of lymphoedema based on the results from the three validity tests. However, visual 
feedback has the potential to confirm limb volume outcomes. This will be discussed further in Section 6.5.2.

Table 36: Oedema maps matching CTM, perometry and 3DCI \%VD over time

\begin{tabular}{|c|c|c|c|c|c|c|c|c|c|}
\hline \multirow{2}{*}{$\begin{array}{c}\text { Participant } \\
\text { Number }\end{array}$} & \multicolumn{2}{|c|}{ CTM } & \multicolumn{2}{|c|}{ Perometer } & \multicolumn{2}{|c|}{ 3DCI } & \multicolumn{3}{|c|}{ Oedema Map } \\
\hline & Yes & No & Yes & No & Yes & No & Yes & Unsure & No \\
\hline 1 & $\checkmark$ & & & $\checkmark$ & $\checkmark$ & & & $\checkmark$ & \\
\hline 2 & $\checkmark$ & & $\checkmark$ & & $\checkmark$ & & & $\checkmark$ & \\
\hline 3 & $\checkmark$ & & $\checkmark$ & & $\checkmark$ & & & & $\checkmark$ \\
\hline 4 & $\checkmark$ & & $\checkmark$ & & $\checkmark$ & & $\checkmark$ & & \\
\hline 5 & $\checkmark$ & & $\checkmark$ & & $\checkmark$ & & & $\checkmark$ & \\
\hline 6 & \multicolumn{2}{|c|}{ No Image } & \multicolumn{2}{|c|}{ No Image } & \multicolumn{2}{|c|}{ No Image } & \multicolumn{3}{|c|}{ No Image } \\
\hline 7 & $\checkmark$ & & & $\checkmark$ & $\checkmark$ & & & $\checkmark$ & \\
\hline 8 & $\checkmark$ & & $\checkmark$ & & $\checkmark$ & & $\checkmark$ & & \\
\hline 9 & & $\checkmark$ & & $\checkmark$ & & $\checkmark$ & & & $\checkmark$ \\
\hline 10 & \multicolumn{2}{|c|}{ No Image } & \multicolumn{2}{|c|}{ No Image } & \multicolumn{2}{|c|}{ No Image } & \multicolumn{3}{|c|}{ No Image } \\
\hline 11 & $\checkmark$ & & $\checkmark$ & & $\checkmark$ & & & & $\checkmark$ \\
\hline 12 & $\checkmark$ & & $\checkmark$ & & $\checkmark$ & & $\checkmark$ & & \\
\hline 13 & $\checkmark$ & & $\checkmark$ & & $\checkmark$ & & & & $\checkmark$ \\
\hline 14 & $\checkmark$ & & $\checkmark$ & & $\checkmark$ & & $\checkmark$ & & \\
\hline 15 & & $\checkmark$ & & $\checkmark$ & $\checkmark$ & & $\checkmark$ & & \\
\hline 16 & & $\checkmark$ & & $\checkmark$ & $\checkmark$ & & $\checkmark$ & & \\
\hline 17 & $\checkmark$ & & & $\checkmark$ & $\checkmark$ & & & $\checkmark$ & \\
\hline 18 & $\checkmark$ & & & $\checkmark$ & $\checkmark$ & & $\checkmark$ & & \\
\hline 19 & $\checkmark$ & & $\checkmark$ & & $\checkmark$ & & $\checkmark$ & & \\
\hline 20 & \multicolumn{2}{|c|}{ No Image } & \multicolumn{2}{|c|}{ No Image } & \multicolumn{2}{|c|}{ No Image } & \multicolumn{3}{|c|}{ No Image } \\
\hline
\end{tabular}

\subsubsection{Objective 2.2: Ascertain 3DCI as a Sensitive Measure of Change in} Lymphoedema

As for Phase One, $2 \times 2$ crosstabulations were used to compare the R-values to the $\% V D$ for the three methods of measurements.

\subsubsection{Objective 2.2a) Sensitivity to Change in Limb Volume Over Time}

Section 5.5.3 showed VD measured by 3DCI to have good sensitivity when compared to CTM (79.4\%) and perometry $(73.5 \%)$. Thus, 3DCI volume measurements demonstrate sensitivity to change in limb volume over time.

\subsubsection{Objective 2.2b) Sensitivity to Change in Limb Shape Over Time}

The R-value was compared to the $\% V D$ for 3DCI, CTM and perometry to determine whether limb shape value had similar sensitivity over time (Table 37). The results from the $2 \times 2$ crosstabulations indicated poor to moderate sensitivity between R-value and perometry (28.6\%), CTM (45.5\%) and 3DCI (50.0\%). Specificity was moderate to excellent compared to perometry $(60.0 \%)$, CTM $(83.3 \%)$ and 3DCI (100\%). 
Table 37: $2 \times 2$ Crosstabulation of sensitivity and specificity between R-Value, 3DCI, CTM and perometry

\begin{tabular}{|c|c|c|c|c|c|c|c|}
\hline \multirow{2}{*}{} & \multicolumn{2}{c|}{$3 \mathrm{DCl}$} & \multicolumn{2}{c|}{ CTM } & \multicolumn{2}{c|}{ Perometer } \\
\cline { 3 - 8 } & Negative & Positive & Negative & Positive & Negative & Positive \\
\hline \multirow{2}{*}{\begin{tabular}{c} 
R-Value \\
\cline { 2 - 8 }
\end{tabular}} & Negative & $100 \%$ & $50.0 \%$ & $83.3 \%$ & $54.5 \%$ & $60.0 \%$ & $71.4 \%$ \\
\cline { 2 - 8 } & Positive & $0.0 \%$ & $50.0 \%$ & $16.7 \%$ & $45.5 \%$ & $40.0 \%$ & $28.6 \%$ \\
\hline
\end{tabular}

Key:

Green highlight $=$ Sensitivity $($ true positive) $\quad$ Purple highlight $=$ Specificity (true negative)

When reflecting on the oedema maps (Appendix 14) and Table 36, oedema maps do reflect sensitive change over time that follow the trends of $\% V D$. Thus, shape redundancy does not always reflect the oedema map's visual interpretations. 
Chapter 6:

Discussion and

Conclusion 
This study aimed to determine the validity, reliability and accuracy of upper limb volume results obtained from 3DCI compared to CTM and perometry. This was achieved by proving the criterion validity and intra-rater reliability of 3DCI against CTM and perometry, and was found to be similar to the current literature. The accuracy of 3DCI volumes demonstrated good sensitivity, but moderate specificity. This was the first study to report sensitivity and specificity, and more research with a bigger cohort is encouraged to add more value to this data. The study also sought to discover whether limb shape is a feasible alternative to measure lymphoedema. As the use of limb shape to assess for lymphoedema is a new concept, not only does it need to be 'a feasible alternative' to therapists and patients, it has to prove that it offers more detailed information about the lymphoedema. This proved to be more challenging as comparing volume to shape, or a value of shape (R-value), is in itself a fundamentally different construct of measurement to volume. Comparing each oedema map to the $\% V D$ of each measurement showed good similarity, but more research is needed to define the shape objectively for comparison purposes.

Based on the results, 3DCI could be used within a clinical setting, as it provides limb volume information as well as images in the form of oedema maps. Through the information produced from limb shape, both the patient and therapist could gain additional information to the standard limb volume data by clearly visualising and highlighting the problem areas of the lymphoedema using colour coding. This would inform the localisation and extent of the problem, thus allowing for improved decision making in terms of the type of treatment options that could be made available. For example, adding soft textured foam to the problem area, or introducing alternative massage techniques to the localised swelling, thus providing specific, individualised treatment for each patient's symptoms. Patients may be more receptive to this method of measurement where their lymphoedema can be visualised, thus potentially improving their compliance with the treatment.

\subsection{Cohort Demographics}

The calculated average age of breast cancer cases from 2015-2017 was 63.5 years (range 17 to $90+$ ), and the most prevalent age group for breast cancer for this period was $65-70$ years (Cancer Research UK, 2020). As about $21 \%$ of women who have breast cancer 
treatment develop lymphoedema (DiSipio et al., 2013), it would be logical for a BCRL cohort to be of a similar age group. Unfortunately, no data exists to report on the average age of BCRL cohorts. For this study, the mean age was slightly lower than the national breast cancer average at 58.9 years.

The literature reports that BMI affects limb volume (Ancukiewicz et al., 2012; Buffa et al., 2015); conversely, Wang et al.'s (2017) study contradicted this; however, their cohort was all in the healthy BMI range $\left(20-24.9 \mathrm{~kg} / \mathrm{m}^{2}\right)$, whereas all other papers reported on a range of healthy to obese $\left(\mathrm{BMI}>30 \mathrm{~kg} / \mathrm{m}^{2}\right)$ participants. This study's participants had an average BMI of $32.2 \mathrm{~kg} / \mathrm{m}^{2}$ which is equivalent to the obese class I category (World Health Organization, 2021). As in the literature, this study's BMI ranged from the healthy to obese class III $\left(23.2-49.2 \mathrm{~kg} / \mathrm{m}^{2}\right)$.

From a recent online report published by McCarthy (2020), 12.2\% of the UK population are left-handed. This study, therefore, had a representative sample with $10 \%$ of the participants being left-handed. There was a good split between affected dominant, and affected non-dominant arm (45:55), thus this sample had a good representation for hand dominance as affected or unaffected. Although Wang et al. (2017) found hand dominance influenced CTM and limb volume as the dominant arm measured significantly bigger than the non-dominant arm, Ancukiewicz et al. (2011) did not find hand dominance to affect relative volume change using the perometer. To date, no research using 3DCI has examined how much hand dominance may affect limb volume measurements.

It is recognised that cancer treatment is a cause of secondary lymphoedema (Lymphoedema Framework, 2006). Tsai et al. (2018) report that surgical methods were found to contribute to the development of arm lymphoedema, with mastectomy and axillary node clearance being a higher risk than a wide local excision and sentinel lymph node biopsy. The main surgery for this study's cohort was mastectomy $(n=12)$ and axillary node clearance $(n=16)$, thus is representative of this higher risk group for developing lymphoedema secondary to breast cancer treatment.

Alcohol consumption is considered a causative factor of breast cancer (NHS UK, 2020). From this cohort, $75 \%$ of the participants drank alcohol ranging from occasionally to 10 
units per week, and 15\% of the participants smoked. Alcohol consumption has not been confirmed to be a cause of lymphoedema, and none of the participants reported alcohol consumption as an exacerbating factor. Smoking is known to be harmful to health, including the circulatory system (Babizhayev \& Yegorov, 2011); however, no study to date has found an effect of smoking on the lymphatic system. Interestingly, Bedi et al. (2015) report that smoking negatively predicted lymphoedema in their study following treatment for Sarcoma. At this time, until there is a definitive causative link between lymphoedema and alcohol/smoking, the relevance of collecting this data has to be questioned. A study comparing measurements before and after alcohol consumption or smoking could be considered, but it is recognised that there may be ethical concerns due to these being identified risk factors to ill health.

\subsection{Validity and Reliability of 3DCI}

\subsubsection{Concurrent Validity}

This study demonstrated good concurrent (criterion) validity of 3DCI with CTM $(\mathrm{r}=0.86)$, and perometry $(\mathrm{r}=0.82)$. These data are lower than the average reported in the systematic literature review (0.94) but falls within the reported range of 0.74-0.99.

Nevertheless, a high correlation does not necessarily mean that the methods have good agreement (Montenij et al., 2016; Doğan, 2018). Criterion validity was further determined through the use of Bland-Altman plots which looked at the agreement of the limb volume differences measured by 3DCI, CTM and perometry. The Bland-Altman plots showed a level of agreement between 3DCI and CTM and perometry, with no proportional bias. The low mean volume differences between the methods indicate a good agreement between the methods as the mean volume difference between the volume differences of 3DCI and CTM was $19 \mathrm{ml}$ (SD $141 \mathrm{ml})$, and between 3DCI and perometry it was $37 \mathrm{ml}$ (SD $163 \mathrm{ml}$ ). Lu \& Dixon (2019) also constructed Bland-Altman plots comparing 3DCI to perometry that showed a low mean volume difference of $6 \mathrm{ml}$ (SD $133 \mathrm{ml}$ ), again supporting good agreement between the methods of measurement.

Results from this study show that 3DCI underestimated limb volume compared to CTM by $278 \mathrm{ml}$. This outcome is similar to Karakashian et al. (2018) who found 3DCI to underestimate CTM by $\approx 100 \mathrm{ml}$, however, it is in contrast to both Cau et al.'s (2016; 
2018) papers who found 3DCI to overestimate limb volume compared to CTM. The large difference between the volumes of this study and Karakashian et al.'s (2018) can be explained by not matching limb length (systematic error) between 3DCI and CTM in this study, whereas Karakashian et al. (2018) matched arm lengths, but these did not extend to the axilla. The difference in volumes is also thought to be due to the geometric approximations used when calculating volume by CTM to be inaccurate, i.e. the truncated cone formula postulates that every segment of the limb represents a perfect circle and that the walls of the cone are rectilinear (De Vrieze et al., 2019). Furthermore, 3DCI accounts for the volume of the actual shape of the arm, as opposed to the measured segments used in CTM. That is, 3DCI measures every millimetre circumferentially, whereas CTM uses a range from $4 \mathrm{~cm}$ to $10 \mathrm{~cm}$ spacing between measured circumferential points. Thus, results from 3DCI could be considered as the more accurate method for measuring limb volume.

This study also found 3DCI to underestimate limb volume compared to perometry by an average of $364 \mathrm{ml}$. Conversely, three papers reported 3DCI to overestimate volume compared to perometry (Lu et al., 2013; Lu et al., 2014; Lu \& Dixon, 2019), with Lu \& Dixon (2019) quoting a volume overestimation of $118 \mathrm{ml}$. Lu et al. (2014) report that the discrepancies between 3DCI and perometry may be due to two factors, namely arm length not being identical for both methods (systematic error), and the deformation of the arm during the scan (random error), i.e. any slight movement will affect volume calculations. This study and Lu et al.'s (2014) study used similar methodologies in terms of capturing images, i.e. the perometer was captured identically (apart from the patient position being sitting versus standing) and the 3DCI had arms held to the side unsupported at shoulder-height. Of note, all of Lu's studies used the Microsoft Kinect ${ }^{\mathrm{TM}}$ camera, and this study utilised a similar infrared camera called Asus Xtion Pro (Gonzalez-Jorgea, Riveirob, Vazquez-Fernandezc, Martinez-Sáncheza, \& Ariasa, 2013). Due to the errors in capturing the images in this study, thought to be due to slight movement of the unsupported arm, it is recommended that a platform, similar to that used in perometry, be designed for 3DCI to offer more stability to the arm whilst the measurement is being taken.

Similarly to CTM, 3DCI is thought to be more accurate than perometry due to volume measurements being taken every $1 \mathrm{~mm}$ as opposed to $3.1 \mathrm{~mm}$ or $4.7 \mathrm{~mm}$. The perometer 
also uses cylinder type formulae, and although the gaps between measurements are not as significant as for CTM, the irregularity in limb shape cannot be accounted for. Also, 3DCI utilises an algorithm based on the actual surface area of the limb as opposed to circumferential points; thus, all shape distortion is included in the calculation. Furthermore, the engineers at Swansea University question the accuracy of the perometer as a rectangular frame is taking the image of an oval or circular object. Thus, the corners of the perometer frame could be distorting the limb image, resulting in a less accurate volume measure for the limb. Therefore, to understand all the discrepancies described, further research is required with stricter controls of the participant's positioning, identifying the limb length before taking measurements, and testing the perometer and 3DCI with a known volume object, such as a tube, for accuracy and calibration.

Most of the other studies in the systematic literature review compared 3DCI to water displacement, which found it to correlate well (range 0.98 to 0.99) (Landau et al., 2018; Preu $\beta$ et al., 2018; Hameeteman et al., 2016; Erends et al., 2014; Lu et al., 2014; Öhberg et al., 2014). There was some disagreement as to whether 3DCI over or underestimates volume compared to water displacement. However, from this thesis' systematic literature review, most papers report 3DCI to overestimate volume compared to water displacement. This could be due to arm length discrepancies in either measurement or motion of the arm during 3DCI measurement.

From the results of this study and the literature review, volumetric methods of measurements should not be used interchangeably as they produce different and incompatible total limb volumes.

\subsubsection{Intra-Rater Reliability}

This study investigated whether 3DCI was a reliable method of measurement compared to CTM and perometry. As the study consisted of one researcher performing the measurements at each appointment, only intra-rater data analysis was possible. The intra-rater correlation coefficient of 3DCI was statistically significant $(\mathrm{p}<0.00)$ with a

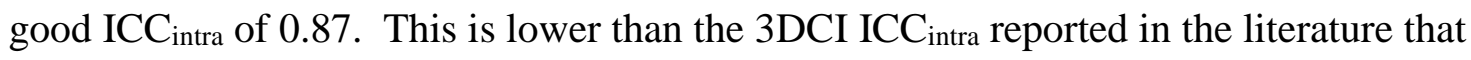
ranged from 0.95 to 1.00. Mehta et al. (2018) explain that once a cohort threshold of $\mathrm{n}=80$ is achieved, larger groups do not seem to influence intra-rater correlation 
coefficient results. Interestingly, all studies reporting on intra-rater correlation coefficient values for 3DCI had cohorts smaller than 40. This may account for the differences in the reported correlations for this study as the participant number was only 20, lower again than the recommended 30 in the literature, and is explained in the limitations of this study.

Furthermore, 3DCI demonstrated good intra-rater reliability, as the results from the testretest data are more consistent due to the lower differences in volume between measurements and smaller standard deviations when compared to other measurements. 3DCI had the lowest mean limb volume for both the affected and unaffected arms over the study period, as well as the smallest $V D(214 \mathrm{ml})$ between arms, the lowest SD (274 ml) and SEM (33.8) values with all data significant at $\mathrm{p}=0.00$. However, the lowest \%VD was with CTM (8.1\%), and the highest was 3DCI with $8.6 \%$. 3DCI had an SEM of $1.4 \%$ and SDC of $3.9 \%$; compared to the systematic literature review, these values are slightly lower (SEM 1.7\%; SDC 4.7\%), indicating similarity in results.

Results from this study indicate that 3DCI has good criterion validity for the measurement of limb volume as it correlates highly to CTM and perometry, and there is an agreement between 3DCI and the other two measurements with no proportional bias seen from the Bland-Altman plots. Furthermore, when determining the reliability of 3DCI compared to CTM and perometry in upper limb lymphoedema, the good intrarater correlation coefficient value, lower standard deviation and the smaller standard error of measurement all indicate 3DCI to be a reliable tool for the measurement of upper limb volume. Compared to the literature, this study supports the findings that 3DCI is a valid tool for the measurement of lymphoedema; however, without a gold standard measure against which to compare 3DCI, its validity remains inconclusive.

\subsection{Establish the Accuracy of 3DCI Compared to CTM and Perometry}

Bossuyt, Irwig, Craig, \& Glasziou (2006) posit that where studies are analysing comparative accuracies, they could compare the new test with existing tests and verify test results against the same reference standard. A simple estimate of a new test's 
sensitivity and specificity is required to determine whether it can serve as a replacement, alternative or adjunct, i.e. a test's ability to detect true positives and true negatives.

From the results of this study, 3DCI demonstrated good levels of accuracy in that $92 \%$ of lymphoedema cases compared to CTM (sensitivity) and $100 \%$ when compared to perometry were positively detected, with low false-negative rates less than $8 \%$. However, 3DCI's accuracy at detecting non-lymphoedema cases was moderate in that its specificity compared to CTM was $67 \%$ and perometry $50 \%$, with false positives up to $50 \%$. In clinical practice, with the higher rate of over-diagnosing lymphoedema, it is even more important to ensure the examination includes visual and palpation assessment in conjunction with the patient's reported symptoms when using 3DCI. As lymphoedema is the second most feared side effect of cancer treatment among breast cancer patients (Cornelissen et al., 2018), it stands to reason that over-diagnosing lymphoedema will further increase existent distress (Alcorso \& Sherman, 2015), therefore it is vital that an accurate diagnoses be made when measuring for lymphoedema. A larger cohort may present different outcomes, and with no papers (Lu et al., 2013; Öhberg et al., 2014; Lu et al., 2014; Erends et al., 2014; Buffa et al., 2015; Hoevenaren et al., 2016; Hameeteman et al., 2016; Cau et al., 2016; Levenhagen et al., 2017; Verhulst et al., 2017; Preuß et al., 2018; Landau et al., 2018; Karakashian et al., 2018; Cau et al., 2018; Lu \& Dixon, 2019; Karakashian et al., 2019; Binkley et al., 2020) reporting on 3DC's sensitivity or specificity against other methods, further research and analysis is needed.

In Wales, a diagnosis of lymphoedema is made when there is $\geq 5 \%$ limb volume difference between the affected and unaffected limbs (LNW, 2020). The diagnosis must be supported by both the clinical report and presentation from the patient as well. This 5\% threshold was utilised in this study. Within the systematic literature review, Asim et al. (2012) and Ancukiewicz et al. (2012) both report that $7.5 \%$ may be the ideal thresholds for diagnosing lymphoedema. However, until the method of diagnosing lymphoedema through the application of thresholds for limb volume is resolved, research will continue to report the vast discrepancies of lymphoedema incidence, and due to the differing thresholds, makes lymphoedema research continually challenging. Nevertheless, with 3DCI being an accurate and sensitive method of volume measurement, the lower threshold of 5\% volume difference between arms would reflect 
a more sensitive change over time. Further research to determine the most appropriate threshold for diagnosing lymphoedema using 3DCI should therefore, be considered.

\subsection{Ascertain Whether Limb Shape Derived from 3DCI is a Potential New Method of Lymphoedema Measurement}

The reliability, validity and accuracy of shape versus volumetric measurement were determined, and the results highlighted that by using the R-value, there was a statistically significant but moderate intra-rater correlation of $0.71(\mathrm{p}=0.01)$. This is similar to Gillies, Kinahan, \& Hricak's (2016) findings, where they found converting image data into quantitative results helped with making a diagnosis and then deciding upon treatment options.

The criterion validity of shape redundancy showed a poor correlation with limb volume measures (Pearson's correlations coefficient $\leq 0.39$ ) that was confirmed visually using box- and violin plots. There was also no agreement between the methods as BlandAltman plots were not possible, given that the R-value were statistically significant $(\mathrm{p}<0.05)$ for the $t$-test between the methods of measurement.

The accuracy of shape redundancy demonstrated poor to moderate sensitivity (range $29 \%$ to $50 \%$ ), but moderate to excellent specificity (range $60 \%$ to $100 \%$ ). Thus using shape redundancy has a low ability to detect lymphoedema, but a good probability to determine the absence of lymphoedema. More research is required to build the evidence base for this unique concept of lymphoedema measurement by quantifying shape.

Further analysis of shape was done in the form of visual interpretation of the oedema maps. The colours towards red indicate a stronger deviation from the 'norm' (unaffected side), whereas cyan indicates a closer relationship with the 'norm'. From this study, 94\% of the oedema maps matched the findings from $\% V D$ as recorded by 3DCI, $82 \%$ compared to CTM and $65 \%$ to perometry. A literature review by van de Water et al. (2018) concluded that patients prefer to receive positively framed risk information and that they prefer to receive graphical over textual information. The current visual interpretation of the oedema maps may be subjective for scientific purposes; however, 
for a patient, it may help confirm where the worst of their lymphoedema problem may be and add to the understanding of the need for bespoke treatment.

Thus, the use of oedema maps has the potential to be used as a new and alternative measure of lymphoedema. With the oedema maps being sensitive to small changes in volume and shape, this method may be useful as a risk reduction measurement tool; however, further research with this specific cohort of patients would be needed. Also, from this study's results, 3DCI has the potential to replace both CTM and perometry as not only does this method of measurement provide limb volume data, it also supports findings through the use of oedema maps that offer more information in terms of localised swelling, which CTM and perometry are not sensitive enough to detect and report. Clinical research regarding the use of oedema maps with different stages of lymphoedema is needed. Also, a study determining the effect of oedema maps on people less compliant with their lymphoedema self-management would be of interest to understand whether visual feedback of their lymphoedema changes their approach to treatment. Furthermore, utilisation of deep infrared thermograms (heat maps) should be considered as according to Belgrado et al. (2010) the use of infrared thermal cameras can obtain surface thermograms of high precision and excellent resolution depicting dermal backflow. These thermal images could then be compared to the 3DCI oedema maps to determine similarities in the detection of lymphoedema and localised swelling.

Shape redundancy (R-value), however, does not currently seem to add much value to limb shape as an assessment tool as, even though it has moderate reliability, it has poor validity with limb volume measurements. Further research into the application of oedema maps in clinical practice is needed to explore whether R-value could be used as an additional tool measuring upper limb shape changes over time.

\subsection{Potential Benefits of 3DCI in Clinical Practice}

\subsubsection{Timing of Measurements}

Timing for CTM measurements in this study varied greatly from 3:49 min to 8:49 min. In clinical practice, measuring limbs using CTM can fluctuate due to the need to repeat measures as a result of errors in marking the limb, positioning the limb, or writing the measured values incorrectly on the datasheet. Furthermore, experienced therapists are 
likely to perform the measurements more quickly than newly trained staff. CTM is the only hands-on method of measuring limb volume, and as such is a personal procedure to perform for patients. Often patients like to converse while measurements are being taken, which lengthens the process even more. Furthermore, as the therapist's hands are in close contact to the skin during measuring, it is easy for the therapist to palpate the skin and tissue, enabling them to detect changes in tissue consistency and determine the tissue's responsiveness to touch and massage (Johnson, Kennedy, \& Henry, 2014). However, this also adds contact infection risk as previously discussed.

There was less variation in the timings for perometry (range 1:17 min to 3:08 min) and 3DCI (range 1:27 min to 2:38 min) compared to CTM. This would be due to the methods utilising technology that requires distancing of the therapist from the patient. The tests are quick to perform, thus thwarting the opportunity for a patient to start a discussion during the test as their body position needs changing to measure the opposite limb. Perometry still requires close contact between the patient and therapist who has to slide the frame back and fore the perometer stand. The time saved performing these tests allows for more discussion time for when the results of the tests are shared with the patient during their allocated appointment time. An unexpected benefit of using this study's 3DCI method over perometry is that the limb can be measured supporting the COVID-19 social distancing regulations as there is a two-meter gap between the patient and the therapist who is standing behind the camera stand.

Three papers report on the timing of the tests in their study, which are summarised in Table 38. Including this study, there is a large discrepancy in times recorded due to differences in methods of timing the tests. This study timed the setup and performance of the test, while Landau et al. (2018) included the setup time, performance of the test, and the time taken to calculate the results. Buffa et al. (2015) compared their 3DCI system (SkanLab system) to water displacement and timed the duration of the measurements. They found their 3DCI system to be faster than water displacement by 2:47 min (Buffa et al., 2015). Landau et al. (2018) timed their three methods of measurement which were 3DCI (Vectra 3D system), CTM and water displacement. They report that their 3DCI system was on average 9:48 min faster to perform than CTM, and 19:00 min faster than water displacement (Landau et al., 2018). Thus the consensus 
from the studies reporting on 3DCI in Table 38 is that 3DCI is faster to perform than current methods of lymphoedema measurement.

Table 38: Timing of methods of measurement between Buffa et al. (2015), Landau et al. (2018), De Vrieze et al. (2019) and Thesis Study

\begin{tabular}{|l|c|c|c|c|}
\hline $\begin{array}{l}\text { Method of } \\
\text { Measurement }\end{array}$ & $\begin{array}{c}\text { Buffa et al. } \\
(\mathbf{2 0 1 5}) \\
\text { Time (min) }\end{array}$ & $\begin{array}{c}\text { Landau et al. } \\
\mathbf{( 2 0 1 8 )} \\
\text { Time (min) }\end{array}$ & $\begin{array}{c}\text { De Vrieze et al. (2019) } \\
\text { Time (min) }\end{array}$ & $\begin{array}{c}\text { Thesis Study } \\
\text { (2020) } \\
\text { Time (min) }\end{array}$ \\
\hline 3DCI & $0: 42 \pm 0: 11$ & $5: 54 \pm 2: 18$ & - & $1: 50 \pm 0: 17$ \\
\hline CTM & & $15: 42 \pm 3: 06$ & $4: 24 \pm 0: 44$ & $5: 54 \pm 1: 17$ \\
\hline $\begin{array}{l}\text { Water } \\
\text { Displacement }\end{array}$ & $2: 03 \pm 0: 29$ & $24: 54 \pm 4: 06$ & $\begin{array}{c}\text { Type 1: } 10: 41 \pm 1: 49 \\
\text { Type 2: 5:35 } \pm 0: 19\end{array}$ & \\
\hline Perometry & & & $1: 43 \pm 0: 43$ & $1: 56 \pm 0: 35$ \\
\hline
\end{tabular}

From a Value-Based Healthcare perspective reviewing outcomes, quality and cost (Porter, Pabo, \& Lee, 2013; Gray, 2017), 3DCI is a time and cost-saving method of measurement that has good reliability and validity against CTM and perometry.

\subsubsection{Oedema Maps}

The interpretation of shape through oedema maps will provide information that may help people who understand visual images better than numbers. Edwards, Elwyn, \& Mulley (2002) begin by explaining that people interpret words and numbers differently, for example, 'likely' might be a 1 in 10 chance for one person, but for another, it may be a 1 in 2. Yamagishi's (1997) paper highlighted how people interpreted 1286 out of 10,000 death rates as riskier than 24.14 out of 100. It was recognised that standardisation for explaining risk was required which resulted in a pictorial explanation of risk ranging from 1 in 1 , to 1 in $1,000,000,000,000$ and comparing it to everyday familiar risks (Edwards, Elwyn, \& Mulley, 2002). A study by Garcia-Retamero \& Galesic (2010) with participants from both America and Germany, revealed that by using visual aids that do not require high levels of numeracy, the information given to patients improved their medical decision-making based on risk to treatment.

Framing language also has effects such as the persuasive or positive techniques used to encourage greater uptake of procedures or examinations such as mammograms. Van de Water et al. (2018) established that patient satisfaction was higher when verbal information was combined with numerical information. A more recent study by Kim, Trinidad, Mikesell, \& Aakhus (2020) found that an appropriate visual format in the 
delivery of prognosis information can reduce potential negative effects in conveying data, as well as helping patients stay positive and motivated. The use of images has been essential during the COVID-19 pandemic in making sure people understand social distancing, as opposed to just using numbers, i.e. the 2-meter social distancing has been depicted as the length of a door, or the length of two German Shepherds (Figure 37), or the length of an SUV car.

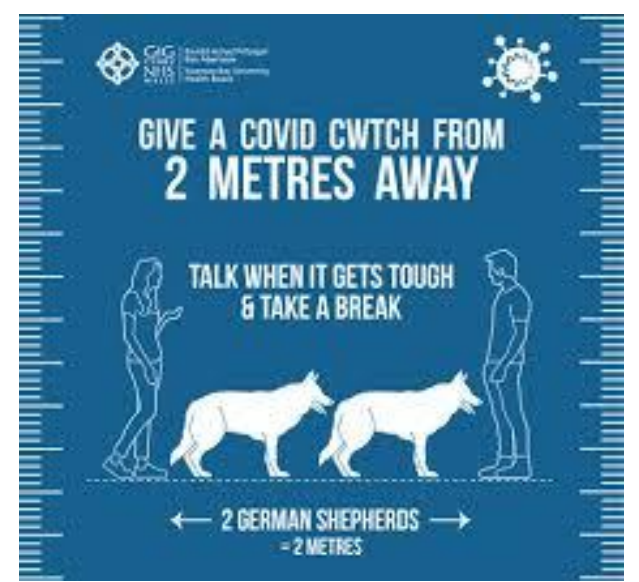

Figure 37: NHS Wales COVID-19 Social Distancing Guidance

However, Edwards et al. (2002) go on to explain that this does not always allow for a fully informed decision to be made as information may be withheld. They go on to explain that the use of images has been explored, and some people may prefer numbers, but others may like the use of bar charts, crowd figures, pie charts, thermometer scales, or survival curves. They conclude that a synthesis of current evidence on patients' preferences for different information formats is required, together with an assessment of the effect of the various formats. Currently there is no evidence to suggest which method of assessment is preferred by patients, however, one could hypothesise that visual images could provide more understanding for some.

3DCI, by its very nature, can offer more than just limb volume information due to the type of data it collects. Imaging provides data that circumferential measurements cannot, i.e. a visual picture or illustration of the problem area. Cau et al. (2016) first suggested the potential of 3DCI to measure geometric parameters that would enable an alternative method of measurement using limb shape. Karakashian et al. (2018) later developed swelling maps (oedema maps) utilising colour codes to visualise the areas of deviance between the affected and unaffected arm. This is something that CTM and 
perometry cannot offer as these produce volumes over a whole limb resulting in localised oedema areas being absorbed into the whole limb volume. The use of the 3DCI camera in this study, the Asus Xtion Pro camera, allowed for images of high quality and pixilation to be taken, making it possible to utilise and manipulate the data images into the oedema maps.

Given the outcomes of this study, it stands to reason that patients could benefit from a visual aid that interprets the severity of their lymphoedema in addition to volumes and percentages that are current practice. The use of colour codes focuses attention on the problem areas, and could add value to a lymphoedema consultation in terms of enabling better-targeted treatment strategies to manage and reduce the lymphoedema. Consequently, for both the patient and therapist, the oedema maps could help identify where the lymphoedema is located, and how it changes with the advised treatment, such as targeted massage and specific compression therapy.

Gillies et al. (2016) extrapolated data from radiologic examinations and converted the images into quantitative data. This process has been named 'radiomics', and they were able to make a diagnosis and decisions regarding treatment options from the interpreted radiological images. They found this method to be highly accurate and anticipate it to replace the traditional practice of treating medical images as pictures intended solely for visual interpretation. Thus, there is not only value in images for diagnostic purposes, but also the ability to extrapolate quantitative data from image data. Watson et al. (Submitted) attempted to develop a mathematical equation to provide a quantitative value for the oedema maps. This led to the development of 'shape redundancy' (Rvalue) used in this study. Given Gillies et al.'s (2016) success in converting images into quantitative data, further refinement of the R-value is needed to develop meaningful quantification of the oedema maps to aid more accurate recording. Once refinement is made, more qualitative research is required to understand patient perspectives.

\subsubsection{Educational Tool}

3DCI could also be used as an educational tool for the patient, their carers or families and, the broader professional community to have a greater understanding of the lymphoedema severity and locations. This may have the potential to motivate a patient to continue with their treatment, or to consider a change in treatment if required, where 
the visual oedema maps are not improving, which is suggested by the findings from Kim et al.'s (2020) study. Using the colour spectrum, a patient can visualise how and where their lymphoedema is changing, which can help explain some of the sensory alterations they may experience such as pain, tightness and heaviness. They would be able to see that if the oedema map has a more extensive and darker area of orange or red, it might explain why their arm has been feeling heavier, and make them feel validated in their description of their symptoms. Conversely, if the picture shows an increasing area of blue, it would explain why the pain in their arm has been lessening. Thus, oedema maps may help patients understand their lymphoedema and related symptoms better, thus educating them on the importance of adhering to their lymphoedema self-management plan.

However, no study yet exists to determine whether patients would value this new technology or if they would find interpreting images easier than current volumes and percentages.

\subsubsection{Practicability}

This study was performed within a clinical setting as part of a patient's normal lymphoedema care pathway. This study has therefore demonstrated the practical set up and application within the lymphoedema examination rooms without disrupting the service. The device can be collapsed to be stored in the corner of a room or a cupboard. The equipment is lightweight and easily portable, thus has the potential to be utilised in the community. 3DCI is a non-invasive method of measuring that is not labour intensive; thus, there are no health \& safety or infection control concerns regarding its use within a clinic or community setting. The work in this study focussed entirely on the upper limb. Studies exist where 3DCI has examined the hand (Hoevenaren et al., 2015; Vicini et al., 2012), breast (Henseler et al., 2012; Henseler et al., 2014) and facial oedema (Aldridge et al., 2005; Plooij et al., 2009; Maal et al., 2010; van der Meer et al., 2014); however, none have looked at measuring the full lower limb. If a protocol could be developed to measure the lower limb, interest and uptake of 3DCI in a clinical setting would increase enormously, as the current limitations would be less. 


\subsection{Limitations of the Study}

The limitations of the study included key factors in the recruitment protocol, unavailability of oedema maps, measuring protocol, the timing of the follow-up sessions, and equipment failure.

\subsubsection{Recruitment Protocol}

The participants came from only one lymphoedema service in Wales. Although one participant was Asian, there were no other ethnic groups in this study; thus, it was not truly representative of the Welsh population. This cohort was also mainly women of middle age; thus, younger (aged less than 40) and older (aged over 70) age groups were not adequately represented. The cohort was small with 20 participants and a $15 \%$ attrition rate $(n=3)$ resulting in less data collected. Shrout and Fleiss (1979) advocate at least 30 heterogeneous participants are recruited for reliability studies. However, it is recognised that before undertaking research, it is prudent to perform a Power of Analysis test to determine the required sample size for the study and recruit to that. This study however performed a retrospective Power of Analysis showing a moderate effect (0.73) to disprove the null hypothesis of this thesis; however, the sample of 30 as reported by Field (2013) would have a high effect (0.99). The first null hypothesis - 3DCI is not a valid and reliable method of measurement for lymphoedema - was disproved as 3DCI demonstrated good validity and reliability compared to CTM and perometry, and was comparable to the existent literature. The second null hypothesis - shape entropy derived from 3DCI is not an alternative method that correlates to arm volume measurement was found to be true as the R-value demonstrated poor validity and moderate reliability compared to volume measurements. However, this could be due to the R-value and volume being completely different concepts of measurements, making them incomparable to one another. Due to the moderate effect of this study's sample size as determined by the Power of Analysis results, more research will be needed to provide a stronger effect to prove/disprove these null hypotheses. Furthermore, research into the use of the R-value as an adjunct to limb volume and oedema maps could be investigated so that these metrics are not used in isolation, but integrated to build a better interpretation of the lymphoedema. 
A further limitation of the study was that the author was the only researcher for the duration of the study; thus, no inter-rater correlations were calculable. It is a strong recommendation that future studies include more than one researcher to provide further evidence of the inter-rater reliability of 3DCI between users. Also, there was strong reliance on a third party for recruitment into the study, which may have influenced the length of time it took to enlist the required number of participants.

\subsubsection{Unavailability of Oedema Maps}

A significant limitation was the software being unable to produce the oedema maps at the appointment. This was due to the software not being fully developed at the time of the study. Thus, participants did not get the opportunity to see their oedema maps and to visualise the colour coding identifying their lymphoedema along with the localised swelling that was present in many of the cases. This would be important in future studies where patient and therapist perceptions could be captured to compare to the quantitative data results.

\subsubsection{Measuring Protocol}

The positioning of the participant will need stricter control for future studies. A handrest is recommended to reduce the risk of movement while capturing the image using 3DCI. This will also reduce the risk of the arm being flexed when the image is captured. Stricter control in terms of arm length across all methods of measurement needs to be considered to determine whether there is a change in the validity and accuracy outcomes.

Participants who were in the obese class 3 group $\left(\mathrm{BMI} \geq 40 \mathrm{~kg} / \mathrm{m}^{2}\right)$ and had large limb volumes (upper arm $>45 \mathrm{~cm}$ circumference) were not able to be captured with 3DCI as the arm could not be isolated from the trunk, i.e. the upper arm was resting on the trunk at shoulder height position. Currently, CTM can measure these limbs as the tape can be manipulated between the soft tissues; however, it is recognised that the limb will be distorted where it is resting on the trunk thus the readings may be inaccurate. The frame of the perometer was able move some of the truncal tissue exposing most of the upper arm, but again the length of the arm captured through perometry differed from that of CTM, thus making a comparison between the two methods challenging. 
People with reduced shoulder range of movement, i.e. those unable to elevate their arm to shoulder height, can currently not be examined using either perometry or 3DCI, as both systems require central positioning of the limb to the scanning area. Thus, further research regarding alternative positional considerations is needed in the future.

\subsubsection{Follow-up Timescales}

With three participants failing to complete the study, a review of the timescales between sessions may be needed. The usual follow up time for newly assessed patients at the lymphoedema service is between two and four months from their initial assessment. From this study, two-monthly reviews for six months may be considered onerous to patients in terms of travel time required, time off work (for those in employment) and the stress of finding parking at a hospital location. Future longitudinal studies should consider longer review timescales between sessions as part of their study protocol; alternatively, two sessions within quick succession (two to four weeks) could also be considered.

\subsubsection{Equipment Failure}

Problems identified with the apparatus were due to software problems for both the perometer and 3DCI. It is unclear why the software systems failed on occasion, but it is thought that there was some incompatibility with the organisation's IT systems. This will need to be resolved to allow for seamless capturing of data within the clinical setting to ensure data is captured for the assessment of lymphoedema, the implementation of its management and the tracking of treatment outcomes. Improved IT services would also allow for more data-rich studies in the future.

\subsection{Recommendations for Further Research}

From the limitations and opportunities identified, recommendations for future studies can be made. This next section outlines these recommendations.

\subsubsection{Cohort}

It would be ideal for the cohort to be more reflective of a clinical caseload that would include both men and women, younger and older age groups, and people of different ethnicity. This would ensure evidence of the outcomes that are translatable to all patients 
attending a lymphoedema clinic for assessment. It would also give clinicians more confidence in the results applying to their lymphoedema caseloads.

Lymphoedema, due to other causes such as primary, secondary to melanoma treatment and vascular insufficiency, needs to be included to capture the different distributions of the swelling resulting from the different aetiologies. This study happened to recruit participants who had lymphoedema secondary to breast cancer only. It is known that other causes of lymphoedema respond differently to treatment, and it would be of benefit to ascertain whether 3DCI would be more sensitive in detecting the different types of changes that people report from other lymphoedema causes compared to current methods of measurement and assessment.

Due to the length of time taken to recruit the 20 participants who were new patients to the lymphoedema service, it would be worth altering the protocol to allow for more recruitment potential by considering existent patients within the lymphoedema service. Furthermore, a multi-site study could be considered as it would allow for larger cohorts and richer data for the analysis of both intra- and inter-rater reliability. Posters could be put up in clinics and letters to all patients with unilateral limb lymphoedema could be sent inviting them to the study. This would ensure an increased chance of recruiting both men and women from a larger geographical area, as well as a broader range of lymphoedema types.

\subsubsection{Extend to Lower Limb and Mid-Line Lymphoedema}

Working jointly with educational and third sector research partners may further expand and explore the use of 3D technology to assess lymphoedema for other parts of the body. There exists a wealth of research related to upper limb lymphoedema secondary to breast cancer; however, the number of patients suffering from lower limb lymphoedema is growing, and in Wales, there are more people with lower limb than upper limb lymphoedema. This is due to health professionals becoming more aware of lymphoedema from other causes of swelling apart from cancer; thus, a suitable imaging system needs to be developed for the lower limbs. The use of limb shape for lower limb lymphoedema would be of particular interest to determine where patterns of localised oedema occur for the different causes of leg lymphoedema. 
Imaging of the lower limb in obese patients could be challenging as the inner thighs resting against each other will distort the shape and potentially preclude the capturing of a full 3D image; thus the actual volume of the limb will be difficult to determine. Currently, CTM can take some measurements as the tape can be manipulated between the thighs, but for some people, even the perometer would not be able to take full leg measurements to the groin due to the excess tissues.

Mid-line oedema (head and neck, breast, genital) and extremities (hands and feet) are often neglected with new methods of measurement, and there is further research to be done with 3DCI to provide a better objective assessment of these lymphoedema sites. Head, neck, and genital lymphoedema may need to be incorporated with heat sensor technology, as these areas do not have an opposite side to measure as a reference; however, longitudinal data would be able to capture change over time to demonstrate improvement or deterioration from the first appointment. There is thus scope for some truly innovative technological research for the future.

\subsubsection{Patient Perspectives}

The use of oedema maps in clinical practice could be further explored with clinicians and patients to determine their interpretations of the images, acceptability of their use in assessing lymphoedema, the usefulness of the information they portray, and whether they influence adherence to treatment. Thus there is potential not only for quantitative research but also for exploring the qualitative aspect of the use of $3 \mathrm{D}$ technology in lymphoedema to determine how staff and patients feel about the use of this new technology in their service. Exploring how people interpret the oedema maps, whether they are easier to interpret as opposed to limb volumes, and how they would utilise them in clinical practice will add further depth to the benefits of this new technological assessment. Further research should investigate whether the use of 3D technology with oedema maps could enhance patient compliance due to their improved understanding of the measurements taken.

\subsubsection{Hardware}

Research into the use of alternative 3D cameras is required as both the Asus Xtion Pro 3D and Microsoft Kinect ${ }^{\mathrm{TM}}$ are no longer available on the market. However, the relevance of the 3DCI data from this study is transferable to other systems able to 
produce high-quality pixelated images. When reviewing the development of 3D imaging from the systematic literature review, it is clear that 3D technology is evolving at a rapid pace. From the proof of concept by Lu et al (2013) to the most recent study by Binkley et al. (2020), 3D camera technology has changed significantly. This is evident from the use of static cameras of various size and design to the more mobile Microsoft Kintect $^{\mathrm{TM}}$ and Asus Xtion Pro, and more recently to the development of a handheld device in the form of a modified iPad - LymphaTech (Binkley et al., 2020).

The evidence of the changing technology is obvious in the everyday use of smartphone technology. People no longer buy a mobile phone for its communication function alone, but its camera clarity and function. Recognising these fast-paced changes in the world of technology, the search for a camera that can capture images compatible with the conversion software is critical. Smaller devices may make manipulation of the camera even easier, the potential of hands-free systems such as micro-drones, and the possibility of blue-tooth technology for the images to be fed directly into the computer software should be a possibility with current technological advances.

The challenge lies in finding a camera that provides the same or better quality imaging for data extraction and manipulation. Failing that, the development of a new alternative camera that is compact, hands-free, compatible with the software and offers blue-tooth options, would be a unique challenge for a technology student looking for a research project to advance medical care. The software systems exist; the algorithms for the oedema maps are transferable; the challenge now is finding the hardware to feed into the software to produce the oedema maps and build on the current research outcomes, pushing its use into clinical practice.

\subsection{Summary and Conclusion}

This is the first study to determine whether limb shape could be a feasible measurement tool for lymphoedema. The outcome of this study is that 3DCI does indeed provide new information or data in the form of oedema maps and shape redundancy compared to the outcomes from CTM and perometry, however, refinement of the R-value is needed. 3DCI compared to the usual methods of measurements (CTM and perometry) was shown to be reliable, valid and accurate as a measure of upper limb volume. Limb shape 
demonstrated good similarities to limb volume outcomes and with the use of the oedema maps, was able to identify localised lymphoedema and the severity of the focal swelling, something that neither CTM nor perometry can do.

Change to practice can be challenging and introducing new methods for the assessment of lymphoedema even more so. Without more robust, large scale, multi-site studies, preferably performed within a clinical setting, clinicians are less likely to consider new and alternative methods of measurement, especially when current methods seem to be functional. Consideration of cost for devices needs to be outweighed by strong evidence supporting the need for change, and the benefits of the new method need to be promoted. There was much interest in the production of the oedema maps by the participants. Thus, both patients and therapists could find oedema maps helpful in identifying where the lymphoedema is located, how it has changed over time, whether the treatment is effective, and highlighting areas where targeted treatment and garments are needed through the colour grading system. However, more robust research is required to prove that it is a financially and clinically feasible alternative to current practice.

Newer technologies offer the chance of better options for capturing the 3D data that can be further researched on a larger scale to add to the body of evidence of this study. Qualitative research must be undertaken to determine the use of limb shape as a teaching aid, to determine how patients and staff interpret the results, and whether it is easier to interpret results from limb shape as opposed to limb volume and percentages.

Finally, in addressing the thesis title, 'can 3D camera imaging provide improved information to assess and manage lymphoedema in clinical practice', the answer is positive. Using oedema maps will not only improve the assessment of lymphoedema, but the potential for targeted treatment based on the visualised, localised lymphoedema is also greatly enhanced. 
Glossary of Terms 
Absolute Volume It is the difference in the current volume of a limb compared to

Difference (AVD) its volume at the initial assessment. It is calculated by subtracting the initial limb volume $\left(V_{I A}\right)$ from the current limb volume of the same arm where a negative value indicates a reduction in limb volume. Equation:

$$
A V D=V-V_{I A}
$$

Accuracy

The ability of a test to differentiate between healthy and pathologic cases. This should include testing of sensitivity and specificity of the test:

- Sensitivity - the ability of a test to identify true positive cases

- Specificity - the ability of a test to identify healthy cases correctly, i.e. true negative cases

Circumferential Most common technique for determining limb volume using a Tape Measurement tape measure. The two more commonly used methods are $4 \mathrm{~cm}$ (CTM)

CTM and 5-point CTM, where $4 \mathrm{~cm}$ CTM measures the arm at $4 \mathrm{~cm}$ intervals from the wrist to the axilla. The 5-point CTM method measures five set points up the arm, i.e. the wrist, widest part of the forearm, the elbow, widest part of the upper arm and the point nearest the axilla. Limb volumes are calculated using either a cylinder or a frustum formula (see section 3.4).

Intraclass

Correlation

ICC is a reliability index in test-retest, intra-rater and inter-rater

Coefficient (ICC)

Lymphoedema

Percentage

Absolute Volume

Difference

(\%AVD)

reliability analysis with values closer to 1.00 indicating excellence.

Lymphoedema is a persistent dysfunction of the lymphatic system resulting in swelling that can affect any part of the body, but is most commonly seen in the limbs. Lymphoedema can be either primary (congenital or genetic) or secondary (acquired).

Percentage Volume In unilateral limb lymphoedema is the difference in between the

Difference $(\% \mathrm{VD}) \quad$ volumes of the oedema $\left(V_{O}\right)$ and Normal $\left(V_{N}\right)$ sides, divided by the Normal $\left(V_{N}\right)$ side and multiplied by 100. Equation:

$$
\% V D=\left(\frac{V_{O-V_{N}}}{V_{N}}\right) * 100
$$

In clinical practice, patients have a better understanding of limb volume difference expressed as both a volume (cups or pints) and percentage of the lymphoedema arm compared to their 'normal' (unaffected) arm 
Perometer

Reliability

Standard Error of

Measurement

(SEM)

Shape Redundancy

Value (R-value)

Sensitivity

Specificity

Three-dimensional

Camera Imaging

(3DCI)

Validity
It is a technology-based circumferential measurement. It is an infrared optoelectric imaging system that is used for the indirect measurement of arm or leg volume. It offers information on circumferences, contours and provides schematic representations of the cross-sectional area of the limb (see section 3.5).

The ability of a measure or instrument to produce consistent outcomes across different situations. There exists two main types of reliability:

- Repeatability or test-retest reliability - evaluates the stability of the measure administered using the same protocol at different times to a person

- Inter-rater reliability - when different researchers apply the same test.

SEM quantifies the amount of variability in a test and is measured in the same units as the original measurement with values closer to 0 indicating better reliability

A single value that describes the shape of the arm (the concept finds its origins in computer graphics) whereby the swollen arm is considered to be more 'round' and 'stretched', thus lacking in definition (see section 5.5.7.2).

The proportion of true-positives that are correctly identified by a test, i.e. the test identifies the presence of a condition The proportion of true-negatives that are correctly identified by a test, i.e. the test identifies the absence of a condition

A newer method of calculating limb volume using 3D modelling and is derived from a variety of methods including 3D stereophotogrammetry, Microsoft Kinect ${ }^{\mathrm{TM}}$, Asus Xtion infrared sensors and a modified iPad tablet (see section 3.8 for more details on the different types of $3 \mathrm{D}$ equipment used in research)

The extent to which an instrument measures what it purports to measure. There are three types of validity:

- Content Validity - determines the logical link between the objectives and the research questions

- Criterion Validity - where a new measure is compared to an existing or comparable measure to determine the same or similar outcomes. Two main types:

- Concurrent validity: the simultaneous accuracy of measurment evidenced by comparing with results from a previously valudated test

- Predictive validity: the degree to which a test accurately predicts a criterion that will occur in the future 
- Construct Validity - is a more sophisticated technique where the underlying construct or idea behind an instrument is validated by statistical procedures. Has two subcategories:

- Convergent validity: measures that are related should have high correlation

- Discriminant validity: the degree to which two measures have independent unique variances, i.e. no correlation 
References 
Adriaenssens, N., Buyl, R., Lievens, P., Fontaine, C., \& Lamote, J. (2013). Comparitive study between mobile infrared optoelectronic volumetry with a perometer and two commonly used methods for the evaluation of arm volume in patients with breast cancer related lymphedema of the arm. Lymphology, 46:132-143.

Akobeng, A. K. (2007). Understanding diagnostic tests 3: receiver operating characteristic curves. Acta Paediatrica, 96:664-647.

Alcorso, J., \& Sherman, K. A. (2015). Factors associated with psycohological distress in women with breast cancer-related lymphoedema. Psycho-Oncology, 25(7):749-880.

Aldridge, K., Boyadjiev, S. A., Capone, G. T., DeLeon, V. B., \& Richtsmeier, J. T. (2005). Precision and Error of Three-dimensional Phenotypic Measures Acquired from 3dMD Photogrammatric Images. American Journal of Medical Genetics Part A, 15(3):247-253.

Allied Health Professions (AHP) Outcome Measures UK Working Group. (2019). Key questions to ask when selecting outcomes measures: a checklist for allied health professionals. London: Royal College of Speech and Language Therapists.

Al-Niaimi, F., \& Cox, N. (2009). Cellulitis and Lymphoedema: a vicious cycle. Journal of Lymphoedema, 4(2):38-42.

Altman, D. G., \& Bland, J. M. (1994). Diagnostic tests 1: senstivity and specificity. BMJ, 308:1552.

Ancukiewicz, M., Miller, C. L., Skolny, M., O'Toole, J., Warren, L. E., Jammallo, L. S., Michelle, C., \& Taghian, A. G. (2012). Comparison of relative versus absolute arm size change as criteria for quantifying breast cancer-related lymphedema: the flaws in current studies and need for universal methodology. Breast Cancer Research and Treatment, 135:145-152.

Ancukiewicz, M., Russell, T. A., Otoole, J., Specht, M., Singer, M., Kelada, A., Murphy, C. D., Pogachar, J., Gioioso, V., Patel, M., Skolny, M., Smith, B. L., \& Taghian, A. G. (2011). Standardised Method for Quantification of Developing Lymphedema in Patients Treated for Breast Cancer. International Journal of Radiation Oncology Biology Physics, 79(5):1436-1443.

Antwi, S. K., \& Hamza, K. (2015). Qualitative and Quantitative Research Paradigms in Business Research: A Philosophical Reflection. European Journal of Business and Management, 7(3):217-225. 
Arinaga, Y., Piller, N., \& Sato, F. (2016). How can we know the true magnitude of any breast cancer-related lymphoedema if we do not know which is the true dominant arm? Journal of Lymphoedema, 11(1):27-34.

Asim, M., Cham, A., Banerjee, S., Nancekivell, R., Dutu, G., McBride, C., Cavanagh, S., Lawrenson, R., \& Campbell, I. (2012). Difficulties with defining lymphoedema after axillary dissection for breast cancer. The New Zealand Medical Journal, 125(1351):29-39.

Åström, K. G., Abdsaleh, S., Brenning, G. C., \& Ahlström, K. H. (2001). MR imaging of primary, secondary, and mixed forms of lymphedema. SAGE Journals, 42(4):409-416.

Atieno, O. P. (2009). An Analysis of the Strengths and Limitation of Qualitative and Quantitative Research Paradigms. Problems of Education in the 21st Century, 13:13-18.

Babizhayev, M. A., \& Yegorov, Y. E. (2011). Smoking and health: association between telomere length and factors impacting on human disease, quality of life and life span in a large population-based cohort under the effect of smoking duration. Fundamental and Clinical Pharmacology, 25(4):425-442.

Bakar, Y., Tuğral, A., \& Üyetürk, Ü. (2018). Measurement of Local Tissue Water in Patients with Breast Cancer-Related Lymphedema. Lymphatic Research and Biology, 16(2):160-164.

Barrio, A. V., Eaton, A., \& Frazier, T. G. (2015). A Prospective Validation Study of Bioimpedance with Volume Displacement in Early-Stage Breast Cancer Patients at Risk for Lymphoedema. Annals if Surgical Oncology, 22:S370-S375.

Batista, B. N., Baiocchi, J. M., Campanholi, L. L., Bergmann, A., \& Duprat, J. P. (2018). Agreement between Perometry and Sequential Arm Circumference Meassurements in Objective Determination of Arm Volume. Journal of Reconstructive Microsurgery, 34(1):29-34.

Beauchamp, T. L., \& Childress, J. F. (2012). Principles of Biomedical Ethics. 7th Edition. New York: Oxford University Press.

Bedi, M., King, D., Whitfield, R., Hackbarth, D., Neilson, J., Charlson, J., \& Wang, D. (2015). The Effect of Smoking and Major Vein Resection on Post-therapy Lymphedema in Soft Tissue Sarcomas Treated With Neoadjuvant Radiation and Limb-salvage Surgery. American Journal of Clinical Oncology, 38(2):184188(5). 
Belgrado, J. P., Bracale, P., Bates, J., Roh, N., Rosiello, R., Cangiano, A., \& Moraine, J. J. (2010). Lymphoedema: What can be measured and how... Overview. The European Journal of Lymphology, 21(61):3-9.

Berlit, S., Brade, J., Tuschy, B., Hornemann, A., Leweling, H., Eghardt, V., \& Sutterlin, M. (2012). Comparing Bioelectrical Impedance Values in Assessing Early Upper Limb Lymphedema after Breast Cancer Surgery. in vivo, 26:863-868.

Berlit, S., Brade, J., Tuschy, B., Hornemann, A., Leweling, H., Eghardt, V., \& Sutterlin, M. (2013). Whole-body Bioelectrical Impedance Analysis in Assessing Upperlimb Lymphedema After Breast Cancer Therapy. Anticancer Research, 33:44534556.

Bilir, S. P., DeKoven, M. P., \& Munakata, J. (2012). Economic Benefits of BIS-Aided Assessment of Post-BC Lymphedema in the United States. American Journal of Managed Care, 18(5):234-241.

Binkley, J. M., Weiler, M. J., Frank, N., Bober, L., Dixon, J. B., \& Stratford, P. W. (2020). Assessing Arm Volume in People During an After Treatment for Breast Cancer: Reliability and Convergent Validity of the LymphaTech System. Physical Therapy, 100(3):1-11.

Blaney, J. M., McCollum, G., Lorimer, J., Bradley, J., Kennedy, R., \& Rankin, J. P. (2015). Prospective surveillance of breast cancer-related lymphoedema in the first-year post-surgery: feasibility and comparison of screening measures. Supportive Care in Cancer, 23:1549-1559.

BLS \& LSN. (2016, December). Consensus Document on the Management of Cellulitis in Lymphoedema. London: British Lymphology Society \& Lymphoedema Support Network.

Bodystat. (2017). www.bodystat.com/products/. Retrieved from www.bodystat.com: https://www.bodystat.com/products/ on 29 June 2017

Bossuyt, P. M., Irwig, L., Craig, J., \& Glasziou, P. (2006). Comparative accuracy: assessing new tests against existing diagnostic pathways. British Medical Journal, 332(7549):1089-1092.

Bossuyt, P. M., Reitsma, J. B., Bruns, D. E., Gatsonis, C. A., Glasziou, P. P., Irwig, L., Lijmer, J. G., Moher, D., Rennie, D., de Vet, H. C. W., Kressel, H. Y., Rifai, N., Golub, R. M., Altman, D. G., Hooft, L., Korevaar, D. A., \& Cohen, J. F. (2015). STARD 2015: An Updated List of Essential Items for Reporting Diagnostic Accuracy Studies. Radiology, 277(3):826-832. 
British Lymphology Society. (2016). Population Needs Assessment. Retrieved from British Lymphology Society: https://thebls.com/documents-library on 13 July 2017

Brorson, H., \& Hoijer, P. (2012). Standardised measurements used to order compression garments can be used to calculate arm volumes to evaluate lymphoedema treatment. Journal of Plastic and Surgical Hand Surgery, 46:410-415.

Buendia, R., Essex , T., Kilbreath, S. L., Czerniec, S., Dylke, E., \& Ward, L. C. (2018). Estiamtion of Arm Adipose Tissue Quotient Using Segmental Bioimpedance Spectroscopy. Lymphatic Research and biology, 16(4):377-384.

Buffa, R., Mereu, E., Lussu, P., Succa, V., Pisanu, T., Buffa, F., \& Marini, E. (2015). A New, Effective and Low-Cost Three-Dimensional Approach for the Estimation of Upper-Limb Volume. Sensors, 15(6):12342-12357.

Bulley, C., Coutts, F., \& Tan, C. W. (2013). Perometry limb volume measurement: protocol development and reliability. European Journal of Physiotherapy, 15:193-200.

Bundred, N., Foden, P., Todd, C., Morris, J., Watterson, D., Purushotham, A., Bramley, M., Riches, K., Hodgkiss, T., Evans, A., Skene, A., Keeley, V., \& the investigators of BEA/PLACE studies. (2020). Increases in arm volume predict lymphoedema and quality of life deficits after axillary surgery: a prospective cohort study. Brisith Journal of Cancer, DOI: 10.1038/s41416-020-0844-4.

Cancer Research UK. (2020). Lymphoedema after breast cancer treatment. Retrieved from Cancer Research UK: https://www.cancerresearchuk.org/aboutcancer/breast-cancer/living-with/lymphoedema-after-treatment on 30 May 2020

Cannon, J., Dyer, J., Carapetis, J., \& Manning, L. (2018). Epidemiology and risk factors for recurrent severe lower limb cellulitis: a longitudinal cohort study. Clinical Microbiology and Infection, 24(10):1084-1088.

Carron, J. (2016). Violin Plots 101: Visualizing Distribution and Probability Density. Retrieved from Mode Blog: https://mode.com/blog/violin-plot-examples on 02 February 2020

Caruana, E. J., Roman, M., Hernández-Sánchez, J., \& Solli, P. (2015). Longitudianl Studies. Journal of Thoracic Disease, 7(11):E537-E540.

Casley-Smith, J. R. (1994). Measuring and Representing Peripheral Oedema and Its Alterations. Lymphology, 27:56-70. 
Cau, N., Galli, M., Cimolin, V., Aranci, M., Caraceni, A., \& Balzarini, A. (2016). Comparative study between circumferential method and laser scanner 3D method for the evaluation of arm volume in healthy subjects. Journal of Vascular Surgery: Venous and Lymphatic Disorders, 4(1):64-72.

Cau, N., Galli, M., Cimolin, V., Grossi, A., Battarin, I., Puleo, G., Balzarini, A., \& Caraceni, A. (2018). Quantitative comparison between the laser scanner threedimensional method and the circumferential method for evaluation of arm volume in patients with lymphedema. Journal of Vascular Surgery: Venous and Lymphatic Disorders, 6(1):96-103.

Centers for Disease Control and Prevention. (2019). Alcohol Use and Your Health. Retrieved from CDC: https://www.cdc.gov/alcohol/fact-sheets/alcohol-use.htm on 06 July 2020

Centre for Evidence-Based Medicine (CEBM). (2014). cebm study designs. Retrieved from Centre for Evidence-Based Medicine (CEBM): https://www.cebm.net /2014/04/study-designs/ on 24 June 2019

Chang, D. W., Soami, H., \& Skoracki, R. (2013). A Prospective Analysis of 100 Consecutive Lymphovenous Bypass Cases for Treatment of Extremity Lymphedema. Plastic and Reconstructive Surgery, 132:1305-1314.

Chartered Society of Physiotherapy (CSP). (2011). Code of Member's Professional Values and Behaviour. London: Chartered Society of Physiotherapy.

Chen, Y.-W., Tsai, H.-J., Hung, H.-C., \& Tsauo, J.-Y. (2008). Reliability Study of Measurements for Lymphedema in Breast Cancer Patients. American Journal of Physical Medicine and Rehabilitation, 87(1):33-38.

Chiewvit, S., \& Kumnerdnakta, S. (2017). Lymphosctintigraphic findings that predict favorable outcome after lymphaticovenous anastomosis. Lymphology, 50(1):18.

Cohen, M. X. (2018). How to interpret and create violin plots. Retrieved from youtube.com: https://www.youtube.com/watch?v=M6Nu59Fsyyw on 28 September 2019

Cook, T. S., Couch, G., Couch, T. J., Kim, W., \& Boonn, W. W. (2013). Using the Microsoft Kinect for Patient Size Estimation and Radiation Dose Normalization: Proof of Concept and Initial Validation. Journal of Digital Imaging, 26: 657662. 
Cornelissen, A. J., Kool, M., Keuter, X. H., Heuts, E. M., Piatkowski de Grzymala, A. A., van der Hulst, R. R., \& Qui, S. S. (2018). Quality of Life Questionnaires in Breast Cancer-Related Lymphedema Patients: Review of the Literature. Lymphatic Research and Biology, 16(2):134-139.

Cornish, B. H., Ward, L. C., Thomas, B. J., \& Bunce, I. H. (1998). Quantification of Lymphoedema using Multi-frequency Bioimpedance. Applied Radiation and Isotopes, 651-652.

Critical Appraisal Skills Programme. (2017). CASP Checklists. Retrieved from Critical Appraisal Skills Programme (CASP): http://www.casp-uk.net/casp-toolschecklists; accessed 20 January 2018

Currell, G. (2015). Transforming for Normality. Retrieved from Scientific Data Analysis, Oxford University Press: https://www.youtube.com/watch?v= alC9rZr1rG8 accesses on 12 April 2021

Czerniec, S. A., Ward, L. C., \& Kilbreath, S. L. (2015). Assessment of Breast CancerRelated Lymphedema: A Comparison of Moisture Meter and Spot Bioimpedane Measurement. Lymphatic Research and Biology, 13(1):10-19.

Czerniec, S. A., Ward, L. C., Lee, M.-J., Refshuage, K. M., Beith, J., \& Kilbreath, S. L. (2011). Segmental measurement of breast cancer-related arm lymphoedema using perometry and bioimpedance spectroscopy. Supportive Care in Cancer, 19:703-710.

Czerniec, S. A., Ward, L. C., Refshauge, K. M., Beith, J., Lee, M. J., York, S., \& Kilbreath, S. L. (2010). Assessment of Breast Cancer-Related Arm Lymphedema - Comparison of Physical Measurement Methods and Self-Report. Cancer Investigation, 28:54-62.

Data Protection Act. (2018). The National Archives. Retrieved from legislation.gov.uk: http://www.legislation.gov.uk/ukpga/2018/12/pdfs/ukpga_20180012_en.pdf on 01 April 2019

Daya, S. (1996). Study Design for the Evaluation of Diagnostic Tests. Seminars in Reproductive Endocrinology, 14(2):101-109.

de Vet, H. C., \& Terwee, C. B. (2010). The minimal detectable change should not replace the minimal important difference. Journal fo Clinical Epidemiology, 63:804-805.

De Vrieze, T., Gebruers, N., Nevelsteen, I., De Groef, A., Tjalma, W. A., Thomis, S., Dams, L., Van der Gucht, E., Penen, F., \& Devoogdt, N. (2020). Reliability of 
the MoistureMeterD Compact Device and the Pitting Test to Evaluate Local Tissue Water in Subjects with Breast Cancer-Related Lymphedema. Lymphatic Research and Biology, 18(2):116-128.

De Vrieze, T., Gebruers, N., Tjalma, W. A., Wiebren, A. A. Nevelsteen, I., Thomis, S., De Groef, A., Dams, L., Van der Gucht, E., Belgrado, J. P., Vandermeeren, L., \& Devoogdt, N. (2019). What is the best method to determine excessive arm volume in patients with breast cancer-related lymphoedema in clinical practice? Reliability, time efficiency and clinical feasibility of five different methods. Clinical Rehabilitation, DOI: 10.1177/0269215519835907.

Delfin Technologies. (2017). delfintech.com/en/product_information/moisturemeterd_ compact/. Retrieved from www.delfintech.com: http://www.delfintech.com/ en/product_information/moisturemeterd_compact/ on 29 June 2017

Delfin Technologies Ltd. (2014). MoistureMeterD Compact User Manual. Kuopio, Finland: Delfin Technologies Ltd.

DeSnyder, S. M., Khietkhah, A., Travis, M. L., Lilly, S. E., Bedrosian, I., Buchholz, T. A., Schaverien, M. V., \& Shaitelman, S. F. (2019). Optimizing Patient Positioning to Reduce Variation in the Measurement of breast Cancer-Related Lymphedema. Lymphatic Research and Biology, 17(4):440-446.

Devoogdt, N., Lemkens, H., Geraerts, I., van Nuland, I., Flour, M., Coremans, T., Christiaens, M. R., \& van Kampen, M. (2010). A new device to measure upper limb circumferences: validity and reliability. International Angiology, 29(5):401-407.

Devoogdt, N., Van Kampen, M., Geraerts, I., Coremans, T., \& Christiaens, M.-R. (2011). Lymphoedema Functioning, Disability and Health Questionnaire (Lymph-ICF): Reliability and Validity. Physical Therapy, 91(6):944-957.

di Ruffano, L. F., Hyde, C. J., McCaffery, K. J., Bossuyt, P. M., \& Deeks, J. J. (2012).

Research Methods \& Reportng: Assessing the value of diagnostic tests: a framework for designing and evaluating trials. BMJ, 344:e686 doi: 10.1136/bmj.e686.

DiSipio, T., Rye, S., Newman, B., \& Hayes, S. (2013). Incidence of unilateral lymphoedema after breast cancer: a systematic review and meta-analysis. The Lancet Oncology, 14(6):500-515.

Doğan, N. Ö. (2018). Bland-Altman analysis: A paradigm to understand correlation and agreement. Turkish Journal of Emergency Medicine, 18:139-141. 
Dontje, M. L., Dall, P. M., Skelton, D. A., Gill, J. M., Chastin, S. F., \& Team, O. B. (2018). Reliability, minimal detectable change and responsiveness to change: Indicators to select the best method to measure sedentary behaviour in older adults in different study designs. PLoS ONE, 13(4):e0195424 DOI:10.1371/journal.pome.0195424.

Dylke, E. S., Schembri, G. P., Bailey, D. L., Bailey, E., Ward, L. C., Refshauge, K., Beith, J., Black, D., \& Kilbreath, S. L. (2016). Diagnosis of upper limb lymphedema: development of an evidence-based approach. Acta Oncologica, 55(12):1477-1483.

Dylke, E. S., Yee, J., Ward, L. C., Foroughi, N., \& Kilbreath, S. L. (2012). Normative Volume Differences Between the Dominant and Nondominant Upper Limbs in Healthy Older Women. Lymphatic Research and Biology, 10(4):182-188.

Edwards, A., Elwyn, G., \& Mulley, A. (2002). Explaining risks: turning numerical data into meaningful pictures. British Medical Journal, 324:827-830.

Erends, M., van der Aa, T., van der Hulst, R., \& de Grzymala, A. P. (2014). Validity and Reliability of Three-Dimensional Imaging for Measuring the Volume of the Arm. Lymphatic Research and Biology, 12(4): 275-281.

EuroQol. (2017). EQ-5D. Retrieved from www.euroqol.org: https://euroqol.org/eq-5dinstruments/eq-5d-51-about/on 16 May 2017

Field, A. (2013). Discovering Statistics Using IBM SPSS Statistics; 4th edition. London: SAGE Publication Ltd.

Fife, C. E., Farrow, W., Hebert, A. A., Armer, N. C., Stewart, B. R., Cormier, J. N., \& Armer, J. M. (2017). Skin and Wound Care in Lymphedema Patients: A Toxonomy, Primer, and Literature Review. Advances in Skin \& Wound Care, 30(7):305-318.

Finnane, A., Janda, M., \& Hayes, S. C. (2015). Does teatment alleviate lympedema symptoms? A cross-sectional study evaluating patient perspectives. Lymphology, 48: 110-120.

Foroughi, N., Dylke, E. S., Paterson, R. D., Sparrow, K. A., Fan, J., Warwick, E. B., \& Kilbreath, S. L. (2011). Inter-Rater Reliability of Arm Circumference Measurement. Lymphatic Research and Biology, 9(2):101-107.

Fu, M. R., Cleland, C. M., Guth, A. A., Kayal, M., Haber, J., Cartwright, F., Kleinman, R., Kang, Y., Scaglioa, J., \& Axelrod, D. (2013). L-Dex Ratio in Detectng Breast 
Cancer-Related Lymphedema: Rliability, Sensitivity, and Specificity. Lymphology, 46:85-96.

Garcia-Retamero, R., \& Galesic, M. (2010). Who profits from visual aids: Overcoming challenges in people's understandingof risks. Social Science and Medicine, 70:1019-1025.

Gebruers, N., Verbelen, H., De Vrieze, T., Vos, L., Devoogdt, N., Fias, L., \& Tjalma, W. (2017). Current and future perspectives on the evaluation, prevention and conservative management of breast cancer related lymphoedema: A best practice guideline. European Journal of Obstetrics \& Gynecology and Reproductive Bioloy, 216:245-253.

Gillies, R. J., Kinahan, P. E., \& Hricak, H. (2016). Radiomics: Images Are More than Pictures, They Are Data. Radiology, 278(2): 563-577.

Glasser, S. P. (2008). Essentials of Clinical Research. Birmingham: Springer.

Glen, S. (2015a). Concurrent Validity Definition and Examples. Retrieved from StatisticsHowTo.com: Elementary Statistics for the rest of us!: https://www.statisticshowto.com/concurrent-validity/ accessed on 07 April 2021

Glen, S. (2015b). Box Cox Transformation. Retrieved from StatisticsHowTo.com: https://www.statisticshowto.com/box-cox-transformation/ accessed on 12 April 2021

Glen, S. (2017). Chi-Square Test for Normality. Retrieved from Statistics How To: https://www.statisticshowto.datasciencecentral.com/chi-square-test-normality/ on 02 February 2020

Gonzalez-Jorgea, H., Riveirob, B., Vazquez-Fernandezc, E., Martinez-Sáncheza, J., \& Ariasa, P. (2013). Metrological evaluation of Microsfot Kinect and Asus Xtion sensors. Measurement, 46(6):1800-1806.

Good, O. S. (2017). Kinect is officially dead. Really. Officially. It's dead. Retrieved from www.polygon.com: https://www.polygon.com/2017/10/25/16543192/kinectdiscontinued-microsoft-announcement on 28 October 2017

Grada, A. A., \& Phillips, T. J. (2017). Lymphedema: Pathophysiology and clinical manifestations. Journal of the American Acadamy of Dermatology, 77(6):10091020.

Gray, M. (2017). Value based healthcare. British Medical Journal, 356:j437 DOI: 10.1136/bmj.j437. 
Greene, A., Grant, F., Slavin, S., \& Maclellan, R. (2015). Obesity-Induced Lymphedema: Clinical and Lymphoscintigraphic Features. Plastic and Reconstructive Surgery, 135(6):1715-1719.

Groenlund, J. H., Telinius, N., Skov, S. N., \& Hjortdal, V. (2017). A Validation Study of Near-Infrared Fluorescence Imaging of Lymphatic Vessels in Humans. Lymphatic Research and Biology, 15(3):22-234.

Guermazi, A., Brice, P., Hennequin, C., \& Sarfati, E. (2003). Lymphography: An Old Technique Retains Its Usefulness. RadioGraphics, 23:1541-1560.

Hadamitzky, C., Pabst, R., Gordon, K., \& Vogt, P. M. (2014). Surgical procedures in lymphedema management. Journal of Vascular Surgery, Venous and Lymphatic Disorders, 2(4):461-468.

Hameeteman, M., Verhulst, A. C., Vreeken, R. D., Maal, T. J., \& Ulrich, D. J. (2016). 3D stereophotogrammetry in upper-extremity lymphedema: An accurate diagnostic method. Journal of Plastic, Reconstructive \& Aesthetic Surgery, 69(2):241-247.

Hammerberg, K., Kirkman, M., \& de Lacey, S. (2016). Qualitative research methods: when to use them and how to judge them. Human Reproduction, 31(3):498-501.

Hara, H., Mihara, M., Seki, Y., Todokoro, T., Iida, T., \& Koshima, I. (2013). Comparison of Indocyanine Green Lymphographic Findings with the Conditions of Collecting Lymphatic Vessles of Limbs in Patients with Lymphedema. Plastic and Reconstructive Surgery, 132: 1612-1618.

Harrington, S., Gilchrist, L., \& Sander, A. (2014). Breast Cancer Edge Task Force Outcomes: Clinical Measures of Pain. Rehabilitation Oncology, 32(1):13-21.

Health \& Care Professions Council (HCPC). (2016). Standards of Conduct, Performance and Ethics. Retrieved from hcpc-uk standards of conduct performance and ethics: https://www.hcpc-uk.org/standards/standards-ofconduct-performance-and-ethics/ on 07 July 2019

Henseler, H., Kuznetsova, A., Vogt, P., \& Rosenhahn, B. (2014). Validation of the Kinect device as a new portable imaging system for three-dimensional breast assessment. Journal of Plastic, Reconstructive \& Aesthetic Surgery, 67: 483-488.

Henseler, H., Smith, J., Bowman, A., Khambay, B. S., Ju, X., Ayoub, A., \& Ray, A. K. (2012). Investigation into variation and errors of a three-dimensional breast imaging system using multiple stereo cameras. Journal of Plastic, Reconstructive \& Aesthetic Surgery, 65:332-337. 
Hidding, J. T., Viehoff, P. B., Beurskens, C. H., van Laarhoven, H. W., Nijhuis-van der Sanden, M. W., \& van der Wees, P. J. (2016). Measurement Properties of Instruments for Measuring of Lymphedema: Systematic Review. Physical Therapy, 96(12):1965-1981.

Hoevenaren, I. A., Maal, T. J., Krikken, E., de Haan, A. F., Berge, S. J., \& Ulrich, D. J. (2015). Development of a three-dimensional hand model using 3D stereophotogrammetry: Evaluation of landmark reproducibility. Journal of Plastic, Reconstructive \& Aesthetic Surgery, 68:709-716.

Hoevenaren, I. A., Verhulst, A. C., Hameeteman, M., Vreeken, R. D., Maal, T. J., \& Ulrich, D. J. (2016). Three-dimensional stereophotogrammetry as an accurate tool for analyzing lymphedema of the hand. Journal of Plastic, Reconstructive and Aesthetic Surgery, 10:40-46.

Howick, J. (2013). cebm.net/wp-content/uploads/2014/06/CEBM-study-design-april20131.pdf. Retrieved from cebm.net: https://www.cebm.net/wpcontent/uploads/2014/06/CEBM-study-design-april-20131.pdf on 24 June 2019

Huang, J. L., \& Leong, F. T. (2016). Standard Error of Measurement. Retrieved from Britannica: https://www.britannica.com/science/standard-error-of-measurement /additional-info\#contributors on 25 March 2021

Hvidsten, S., Toyserkani, N. M., Sørensen , J. A., Høilund-Carlsen, P. F., \& Simonsen, J. A. (2018). A Scintigraphic for Quantitation of Lymphatic Function in Arm Lymphedema. Lymphatic Research and Biology, DOI:10.1089/lrb.2017.0054.

ImpediMed Ltd. (2017). www.impedimed.com/products/l-dex-u400/. Retrieved from www.impedimed.com: https://www.impedimed.com/products/l-dex-u400/ on 29 June 2017

International Lymphoedema Framework. (2012). Position Document: Best Practice for the management of lymphoedema - 2nd edition. Compression Therapy: A position document on compression bandaging. Saint-Etienne, France: The International Lymphoedema Framework in association with the World Alliance for Wound and Lymphoedema Care.

International Society of Lymphology [ISL]. (2013). The diagnosis and treatment of peripheral lymphedema: 2013 consenus document of the International Society of Lymphology. Lymphology 46, 1-11. 
International Society of Lymphology [ISL]. (2016). The Diagnosis and Treatment of Peripheral Lymphedema: 2016 Consensus Document of the International Society of Lymphology. Lymphology, 49:170-184.

Iyigun, Z. E., Selamoglu, D., Alco, G., Pilanci, K. N., Ordu, C., Agacayak, F., Elbuken, F., Bozdogan, A., Ilgun, S., Uysal, F. G., \& Ozmen, V. (2015). Bioelectrical Impedance for Detecting and Monitoring Lymphedema in Patients with Breast Cancer. Preliminary Results of the Florence Nightingale Breast Study Group. Lymphatic Research and Biology, 13(1):40-45.

Jain, M. S., Danoff, J. V., \& Paul, S. M. (2010). Correlation between bioelectrical spectroscopy and perometry in assessment of upper extremity swelling. Lymphology, 43:85-94.

Johnson, K. C., Kennedy, A. G., \& Henry, S. M. (2014). Clinical Measurements of Lymphedema. Lymphatic Research and Biology, 12(4):216-221.

Jørgensen, M. G., Toyserkani, N. M., \& Sørensen, J. (2017). The effect of prophylactic lymphovenous anstomosis and shunts for preventing cancer-related lymphedema: a systematic review and meta-analysis. Microsurgery, 1-10.

Jung, M., Jeon, J. Y., Yun, G. J., Yang, S., Kwon, S., \& Seo, Y. S. (2018). Reference values of bioelectrical impedance analysis for detecting breast cancer-related lymphedema. Medicine, 97(44):e12945 DOI: 10.1097/MD.0000000000012945.

Kang, H. (2013). The prevention and handling of the missing data. Korean Journal of Anesthesiology, 64(5):402-406.

Karakashian, K., Pike, C., \& van Loon, R. (2019). Computational investigation of the Laplace law in compression therapy. Journal of Biomechanics, 85:6-17.

Karakashian, K., Shaban, L., Pike, C., \& van Loon, R. (2018). Investigation of Shape with Patients Suffering from Unilateral Lymphoedema. Annals of Biomedical Engineering, 46(1):108-121.

Katz-Leurer, M., \& Bracha, J. (2012). Test-retest reliability of arm volume measurement in women with breast cancer-related lymphoedema. Journal of Lymphoedema, $7(2): 8-13$.

Keeley, V. (2006). The use of lymphoscintigraphy in the management of chronic oedema. Journal of Lymphology, 1(1):42-57.

Keeley, V., Crooks, S., Locke, J., Veigas, D., Riches, K., \& Hilliam, R. (2010). A quality of life measure for limb lymphoedema (LYMQOL). Journal of Lymphoedema, $5(1)$. 
Kim, S., Trinidad, B., Mikesell, L., \& Aakhus , M. (2020). Improving prognosis communication for patients facing complex medical treratment: A user-centered design approach. International Journal of Medical Informatics, 141, no pagination DOI: 10.1016/j.ijmedinf.2020.104147 .

Kim, L., Jeon, J. Y., Sung, I. Y., Jeong, S. Y., \& Do, H. J. (2011). Prediction of Treatment Outcome with Bioimpedance Measurements in Breast Cancer Related Lymphedema Patients. Annals of Rehabilitation Medicine, 35:687-693.

Kim, W., Chung, S. G., Kim, T. W., \& Seo, K. S. (2008). Measurements of the soft tissue compliance with pressure using ultrasonography. Lymphology, 41:167-77.

Kimberlin, C. L., \& Winterstein, A. G. (2008). Validity and reliability of measurement instruments used in research. American Journal of Health-System Pharmacy, 65:2276-2284.

Klernäs, P., Johnsson, A., Horstmann, V., Kristjanson, L. J., \& Johansson, K. (2015). Lymphedema Quality of Life Inventory (LyQLI) - Development and investigation of validity and reliability. Quality of Life Research, 24(2):427-439.

Knottnerus, J. A., \& Muris, J. W. (2003). Assessment of the accuracy of diagnostic tests: the cross-sectional study. Journal of Clinical Epidemiology, 56:1118-1128.

Koo, T. K., \& Li, M. Y. (2016). A Guideline if Selecting and Reporting Intraclass Correlation Coefficients for Reliability Research. Journal of Chiropractic Medicine, 15:155-163.

Kumar, R. (2005). Research Methodology second edition. London: SAGE Publications Ltd.

Laerd Statistics. (2018a). Two-way repeated measures ANOVA using SPSS Statistics. Retrieved from Laerd Statistics: https://statistics.laerd.com/spss-tutorials/twoway-repeated-measures-anova-using-spss-statistics.php\#targetText=A\%20two $\% 2$ Dway\%20repeated $\% 20$ measures,also $\% 20$ known $\% 20$ as $\% 20$ independent $\% 2$ 0variables) on 02 October 2019

Laerd Statistics. (2018b). Testing for Normality using SPSS Statistics. Retrieved from Laerd Statistics: https://statistics.laerd.com/spss-tutorials/testing-for-normalityusing-spss-statistics.php on 21 October 2019

Lahtinen, T., Seppala, J., Viren, T., \& Johansson, K. (2015). Experimental and Alanlytical Comparisons of Tissue Dielectric Constant (TDC) and Bioimpedance Spectroscopy (BIS) in Assessment of Early Arm Lymphedema in 
Breast Cancer Patients after Axillary Surgery and Radiotherapy. Lymphatic Research and Biology, 13(3):176-185.

Lam, R., Wallace, A., Burbidge, B., Franks, P., \& Moffatt, C. J. (2006). Experiences of patients with lymphoedema. Journal of Lymphoedema, 1(1):16-21.

Landau, M. J., Kim, J. S., Gould, D. J., \& Patel, K. M. (2018). Vectra 3D Imaging for Quantitative Volumetric Analysis of the Upper Limb: A Feasibility Study for Tracking Outcomes of Lymphedema Treatment. Plastic and Reconstructive Surgery, 141(1):80e-84e.

Launois, R., Mègnigbêto, A. C., Pocquet, K., \& Alliot, F. (2002). A specific quality of life scale in upper limb lymphoedema: the ULL-27 questionnaire. Lymphology, 35(Supplement):1-760:181-187.

Leddy, A. L., Crowner, B. E., \& Earhart, G. M. (2011). Functional Gait Assessment and Balance Evaluation System Test: Reliability, Validity, Sensitivity, and Specificity for Identifying Individuals With Parkinson Disease Who Fall. Physical Therapy, 91(1):102-113.

Lee, B. B., Andrade, M., Antignani, P. L., Boccardo, F., Bunke, N., Campisi, C., Damstra, R., Flour, M., Forner-Cordero, I., Gloviczki, P., Laredo, J., Partsch, H., Piller, N., Michelini, S., Mortimer, P., Rabe, E., Rockson, S., Scuderi, A., Szolnoky, G., \& Villavicencio, J. L. (2013). Diagnosis and Treatment of Primary Lymphedema Consensus Document of the International Union of Phlebology (IUP)-2013. International Angiology, 32(6):541-574.

Lee, M.-J., Boland, R. A., Czerniec, S., \& Kilbreath, S. L. (2011). Reliability and Concurrent Validity of the Perometer for Measuring Hand Volume in Women With and Without Lymphedema. Lymphatic Research and Biology, 9(1):13-18.

Levenhagen, K., Davies, C., Perdomo, M., Ryans, K., \& Gilchrist, L. (2017). Diagnosis of Upper-Quadrant Lymphedema Secondary to Cancer: Clinical Practice Guideline From the Oncology Section of APTA. Rehabilitation Oncology, 35:E1-E18.

Lu, G., DeSouza, G. N., Armer, J., Anderson, B., \& Shyu, C.-R. (2013). A System for Limb-Volume Measurement using 3D Models from an Infrared Depth Sensor. IEEE Symposium on Computational Intelligence in Healthcare and e-health (CICARE), Singapore, 64-69.

Lu, G., Han, K., DeSouza, G. N., Armer, J., \& Shyu, C.-R. (2014). A new algorithm for $3 \mathrm{D}$ registration and its application in self-monitoring and early detection of 
lymphedema. Innovation and Research in BioMedical Engineering (IRBM), $35: 370-384$.

Lu, I. M., \& Dixon, J. B. (2019). Assessment of Upper Extremity Swelling Among Breast Cancer Survivors wth a Commercial Infrared Sensor. Lymphatic Research and Biology, DOI: 10.1089/1rb.2018.0010.

Lymphoedema Framework. (2006). Best Practice for the Management of Lymphoedema. International Consensus. London: MEP Ltd.

Lymphoedema Network Wales. (2017). All Wales Lymphoedema Guidance: Measuring Volumes to Determine Lymphoedema Outcome. Neath: Lymphoedema Network Wales.

Lymphoedema Network Wales. (2020a). LNW End of Year Report 2019-2020. Swansea: Lymphoedema Network Wales.

Lymphoedema Network Wales. (2020b). Lymphoedema Treatment Outcomes. Swansea: Lymphoedema Network Wales.

Maal, T. J., van Loon, B., Plooij, J. M., Rangel, F., Ettema, A. M., Borstlap, W. A., \& Berge, S. J. (2010). Registration of 3-Dimensional Facial Photographs for Clinical Use. American Journal of Oral Maxillofacial Surgery, 68:2391-2401.

Maclellan, R. A., \& Greene, A. K. (2014). Lymphedema. Seminars in Pediatric Surgery 23, 191-197.

Makoto, M., Hayashi, Y., Hara, H., Iida, T., Narushima, M., Yamamoto, T., Todokoro, T., Murai, N., \& Koshima, I. (2014). High-accuracy Diagnosis and Regional Classification of Lymphedema Using Indocyanine Green Fluorescent Lymphography After Gynecologic Cancer Treatment. Annals of Plastic Surgery, 72: 204-208.

Mason, J. M., Thomas, K. S., Crook, A. M., Foster, K. A., Chalmers, J. R., Nunn, A. J., \& Williams, C. W. (2014). Prophylactic Antibiotics to Prevent Cellulitis of the Leg: Economic Analysis of the PATCH I \& II Trials. PLoS ONE, 9(2): e82694 DOI: 10.137/journal.pone.0082694.

Mayrovitz, H. N. (2010). Local tissue water assessed by measuring forearm skin dielectric constant: dependence on measurerment depth, age and body mass index. Skin Research and Technology, 16:16-22.

Mayrovitz, H. N., Arzanova, E., Somarriba, S., \& Eisa, S. (2018b). Reference Values for Assessing Localized Hand Lymphedema Using Interhand Tissue Dielectric Constant Ratios. Lymphatic Research and Biology, 16(5):442-445. 
Mayrovitz, H. N., Carson, S., \& Luis, M. (2010). Male-female differences in forearm skin tisue dielectric constant. Clinical Physiology and Functional Imaging, 30:328-332.

Mayrovitz, H. N., Corbitt, K., Grammenos, A., Abello, A., \& Mammino, J. (2017a). Skin indentation firmness and tissue dielectric constant assessed in face, neck, and arm skin of young healthy women. Skin Research and Technology, 23:112120.

Mayrovitz, H. N., Davey, S., \& Shapiro, E. (2008). Local tissue water assessed by tissue dielectric constant: anatomical site and depth dependence in women prior to breast cancer treatment-related surgery. Clinical Physiology and Functional Imaging, 28(5):337-342.

Mayrovitz, H. N., Davey, S., \& Shapiro, E. (2009a). Suitability of single tissue dielectric constant measurements to assess local tissue water in normal and lymphedematous skin. Clinical Physiology and Functional Imaging, 29:123127.

Mayrovitz, H. N., Fasen, M., Spagna, P., \& Wong, J. (2018a). Role of handedness on forearm skin tissue dielectric constant (TDC) in relation to detection of earlystage breast cancer-related lymphedema. Clinical Physiology and Functional Imaging, 38(4):670-675.

Mayrovitz, H. N., Grammenos, A., Corbitt, K., \& Bartos, S. (2017b). Age-related changes in male forearm skin-to-fat tissue dielectric constant at $300 \mathrm{MHz}$. Clinical Physiology and Functional Imaging, 37:198-204.

Mayrovitz, H. N., Macdonald, J., Davey, S., Olson, K., \& Washington, E. (2007). Measurement Decisions for Clinical Assessment of Limb Volume Changes in Patients With Bilateral and Unilateral Limb Edema. Physical Therapy, 87(10):1362-1368.

Mayrovitz, H. N., Weingrad, D. N., \& Davey, S. (2009b). Local Tissue Water in AtRisk and Contralateral Forearms of Women with and without Breast Cancer Treatment-Related Lymphedema. Lymphatic Research and Biology, 7(3):153158.

Mayrovitz, H. N., Weingrad, D. N., \& Lopez, L. (2015). Patterns of Temporal Changes in Tissue Dielectric Constant as Indices of Localized Skin Water Changes in Women Treated for Breast Cancer: A Pilot Study. Lymphatic Research and Biology, 13(1):20-32. 
Mayrovitz, H. N., Wiengrad, D. N., \& Lopez, L. (2016). Tissue Dielectric Contstant (TDC) as an index of skin water in women with and without breast cancer: upper limb assessment via a self-contained compact measurement device. Lymphology, 49:27-35.

McCarthy, N. (2020). The Countries With The Most Left-Handed People. Retrieved from Statista: https://www.statista.com/chart/20708/rate-of-left-handedness-inselected-countries/ on 30 June 2020

McLeod, S. (2019). What does a box plot tell you? Retrieved from Simply Psychology: https://www.simplypsychology.org/boxplots.html on 10 April 2021

Mehrara, B. J., \& Greene, A. K. (2014). Lymphedema and Obesity: Is There a Link? Plastic and Reconstructive Surgery, 134(1): 154e-160e.

Mehta, S., Bastero-Caballero, R. F., Sun, Y., Zhu, R., Murphy, D. K., Hardas, B., \& Koch, G. (2018). Performance of intraclass correlation coefficient (ICC) as a reliability index under various distributions in scale reliability studies. Statistics in Medicine, 37(18): 2734-2752.

Mihara, M., Hayashi , Y., Hara, H., Iida, T., Narushima, M., Yamamoto, T., Todokoro, T., Murai, N., \& Koshimo, I. (2014). High-accuracy Diagnosis and Regional Classification of Lymphedema Using Indocyanine Green Fluorescent Lymphography After Gynecologic Cancer Treatment. Annals of Plastic Surgery, 72(2):204-208.

Moffatt, C. J., Doherty, D. C., Franks, P. J., \& Mortimer, P. S. (2018). CommunityBased Treatment for Chronic Edema: An Effective Service Model. Lymphatic Research and Biology, 16(1):92-99.

Moffatt, C. J., Franks, P. J., Doherty, D. C., Williams, A. F., Badger, C., Feffs, E., Bosanquet, N., \& Mortimer, P. S. (2003). Lymphoedema: an underestimated health problem. QJM 96(10), 731-738.

Moffatt, C. J., Keeley, V., Franks, P. J., Rich, A., \& Pinnington, L. L. (2017). Chronic oedema: a prevalent health care problem for UK health services. International Wound Journal, 14:772-781.

Monnin-Delhom, E. D., Gallix, B. P., Achard, C., Bruel, J. M., \& Janbon, C. (2002). High resolution unenhanced computed tomography in patients with swollen legs. Lymphology, 35:121-128.

Montenij, L. J., Buhre, W. F., Jansen, J. R., Kruitwagen, C. L., \& de Waal, E. E. (2016). Methodology of method comparison studies evaluating the validity of cardiac 
output monitors: a stepwise approach and checklist. British Journal of Anaesthesia, 116(6):750-758.

Mori, T., Lustman, A., \& Katz-Leurer, M. (2015). Self-measurement of upper extermity volume in women post-breast cancer: reliability and validity study. Physiotherapy Theory and Practice, 31(4):283-287.

Mortimer, P. S., \& Levick, J. R. (2004). Chronic peripheral oedema: the critical role of the lymphatic system. Clinical Medicine, 4: 448-453.

Narushima, M., Yamamoto, T., Ogata, F., Yoshimatsu, H., Mihara, M., \& Koshima , I. (2016). Indocyanine Green Lymphography Findings in Limb Lymphoedema. Journal of Reconstructive Microsurgery, 32: 72-79.

National Institute for Clinical Excellence (NICE). (2017). L-Dex U400 for lymphoedema after breast cancer treatment (MIB111). Newcastle and York: National Institute for Health and Care Excellence.

National Institute for Health Research Clinical Research Network (NIHR CRN). (2016). Good Clinical Practice . Retrieved from nihr.ac.uk: https://www.nihr.ac.uk/ourresearch- community/documents/GCP\%20Reference\%20Guide.pdf on 26 March 2019

National Lymphoedema Partnership. (2015). Consensus Statement on the Chronic Oedema - Lymphoedema Interface. Retrieved from British Lymphology Society: https://www.thebls.com/documents-library on 18 April 2017

Ng, M., \& Munnoch, A. (2010). Clinimetrics of volume measurement in upper limb LE. Journal of Lymphoedema, 5(2):62-67.

Nuutinen, J., Ikäheimo, R., \& Lahtinen, T. (2004). Validation of a new dielectric device to assess changes of tissue water in skin and subcuaneous fat. Physiological Measurement, 25; 447-454.

Ochalek, K., Gradalski, T., \& Szygula, Z. (2015). Physical Therapy in Postmastectomy Lymphedema. Lymphatic Research and Biology, 13(1):54-58.

Öhberg, F., Zachrisson, A., \& Holmner-Rocklöv, Å. (2014). Three-Dimensional Camera System for Measuring Arm Volume in Women with Lymphedema Following Breast Cancer Treatment. Lymphatic Research and Biology, 12(4): 267-274.

Pappalardo, M., \& Cheng, M.-H. (2020). Lymphoscintigraphy for the dagnosis of extremity lymphedema: Current controversies regarding protocol, interpretation, and clinical application. Journal of Surgical Oncology, 121:37-47. 
Parahoo, K. (2014). Nursing Research: Principles, Process and Issues. (3rd ed). Basingstoke: Palgrave Macmillan.

Peleg, R., \& Katz-Leurer, M. (2017). Effect of Arm Position on Circumference Measurement of Upper Arms in Healthy and in Women With Breast CancerRelated Lymphedema. Rehabilitation Oncology, 72-80.

Perdomo, M., Davies, C., Levenhagen, K., \& Ryans, K. (2014). Breast Cancer Edge Task Force Outcomes: Assessment Measures of Secondary Lymphedema in Breast Cancer Survivors. Rehabilitation Oncology, 32(1):22-35.

Pero-System Messgeraete GmbH. (2017). How the Perometer works. Retrieved from Pero-System: http://pero-system.de/en/funktionsprinzip-von-perometern/ 02 August 2017

Pillar, N. (2007). To Measaure or Not to Measure? What and When is the Question. Journal of Lymphoedema, 2(2):39-45.

Plooij, J. M., Swennen, G. R., Rangel, F. A., Maal, T. J., Schutsyer, F. A., Bronkhorst, E. M., Kuijpers-Jagtman, A M., \& Berge, S. J. (2009). Evaluation of reproducibility and reliability of 3D soft tissue analysis using 3D stereophotogrammetry. International Journal of Oral Maxillofacial Surgery, 38:267-273.

Porter, M. E., Pabo, E. A., \& Lee, T. H. (2013). Redesigning Primary Care: A Strategic Vision to Improve Value by Organizing Around Patients' Needs. Health Affairs, 32(3):516-525.

Preferred Reporting Items for Systematic Reviews and Meta-Analyses (PRISMA). (2018). Prisma Statement Home Page. Retrieved from www.prismastatement.org: http://www.prisma-statement.org/ on 25 February 2018

Preuß, M., Killaars, R., de Grzymala, A. P., Binnebösel, M., \& Neumann, U. (2018). Validity and Reliability of Three-Dimensional Imaging for Measuring Breast Cancer-Related Lymphedema in the Upper Limb: A Cross-Sectional Study. Lymphatic Research and Biology, 16(6):525-532.

Pusic, A. L., Cernal, Y., Albornoz, C., Klassen, A., Cano, S., Sulimanoff, I., Hernandez, M., Massey, M., Cordeiro, P., Morrow, M., \& Mehrara, B. (2013). Quality of life among breast cancer patients with lymphedema: a systematic review of patient-reported outcome instruments and outcomes. Journal of Cancer Survivorship, 7(1):83-92. 
Rafn, B. S., McNeely, M. L., Camp, P. G., Midtgaard, J., \& Campbell, K. L. (2019). Self-Measured Arm Circumference in Women With Breast Cancer Is Reliable and Valid. Physical Therapy, 99(2):240-253.

Ridner, S. H., Dietrich, M. S., Deng, J., Bonner, C. M., \& Kidd, N. (2009). Bioelectrical Impedance for Detecting Upper Limb Lymphedema in Nonlaboratory Settings. Lymphatic Research and Biology, 7(1):11-15.

Ridner, S. H., Shih, Y.-C. T., Doersam, J. K., Rhoten, B. A., Schultze, B. S., \& Dietrich, M. S. (2014). A Pilot Randomized Trial Evaluating Lymphedema SelfMeasurement with Bioelectrical Impedance, Self-Care Adherence, and Health Outcomes. Lymphatic Research and Biology, 12(4):258-266.

Rincon, K., Shah, P., Ramella-Roman, J., \& Bhansali, S. (2016). A Review of Engineering Approaches for Lymphedema Detection. IEEE Reviews in Biomedical Engineering, 9:79-90.

Rockson, S. G. (2018). Lymphedema after Breast Cancer Treatment. The New England Journal of Medicine, 379:1937-1944.

Rutjes, A. W., Reitsma, J. B., Coomarasamy, A., Khan, K. S., \& Bossuyt, P. M. (2007). Evaluation of diagnostic tests when there is no gold standard. A review of methods. Health Technology Assessment, 11(50):iii, ix-51.

Sadeghi, R., Kazemzadeh, G., \& Keshtgar, M. (2010). Diagnostic application of lymphoscintigraphy in the management of lymphoedema. Hellenic Journal of Nuclear Medicine, 13(1):6-10.

SAGE. (2019). SAGE Research Methods: Sensitivity; Specificity. Retrieved from SAGE Research Methods: http://methods.sagepub.com/reference/encyc-of-researchdesign/.xml on 29 October 2019

Sagen, Å., Kårensen, R., Skaane, P., \& Risberg, M. A. (2009). Validity for the Simplified Water Displacement Instrument to Meassure Arm Lymphedemaas a Result of Breast Cancer Surgery. Archives of Physical Medicine and Rehabilitation, 90:803-809.

Salkind, N. J. (2011). Statistics for People Who (Think They) Hate Statistics: 4th edition. London: SAGE Publications Ltd.

Schaaf, H. (2009). Evolution of photography in maxillofacial surgery: from analog to 3D photography - an overview. Clinical, Cosmetic and Investigational Dentistry, $1: 39-45$. 
Seward, C., Skolny, M., Brunelle, C., Asdourian, M., Salama, L., \& Taghian, A. G. (2016). A Comprehnsive Review of Bioimpedanc Spectroscopy as a Diagnostic Tool for the Detection and Measurement of Breast Cancer-Related Lymphedema. Journal of Surgical Oncology, 114:537-542.

Shah, C., Vicini, F. A., \& Arthur, D. (2016). Bioimpedance Spectroscopy for Breast Cancer Related Lymphedema Assessment: Clinical Practice Guidelines. The Breast Journal, 22(6):645-650.

Shaitelman, S. F., Cromwell, K. D., Rasmussen, J. C., Stout, N. L., Armer, J. M., Lasinski, B. B., \& Cormier, J. N. (2014). Recent Progress in the Treatment ad Prevention of Cancer-Related Lymphedema. CA: A Cancer Journal for Clinicians, 65:55-81.

Shamseer, L., Moher, D., Clarke, M., Ghersi, D., Liberati, A., Petticrew, M., Shekelle, P., \& Stewart, L. A. (2015). Preferred reporting items for systematic review and meta-analysis protocols (PRISMA-P). British Medical Journal, 349:g7647. DOI:10.1136/bmj.g7647.

Sharkey, A. R., King, S. W., Kuo, R. Y., Bickerton, S. B., Ramsden, A. J., \& Furniss, D. (2018). Measuring Limb Volume: Accuracy and Reliability of Tape Measurement Versus Perometer Measurement. Lymphatic Research and Biology, 16(2):182-186.

Shih, H. B., Shakir, A., \& Nguyen, D. H. (2016). Use of indocyanine green-SPY angiography for tracking lymphatic recovery after lymphaticovenous anastomosis. Annals of Plastic Surgery, 76(3):S232-237 DOI: 10.1097/SAP.0000000000000766.

Shinaoka, A., Koshimune, S., Yamada, K., Matsumoto, K., Honda, M., Miyake, M., Furuichi, H., Hongo, A., \& Kimata, Y. (2017). Accelerated Lymph Flow in Early-Stage Secondary Lymphedema Detected by Indocyanine Green Fluorescence Lymphography. Journal of Reconstructive Microsurgery, 33(8):596-602.

Shrout, P. E., \& Fleiss, J. L. (1979). Intraclass correlations: uses in assessing rater reliability. Psychological Bulletin Journal, 86(2):420-428.

Sitzia, J. (1995). Volume measurement in lymphoedema treatment: examination of formulae. European Journal of Cancer Care, 4:11-16. 
Smoot, B. J., Wong, J. F., \& Dodd, M. J. (2011). Comparison of Diagnostic Accuracy of Clinical Measures of Breast Cancer-Related Lymphedema: Area Under the Curve. Archives of Physical Medicine and Rehabilitation, 92(4):603-610.

Smoot, B., Paul, S. M., Aouizerat, B. E., Elboim, C., Levine, J. D., Abrams, G., Hamolsky, D., Neuhaus, J., Schmidt, B., West, C., Topp, K., \& Miaskowski, C. (2014). Side of Cancer Does Not Influence Limb Volumes in Women Prior to Breast Cancer Surgery. Lymphatic Research and Biology, 12(3):189-193.

Spinelli, B., Kallan, M. J., Zhang, X., Cheville, A., Troxel, A., Cohn, J., Dean, L., Sturgeon, K., Evangelista, M., Zhang, Z., Ebaugh, D., \& Schmitz, K. H. (2019). Intra- and Interrater Reliability and Concurrent Validity of a New Tool for Assessment of Breast Cancer-Related Lymphedema of the Upper Extremity. Archives of Physical Medicine and Rehabilitation, 100:315-326.

Stanton, A. W., Badger, C., \& Sitzia, J. (2000). Non-invasive assessment of the lymphoedematous limb. Lymphology, 33:122-135.

Stanton, A. W., Modi, S., Bennet Britton, T. M., Purushotham, A. D., Peters, A. M., Levick, J. R., \& Mortimer, P. S. (2009). Lymphatic drainage in the muscle and subcutis of the arm after breast cancer treatment. Breast Cancer Research \& Treament, 117(3):549-557.

Stanton, A. W., Northfield, J. W., Holroyd, B., Mortimer, P., \& Levick, J. (1997). Validation of an Optoelectronic Limb Volumeter (Perometer). Lymphology, 30; 77-97.

Statistics Solutions. (2021). Missing Values in Data. Retrieved from Statistics Solutions: https://www.statisticssolutions.com/missing-values-in-data/ on 10 April 2021

Sun, F., Hall, A., Tighe, M. P., Brunelle, C. L., Sayegh, H. E., Gillespie, T. C., Daniell, K. M., \& Taghian, A. G. (2018). Perometry versus simulated circumferential tape measurement for the detection of breast cancer-related lymphedema. Breast Cancer Research and Treatment, 172:83-91.

Svensson, B. J., Dylke, E. S., Ward, L. C., \& Kilbreath, S. L. (2015). Segmental Impedance Thresholds for Early Detection of Unilateral Upper Limb Swelling. Lymphatic Research and Biology, 13(4):253-259.

Svensson, B. J., Ward, L. C., Dylke, E. S., \& Kilbreath, S. L. (2017). Segmental Bioimpedance Informs Diagnosis of Breast Cancer-Related Lymphedema. Lymphatic Research and Biology, 15(4):349-355. 
Tashiro, K., Yamashita, S., Saito, T., Iida, T., \& Koshima, I. (2015). Proximal and distal patterns: Different spreading patterns of indocyanine green lymphography in secondary lower extremity lymphedema. Journal of Plastic, Reconstructive \& Aesthetic Surgery, 69:368-375.

Taylor, R., Jayasinghe, U. W., Koelmeyer, L., Ung, O., \& Boyages, J. (2006). Reliability and validity of arm volume measurements for assessment of lymphedema. Physical Therapy, 86(2):205-214.

Tewari, N., Gill, P. G., Bochner, M. A., \& Kollias, J. (2008). Comparison of volume displacement versus circumferential arm measurements for lymphoedema: implications for the SNAC trial. ANZ Journal of Surgery, 78:889-893.

Thomas, R., \& Hamilton , R. (2014). Illustrating the (in)visible: understanding the impact of loss in adults living with secondary lymphedema after cancer. International Journal of Qualitative Studies on Health \& Wellbeing, 9. doi:http://dx.doi.org/10.3402/qhw.v9.24354.

Thomas, M., \& Morgan, K. (2017). The Chronic Oedema 'Wet Leg' (Lymphorrhoea) Pathway. Cimla: Lymphoedema Network Wales.

Thomas, S. (2017). The use of compressions wraps in the management of lymphoedema. Journal of Lymphoedema, 12(1):32-38.

Tidhar, D., Armer, J. M., Deutscher, D., Shyu, C.-R., Azuri, J., \& Madsen, R. (2015). Measurement Issues in Anthropometric Measures of Limb Volume Change in Persons at Risk for and Living with Lymphedema: A Reliability Study. Journal of Personilzed Medicine, 5:341-353.

Timmer, C. Y., Bosman, J., Geertzen, J. H., \& Dijkstra, P. U. (2020). Variation in Measurement Results Using Bioimpedance Spectroscopy to Determine Extracellular Fluid of Upper Extremity. Lymphatic Research and Biology, 18(2):110-115.

Trochim, W. M. (2020). Convergent \& Discriminant Validity. Retrieved from Conjoint.ly: https://conjointly.com/kb/convergent-and-discriminant-validity/ on 07 April 2021

Tsai, R. J., Dennis, L. K., Lynch, C. F., Snetselaar, L. G., Zamba, G., \& Scott-Conner, C. (2018). Lymphedema following breast cancer: The importance of surgical methods and obesity. Frontiers in women's health, 3(2), 10.15761/FWH.1000144. DOI: 10.15761/FWH.1000144. 
UCLA Institute for Digital Research and Education. (2019). Introduction to Power of Analysis. Retrieved from UCLA Institute for Digital Research and Education: https://stats.idre.ucla.edu/other/mult-pkg/seminars/intro-power/ on 02 October 2019

Unno, N., Inuzuka, K., Suzuki, M., Yamamoto, N., Sagara, D., Nishiyama, M., \& Konno, H. (2007). Preliminary experience with a novel fluorescence lymphography using indocyanine green in patients with secondary lymphedema. Journal of Vascular Surgery, 45(5):1016-1021.

Van De Water, L. F., Van Kleef, J. J., Henselman, I., Van Den Boorn, H. G., Vaarzon Morel , N. M., Schut, K. F., Daams, J. G., Smets, E. M. A., \& Van Laarhoven, H. W. (2018). Communicating treatment risks and benefits to cancer patients: A systematic review of different verbal and visual communication methods. Quality of Life Research, Supplement 1:S56.

van der Meer, W. J., Dijkstra, P. U., Visser, A., Vissinl, A., \& Ren, Y. (2014). Reliability and validity of measurement of facial swelling with a stereophotogrammetry optical three-dimensional scanner. British journal of Oran and Maxillofacaial Surgery, 52:922-927.

Verhulst, A. C., Wesselius, T. S., Glas, H. H., Vreeken, R. D., Ulrich, D. J., \& Maal, T. J. (2017). Accuracy and reproducibility of a newly developed tool for volume measurements of the arm using 3D stereophotogrammetry. Journal of Plastic, Reconstructive \& Aesthetic Surgery, 70:1753-1759.

Vicini, F., Shah, C., Lyden, M., \& Whitworth, P. (2012). Bioelectrical Impedance for Detecting and Monitoring Patients for the Development of Upper Limb Lymphedema in the Clinic. Clinical Breast Cancer, 12(2)133-137.

Wang, H., Shen, L., Liu, T., Shao, P., Dylke, E. S., Jia, J., \& Kilbreath, S. L. (2017). Circumference-Based Criteria for Detection of Secondary Arm Lymphedema for Chinese Women. Lymphatic Research and Biology, 15(3):262-267.

Ward, L. (2009). Is BIS ready for prime time as the gold standard measure? Journal of Lymphoedema, 4(2):52-56.

Ward, L. C., Buncet, I. H., Cornish, B. H., Mirolo, B. R., Thomas, B. J., \& Jones, L. C. (1992). Multi-frequency bioelectrical impedance augments the diagnosis and management of lymphoedema in post-mastectomy patients. European Journal of Clinical Investigation, 22:751-754. 
Ward, L. C., Czerniec, S., \& Kilbreath, S. L. (2009b). Operational Equivalence of Bioimpedance Indices and Perometry for the Assessment of Unilateral Arm Lymphedema. Lymphatic Research and Biology, 7(2):81-85.

Ward, L. C., Czerniec, S., \& Kilbreath, S. L. (2009a). Quantitative bioimpedance spectroscopy for the assessment of lymphoedema. Breast Cancer Research and Treatment, 117:541-547.

Ward, L. C., Dylke, E., Czerniec, S., Isenring, E., \& Kilbreath, S. L. (2011). Confirmation of the Reference Impedance Ratios Used for Assessment of Breast Cancer-Related Lymphedema by Bioelectrical Impedance Spectroscopy. Lymphatic Research and Biology, 9(1):47-51.

Watson, D. J., Karakashian, K., Pike, C. E., \& van Loon, R. (2019). Limb shape: a novel metric for lymphoedema.

Welsh Assembly Government. (2009). Strategy for Lymphoedema in Wales: Designed for Lymphoedema. Retrieved from Welsh Government: http://gov.wales/topics/ health/publications/health/strategies/lymphoedema/?lang=en on 18 April 2018

Williams, A. F., Moffatt, C. J., \& Franks, P. J. (2004). A phenomenological study of the lived experiences of people with lymphoedema. International Journal of Palliative Nursing, 10(6):279-286.

World Health Organization. (2021). Body mass index - BMI. Retrieved from World Health Organization: Regional Office for Europe: https://www.euro.who.int/ en/health-topics/disease-prevention/nutrition/a-healthy-lifestyle/body-massindex-bmi accessed on 08 April 2021

Xiong, L., Engel, H., Gazyakan, E., Rahimi, M., Hünerbein, M., Sun, J., Kneser, U., \& Hirche, C. (2014). Current techniques for lymphatic imaging: State of the art and future perspectives. European Journal of Surgical Oncology, 40:270-276.

Yamagishi, K. (1997). When a $12.86 \%$ mortality is more dangerous than $24.14 \%$ : implications for risk communication. Applied Cognitive Psychology, 11:495506.

Yamamoto, N., Yamamoto, T., Hayashi, N., Hayashi, A., Iida, T., \& Koshima, I. (2016). Arm Volumetry Versus Upper Extremity Lymphedema Index: Validity of Upper Extremity Lymphedema Index for Body-Type Corrected Arm Volume Evaluation. Annals of Plastic Surgery, 76(6):697-699.

Yamamoto, T., Matsuda, N., Doi, K., Oshima, A., Yoshimatsu, H., Todokoro, T., Ogata, F., Mihara, M., Narushima, M., Iida, T., \& Koshima, I. (2011b). The earliest 
finding of indocyanine green lymphography in asymptomatic limbs of lower extremity lymphedema patients secondary to cancer treatment: The modified dermal backflow stage and concept of subclinical lymphedema. Plastic and Recosntructive Surgery, 128:314-321.

Yamamoto, T., Yamamoto, N., \& Yoshimatsu, H. (2017). Localized Arm Volume Index: A New Method for Body Typre-Corrected Evaluation of Localized arm Lymphedematous Volume Change. Annals of Plastic Surgery, 79(4):390-392.

Yamamoto, T., Yamamoto, N., Doi, K., Oshima, A., Yoshimatsu, H., Todokoro, T., Ogata, F., Mihara, M., Narushima, M., Iida, T., \& Koshima, I. (2011a). Indocyanine Green-Enhanced Lymphography for Upper Extemity Lymphedema: A Novel Severity Staging System Using Dermal Backflow Patterns. Plastic \& Reconstructive Surgery, 128(4): 941-947.

Yanbaeva, D., Dentener, M., Creutzberg, E., Wesseling, G., \& Wouters, E. (2007). Systemic Effects of Smoking. CHEST, 131(5):1557-1566.

Yoo, J. S., Chung, S. H., Lim, M. C., Kim, Y. J., Kim, K. G., Hwang, J. H., \& Kim, Y. (2017). Computed tomography-based quantitative assessment of the lower extremity lymphedema following treatment for gynecologic cancer. Journal of Gynecologic Oncology, 28(2):e18-e28.

York, S. L., Ward, L. C., Czerniec, S., Lee, M. J., Refshauge, K. M., \& Killbreath, S. L. (2009). Single frequency versus bioimpedance spectroscopy for the assessment of lymphedema. Breast Cancer Research and Treatment, 117:117 doi.org/10.1007/s10549-008-0090-6. 
Appendices 
APPENDIX 1: Lymphoedema Staging

\begin{tabular}{|c|c|c|}
\hline \multicolumn{3}{|c|}{ Lymphoedema Staging Systems } \\
\hline $\begin{array}{l}\text { Clinical } \\
\text { Stage }\end{array}$ & $\begin{array}{l}\text { International Society of } \\
\text { Lymphology (ISL, 2013) }\end{array}$ & $\begin{array}{c}\text { British Lymphology Society } \\
\text { (British Lymphology Society, 2016) }\end{array}$ \\
\hline $\mathbf{0}$ & $\begin{array}{l}\text { Latent or subclinical state; } \\
\text { swelling not yet evident } \\
\text { despite impaired lymph } \\
\text { transport. May exist months } \\
\text { or years before oedema } \\
\text { becomes evident. }\end{array}$ & $\begin{array}{l}\text { May be primary or secondary. No swelling } \\
\text { evident despite impaired lymph transport. } \\
\text { May exist for months or years before } \\
\text { oedema becomes evident. }\end{array}$ \\
\hline $\mathbf{I}$ & $\begin{array}{l}\text { Early accumulation of fluid } \\
\text { which subsides with limb } \\
\text { elevation. Pitting may occur. }\end{array}$ & $\begin{array}{l}\text { Swelling present less than } 3 \text { months; subtle } \\
\text { changes in volume; self-reported } \\
\text { symptoms of heaviness, aching, clothing or } \\
\text { jewellery feeling tighter; swelling relieved } \\
\text { by bed rest or elevation. May have } \\
\text { infection (cellulitis) but no secondary skin } \\
\text { changes. }\end{array}$ \\
\hline II & $\begin{array}{l}\text { Limb elevation rarely } \\
\text { reduces tissue swelling, and } \\
\text { pitting is manifest. } \\
\text { Late Stage II the limb may or } \\
\text { may not pit as excess fat and } \\
\text { fibrosis appear. }\end{array}$ & $\begin{array}{l}\text { Swelling affecting a limb(s); present more } \\
\text { than } 3 \text { months; not relieved by bed rest or } \\
\text { elevation. Subcutaneous tissues are soft } \\
\text { and normal shape of the limb preserved. } \\
\text { Swelling does not affect the head and neck, } \\
\text { trunk or genital regions of the body }\end{array}$ \\
\hline III & $\begin{array}{l}\text { Encompasses lymphostatis } \\
\text { elephantiasis where pitting } \\
\text { can be absent and trophic } \\
\text { skin changes such as } \\
\text { thickening, increased skin } \\
\text { folds, hyperpigmentation, fat } \\
\text { deposits and papillomatosis } \\
\text { develop }\end{array}$ & $\begin{array}{l}\text { 3a) One or more of the following } \\
\text { secondary skin changes are present - } \\
\text { fibrosis, hyperkeratosis, papillomatosis, } \\
\text { recurrent cellulitis/ fungal infections or } \\
\text { lymphorrhea. Shape of the limb is } \\
\text { significantly distorted with skin folds; limb } \\
\text { is heavily impacting on activities of daily } \\
\text { life; may have psychosocial consequences } \\
\text { 3b) Swelling is extensive and affects more } \\
\text { than one site of the body with volume. } \\
\text { Swelling may affect the limbs, head \& } \\
\text { neck, trunk or genitals. Secondary skin } \\
\text { changes present. Distorted shape, skin } \\
\text { folds and heaviness affecting 'normal' } \\
\text { function. Activities of daily life and } \\
\text { psychosocial health are severely affected } \\
\text { 3c) Head and neck intervention/ Genital } \\
\text { oedema such as associated with anogenital } \\
\text { granulomatosis }\end{array}$ \\
\hline
\end{tabular}




\begin{tabular}{|c|c|}
\hline \multicolumn{2}{|c|}{$\begin{array}{l}\text { Oedema at the end of life and in advanced cancer (British Lymphology Society, } \\
\text { 2016) }\end{array}$} \\
\hline Classification & $\begin{array}{l}\text { Description of end of life and advanced cancer oedema/ } \\
\text { lymphoedema }\end{array}$ \\
\hline At-risk & $\begin{array}{l}\text { Risk factors: Previous cancer treatment; active or advanced disease } \\
\text { in the abdomen, groin, chest/breast or head and neck regions; active } \\
\text { or previous thrombosis; reduced mobility; abnormal liver, renal or } \\
\text { thyroid function; reduced function or paralysis of a limb(s); } \\
\text { cellulitis or recurrent infection. No swelling evident but at higher } \\
\text { risk of developing swelling }\end{array}$ \\
\hline Mild oedema & $\begin{array}{l}\text { Swelling may be transient. Affects the limb(s) only and is pitting. } \\
\text { May reduce with bed rest and elevation. Subcutaneous tissues are } \\
\text { soft, with no evidence of fibrosis. The skin is intact; however, there } \\
\text { is a risk of cellulitis and/ or lymphorrhoea through accidental } \\
\text { trauma. Swelling may be due to thrombosis and should be } \\
\text { considered as part of examination }\end{array}$ \\
\hline $\begin{array}{l}\text { Moderate } \\
\text { oedema }\end{array}$ & $\begin{array}{l}\text { May affect the trunk, genitals, head and neck or limb(s) causing } \\
\text { discomfort. Is not relieved by bed rest, elevation or light } \\
\text { compression. There is evidence of secondary skin changes such as } \\
\text { fibrosis, hyperkeratosis and papillomatosis. May have been present } \\
\text { before exacerbation but is more pronounced with advanced disease } \\
\text { or deterioration of condition. Function of the affected limb/region } \\
\text { of the body is affected. Lymphorrhoea may be present, and there is } \\
\text { a high risk of cellulitis and further maceration of the skin }\end{array}$ \\
\hline Severe oedema & $\begin{array}{l}\text { Severe obstruction of the lymphatic and/or vascular pathways } \\
\text { through advanced cancer rendering limbs heavy and severely } \\
\text { impacting function (i.e. massive disease in the axillary lymph nodes } \\
\text { which affects the brachial plexus or a fungating tumour) } \\
\text { and/or } \\
\text { Severe obstruction of the lymphatic pathways of the head and neck } \\
\text { affecting swallow, airway or vision requiring medical intervention } \\
\text { (pharmacological, surgical, specialist assessment) } \\
\text { and/or } \\
\text { Pre-existing lymphoedema considerably worsened. Extensive and } \\
\text { widespread swelling of the affected areas causing shape distortion } \\
\text { and secondary skin changes including skin folds, fibrosis and } \\
\text { pigmentation changes } \\
\text { Extensive generalised oedema affecting more than half of the body, } \\
\text { which may be due to multiple factors such as immobility, advanced } \\
\text { disease and hypoproteinaemia. Typically this oedema is present in } \\
\text { the final days of life and can cause considerable psychological and } \\
\text { physical distress. }\end{array}$ \\
\hline
\end{tabular}




\begin{tabular}{|c|c|c|}
\hline Arm Dermal Backflow Sta & ging (Yamamoto et al., 2011 & \\
\hline ADB stage 0 & ADB stage I & ADB stage II \\
\hline DB pattern (-) & Splash (+) & Stardust (+) \\
\hline $\begin{array}{l}\text { Stage } 0: \text { no oedema or } \\
\text { dermal backflow detected }\end{array}$ & $\begin{array}{l}\text { Stage I: splash pattern } \\
\text { observed around the axilla }\end{array}$ & $\begin{array}{l}\text { Stage II: stardust pattern is } \\
\text { limited to proximal to the } \\
\text { olecranon }\end{array}$ \\
\hline ADB stage III & ADB stage IV & ADB stage $V$ \\
\hline Stardust $(++)$ & Stardust $(++)$ & Diffuse $(+)$ \\
\hline $\begin{array}{l}\text { Stage III: stardust pattern } \\
\text { extends distal to the } \\
\text { olecranon }\end{array}$ & $\begin{array}{l}\text { Stage IV: stardust pattern } \\
\text { is seen throughout the } \\
\text { entire limb, including the } \\
\text { hand }\end{array}$ & $\begin{array}{l}\text { Stage } V \text { : diffuse pattern } \\
\text { evident with the presence } \\
\text { of stardust pattern }\end{array}$ \\
\hline
\end{tabular}

${ }^{6}$ Yamamoto et al., Indocyanine Green-Enhanced Lymphography for Upper Extremity Lymphedema: A Novel Severity Staging System Using Dermal Backflow Patterns, Plastic and Reconstructive Surgery, volume 128, issue 4, pages 941-947, Copyright (2011a), https://journals.lww.com/plasreconsurg/fulltext/ 2011/10000/Indocyanine_Green_Enhanced_Lymphography_for_Upper.26.aspx 


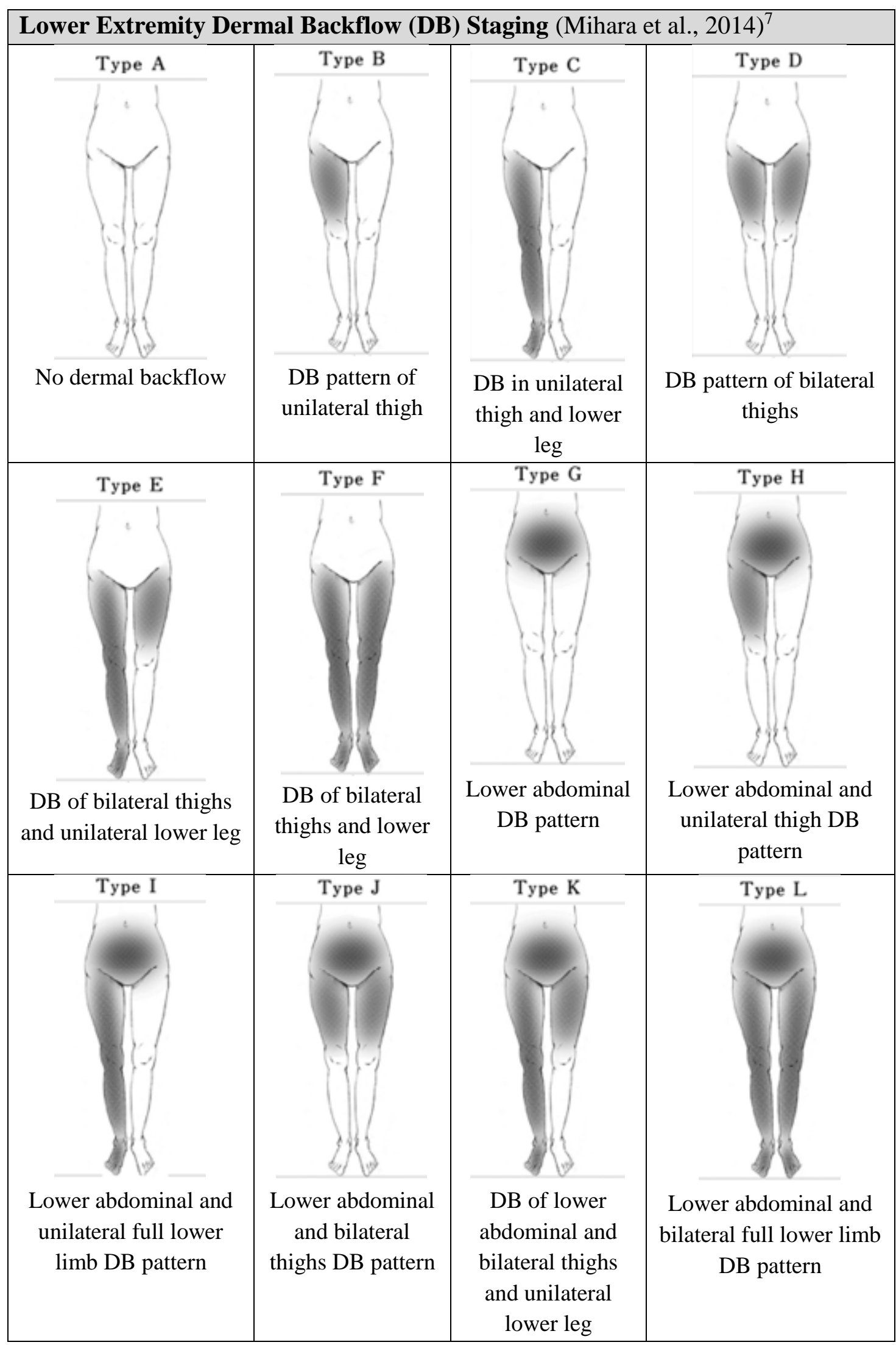

${ }^{7}$ Mihara et al., High-accuracy Diagnosis and Regional Classification of Lymphedema Using Indocyanine Green Fluorescent Lymphography After Gynecologic Cancer Treatment, Annals of Plastic Surgery, volume 72, issue 2, pages 204-208, Copyright (2014), https://journals.lww.com/annalsplasticsurgery/ fulltext/2014/02000/High_accuracy_Diagnosis_and_Regional.19.aspx 


\section{APPENDIX 2: Types of Lymphoedema Medical Imaging}

\section{Lymphangiography and Lymphography}

Lymphangiography is one of the only direct imaging techniques whereby an iodinated contrast agent is directly injected into an isolated lymphatic channel. The dye lasts for months to years in the body, enabling long-term follow-up. This technique is rarely performed due to it being invasive, technically challenging to cannulate, and some lifethreatening complications such as contrast-induced nephropathy having been reported (Xiong et al., 2014).

Lymphography (Figure 5) refers to a contrast agent or dye that is injected interstitially and drains to the lymphatic vessels (Xiong et al., 2014). It is considered diagnostically more accurate than Computed Tomography (CT) scan as it is capable of demonstrating the internal architectural anomalies within normal-sized lymph nodes, and due to it opacifying the lymphatic channels, it can detect lymphatic fistulas and lymphatic leakage (Guermazi, Brice, Hennequin, \& Sarfati, 2003).

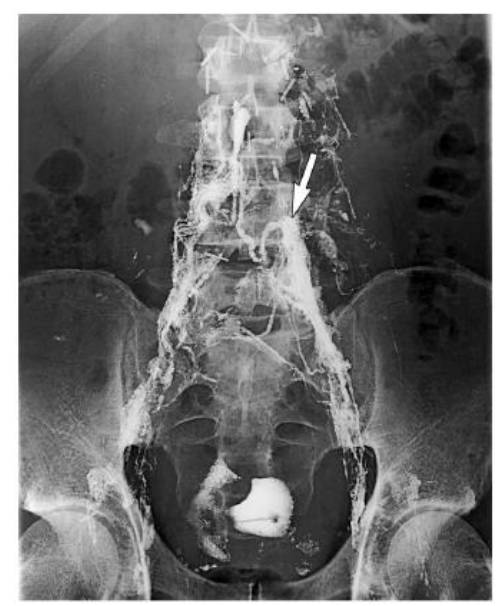

Lymphogram (Guermazi et al., 2003)

The dye and contrast materials used with these techniques cause the main complications as a result of lymphography rather than the technique used (Guermazi et al., 2003). The most common complication is a pulmonary oil embolism which generally remains asymptomatic. Other complications include hypersensitivity to the dye or oil, intraalveolar haemorrhage with complete reversibility, hypothyroidism and systemic arterial

${ }^{8}$ Guermazi, Brice, Hennequin, \& Sarfati, RadioGraphics, 2003, volume 23, pages 1541-1560, with permission from the Radiological Society of North America (RSNA ${ }^{\circledR}$ ) 
embolisation to the brain or kidney (rare) (Guermazi et al., 2003). Due to these complications, lymphography is reserved for complex conditions such as chylous reflux syndrome and thoracic duct injury, which are difficult to diagnose, and will require medical or surgical intervention (International Society of Lymphology [ISL], 2016).

\section{Lymphoscintigraphy}

Lymphoscintigraphy (Figure 6) was introduced in the 1950s (Sadeghi, Kazemzadeh, \& Keshtgar, 2010; Shinaoka et al., 2017) and remains the 'gold-standard' for imaging and evaluating lymphatic function (Keeley, 2006; Pillar, 2007; Hvidsten, Toyserkani, Sørensen, Høilund-Carlsen, \& Simonsen, 2018; Pappalardo \& Cheng, 2020). According to Sadeghi, Kazemzadeh, \& Keshtgar (2010) and Pappalardo \& Cheng (2020), it is a non-invasive procedure, which is in contradiction to Chiewvit \& Kumnerdnakta (2017) who correctly acknowledge it is an invasive procedure as a dye is injected into the foot or hand. A radiolabelled colloid is injected into the interstitial space at the first web space of each foot or hand using a radiopaque contrast dye. An external gamma camera tracks it as it travels up the lymphatics (Keeley, 2006; Lymphoedema Framework, 2006; Maclellan \& Greene, 2014; Pappalardo \& Cheng, 2020). It displays the deep pathways of lymph flow and lymph nodes in a quantitative and timed manner (Hvidsten et al., 2018).

Lymphoscintigraphy has mostly replaced the conventional oil contrast lymphography for visualising the lymphatic network (Chiewvit \& Kumnerdnakta, 2017). Although lymphoscintigraphy has not been strictly standardised, the images are easily repeatable and afford a good insight into lymphatic structural abnormalities and (dys)function (ISL, 2016; Pappalardo \& Cheng, 2020).

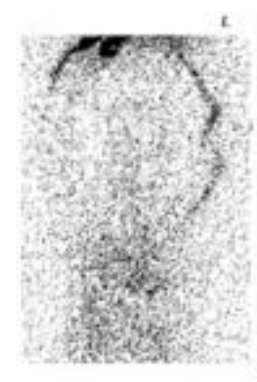

$5 \mathrm{~min}$

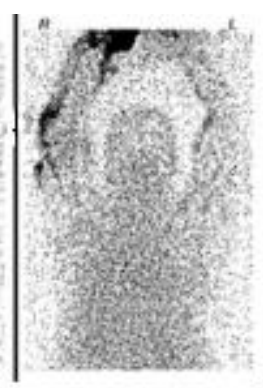

$15 \mathrm{~min}$

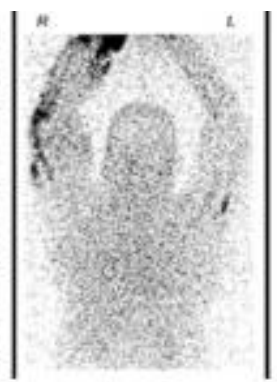

$30 \mathrm{~min}$

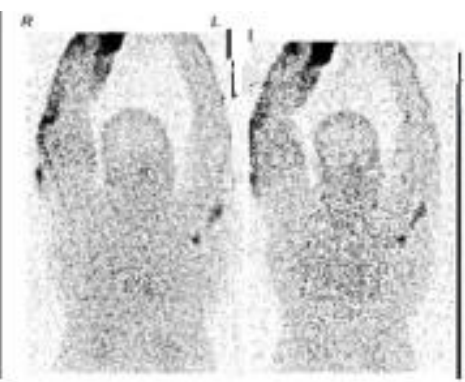

$45 \mathrm{~min}$

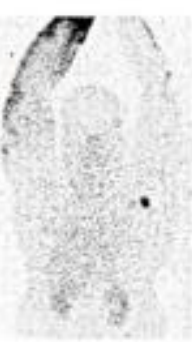

4 hour

Lymphoscintigraphy of the upper limbs (Chiewvit \& Kumnerdnakta, 2017) 
Lymphoedema is diagnosed by asymmetric visualisation of and/or delayed transit time to the regional lymph nodes, dermal backflow, the formation of collateral lymphatic channels and a reduced number of regional nodes (Sadeghi et al., 2010; Chiewvit \& Kumnerdnakta, 2017). However, Shaitelman et al. (2014) identify limiting factors for its use including a relatively poor spatial resolution that limits visualisation of small lymphatic vessels, and a long integration time that precludes direct imaging of contractile lymphatic pumping. It is a rarely performed diagnostic procedure due to the complexity of the procedure, the potential associated complications and the reliance on an experienced radiographer who is practised in reading the images to provide the clinician with useful data on which to construct a treatment plan (Maclellan \& Greene, 2014).

\section{Indocyanine Green Lymphography}

The newest method for imaging lymphatics is through Indocyanine Green (ICG) Lymphography (Narushima et al., 2016) or near-infrared fluorescence imaging (Groenlund, Telinius, Skov, \& Hjortdal, 2017). It differs from lymphoscintigraphy in that it images the superficial lymphatics up to a depth of approximately two centimetres, it visualises the lymphatic activity in real-time, and the results of the scan can be given immediately (ISL, 2016; Groenlund et al., 2017). Makoto et al. (2014) state that the use of ICG lymphography provides high-sensitivity detection of lymphoedema and is capable of evaluating the pathologic condition over a wide area. Groenlund, Telinius, Skov, \& Hjortdal (2017) validated the use of ICG lymphography stating that it has the sensitivity to detect changes in lymphatic activity by local hyperthermia and exercise, and that pumping pressures demonstrated good repeatability. Shinaoka et al. (2017) found that lymph flow and lymphatic function could be evaluated and that accelerated lymph flow in early-stage secondary lymphoedema is detectable.

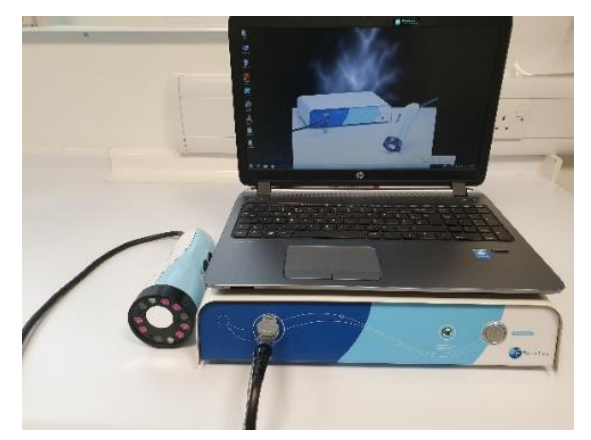

Fluobeam ${ }^{\circledR}$ Near-Infrared Camera 
ICG was primarily used in angiography until Unno et al. (2007) introduced ICG lymphography. It is a minimally invasive procedure whereby $0.2 \mathrm{ml}$ of dilute concentration ICG dye $(5 \mathrm{mg} / \mathrm{ml})$ is injected into, or near to, the webspace of both feet or hands (Narushima et al., 2016) and is tracked using a near-infrared camera such as the Fluobeam ${ }^{\circledR}$ (Figure 7). The dye binds to protein molecules in the lymph fluid and emits energy in the near-infrared region between 750 and $810 \mathrm{~nm}$ (Chang, Soami, \& Skoracki, 2013). Once this fluid enters the lymphatic vessels, ICG lymphography can detect this near-infrared light and reveals the pathway of the lymphatic vessels, rate of flow and function of the superficial lymphatics via software onto a computer screen. The advantage of using ICG lymphography over lymphoscintigraphy is that the images are of higher resolution, and there is no radiation exposure (Shinaoka et al., 2017).

ICG lymphography can be administered by a medic or qualified Health Care Professional within a legal framework known as a Patient Group Directive. Image patterns (Figure 8) are classified as linear, splash, stardust or diffuse (Yamamoto et al., 2011b; Hara et al., 2013; Makoto et al., 2014) and can be interpreted to inform lymphoedema progression, conservative treatment and surgical options. ICG lymphography is also being used to assess the patency of performed lymphaticvenous anastomoses (Shih, Shakir, \& Nguyen, 2016; Jørgensen, Toyserkani, \& Sørensen, 2017) and early onset of lymphoedema (Yamamoto et al., 2011a; Jørgensen et al., 2017).

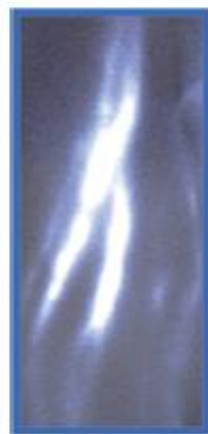

Linear

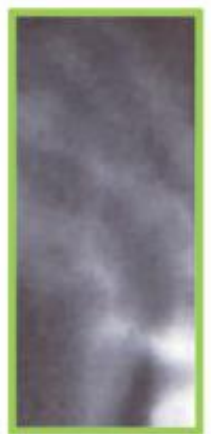

Splash

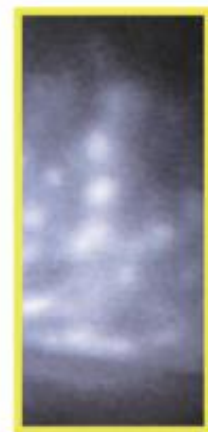

Stardust

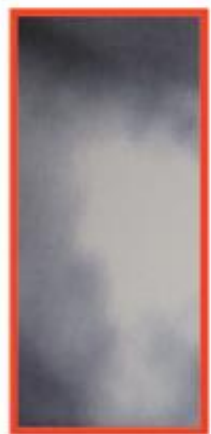

Diffuse

Characteristic findings of ICG Green Fluorescent Lymphography (Yamamoto et al., 2011a) ${ }^{9}$

\footnotetext{
${ }^{9}$ Yamamoto et al., Indocyanine Green-Enhanced Lymphography for Upper Extremity Lymphedema: A Novel Severity Staging System Using Dermal Backflow Patterns, Plastic and Reconstructive Surgery, volume 128, issue 4, pages 941-947, Copyright (2011a), https://journals.lww.com/plasreconsurg/fulltext/ 2011/10000/Indocyanine_Green_Enhanced_Lymphography_for_Upper.26.aspx
} 
The most significant limitation with ICG lymphography is its inability to image the deeper lymphatic system due to tracer detection being limited to a few centimetres beneath the skin (ISL, 2016; Groenlund et al., 2017). Where it is clinically indicated, a lymphoscintigraphy may be requested in combination with ICG lymphography to understand the presenting lymphoedema better, especially where surgery for the lymphoedema is being considered.

There are some non-invasive imaging modalities including magnetic resonance imaging, computed tomography (CT scan), and ultrasonography; but, these are neither sensitive nor specific enough for diagnosing lymphoedema, or they are cost-prohibitive.

\section{Magnetic Resonance Imaging (MRI)}

Magnetic Resonance Imaging (MRI) has the advantage of assessing an entire limb without exposure to radiation or drug administration; however, it does not allow for lymphatic vessel function to be assessed in real-time (Tashiro, Yamashita, Saito, Iida, \& Koshima, 2015). It is useful in detecting honeycomb patterns in the subcutaneous tissues indicative of fluid or fibrosis, subfascial fluid accumulation and dermal oedema (Åström, Abdsaleh, Brenning, \& Ahlström, 2001).

\section{Computed Tomography (CT-Scan)}

Computed Tomography (CT scan) provides an image (Figure 9) whereby subcutaneous tissue structures and volume can be measured. Classic signs visualised include peculiar trabecular areas of honeycombed patterning in the subcutaneous tissue, thickening of the skin, epifascial fluid lakes and the absence of oedema within muscular compartments (Yoo et al., 2017).

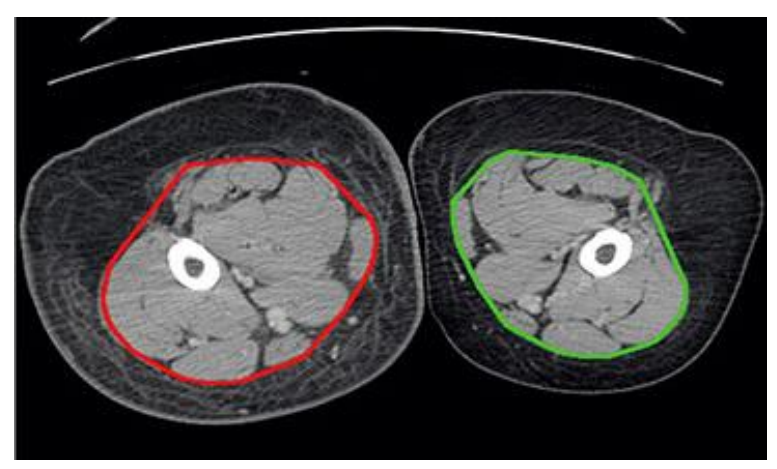

Computed Tomography (CT scan) cross-section scan of thighs (Yoo et al., 2017) 
Monnin-Delhom, Gallix, Achard, Bruel and Janbon (2002) demonstrated that CT imaging has a high sensitivity (93\%) and specificity (100\%) in confirming the diagnosis of lymphoedema; however, due to the high cost of the procedure and the radiation exposure to the patient (albeit small), this technique is rarely used in the diagnosis of lymphoedema.

\section{Ultrasonography}

Ultrasonography (Figure 10) is the most effective method of measuring the structural status of the lymphatic system and of the limb itself. It is a clinically convenient tool, and measurement of soft tissues can be performed within the clinical setting. It can provide information about changes to the thickness of the cutaneous, epifascial and subfascial tissue compartments and, identify fluid collection and fibrosis (Kim, Chung, Kim, \& Seo, 2008). It is more cost-effective than MRI or CT scans even though these provide greater accuracy and certainty (Pillar, 2007).

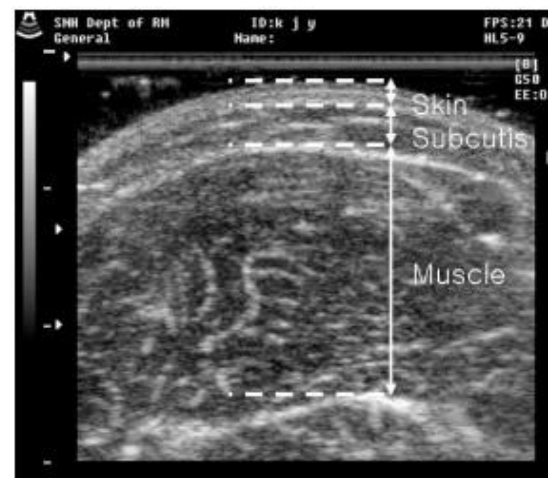

Ultrasonography of the upper arm (Kim, Chung, Kim, \& Seo, 2008) 
APPENDIX 3: Evidence Table of the Literature Reviewed on CTM, Perometry and 3DCI for Chapter 4

\begin{tabular}{|c|c|c|c|c|c|c|}
\hline Citation & Purpose & $\begin{array}{l}\text { Research } \\
\text { Design }\end{array}$ & Sample & Validity of Study & Study Results & Clinical Benefit \\
\hline $\begin{array}{l}\text { Chen, Tsai, } \\
\text { Hung, \& } \\
\text { Tsauo, } 2008\end{array}$ & $\begin{array}{l}\text { Investigate the } \\
\text { reliability of, and to } \\
\text { define the limits for } \\
\text { clinical change } \\
\text { indicative of clinical } \\
\text { improvement, for } \\
\text { water displacement, } \\
\text { circumference } \\
\text { measurement and } \\
\text { tissue tonometry }\end{array}$ & $\begin{array}{l}\text { Diagnostic Test } \\
\text { Study }\end{array}$ & $\begin{array}{l}14 \text { for limb } \\
\text { volume; } 17 \text { for } \\
\text { tissue tonometry }\end{array}$ & $\begin{array}{l}\text { Two groups of people with } \\
\text { lymphoedema: one group } \\
\text { (n=14) for limb volume by } \\
\text { water displacement and } 3 \\
\text { site circumference } \\
\text { measurement; the other } \\
\text { group ( } \mathrm{n}=17 \text { ) for tissue } \\
\text { tonometry only. The } \\
\text { protocol described two } \\
\text { therapists independently and } \\
\text { alternately performing the } \\
\text { tests for each method } 10 \text { min } \\
\text { apart. Mean of each test was } \\
\text { used to determine interrater } \\
\text { (ICC }[3,1] \text { ) reliability. } \\
\text { Reliability was determined } \\
\text { for agreement of results } \\
\text { according to criteria by } \\
\text { Fleiss between raters } \\
\text { (ICC[2,1] for intra-rater) or } \\
\text { trials (Bland-Altman } \\
\text { analysis), presence of } \\
\text { systematic changes in mean, } \\
\text { size of measurement error } \\
\text { (standard error of } \\
\text { measurement [SEM]) and } \\
\text { presence of clinically } \\
\text { significant changes (smallest } \\
\text { real difference [SRD]). }\end{array}$ & 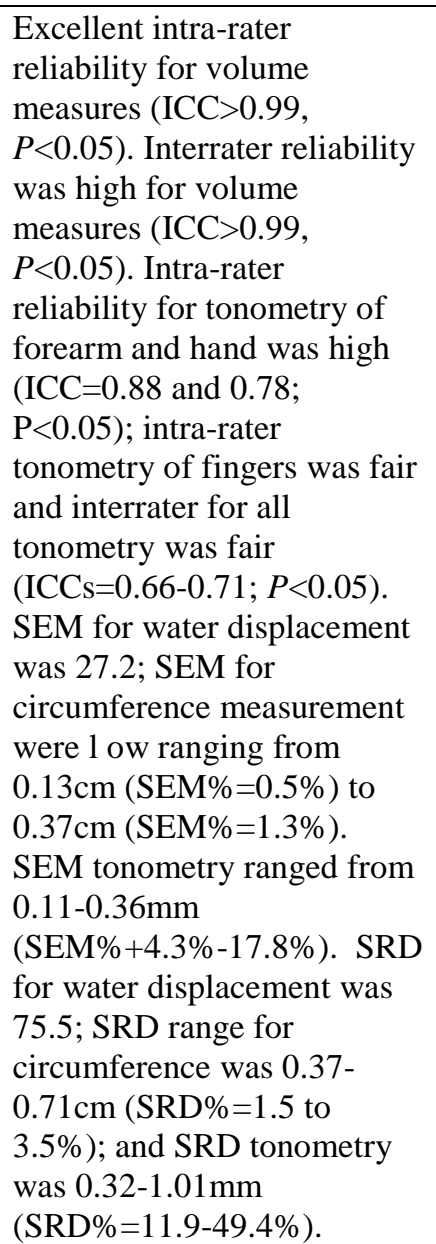 & $\begin{array}{l}\text { Water displacement and } \\
\text { circumference measure } \\
\text { are reliable techniques } \\
\text { for assessment of } \\
\text { lymphoedema; } \\
\text { tonometry is less } \\
\text { reliable. }\end{array}$ \\
\hline
\end{tabular}




\begin{tabular}{|c|c|c|c|c|c|c|}
\hline Citation & Purpose & $\begin{array}{l}\text { Research } \\
\text { Design }\end{array}$ & Sample & Validity of Study & Study Results & Clinical Benefit \\
\hline $\begin{array}{l}\text { Tewari, Gill, } \\
\text { Bochner, \& } \\
\text { Kollias, } 2008\end{array}$ & $\begin{array}{l}\text { Assess the accuracy } \\
\text { of circumferential } \\
\text { arm measurement } \\
\text { compared with } \\
\text { volume displacement } \\
\text { for the SNAC trial }\end{array}$ & $\begin{array}{l}\text { Diagnostic Test } \\
\text { Study }\end{array}$ & 87 women & $\begin{array}{l}\text { Ethics was granted. All } \\
\text { patients had the same tests } \\
\text { performed: circumferential } \\
\text { measurement with narrow } \\
\text { tape, circumferential } \\
\text { measurement with wide tape } \\
\text { and water displacement. A } \\
\text { clear protocol was followed; } \\
\text { however, no mention is made } \\
\text { of blinding or independent } \\
\text { testing. }\end{array}$ & $\begin{array}{l}\text { A significant correlation } \\
\text { between wide and narrow } \\
\text { tape volume estimations was } \\
\text { found using Pearson's } \\
\text { correlation coefficient } 0.95 \text {, } \\
\text { p }<0.0001 \text {. A significant } \\
\text { correlation between the } \\
\text { narrow tape and water } \\
\text { displacement was found with } \\
\text { a Pearson's correlation } \\
\text { coefficient of } 0.92 \text {, (CI } 0.89 \text { - } \\
0.94, \text { p }<0.0001) \text {. Pearson's } \\
\text { correlation coefficient } \\
\text { between the wide tape and } \\
\text { water displacement was } 0.88 \\
\text { (CI } 0.84-0.91, \text { p }<0.0001) \text {. } \\
\text { Further comparison between } \\
\text { BMI groups demonstrates } \\
\text { the highest correlation with } \\
\text { volume displacement for } \\
\text { overweight (BMI } 25-29.9 \text { ) } \\
\text { women using the narrow } \\
\text { tape. Lowest correlation was } \\
\text { for obese women (BMI } \geq 30 \text { ) } \\
\text { using the wide tape. }\end{array}$ & $\begin{array}{l}\text { Use of the narrow tape } \\
\text { measure to estimate limb } \\
\text { volume is similar to } \\
\text { water displacement. } \\
\text { This outcome is useful } \\
\text { in clinical practice as } \\
\text { water displacement is a } \\
\text { time-consuming, messy } \\
\text { system that is unsuitable } \\
\text { with some skin problems } \\
\text { and healing wounds due } \\
\text { to the risk of cross- } \\
\text { contamination. Use of } \\
\text { the narrow tape measure, } \\
\text { which is readily } \\
\text { available, is subject to } \\
\text { potential errors, and it is } \\
\text { recognised that a } \\
\text { standardised method is } \\
\text { required. To reduce } \\
\text { inter-observer variation, } \\
\text { it is advised that the } \\
\text { same therapist review } \\
\text { the patient to ensure the } \\
\text { degree of tension } \\
\text { applied to the measuring } \\
\text { tape on the limb needs is } \\
\text { consistent. }\end{array}$ \\
\hline $\begin{array}{l}\text { Czerniec et al., } \\
2010\end{array}$ & $\begin{array}{l}\text { Determine the } \\
\text { relationship between } \\
\text { physical methods of } \\
\text { measuring } \\
\text { lymphoedema and } \\
\text { self-reported }\end{array}$ & $\begin{array}{c}\text { Diagnostic Test } \\
\text { Study }\end{array}$ & $\begin{array}{l}33 \text { women with } \\
\text { unilateral arm } \\
\text { lymphoedema } \\
\text { and control } \\
\text { group of } 18 \\
\text { women }\end{array}$ & $\begin{array}{l}\text { Ethics approval and consent } \\
\text { was obtained. } \\
\text { Measurements taken } \\
\text { included self-report on a } \\
\text { visual analogue scale (VAS), } \\
\text { circumferential measures at }\end{array}$ & $\begin{array}{l}\text { Very strong agreement }(0.89 \\
\text { to } 0.99) \text { between measures } \\
\text { obtained by tape measure, } \\
\text { perometer and BIS methods } \\
\text { with the strongest } \\
\text { relationship between limb }\end{array}$ & $\begin{array}{l}\text { All physical methods of } \\
\text { assessment show } \\
\text { excellent reliability, } \\
\text { whereas self-report only } \\
\text { has moderate reliability. }\end{array}$ \\
\hline
\end{tabular}




\begin{tabular}{|c|c|c|c|c|c|c|}
\hline Citation & Purpose & $\begin{array}{c}\text { Research } \\
\text { Design }\end{array}$ & Sample & Validity of Study & Study Results & Clinical Benefit \\
\hline & $\begin{array}{l}\text { swelling, their } \\
\text { reliability, and their } \\
\text { standard error of } \\
\text { measurement }\end{array}$ & & & $\begin{array}{l}\text { 10cm intervals, perometer } \\
\text { volume measures and } \\
\text { bioimpedance spectroscopy. } \\
\text { The same assessor performed } \\
\text { all measurements on both } \\
\text { arms and at two occasions, } \\
\text { four weeks apart. } \\
\text { Limits of agreement analysis } \\
\text { and reliability and SEM were } \\
\text { considered. }\end{array}$ & $\begin{array}{l}\text { volume difference by the } \\
\text { truncated cone method (for } \\
\text { tape measure) and perometer } \\
\left(\mathrm{r}_{\mathrm{c}}=0.99\right) \text {. The weakest } \\
\text { relationship was between } \\
\text { BIS and truncated cone } \\
\text { method }\left(\mathrm{r}_{\mathrm{c}}=0.89 \text { ). }\right. \\
\text { Correlation between VAS } \\
\text { score and limb volume was } \\
\text { moderate (tape: } \mathrm{r}_{\mathrm{c}}=0.66 \text {; } \\
\left.\text { perometer: } \mathrm{r}_{\mathrm{c}}=0.65\right) \text {; whereas } \\
\text { correlation between VAS } \\
\text { and BIS was higher } \\
\left(\mathrm{r}_{\mathrm{c}}=0.71\right) \text {. } \\
\text { Truncated cone method } \\
\text { underestimated limb volume } \\
\text { compared to perometer. } \\
\text { All three physical tests } \\
\text { demonstrated excellent intra- } \\
\text { rater reliability (ICC }=0.94 \\
\text { for volume and ICC }=1.00 \text { for } \\
\text { BIS), with self-reported } \\
\text { swelling moderately reliable } \\
\text { (ICC }=0.70) \text {. } \\
\text { All physical methods showed } \\
\text { excellent inter-rater } \\
\text { reliability with tape } \\
\text { ICC }=0.98 ; \text { perometer } \\
\text { ICC }=1.00 \text { and BIS } \\
\text { ICC }=0.99 \text {. } \\
\text { Limitations to study } \\
\text { identified. }\end{array}$ & $\begin{array}{l}\text { To note, even though } \\
\text { there was a high level of } \\
\text { agreement between the } \\
\text { physical methods, they } \\
\text { are not interchangeable. }\end{array}$ \\
\hline
\end{tabular}




\begin{tabular}{|c|c|c|c|c|c|c|}
\hline Citation & Purpose & $\begin{array}{c}\text { Research } \\
\text { Design }\end{array}$ & Sample & Validity of Study & Study Results & Clinical Benefit \\
\hline $\begin{array}{l}\text { Devoogdt et } \\
\text { al., } 2010\end{array}$ & $\begin{array}{l}\text { Examine the } \\
\text { reliability of a new } \\
\text { self-developed } \\
\text { measurement device } \\
\text { that counters the } \\
\text { disadvantages of } \\
\text { traditional methods } \\
\text { of arm } \\
\text { circumference } \\
\text { measurements }\end{array}$ & $\begin{array}{c}\text { Prospective } \\
\text { Diagnostic Test } \\
\text { Study }\end{array}$ & $\begin{array}{l}\text { Data from } 112 \\
\text { patients were } \\
\text { available for } \\
\text { reliability } \\
\text { analysis. }\end{array}$ & $\begin{array}{l}\text { Ethical approval gained. } \\
\text { Measurements taken pre-op } \\
\text { and between } 3 \text { - } 9 \text { days post- } \\
\text { op on the unaffected side } \\
\text { only. Measurements taken } \\
\text { by } 3 \text { trained physical } \\
\text { therapists who were blinded } \\
\text { to each other. } \\
\text { Measurements included the } \\
\text { use of the new device: steel } \\
\text { bar with a tape measure } \\
\text { every 4cm with weighted } \\
\text { 20g at the end of the tape } \\
\text { with truncated cone } \\
\text { (frustrum formula) } \\
\text { calculation; and water } \\
\text { displacement. Statistical } \\
\text { analysis includes Intraclass } \\
\text { Correlation Coefficients } \\
\text { (ICCs) using Pearson } \\
\text { correlation coefficient to } \\
\text { determine any relationships } \\
\text { between the new device and } \\
\text { water displacement; Bland- } \\
\text { Altman analysis was used to } \\
\text { determine interchangeability } \\
\text { between methods of } \\
\text { measure, SEM, and Small } \\
\text { Real Difference (SRD) } \\
\text { analysis was also } \\
\text { undertaken. }\end{array}$ & $\begin{array}{l}\text { ICC for intra-rater reliability } \\
\text { of the new device ranged } \\
\text { from } 0.977 \text { (95\% CI } 0.960- \\
0.986 \text { ) at the lower arm to } \\
0.996 \text { (95\% CI } 0.992-0.998) \\
\text { for the upper arm. } \\
\text { ICC for interrater reliability } \\
\text { of the new device ranged } \\
\text { from } 0.942 \text { (95\% CI 0.828- } \\
0.975) \text { for the lower arm and } \\
0.994 \text { (95\% CI 0.990-0.997) } \\
\text { for the upper arm. } \\
\text { SEM for circumference } \\
\text { measurement was low } \\
(0.8 \%) \text {, and SEM for } \\
\text { calculated arm volume was } \\
\text { low (2.0\%). } \\
\text { SRD for circumferential } \\
\text { measurements was } 3.4 \%, \text { and } \\
\text { SRD for calculated arm } \\
\text { volume was } 3.9 \% \text {. } \\
\text { Volumes calculated from } \\
\text { both methods were highly } \\
\text { correlating (r=0.973). } \\
\text { However, as there was a } \\
\text { wide } 95 \% \text { confidence } \\
\text { interval, it suggests that these } \\
\text { two methods are not } \\
\text { interchangeable. }\end{array}$ & $\begin{array}{l}\text { Study weakness was that } \\
\text { no measures done on } \\
\text { lymphoedema side. } \\
\text { Strengths of the study } \\
\text { are high sample number, } \\
\text { age and BMI } \\
\text { representative of the } \\
\text { population, } \\
\text { measurements } \\
\text { performed in 'the field' } \\
\text { thus reflective of clinical } \\
\text { setting (guarantees the } \\
\text { authenticity of results). } \\
\text { Measurements were } \\
\text { taken using bony } \\
\text { reference point as } \\
\text { opposed to distance } \\
\text { from fingertip. } \\
\text { In follow up of patient, } \\
\text { always use the same } \\
\text { method of measure, as } \\
\text { methods are not } \\
\text { interchangeable. }\end{array}$ \\
\hline
\end{tabular}




\begin{tabular}{|c|c|c|c|c|c|c|}
\hline Citation & Purpose & $\begin{array}{c}\text { Research } \\
\text { Design }\end{array}$ & Sample & Validity of Study & Study Results & Clinical Benefit \\
\hline $\begin{array}{l}\text { Ancukiewicz et } \\
\text { al., } 2011\end{array}$ & $\begin{array}{l}\text { To develop a simple } \\
\text { and practical formula } \\
\text { for quantifying upper } \\
\text { limb lymphoedema, } \\
\text { accounting for both } \\
\text { the asymmetry of the } \\
\text { extremities' volumes } \\
\text { and their temporal } \\
\text { changes }\end{array}$ & $\begin{array}{c}\text { Prospective } \\
\text { Diagnostic Test } \\
\text { Study }\end{array}$ & $\begin{array}{l}677 \text { consecutive } \\
\text { unilateral breast } \\
\text { cancer patients }\end{array}$ & $\begin{array}{l}\text { Approval gained from the } \\
\text { Partners Institute Review } \\
\text { Board. Prospective collection } \\
\text { of repeated bilateral arm } \\
\text { volume measurements by } \\
\text { perometry of breast cancer } \\
\text { patients was collected } \\
\text { preoperatively (baseline) and } \\
\text { post-operatively three to six } \\
\text { times (time-dependent). }\end{array}$ & $\begin{array}{l}\text { No statistically significant } \\
\text { difference between baseline } \\
\text { measurements of the tumour- } \\
\text { ipsilateral and contralateral } \\
\text { arms (p=0.42). } \\
\text { Baseline log-transformed } \\
\text { ratio ipsilateral/contralateral } \\
\text { (A/U) most closely } \\
\text { approximates normal } \\
\text { distribution (skewness } \\
\text { coefficient of } 0.34 \text { ). Log- } \\
\text { normal distribution of the } \\
\text { ratio of A/U ratios over time } \\
\text { proved statistically } \\
\text { significant (p=0.08). } \\
\text { Analysis of longitudinal } \\
\text { variation of Relative Volume } \\
\text { Change (RVC), weight was } \\
\text { the only covariate } \\
\text { significantly associated with } \\
\text { an expected value of RVC } \\
\text { (p=0.007); patients with } \\
\text { higher baseline weight } \\
\text { tended to develop higher } \\
\text { RVC in the follow-up. } \\
\text { Constant arm length during a } \\
\text { test was used for volume } \\
\text { calculation even though there } \\
\text { was a slight variance in } \\
\text { length between visits. The } \\
\text { effect of the arm length on } \\
\text { RVC was not statistically } \\
\text { significant (p=0.466). }\end{array}$ & $\begin{array}{l}\text { The researchers propose } \\
\text { the use of their protocol } \\
\text { and formula for } \\
\text { quantification of } R V C \\
\left(=\left(\mathrm{A}_{2} \mathrm{U}_{1}\right) /\left(\mathrm{U}_{2} \mathrm{~A}_{1}\right)-1\right) \text { to } \\
\text { enable standardised } \\
\text { reporting of volumetric } \\
\text { monitoring of } \\
\text { lymphoedema. } \\
\text { The researchers have } \\
\text { devised a 'Perometer } \\
\text { Measurement Protocol' } \\
\text { for the positioning of the } \\
\text { patient and the use of the } \\
\text { perometer. }\end{array}$ \\
\hline
\end{tabular}




\begin{tabular}{|c|c|c|c|c|c|c|}
\hline Citation & Purpose & $\begin{array}{l}\text { Research } \\
\text { Design }\end{array}$ & Sample & Validity of Study & Study Results & Clinical Benefit \\
\hline $\begin{array}{l}\text { Foroughi et al., } \\
2011\end{array}$ & $\begin{array}{l}\text { To assess the inter- } \\
\text { reliability and } \\
\text { coherence of a } \\
\text { protocol for } \\
\text { measuring arm } \\
\text { circumference by } \\
\text { untrained people } \\
\text { against perometry }\end{array}$ & $\begin{array}{c}\text { Cross-sectional } \\
\text { study }\end{array}$ & $\begin{array}{l}57 \text { community- } \\
\text { dwelling adults } \\
\text { over the age of } \\
40 \text { years }\end{array}$ & $\begin{array}{l}\text { Ethics and consent gained. } \\
\text { Inclusion and exclusion } \\
\text { criteria identified. Measuring } \\
\text { protocol posted out together } \\
\text { with a tape measure to } \\
\text { participants' homes to } \\
\text { measure } 10 \mathrm{~cm} \\
\text { circumferences of both arms } \\
\text { starting at the wrist. } \\
\text { Participant then seen within } \\
\text { two weeks at the laboratory } \\
\text { for } 10 \mathrm{~cm} \text { circumferential } \\
\text { measurement and perometry. } \\
\text { Anthropometric data } \\
\text { collected. Reliability at level } \\
0.8 \text { showed a sufficient } \\
\text { sample size of } 50 \text { people. } \\
\text { Data expressed as mean and } \\
\text { standard deviation (SD) or } \\
\text { median and range once } \\
\text { tested for normality } \\
\text { (skewness-1 } 1 \text { ). Lin's } \\
\text { concordance correlation and } \\
\text { limits of agreement analysis } \\
\text { used to compare participant's } \\
\text { measurement to the } \\
\text { laboratory's two } \\
\text { measurements. Dominant } \\
\text { arm used for analysis. }\end{array}$ & $\begin{array}{l}\text { Medium to high concordance } \\
\text { between arm circumferences } \\
\text { by participant and laboratory } \\
\text { and ranged from } r_{c}=0.68 \text { at } \\
10 \mathrm{~cm} \text { to } r_{c}=0.94 \text { at } 20 \mathrm{~cm} \text {. } \\
\text { Measurement concordance } \\
\text { between laboratory } \\
\text { circumference measure and } \\
\text { perometry ranged from } \\
r_{c}=0.83 \text { for } 10 \mathrm{~cm} \text { measure } \\
\text { and } r_{c}=0.94 \text { for } 40 \mathrm{~cm} \text {. } \\
\text { Measure concordance of } \\
\text { participant circumference, } \\
\text { and perometer ranged from } \\
r_{c}=0.76 \text { at } 20 \mathrm{~cm} \text { to } r_{c}=0.93 \text { at } \\
10 \mathrm{~cm} \text {. Bias between methods } \\
\text { minimal, and ranged from - } \\
5.5 \% \text { at } 40 \mathrm{~cm} \text { to } 1.5 \% \text { at } \\
10 \mathrm{~cm} \text { taken by laboratory } \\
\text { compared to perometer, and } \\
\text { from }-2.4 \% \text { at } 40 \mathrm{~cm} \text { to } 4.0 \% \\
\text { at wrist taken by laboratory } \\
\text { compared to participants. }\end{array}$ & $\begin{array}{l}\text { Self-monitoring by tape } \\
\text { measure using given } \\
\text { protocol is a cost- } \\
\text { efficient, user-friendly } \\
\text { tool for patients to use at } \\
\text { home. }\end{array}$ \\
\hline $\begin{array}{l}\text { Lee, Boland, } \\
\text { Czerniec, \& } \\
\text { Kilbreath, } 2011\end{array}$ & $\begin{array}{l}\text { Investigate the } \\
\text { criterion validity and } \\
\text { reliability of } \\
\text { Perometry for } \\
\text { measuring hand } \\
\end{array}$ & $\begin{array}{c}\text { Diagnostic Test } \\
\text { Study }\end{array}$ & $\begin{array}{l}40 \text { participants: } \\
20 \text { with breast } \\
\text { cancer-related } \\
\text { lymphoedema }\end{array}$ & $\begin{array}{l}\text { Ethics and consent gained. A } \\
\text { within-session study } \\
\text { consisting of a test-retest } \\
\text { protocol to determine inter- } \\
\text { and intra-reliability; the }\end{array}$ & $\begin{array}{l}\text { Measurements using } \\
\text { perometer demonstrated } \\
\text { excellent inter- and intra- } \\
\text { rater reliability. Intra-rater } \\
\text { ICC }_{2,1} \text { was } 0.989 \text { (95\% CI: }\end{array}$ & $\begin{array}{l}\text { Perometer offers an } \\
\text { alternative to water } \\
\text { displacement for } \\
\text { measurement of hand } \\
\text { volume. Methods are not }\end{array}$ \\
\hline
\end{tabular}




\begin{tabular}{|c|c|c|c|c|c|c|}
\hline Citation & Purpose & $\begin{array}{c}\text { Research } \\
\text { Design }\end{array}$ & Sample & Validity of Study & Study Results & Clinical Benefit \\
\hline & $\begin{array}{l}\text { volume using } \\
\text { samples of } \\
\text { symptomatic and } \\
\text { asymptomatic } \\
\text { participants. }\end{array}$ & & $\begin{array}{l}\text { (BCRL) and } 20 \\
\text { non-LO }\end{array}$ & $\begin{array}{l}\text { validity of perometer for } \\
\text { hand volume measurement } \\
\text { compared to water } \\
\text { displacement. Inclusion and } \\
\text { exclusion criteria identified. } \\
\text { LO arm for BCRL and } \\
\text { dominant in non-LO } \\
\text { measured by perometer twice } \\
\text { by one rater and once by the } \\
\text { second rater; and then once } \\
\text { by water displacement. } \\
\text { Garments removed } 2 \text { hrs } \\
\text { before testing. Tip of the } \\
\text { third finger to distal palmer } \\
\text { crease measured. Descriptive } \\
\text { statistics calculated; ICC }{ }_{2,1} \\
\text { with } 95 \% \text { confidence } \\
\text { intervals and Percent Close } \\
\text { Agreement (PCA) used to } \\
\text { determine intra- and inter- } \\
\text { rater reliability, paired } t \text {-test } \\
\text { performed to determine the } \\
\text { difference between means of } \\
\text { test-retest data. Reliability } \\
\text { poor when ICC } 2,1<0.40 ; \\
\text { moderate } 0.40-0.75 ; \\
\text { substantial } 0.75-0.90 \text { and } \\
\text { excellent }>0.90 . \text { Limits of } \\
\text { agreement analysis and Lin's } \\
\text { concordance correlation used } \\
\text { to evaluate the relationship } \\
\text { between measurements taken } \\
\text { with both methods. }\end{array}$ & $\begin{array}{l}0.979-0.994) \text {; Inter-rater } \\
\text { ICC }_{2,1} \text { was } 0.993 \text { ( } 0.986- \\
0.996) . \text { Paired } t \text {-test showed } \\
\text { no significant differences in } \\
\text { hand volume taken by } \\
\text { different raters. PCA showed } \\
\text { less than } 9 \mathrm{ml} \text { difference in } \\
\text { hand volume for } 80 \% \text { of } \\
\text { measurements taken by two } \\
\text { raters and } 73 \% \text { by a single } \\
\text { rater. Strong concordance } \\
\text { between both methods } \\
\left(\mathrm{r}_{\mathrm{c}}=0.88\right) \text {; limits of } \\
\text { agreement showed perometer } \\
\text { to overestimate the volume } \\
\text { of hand obtained from water } \\
\text { displacement (bias: } 7.5 \%) .\end{array}$ & $\begin{array}{l}\text { interchangeable. } \\
\text { Standardise } \\
\text { measurement and } \\
\text { position fingers together } \\
\text { (including thumb) for } \\
\text { perometer to reduce } \\
\text { spaces being interpreted } \\
\text { as volume. The benefit } \\
\text { of perometer over water } \\
\text { displacement: (i) } \\
\text { convenient and quick } \\
\text { with reduced set-up time } \\
\text { and shorter measurement } \\
\text { time, (ii) excellent inter- } \\
\text { and intra-rater reliability } \\
\text { means patient only } \\
\text { needs to be measured } \\
\text { once }\end{array}$ \\
\hline
\end{tabular}




\begin{tabular}{|c|c|c|c|c|c|c|}
\hline Citation & Purpose & $\begin{array}{l}\text { Research } \\
\text { Design }\end{array}$ & Sample & Validity of Study & Study Results & Clinical Benefit \\
\hline $\begin{array}{l}\text { Ancukiewicz et } \\
\text { al., } 2012\end{array}$ & $\begin{array}{l}\text { To evaluate the } \\
\text { effect of an absolute } \\
\text { change in arm size of } \\
200 \mathrm{ml} \text { or } 2 \mathrm{~cm} \\
\text { compared with } \\
\text { relative arm volume } \\
\text { change as criteria for } \\
\text { defining BCRL }\end{array}$ & $\begin{array}{c}\text { Diagnostic Test } \\
\text { Study }\end{array}$ & $\begin{array}{l}677 \text { patients } \\
\text { undergoing } \\
\text { surgery for } \\
\text { unilateral breast } \\
\text { cancer }\end{array}$ & $\begin{array}{l}\text { Ethics gained. Bilateral } \\
\text { perometer arm } \\
\text { measurements taken pre- and } \\
\text { post-operatively. Using pre- } \\
\text { operative arm measurements, } \\
\text { the calculated effect of } \\
\text { 200ml arm volume change } \\
\text { compared with relative arm } \\
\text { volume change, assuming } \\
\text { the volume of the opposite } \\
\text { arm remains unchanged. } \\
\text { Also calculated the effect of } \\
\text { circumference increase of } \\
2 \text { cm in the middle of the arm } \\
\text { in a sample of } 45 \text { patients } \\
\text { compared with relative arm } \\
\text { volume change. Analysed } \\
\text { the within-patient variation } \\
\text { of the unaffected arm } \\
\text { volumes throughout follow- } \\
\text { up in a subset of } 124 \text { patients } \\
\text { with 6-13 sequential } \\
\text { measurements. Histograms } \\
\text { were used; standard } \\
\text { deviation and range looked } \\
\text { at within-patient temporal } \\
\text { variation. Kendall's } \\
\text { correlation coefficients were } \\
\text { calculated and Kendall's test } \\
\text { used as a non-parametric test } \\
\text { of correlation. }\end{array}$ & $\begin{array}{l}\text { Pre-op unaffected arm } \\
\text { volumes ranged from } 1,270 \\
\text { to } 6,873 \text { ml ( } 5.4 \text {-fold } \\
\text { variation). Strong correlation } \\
\text { of unaffected arm volume } \\
\text { with bodyweight (Kendall's } \\
\mathrm{T}=0.65, \mathrm{p}<0.00 \text { ) and BMI } \\
\text { (Kendall's T }=0.55, \mathrm{p}<0.00) \text {. } \\
\text { Absolute volume change of } \\
200 \text { ml corresponded to } \\
\text { relative arm volume change } \\
\text { of } 15.7 \% \text { for pre-op arm } \\
\text { volume of } 1,270 \text { ml and } \\
2.9 \% \text { for arm volume of } \\
6,873 \text { ml. Median relative } \\
\text { arm volume change for } 200 \\
\text { ml increase was } 7.7 \% . \text { In a } \\
\text { subset of } 45 \text { patients, } \\
\text { increase of } 2 \text { cm } \\
\text { circumference corresponded } \\
\text { to relative arm volume } \\
\text { change of } 9.8 \% \text { for a pre-op } \\
\text { volume of } 1,799 \text { ml and } \\
6.0 \% \text { for arm volume of } \\
4,376 \text { ml. Median relative } \\
\text { arm volume change for } 2 \text { cm } \\
\text { increase was } 7.5 \% \text {. In subset } \\
\text { of } 124 \text { patients with } 6 \text { or } \\
\text { more sequential } \\
\text { measurements, range of } \\
\text { longitudinal variation of } \\
\text { absolute volume change of } \\
\text { unaffected arm correlated } \\
\text { with pre-op arm volume }\end{array}$ & $\begin{array}{l}\text { Relative arm volume } \\
\text { change is independent of } \\
\text { body size and should be } \\
\text { used as a standard } \\
\text { criterion for the } \\
\text { diagnosis of BCRL. } \\
\text { Changes in the size of } \\
\text { the contralateral arm } \\
\text { should be used as a } \\
\text { control arm to account } \\
\text { for fluctuations in BMI. }\end{array}$ \\
\hline
\end{tabular}




\begin{tabular}{|c|c|c|c|c|c|c|}
\hline Citation & Purpose & $\begin{array}{c}\text { Research } \\
\text { Design }\end{array}$ & Sample & Validity of Study & Study Results & Clinical Benefit \\
\hline & & & & & $\begin{array}{l}\text { (Kendall's } \mathrm{T}=0.34, \mathrm{p}<0.00) \text {, } \\
\text { patient weight }(\text { Kendall's T = } \\
0.41, \mathrm{p}<0.00) \text { and } \mathrm{BMI} \\
(\text { Kendall's } \mathrm{T}=0.36, \mathrm{p}<0.00) \text {. } \\
\text { In contrast, the range of } \\
\text { variation in relative arm } \\
\text { volume changes was not } \\
\text { correlated with pre-op arm } \\
\text { volume, weight or BMI } \\
\text { (p>0.05 for all) - even } \\
\text { though substantial magnitude } \\
\text { resent (median range } 10.5 \%) \text {. } \\
\text { SD of longitudinal variation: } \\
\text { for absolute volume changes } \\
\text { correlate with pre-op arm } \\
\text { volume, weight and BMI } \\
\text { (p<0.00), but no correlation } \\
\text { for relative arm volume } \\
\text { changes (p }>0.05 \text { ). The } \\
\text { asymmetry between arms } \\
\text { pre-op observed as } 100 \mathrm{ml} \text { in } \\
30.0 \% \text { and } 200 \text { ml in } 8.1 \% \text { of } \\
\text { the cohort. }\end{array}$ & \\
\hline $\begin{array}{l}\text { Asim et al., } \\
2012\end{array}$ & $\begin{array}{l}\text { Primary aims were } \\
\text { to ascertain the local } \\
\text { prevalence of } \\
\text { lymphoedema after } \\
\text { Axillary Node } \\
\text { Dissection (AND) } \\
\text { and to compare the } \\
\text { relationship between } \\
\text { objective measures } \\
\text { and subjective } \\
\text { symptoms. }\end{array}$ & $\begin{array}{c}\text { Diagnostic Test } \\
\text { Study }\end{array}$ & 193 women & $\begin{array}{l}\text { Consent gained (no mention } \\
\text { of ethics). Inclusion and } \\
\text { exclusion criteria described. } \\
\text { Four staff performed } \\
\text { assessments. The assessment } \\
\text { consisted of a questionnaire } \\
\text { and circumferential limb } \\
\text { measurements at } 3 \text { points } \\
\text { (15cm above and } 10 \mathrm{~cm} \\
\text { below olecranon, and around } \\
\text { the hand); } 73 \text { patients had }\end{array}$ & $\begin{array}{l}\text { LO defined as }>7.5 \% \\
\text { increase in any } \\
\text { circumference on the } \\
\text { operated side }(=15.5 \% \\
\text { increase in volume })-23.3 \% \\
\text { of cohort positive for LO. In } \\
\text { a subset of } 73 \text { patients, LO } \\
\text { prevalence was } 8.2 \%, 9.6 \% \\
\text { and } 19.1 \% \text { for the forearm, } \\
\text { upper arm and hand } \\
\text { respectively. Prevalence }\end{array}$ & $\begin{array}{l}\text { Arm circumference } \\
\text { measure is easy and } \\
\text { convenient. Threshold } \\
\text { increase at a single point } \\
\text { may be more indicative } \\
\text { of LO than global } \\
\text { measure. Using ten } \\
\text { percentage increase } \\
\text { rather than absolute } \\
\text { measure of } 2 \mathrm{~cm} \text { takes }\end{array}$ \\
\hline
\end{tabular}




\begin{tabular}{|c|c|c|c|c|c|c|}
\hline Citation & Purpose & $\begin{array}{c}\text { Research } \\
\text { Design }\end{array}$ & Sample & Validity of Study & Study Results & Clinical Benefit \\
\hline & $\begin{array}{l}\text { Secondary aims were } \\
\text { to determine local } \\
\text { risk factors affecting } \\
\text { rate of LO, measure } \\
\text { the morbidity } \\
\text { associated with LO } \\
\text { using QoL } \\
\text { questionnaires and to } \\
\text { establish easy and } \\
\text { convenient ways to } \\
\text { detect LO. }\end{array}$ & & & $\begin{array}{l}\text { another seven measurements } \\
\text { taken starting at the base of } \\
\text { the middle finger, then } 10 \mathrm{~cm} \\
\text { up to the top of the arm. } \\
\text { Limb volume calculated } \\
\text { using the truncated cone } \\
\text { method. Statistical analysis: } \\
\text { data entered into the } \\
\text { Mircosoft Access database. } \\
\text { A multivariate logistic } \\
\text { regression method for } \\
\text { analysis of LO risk factors; a } \\
\text { binary variable was the } \\
\text { dependent variable. } \\
\text { GraphPad Prism analysed the } \\
\text { Activities of Daily Living } \\
\text { questionnaire. Continuous } \\
\text { variables were compared } \\
\text { using the Mann-Whitney } \\
\text { test. Arm dominance } \\
\text { correction applied as } 1.4 \% \\
\text { for the forearm, } 1.2 \% \text { for } \\
\text { hand and } 0 \% \text { for the upper } \\
\text { arm. }\end{array}$ & $\begin{array}{l}\text { using } \geq 2 \mathrm{~cm} \text { increase at any } \\
\text { point on the operated side } \\
\text { was } 25.3 \% \text {. Prevalence using } \\
\text { subjective method was } \\
10.8 \% \text { ( } 3+\text { score) and } 41.9 \% \\
(2+\text { score). Sensitivity and } \\
\text { specificity in arm } \\
\text { circumference increase of } \\
\geq 7.5 \text { and } \geq 10 \% \text { was } 83 \% \text { and } \\
81 \% \text {, and } 66 \% \text { and } 89 \% \\
\text { respectively. Poor } \\
\text { concordance between } \\
\text { objective measure and } \\
\text { subjective symptoms at } \\
\geq 7.5 \% \text { threshold; with more } \\
\text { concordance at } \geq 10 \% \\
\text { threshold. }\end{array}$ & $\begin{array}{l}\text { into account varying } \\
\text { body sizes. }\end{array}$ \\
\hline $\begin{array}{l}\text { Brorson \& } \\
\text { Hoijer, } 2012\end{array}$ & $\begin{array}{l}\text { To estimate the } \\
\text { correlation between } \\
\text { water displacement } \\
\text { and circumference } \\
\text { measurements are } \\
\text { taken either at } 4 \mathrm{~cm} \\
\text { circumference } \\
\text { measurements or } \\
\text { five-set } \\
\text { circumference point }\end{array}$ & $\begin{array}{l}\text { Diagnostic Test } \\
\text { Study }\end{array}$ & $\begin{array}{l}10 \text { women with } \\
\text { unilateral BCRL }\end{array}$ & $\begin{array}{l}\text { Consent was gained, and } \\
\text { study followed Good } \\
\text { Clinical Practice (GCP) } \\
\text { guidelines. Water } \\
\text { displacement and } \\
\text { circumference measures } \\
\text { taken of both arms as part of } \\
\text { routine follow-up in } \\
\text { lymphoedema clinic. } \\
\text { Calculation of coefficient }\end{array}$ & $\begin{array}{l}\text { CV\% for volume } \\
\text { calculations was } 0.63 \% \text { for } \\
4 \mathrm{~cm} \text { CTM, } 0.63 \% \text { for } 5 \text { point } \\
\text { CTM and } 0.61 \% \text { for water } \\
\text { displacement. Affected arm: } \\
\text { water displacement had } \\
\text { larger mean value due to the } \\
\text { inclusion of hand at } 3138 \mathrm{ml} \text {, } \\
4 \mathrm{~cm} \text { was } 2972 \mathrm{ml} \text {, and } 5- \\
\text { point was } 2916 \mathrm{ml} \text {. Mean }\end{array}$ & $\begin{array}{l}\text { Can use 5-point as for } \\
\text { measuring for made-to- } \\
\text { measure garments to } \\
\text { gain clinical outcomes } \\
\text { of treatment effect. }\end{array}$ \\
\hline
\end{tabular}




\begin{tabular}{|c|c|c|c|c|c|c|}
\hline Citation & Purpose & $\begin{array}{c}\text { Research } \\
\text { Design }\end{array}$ & Sample & Validity of Study & Study Results & Clinical Benefit \\
\hline & $\begin{array}{l}\text { - the latter is used to } \\
\text { measure for made- } \\
\text { to-measure } \\
\text { compression } \\
\text { garments. }\end{array}$ & & & $\begin{array}{l}\text { variation (CV\%) was done } \\
\text { for each method by } \\
\text { researcher performing a test } \\
\text { on colleague ten times. } \\
\text { Truncated cone method used } \\
\text { for volume calculation for } \\
\text { circumferential method, hand } \\
\text { excluded. Water } \\
\text { displacement measured hand } \\
\text { as well. Measurements } \\
\text { presented as mean, standard } \\
\text { deviation (SD) and range. } \\
\text { Student's } t \text {-test for paired } \\
\text { observations used to assess } \\
\text { significant differences; non- } \\
\text { parametric calculations } \\
\text { performed finding no } \\
\text { difference in statistical } \\
\text { significance; linear } \\
\text { regression analysis used to } \\
\text { obtain the coefficient of } \\
\text { regression }(\square) \text { with } 95 \% \\
\text { confidence interval, and } \\
\text { correlation coefficient }(r) \text {. }\end{array}$ & $\begin{array}{l}\text { difference between } 4 \mathrm{~cm} \text { and } \\
5 \text {-point methods was } 56 \mathrm{ml} \\
\text { (range=179-308 ml) } \\
\text { indicating fewer segments } \\
\text { give smaller volume, but not } \\
\text { statistically significant. A } \\
\text { high correlation between all } \\
\text { methods with a coefficient of } \\
\text { correlation }>0.88 \text { and } \\
\text { coefficient regression close } \\
\text { to } 1 . \text { Unaffected arm: water } \\
\text { displacement mean volume } \\
\text { was } 2498 \text { ml, } 4 \mathrm{~cm} \text { was } 2390 \\
\text { ml, and 5-point was } 2363 \mathrm{ml} . \\
\text { Mean difference between } \\
4 \mathrm{~cm} \text { and 5-point was } 26 \mathrm{ml} \\
\text { (range=224-210 ml). } \\
\text { Statistical difference } \\
\text { between water displacement } \\
\text { and 5-point method } \\
\text { (p<0.05); a high correlation } \\
\text { between all methods with a } \\
\text { coefficient of correlation } \\
>0.81 \text { and coefficient of } \\
\text { regression close to } 1 . \text { Excess } \\
\text { Volume: water displacement } \\
\text { mean was 641 ml, 4cm was } \\
582 \text { ml, and 5-point was } 553 \\
\text { ml. Mean difference between } \\
4 \mathrm{~cm} \text { and 5-point was } 29 \mathrm{ml} \\
\text { (range= } 82-164 \text { ml) which } \\
\text { was not statistically } \\
\text { significant. Statistical } \\
\text { significance between water }\end{array}$ & \\
\hline
\end{tabular}




\begin{tabular}{|c|c|c|c|c|c|c|}
\hline Citation & Purpose & $\begin{array}{c}\text { Research } \\
\text { Design }\end{array}$ & Sample & Validity of Study & Study Results & Clinical Benefit \\
\hline & & & & & $\begin{array}{l}\text { displacement and } 4 \mathrm{~cm} \\
\text { method was } \mathrm{p}<0.05 \text { and the } \\
5 \text {-point method was } \mathrm{p}<0.00 \text {. } \\
\text { A high correlation between } \\
\text { all methods with a } \\
\text { correlation coefficient of } \\
>0.93 \text { and regression } \\
\text { coefficient close to } 1 .\end{array}$ & \\
\hline $\begin{array}{l}\text { Dylke, Yee, } \\
\text { Ward, } \\
\text { Foroughi, \& } \\
\text { Kilbreath, } 2012\end{array}$ & $\begin{array}{l}\text { To describe normal } \\
\text { inter-limb variance } \\
\text { and to determine } \\
\text { statistically-based } \\
\text { significant cut-offs } \\
\text { for both } \\
\text { circumference and } \\
\text { volume } \\
\text { measurements using } \\
\text { a perometer in } \\
\text { healthy older women }\end{array}$ & $\begin{array}{c}\text { Diagnostic Test } \\
\text { Study }\end{array}$ & $\begin{array}{l}204 \text { healthy } \\
\text { participants (no } \\
\text { LO) }\end{array}$ & $\begin{array}{l}\text { Ethics and consent gained. } \\
\text { Inclusion and exclusion } \\
\text { criteria identified. Four age } \\
\text { groups: } 40-49 \text { years, } 50-59 \\
\text { years, } 60-69 \text { years and } 70+ \\
\text { years. Descriptive analysis of } \\
\text { groups was presented. A } \\
\text { single assessment where both } \\
\text { arms were measured using } \\
\text { the perometer. } \\
\text { Circumference } \\
\text { measurements were gained } \\
\text { manually from the Peroplus } \\
\text { software; limb volumes were } \\
\text { done manually using the } \\
\text { perometer value and both the } \\
\text { truncated cone method and } \\
\text { the cylinder method. All data } \\
\text { were presented as means } \pm \\
\text { standard deviation. Volume } \\
\text { data for each method was } \\
\text { compared using concordance } \\
\text { correlation and limits of } \\
\text { agreement; paired } t \text {-tests } \\
\text { were used to determine } \\
\text { whether the circumferences }\end{array}$ & $\begin{array}{l}\text { Good concordance and high } \\
\text { correlation between volume } \\
\text { by perometer truncated cone } \\
\text { and cylinder methods noted; } \\
\text { however, small but } \\
\text { significant differences were } \\
\text { presented. For total limb } \\
\text { volumes, the mean bias } \\
\text { between methods was } 2.9 \% \\
\text { with the largest bias between } \\
\text { perometer and cylinder } \\
\text { method for dominant limb } \\
\text { (5.0\%). The limits of } \\
\text { agreement (2SD) between } \\
\text { methods were larger when } \\
\text { perometer compared to } \\
\text { calculated volume methods } \\
\text { than calculated methods } \\
\text { compared to each other. The } \\
\text { largest limit of agreement } \\
\text { was -1.0\% to } 10.0 \% \text { for } \\
\text { perometer compared to the } \\
\text { truncated cone for the } \\
\text { dominant limb. Paired } t \text {-tests } \\
\text { between dominant and non- } \\
\text { dominant circumference }\end{array}$ & $\begin{array}{l}\text { Current criteria for } \\
\text { diagnosing } \\
\text { lymphoedema is based } \\
\text { on convenience and not } \\
\text { normative differences, } \\
\text { as presented in this } \\
\text { paper. It highlights the } \\
\text { need for pre-operative } \\
\text { measurements due to } \\
\text { high inter-limb } \\
\text { differences. Within } \\
\text { clinical setting can be } \\
\text { cumbersome to } \\
\text { remember values; } \\
\text { however, it does } \\
\text { highlight need to } \\
\text { consider arm } \\
\text { dominance. }\end{array}$ \\
\hline
\end{tabular}




\begin{tabular}{|c|c|c|c|c|c|c|}
\hline Citation & Purpose & $\begin{array}{c}\text { Research } \\
\text { Design }\end{array}$ & Sample & Validity of Study & Study Results & Clinical Benefit \\
\hline & & & & $\begin{array}{l}\text { or volumes of the dominant } \\
\text { limb were significantly } \\
\text { different from the non- } \\
\text { dominant limb. Inter-limb } \\
\text { differences were used to } \\
\text { determine diagnostic cut- } \\
\text { offs. Mean and standard } \\
\text { deviations of the difference } \\
\text { for each variable were } \\
\text { determined and cut-offs } \\
\text { established as means plus } \\
\text { three times the standard } \\
\text { deviation. Regression } \\
\text { analysis to determine if age, } \\
\text { side of dominance, weight, } \\
\text { height, or BMI were related } \\
\text { to inter-limb differences in } \\
\text { circumference or volume. }\end{array}$ & $\begin{array}{l}\text { measurements revealed } \\
\text { significant differences due to } \\
\text { dominance esp. for } 10 \mathrm{~cm}, \\
20 \mathrm{~cm} \text { and } 40 \mathrm{~cm} \text { points. No } \\
\text { significant difference at } \\
30 \mathrm{~cm} \text {. Paired t-tests for both } \\
\text { perometer and calculated } \\
\text { volume methods: all } \\
\text { perometer determined } \\
\text { segments were significantly } \\
\text { different (p }<0.03) \text {. The cut- } \\
\text { off for diagnosis was based } \\
\text { on three standard deviations } \\
\text { above mean difference which } \\
\text { for the dominant arm was } \\
1.3 \mathrm{~cm}, 2.4 \mathrm{~cm}, 2.5 \mathrm{~cm}, 2.7 \mathrm{~cm} \\
\text { and } 3 \mathrm{~cm}, \text { respectively, and } \\
\text { for the non-dominant arm, it } \\
\text { was } 1.2 \mathrm{~cm}, 1.9 \mathrm{~cm}, 1.9 \mathrm{~cm}, \\
2.7 \mathrm{~cm} \text { and } 2.7 \mathrm{~cm} \text {. Cut-offs } \\
\text { for dominant and non- } \\
\text { dominant arm for perometer } \\
\text { was higher at } 370 \text { ml and } \\
210 \text { ml; and } 290 \text { ml and } 240 \\
\text { ml for calculated methods. } \\
\text { Regression analysis found a } \\
\text { small but significant } \\
\text { relationship between inter- } \\
\text { limb volume difference and } \\
\text { age irrespective of the } \\
\text { method used. Hand } \\
\text { dominance, height, weight } \\
\text { and BMI showed no } \\
\text { relationship. }\end{array}$ & \\
\hline
\end{tabular}




\begin{tabular}{|c|c|c|c|c|c|c|}
\hline Citation & Purpose & $\begin{array}{l}\text { Research } \\
\text { Design }\end{array}$ & Sample & Validity of Study & Study Results & Clinical Benefit \\
\hline $\begin{array}{l}\text { Katz-Leurer \& } \\
\text { Bracha, } 2012\end{array}$ & $\begin{array}{l}\text { To determine the } \\
\text { test-retest reliability } \\
\text { of total arm volume } \\
\text { from circumference } \\
\text { measurements and } \\
\text { arm segment volume } \\
\text { measurement }\end{array}$ & $\begin{array}{c}\text { Diagnostic Test } \\
\text { Study }\end{array}$ & $\begin{array}{l}16 \text { women with } \\
\text { arm } \\
\text { lymphoedema }\end{array}$ & $\begin{array}{l}\text { Ethics and consent gained. } \\
\text { All subjects had both arms } \\
\text { measured by the same } \\
\text { therapist at the same time } \\
\text { one week apart. } \\
\text { Measurements were taken } \\
\text { according to a predefined } \\
\text { protocol. The calculation } \\
\text { used the truncated cone } \\
\text { formula. } \\
\text { Paired } t \text {-test, intra-class } \\
\text { correlation coefficient, } \\
\text { standard of error } \\
\text { measurements and minimal } \\
\text { detectable change for total } \\
\text { and segmental volumes in } \\
\text { both arms were performed. } \\
\text { Composite reliability using } \\
\text { Cronbach and Bland \& } \\
\text { Altman was also done. }\end{array}$ & $\begin{array}{l}\text { Lymphoedema arm } \\
\text { compared to 'normal' was } \\
\text { statistically significant at } 7 \% \\
\text { ( }<<0.01 \text { ). } \\
\text { Test-retest reliability was } \\
\text { high for the lymphoedema } \\
\text { arm with ICC }=0.96-0.99 \text { and } \\
\text { the non-lymphoedema arm } \\
\text { where ICC }=0.98-1.00 \text {. } \\
\text { However, the reliability test- } \\
\text { retest for the lymphoedema } \\
\text { hand was only good with } \\
\text { ICC=0.60-0.93 compared to } \\
\text { ICC=0.79-0.91 for the non- } \\
\text { lymphoedema hand. } \\
\text { The SEM for total volume } \\
\text { was } 47.1 \text { ml for non- } \\
\text { lymphoedema arm versus } \\
78.8 \text { ml for the lymphoedema } \\
\text { arm. SEM for segmental } \\
\text { volumes resulted in an } \\
\text { increase of } 66.6 \mathrm{ml} \text { for the } \\
\text { non-lymphoedema arm but a } \\
\text { decrease of } 45.0 \mathrm{ml} \text { for the } \\
\text { lymphoedema arm. }\end{array}$ & $\begin{array}{l}\text { Arm volume determined } \\
\text { by circumferential } \\
\text { measurements } \\
\text { demonstrates high } \\
\text { reliability in the arm but } \\
\text { less for the hand. } \\
\text { Minimal detectable } \\
\text { change for each segment } \\
\text { may be used to assess } \\
\text { real change due to } \\
\text { treatment effect in these } \\
\text { segments but may } \\
\text { overestimate total arm } \\
\text { volume if segments are } \\
\text { summated. }\end{array}$ \\
\hline $\begin{array}{l}\text { Adriaenssens, } \\
\text { Buyl, Lievens, } \\
\text { Fontaine, \& } \\
\text { Lamote, } 2013\end{array}$ & $\begin{array}{l}\text { Compare perometry } \\
\text { with water } \\
\text { displacement and } \\
\text { circumferential } \\
\text { measurements for } \\
\text { the evaluation of arm } \\
\text { volume in women } \\
\text { with and without } \\
\text { lymphoedema }\end{array}$ & $\begin{array}{c}\text { Diagnostic Test } \\
\text { Study }\end{array}$ & $\begin{array}{l}80 \text { participants: } \\
31 \text { healthy } \\
\text { (control); } 49 \\
\text { breast cancer }\end{array}$ & $\begin{array}{l}\text { Ethics and consent gained. } \\
\text { Description of recruitment } \\
\text { given. Measurements taken } \\
\text { include water displacement, } \\
4 \mathrm{~cm} \text { circumferential tape } \\
\text { measure ( } 11 \text { points) using } 3 \\
\text { methods of calculation, and } \\
\text { perometry was done three } \\
\text { times for each side }(0.47 \mathrm{~cm}\end{array}$ & $\begin{array}{l}\text { When comparing dominant } \\
\text { and non-dominant arms in } \\
\text { the control group, the } \\
\text { volume difference was } \\
\text { significantly higher for the } \\
\text { dominant arm (ave } 2.24 \% \text { ). } \\
\text { Perometer ICC for control } \\
\text { group dominant arm was } \\
0.998 \text {; for non-dominant }\end{array}$ & $\begin{array}{l}\text { Perometer should be } \\
\text { used due to ease of use } \\
\text { and reproducibility. } \\
\text { Limb volume change of } \\
\geq 10 \% \text { could be used as } \\
\text { diagnostic threshold } \\
\text { criteria for diagnosis of } \\
\text { BCRL. Single frustrum } \\
\text { method should not be }\end{array}$ \\
\hline
\end{tabular}




\begin{tabular}{|c|c|c|c|c|c|c|}
\hline Citation & Purpose & $\begin{array}{l}\text { Research } \\
\text { Design }\end{array}$ & Sample & Validity of Study & Study Results & Clinical Benefit \\
\hline & & & & $\begin{array}{l}\text { circumference intervals) - } \\
\text { mean calculated as the } \\
\text { absolute volume of arm. } \\
\text { Descriptive statistics, } \\
\text { independent samples t-tests, } \\
\text { paired-samples t-tests and } \\
\text { repeated measures analysis } \\
\text { of variance were performed. } \\
\text { Intra-class correlation } \\
\text { coefficient (ICC) for } \\
\text { absolute agreement was used } \\
\text { to check intra-rater reliability } \\
\text { of the three perometer } \\
\text { measurements. }\end{array}$ & $\begin{array}{l}\text { 0.997. For oedema group, } \\
\text { ICC of oedema arm was } \\
0.999 \text { and for non-oedema } \\
\text { arm } 0.998 \text {. Comparing all } \\
\text { methods: frustrum and disc } \\
\text { model gave the highest } \\
\text { volume estimation; water } \\
\text { displacement gave the lowest } \\
\text { volume estimation; } \\
\text { perometer was in-between. } \\
\text { Control group had a } \\
\text { significant effect in } \\
\text { measurement method } \\
\text { ( } p<0.001 \text { ) and in arm } \\
\text { dominance (p=0.002) for } \\
\text { volume estimation. No } \\
\text { significant interaction effect } \\
\text { ( } p>0.05 \text { ) on arm volume } \\
\text { estimation. BCRL group had } \\
\text { a significant effect of } \\
\text { measurement method } \\
\text { ( } p<0.001 \text { ), side of surgery } \\
\text { ( } p<0.001 \text { ) and interaction } \\
\text { ( } p<0.001 \text { ) between } \\
\text { measurement method and } \\
\text { side of surgery on arm } \\
\text { volume estimation. }\end{array}$ & $\begin{array}{l}\text { used for volume } \\
\text { estimation. }\end{array}$ \\
\hline $\begin{array}{l}\text { Bulley, Coutts, } \\
\& \text { Tan, } 2013\end{array}$ & $\begin{array}{l}\text { Develop a protocol } \\
\text { for the perometer } \\
\text { and determine the } \\
\text { inter- and intra-rater } \\
\text { reliability of the } \\
\text { protocol }\end{array}$ & $\begin{array}{c}\text { Diagnostic Test } \\
\text { Study }\end{array}$ & $\begin{array}{l}30 \text { volunteers: } 7 \\
\text { men; } 23 \text { women }\end{array}$ & $\begin{array}{l}\text { Consent and ethics gained. } \\
\text { Inclusion and exclusion } \\
\text { criteria identified. Protocol } \\
\text { development referred to } 5 \\
\text { questions: } 1 \text { ) What are the } \\
\text { optimal conditions for } \\
\text { standardising measurements? }\end{array}$ & $\begin{array}{l}\text { The high ICC for inter- and } \\
\text { intra-rater reliability in upper } \\
\text { limbs }(0.953-0.989) \text {. } \\
\text { Standard deviation and CoV } \\
\text { were moderate }(\mathrm{SD}=281- \\
309 ; \mathrm{CoV}=18-20) \text {. Limits of } \\
\text { agreement were less positive }\end{array}$ & $\begin{array}{l}\text { Protocol developed to } \\
\text { enable greater } \\
\text { standardisation in the } \\
\text { measurement of limbs; } \\
\text { however, further testing } \\
\text { of the protocol is } \\
\text { required. }\end{array}$ \\
\hline
\end{tabular}




\begin{tabular}{|c|c|c|c|c|c|c|}
\hline Citation & Purpose & $\begin{array}{c}\text { Research } \\
\text { Design }\end{array}$ & Sample & Validity of Study & Study Results & Clinical Benefit \\
\hline & & & & $\begin{array}{l}\text { 2) How can a standard } \\
\text { proportion of the limb be } \\
\text { measured in order to allow } \\
\text { comparisons between and } \\
\text { within individuals? 3) What } \\
\text { is the optimal limb position } \\
\text { within the perometer frame? } \\
\text { 4) Are there any } \\
\text { considerations relating to the } \\
\text { speed of the perometer frame } \\
\text { movement? and 5) Are there } \\
\text { any considerations relating to } \\
\text { the generation or } \\
\text { interpretation of } \\
\text { measurements? Final } \\
\text { protocol tested for inter- and } \\
\text { intra-rater reliability: } 2 \\
\text { researchers took two } \\
\text { recordings on each volunteer } \\
\text { on two occasions, 24-48hrs } \\
\text { apart. Both raters were } \\
\text { blinded to measurement } \\
\text { recordings. Landmarks on } \\
\text { limbs identified and marked. } \\
\text { Dominant limbs were } \\
\text { measured. Shapiro-Wilk test } \\
\text { showed data not normally } \\
\text { distributed for upper limb } \\
\text { (p=0.01-0.02). Parametric } \\
\text { analysis included ICC and } \\
95 \% \text { confidence interval } \\
\text { calculations for both inter- } \\
\text { and intra-rater reliability. } \\
\text { Coefficient of variation }\end{array}$ & $\begin{array}{l}\text { with upper limit range } 85 \text { to } \\
156 \text {, and lower limit range - } \\
85 \text { to }-135 \text {. }\end{array}$ & \\
\hline
\end{tabular}




\begin{tabular}{|c|c|c|c|c|c|c|}
\hline Citation & Purpose & $\begin{array}{c}\text { Research } \\
\text { Design }\end{array}$ & Sample & Validity of Study & Study Results & Clinical Benefit \\
\hline & & & & $\begin{array}{l}(\mathrm{CoV}) \text { was calculated, and } \\
\text { limits of agreement analysis } \\
\text { were conducted. }\end{array}$ & & \\
\hline $\begin{array}{l}\text { Lu, DeSouza, } \\
\text { Armer, } \\
\text { Anderson, \& } \\
\text { Shyu, } 2013\end{array}$ & $\begin{array}{l}\text { Infra-red imaging } \\
\text { (Microsoft Kinect) } \\
\text { as a method of } \\
\text { measuring limb } \\
\text { volume for the early } \\
\text { detection of } \\
\text { lymphoedema }\end{array}$ & $\begin{array}{c}\text { Diagnostic Test } \\
\text { Study }\end{array}$ & $\begin{array}{l}6 \text { subjects: } \\
5 \text { healthy } \\
1 \text { with } \\
\text { lymphoedema; } \\
\text { although } 3 \\
\text { healthy and one } \\
\text { lymphoedema } \\
\text { case reported }\end{array}$ & $\begin{array}{l}\text { No ethics approval or consent } \\
\text { gained reported. Each subject } \\
\text { was scanned with the } \\
\text { perometer and the 3D } \\
\text { camera (Microsoft Kinect) as } \\
\text { the first experiment; then } \\
\text { accuracy to detect localised } \\
\text { swelling was tested as the } \\
\text { second experiment. } \\
\text { Volumes and percentage } \\
\text { volume difference were } \\
\text { calculated for both } \\
\text { experiments. }\end{array}$ & $\begin{array}{l}\text { Perometry shows a } \\
\text { percentage discrepancy of } \\
\text { less than } 13 \% \text { to the 3D } \\
\text { camera - perometer } \\
\text { underestimates volumes } \\
\text { compared to 3D camera. 3D } \\
\text { camera was able to detect a } \\
\text { small change in volume by } \\
\text { detecting } 80 \% \text { of a pen taped } \\
\text { to the forearm of the subject. } \\
\text { Limitation of the study is the } \\
\text { very small cohort }\end{array}$ & $\begin{array}{l}\text { 3D camera can } \\
\text { accurately model the } \\
\text { human arm and can } \\
\text { detect small, localised } \\
\text { differences in limb } \\
\text { volume. }\end{array}$ \\
\hline $\begin{array}{l}\text { Erends, van der } \\
\text { Aa, van der } \\
\text { Hulst, \& de } \\
\text { Grzymala, } \\
2014\end{array}$ & $\begin{array}{l}\text { Assess the validity } \\
\text { and reliability of } 3 \mathrm{D} \\
\text { volume } \\
\text { measurements of the } \\
\text { upper limb compared } \\
\text { to water } \\
\text { displacement }\end{array}$ & $\begin{array}{c}\text { Diagnostic Test } \\
\text { Study }\end{array}$ & $\begin{array}{l}33 \text { subjects with } \\
\text { no } \\
\text { lymphoedema: } \\
20 \text { female; } 13 \\
\text { male }\end{array}$ & $\begin{array}{l}\text { No ethics approval or consent } \\
\text { gained reported. Each subject } \\
\text { was measured by 3D camera } \\
\text { (Vectra XT) and water } \\
\text { displacement twice by two } \\
\text { researchers. Study protocol } \\
\text { described wherein hand was } \\
\text { excluded. Pearson } \\
\text { correlation coefficient, } \\
\text { paired sample } t \text {-test or } \\
\text { Wilcoxon signed-rank test } \\
\text { and Bland and Altman plots } \\
\text { were used. }\end{array}$ & $\begin{array}{l}\text { 3DCI on average } \\
\text { overestimated volume by } \\
13.8 \mathrm{cc} \text { compared to water } \\
\text { displacement; however, there } \\
\text { was a high correlation }(0.98) \\
\text { between the two tests and no } \\
\text { significant difference } \\
\text { between them (p=0.192). } \\
\text { Intra-rater reliability for both } \\
\text { measures was } 0.99 \text {; however, } \\
\text { no significant difference } \\
\text { between the two 3DCI was } \\
\text { noted (p=0.673); but for } \\
\text { water displacement, there } \\
\text { was a significant difference } \\
(\mathrm{p}<0.001) . \text { There was a high } \\
\text { inter-rater reliability of the } \\
\text { 3DCI of } 0.99\end{array}$ & $\begin{array}{l}\text { 3DCI has good validity } \\
\text { and reliability and was } \\
\text { shown to be more } \\
\text { reliable than water } \\
\text { displacement. } \\
\text { Advantage of 3DCI is } \\
\text { that it is suitable for } \\
\text { people with skin lesions, } \\
\text { and there are no contra- } \\
\text { indications for its use. } \\
\text { Currently unsuitable for } \\
\text { measuring the hand due } \\
\text { to the web spaces. } \\
\text { Needs training for this } \\
\text { camera's use due to } \\
\text { specific landmark } \\
\text { notches required. The }\end{array}$ \\
\hline
\end{tabular}




\begin{tabular}{|c|c|c|c|c|c|c|}
\hline Citation & Purpose & $\begin{array}{l}\text { Research } \\
\text { Design }\end{array}$ & Sample & Validity of Study & Study Results & Clinical Benefit \\
\hline & & & & & & $\begin{array}{l}\text { equipment is expensive } \\
\text { and not widely available. }\end{array}$ \\
\hline $\begin{array}{l}\text { Lu, Han, } \\
\text { DeSouza, } \\
\text { Armer, \& } \\
\text { Shya, } 2014\end{array}$ & $\begin{array}{l}\text { Specific research } \\
\text { questions: 1) how } \\
\text { well does the } \\
\text { Iterative Clustered } \\
\text { Closest Points } \\
\text { (ICCP) algorithm } \\
\text { handle smooth } \\
\text { surfaces; 2) how } \\
\text { reliable is the } \\
\text { method in terms of } \\
\text { detecting small, } \\
\text { localised swellings; } \\
\text { and 3) to what extent } \\
\text { are the proposed } \\
\text { method and water } \\
\text { displacement } \\
\text { correlated? }\end{array}$ & $\begin{array}{c}\text { Diagnostic Test } \\
\text { Study }\end{array}$ & $\begin{array}{l}6 \text { human } \\
\text { subjects (12 } \\
\text { arms) for } \\
\text { perometer test; } 7 \\
\text { test subjects for } \\
\text { water } \\
\text { displacement } \\
\text { test }\end{array}$ & $\begin{array}{l}\text { No mention of consent or } \\
\text { ethics. 3D camera calibrated, } \\
\text { algorithm tested and } \\
\text { validated on PVC pipes. } \\
\text { Perometer and 3DCI taken of } \\
\text { both arms, then 3D repeated } \\
\text { with a pen taped to anterior } \\
\text { elbow to detect small } \\
\text { changes in volume. Both } \\
\text { arms tested on } 7 \text { subjects for } \\
\text { water displacement and } \\
\text { repeated after } 2 \text { hours. } \\
\text { Concurrently, six } \\
\text { consecutive measurements } \\
\text { by 3D camera taken of both } \\
\text { arms and averaged for the } \\
\text { corresponding arm. Pearson } \\
\text { product-moment correlations } \\
\text { performed for water } \\
\text { displacement and 3D image; } \\
\text { also mean }+ \text { SD }\end{array}$ & $\begin{array}{l}\text { Differences in volume } \\
\text { between perometer and 3D } \\
\text { are due to differences in arm } \\
\text { length measured and arm } \\
\text { movement and/or position } \\
\text { during the scan (bend at the } \\
\text { elbow). 3D image was able } \\
\text { to pick up the volume of pen } \\
\text { equivalent to less than } 1 \% \text { of } \\
\text { arm volume. A high } \\
\text { correlation between water } \\
\text { displacement and 3D image } \\
\text { of } 0.98317 \text {; with mean for } \\
\text { water displacement at } \\
1560.06 \pm 387.01 ; \text { and for 3D } \\
\text { image, it was } \\
1568.41 \pm 374.66\end{array}$ & $\begin{array}{l}\text { 3D method promising as } \\
\text { correlates well with } \\
\text { water displacement and } \\
\text { picks up the small } \\
\text { localised change in } \\
\text { volume. Requires further } \\
\text { research to address arm } \\
\text { movement during the } \\
\text { scan. Proposed research } \\
\text { to look at breast cancer } \\
\text { cohort to detect an early } \\
\text { change in volume for } \\
\text { diagnosis of } \\
\text { lymphoedema. }\end{array}$ \\
\hline $\begin{array}{l}\text { Öhberg, } \\
\text { Zachrisson, \& } \\
\text { Holmner- } \\
\text { Rocklöv, } 2014\end{array}$ & $\begin{array}{l}\text { Assess the } \\
\text { performance of 3D- } \\
\text { technology in } \\
\text { comparison to } \\
\text { circumference } \\
\text { measurement and } \\
\text { water displacement }\end{array}$ & $\begin{array}{c}\text { Diagnostic Test } \\
\text { Study }\end{array}$ & $\begin{array}{l}25 \text { people with } \\
\text { lymphoedema }\end{array}$ & $\begin{array}{l}\text { Ethics and consent gained. } \\
\text { Power analysis performed } \\
\text { before the study. } \\
\text { Volume of affected and } \\
\text { unaffected arms taken using } \\
\text { CTM, water displacement } \\
\text { and 3D-camera by the same } \\
\text { therapist. The volume of } \\
\text { hand was excluded. } \\
\text { Study protocol described. }\end{array}$ & $\begin{array}{l}\text { 3D camera overestimates } \\
\text { volume compared to water } \\
\text { displacement by } 45.25 \mathrm{ml} \\
(\mathrm{CI}=-36.31-126.82 ; \mathrm{p}=0.27) \\
\mathrm{CTM} \text { underestimates volume } \\
\text { compared to water } \\
\text { displacement } 24.28 \mathrm{ml} \\
(\mathrm{CI}=-99.78-51.22 ; \mathrm{p}=0.52) \\
\text { No statistical significance } \\
\text { found between the three } \\
\text { methods. }\end{array}$ & $\begin{array}{l}\text { Microsoft Kinect camera } \\
\text { offers an alternative to } \\
\text { CTM to measure the } \\
\text { volume of the upper } \\
\text { limb. } \\
\text { Could be useful as an } \\
\text { educational tool for the } \\
\text { patient: graphical } \\
\text { representation of shape } \\
\text { illustrating change over } \\
\text { time could be used as a }\end{array}$ \\
\hline
\end{tabular}




\begin{tabular}{|c|c|c|c|c|c|c|}
\hline Citation & Purpose & $\begin{array}{c}\text { Research } \\
\text { Design }\end{array}$ & Sample & Validity of Study & Study Results & Clinical Benefit \\
\hline & & & & $\begin{array}{l}\text { Two separate mixed-design } \\
\text { ANOVA 's were used, and } \\
\text { Bland-Altman plots. }\end{array}$ & & $\begin{array}{l}\text { motivator to wear a } \\
\text { compression garment. }\end{array}$ \\
\hline $\begin{array}{l}\text { Buffa et al., } \\
2015\end{array}$ & $\begin{array}{l}\text { Validate new } \\
\text { procedure (SkanLab) } \\
\text { for 3-dimensional } \\
\text { estimation of total } \\
\text { arm volume }\end{array}$ & $\begin{array}{l}\text { Diagnostic Test } \\
\text { Study }\end{array}$ & $\begin{array}{l}12 \text { plastic } \\
\text { cylinders; } \\
30 \text { adult's right } \\
\text { arms ( } 15 \text { men } \\
\text { and } 15 \text { women })\end{array}$ & $\begin{array}{l}\text { Ethics and consent gained. } \\
\text { Subjects recruited by } \\
\text { convenience sampling; } \\
\text { exclusion criteria } \\
\text { incorporated. Study protocol } \\
\text { described comparing } \\
\text { SkanLab volumes to water } \\
\text { displacement volumes by } \\
\text { two raters and two } \\
\text { replications. } \\
\text { The statistical analysis } \\
\text { looked at bias and limits of } \\
\text { agreement between } \\
\text { techniques which was } \\
\text { determined by Bland-Altman } \\
\text { method; intra- and inter-rater } \\
\text { reliability was determined by } \\
\text { ICC and SEM, and Student's } \\
\text { t-test assessed duration of } \\
\text { both tests. }\end{array}$ & $\begin{array}{l}\text { Bias of SkanLab was - } \\
21.9 \mathrm{ml}(-5.7 \%) \text { when } \\
\text { measuring cylinder; and for } \\
\text { arm volumes was }-9.9 \mathrm{ml} \\
(-0.6 \%) \text {. No significant } \\
\text { relationship between total } \\
\text { arm volume and accuracy; } \\
\text { however, the relationship } \\
\text { between BMI and accuracy } \\
\text { was highly significant } \\
\text { (p=0.004); high BMI can } \\
\text { result in overestimation of } \\
\text { total arm volume with } \\
\text { SkanLab. Intra- and inter- } \\
\text { rater reliabilities were very } \\
\text { high (ICC >099). Mean } \\
\text { duration for water } \\
\text { displacement was } 2 \text { min } 3 \mathrm{sec} \text {, } \\
\text { and for SkanLab } 42 \mathrm{sec} \text {. } \\
\text { Duration for data cleaning } \\
\text { using MeshLab was } 8 \text { min } \\
\text { 35sec. }\end{array}$ & $\begin{array}{l}\text { SkanLab is a fast, safe } \\
\text { transportable, hygienic } \\
\text { and low-cost method for } \\
\text { assessing total arm } \\
\text { volume. It has a high } \\
\text { level of accuracy and } \\
\text { reliability; thus has } \\
\text { potential as a future } \\
\text { clinical tool. } \\
\text { Due to high accuracy } \\
\text { can be used for persons } \\
\text { at risk of lymphoedema } \\
\text { to monitor for early } \\
\text { intervention. }\end{array}$ \\
\hline $\begin{array}{l}\text { Mori, Lustman, } \\
\text { \& Katz-Leurer, } \\
2015\end{array}$ & $\begin{array}{l}\text { To determine if } \\
\text { people with BCRL } \\
\text { can perform self- } \\
\text { measurement of arm } \\
\text { volume reliably and } \\
\text { validly by using an } \\
\text { adaptive paper tape } \\
\text { with a loop which }\end{array}$ & $\begin{array}{c}\text { Diagnostic Test } \\
\text { Study }\end{array}$ & $\begin{array}{l}17 \text { BCRL } \\
\text { women }\end{array}$ & $\begin{array}{l}\text { Ethics and consent gained. } \\
\text { Inclusion and exclusion } \\
\text { criteria identified. Limb } \\
\text { volume measured by water } \\
\text { displacement and arm } \\
\text { circumference at six arm } \\
\text { levels (including hand) using } \\
\text { a plastic tape measure and } \\
\text { then paper tape with loop }\end{array}$ & $\begin{array}{l}\text { Water displacement volume } \\
\text { difference on day } 1 \text { was } \\
183,2 \mathrm{ml}(\mathrm{p}<0.01) \text { with a } \\
\text { range of } 1.8-40.0 \% \text { excess } \\
\text { limb volume. Self-measure } \\
\text { reliability analysis: ICC } \\
\text { show excellent values (ICC } \\
\text { LO side } 0.97-0.99 ; \text { ICC non- } \\
\text { LO side } 0.96-0.99) \text {. Self- }\end{array}$ & $\begin{array}{l}\text { Self-measurement by } \\
\text { paper tape is a valid and } \\
\text { reliable method. }\end{array}$ \\
\hline
\end{tabular}




\begin{tabular}{|c|c|c|c|c|c|c|}
\hline Citation & Purpose & $\begin{array}{l}\text { Research } \\
\text { Design }\end{array}$ & Sample & Validity of Study & Study Results & Clinical Benefit \\
\hline & $\begin{array}{l}\text { can be tightened } \\
\text { with one hand. }\end{array}$ & & & $\begin{array}{l}\text { form - volume calculated } \\
\text { using the frustrum method. } \\
\text { Procedure described. } \\
\text { Descriptive analysis means } \\
( \pm \text { SD) for continued } \\
\text { variables and percentage for } \\
\text { category variables. Self- } \\
\text { measurement, plastic tape } \\
\text { and water displacement had } \\
\text { test and retest reliability } \\
\text { assessed within a day (day } 1) \\
\text { and between days (day } 1 \text { and } \\
\text { day } 10) \text { using ICC measure. } \\
\text { Pearson correlations used to } \\
\text { assess criterion-related } \\
\text { concurrent validity between } \\
\text { methods. }\end{array}$ & $\begin{array}{l}\text { measure validity analysis: } \\
\text { Pearson correlation } \\
\text { coefficient between self- } \\
\text { measure and water } \\
\text { displacement was } 0.59-0.68 \\
(\mathrm{p}<0.01) \text {; and between self- } \\
\text { measure and the plastic tape } \\
\text { was } 0.88-0.95 \text { ( }<<0.01) \text {. }\end{array}$ & \\
\hline $\begin{array}{l}\text { Tidhar et al., } \\
2015\end{array}$ & $\begin{array}{l}\text { Determine Standard } \\
\text { of Error } \\
\text { Measurement (SEM) } \\
\text { for measuring limb } \\
\text { volume; determine if } \\
\text { there is a difference } \\
\text { between SEM of } \\
\text { healthy versus } \\
\text { lymphoedema limbs } \\
\text { and to examine the } \\
\text { reliability between } \\
\text { therapists when } \\
\text { measuring limbs }\end{array}$ & $\begin{array}{l}\text { Diagnostic Test } \\
\text { Study }\end{array}$ & $\begin{array}{l}41 \text { physio- } \\
\text { therapists; } \\
5 \text { patients }\end{array}$ & $\begin{array}{l}\text { Ethical approval gained; } \\
\text { participants volunteered - no } \\
\text { record of consent gained } \\
\text { from physiotherapists but } \\
\text { consent gained from patients. } \\
\text { Each therapist using } 6 \\
\text { points/landmarks on the limb } \\
\text { measured both limbs three } \\
\text { times. Volume calculated } \\
\text { using the truncated cone } \\
\text { formula. The SEM and } \\
\text { mean scaled SEM were } \\
\text { calculated, and differences } \\
\text { were analysed by Kruskal- } \\
\text { Wallis non-parametric test. } \\
\text { True mean differences of } \\
\text { each therapist's }\end{array}$ & $\begin{array}{l}\text { Average SEM for arm }= \\
27.5 \mathrm{ml} \text {; for leg }=83.6 \mathrm{ml} \text {. } \\
\text { Mean scaled SEM for arm } \\
\text { was } 0.82 \% \text { and for leg } \\
0.64 \% \text {. No statistical } \\
\text { difference in scaled SEM for } \\
\text { arm found ( } \mathrm{p}=0.847) \text {; nor in } \\
\text { leg (p-0.598). } \\
\text { No statistical difference } \\
\text { between healthy and LO } \\
\text { limb in both arm (p=0.945) } \\
\text { and legs (p=0.533). } \\
56 \% \text { of the first } \\
\text { measurement of arms was } \\
\text { within 5\% of true mean } \\
\text { value; } 80.5 \% \text { for legs. }\end{array}$ & $\begin{array}{l}\text { Importance of using } \\
\text { measured points as } \\
\text { opposed to landmarks } \\
\text { for measuring may result } \\
\text { in higher inter-rater } \\
\text { reliability. }\end{array}$ \\
\hline
\end{tabular}




\begin{tabular}{|c|c|c|c|c|c|c|}
\hline Citation & Purpose & $\begin{array}{l}\text { Research } \\
\text { Design }\end{array}$ & Sample & Validity of Study & Study Results & Clinical Benefit \\
\hline & & & & $\begin{array}{l}\text { measurements were } \\
\text { calculated and differences } \\
\text { calculated using Wilcoxon } \\
\text { Rank Sum test. }\end{array}$ & $\begin{array}{l}\text { Study limitations identified: } \\
\text { not all patients were } \\
\text { measured by each therapist, } \\
\text { thus reducing external } \\
\text { validity of study; participants } \\
\text { not assessed within their } \\
\text { clinical teams may have } \\
\text { influenced arm measurement } \\
\text { results between groups. }\end{array}$ & \\
\hline Cau et al., 2016 & $\begin{array}{l}\text { Develop a protocol } \\
\text { for limb volume } \\
\text { assessment using } \\
\text { laser scanner 3D } \\
\text { (LS3D), and } \\
\text { compare LS3D to } \\
\text { circumferential tape } \\
\text { measure. }\end{array}$ & $\begin{array}{c}\text { Diagnostic Test } \\
\text { Study }\end{array}$ & 12 healthy adults & $\begin{array}{l}\text { Ethics and consent gained. } \\
\text { Circumference } \\
\text { measurements (CM) } \\
\text { performed by tape twice by } \\
\text { both expert LO therapists } \\
\text { and volume calculated using } \\
\text { Frustrum method, and LS3D } \\
\text { (taking 2-3min) by two } \\
\text { different operators } \\
\text { identifying same marks used } \\
\text { during CM. Kolmogorov- } \\
\text { Smirnov test done to verify } \\
\text { normal parameters; Mean } \\
\text { and standard deviation (SD) } \\
\text { or median and interquartile } \\
\text { range done depending on } \\
\text { data distribution; Wilcoxon } \\
\text { test for intra-reliability and } \\
\text { inter-reliability by Mann- } \\
\text { Whitney test. Pearson } \\
\text { correlation coefficient; } \\
\text { Bland-Altman plots and non- } \\
\text { parametric tests performed. }\end{array}$ & $\begin{array}{l}\text { CM shows very good intra- } \\
\text { and inter-reliability. } \\
\text { Excellent intra-and inter- } \\
\text { observer reliability with } \\
\text { LS3D. Very high correlation } \\
\text { between CM and LS3D } \\
\left(\mathrm{R}^{2}=0.923 ; P<0.05\right) \text {. Study } \\
\text { limitations identified. }\end{array}$ & $\begin{array}{l}\text { LS3D suitable for limb } \\
\text { assessment as provides a } \\
\text { quantitative objective } \\
\text { evaluation of the } \\
\text { diameters and volume of } \\
\text { the arm quickly. LS3D } \\
\text { can quantify treatment } \\
\text { outcomes based on an } \\
\text { accurate method. LS3D } \\
\text { provides a representation } \\
\text { of the upper limb - } \\
\text { useful in detecting shape } \\
\text { alteration. }\end{array}$ \\
\hline
\end{tabular}




\begin{tabular}{|c|c|c|c|c|c|c|}
\hline Citation & Purpose & $\begin{array}{l}\text { Research } \\
\text { Design }\end{array}$ & Sample & Validity of Study & Study Results & Clinical Benefit \\
\hline $\begin{array}{l}\text { Hameeteman, } \\
\text { Verhulst, } \\
\text { Vreeken, Maal, } \\
\text { \& Ulrich, } 2016\end{array}$ & $\begin{array}{l}\text { Evaluate the use of } \\
\text { 3D stereo- } \\
\text { photogrammetry in } \\
\text { volume } \\
\text { measurements in } \\
\text { people with upper } \\
\text { limb lymphoedema }\end{array}$ & $\begin{array}{c}\text { Prospective } \\
\text { Diagnostic Test } \\
\text { Study }\end{array}$ & $\begin{array}{l}11 \text { patients (22 } \\
\text { arms including } \\
\text { hands) }\end{array}$ & $\begin{array}{l}\text { No mention of ethical } \\
\text { approval or patient consent. } \\
\text { Inclusion and exclusion } \\
\text { criteria identified. Two } \\
\text { measurements were taken of } \\
\text { both arms by 3D stereo- } \\
\text { photogrammetry and water } \\
\text { displacement. Study } \\
\text { protocol described. } \\
\text { Mean difference, variance } \\
\text { and standard deviation for } \\
\text { both methods were } \\
\text { calculated. Variance } \\
\text { component analysis was } \\
\text { performed to determine the } \\
\text { variance in both methods of } \\
\text { measurement. } \\
\text { Pearson's correlation } \\
\text { coefficient determined the } \\
\text { correlation between methods, } \\
\text { and a Bland-Altman plot was } \\
\text { computed. }\end{array}$ & $\begin{array}{l}\text { Mean volume difference in } \\
\text { 3D measure was } 13 \mathrm{ml} \text { and } \\
\text { for water displacement } 35 \mathrm{ml} \\
\text { Paired sample } t \text {-test showed } \\
\text { no significant difference } \\
\text { between the two 3D } \\
\text { measures ( } \mathrm{p}=0.33 \text { ) and the } \\
\text { two water displacement } \\
\text { measures ( } \mathrm{p}=0.84 \text { ). } \\
\text { There was a high correlation } \\
\text { between the means with } \\
\text { Pearson's correlation of } 0.99 \\
\text { (p=0.01) but does not signify } \\
\text { the degree of agreement } \\
\text { between the measurements. } \\
\text { Delta values indicate that } 3 \mathrm{D} \\
\text { measure overestimates the } \\
\text { volume measured by water } \\
\text { displacement by } 11 \mathrm{ml} \text {. } \\
\text { Variances between measures } \\
\text { had } \mathrm{p}<0.00 \text {. High ICC for } \\
\text { both water displacement } \\
\text { (ICC }=0.997 ; \mathrm{p}<0.00 \text { ) and } 3 \mathrm{D} \\
\text { volume (ICC }=1.00 ; \mathrm{p}<0.00 \text { ) } \\
\text { measurements. }\end{array}$ & $\begin{array}{l}3 \mathrm{D} \\
\text { stereophotogrammetry is } \\
\text { an accurate and reliable } \\
\text { method for determining } \\
\text { upper limb volume } \\
\text { measurements. Results } \\
\text { show a statistically } \\
\text { lower variance } \\
\text { compared to water } \\
\text { displacement. }\end{array}$ \\
\hline $\begin{array}{l}\text { Hoevenaren et } \\
\text { al., } 2016\end{array}$ & $\begin{array}{l}\text { Investigating } \\
\text { whether } 3 \mathrm{D} \text { imaging } \\
\text { can be used as a } \\
\text { reproducible and } \\
\text { useful tool for } \\
\text { qualifying and } \\
\text { quantifying } \\
\text { lymphoedema of the } \\
\text { hand }\end{array}$ & $\begin{array}{l}\text { Diagnostic Test } \\
\text { Study - pilot } \\
\text { study }\end{array}$ & $\begin{array}{l}18 \text { people with } \\
\text { unilateral upper } \\
\text { limb } \\
\text { lymphoedema: } \\
9 \text { with hand } \\
\text { oedema, } 9 \\
\text { without hand } \\
\text { oedema; plus }\end{array}$ & $\begin{array}{l}\text { Ethics not obtained as review } \\
\text { board had previously } \\
\text { declared the tool as not } \\
\text { harmful or invasive, but the } \\
\text { study was done in } \\
\text { compliance with } \\
\text { international medical } \\
\text { research ethics. Inclusion }\end{array}$ & $\begin{array}{l}\text { Mean volume difference } \\
\text { between oedema hand and } \\
\text { normal hand was } 73 \mathrm{ml} \text {; } \\
\text { mean difference between } \\
\text { hands without oedema was } 4 \\
\text { ml, mean difference in the } \\
\text { control group was } 8 \mathrm{ml} . \\
\text { Reproducibility showed an } \\
\text { average difference of } 5.2 \mathrm{ml} \text {. }\end{array}$ & $\begin{array}{l}\text { Tool effective and } \\
\text { sensitive in measuring } \\
\text { hand oedema; however, } \\
\text { no comparison made to } \\
\text { other methods of volume } \\
\text { measurement such as } \\
\text { water displacement. }\end{array}$ \\
\hline
\end{tabular}




\begin{tabular}{|c|c|c|c|c|c|c|}
\hline Citation & Purpose & $\begin{array}{l}\text { Research } \\
\text { Design }\end{array}$ & Sample & Validity of Study & Study Results & Clinical Benefit \\
\hline & & & $\begin{array}{l}15 \text { healthy } \\
\text { women }\end{array}$ & $\begin{array}{l}\text { and exclusion criteria } \\
\text { identified. } \\
\text { Both hands captured with 3D } \\
\text { imaging. } \\
\text { Study design described. } \\
\text { Mean difference in hand } \\
\text { volume calculated. Kruskal- } \\
\text { Wallis test with post hoc } \\
\text { analysis performed to } \\
\text { determine significant } \\
\text { differences between groups. } \\
\text { Patient consent gained. }\end{array}$ & $\begin{array}{l}\text { Kruskal-Wallis analysis } \\
\text { showed a statistically } \\
\text { significant difference in hand } \\
\text { volume between groups (chi- } \\
\text { square }=1.89 ; \mathrm{p}<0.00 \text { ). Post } \\
\text { hoc analysis showed a } \\
\text { significant difference } \\
\text { between people with hand } \\
\text { oedema and control group } \\
\text { (p<0.00), and between } \\
\text { people with and without } \\
\text { hand oedema ( }<0.00) . \text { No } \\
\text { significant difference } \\
\text { between non-oedema hand } \\
\text { and control group }(p=0.77)\end{array}$ & \\
\hline $\begin{array}{l}\text { Yamamoto et } \\
\text { al., } 2016\end{array}$ & $\begin{array}{l}\text { Compare arm } \\
\text { volumetry and upper } \\
\text { extremity } \\
\text { lymphoedema (UEL) } \\
\text { index regarding } \\
\text { body-type correction } \\
\text { in non-oedematous } \\
\text { arms }\end{array}$ & $\begin{array}{c}\text { Diagnostic Test } \\
\text { Study }\end{array}$ & $\begin{array}{l}35 \text { participants } \\
\text { with no history } \\
\text { of lymphoedema } \\
\text { or breast cancer }\end{array}$ & $\begin{array}{l}\text { Ethics and consent gained. } \\
\text { Participants' height, weight } \\
\text { and BMI recorded; } \\
\text { measurements at set points } \\
\text { on both arms were taken and } \\
\text { truncated cone volume } \\
\text { method used. } \\
\text { The UEL index was } \\
\text { calculated using } \\
\text { circumferences and BMI. } \\
\text { Arm volume and UEL index } \\
\text { were compared with } \\
\text { corresponding BMI groups. } \\
\text { Statistical analysis used an } \\
\text { analysis of variance. }\end{array}$ & $\begin{array}{l}\text { Mean arm volume was } \\
\text { 1090.9 ml and UEL index } \\
\text { 96.9. Statistically significant } \\
\text { differences (p<0.001) in arm } \\
\text { volume between BMI groups } \\
\text { noted: Low-BMI }(<20) \text { vs } \\
\text { middle-BMI }(20-25) \text {; low- } \\
\text { BMI vs high-BMI ( }>25) \text {; and } \\
\text { middle-BMI vs high-BMI. } \\
\text { Mean UEL indices in low- } \\
\text { BMI= 97.2; middle-BMI= } \\
\text { 96.6 and high-BMI=96.7; no } \\
\text { statistical significance } \\
\text { between groups. } \\
\text { Limitations included } \\
\text { Japanese women only in } \\
\text { study and water } \\
\text { displacement not used for } \\
\text { volume. }\end{array}$ & $\begin{array}{l}\text { Upper extremity } \\
\text { lymphoedema (UEL) } \\
\text { index allow for better } \\
\text { body-type corrected arm } \\
\text { volume evaluation } \\
\text { compared with arm } \\
\text { volumetry. }\end{array}$ \\
\hline
\end{tabular}




\begin{tabular}{|c|c|c|c|c|c|c|}
\hline Citation & Purpose & $\begin{array}{c}\text { Research } \\
\text { Design }\end{array}$ & Sample & Validity of Study & Study Results & Clinical Benefit \\
\hline $\begin{array}{l}\text { Peleg \& Katz- } \\
\text { Leurer, } 2017\end{array}$ & $\begin{array}{l}\text { To assess the effect } \\
\text { of position on arm } \\
\text { volume } \\
\text { measurement }\end{array}$ & $\begin{array}{l}\text { Case-control } \\
\text { study }\end{array}$ & $\begin{array}{l}14 \text { women with } \\
\text { BCRL } \\
16 \text { healthy } \\
\text { volunteers } \\
\text { (control) }\end{array}$ & $\begin{array}{l}\text { Sample size was calculated } \\
\text { to } 14 \text { subjects. No ethics } \\
\text { approval or consent gained } \\
\text { reported. } \\
\text { Study protocol described. 5- } \\
\text { point measurements of both } \\
\text { arms taken and volume } \\
\text { calculated with the truncated } \\
\text { cone method. Calculation } \\
\text { corrections for handedness } \\
\text { were taken into account. } \\
\text { Two arm positions were } \\
\text { measured: } 90^{\circ} \text { shoulder } \\
\text { flexion (horizontal) and arm } \\
\text { dependent (vertical) } \\
\text { alongside the body. Three } \\
\text { measurements were taken in } \\
\text { each position. } \\
\text { Kolmogorov-Smirnov test } \\
\text { for normalcy was performed } \\
\text { for volume outcome } \\
\text { measures. Repeated- } \\
\text { measures analysis of } \\
\text { variance used to assess } \\
\text { differences in volumes in } \\
\text { different positions; as well as } \\
\text { paired } t \text {-test to emphasise } \\
\text { absolute and relative volume } \\
\text { differences. Intra-class } \\
\text { correlation coefficient used } \\
\text { for test-retest reliability. } \\
\text { SEM used to measure } \\
\text { absolute reliability. }\end{array}$ & $\begin{array}{l}\text { ICC values were excellent } \\
\text { for both groups in both arm } \\
\text { positions (ICC } \geq 0.997) \text {. } \\
\text { BCRL group had a mean } \\
\text { volume difference of } 280 \mathrm{ml} \\
\text { in horizontal position and } \\
275 \mathrm{ml} \text { in the vertical } \\
\text { position; the healthy group } \\
\text { had } 84 \mathrm{ml} \text { in horizontal and } \\
108 \mathrm{ml} \text { in vertical positions. } \\
\text { Non-significant interaction } \\
\text { was found between arm size, } \\
\text { position and group on arm } \\
\text { volumes ( } \mathrm{F}_{1,28}=3.30, \\
\mathrm{p}=0.08) \text { Significant } \\
\text { interaction noted in arm } \\
\text { volume between positions } \\
\text { and between groups } \\
\left(\mathrm{F}_{1,28}=4.42 ; \mathrm{p}=0.04\right) \text { : volume } \\
\text { difference with arm position } \\
\text { change more significant in } \\
\text { the healthy group than } \\
\text { BCRL; significant } \\
\text { interaction effect noted in } \\
\text { arm volume between arms } \\
\text { and groups }\left(\mathrm{F}_{1,28}=10.45,\right. \\
\mathrm{p}=0.003) \text { with the difference } \\
\text { in BCRL group larger. } \\
\text { In BCRL group, larger arms } \\
\text { had less volume shift } \\
(1.37 \%) \text { in position change } \\
\text { than small arms }(1.61 \%) ; \\
\text { however, in the normal }\end{array}$ & $\begin{array}{l}\text { Measurements should be } \\
\text { performed in the same } \\
\text { positions. }\end{array}$ \\
\hline
\end{tabular}




\begin{tabular}{|c|c|c|c|c|c|c|}
\hline Citation & Purpose & $\begin{array}{l}\text { Research } \\
\text { Design }\end{array}$ & Sample & Validity of Study & Study Results & Clinical Benefit \\
\hline & & & & $\begin{array}{l}\text { Ethical approval and } \\
\text { informed consent gained. }\end{array}$ & $\begin{array}{l}\text { group this was the opposite } \\
\text { (large }=3.36 \% \text {; small }=2.46 \% \text { ) } \\
\text { Limitations included stage of } \\
\text { BCRL limited to stage } 2 \text {, and } \\
\text { only two arm positions were } \\
\text { studied. }\end{array}$ & \\
\hline $\begin{array}{l}\text { Verhulst et al., } \\
2017\end{array}$ & $\begin{array}{l}\text { Investigate the } \\
\text { reproducibility of } \\
\text { volume } \\
\text { measurements of the } \\
\text { hand and forearm } \\
\text { with the use of a } \\
\text { newly developed } \\
\text { device for landmark } \\
\text { placement, and the } \\
\text { effect of hand } \\
\text { position during } \\
\text { imaging }\end{array}$ & $\begin{array}{l}\text { Diagnostic Test } \\
\text { Study }\end{array}$ & $\begin{array}{l}10 \text { healthy } \\
\text { subjects } \\
\text { ( } 5 \text { male, } 5 \\
\text { female })\end{array}$ & $\begin{array}{l}\text { Ethics and consent gained. } \\
\text { Exclusion criteria identified. } \\
\text { The study protocol is } \\
\text { described. After landmarks } \\
\text { were placed, the hand was } \\
\text { measured twice in neutral, } \\
\text { and then } 45^{\circ} \text { flexion and } 45^{\circ} \\
\text { extension. } \\
\text { Paired sample } t \text {-test used to } \\
\text { determine intra- and inter- } \\
\text { rater comparison once mean } \\
\text { differences were calculated } \\
\text { for consecutive } \\
\text { measurements. } \\
\text { ICC was calculated to } \\
\text { determine intra- and inter- } \\
\text { rater reliability. } \\
\text { One-way ANOVA used to } \\
\text { determine hand position } \\
\text { during 3D imaging. }\end{array}$ & $\begin{array}{l}\text { Paired sample } t \text {-test showed } \\
\text { no significant difference } \\
\text { between measurements } \\
\text { ( } \mathrm{p}=0.75 \text { ); no significant } \\
\text { difference between intra- } \\
\text { rater ( } \mathrm{p}=0.67 \text { ) and inter-rater } \\
\text { ( } \mathrm{p}=0.50) \text { measures. } \\
\text { Both intra- and inter-rater } \\
\text { reliability was excellent } \\
\text { (ICC }=0.99 \text { ). } \\
\text { No significant difference in } \\
\text { hand volume with position } \\
\text { neutral compared to } \\
\text { extension }(\mathrm{p}=0.31 \text { ) and } \\
\text { flexion }(\mathrm{p}=0.22) \text {. }\end{array}$ & $\begin{array}{l}\text { Landmark allows for } \\
\text { better reproducibility. } \\
\text { Although 3D imaging is } \\
\text { fast and low cost; it } \\
\text { requires a highly skilled } \\
\text { 3D specialist to process } \\
\text { the image data. }\end{array}$ \\
\hline $\begin{array}{l}\text { Wang et al., } \\
2017\end{array}$ & $\begin{array}{l}\text { To determine: i) } \\
\text { normative-based } \\
\text { inter-limb } \\
\text { circumference and } \\
\text { volume differences, } \\
\text { ii) impact of age and } \\
\text { body weight on } \\
\text { inter-limb difference, }\end{array}$ & $\begin{array}{l}\text { Diagnostic Test } \\
\text { Study }\end{array}$ & $\begin{array}{l}484 \text { healthy } \\
\text { women of ages: } \\
20-39 \text { yrs }=55 ; \\
40-54 \text { yrs }=79 ; \\
54-64 \text { yrs }=203 ; \\
65-74 \text { yrs }=125 ; \\
75+\text { yrs }=22\end{array}$ & $\begin{array}{l}\text { Ethics and consent gained. } \\
\text { Inclusion and exclusion } \\
\text { criteria identified. A single } \\
\text { session with height, weight } \\
\text { (for BMI) and arm } \\
\text { circumference measured at } \\
10 \mathrm{~cm} \text { intervals and used the } \\
\text { truncated cone method for }\end{array}$ & $\begin{array}{l}\text { The extent of dominance and } \\
\text { location: Dominant limb } \\
\text { circumference significantly } \\
\text { larger than non-dominant } \\
\text { limb and increased } \\
\text { significantly from wrist to } \\
40 \mathrm{~cm} \text { location (Limb } \\
\mathrm{F}_{1,4796}=9.2, \mathrm{p}=0.002 ;\end{array}$ & $\begin{array}{l}\text { Revised thresholds are } \\
\text { appropriate for detection } \\
\text { of LO for western and } \\
\text { eastern women. }\end{array}$ \\
\hline
\end{tabular}




\begin{tabular}{|c|c|c|c|c|c|c|}
\hline Citation & Purpose & $\begin{array}{c}\text { Research } \\
\text { Design }\end{array}$ & Sample & Validity of Study & Study Results & Clinical Benefit \\
\hline & $\begin{array}{l}\text { iii) population- } \\
\text { specific thresholds } \\
\text { for Chinese women, } \\
\text { and iv) if specific } \\
\text { population-based } \\
\text { thresholds are } \\
\text { required }\end{array}$ & & & $\begin{array}{l}\text { volume calculation. Segment } \\
\text { A=ulnar styloid to } 10 \mathrm{~cm} ; \\
\text { Segment B=10-20cm; } \\
\text { Segment } C=20-30 \mathrm{~cm} \text {, and } \\
\text { Segment } D=30-40 \mathrm{~cm} \text {. Data } \\
\text { presented as mean } \pm \text { SD for } \\
\text { both arms and segments. } \\
\text { Inter-limb differences } \\
\text { expressed as mean }(95 \% \\
\text { confidence interval [CI]). } \\
\text { ANOVA determined } \\
\text { whether limb and location } \\
\text { along limb affected } \\
\text { circumference or volume } \\
\text { measurements. Duncan's post } \\
\text { hoc tests identified where } \\
\text { significant differences } \\
\text { occurred. Absolute (ml) and } \\
\text { relative (ratio) inter-limb } \\
\text { differences calculated for } \\
\text { volume and circumference. } \\
\text { Inter-limb differences tested } \\
\text { for outliers using Tukey "far } \\
\text { out" test. Univariate } \\
\text { ANOVA determined } \\
\text { differences between absolute } \\
\text { and relative measures; } \\
\text { Duncan's post hoc analysis } \\
\text { used to determine where } \\
\text { differences occurred. } \\
\text { Regression analysis used to } \\
\text { determine if age or BMI } \\
\text { related to inter-limb } \\
\text { differences in circumference }\end{array}$ & $\begin{array}{l}\text { Location } \mathrm{F}_{4,4796}=4820.3, \\
\text { p }<0.001) \text { with no interaction } \\
\text { identified. Duncan's post hoc } \\
\text { analysis showed a significant } \\
\text { difference between } 5 \\
\text { locations. Limb volumes } \\
\text { larger along length of limb } \\
\text { (Limb } \mathrm{F}_{1,3840}=6.6, \mathrm{p}=0.01 ; \\
\text { Location } \mathrm{F}_{3,3840}=2598.2, \\
\text { p }<0.001) \text { with no interaction } \\
\text { identified. Duncan's post hoc } \\
\text { analysis showed that each } \\
\text { location volume differed } \\
\text { significantly to each other. } \\
\text { Absolute and relative } \\
\text { differences: } 8 \text { cases removed } \\
\text { in outlier test. Absolute mean } \\
\text { inter-limb differences in } \\
\text { circumference were } \\
\text { significantly different } \\
\text { ranging from } 0.06 \mathrm{~cm} \text { at wrist } \\
\text { to 0.32cm at } 40 \mathrm{~cm} \text { location. } \\
\text { Post hoc analysis showed no } \\
\text { significant difference at } \\
\text { locations. Absolute inter- } \\
\text { limb volume difference } \\
\text { among segments differed } \\
\text { with least mean volume } \\
\text { difference in Seg A ( } 3.1 \mathrm{ml} \text {; } \\
95 \% \text { CI: } 1.6-4.7 \mathrm{ml} \text { and } \\
\text { greatest in Seg D (10.2ml; } \\
95 \% \text { CI: } 7.4-13.0 \mathrm{ml}) \text {. Post } \\
\text { hoc analysis showed no } \\
\text { significant difference }\end{array}$ & \\
\hline
\end{tabular}




\begin{tabular}{|c|c|c|c|c|c|c|}
\hline Citation & Purpose & $\begin{array}{c}\text { Research } \\
\text { Design }\end{array}$ & Sample & Validity of Study & Study Results & Clinical Benefit \\
\hline & & & & $\begin{array}{l}\text { or volume. Thresholds for } \\
\text { classifying women with LO } \\
\text { determined for } \\
\text { circumference and volume } \\
\text { with diagnostic cut off at } \\
\text { mean plus } 2 \text { and } 3 \text { times SD. }\end{array}$ & $\begin{array}{l}\text { between Seg B and C and } \\
\text { between Seg C and D. Mean } \\
\text { inter-limb relative } \\
\text { circumference ratios differed } \\
\text { significantly ranging from } \\
1.01(95 \% \text { CI: } 1.00-1.01) \text { at } \\
\text { the wrist to } 1.01 \text { ( } 95 \% \text { CI: } \\
1.01-1.02) \text { at } 40 \mathrm{~cm} \mathrm{location.} \\
\text { Post hoc analysis showed } \\
\text { wrist significantly different } \\
\text { to } 40 \mathrm{~cm} \text { location but not to } \\
\text { others. Volume ratios not } \\
\text { significantly different } \\
\text { ranging from } 1.01 \text { ( } 95 \% \mathrm{CI} \text { : } \\
1.01-1.02) \text { to } 1.02(95 \% \mathrm{CI} \\
1.02-1.03) \text {. Effect of age and } \\
B M I \text { : Age and BMI did not } \\
\text { affect any measures. } \\
\text { Thresholds for detection of } \\
L O \text { : No significant } \\
\text { differences between inter- } \\
\text { limb circumference } \\
\text { differences at } 10,20 \text { and } \\
30 \mathrm{~cm} \text {; these were collapsed } \\
\text { for a single cut-off point. } \\
2 S D \text { cut-offs for the } \\
\text { dominant side were } 0.7 \mathrm{~cm} \text { at } \\
\text { the wrist, } 1.8 \mathrm{~cm} \text { for } \\
\text { collapsed sites and } 2.3 \mathrm{~cm} \text { for } \\
40 \mathrm{~cm} \text { location. } 2 S D \text { cut-offs } \\
\text { for the non-dominant side } \\
\text { were } 0.6,1.4 \text { and } 1.6 \mathrm{~cm} \\
\text { respectively. }\end{array}$ & \\
\hline
\end{tabular}




\begin{tabular}{|c|c|c|c|c|c|c|}
\hline Citation & Purpose & $\begin{array}{l}\text { Research } \\
\text { Design }\end{array}$ & Sample & Validity of Study & Study Results & Clinical Benefit \\
\hline $\begin{array}{l}\text { Yamamoto, } \\
\text { Yamamoto, \& } \\
\text { Yoshimatsu, } \\
2017\end{array}$ & $\begin{array}{l}\text { Evaluate the } \\
\text { feasibility of } \\
\text { localised arm } \\
\text { volume index } \\
\text { (LAVI) compared } \\
\text { with localised arm } \\
\text { volumetry }\end{array}$ & $\begin{array}{l}\text { Diagnostic Test } \\
\text { Study }\end{array}$ & $\begin{array}{l}53 \text { healthy } \\
\text { volunteers } \\
1 \text { bilateral upper } \\
\text { limb } \\
\text { lymphoedema } \\
\text { patient }\end{array}$ & $\begin{array}{l}\text { Ethical approval gained. } 5 \\
\text { point limb circumference } \\
\text { measurements were taken } \\
(k=1-5) \text { by two researchers. } \\
\text { LAVI is defined as the } \\
\text { percentage proportion of 1- } \\
\text { UELI } \text { (upper extremity } \\
\text { lymphoedema index = arm } \\
\text { circumference and } \\
\text { length/BMI) compared with } \\
\text { s1-UELI }(\text { standard localised } \\
\text { UELI). } \\
\text { Intra- and Pearson's } \\
\text { correlation coefficient tested } \\
\text { inter-rater reliability. } \\
\text { Mann-Whitney } U \text { test } \\
\text { analysed variability in body } \\
\text { physique (high and low BMI } \\
\text { groups). }\end{array}$ & $\begin{array}{l}\text { Interrater reliability of } \\
\mathrm{LAVI}_{k} \text { ranged from } 0.989 \text { to } \\
0.997 . \text { Intra-rater reliability } \\
\text { of } \mathrm{LAVI}_{k} \text { ranged from } 0.983 \text { - } \\
0.998 \text {. } \\
\text { LAVI is less affected by } \\
\text { body physique (BMI) } \\
\text { compared with volumetry. } \\
\text { Limitations include using } \\
\text { circumferential tape measure } \\
\text { as opposed to water } \\
\text { displacement; women only } \\
\text { and Japanese population } \\
\text { only. }\end{array}$ & $\begin{array}{l}\text { Localised arm volume } \\
\text { index is calculated only } \\
\text { with } \mathrm{C}_{k} \text { and BMI. It is a } \\
\text { useful tool to detect } \\
\text { localised arm volume } \\
\text { change (even for } \\
\text { bilateral BCRL). When } \\
\text { LAVI is higher than } \\
100 \% \text {, the region is } \\
\text { considered larger than } \\
\text { standard volume. }\end{array}$ \\
\hline $\begin{array}{l}\text { Batista, } \\
\text { Baiocchi, } \\
\text { Campanholi, } \\
\text { Bergmann, \& } \\
\text { Duprat, 2018 }\end{array}$ & $\begin{array}{l}\text { Evaluate how two } \\
\text { commonly used } \\
\text { techniques for } \\
\text { measuring limb } \\
\text { volume, } \\
\text { circumferential } \\
\text { measurement (CM) } \\
\text { and perometry, relate } \\
\text { to each other when } \\
\text { performed in the } \\
\text { same subject. }\end{array}$ & $\begin{array}{c}\text { Diagnostic Test } \\
\text { Study }\end{array}$ & $\begin{array}{l}\text { Convenient } \\
\text { sample of } 91 \\
\text { patients: } 52 \\
\text { men, } 39 \text { women }\end{array}$ & $\begin{array}{l}\text { Ethics and consent gained. } \\
\text { Sample characteristics } \\
\text { described. Both arms were } \\
\text { measured using CM and } \\
\text { perometry for a length of } \\
40 \mathrm{~cm} \text { from the wrist. CM } \\
\text { was measured once by the } \\
\text { same investigator, had five } \\
\text { girth measurements taken } \\
\text { and used the truncated cone } \\
\text { formula. Perometer was } \\
\text { taken three times, and the } \\
\text { mean of the limb volumes } \\
\text { was utilised. Continuous } \\
\text { variables are presented by }\end{array}$ & $\begin{array}{l}\text { A very strong correlation } \\
\text { between the methods exists } \\
\text { with Pearson's correlation } \\
\text { coefficients of rV=0.99, } \\
\text { rVD }=0.88 \text { and rPEV }=0.86 \text {. } \\
\text { Bland-Altman plots display a } \\
\text { visual confirmation of the } \\
\text { above. On average, the } \\
\text { largest arm by perometry } \\
\text { was } 10.6 \mathrm{ml} \text { smaller than that } \\
\text { of CM. LOA ranged from- } \\
202 \mathrm{ml} \text { to } 181 \text { ml. LOA for } \\
\text { volume difference of the } \\
\text { same subject was }-101 \text { to } \\
141 \mathrm{ml}, \text { with a mean }\end{array}$ & $\begin{array}{l}\text { Considerable } \\
\text { measurement bias was } \\
\text { found between the two } \\
\text { methods; thus, a direct } \\
\text { comparison of the two } \\
\text { method's results should } \\
\text { be made with caution. } \\
\text { Limb volume is subject } \\
\text { to change with time of } \\
\text { day, temperature, } \\
\text { hydration status and use } \\
\text { of compression - } \\
\text { challenging to control in } \\
\text { clinical studies. }\end{array}$ \\
\hline
\end{tabular}




\begin{tabular}{|c|c|c|c|c|c|c|}
\hline Citation & Purpose & $\begin{array}{c}\text { Research } \\
\text { Design }\end{array}$ & Sample & Validity of Study & Study Results & Clinical Benefit \\
\hline & & & & $\begin{array}{l}\text { their median and } \\
\text { interquartile ranges, and } \\
\text { categorical variables by their } \\
\text { absolute and relative } \\
\text { distributions. Absolute } \\
\text { volume (V) of the biggest } \\
\text { arm, volume difference (VD) } \\
\text { between arms and relative } \\
\text { difference (PEV) between } \\
\text { two arms were estimated for } \\
\text { each subject. The agreement } \\
\text { between CM and perometry } \\
\text { was compared for these three } \\
\text { outcomes using Pearson's } \\
\text { correlation and Bland- } \\
\text { Altman's method. Limits of } \\
\text { agreement (LOA) were } \\
\text { calculated to 95\%. }\end{array}$ & $\begin{array}{l}\text { difference of } 19.9 \mathrm{ml} \text {, while } \\
\text { PEV had a mean difference } \\
\text { of } 0.9 \% \text {, with LOA ranging } \\
\text { from }-5 \text { to } 6.8 \% \text {. Study } \\
\text { limitations reported include } \\
10 \mathrm{~cm} \text { girth measurements as } \\
\text { opposed to } 4 \mathrm{~cm}, \mathrm{CM} \\
\text { measurement taken once, } \\
\text { sample mainly non- } \\
\text { lymphoedema subjects, and } \\
\text { excludes the hand in the } \\
\text { assessment. }\end{array}$ & \\
\hline Cau et al., 2018 & $\begin{array}{l}\text { Comparison of } \\
\text { circumferential arm } \\
\text { measurements (CM) } \\
\text { by tape to } \\
\text { measurements } \\
\text { obtained by laser } \\
\text { scanner 3D (LS3D). }\end{array}$ & $\begin{array}{l}\text { Prospective } \\
\text { Cohort Study }\end{array}$ & $\begin{array}{l}200 \text { women with } \\
\text { upper limb } \\
\text { lymphoedema }\end{array}$ & $\begin{array}{l}\text { Ethics and consent gained. } \\
\text { Inclusion and exclusion } \\
\text { criteria mentioned. Both } \\
\text { arms measured by 4cm CM } \\
\text { and volume calculated using } \\
\text { Frustrum method, and LS3D. } \\
\text { Kolmogorov-Smirnov test } \\
\text { done to verify normal } \\
\text { parameters; Mean and } \\
\text { standard deviation (SD) or } \\
\text { median and interquartile } \\
\text { range done depending on } \\
\text { data distribution; Spearman } \\
\text { correlation coefficient; } \\
\text { Bland-Altman plots and } \\
\text { Wilcoxon tests performed. }\end{array}$ & $\begin{array}{l}\text { Spearman correlation } \\
\text { coefficient between volumes } \\
\text { determined for } \mathrm{LO} \text { arm was } \\
\text { good }\left(\mathrm{R}^{2}=0.738 ; P<0.05\right) ; \\
\text { for 'normal' arm was good } \\
\left(\mathrm{R}^{2}=0.762 ; P<0.05\right) .\end{array}$ & $\begin{array}{l}\text { LS3D could be a reliable } \\
\text { and valid method to } \\
\text { asses limb } \\
\text { circumference and } \\
\text { volume within LO } \\
\text { clinic. Has the ability to } \\
\text { measure geometric and } \\
\text { shape parameters. Is } \\
\text { less time consuming to } \\
\text { perform than CM. }\end{array}$ \\
\hline
\end{tabular}




\begin{tabular}{|c|c|c|c|c|c|c|}
\hline Citation & Purpose & $\begin{array}{l}\text { Research } \\
\text { Design }\end{array}$ & Sample & Validity of Study & Study Results & Clinical Benefit \\
\hline $\begin{array}{l}\text { Karakashian, } \\
\text { Shaban, Pike, } \\
\text { \& van Loon, } \\
2018\end{array}$ & $\begin{array}{l}\text { Examine the use of } \\
\text { 3D cameras to scan } \\
\text { upper limbs and } \\
\text { capture shape } \\
\text { information; identify } \\
\text { shape-related metrics } \\
\text { to diagnose and } \\
\text { monitor } \\
\text { lymphoedema }\end{array}$ & $\begin{array}{c}\text { Service } \\
\text { evaluation }\end{array}$ & $\begin{array}{l}24 \text { BCRL } \\
\text { women } \\
7 \text { healthy } \\
\text { subjects }\end{array}$ & $\begin{array}{l}\text { Inclusion and exclusion } \\
\text { criteria identified. R\&D } \\
\text { approval gained for service } \\
\text { evaluation and informed } \\
\text { consent obtained. } \\
\text { Compared 4cm } \\
\text { circumferential volume } \\
\text { measurements to 3D } \\
\text { imaging. Study protocol } \\
\text { described. } \\
\text { k-means clustering used to } \\
\text { achieve patient grouping. } \\
\text { Repeatability, ICC using } \\
\text { McGraw and Wong } \\
\text { formulations were analysed, } \\
\text { and paired-sample } t \text {-test was } \\
\text { used to distinguish healthy } \\
\text { and affected arms using } \\
\text { circumference and circularity } \\
\text { measurements. }\end{array}$ & $\begin{array}{l}\text { Repeatability of 3D imaging } \\
\text { demonstrated a } 2.52 \mathrm{~L} \\
\text { standard deviation (SD) with } \\
\text { a coefficient variation of } \\
0.99 \% \text {. A test-retest analysis } \\
\text { of healthy subjects (ICC) } \\
\text { was } 0.95 \text {. Low-resolution } \\
\text { scans produced increased } \\
\text { volumes of 5.76\%; thus, } \\
\text { high-resolution 3D imaging } \\
\text { is advised. Shape related } \\
\text { metric such as circumference } \\
\text { and circularity were used to } \\
\text { distinguish between healthy } \\
\text { and affected arms (p } \leq 0.05 \text { ). } \\
\text { Swelling maps were created } \\
\text { to identify the distribution of } \\
\text { swelling on arms which } \\
\text { could be used for diagnosis } \\
\text { and monitoring of } \\
\text { lymphoedema. }\end{array}$ & $\begin{array}{l}\text { Shape as a measure of } \\
\text { lymphoedema provides } \\
\text { the potential for detailed } \\
\text { additional information in } \\
\text { diagnosing and } \\
\text { monitoring } \\
\text { lymphoedema. } \\
\text { Current methods of } \\
\text { calculating limb volume } \\
\text { assume arm is circular; } \\
\text { as this is untrue, these } \\
\text { methods could be } \\
\text { overestimating limb } \\
\text { volumes by } \approx 100 \mathrm{ml} \text {. } \\
\text { 3D scan takes about } 20 \\
\text { to } 30 \text { seconds to } \\
\text { perform; therefore is } \\
\text { time-efficient. }\end{array}$ \\
\hline $\begin{array}{l}\text { Landau, Kim, } \\
\text { Gould, \& Patel, } \\
2018\end{array}$ & $\begin{array}{l}\text { Determine the } \\
\text { feasibility of the } \\
\text { Vectra 3D system to } \\
\text { quantify volume in } \\
\text { BCRL }\end{array}$ & $\begin{array}{c}\text { Diagnostic Test } \\
\text { Study }\end{array}$ & $\begin{array}{l}11 \text { women with } \\
\text { BCRL }\end{array}$ & $\begin{array}{l}\text { Informed consent gained; no } \\
\text { mention of ethical approval. } \\
\text { 3D imaging of fist, forearm } \\
\text { and upper arm to } 15 \mathrm{~cm} \\
\text { proximal to the olecranon. } \\
\text { Circumferential } \\
\text { measurements were taken } \\
\text { every } 4 \mathrm{~cm} \text { from ulnar styloid } \\
\text { process to } 15 \mathrm{~cm} \text { above } \\
\text { olecranon, and water } \\
\text { displacement (WD) } \\
\text { performed up to } 15 \mathrm{~cm} \text { mark. } \\
\text { Each technique determined }\end{array}$ & $\begin{array}{l}\text { All measurements showed } \\
\text { good agreement. 3D had } \\
\text { excellent correlation with } \\
\text { WD (R2 }=0.987) \text { with an } \\
\text { average difference of } 2 \% .3 \mathrm{D} \\
\text { had excellent correlation } \\
\text { with circumferential volume } \\
(0.991) \text { with an average } \\
\text { difference of } 2.8 \% \text {. Time } \\
\text { taken to do 3D measure }= \\
5.9 \mathrm{~min} \text {; circumference }= \\
15.7 \mathrm{~min} \text { and } \mathrm{WD}=24.9 \mathrm{~min}\end{array}$ & $\begin{array}{l}\text { 3D imaging potential for } \\
\text { clinical use as is fast, } \\
\text { accurate, precise and } \\
\text { reliable for measuring } \\
\text { upper limb volume }\end{array}$ \\
\hline
\end{tabular}




\begin{tabular}{|c|c|c|c|c|c|c|}
\hline Citation & Purpose & $\begin{array}{c}\text { Research } \\
\text { Design }\end{array}$ & Sample & Validity of Study & Study Results & Clinical Benefit \\
\hline & & & & $\begin{array}{l}\text { limb volume. Microsoft } \\
\text { Excel was used to calculate } \\
\text { the averages, and standard } \\
\text { deviations and } \mathrm{R}^{2} \text { values } \\
\text { were calculated using } \\
\text { standard linear regression. }\end{array}$ & & \\
\hline $\begin{array}{l}\text { Preuß, Killaars, } \\
\text { Piatkowski de } \\
\text { Grzymala, } \\
\text { Binnebösel, \& } \\
\text { Neumann, } \\
2018\end{array}$ & $\begin{array}{l}\text { To investigate the } \\
\text { reliability of Vectra } \\
\text { XT for the } \\
\text { measurement of arm } \\
\text { volume in BCRL } \\
\text { patients and to } \\
\text { compare these } \\
\text { results to volumes } \\
\text { obtained by water } \\
\text { displacement (WD) }\end{array}$ & $\begin{array}{l}\text { Cross-sectional } \\
\text { Diagnostic Test } \\
\text { Study }\end{array}$ & $\begin{array}{l}37 \text { women with } \\
\text { unilateral BCRL }\end{array}$ & $\begin{array}{l}\text { Ethics and consent gained. } \\
\text { All participants had both } \\
\text { arms measured twice by both } \\
\text { WD and Vectra 3D imaging. } \\
\text { Arm length is taken from } \\
30 \% \text { to } 80 \% \text { of total arm } \\
\text { length (measurement } \\
\text { excluded hand volume). } \\
\text { Power of analysis, p<0.05 } \\
\text { determined } 33 \text { participants } \\
\text { required for the study. } \\
\text { Participant characteristics } \\
\text { and results were described } \\
\text { using descriptive statistics. } \\
\text { Pearson correlation } \\
\text { coefficient to assess } \\
\text { associations between WD } \\
\text { and 3D imaging. Paired } t \text {-test } \\
\text { applied to determine the } \\
\text { mean volume differences } \\
\text { between the measurements. } \\
\text { Bland-Altman plots used to } \\
\text { analyse agreement between } \\
\text { the methods. Intra-rater } \\
\text { reliability of WD and 3D } \\
\text { imaging determined by ICC. } \\
\text { Inter-rater reliability not } \\
\text { assessed as has been }\end{array}$ & $\begin{array}{l}\text { Mean arm length was } \\
70.6 \mathrm{~cm} \text { (SD 3.1) for both } \\
\text { arms. Validity: High } \\
\text { correlation shown between } \\
\text { WD and 3D for healthy arms } \\
(0.996) \text { and BCRL arms } \\
(0.998) \text {. WD measured } \\
\text { higher limb volumes than 3D } \\
\text { but was not significant for } \\
\text { healthy arms (p=0.058), but } \\
\text { was significant for BCRL } \\
\text { arms (p<0.001). Bland- } \\
\text { Altman plots show high } \\
\text { agreement between } \\
\text { measures. In healthy and } \\
\text { BCRL arms, } 86.5 \% \text { and } \\
\text { 89.2\% of the mean volume } \\
\text { difference was less than } \\
\text { 50ml. Reliability: Intra-rater } \\
\text { reliability for WD was high } \\
\text { for both arms (ICC=0.998); } \\
\text { for 3D, both arms had ICC of } \\
\text { 0.999. No limitations } \\
\text { identified. }\end{array}$ & $\begin{array}{l}\text { 3D imaging by Vectra } \\
\text { XT is safe, reliable and } \\
\text { valid; but time- } \\
\text { consuming. }\end{array}$ \\
\hline
\end{tabular}




\begin{tabular}{|c|c|c|c|c|c|c|}
\hline Citation & Purpose & $\begin{array}{l}\text { Research } \\
\text { Design }\end{array}$ & Sample & Validity of Study & Study Results & Clinical Benefit \\
\hline & & & & $\begin{array}{l}\text { previously determined. A } \\
\text { significance level of } \leq 0.05 \\
\text { considered statistically } \\
\text { significant. }\end{array}$ & & \\
\hline $\begin{array}{l}\text { Sharkey et al., } \\
2018\end{array}$ & $\begin{array}{l}\text { To assess the intra- } \\
\text { and interclass } \\
\text { correlation of } \\
\text { circumferential tape } \\
\text { measure and } \\
\text { perometry to } \\
\text { establish the most } \\
\text { appropriate method } \\
\text { for clinical practice } \\
\text { and research, and to } \\
\text { compare the } \\
\text { accuracy and } \\
\text { reliability of tape } \\
\text { measurement against } \\
\text { perometry }\end{array}$ & $\begin{array}{c}\text { Diagnostic Test } \\
\text { Study }\end{array}$ & $\begin{array}{l}35 \text { arm and } 26 \\
\text { leg volunteers: } \\
\text { Student group } \\
\text { measured } 23 \\
\text { arms and } 22 \\
\text { legs; Expert } \\
\text { group measured } \\
12 \text { arms and } 14 \\
\text { legs }\end{array}$ & $\begin{array}{l}\text { Ethics gained. Tape measure } \\
\text { calculation used was the } \\
\text { summation of } 4 \mathrm{~cm} \text { points } \\
\text { using } \pi \mathrm{r}^{2} \mathrm{~h} \text {. Perometer takes } \\
\text { measurements at every } \\
4.7 \mathrm{~mm} \text {. The student group } \\
\text { was shown a video } \\
\text { demonstrating measuring } \\
\text { techniques and then } \\
\text { measured the right arm or leg } \\
\text { of a healthy volunteer twice. } \\
\text { The expert group performed } \\
\text { two measurements of each } \\
\text { method. For inter-observer } \\
\text { reliability, the mean and 95\% } \\
\text { confidence intervals (CI) } \\
\text { were calculated. For intra- } \\
\text { observer reliability, } \\
\text { Cronbach's alpha was used to } \\
\text { determine the correlation } \\
\text { between measures. }\end{array}$ & $\begin{array}{l}\text { Tape measure: SE was lower } \\
\text { in the expert group (expert } \\
\mathrm{SE}=25 \text {; student } \mathrm{SE}=29.55) \text {; } \\
\text { expert CI was narrower. } \\
\text { Perometer: lower SE and CI } \\
\text { for both groups. SE } \\
\text { perometer for both groups = } \\
24.88 \text { vs tape SE average = } \\
28.04 \text { for arms and SE } \\
\text { Perometer = } 22 \text { vs tape SE = } \\
114.10 \text { for legs. However } \\
\text { expert group arms tape SE = } \\
25 \text { vs Perometer SE = 38.99. } \\
\text { Expert data shows excellent } \\
\text { internal consistency with } \\
\text { Perometer (Cronbach's } \\
\text { alpha= } 0.90) \text { and tape } \\
\text { (Cronbach's alpha = 0.94). } \\
\text { Broadly, Perometer more } \\
\text { accurate as had smaller SE } \\
\text { and narrower CIs than tape. } \\
\text { Major limitation is low } \\
\text { numbers of participants; } \\
\text { thus, no conclusive outcome } \\
\text { made. }\end{array}$ & $\begin{array}{l}\text { The expertise of value } \\
\text { for consistency of } \\
\text { measurements. } \\
\text { Perometer indicative of } \\
\text { being more accurate } \\
\text { than tape. }\end{array}$ \\
\hline Sun et al., 2018 & $\begin{array}{l}\text { Use a method of } \\
\text { simulated } \\
\text { circumferential } \\
\text { measurement to }\end{array}$ & $\begin{array}{c}\text { Diagnostic Test } \\
\text { Study }\end{array}$ & $\begin{array}{l}\text { Random } \\
\text { selection of } 287 \\
\text { women with } \\
\text { unilateral breast }\end{array}$ & $\begin{array}{l}\text { Ethical approval or consent } \\
\text { not reported. All participants } \\
\text { have both arms prospectively } \\
\text { measured with perometry as }\end{array}$ & $\begin{array}{l}\text { The correlation coefficient } \\
\text { was consistently }>0.98 \text { for all } \\
\text { measurement methods when } \\
\text { compared to perometry. }\end{array}$ & $\begin{array}{l}\text { Landmark measurement } \\
\text { superior to interval- } \\
\text { based method; however, } \\
\text { it underestimates the }\end{array}$ \\
\hline
\end{tabular}




\begin{tabular}{|c|c|c|c|c|c|c|}
\hline Citation & Purpose & $\begin{array}{c}\text { Research } \\
\text { Design }\end{array}$ & Sample & Validity of Study & Study Results & Clinical Benefit \\
\hline & $\begin{array}{l}\text { compare perometry } \\
\text { to volumetric tape } \\
\text { measurement for the } \\
\text { detection of BCRL, } \\
\text { utilising both } \\
\text { anatomic landmark- } \\
\text { and interval-based } \\
\text { techniques. }\end{array}$ & & $\begin{array}{l}\text { cancer stratified } \\
\text { by BMI }\end{array}$ & $\begin{array}{l}\text { part of a local screening } \\
\text { programme. Utilising these } \\
\text { perometer data, simulated } \\
\text { points were determined for } \\
4 \mathrm{~cm} \text { intervals, anatomical } \\
\text { landmarks and } 10 \mathrm{~cm} \\
\text { proximal and distal to the } \\
\text { elbow. Calculations of these } \\
\text { points used the frustrum } \\
\text { method and were compared } \\
\text { to the perometer calculated } \\
\text { volumes. The volume of the } \\
\text { hand was excluded. } \\
\text { Perometer measurements } \\
\text { were treated as the reference } \\
\text { technique for the analysis. } \\
\text { The Bland-Altman method } \\
\text { used to compute the mean } \\
\text { difference with 95\% } \\
\text { confidence interval. Two-by- } \\
\text { two confusion matrix } \\
\text { analysis performed for each } \\
\text { simulated measurement } \\
\text { against perometry, first for } \\
\text { full cohort, then stratified by } \\
\text { BMI. }\end{array}$ & $\begin{array}{l}\text { There was no significant } \\
\text { difference in total arm } \\
\text { volumes between all } \\
\text { methods used and perometry. } \\
\text { Anatomical landmark and } \\
10 \mathrm{~cm} \text { either side of elbow } \\
\text { significantly underestimated } \\
\text { upper arm volume compared } \\
\text { to perometry; and } \\
\text { overestimation of the } \\
\text { forearm. For lymphoedema } \\
\text { screening, the landmark and } \\
10 \mathrm{~cm} \text { method had greater } \\
\text { sensitivity ( } 93.1 \text { and } 90.3 \% \\
\text { vs. } 81.9 \text { and } 77.8 \%) \\
\text { specificity (93.5\% vs. } 68.4 \\
\text { and } 92.6 \%), \text { positive } \\
\text { predictive value (82.7 and } \\
82.3 \% \text { vs. } 46.5 \text { and } 77.8 \%), \\
\text { and negative predictive value } \\
\text { (97.6 and } 96.6 \% \text { vs. } 91.9 \text { and } \\
92.6 \% \text { compared to } 4 \mathrm{~cm} \\
\text { interval method. Methods } \\
\text { comparable to perometry at } \\
\text { relative volume change } \\
\text { (RVC) of } \geq 10 \% \text {; but } \\
\text { sensitivity reduced to 63.2- } \\
66.7 \% \text { for RVC 5-10\%. BMI } \\
\text { did not affect confusion } \\
\text { matrixes. Limitations } \\
\text { identified include the } \\
\text { inability to account for test- } \\
\text { retest reliability and human } \\
\text { error of tape measurement }\end{array}$ & $\begin{array}{l}\text { upper arm volumes and } \\
\text { overestimates forearm } \\
\text { volumes compared to } \\
\text { perometry. }\end{array}$ \\
\hline
\end{tabular}




\begin{tabular}{|c|c|c|c|c|c|c|}
\hline Citation & Purpose & $\begin{array}{c}\text { Research } \\
\text { Design }\end{array}$ & Sample & Validity of Study & Study Results & Clinical Benefit \\
\hline & & & & & $\begin{array}{l}\text { due to simulation of the } \\
\text { circumferences; and the } \\
\text { exclusion of the hand due to } \\
\text { limited research in } \\
\text { perometry. }\end{array}$ & \\
\hline $\begin{array}{l}\text { De Snyder et } \\
\text { al., } 2019\end{array}$ & $\begin{array}{l}\text { To improve } \\
\text { positioning during } \\
\text { perometry to reduce } \\
\text { perometer-acquired } \\
\text { arm volume } \\
\text { measurements } \\
\text { variability }\end{array}$ & $\begin{array}{c}\text { Diagnostic Test } \\
\text { Study }\end{array}$ & $\begin{array}{l}30 \text { patients and } 5 \\
\text { healthy } \\
\text { volunteers }\end{array}$ & $\begin{array}{l}\text { Board-level approval gained. } \\
\text { No consent reported. } \\
\text { Baseline calibration: Two } \\
\text { operators measured both } \\
\text { arms of } 30 \text { patients three } \\
\text { times. Lymphoedema } \\
\text { diagnosed as volume } \\
\text { difference of } \geq 10 \% \text {. } \\
\text { Repeatability, reproducibility } \\
\text { and gage precision/tolerance } \\
\text { (P/T) ratio were calculated. } \\
\text { Regression analysis used to } \\
\text { determine the association } \\
\text { between BMI, height, or } \\
\text { weight and variability in } \\
\text { measurements. Type I } \\
\text { (overdiagnosis) and type II } \\
\text { (underdiagnosis) errors were } \\
\text { calculated. } \\
\text { Larger group assessment: } 23 \\
\text { therapists of mixed skill } \\
\text { level were divided into } \\
\text { cohorts of 5-6 therapists who } \\
\text { measured five healthy } \\
\text { volunteers. Type I and Type } \\
\text { II errors were calculated for } \\
\text { each of the cohorts. } \\
\text { Optimisation of patient } \\
\text { positioning: Handlebars and }\end{array}$ & $\begin{array}{l}\text { Baseline calibration: P/T } \\
\text { ratio was } 19.9 \%, \\
\text { repeatability } 65 \% \text {, } \\
\text { reproducibility } 19 \% \text {. Type I } \\
\text { error was } 3.5 \% \text { and type II } \\
2.3 \% \text { based on } 10 \% \text { volume } \\
\text { difference; for } 5 \% \text { volume } \\
\text { difference, it was } 18.2 \% \text { for } \\
\text { type I and } 17.0 \% \text { for type II } \\
\text { error. Univariate analysis } \\
\text { showed significance for BMI } \\
\text { (p<0.001), height (p=0.003) } \\
\text { and weight (p<0.001). } \\
\text { Multiple regression showed } \\
\text { height and BMI with the } \\
\text { highest R-squared values } \\
\text { (R }{ }^{2}=16.27 \text { ) } \\
\text { Larger group: Type I error } \\
\text { was } 9.8 \% \text { and type II } 7.8 \% \\
\text { based on } 10 \% \text { volume } \\
\text { difference, and for } 5 \% \\
\text { volume difference it was } \\
25.9 \% \text { for type I and } 24.9 \% \\
\text { for type II errors. } \\
\text { Optimisation: Reduced } \\
\text { variation in limb volume by } \\
28 \% \text { (p=0.02). } \\
\text { Larger group: With } \\
\text { experienced therapists, the }\end{array}$ & $\begin{array}{l}\text { Space for and cost of a } \\
\text { perometer are } \\
\text { prohibitive to many } \\
\text { clinics. Standardisation } \\
\text { of perometry procedure } \\
\text { is critical to minimise } \\
\text { variability. }\end{array}$ \\
\hline
\end{tabular}




\begin{tabular}{|c|c|c|c|c|c|c|}
\hline Citation & Purpose & $\begin{array}{l}\text { Research } \\
\text { Design }\end{array}$ & Sample & Validity of Study & Study Results & Clinical Benefit \\
\hline & & & & $\begin{array}{l}\text { wall bars were installed. One } \\
\text { operator measured both arms } \\
\text { of } 20 \text { patients three times in } \\
\text { original and new position. } \\
\text { Variation calculated by the } \\
\text { standard deviation of the } \\
\text { three measurements divided } \\
\text { by the average of the mean } \\
\text { of the three arm } \\
\text { measurements. Student's } t \text { - } \\
\text { test used to compare two } \\
\text { positions. } \\
\text { New position larger group: } 8 \\
\text { therapists measured eight } \\
\text { volunteers three times each. } \\
\text { Type I and type II errors } \\
\text { were quantified. } \\
\text { CTM measurement: Four } \\
\text { therapists measured both } \\
\text { arms of } 3 \text { volunteers at } 5 \\
\text { points. Gag P/T ratio was } \\
\text { calculated for: } \\
\text { 1) } 2 \text { cm circumferential } \\
\text { difference } \\
\text { 2) } 10 \% \text { volume difference }\end{array}$ & $\begin{array}{l}\text { gage } \mathrm{P} / \mathrm{T} \text { ratio was } 6.5 \%, \\
\text { repeatability } 2.4 \% \text { and } \\
\text { reproducibility } 6.1 \% \text {. Type I } \\
\text { and type II errors were } 0 \% \\
\text { for } 10 \% \text { volume difference, } \\
\text { and for } 5 \% \text { volume } \\
\text { difference type I error was } \\
0.04 \% \text {, and type II was } \\
0.02 \% \text {. With a mixed skill } \\
\text { group, the gage P/T ratio was } \\
18.7 \% \text {, repeatability } 15.0 \% \\
\text { and reproducibility } 11.0 \% \text {. } \\
\text { CTM: Arm volume gage P/T } \\
\text { ratio was } 9.7 \%\end{array}$ & \\
\hline $\begin{array}{l}\text { De Vrieze et } \\
\text { al., } 2019\end{array}$ & $\begin{array}{l}\text { To investigate the } \\
\text { reliability, time } \\
\text { efficiency and } \\
\text { clinical feasibility of } \\
\text { five commonly used } \\
\text { methods for } \\
\text { assessing excessive } \\
\text { arm volume in } \\
\text { patients with BCRL }\end{array}$ & $\begin{array}{l}\text { Cross-Sectional } \\
\text { Study }\end{array}$ & $\begin{array}{l}30 \text { women with } \\
\text { unilateral BCRL }\end{array}$ & $\begin{array}{l}\text { Ethics and consent gained. } \\
\text { Inclusion and exclusion } \\
\text { criteria identified. Five } \\
\text { methods of excessive arm } \\
\text { volume determined: M1) } \\
\text { Traditional volumetry with } \\
\text { overflow, overflow is } \\
\text { weighed; M2) Volumetry } \\
\text { without overflow; upward }\end{array}$ & $\begin{array}{l}\text { Mean age was } 65 \text { years, and } \\
\text { mean BMI was } 28 \text {. Intra- } \\
\text { rater reliability showed ICCs } \\
\text { ranging from } 0.777 \text { to } 0.987 \text {. } \\
\text { M5 had the highest ICC } \\
\text { value. Inter-rater reliability } \\
\text { had ICCs ranging from } 0.791 \\
\text { to } 0.984 \text {, with M5 at highest } \\
\text { ICC value. Set up time: M } 2\end{array}$ & $\begin{array}{l}\text { CTM considered as the } \\
\text { most appropriate method } \\
\text { for clinical practice. } \\
\text { Limitations include a } \\
\text { relatively small cohort, } \\
\text { and a lower limb } \\
\text { optoelectric device being } \\
\text { utilised; this was }\end{array}$ \\
\hline
\end{tabular}




\begin{tabular}{|c|c|c|c|c|c|c|}
\hline Citation & Purpose & $\begin{array}{c}\text { Research } \\
\text { Design }\end{array}$ & Sample & Validity of Study & Study Results & Clinical Benefit \\
\hline & & & & $\begin{array}{l}\text { displaced water weighed } \\
\text { when arm submerged; M3) } \\
\text { Inverse water volumetry; } \\
\text { shortness of water is } \\
\text { measured; M4) Perometry } \\
\text { (no hand volume); M5) CTM } \\
\text { using truncated cone method } \\
\text { (no hand volume). Both } \\
\text { arms measured at one visit } \\
\text { and all measures repeated } \\
\text { three times by two different } \\
\text { raters. VD calculated by } \\
\text { subtracting non-oedematous } \\
\text { from oedematous arm } \\
\text { volumes. Blinding was } \\
\text { preserved. The timing was } \\
\text { captured as 1) preparation; 2) } \\
\text { execution and 3) total time } \\
\text { taken. Data analysis included } \\
\text { descriptive statistics with } \\
\text { mean } \pm \text { SD and median and } \\
\text { interquartile ranges. Intra- } \\
\text { class correlation coefficients } \\
\text { used to examine inter- and } \\
\text { intra-rater reliability } \\
\text { (interpretations of outcomes } \\
\text { described). SEM and paired- } \\
\text { samples } t \text {-test were applied. } \\
\text { One-way ANOVA assisted } \\
\text { with post hoc analyses used. } \\
\text { Data used to compile a } \\
\text { ranking table to include } \\
\text { reliability and timing of each } \\
\text { method }\end{array}$ & $\begin{array}{l}\text { required the least time ( } 4 \mathrm{~min} \\
\text { 40sec); with a statistically } \\
\text { significant difference } \\
\text { between M1 and M2 } \\
\text { (p<0.01). Execution time: } \\
\text { M2 had the lowest time ( } 56 \\
\text { sec), M3 had the highest } \\
\text { time (5min } 34 \mathrm{sec})(\mathrm{p}<0.01) \text {. } \\
\text { Total time: M4 fastest ( } 1 \mathrm{~min} \\
\text { 43sec); all others statistically } \\
\text { significantly different } \\
\text { (p<0.05). Ranking table: M5 } \\
\text { scored the highest overall } \\
\text { rank }\end{array}$ & $\begin{array}{l}\text { remedied through a strict } \\
\text { positioning protocol }\end{array}$ \\
\hline
\end{tabular}




\begin{tabular}{|c|c|c|c|c|c|c|}
\hline Citation & Purpose & $\begin{array}{c}\text { Research } \\
\text { Design }\end{array}$ & Sample & Validity of Study & Study Results & Clinical Benefit \\
\hline $\begin{array}{l}\text { Karakashian, } \\
\text { Pike, \& van } \\
\text { Loon, } 2019\end{array}$ & $\begin{array}{l}\text { To use } \\
\text { computational } \\
\text { methods for } \\
\text { elucidating the effect } \\
\text { of limb shape on } \\
\text { sub-garment and } \\
\text { subcutaneous } \\
\text { pressures, stresses } \\
\text { and strains }\end{array}$ & $\begin{array}{l}\text { Diagnostic Test } \\
\text { Study }\end{array}$ & $\begin{array}{l}23 \text { women with } \\
\text { unilateral BCRL }\end{array}$ & $\begin{array}{l}\text { R\&D approval and consent } \\
\text { gained. 3DCI images of } \\
\text { lymphoedematous arms were } \\
\text { utilised for the simulation of } \\
\text { mathematical geometries } \\
\text { looking at the effects of sub- } \\
\text { garment pressures, } \\
\text { subcutaneous stresses and } \\
\text { strains. The forearm segment } \\
\text { of the lymphoedema arm } \\
\text { was used. Equations are } \\
\text { provided for each simulation. }\end{array}$ & $\begin{array}{l}\text { Delivered pressure and } \\
\text { Laplace Law: due to non- } \\
\text { circularity of the forearm, } \\
\text { higher pressures are recorded } \\
\text { at the mid-forearm - higher } \\
\text { than the wrist (i.e. negative } \\
\text { gradient pressure). Higher } \\
\text { mean maximum pressures } \\
\text { were recorded at } 5100 \mathrm{~Pa} \text {, } \\
\text { even though intended } \\
\text { pressure from manufacturer } \\
\text { is } 2500 \text { Pa. Subcutaneous } \\
\text { stresses and strains: adipose } \\
\text { tissues follow garment } \\
\text { pressure patterns more } \\
\text { closely than stiffer tissues. } \\
\text { Ironically there was a } \\
\text { positive gradient pressure in } \\
\text { the subcutaneous tissues } \\
\text { under the garment pressures. }\end{array}$ & $\begin{array}{l}\text { Limitation identified } \\
\text { was that garment } \\
\text { properties and } \\
\text { geometries were not } \\
\text { included in this work. } \\
\text { This mathematical } \\
\text { modelling informs } \\
\text { clinical effect and use of } \\
\text { compression garments } \\
\text { and can inform a } \\
\text { garment's design, } \\
\text { leading to optimal } \\
\text { treatment plans. }\end{array}$ \\
\hline $\begin{array}{l}\text { Lu \& Dixon, } \\
2019\end{array}$ & $\begin{array}{l}\text { To demonstrate that } \\
\text { a single stationary } \\
\text { Kinect for Windows } \\
\text { v2 IR sensor, in } \\
\text { conjunction with a } \\
\text { custom image } \\
\text { processing } \\
\text { algorithm, is a tool } \\
\text { that can be } \\
\text { implemented in a } \\
\text { clinical setting to } \\
\text { calculate arm } \\
\text { volume in BCRL }\end{array}$ & $\begin{array}{l}\text { Diagnostic Test } \\
\text { Study }\end{array}$ & $\begin{array}{l}73 \text { breast cancer } \\
\text { survivors; } 30 \\
\text { with } \\
\text { lymphoedema }\end{array}$ & $\begin{array}{l}\text { Consent gained. Inclusion } \\
\text { and exclusion criteria } \\
\text { identified. Protocol for } \\
\text { Kinect IR system and } \\
\text { perometer described. Linear } \\
\text { regression analysis was } \\
\text { performed to determine the } \\
\text { level of correlation between } \\
\text { the perometer and Kinect IR } \\
\text { system. A Bland-Altman plot } \\
\text { was used to compare the two } \\
\text { methods with regards to limb } \\
\text { volume difference. Cohen's } \\
\text { kappa agreement for }\end{array}$ & $\begin{array}{l}\text { Arm volumes in relation to } \\
\text { limb length had a correlation } \\
\text { between the perometer and } \\
\text { Kinect IR system of } 0.84 \text {. } \\
\text { With matched arm lengths, } \\
\text { the correlation value } \\
\text { increased to } 0.88 \text {. Percentage } \\
\text { difference correlation was } \\
\text { good with R-squared ranged } \\
\text { at } 0.63-0.71 \text {. The Cohen's } \\
\text { kappa coefficient indicates } \\
\text { fair agreement with } 10 \% \\
\text { volume difference }(\mathrm{k}=0.27) \\
\text { and moderate agreement }\end{array}$ & $\begin{array}{l}\text { The Kinect IR system is } \\
\text { a fast, cost-efficient and } \\
\text { accurate method for the } \\
\text { assessment of } \\
\text { lymphoedema }\end{array}$ \\
\hline
\end{tabular}




\begin{tabular}{|c|c|c|c|c|c|c|}
\hline Citation & Purpose & $\begin{array}{c}\text { Research } \\
\text { Design }\end{array}$ & Sample & Validity of Study & Study Results & Clinical Benefit \\
\hline & $\begin{array}{l}\text { with requisite } \\
\text { clinical accuracy }\end{array}$ & & & $\begin{array}{l}\text { categorical data was utilised } \\
\text { to compare the outcomes of } \\
\text { both modalities using } \\
\text { lymphoedema thresholds. } \\
\text { Mann-Whitney tests were } \\
\text { performed comparing } \\
\text { lymphoedema to non- } \\
\text { lymphoedema groups. }\end{array}$ & $\begin{array}{l}\text { with } 200 \mathrm{ml} \text { difference } \\
\text { (k=0.55). Bland-Altman } \\
\text { analysis of volume } \\
\text { differences indicated a bias } \\
\text { of } 6.02 \mathrm{ml} \text {; thus, a minor } \\
\text { correction factor would be } \\
\text { necessary between the two } \\
\text { modalities. Limitations } \\
\text { identified include the cost of } \\
\text { the system - cheaper than the } \\
\text { perometer; inability to } \\
\text { calculate volume based on an } \\
\text { elliptical cross-section, and } \\
\text { the Kinect sensor has been } \\
\text { discontinued }\end{array}$ & \\
\hline $\begin{array}{l}\text { Rafn, } \\
\text { McNeely, } \\
\text { Camp, } \\
\text { Midtgaard, \& } \\
\text { Campbell, } \\
2019\end{array}$ & $\begin{array}{l}\text { To develop and test } \\
\text { the reliability and } \\
\text { validity of a written } \\
\text { and video-supported } \\
\text { protocol for women } \\
\text { with breast cancer to } \\
\text { self-measure arm } \\
\text { circumference }\end{array}$ & $\begin{array}{l}\text { Cross-sectional } \\
\text { reliability and } \\
\text { validity study }\end{array}$ & $\begin{array}{l}41 \text { participants: } \\
20 \text { healthy; } \\
21 \text { BCRL }\end{array}$ & $\begin{array}{l}\text { Consent gained. Inclusion } \\
\text { criteria identified. Methods } \\
\text { of measurement described: } \\
\text { 1) CTM home self- } \\
\text { measurement, 2) CTM lab } \\
\text { self-measurement, 3) CTM } \\
\text { therapist measurement, and } \\
\text { 4) perometer measurement. } \\
\text { Analysis conducted for each } \\
\text { group of participants, and the } \\
\text { affected and unaffected arms } \\
\text { in BCRL group. Intra- and } \\
\text { interrater correlations (ICC) } \\
\text { were calculated using 95\% } \\
\text { confidence intervals. Pearson } \\
\text { correlation and levels of } \\
\text { agreement between lab self- } \\
\text { measurement and perometer } \\
\text { were calculated to determine }\end{array}$ & $\begin{array}{l}\text { Non-BCRL group: high to } \\
\text { excellent intrarater reliability } \\
\text { between 1) and 2) } \\
(\mathrm{ICC} \geq 0.86,95 \% \text { CI o.64- } \\
0.94, \mathrm{p}<0.001) \text {. Interrater } \\
\text { reliability was high to } \\
\text { excellent between 2) and 3) } \\
\text { (ICC } \geq 0.88,95 \% \text { CI } 0.67- \\
0.96, \mathrm{p}<0.001) \text {. Correlation } \\
\text { between 2) and 4) was } 0.95 \\
\text { (p<0.001). } \\
\text { BCRL group: excellent } \\
\text { intrarater reliability between } \\
\text { 1) and 2) (ICC } \geq 0.93,95 \% \text { CI } \\
0.83-0.97, \text { p }<0.001) \text {. } \\
\text { Interrater reliability between } \\
\text { 2) and 3) was excellent } \\
\text { (ICC }>0.91,95 \% \text { CI } 0.41- \\
0.98, p<0.001) \text {. Correlation }\end{array}$ & $\begin{array}{l}\text { Self-monitoring of limb } \\
\text { size may be a cost- } \\
\text { efficient means of early } \\
\text { identification of BCRL } \\
\text { to commence earlier } \\
\text { treatment and better } \\
\text { prognosis. Those with } \\
\text { BCRL can detect } \\
\text { exacerbations and seek } \\
\text { help more quickly; or } \\
\text { can be used a means of } \\
\text { self-monitoring of the } \\
\text { self-management of their } \\
\text { BCRL. }\end{array}$ \\
\hline
\end{tabular}




\begin{tabular}{|c|c|c|c|c|c|c|}
\hline Citation & Purpose & $\begin{array}{l}\text { Research } \\
\text { Design }\end{array}$ & Sample & Validity of Study & Study Results & Clinical Benefit \\
\hline & & & & $\begin{array}{l}\text { criterion validity. Bland- } \\
\text { Altman plots used for lab } \\
\text { self-measurement and } \\
\text { perometer mean differences } \\
(\%) \text {. Self-reported ease of } \\
\text { performing the measurement } \\
\text { at home versus the lab was } \\
\text { compared using } t \text {-test. }\end{array}$ & $\begin{array}{l}\text { between } 2) \text { and } 4) \text { was } 0.98 \\
(\mathrm{p}<0.001) \text {. } \\
\text { No limitations were } \\
\text { identified. }\end{array}$ & \\
\hline $\begin{array}{l}\text { Spinelli et al., } \\
2019\end{array}$ & $\begin{array}{l}\text { To develop and } \\
\text { assess intra- and } \\
\text { interrater reliability } \\
\text { and validity of a } \\
\text { clinical evaluation } \\
\text { tool for BCRL, for } \\
\text { use in the context of } \\
\text { outcome evaluation } \\
\text { in clinical trials }\end{array}$ & $\begin{array}{c}\text { Blinded } \\
\text { Repeated } \\
\text { Measures } \\
\text { Observational } \\
\text { Study }\end{array}$ & $\begin{array}{l}71 \text { breast cancer } \\
\text { survivors with } \\
\text { and without } \\
\text { BCRL }\end{array}$ & $\begin{array}{l}\text { Ethics and consent gained. } \\
\text { Inclusion and exclusion } \\
\text { criteria defined. The new } \\
\text { BCRL of the Upper } \\
\text { Extremity (CLUE) tool is } \\
\text { validated against perometry } \\
\text { and the Norman } \\
\text { lymphoedema survey. CLUE } \\
\text { consists of 1) obscuration of } \\
\text { the anatomical architecture } \\
\text { which was rated as none, } \\
\text { close inspection, or readily } \\
\text { apparent; } 2 \text { ) anatomical } \\
\text { contour which was rated as } \\
\text { none, readily apparent or } \\
\text { gross deviation; } 3 \text { ) tissue } \\
\text { texture assessment which } \\
\text { was rated as normal, spongy, } \\
\text { firm or hard; and } 4 \text { ) pitting } \\
\text { or non-pitting oedema } \\
\text { present which was rated as } \\
\text { none, non-pitting or pitting. } \\
\text { A sophisticated scoring } \\
\text { system ranges from } 0 \text { to } 72 \text {, } \\
\text { where } 0 \text { indicate no } \\
\text { lymphoedema. }\end{array}$ & $\begin{array}{l}\text { CLUE tool excludes } \\
\text { Stemmers sign and skin folds } \\
\text { due to low presentation } \\
\text { within-study sample. The } \\
\text { intra-rater reliability for } \\
\text { perometry had an ICC of } \\
0.93 \text { (95\% CI, 0.72-0.99); } \\
\text { the ICC for CLUE was } 0.88 \\
\text { (95\% CI, 0.71-0.96). The } \\
\text { interrater reliability for } \\
\text { perometry was } 0.98 \text { ( } 95 \% \\
\text { CI, 0.97-0.99), and for } \\
\text { CLUE the ICC was } 0.90 \\
\text { (95\% CI, 0.79-0.95). Pearson } \\
\text { product-moment correlation } \\
\text { coefficient between CLUE } \\
\text { and perometry was } 0.79 ; \\
\text { between CLUE and Norman } \\
\text { lymphoedema survey ranged } \\
\text { from } 0.40 \text { to } 0.53 \text {. } \\
\text { Limitations included } \\
\text { experienced therapists only } \\
\text { as raters, inability to recruit } \\
\text { sufficiently into all } \\
\text { lymphoedema categories, } \\
\text { and the use of lymphoedema }\end{array}$ & $\begin{array}{l}\text { The CLUE tool aims to } \\
\text { provide an outcomes } \\
\text { score that can be utilised } \\
\text { in clinical trials to } \\
\text { standardise measures of } \\
\text { improvement. CLUE } \\
\text { could be an outcomes } \\
\text { tool in clinical practice } \\
\text { against which to } \\
\text { measure treatment } \\
\text { efficacy. }\end{array}$ \\
\hline
\end{tabular}




\begin{tabular}{|c|c|c|c|c|c|c|}
\hline Citation & Purpose & $\begin{array}{l}\text { Research } \\
\text { Design }\end{array}$ & Sample & Validity of Study & Study Results & Clinical Benefit \\
\hline & & & & $\begin{array}{l}\text { Descriptive data were } \\
\text { derived for the three tests } \\
\text { and reported within a stage } \\
\text { of lymphoedema. ICCs } \\
\text { calculated to determine intra- } \\
\text { and interrater reliability with } \\
\text { a 95\% confidence interval. } \\
\text { Pearson product-moment } \\
\text { correlation coefficients to } \\
\text { determine validity between } \\
\text { the three tests was performed } \\
\text { with scatterplots for graphic } \\
\text { representation. }\end{array}$ & $\begin{array}{l}\text { severity being reduced to a } \\
\text { single score being of value } \\
\text { clinically. }\end{array}$ & \\
\hline $\begin{array}{l}\text { Binkley et al., } \\
2020\end{array}$ & $\begin{array}{l}\text { To estimate the } \\
\text { reliability and } \\
\text { validity of the } \\
\text { LymphaTech for } \\
\text { measuring arm } \\
\text { volume in the } \\
\text { context of women } \\
\text { seeking care in a } \\
\text { speciality breast } \\
\text { cancer rehabilitation } \\
\text { clinic }\end{array}$ & $\begin{array}{l}\text { Cross-sectional } \\
\text { reliability and } \\
\text { convergent } \\
\text { validity study }\end{array}$ & $\begin{array}{l}66 \text { women with } \\
\text { BCRL or at risk } \\
\text { of BCRL: } \\
30 \text { with BCRL, } \\
36 \text { at risk }\end{array}$ & $\begin{array}{l}\text { Informed consent gained. No } \\
\text { ethics approval, but study } \\
\text { approval was granted by the } \\
\text { LymphaTech Institutional } \\
\text { Review Board Protocol for } \\
\text { each device was given. } \\
\text { Reliability Design: A } \\
\text { convenience sample of } 30 \\
\text { participants was measured } \\
\text { twice by each device: two } \\
\text { measurements by two raters } \\
\text { were taken by LymphaTech } \\
\text { device, and perometer had } \\
\text { two measurements by one } \\
\text { rater. } \\
\text { Validity Design: } 66 \\
\text { participants have at least one } \\
\text { paired measurement on each } \\
\text { device. } \\
\text { Analysis included means and } \\
\text { SDs for participant }\end{array}$ & $\begin{array}{l}\text { Participant time of cancer } \\
\text { treatment was current or } \\
\text { within } 3 \text { years of initial } \\
\text { diagnosis and treatment. } \\
\text { Majority had bilateral } \\
\text { mastectomy with } \\
\text { reconstruction, average } \\
\text { number of lymph nodes } \\
\text { removed was eight, and } \\
\text { approximately 50\% had } \\
\text { radiotherapy. } \\
\text { Reliability: LymphaTech and } \\
\text { Perometer had ICC of }>0.99 \\
\text { for a single measure, and } \\
0.97 \text { for limb VD. SEM for } \\
\text { LymphaTech and perometer } \\
\text { single measure was } 28.3- \\
35.8 m 1, \text { and for limb VD it } \\
\text { was } 38.0-40.7 m l . \\
\text { Mean volume for } 66 \text { limbs } \\
\text { was } 2215.9 \text { (SD=637.7)ml }\end{array}$ & $\begin{array}{l}\text { Arms }>3000 \mathrm{ml} \text { has more } \\
\text { error associated with } \\
\text { volume estimations for } \\
\text { both LymphaTech and } \\
\text { perometer. Error is } \\
\text { reduced by averaging } \\
\text { two measurements. } \\
\text { The device is portable, } \\
\text { thus easy to use in } \\
\text { clinical and community } \\
\text { settings. }\end{array}$ \\
\hline
\end{tabular}




\begin{tabular}{|c|c|c|c|c|c|c|}
\hline Citation & Purpose & $\begin{array}{c}\text { Research } \\
\text { Design }\end{array}$ & Sample & Validity of Study & Study Results & Clinical Benefit \\
\hline & & & & $\begin{array}{l}\text { characteristics as continuous } \\
\text { measures, and counts or } \\
\text { percentages for categorical } \\
\text { data. Estimates of all } \\
\text { parameter included } 95 \% \text { CIs. } \\
\text { Analysis performed using } \\
\text { STATA v15.1. Inter-rater } \\
\text { reliability was tested for } \\
\text { LymphaTech and intra-rater } \\
\text { reliability for perometer, and } \\
\text { validation analyses } \\
\text { performed included Bland- } \\
\text { Altman plot and ICC. }\end{array}$ & $\begin{array}{l}\text { for LymphaTech and } 2223.7 \\
\text { (SD=622.2)ml for perometer. } \\
\text { Thus, LymphaTech } \\
\text { underestimates volume } \\
\text { compared to perometry by } \\
7.8 \mathrm{ml} \text {. } \\
\text { Validity: ICC was } 0.99 \text { ( } 95 \% \\
\mathrm{CI}=0.98-0.99) \text { for affected } \\
\text { limb; and ICC was } 0.98 \\
(95 \% \mathrm{CI}=0.97-0.99) \text { for } \\
\text { unaffected limb. Agreement } \\
\text { showed a mixture of either } \\
\text { device providing larger limb } \\
\text { volumes. }\end{array}$ & \\
\hline
\end{tabular}




\section{APPENDIX 4: Ethics and R\&D Approval}

Hewitt J.L. $\quad$ Q Cheryl Pike (ABM ULHB - Lymphoedema); $\square$ Terry K. .

RE: CHHS and CoM Research Ethics Committee

(i) Follow up. Completed on 03 May 2016.

You forwarded this message on 15/03/2016 08:06.

\section{From:}

Sent: 11 March 2016 13:09

To: Cheryl Pike (ABM ULHB - Lymphoedema)

Cc:

Subject: RE: CHHS and CoM Research Ethics Committee

Dear Cheryl

Approval for your study is now granted.

\section{Best Wishes}

Jeanette

Dr. J.L.Hewitt

Associate Professor / Athro Cynorthwyol

Chair, CHHS and College of Medicine Research Ethics Committee

www.swansea.ac.uk/humanandhealthsciences/research/research-ethics-committee

Department of Philosophy, History \& Law / Uwch Ddarlithydd, Adran Athroniaeth, Hanes a'r Gyfraith

College of Human and Health Sciences / Coleg y Gwyddorau Dynol ac Iechyd

Swansea University / Prifysgol Abertawe

Singleton Park / Parc Singleton

Swansea / Abertawe

Wales / Cymru

SA2 8PP

www.swansea.ac.uk $\perp$ www.abertawe.ac.uk

E-mail/ Ebost:

Editorial Board, Ethics and Social Welfare

http://www.tandf.co.uk/journals/cfp/reswcfp.pdf

Board Member, The European Association of Centres of Medical Ethics (EACME)

http://www.eacmeweb.com/

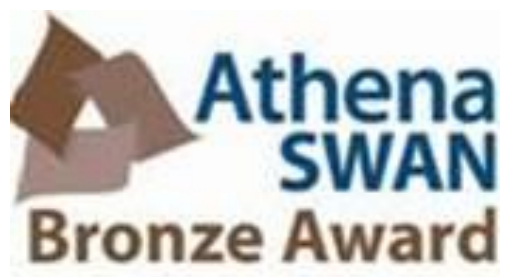


From: Cheryl Pike (ABM ULHB - Lymphoedema) [mailto:

Sent: 11 March 2016 12:54

To:

Cc:

Subject: RE: CHHS and CoM Research Ethics Committee

Dear Dr. Hewitt,

Many thanks for taking the time to talk through the points for clarification.

1) I am in the process of submitting the research project for NRES approval and am working closely with Anne-Claire Owen from ABMUHB Research and Development department to ensure all processes are followed.

2) As advised by yourself, the debrief summary is no longer required as the queries below will answer any concerns raised regarding the normal processes that take place in a lymphoedema clinic and lymphoedema assessment.

3) I have attached a revised version of the consent form that I hope will be less cumbersome to read and will be clearer for a patient to understand what they will be agreeing/ consenting to. I have thus changed the 'wordy' paragraph into two points for consent.

4) Appointments at 2, 4 and 6 months are not part of the normal process. The 'normal' lymphoedema pathway for a patient is to be seen 3 months following their initial assessment. At this time the clinician and patient can decide upon a further review between 6 and 12 months. For purposes of the research study we need to gain as much data as possible to ensure the data being collected from the new 3D camera is as accurate as the 'research gold standard' volume measurements utilising the perometer, and is comparable to the circumferential measurements that are standard practice within a lymphoedema clinic. We also want to see if the 3D camera is sensitive enough to pick up small changes over time.

5) The sensitive questions regarding sexuality and anxiety is normal practice and is integral to the normal lymphoedema assessment. As therapists we need to understand how the lymphoedema is affecting the patient on an emotional level so that they can be referred for emotional / psychological support as needed. The reason for it being repeated in the last session is to determine whether or not the treatment that has been agreed between the patient and the lymphoedema therapist is helping them or whether it is adversely affecting them emotionally - again to determine whether the patient needs more support outside of the lymphoedema clinic.

Should you require further clarification on the above; or if the consent form needs further amending, please do let me know.

Kind regards,

Cheryl Pike

National Macmillan Innovations Lymphoedema Specialist

Lymphoedema Clinic

Cimla Health \& Social Care Services

Cimla

Neath

SA11 3SU

Tel:

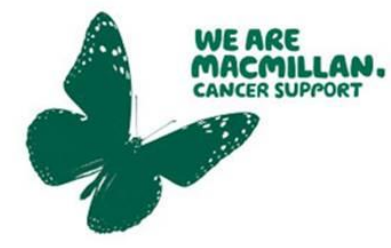




\section{From:}

\section{Sent: 10 March 2016 22:11}

To:

Cc:

Subject: CHHS and CoM Research Ethics Committee

Dear Cheryl

Reference Number: 10316

The CHHS and CoM Research Ethics Committee has met to consider your proposal and asks that you respond to the following queries before approval is granted:

- $\quad$ NRES approval should be sought.

- $\quad$ A debriefing summary is required.

- $\quad$ The last paragraph of the consent form is very wordy and should be revised.

- $\quad$ Are the appointments of 2, 4 and 6 months part of the normal process?

- Is the information collected (questionnaire) part of the normal process? If not, why is the applicant asking about sexuality, anxiety etc... and why only on the first and last session? If this information is not part of the normal assessment process and is being collected for research purposes, then there is a risk of participant distress which should be identified in the application and strategies for dealing with potential distress should be described.

Approval may now be dealt with by Chair's action. Please respond in an email to me including my original text in your reply. Cc Karin Terry.

\section{Best Wishes}

Jeanette

Dr. J.L.Hewitt

Associate Professor / Athro Cynorthwyol

Chair, CHHS and College of Medicine Research Ethics Committee

www.swansea.ac.uk/humanandhealthsciences/research/research-ethics-committee

Department of Philosophy, History \& Law / Uwch Ddarlithydd, Adran Athroniaeth, Hanes a'r

Gyfraith

College of Human and Health Sciences / Coleg y Gwyddorau Dynol ac Iechyd

Swansea University / Prifysgol Abertawe

Singleton Park / Parc Singleton

Swansea / Abertawe

Wales / Cymru

SA2 8PP

www.swansea.ac.uk _www.abertawe.ac.uk

E-mail/ Ebost:

Editorial Board, Ethics and Social Welfare

http://www.tandf.co.uk/journals/cfp/reswcfp.pdf

Board Member, The European Association of Centres of Medical Ethics (EACME)

http://www.eacmeweb.com/ 


\section{WOSRES \\ West of Scotland Research Ethics Service}

Mrs Cheryl E PIke

National Macmilian Innovations Lymphoedema

Speclallst

Abertawe Bro-Morgannwg Unlversity Health Board

Cimla Health \& Soclal Care Centre

Clmla

Neath

SA11 $35 U$

\section{NHS \\ Greater Glasgow and Clyde}

\section{West of Scotland REC 5}

West of Scotisnd Research Eerics Service Weat Glasgow Ambulatory Care Hocplial Dainair stect

Glasgov

G3 $85 \mathrm{~N}$

Date 16 June 2016

Direct line 01412321809

E-mal WosREC5Mogc.scot.nha.uk

\section{Dear Mrs Pike}

study title:

REC reference:

IRAS project ID:
Exploiting 3D scanning technology In lymphoedema for accurate and fast measurements of volume and shape 16 WS $/ 0127$

193784

The Proportionate Review Sub-committee of the West of Scotland REC 5 revlewed the above application on 15 June 2016 .

We plan to publlsh your research summary wording for the above study on the HRA webslte, together with your contact detalls. Publication will be no earler than three months from the date of this favourable opinion letter. The expectation is that this information will be published for all studles that recelve an ethical opinion but should you wish to provide a substitute contact point, wish to make a request to defer, or require further information, please contact the REC Manager Mrs Sharon Macgregor, WoSREC5 $9 g g c . s c o t . n h s . u k$. Under very Imited drcumstances (e.g. for student research which has recelved an unfavourable opinion), it may be possible to grant an exemption to the publication of the study.

\section{Ethical opinion}

On behalf of the committee, the sub-commiltee gave a favourable ethical opinion of the above research on the basis described in the application form, protocol and supporting documentation, subject to the conditions specifled below.

\section{Conditions of the favourable opinion}

The REC favourable opinion Is subject to the following conditions being met prior to the start of the study.

1. In the "Expenses" section of the Participant Information Sheet, the sentence should be changed to "No relmbursement of travel or out of pocket expenses will be provided."

2. In section "What will I have to do?" of the Participant Information Sheet, the word "you're" should be removed from the second bullet point. The following sentence should then be added to the end of the bullet point: "These visits are not part of standard care but 
unfortunately any travel expenses cannot be relmbursed."

3. In the Consent form, it is suggested that the following footer is added to the document: "When completed: 1 for participant; 1 for researcher site fle; 1 (original) to be kept in medical notes."

You should notify the REC once all conditions have been met (except for site approvals from host organlsations) and provide coples of any revised documentation with updated version numbers. Revlsed documents should be submitted to the REC electronically from IRAS. The REC will acknowledge recelpt and provide a final llat of the approved documentation for the study. which you can make avallable to host organlsations to facilltate their permission for the study. Fallure to provide the final versions to the REC may cause delay in obtaining permissions.

Management permission must be obtained from each host organisation prior to the start of the study at the slte concerned.

Management permission should be sought from al NHS organisations Involved in the study in accordance with NHS research governance anrangements. Each NHS organlsatlon must confirm through the signing of agreements and/or other documents that it has given permission for the research to proceed (except where explicitily specined otherwise).

Guldance on applying for HRA Approval (Englano) NHS permission for research is avallable in

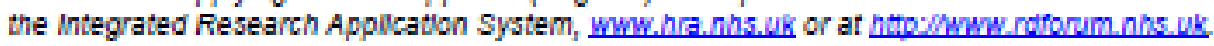

Where a NHS organisation's role in the study is Nmited to identirying and referring potential particlpants to research sites ("participant identincation centre"), guldance should be sought from the RBD office on the information it requires to give permission for this activity.

For non-NHS sites, site management permission should be obtained in accordance with the procedures of the relevant host organisation.

Sponsors are not required to notify the committee of management permissions from host organisations.

\section{Reqistration of Cilnical Trials}

All clinical trials (defined as the first four categorles on the IRAS niter page) must be registered on a publically accessible database. This should be before the first participant is recrulted but no later than 6 weeks after recrultment of the first particlpant.

There is no requirement to separately notify the REC but you should do so at the earllest opportunity e.g. When submitting an amendment. We will audlt the reglstration detals as part of the annual progress reporting process.

To ensure transparency in research, we strongly recommend that al research is registered but for non-clinical trials this is not currently mandatory.

If a sponsor wishes to request a deferral for study registration within the required timeframe, they should contact hra.studyregistrationenhs.net. The expectation is that all clinical trials will be registered, however, in exceptional circumstances non registration may be permissible with prior agreement from the HRA. Guldance on where to register is provided on the HRA website.

It ls the responsibility of the sponsor to ensure that all the conditions are complled with before the start of the study or its Initiation at a particular site (as applicable). 


\section{Ethical review of research sites}

The favourable opinion applles to all NHS sltes taking part in the study, subject to management permission belng obtained from the NHS/HSC R8D office prior to the start of the study (see "Conditions of the favourable opinion").

\section{Summary of dlscussion at the meeting (for Information only)}

\section{Informed consent process and the adequacy and completeness of particlpant} Information

In the Expenses section of the Participant Information Sheet, the use of the word "Incentive" is not appropriate and should be changed to make it clear that no travel expenses will be pald. The document should also be clear that there are extra visits other than standard ones.

A minor error In the "What will I have to do?" section was also noted and needs to be corrected.

In the Consent form, it is suggested that the standard footer regarding duplicate copies is added as a reminder to research staff.

\section{Other general comments}

Although not ethical lssues, a number of spelling mistakes in the application form were noted ("there" Instead of "their" in the ethical issues section; "Dread" Instead of "breed"). The researchers also seemed to have misunderstood question A27-1 about initialy identifying sultable patients so that they could be approached to take part. However, It was agreed that these were minor points and no action was required.

\section{Approved documents}

The documents revlewed and approved were:

\begin{tabular}{|c|c|c|}
\hline Document & |Version & |Date \\
\hline Letter from funder [LRG Grant approval] & & 02 October 2015 \\
\hline Letters of Invitation to participant [Patient Letier] & 1 & 25 January 2016 \\
\hline Partipant consent form [Consent Form] & 3 & 11 March 2016 \\
\hline Partcipant infomation sheet (PIS) [Patient Information Sheet] & 2 & 18 January 2016 \\
\hline REC Application Form [REC_Form_06062016] & & 08 June 2016 \\
\hline $\begin{array}{l}\text { Referee's report or other sclentific critque report [Swansea } \\
\text { Universty Ethics Approval] }\end{array}$ & & 11 March 2016 \\
\hline Research protocol or project proposal [3D scan Protocol] & 1 & 25 January 2016 \\
\hline Summary CV for Chlef Investigator (Cl) [C Plke CV] & & 18 January 2016 \\
\hline
\end{tabular}

\section{Membership of the Proportionate Revlew sub-Committee}

The members of the Sub-Committee who took part in the revlew are listed on the attached sheet.

\section{Statement of compllance}

The Committee is constituted in accordance with the Governance Arrangements for Research Ethics Committees and comples fully with the Standard Operating Procedures for Research 
Ethics Committees in the UK.

\section{After ethical review}

\section{Reporting requirements}

The attached document "After ethical revlew - guldance for researchers" glves detalled guldance on reporting requirements for studles with a favourable opinlon, including:

- Notifying substantlal amendments

- Adding new sites and Investigators

- Notification of serious breaches of the protocol

- Progress and safety reports

- Notifying the end of the study

The HRA website also provides guldance on these toplcs, which is updated in the light of changes in reporting requirements or procedures.

\section{User Feedback}

The Health Research Authority is continually striving to provide a high qualty service to al applicants and sponsors. You are invited to glve your view of the service you have recelved and the application procedure. If you wish to make your vlews known please use the feedoack form avallable on the HRA website: $h$ ttp://www.hra.nhs. uk/about-the-hralgovernancelqualtyassurancel

\section{HRA Training}

We are pleased to welcome researchers and R\&D staff at our training days - see detals at ntto://Www.hra.nhs.uk/hra-training/

With the Committee's best wishes for the success of this project.

Yours sincerely

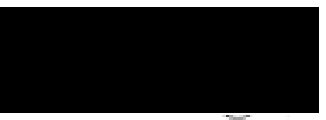

for

Dr stewart Campbell

Chalr

Enclosures: $\quad$ LIst of names and professions of members who took part in the revlew

"After ethical review - guidance for researchers"

Copy to:

Mrs Julle Willams, swansea University M/s Anne-Clalve Owen, Abertawe Bro-Morgannwg Untversity Heath Board 
West of Scotland REC 5

Attendance at PRS Sub-Commltte日 of the REC meeting on 15 June 2016

Commlttee Members:

\begin{tabular}{|c|c|c|c|}
\hline Name & Profession & Present & Notes \\
\hline Dr Stewart Campbell & $\begin{array}{l}\text { Consultant Physiclan } 8 \\
\text { Gastroenterologist (Chair) }\end{array}$ & Yes & \\
\hline Dr James Curran & GP & Yes & Maln reviewer of application \\
\hline Dr Gillan Haroid & Consuftant Radologist & Yes & Maln reviewer of application \\
\hline Mrs Naoml Hickey & Research Nurse & Yes & Maln reviewer of application \\
\hline Dr Gillan Kert & Consultant Physiclan & Yes & \\
\hline Dr Ahmed Khan & Consuftant Psychlatifst & No & \\
\hline Professor Eddle MoKenzle & Statistidan & Yes & \\
\hline Canon Matt McManus & Parlsh Priest (Vice-Chalin) & Yes & Chalred meeting \\
\hline Ms Janis Munro & Key Account Manager & Yes & \\
\hline Mrs June Russel & Retired (Research Chemist) & Yes & \\
\hline Mr Charles Sargent & Retired & Yes & \\
\hline Dr Marcel Strauss & Consưtant Radologist & Yes & \\
\hline Mrs Llz Tregorning & $\begin{array}{l}\text { Retired (Speclal Needs } \\
\text { Teacher) (Altemate Voe- } \\
\text { Chalr) }\end{array}$ & Yes & Maln reviewer of applicaton \\
\hline
\end{tabular}

Also in attendance:

\begin{tabular}{|l|l|} 
Name & Postiton (or reason for attending) \\
\hline Dr Judith Godden & Sclentfic Ofllcer/Manager \\
\hline Mrs Sharon Macgregor & Co-ord nator \\
\hline Ms Amanda Martin & Ooserver (New Member) \\
\hline
\end{tabular}




\section{WOSRES \\ West of Scotland Research Ethics Service}

Mrs cheryl E Plke

National Macmillan Innowations Lymphoedema

Specialst

Abertawe Bro-Morganning Unlverslty Health Board

CImla Health \& Social Care Centre

CImla

Neath

SA113SU
NHS

Greater Glasgow and Clyde

\author{
West of Scotland REC 5 \\ Weat of Ecotiand Research Ethics Benvice \\ Weat Clsogow Ambulatory Care Hoap al \\ Dainsir stret \\ Gluspow \\ $0380 \mathrm{~N}$ \\ Duse \\ 23 June 2016 \\ Direct line \\ E-mal \\ 0141 2321804 \\ WobAEC Hopcrotrha wh
}

\section{Dear Wrs Pre}

study title:

REC reterence:

IRAS project ID:

Exploiting 9D acanning technology In lymphoedema for accurate and fast measurements of volume and shape 1 erws 0127 193784

I can conflm the FEC has recelved the documents llsted below and that these comply with the approwal conditions detalled In our letter dated 16 June 2016

\section{Documante recelved}

The documents recelved were as follows:

\begin{tabular}{|c|c|c|}
\hline Document & Verson & Date \\
\hline Partclpart consert form [Consent Formi' & 4 & 16 . June 2016 \\
\hline Partclpart Irtomation aheet (PIS) [Partc|pant Infomaton Sheet] & 3 & 16 . dune 2016 \\
\hline
\end{tabular}

\section{Approved documents}

The final Ist of approved documentation for the study is therefore as follows:

\begin{tabular}{|c|c|c|}
\hline Document & Version & Date \\
\hline Letter from funder [LRG Grant aporova]] & & Wa octooer 2015 \\
\hline Letters of Inutiation to partlclpant [Patlent Letter] & 1 & 25 danuary 2016 \\
\hline Partclpant consent form [Consent Formi] & 3 & 11 March 2016 \\
\hline Partc|pant consent form [Consent Formi] & 4 & 16 . June 2016 \\
\hline Partcapant Irtomation sheet (PIS) [Patlent Informaton Sheet] & 2 & 16 . January 2016 \\
\hline 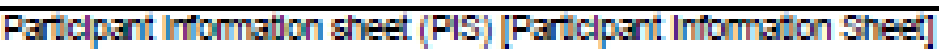 & 3 & 16 June 2016 \\
\hline
\end{tabular}




\begin{tabular}{|c|c|c|}
\hline FEC Appllcation Form [REC_Form_OADE601E] & & Q6 June 2016 \\
\hline $\begin{array}{l}\text { Releree's report or other selentfic contlque repoit [Swanses } \\
\text { Universty Ethics Afprova] }\end{array}$ & & 11 March 2016 \\
\hline Fesearch protocol or proded proposal [3D sean Protoco] & 1 & S January 2016 \\
\hline Sumimary ov for cole' Investlgator (C)] [C Plke CV] & & 16 .January 2016 \\
\hline
\end{tabular}

You should ensure that the sponsor has a copy of the final documentation for the study. It is the sponsor's responsibility to ensure that the documentation is made avalable to FAD offices at all particlpating sltes.

16 WS/0127 Please quote this number on all comrespondence

Yours sincerely

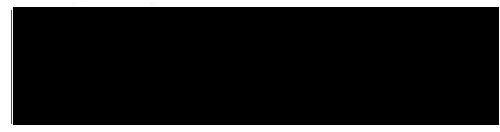

Rose Gallacher Asalsatant Adminlstrator

Copy to: Mrs Juine whams

Ms Anne-Clare Owen, Abertawe Bro-Morgannwg Unversity Heath Board 

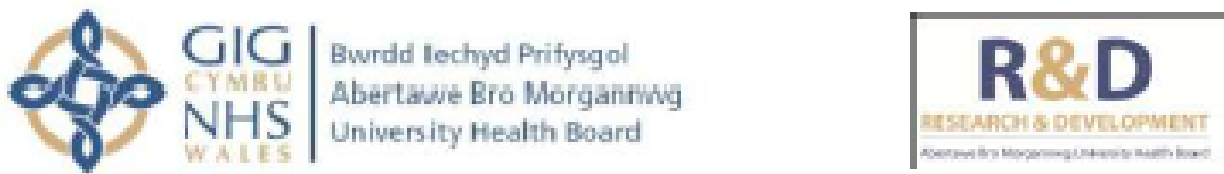

Dyddiad/Date: $31^{*}$ Auguat 2016

Miss Sisn Cement

Macmillan Lymphosdema Cínical Lead

Abertawe Bro-Morgannwg University Heaith Board

Singleton Hospital

Sketty

Swanses

SA2 8QA

3MU Health Board Research \&. Development

Swarcea University

Floor 1, Inctitute of Life Science 2

Singleton Park

Swance:

SA2 $8 \mathrm{PF}$

0179293098

$\triangle$ sbm.rdegwales.rha uk

Desr Miss Clement,

\section{Re: 3D Scanning of Lymphoedema arms}

IRAS Ref: 193784

Sponsor: Abertawe Bro Morgannwg University Health Board

Thank you for submitting the above named research proposal to ABMU Health Bosrd for NHS R8D permiscion. The attached listed documents were reviewed.

Heaith Board R8D Governance checls have been completed and passed. Please sccept this letter as confirmation of local NHS RSD Health Bosrd permission.

As part of Research Governance, you are required to:

1. Achere to the protocol aporoved and inform the RB:D office and the relevant Research Ethics Committes of any changes to the study, including the end date, for review/approval and record update.

2. For Hesith Bosrd Sponsored studies, notify the R\&.D office of serious sdverse events immediately upon knowledge, in accordance with local Standard Operating Procedure on Pharmscovigilance and as outlined in your Study Initiation meeting.

3. For Externally Sponsored studes, the Health Board should only be notified of SAEs or Suspected Unexpected Serious Adverse Reaction (SUSAR) srising in local ABMU Patients.

4. Complete any interim and final reports requested by the R.D office. If sponsored by ABMU Health Board, you will be ssked to complete a 6 monthly progress report for aubmission to the Joint Scientific Review Committee slong with your finsl report at study completion.

5. Ensure that your research complies with sny relevant regulatory requirements and legislation relating to: Cinical Trisls, Data Protection Act 1998, Health \&. Safety, Caldicott Guidelines, the use of Human Tissue for research purposes, Mental Copacity and ICH Good Clinical Practice [GCP]. The R\&D team can sdvise you on applicable regulatory and statutory requirements relevent to your study.

6. Comply with Data Protection requirements, notably no personsl or patient identifiable data should lesve the Heaith Board uniess explicit consent from the individual or patient has been taken and documented. Uniess corcent is present, sl study related documents muat be either fully or linked anonymised. Vdentifioble patient dota includes nome, oddress, ful postcode, date of birth, NHS number and local pationt identifioble codes as wall as photographs, videos, audio tapes or other imoges of potiants. Personal identifíable information includes the momber of staffs name, oddress, full post code, dote of birth, $N$ number and staff number as wail as photogrophs otc' - ABMU Data Protection $s$ Confidentisity Policy, Version 2.1 September 2013.

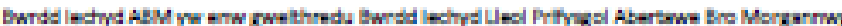

ABM Univeraly Health Bosed in the aperselonsi name of Abertwwe Bro Morgarmwg Univerity Loal Hevith Board

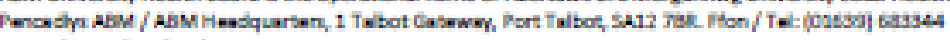

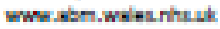


7. Ensure that all training courses requested by the Sporcor are completed by all relevant members of the resesrch team before any resesch sctivity is carried out. All resesrch star undertaking clinical trisle of an imrestigationsl medicinsl product (CTMPs) must be GCP trained, and should continue to update their GCP training every 2 years. Copies of GCP certificates should be filed in the Trial Site File, with a copy forwsrded to the R8.0 Department.

8. Ensure the research is undertaken in complisnce with all Hesith Board Ra.D Standard Operating Procedures (SOPs). The latest versione of all SOPs can be obtained by contacting the RSD Department or from the RSA Intranet pages

9. If the study is sponsored by ABMU Health Board you must notify the RSD Otfice of your intention to open the study in other sites.

10. For ABMU Health Board Sponeored studes, sign a Conditions of Sponeorship Agreement 8 attend a Study Initiation meeting as organised by the RSA Department.

Cinical Research Portfolio Studies

If your study has been sdopted onto the Clinical Resesrch Portfolio (CRP), it will be a condition of our permiscion that the Chief Irvestigator aite uplosds local recruitment dats onto the portfolio datsbese.

For more informstion on the process of uplosding recruitment dats please look at the following link:

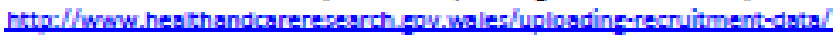

Uploading of recruitment data will enable Health and Care Research Wales to monitor research activity within Health Boards, resulting in NHS R\&D alocations to be driven by activity.

For more information and advice on the Health and Care Research Wales Portfolio plesse email: portfolioewalez.nins.uk

Amendments to the Study

Any changes made to the study after the issue of this letter will be treated as an amendment. Amendments can be 'substantisl' or 'non-substantial'. It is the duty of the Sponsor to classify the amendment and notify all relevant regulatory bodies accordingly, this duty may be delegated to the onief Invest gator or other authorived individusl.

For a subetantial amendment, the Sponsor or delegated individual will be required to submit a Notice of Substantial Amendment form to the REC, the lead permission co-ordinating function for the study snd the MHRA [if applicable]. For all ABMU sponsored studies substantial amendments must first be submitted to the Joirt Study Review Committee [JSAC) for approval prior to submitting to RaC and Hesith and Care Research Wales Permiszions (Research-permissions Qwales.nhs.uk).

For non-substantial smendments, the Sporeor or delegated individusl are required to submit the amendment detsils to the lead permission co-ordinating function for the study. They will then pass the amendment detsils onto sll relevant nations, for Wales this would be Health and Care Research Wale: who will notify ABMU RSD Department for review.

Details of how to classify your amendment as substantisl or non-subatantial are available from Hesith Research Authority - hitto://www.hra.nhs.uk/research-community/during-vour-researchprojectismendmentel

Indemnity Arrangements

The Sponsor indemnifies and holds harmless ABM University Hesith Board, its employees and agents for any harm caused by negligence on behaif of the Sponcor, including sny harm caused to participants by the administration of the investigational product. However, plesse note that the Sponcor will not indemnify ABM University Health Bosrd for any harm caused by negligence on behalf of the research team or other individusl or agent. Resesrchers employed by ABM University Health Board, including

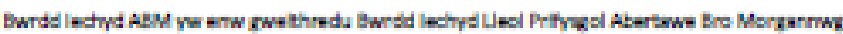

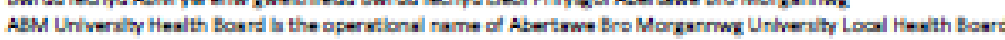

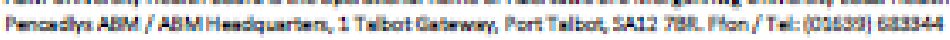

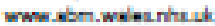


those holding Honorary Controct status are indemnified againet actions for negligent harm via stancard arrangements with Weleh Risk Pool (WRP).

Plesse discuss sny planned use of in-house work inetructions/sops with the Sponsor company during Initiation to enoure localised documents correctly summarise the protocol requirements and this is agreed to, in writing, by the Sporeor Company.

ABM University Heath Bosrd reserves the right to suspend approval of any research study where deviation from appropriate RG \& GCP standarde is uncovered.

Msy I take this opportunity to wish you well in undertaking the research. We will write to you in the future to request updates on the progress of the research and look forward to receiving outcomes of the study.

Yours sincerely,

\section{Protessor J Stephens}

Deputy Assistant Medical Director (RB.D)

ABMU Health Boser

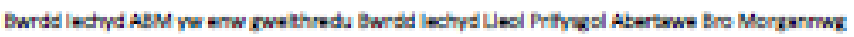

AEM Universily Health Boserd th the aperselonal name of Abertwwe Bro MorgarmwE Univerily Loal Hesth Boerd

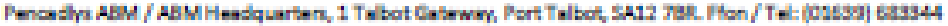

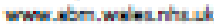

Aesthet 1997st

Base 3 of 6 
Re: 3D Scanning of Lymphoedema arms

IRAS Ref: 193794

Sponsor: Abertawe Bro Morgannwg University Health Board

SSI Documents Received

\begin{tabular}{|c|c|c|}
\hline Document: & Subtitle: & Version: \\
\hline $\begin{array}{l}\text { RB.D Form (Parts A-D) (Pigned/authorised } \\
\text { pdf or hard copy) }\end{array}$ & Signature page - ACO & \\
\hline Site-Specific Information form checkilst & $\begin{array}{l}\text { ABM UHB, Lymphoedems Cínic Singleton } \\
\text { Hospital Swanses }\end{array}$ & \\
\hline $\begin{array}{l}\text { RB.D Form (Parts A-O) (gigned/authorised } \\
\text { pdf or hard copy) }\end{array}$ & Unsigned & \\
\hline $\begin{array}{l}\text { Site-Specific Information Form } \\
\text { [signed/authorised pdt or hard coph] }\end{array}$ & $\begin{array}{l}\text { ABM UHB, Lymphoedems Cínic Singleton } \\
\text { Hospital Swanses }\end{array}$ & 27 Jul 2016 \\
\hline RI.D Application checkist & & \\
\hline
\end{tabular}

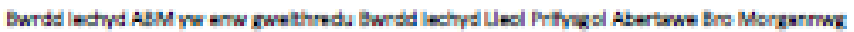

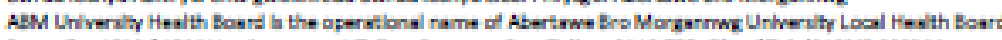

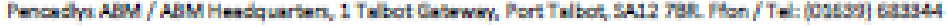

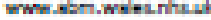

Mestillet: 1937s

Pase 4 of 6 
Re: 3D Scanning of Lymphoedema arms

IRAS Ref: 193784

Sponsor: Abertawe Bro Morgannwg University Health Board

RSD Application Documents Received

\begin{tabular}{|c|c|c|}
\hline Document: & Subtitle: & Version: \\
\hline Letters of invitation to participant & 193784 - Patient letter v1 25.01 .16 & v1, 25.01.16 \\
\hline $\begin{array}{l}\text { Research porticipant informstion sheet } \\
\text { [FIS) }\end{array}$ & Patient information Sheet & v1 \\
\hline Research protocol & & $v 1,25 \operatorname{lan} 2016$ \\
\hline $\begin{array}{l}\text { Research porticipant information sheet } \\
\text { [FIS] }\end{array}$ & & v3 16 Jun 2016 \\
\hline Research porticipant consent form & & v4, 16 Jun 2016 \\
\hline $\begin{array}{l}\text { Confirmation of any other regulatory } \\
\text { spprovals }\left[e_{\text {g. }} \text {. NIGB] and all }\right. \\
\text { correspondence }\end{array}$ & $\begin{array}{l}\text { BLSCC RG response from resesrcher with } \\
\text { further information. }\end{array}$ & $29 \operatorname{sep} 2015$ \\
\hline Summary CV for Chief linvestigator (Cl) & C Pike & \\
\hline $\begin{array}{l}\text { Summary CV for local researchers and } \\
\text { research nurses }\end{array}$ & R Van Loon & \\
\hline other (plesce specity) & $\begin{array}{l}\text { Emsil Request to become a non- } \\
\text { commeroial partner organisation }\end{array}$ & $12 \operatorname{Jan} 2015$ \\
\hline REC form & Checklist & \\
\hline Letter from funder & LRG successful email & 02 Oct 2015 \\
\hline Letter from funder & LRG Application part A & 31 Aug 2015 \\
\hline Letter from funder & LRG Application part B & $01 \operatorname{sep} 2015$ \\
\hline Letter from funder & LRG Application part C & 30 Aug 2015 \\
\hline Letter from funder & emsil RE BLS research grant application & $01 \operatorname{sep} 2015$ \\
\hline $\begin{array}{l}\text { Confirmation of any other regulstory } \\
\text { approvals (e.g. NiGs] and all } \\
\text { correspondence }\end{array}$ & $\begin{array}{l}\text { emsil RE CHHS and CoM Resesrch Ethics } \\
\text { Committee approval }\end{array}$ & 11 Mar 2016 \\
\hline Letter from funder & emsil RE LRG & 29 Oct 2016 \\
\hline $\begin{array}{l}\text { REC opinion letter for amendments } 8 \text { : } \\
\text { correspondence }\end{array}$ & $\begin{array}{l}\text { Ackinowiedgement of receipt of documents } \\
\text { as evidence of compliance }\end{array}$ & 23 Jun 2016 \\
\hline REC form & & 11 Mar 2015 \\
\hline $\begin{array}{l}\text { Summary CV for local researchers and } \\
\text { research nurses }\end{array}$ & R Lewis & \\
\hline Other (olease speciry) & Risk Assessment Tool & \\
\hline Letter from funder & $\begin{array}{l}\text { Lymphoedema Research Grant, request for } \\
\text { further information. }\end{array}$ & $24 \operatorname{sep} 2019$ \\
\hline $\begin{array}{l}\text { Summary CV for Principal Investigator } \\
\text { [FI) }\end{array}$ & 5 Cement & \\
\hline
\end{tabular}

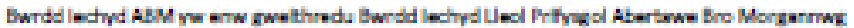

ABM Universly Health Bowrd in the aperselonal name of Abertwwe Bro MorgarmwE Univerilty Loal Heweth Bowrd

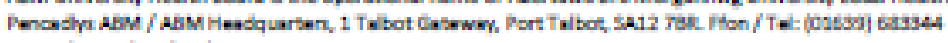

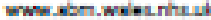

llesthet: 1997s

Baes 5 of 6

To note: page 6 of 6 is blank 


\section{APPENDIX 5: Participant Information Sheet for Study}

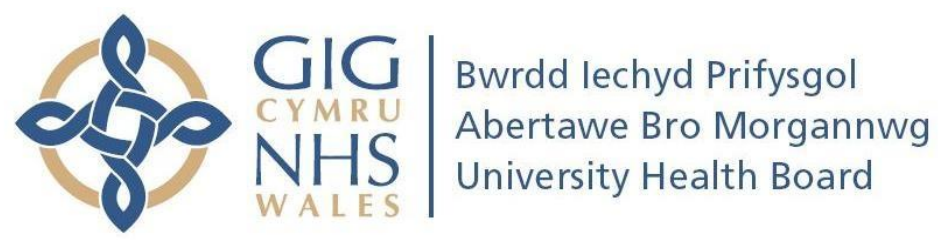

Participant Information Sheet

Version 3

16 June 2016

\section{Study Title}

Exploiting 3D scanning technology in lymphoedema for accurate and fast measurements of volume and shape

\section{Introduction}

You have been invited to join a research study and this sheet tells you about the piece of research and what you would have to do. If you have any other questions please ask any of the persons below for more information - their contact details are at the bottom of the page.

\section{What is the purpose of this study?}

In an attempt to improve how lymphoedema is measured in a person's limbs, we would like to compare three different ways of measuring your arm at your appointment. The standard measurement is performed by using a ruler and/or tape measure to mark $4 \mathrm{~cm}$ on both your arms, and then taking the circumferential measurements at each of these points. These measurements are then entered into a database that calculates how much fluid you have on your affected side compared to your unaffected side.

The two other measurements will include a machine called the perometer and a 3D image. The perometer is a laser scanner that slides up and down your arm which can compute the size of your arms to help the therapist determine how much bigger your affected side is to your unaffected side.

The final measurement is a new 3D camera which takes images of your arms and will then be analysed by a new computer programme. The data will also tell us how much bigger your affected arm is to your unaffected arm, but in addition it will produce images that can help the therapist determine where your problem areas are.

The information we get from all the tests will be used to compare how effective this new $3 \mathrm{D}$ image is compared to the standard measurement (tape measure) and the research validated measure (perometer). 
It is hoped that in the future this new 3D imaging can be used in lymphoedema clinics to help therapists understand your lymphoedema and provide a more individualised treatment programme for you.

\section{Why have I been invited to take part?}

All people who have been referred to the ABMU LHB Lymphoedema Service for assessment and management of their arm lymphoedema will be invited to partake in this research. The assessment will form part of your normal assessment and treatment for your lymphoedema.

\section{Do I have to take part?}

No, participation is voluntary and you can withdraw at any time without your treatment being affected.

\section{Expenses and payments}

No reimbursement of travel or out of pocket expenses will be provided.

\section{What will I have to do?}

If you take part in this study you will be asked to attend for the following visits:

- First/ Initial Assessment. This will be done at Singleton Hospital and will take about 60 to 90 minutes to complete. The appointment will include a full assessment of your lymphoedema, measurements as described above and an explanation of what lymphoedema is and how to manage it.

- 2 month, 4 month and 6 month follow up to include the above measurements to determine how effective the management of your lymphoedema is. These visits are not part of standard care but unfortunately any travel expenses cannot be reimbursed.

\section{What is the time commitment?}

It is anticipated that the assessment will take between 60 to 90 minutes to perform the consent, the person's medical and lymphoedema history, volumetric measurements and commence management of the person's lymphoedema.

Each follow up (2, 4 and 6 months) will take between 40 to 50 minutes to perform the volumetric measurements and ensure that the lymphoedema management is effective for the person.

\section{What are the possible disadvantages and risks of taking part?}

The entire study consists of non-invasive measurements and therefore has no significant risk. 


\section{What are possible benefits of taking part?}

The aim of this study is to validate a new 3D camera image that would offer more accurate measuring of a person's limbs thus offering the potential for a more individualised treatment programme.

\section{What happens when the research stops?}

This study involves the 4 visits described above and afterwards you will continue with the national Lymphoedema Pathway for your lymphoedema management.

\section{What if there is a problem?}

If you feel unwell or suffer any unusual discomfort during the study it is important to inform your study nurse or therapist as soon as possible. Any complaint about the way you have been dealt with during the study or any possible harm you might suffer will be addressed. The normal NHS complaints mechanisms will be available to you.

\section{Will your taking part in this study be kept confidential?}

All information that is collected about you will be kept strictly confidential. All data collected about you during the study will be made anonymous by allocating you a unique number. We will follow ethical and legal practice and all information about you will be handled in confidence. The results of this research might eventually be published and available to the public in an anonymised form.

\section{Contact details:}

Researcher:

Cheryl Pike, National Macmillan Innovations Lymphoedema Specialist

Cimla Health \& Social Care Centre, Neath, SA11 3SU

Tel:

\section{Principal Investigator:}

Dr. Raoul van Loon, Associate Professor in Computational Biomedical Engineering, College of Engineering, Swansea University, Singleton Park, Swansea, SA2 8PP,

Tel:

\section{Lymphoedema Therapist:}

Sian Clement, Macmillan Lymphoedema Clinical Lead, ABMU Health Board, Singleton Hospital, Swansea, SA2 8QA

Tel: 


\section{APPENDIX 6: Patient Letter for Pilot Study}

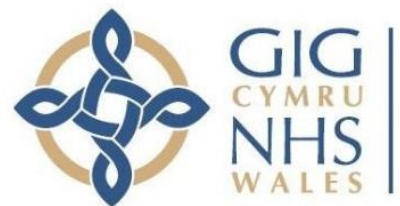

Bwrdd lechyd Prifysgol

Abertawe Bro Morgannwg

University Health Board

Date: $\mathrm{xx} / \mathrm{xx} / \mathrm{xxxx}$

ABMUHB Lymphoedema Service

Singleton Hospital, Swansea

SA2 8QA

Tel:

Patient Letter

Version 1 - 25 January 2016

Dear Sir / Madam

Study Title: Exploiting 3D scanning technology in lymphoedema for accurate and fast measurements of volume and shape

Please find enclosed a 'Patient Information Sheet' in which we explain the purpose and the plan of this study at the ABMUHB Lymphoedema Clinic based at Singleton Hospital.

The study is to look at new means of assessing people who have swelling of their arm to determine if there is a better, more accurate and time saving way of assessing lymphoedema.

Please read the attached information carefully and should you have any queries about the study, please contact myself

( ), for clarification.

) or Sian Clement, Lymphoedema Service Lead

Please note that there is no pressure on you to accept to be a participant in this study and it will not affect your treatment in any way should you choose to join the study and then later withdraw.

Please let us know whether you would like to be a participant or not before your appointment.

At your appointment you will be asked by your therapist whether you have read and understood the 'Patient Information Sheet' and you will be given an opportunity to ask questions. You will then be asked to sign a consent form before any of the study questions and examinations are performed.

Thanking you for your consideration in participating in this study.

Kind regards,

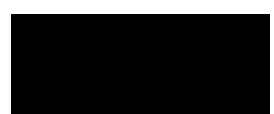

Cheryl Pike

National Macmillan Innovations Lymphoedema Specialist

Tel: 
APPENDIX 7: Lymphoedema Network Wales Referral Form

\begin{tabular}{|c|c|}
\hline \multicolumn{2}{|c|}{$\begin{array}{l}\text { ALL WALES LYMPHOEDEMA SERVICE REFERRAL FORM Lymphoedema Networkifh is } \\
\text { Please complete all sections as much } 38 \text { posilble }\end{array}$} \\
\hline Name \& Ttile: & \begin{tabular}{l|l} 
& HospitalNHS No: \\
\end{tabular} \\
\hline Address: & $\begin{array}{l}\text { Patlents GP: } \\
\text { GP Surgery: }\end{array}$ \\
\hline $\begin{array}{l}\text { Postcode: } \\
\text { Telephone: } \\
\text { Emal address: }\end{array}$ & $\begin{array}{l}\text { Location of patient on referral: } \\
\square \text { Home } \square \text { Hospital \& Ward } \square \text { Other_. }\end{array}$ \\
\hline $\begin{array}{l}\text { Date of Birth: } \\
\text { Gender: } \quad \text { Male } \frac{}{\text { DFemale }}\end{array}$ & $\begin{array}{l}\square \text { Does the patlent require hospital transport } \square \text { No } \\
\square \text { Yes }\end{array}$ \\
\hline \multicolumn{2}{|c|}{ Lymphoedema Hlatory. } \\
\hline \multirow{2}{*}{\multicolumn{2}{|c|}{$\begin{array}{l}\text { Severity of Swelling: } \square \text { Mild } \square \text { Moderate } \square \text { Severe } \square \text { Palliative } \\
\text { Lymphormoea (Leaking) } \square \text { No } \square \text { Yes Duration:_ Referred to Dermatology: } \square \text { No } \square \text { Yes } \\
\text { Ulcers } \quad \square \text { No } \square \text { Yes } \quad \square \text { Venous } \square \text { Arterial } \quad \text { Referred to TVN: } \square \text { No } \square \text { Yes } \\
\text { Cellultils } \square \text { No } \square \text { Yes } \quad \text { No. eplsodes in past } 12 \text { months: }\end{array}$}} \\
\hline & \\
\hline \multicolumn{2}{|c|}{ Cancer Hlatory Related to Lymphoedema $\quad \square$ Not Appllcable } \\
\hline \\
\hline \multicolumn{2}{|c|}{ Surgery $\quad \square$ No $\square$ Yes $\square$ NIA Procedure: } \\
\hline \multicolumn{2}{|c|}{$\begin{array}{l}\text { Chemotherapy } \square \text { No } \square \text { Yes } \square \text { NA Nodes Removed: } \square \text { No } \square \text { Yes Nodes Positive: } \\
\text { Radlotherapy } \square \text { No } \square \text { Yes } \square \text { NA. Advanced Dlsease at Referra: } \square \text { No } \square \text { Yes }\end{array}$} \\
\hline \multicolumn{2}{|c|}{ Past Medical Hetory $\quad$ Detalls / Other Mrdical Conditions } \\
\hline & Vasculan'Arterlal Disease $\square$ No $\square$ Yes \\
\hline \multicolumn{2}{|l|}{ Dlabetes $\quad \square$ No $\square$ Yes } \\
\hline \multicolumn{2}{|l|}{ Psychlatric History $\square$ No $\square$ Yes } \\
\hline \multicolumn{2}{|l|}{ Ooesity $\quad \square$ No $\square$ Yes } \\
\hline Mobillty Problems $\quad \square$ No $\square$ Yes & Stand to transfer $\square$ No $\square$ Yes \\
\hline
\end{tabular}

Please attach prescription medlcation chart Medical Hlatory can continue on additional sheets if required Special Instructions / Cautions: Does the patient have learning dificulties No $\square$ Yes $\square$

Any concerns over patlent's mental capaclty? $\square$ No $\square$ Yes

Does the patient have confimed dagnosis of dementla? $\square$ No $\square$ Yes

Is patlent aware of referral: $\square$ No $\square$ Yes is patlent already known to a Lymphoedema Service: $\square$ No $\square$ Yes PTO for flowchart: $\square$ Routine (includes risk reduction) $\square$ Uigent $\square$ Pallative

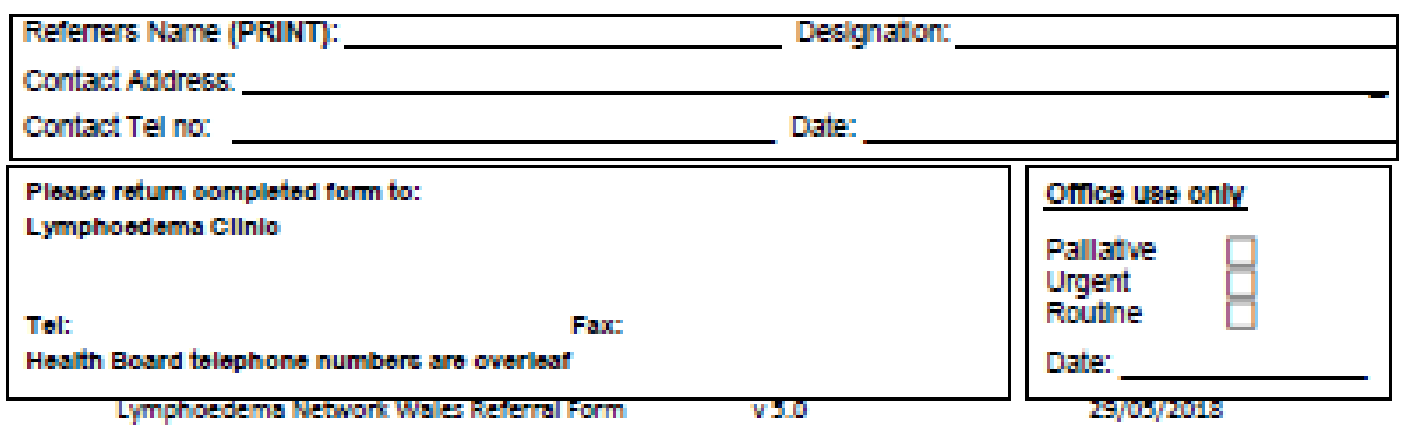




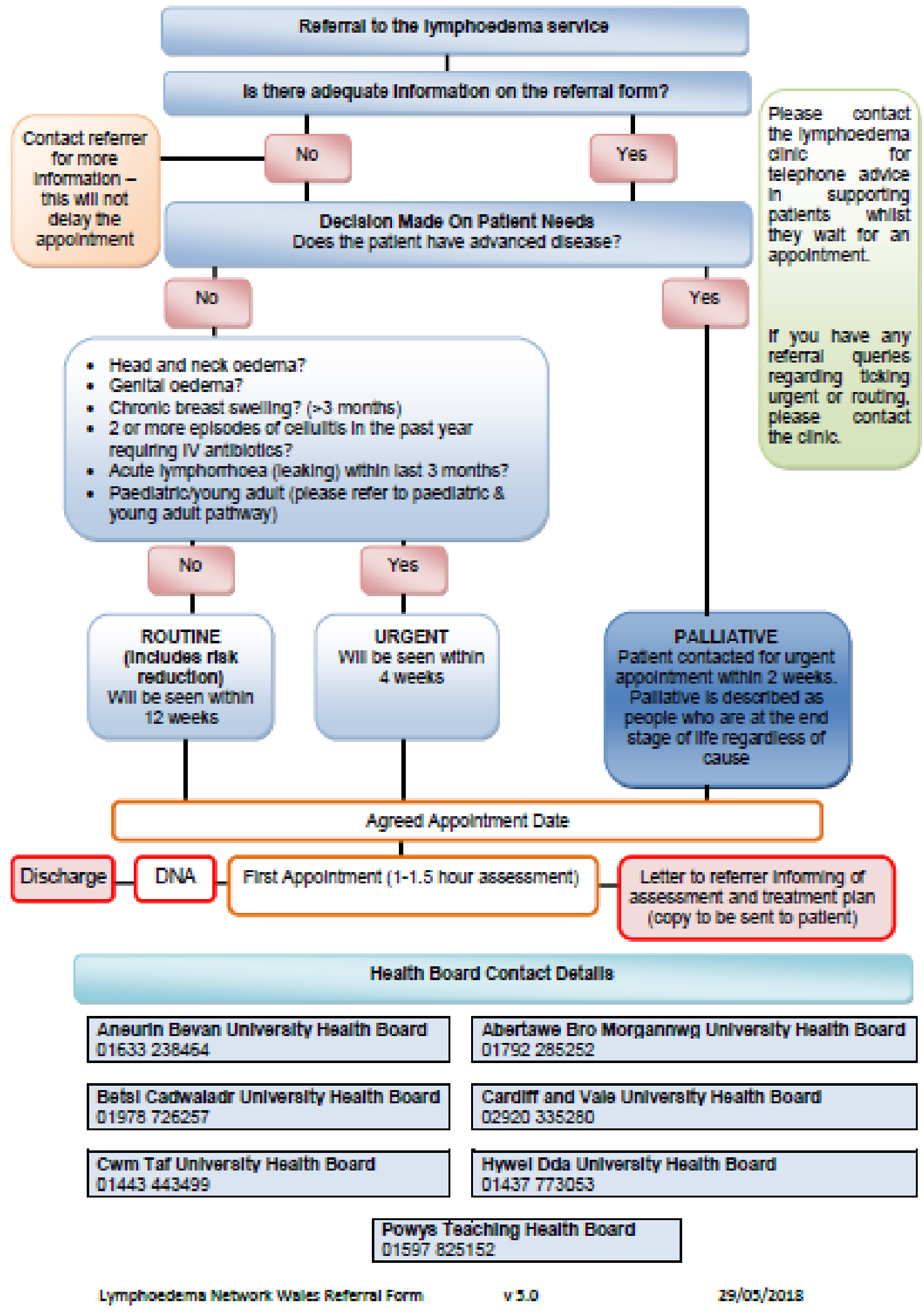




\section{APPENDIX 8: Consent Form}

Patient Identification Number for this trial:

CONSENT FORM (version 4.1, 16 Jun 2016)

Title of Project: Exploiting 3D scanning technology in lymphoedema for accurate and fast measurements of volume and shape

Name of Researchers: Cheryl Pike, Dr Raoul van Loon

Please initial

all boxes

1. I confirm that I have read and understand the information sheet dated 16 June 2016 (Version 3) for the above study. I have had

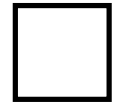
the opportunity to consider the information, ask questions and have had these answered satisfactorily.

2. I understand that my participation is voluntary and that I am free to withdraw at any time without giving any reason, without my medical care or legal rights being affected.

3. I understand that relevant sections of my lymphoedema notes and the data collected during the study may be used by designated individuals from ABMU Health Board and Swansea University for purposes of the research as outlined in the patient information sheet.

4. I give permission for the designated individuals as described in point 3 above to have access to my lymphoedema notes and

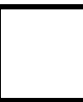
research data.

5. I agree to take part in the above study.

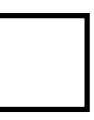

\section{Date}

Date
Signature

Signature

Name of Person taking consent

When completed: 1 for participant; 1 for researcher site file; 1 (original) to be kept in medical notes. 


\section{Summary of the Risk Assessment Tool}

\begin{tabular}{|c|c|c|}
\hline $\begin{array}{c}\text { Chief Investigator/Principal } \\
\text { Investigator NAME }\end{array}$ & \multicolumn{2}{|c|}{ STUDY TITLE } \\
\hline $\begin{array}{c}\text { Cheryl Pike (CI) } \\
\text { Sian Clement (PI) }\end{array}$ & $\begin{array}{l}\text { Exploiting 3D scanning technology in lymphoedema for } \\
\text { accurate and fast measurements of volume and shape }\end{array}$ \\
\hline \multicolumn{3}{|c|}{} \\
\hline STUDY ReDA NUMBER & STUDY SPONSOR & RISK ASSESSMENT COMPLETED BY \\
\hline IRAS Project ID: 193784 & ABMUHB R\&D & Cheryl Pike \\
\hline
\end{tabular}

1. SCALE OF RESEARCH:

\begin{tabular}{|l|c|l|l|}
\hline $\begin{array}{c}\text { Number of Participants to } \\
\text { be Recruited }\end{array}$ & $\begin{array}{c}\text { To be completed } \\
\text { by investigator } \\
\text { Tick as } \\
\text { appropriate }\end{array}$ & Risk Level: & Comments: \\
\hline $0-50$ & $\sqrt{ }$ & Minor Risk & $\begin{array}{l}\text { Maximum 20 participants to } \\
\text { be recruited }\end{array}$ \\
\hline
\end{tabular}

2. PATIENT POPULATION:

\begin{tabular}{|l|c|l|l|}
\hline $\begin{array}{l}\text { Patient Population } \\
\text { Involved in Study: }\end{array}$ & $\begin{array}{l}\text { To be completed } \\
\text { by investigator } \\
\text { Tick as } \\
\text { appropriate }\end{array}$ & Risk Level: & Comments: \\
\hline $\begin{array}{l}\text { Subjects able to give } \\
\text { consent - may benefit from } \\
\text { taking part }\end{array}$ & $\sqrt{ }$ & Minor Risk & $\begin{array}{l}\text { The new 3D scan will provide } \\
\text { information about the extent } \\
\text { of lymphoedema and the } \\
\text { areas most affected }\end{array}$ \\
\hline
\end{tabular}

3. INTERVENTION/ LITIGATION:

\begin{tabular}{|l|c|l|l|}
\hline $\begin{array}{l}\text { Procedures/Interventions } \\
\text { Involved in the Study: }\end{array}$ & $\begin{array}{c}\text { To be completed } \\
\text { by investigator } \\
\text { Tick as } \\
\text { appropriate }\end{array}$ & Risk Level: & Comments: \\
\hline Non invasive procedures & $\sqrt{ }$ & No Risk & \\
\hline
\end{tabular}




\section{INVESTIGATOR:}

\begin{tabular}{|l|c|l|l|}
\hline $\begin{array}{l}\text { Investigator Involvement and } \\
\text { Experience/Suitability: }\end{array}$ & $\begin{array}{c}\text { To be } \\
\text { completed by } \\
\text { investigator } \\
\text { Tick as }\end{array}$ & Risk Level: & Comments: \\
\hline $\begin{array}{l}\text { Study team have up to date } \\
\text { training in Good Clinical }\end{array}$ & $\sqrt{ }$ & Minor risk & \\
\hline
\end{tabular}

5. MONITORING ARRANGEMENT:

\begin{tabular}{|l|c|l|l|}
\hline $\begin{array}{l}\text { Study type and Monitoring } \\
\text { Arrangements: }\end{array}$ & $\begin{array}{c}\text { To be completed } \\
\text { by investigator } \\
\text { Tick as } \\
\text { appropriate }\end{array}$ & Risk Level: & Comments: \\
\hline $\begin{array}{l}\text { GCP compliance monitoring } \\
\text { undertaken Quality Assurance } \\
\text { Officer/funder/external } \\
\text { sponsor as part of the protocol }\end{array}$ & $\sqrt{ }$ & Minor risk & \\
\hline
\end{tabular}

6. INFORMATION/PERSONAL DATA:

\begin{tabular}{|l|c|l|l|}
\hline Nature and Use of Data: & $\begin{array}{c}\text { To be completed } \\
\text { by investigator } \\
\text { Tick as } \\
\text { appropriate }\end{array}$ & Risk Level: & Comments: \\
\hline $\begin{array}{l}\text { Data anonymised or } \\
\text { pseudonymised }\end{array}$ & $\sqrt{ }$ & Minor risk & \\
\hline No data sent outside EU & $\sqrt{ }$ & Minor risk & \\
\hline Clear provision for archiving & $\sqrt{ }$ & Minor risk & \\
\hline $\begin{array}{l}\text { Clear processes for result } \\
\text { dissemination }\end{array}$ & $\sqrt{ }$ & Minor risk & \\
\hline Data stored in secure site & $\sqrt{ }$ & Minor risk & \\
\hline
\end{tabular}

7. PROTOCOL:

\begin{tabular}{|l|c|l|l|}
\hline $\begin{array}{l}\text { Protocol Detail and } \\
\text { Quality: }\end{array}$ & $\begin{array}{c}\text { To be completed } \\
\text { by investigator } \\
\text { Tick as } \\
\text { appropriate }\end{array}$ & Risk Level: & Comments: \\
\hline $\begin{array}{l}\text { Clear complete rationale } \\
\text { and scientific justification }\end{array}$ & $\sqrt{ }$ & Minor risk & \\
\hline Clearly defined proposal & $\sqrt{ }$ & Minor risk & \\
\hline $\begin{array}{l}\text { Clear guidance for protocol } \\
\text { violation }\end{array}$ & $\sqrt{ }$ & Minor risk & \\
\hline
\end{tabular}


8. FINANCE: Investigator to discuss with R\&D Finance Manager

\begin{tabular}{|l|c|l|l|}
\hline Financial Details: & $\begin{array}{c}\text { To be completed } \\
\text { by investigator } \\
\text { Tick as } \\
\text { appropriate }\end{array}$ & Risk Level: & Comments: \\
\hline $\begin{array}{l}\text { Partially funded research } \\
\text { with directorate picking up }\end{array}$ & $\sqrt{ }$ & Minor risk & \\
\hline
\end{tabular}

9. CONSENT:

\begin{tabular}{|l|c|l|l|}
\hline $\begin{array}{l}\text { Consenting Process, } \\
\text { Quality and Information: }\end{array}$ & $\begin{array}{c}\text { To be completed } \\
\text { by investigator } \\
\text { Tick as } \\
\text { appropriate }\end{array}$ & Risk Level: & Comments: \\
\hline $\begin{array}{l}\text { Clearly defined process for } \\
\text { informed consent with } \\
\text { named designation of }\end{array}$ & $\sqrt{ }$ & Minor risk & Minor risk \\
\hline $\begin{array}{l}\text { Clear defined recruitment } \\
\text { process }\end{array}$ & $\sqrt{ }$ & Minor risk & \\
\hline $\begin{array}{l}\text { Patient given at least 48 } \\
\text { hours to consider taking } \\
\text { part }\end{array}$ & $\sqrt{ }$ & Minor risk & \\
\hline $\begin{array}{l}\text { Clearly identified risk and } \\
\text { benefit }\end{array}$ & $\sqrt{ }$ & Minor risk & \\
\hline $\begin{array}{l}\text { Clear and concise consent } \\
\text { form and } \\
\text { information sheet }\end{array}$ & & & \\
\hline
\end{tabular}

Overall Assessment Score: To be completed by investigator

To assess overall scores please count amount of ticks under each risk level (ie 1 Scale of Research, 2 Patient Population etc.) and enter in the columns below

\begin{tabular}{|l|c|c|c|c|c|}
\hline & No Risk & Minor Risk & $\begin{array}{c}\text { Moderate } \\
\text { Risk }\end{array}$ & High Risk & Major Risk \\
\hline $\begin{array}{l}\text { 1. Scale of } \\
\text { Research }\end{array}$ & & 1 & & & \\
\hline $\begin{array}{l}\text { 2. Patient } \\
\text { Population }\end{array}$ & 1 & 1 & & & \\
\hline $\begin{array}{l}\text { 3. Intervention } \\
\text { 4. Investigator }\end{array}$ & 1 & & & \\
\hline $\begin{array}{l}\text { 5. Monitoring } \\
\text { arrangement }\end{array}$ & & 1 & & & \\
\hline $\begin{array}{l}\text { 6. Personal } \\
\text { Data/Information }\end{array}$ & & 5 & & & \\
\hline
\end{tabular}




\begin{tabular}{|l|c|c|l|l|l|}
\hline 7. Protocol & & 3 & & & \\
\hline 8. Finance & & 1 & & & \\
\hline 9. Consent & 1 & 18 & & & \\
\hline $\begin{array}{l}\text { TOTAL } \\
\text { (NUMBER)** }\end{array}$ & 1 & 5 & & & \\
\hline
\end{tabular}

\section{PROJECT GRADED AS (highest number)}

(Eg Total (number)** - No risk $=0$, Minor risk $=3$, Moderate risk $=10$, High risk $=9$, Major risk = 3 then the study is scored as "Moderate Risk")

$\square$ No Risk $\quad \square$ Minor Risk $\square$ Moderate Risk $\square$ High Risk
$\square$ Major Risk

$R \& D$ Office use only

Monitoring: $\square$ Yes $\square$ No

Comments:

Sign:

Print:

Date: 
APPENDIX 10: 3D Study Assessment Form

\section{Lymphoedema History}

Date of lymphoedema diagnosis:

Type of Lymphoedema: $\square$ Primary $\quad \square$ Secondary

Cause of Primary: $\quad \square$ Milroy's Disease $\quad \square$ Meige's Syndrome $\square$ Unknown

$\square$ Vascular Malformation $\quad \square$ Klippel-Trenaunay-Weber Syndrome

$\square$ Turner's Syndrome $\quad \square$ Lymphoedema Distichiasis

$\square$ Other:

Cause of Secondary: $\square$ Trauma (not surgery) $\quad \square$ Infection $\square$ Inflammation

$\square$ Cancer (malignant/tumour) $\square$ Cancer (treatment) $\quad \square$ Venous Disease

$\square$ Obesity $\quad \square$ Lipoedema $\quad \square$ Factitious

$\square$ Immobility \& Dependence $\square$ Other:

Upper Limb: $\square$ Upper arm $\quad \square$ Forearm $\quad \square$ Wrist $\square$ Hand

Affected side: $\square$ Left $\quad \square$ Right $\quad$ Dominant side: $\square$ Left $\square$ Right

Exacerbating factors:

Improving factors:

Family History of Lymphoedema: $\quad \square$ Yes $\quad \square$ No $\quad \square$ N/A

Medical History

Cancer History: $\quad \square$ Yes $\square$ No $\quad$ Type:

Surgery (date / type):

Lymph Node Excision: $\square$ Yes $\square$ No $\quad$ No. removed: .................. No. positive:

Radiotherapy (date / location):

Chemotherapy: $\square$ Yes $\square$ No $\quad$ Hormone Therapy: $\square$ Yes $\square$ No

Other Medical History

CVA / TIA $\quad \square$ Yes $\square$ No Detail:

Cardiac/Respiratory $\square$ Yes $\square$ No Detail:

Vascular/Arterial $\square$ Yes $\square$ No Detail:

Diabetes $\quad \square$ Yes $\square$ No Detail:

Thyroid $\quad \square$ Yes $\square$ No Detail:

Skin Condition $\quad \square$ Yes $\square$ No Detail:

Weight History $\quad \square$ Yes $\square$ No Detail:

Arthritis $\quad \square$ Yes $\square$ No Detail:

Mobility Problems $\square$ Yes $\square$ No Detail:

Cellulitis Episodes: $\quad \square$ Yes $\square$ No No. of episodes within last year:

Prophylactic antibiotics prescribed: $\square$ Yes $\square$ No Detail: 
Other medical conditions:

Medication:

Allergies:

Penicillin: $\quad \square$ Yes $\square$ No $\quad$ Latex: $\square$ Yes $\square$ No

Elastoplast: $\square$ Yes $\square$ No $\quad$ Other:

Social History:

Occupation:

Hobbies:

Smoke $\square$ Yes $\square$ No Detail:

Alcohol $\square$ Yes $\square$ No Detail:

Mobility $\square$ Independent $\quad \square$ Aids

Wheelchair $\square$ Bed bound

Lymphoedema Management - Lymphoedema treatment received (tick all relevant):
No treatment
$\square$ Skin Care Advice
Lymphoedema Exercises
$\square$ Simple Lymph Drainage
$\square$ Compression Garment
$\checkmark$ Weight Management
$\square$ Manual Lymph Drainage
$\square$ MLLB (including 3M)
Lymphassist
Deep Oscillation Therapy
$\square$ Kinesiotape $\square$ Laser
Other:

Please indicate type if previously had compression garment:

$\square$ Ready to wear $\quad \square$ Made to measure $\quad \square$ N/A

Make of compression garment:

Material of garment:

$\checkmark$ Activa

$\square$ Circular Knit

BSN

Flat Knit

$\square$ Haddenham Healthcare

$\square$ Juzo

Compression Class (RAL):

MediUK

$\checkmark \mathrm{ccl} 1$

$\checkmark$ Sigvaris

ccl 2

Venosan

ccl 3

Other:

ccl 4 
No. of days per week you wear your garment:
a. One to two days
b. Three to four days
C. Five to six days
d. Every day
e. Not wearing garment
f. Not applicable

No. of hours you wear your garment:
a. Nil
b. 1-5 hours
C. 6-12 hours
d. 13 plus hours
e. Not applicable

\section{Impact of Lymphoedema}

Distress Thermometer

$\begin{array}{lll}\begin{array}{l}\text { Extreme } \\ \text { distress }\end{array} & \begin{array}{l}\text { Using the thermometer, write down the number (0 to 10) } \\ \text { that best describes how much distress }{ }^{*} \text { you have felt in the }\end{array} \\ \text { distress } & \text { past week, including today: }\end{array}$

a. How anxious does your lymphoedema make you feel?

b. Does your lymphoedema affect your body image?

c. Does your lymphoedema affect your sexuality?

d. What effect does lymphoedema have on your hobbies?

e. What effect does lymphoedema have on your work?

f. What effect does lymphoedema have on you when shopping for clothes?

g. What effect does wearing a compression garment have on your life?

h. Have you been referred for support for anxiety? $\square$ Yes $\quad \square$ No $\quad \square$ N/A 


\section{Initial Assessment}

\section{Subjective Assessment}

Visual analogue scale (0-10). Rate your pain and then heaviness of your lymphoedema arm using the scale below:

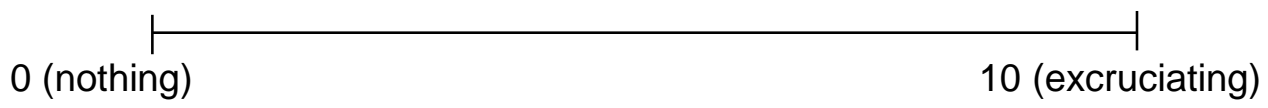

Pain (0-10):

Heaviness (0-10):

\section{Objective Assessment}

Skin Assessment:

Discolouration: $\square$ No $\square$ Yes $\quad$ Detail:

Temperature: $\square$ Normal $\quad \square$ Cold $\quad \square$ Hot

Skin: $\quad \square$ Normal $\quad \square$ Dry $\quad \square$ Fragile $\quad \square$ Blisters

$\square$ Taut $\quad \square$ Shiny $\quad \square$ Ulcers $\quad \square$ Hyperkeratosis

$\square$ Skin folds $\square$ Eczema $\square$ Fungal infection $\square$ Lymphorrhoea

$\square$ Papillamatosis $\quad \square$ Other:

Tissue: $\quad \square$ Pitting $\quad \square$ Non-pitting $\square$ Fibrosis $\quad \square$ Fatty

$\square$ Hard/solid $\square$ Firm $\quad \square$ Soft

Shape : $\quad \square$ Normal $\quad \square$ Distorted Detail:

Stemmer's Sign (affected hand): $\square$ Positive $\square$ Negative

Range of Movement:

\begin{tabular}{|l|l|l|l|l|l|l|l|l|l|l|}
\hline \multirow{2}{*}{} & \multicolumn{7}{|c|}{ RIGHT } & \multicolumn{5}{c|}{ LEFT } \\
\cline { 2 - 11 } & $100 \%$ & $75 \%$ & $50 \%$ & $25 \%$ & $0 \%$ & $100 \%$ & $75 \%$ & $50 \%$ & $25 \%$ & $0 \%$ \\
\hline Shoulder & & & & & & & & & & \\
\hline Elbow & & & & & & & & & & \\
\hline Wrist & & & & & & & & & & \\
\hline Fingers & & & & & & & & & & \\
\hline Additional Info: & & & & & & & & \\
\hline
\end{tabular}




\section{Staging of Lymphoedema}

ISL Staging of Lymphoedema:

$\square$ Stage 0 - Latent

$\square$ Stage I - Reversible

$\square$ Stage II a - Pitting

$\square$ Stage II b - late stage II - Non-pitting

$\square$ Stage III - Elephantatic

BLS Grouping of Lymphoedema:

$\square$ Group 0 - Latent (high risk)

$\square$ Group 1 - Early Lymphoedema (LO)

$\square$ Group 2 - Uncomplicated (established) LO

$\square$ Group 3a-Complex LO: one limb

$\square$ Group 3b - Complex LO: multiple limbs

$\square$ Group 3c - Complex Midline Lymphoedema

$\square$ (Group 4 - Palliative)

\section{Tests:}

3D photo: $\square$ Left arm $\square$ Right arm

Perometer: $\square$ Left arm $\square$ Right arm

Limb volume measurements: $\square$ Measure 1

$\square$ Measure 2
Time taken:

Time taken:

Time taken:

Time taken: 


\begin{tabular}{|c|c|c|c|c|c|c|}
\hline & \multicolumn{2}{|c|}{ Limb Volume 1} & & \multicolumn{2}{|c|}{ Limb Volume 2} \\
\hline & & Right & Left & & Right & Left \\
\hline \multicolumn{2}{|l|}{ Hand } & & & Hand & & \\
\hline \multirow{2}{*}{$\begin{array}{l}\text { First Mark: } \\
\quad \ldots \ldots \ldots \ldots \ldots \ldots\end{array}$} & 1 & & & 1 & & \\
\hline & 2 & & & 2 & & \\
\hline \multirow{3}{*}{ Height (cm): } & 3 & & & 3 & & \\
\hline & 4 & & & 4 & & \\
\hline & 5 & & & 5 & & \\
\hline \multirow[t]{2}{*}{ Weight (kg): } & 6 & & & 6 & & \\
\hline & 7 & & & 7 & & \\
\hline \multirow{3}{*}{ BMI: } & 8 & & & 8 & & \\
\hline & 9 & & & 9 & & \\
\hline & 10 & & & 10 & & \\
\hline \multicolumn{2}{|c|}{ Distal Volume mls } & & & Distal & & \\
\hline & 1 & & & 1 & & \\
\hline & 2 & & & 2 & & \\
\hline & 3 & & & 3 & & \\
\hline & 4 & & & 4 & & \\
\hline & 5 & & & 5 & & \\
\hline & 6 & & & 6 & & \\
\hline & 7 & & & 7 & & \\
\hline & 8 & & & 8 & & \\
\hline & 9 & & & 9 & & \\
\hline & 10 & & & 10 & & \\
\hline \multicolumn{2}{|c|}{ Proximal Volume mls } & & & Prox & & \\
\hline \multicolumn{2}{|c|}{ Total Volume mls } & & & Total & & \\
\hline \multicolumn{2}{|l|}{ Excess mls } & & & Excess ml & & \\
\hline \multicolumn{2}{|l|}{ Excess \% } & & & Excess \% & & \\
\hline \multicolumn{2}{|c|}{ Distal Volume Diff } & $\mathrm{ml}$ & $\%$ & Distal & $\mathrm{ml}$ & $\%$ \\
\hline \multicolumn{2}{|c|}{ Proximal Volume Diff } & $\mathrm{ml}$ & $\%$ & Prox & $\mathrm{ml}$ & $\%$ \\
\hline \multicolumn{2}{|l|}{ P/D Ratio } & & & $P / D$ & & \\
\hline
\end{tabular}




\section{Problem List}

$\square$ Severe complicated swelling

$\square$ Moderate swelling

$\square$ Mild swelling

$\square$ Prevention of LO

Dry skin

Decreased ROM

$\square$ Scar problems

$\square$ At Risk

$\square$ Excess Body Weight

$\square$ Mobility Problems

Psychosocial

Other:

\section{Treatment Plan}

$\begin{array}{ll}\square \text { No treatment } \quad \square \text { Skin Care Advice } \quad \square \text { Lymphoedema Exercises } \\ \square \text { Simple Lymph Drainage } \quad \square \text { Compression Garment } \quad \square \text { Weight Management } \\ \square \text { Manual Lymph Drainage } \quad \square \text { MLLB (including 3M) } \quad \square \text { Lymphassist } \\ \square \text { Deep Oscillation Therapy } \quad \square \text { Kinesiotape } & \square \text { Laser }\end{array}$

Other:

Type of garment: $\square$ Ready to wear $\quad \square$ Made to measure $\quad \square$ N/A

Garment: $\square$ Issued by clinic $\quad \square$ Prescription issued

$\begin{array}{ll}\text { Make of compression garment: } & \text { Material of garment: } \\ \square \text { Activa } & \square \text { Circular Knit } \\ \square \text { BSN } & \square \text { Flat Knit } \\ \square \text { Haddenham Healthcare } & \\ \square \text { Juzo } & \text { Compression Class (RAL): } \\ \square \text { MediUK } & \square \mathrm{ccl} \mathrm{1} \\ \square \text { Sigvaris } & \square \mathrm{ccl} 2 \\ \square \text { Venosan } & \square \mathrm{ccl} \mathrm{3} \\ \square \text { Other: } & \square \mathrm{ccl} 4\end{array}$


Lymphoedema Management - Lymphoedema treatment received (tick all relevant):

$\square$ No treatment $\quad \square$ Skin Care Advice $\quad \square$ Lymphoedema Exercises
$\square$ Simple Lymph Drainage $\quad \square$ Compression Garment $\quad \square$ Weight Management
$\square$ Manual Lymph Drainage $\quad \square$ MLLB (including 3M) $\quad \square$ Lymphassist
$\square$ Deep Oscillation Therapy $\quad \square$ Kinesiotape
$\square$ Other:

Problem acquiring garment? $\square$ Yes $\square$ No $\square$ N/A Details:

No. of days per week you wear your garment:
a. One to two days
b. Three to four days
c. Five to six days
d. Every day
e. Not wearing garment
f. Not applicable

No. of hours you wear your garment:
a. Nil
b. 1-5 hours
c. 6-12 hours
d. 13 plus hours
e. Not applicable 
Follow-Up Assessment (2, 4, 6 month)

\section{Subjective Assessment}

Visual analogue scale (0-10). Rate your pain and then heaviness of your lymphoedema arm using the scale below:

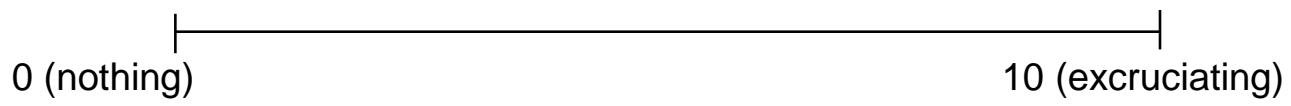

Pain (0-10):

Heaviness (0-10):

\section{Objective Assessment}

Skin Assessment:

Discolouration: $\square$ No $\square$ Yes $\quad$ Detail:

Temperature: $\square$ Normal $\quad \square$ Cold $\quad \square$ Hot

Skin: $\quad \square$ Normal $\quad \square$ Dry $\quad \square$ Fragile $\quad \square$ Blisters

$\square$ Taut $\quad \square$ Shiny $\quad \square$ Ulcers $\quad \square$ Hyperkeratosis

$\square$ Skin folds $\square$ Eczema $\quad \square$ Fungal infection $\square$ Lymphorrhoea

$\square$ Papillamatosis $\quad \square$ Other:

Tissue: $\quad \square$ Pitting $\quad \square$ Non-pitting $\square$ Fibrosis $\quad \square$ Fatty

$\square$ Hard/solid $\square$ Firm $\quad \square$ Soft

Shape : $\quad \square$ Normal $\quad \square$ Distorted Detail:

Stemmer's Sign (affected hand): $\square$ Positive $\square$ Negative

Range of Movement:

\begin{tabular}{|l|l|l|l|l|l|l|l|l|l|l|}
\hline \multirow{2}{*}{} & \multicolumn{7}{|c|}{ RIGHT } & \multicolumn{5}{c|}{ LEFT } \\
\cline { 2 - 11 } & $100 \%$ & $75 \%$ & $50 \%$ & $25 \%$ & $0 \%$ & $100 \%$ & $75 \%$ & $50 \%$ & $25 \%$ & $0 \%$ \\
\hline Shoulder & & & & & & & & & & \\
\hline Elbow & & & & & & & & & & \\
\hline Wrist & & & & & & & & & & \\
\hline Fingers & & & & & & & & & & \\
\hline Additional Info: & & & & & & & & \\
\hline
\end{tabular}

\section{Tests:}

3D photo: $\square$ Left arm $\quad \square$ Right arm

Perometer: $\square$ Left arm $\square$ Right arm

Limb volume measurements: $\square$ Measure $1 \square$ Measure 2 


\begin{tabular}{|c|c|c|c|c|c|c|}
\hline & \multicolumn{2}{|c|}{ Limb Volume 1} & & \multicolumn{2}{|c|}{ Limb Volume 2} \\
\hline & & Right & Left & & Right & Left \\
\hline \multicolumn{2}{|l|}{ Hand } & & & Hand & & \\
\hline \multirow{2}{*}{$\begin{array}{l}\text { First Mark: } \\
\mathrm{cm}\end{array}$} & 1 & & & 1 & & \\
\hline & 2 & & & 2 & & \\
\hline \multirow{3}{*}{ Height $(\mathrm{cm})$ : } & 3 & & & 3 & & \\
\hline & 4 & & & 4 & & \\
\hline & 5 & & & 5 & & \\
\hline \multirow[t]{2}{*}{ Weight $(\mathrm{kg})$ : } & 6 & & & 6 & & \\
\hline & 7 & & & 7 & & \\
\hline \multirow{3}{*}{ BMI: } & 8 & & & 8 & & \\
\hline & 9 & & & 9 & & \\
\hline & 10 & & & 10 & & \\
\hline \multicolumn{2}{|c|}{ Distal Volume mls } & & & Distal & & \\
\hline & 1 & & & 1 & & \\
\hline & 2 & & & 2 & & \\
\hline & 3 & & & 3 & & \\
\hline & 4 & & & 4 & & \\
\hline & 5 & & & 5 & & \\
\hline & 6 & & & 6 & & \\
\hline & 7 & & & 7 & & \\
\hline & 8 & & & 8 & & \\
\hline & 9 & & & 9 & & \\
\hline & 10 & & & 10 & & \\
\hline \multicolumn{2}{|c|}{ Proximal Volume mls } & & & Prox & & \\
\hline \multicolumn{2}{|c|}{ Total Volume mls } & & & Total & & \\
\hline \multicolumn{2}{|l|}{ Excess mls } & & & Excess $\mathrm{ml}$ & & \\
\hline \multicolumn{2}{|l|}{ Excess \% } & & & Excess \% & & \\
\hline \multicolumn{2}{|c|}{ Distal Volume Diff } & $\mathrm{ml}$ & $\%$ & Distal & $\mathrm{ml}$ & $\%$ \\
\hline \multicolumn{2}{|c|}{ Proximal Volume Diff } & $\mathrm{ml}$ & $\%$ & Prox & $\mathrm{ml}$ & $\%$ \\
\hline \multicolumn{2}{|l|}{ P/D Ratio } & & & $P / D$ & & \\
\hline
\end{tabular}




\section{Treatment Plan}

$\square$ No treatment

$\square$ Skin Care Advice $\quad \square$ Lymphoedema Exercises

$\square$ Simple Lymph Drainage $\quad \square$ Compression Garment $\quad \square$ Weight Management

$\square$ Manual Lymph Drainage $\quad \square$ MLLB (including 3M) $\quad \square$ Lymphassist

$\square$ Deep Oscillation Therapy $\quad \square$ Kinesiotape $\quad \square$ Laser

$\square$ Other:

Change in garment needed?: $\square$ Yes $\square$ No $\square$ N/A Detail:

If garment changed please complete:

Garment: $\square$ Issued by clinic $\quad \square$ Prescription issued

Make of compression garment:

$\square$ Activa

Material of garment:

$\square$ BSN

Circular Knit

$\square$ Haddenham Healthcare

$\square$ Juzo

Compression Class (RAL):

$\square$ MediUK

$\square \operatorname{ccl} 1$

$\square$ Sigvaris

$\square \mathrm{ccl} 2$

$\square$ Venosan

$\square \mathrm{ccl} 3$

$\square$ Other:

$\mathrm{ccl} 4$ 


\section{APPENDIX 11: Data results from the measurements}

Summary of the Limb Volume, Volume Difference (VD) and Percentage Volume Difference (\%VD) for CTM over time

\begin{tabular}{|c|c|c|c|c|c|c|c|c|c|c|c|c|c|c|c|c|c|}
\hline \multirow{2}{*}{$\begin{array}{l}\text { ID } \\
\#\end{array}$} & \multicolumn{5}{|c|}{ First Assessment } & \multicolumn{4}{|c|}{ Follow Up 1} & \multicolumn{4}{|c|}{ Follow Up 2} & \multicolumn{4}{|c|}{ Follow Up 3} \\
\hline & $\begin{array}{c}\text { Right } \\
\text { Vol }\end{array}$ & $\begin{array}{l}\text { Left } \\
\text { Vol }\end{array}$ & VD & $\% \mathrm{VD}$ & TIME & $\begin{array}{c}\text { Right } \\
\text { Vol }\end{array}$ & $\begin{array}{l}\text { Left } \\
\text { Vol }\end{array}$ & VD & $\% \mathrm{VD}$ & $\begin{array}{c}\text { Right } \\
\text { Vol }\end{array}$ & $\begin{array}{l}\text { Left } \\
\text { Vol }\end{array}$ & VD & $\% \mathrm{VD}$ & $\begin{array}{c}\text { Right } \\
\text { Vol }\end{array}$ & $\begin{array}{l}\text { Left } \\
\text { Vol }\end{array}$ & VD & $\% \mathrm{VD}$ \\
\hline 1 & 2130.5 & 2044.5 & 86 & $4 \%$ & 07:52 & 2064 & 2077.5 & -13.5 & $-1 \%$ & 2323 & 2295 & 28 & $1 \%$ & 2265.5 & 2240.5 & 25 & $1 \%$ \\
\hline 2 & 2884 & 2657.5 & 226.5 & $9 \%$ & 04:00 & 3371 & 2899.5 & 471.5 & $16 \%$ & 3171 & 2794.5 & 376.5 & $14 \%$ & 3321.5 & 2873.5 & 448 & $16 \%$ \\
\hline 3 & 2159 & 2092.5 & 66.5 & $3 \%$ & 07:00 & 2148.5 & 2155 & -6.5 & $0 \%$ & 2162.5 & 2190 & -27 & $-1 \%$ & 2191.5 & 2115.5 & 76 & $4 \%$ \\
\hline 4 & 2552.5 & 2483 & 69.5 & $3 \%$ & $05: 17$ & 2353.5 & 2415.5 & -62 & $-3 \%$ & 2493.5 & 2443.5 & 50 & $2 \%$ & 2388 & 2445.5 & -57.5 & $-2 \%$ \\
\hline 5 & 3969 & 3273.5 & 695.5 & $22 \%$ & 05:59 & 3998.5 & 3403 & 595.5 & $18 \%$ & 4015 & 3679.5 & 335.5 & $9 \%$ & 4191 & 3805.5 & 385.5 & $10 \%$ \\
\hline 6 & 6715.5 & 5049 & 1666.5 & $33 \%$ & $07: 47$ & 6085 & 4836.5 & 1248.5 & $26 \%$ & 6264 & 4753 & 1511 & $32 \%$ & 6449 & 4758.5 & 1690.5 & $36 \%$ \\
\hline 7 & 4506 & 4319.5 & 186.5 & $5 \%$ & 04:56 & 4215 & 4051.5 & 163.5 & $4 \%$ & 3819.5 & 3871.5 & -52 & $-1 \%$ & 4114 & 4058 & 56 & $2 \%$ \\
\hline 8 & 3135 & 2850 & 286 & $10 \%$ & 05:08 & 2994.5 & 2898 & 96.5 & $4 \%$ & 2824.5 & 2843.5 & -19 & $-2 \%$ & 2851.5 & 2805.5 & 46 & $2 \%$ \\
\hline 9 & 2400 & 2310 & 90 & $4 \%$ & $05: 33$ & 2449 & 2274.5 & 174.5 & $8 \%$ & 2327.5 & 2249.5 & 78 & $4 \%$ & 2514 & 2429 & 85 & $4 \%$ \\
\hline 10 & 2684 & 2237.5 & 447 & $20 \%$ & 03:49 & \multicolumn{4}{|c|}{ Failed to Attend } & \multicolumn{4}{|c|}{ Failed to Attend } & \multicolumn{4}{|c|}{ Failed to Attend } \\
\hline 11 & 2631.5 & 2516 & 116 & $5 \%$ & 05:39 & 2610.5 & 2503 & 107.5 & $5 \%$ & 2466 & 2438.5 & 27.5 & $2 \%$ & 2494.5 & 2484 & 11 & $1 \%$ \\
\hline 12 & 3149 & 3172.5 & -23.5 & $-1 \%$ & 06:06 & 3425 & 3535.5 & -110.5 & $-3 \%$ & 1825 & 1742 & -83 & $-3 \%$ & 3300 & 3392 & -92 & $-3 \%$ \\
\hline 13 & 3002 & 2675 & 327.5 & $13 \%$ & $06: 30$ & 2862 & 2632.5 & 229.5 & $9 \%$ & 2892 & 2625 & 267 & $11 \%$ & 2934.5 & 2601 & 333.5 & $13 \%$ \\
\hline 14 & 4077 & 3093.5 & 983.5 & $32 \%$ & 06:49 & 4179.5 & 3105.5 & 1074 & $35 \%$ & 3969 & 3064 & 905 & $30 \%$ & 3812 & 2939 & 873 & $30 \%$ \\
\hline 15 & 2256 & 2108.5 & 147.5 & $7 \%$ & $05: 48$ & 2258.5 & 2101 & 158 & $8 \%$ & 2279 & 2160 & 119 & $6 \%$ & 2295 & 2168.5 & 126.5 & $6 \%$ \\
\hline 16 & 2068.5 & 1817.5 & 252 & $14 \%$ & 06:00 & 2038.5 & 1871.5 & 167.5 & $9 \%$ & 1986.5 & 1818 & 168.5 & $9 \%$ & 1955 & 1803.5 & 151.5 & $9 \%$ \\
\hline 17 & 2262 & 2986 & 276 & $10 \%$ & 05:30 & 3127 & 3037.5 & 89.5 & $3 \%$ & 3033.5 & 2910.5 & 123 & $4 \%$ & 3070 & 2946.5 & 123.5 & $5 \%$ \\
\hline 18 & 3762.5 & 3564.5 & 198 & $6 \%$ & 08:49 & \multicolumn{4}{|c|}{ Failed to Attend } & 3538.5 & 3625.5 & 87.5 & $3 \%$ & \multicolumn{4}{|c|}{ Failed to Attend } \\
\hline 19 & 2502.5 & 2558 & 55.5 & $-2 \%$ & $04: 24$ & 1593.5 & 2577 & 27 & $-1 \%$ & 1503 & 2443 & 11.5 & $-1 \%$ & \multicolumn{4}{|c|}{ Failed to Attend } \\
\hline 20 & 2970 & 2777.5 & 192 & $7 \%$ & $05: 10$ & 1882.5 & 2933.5 & 71 & $3 \%$ & 1853.5 & 2898.5 & 12.5 & $1 \%$ & 1846 & 2896.5 & 61 & $2 \%$ \\
\hline
\end{tabular}


Summary of the Limb Volume, Volume Difference (VD) and Percentage Volume Difference (\%VD) for Perometry over time

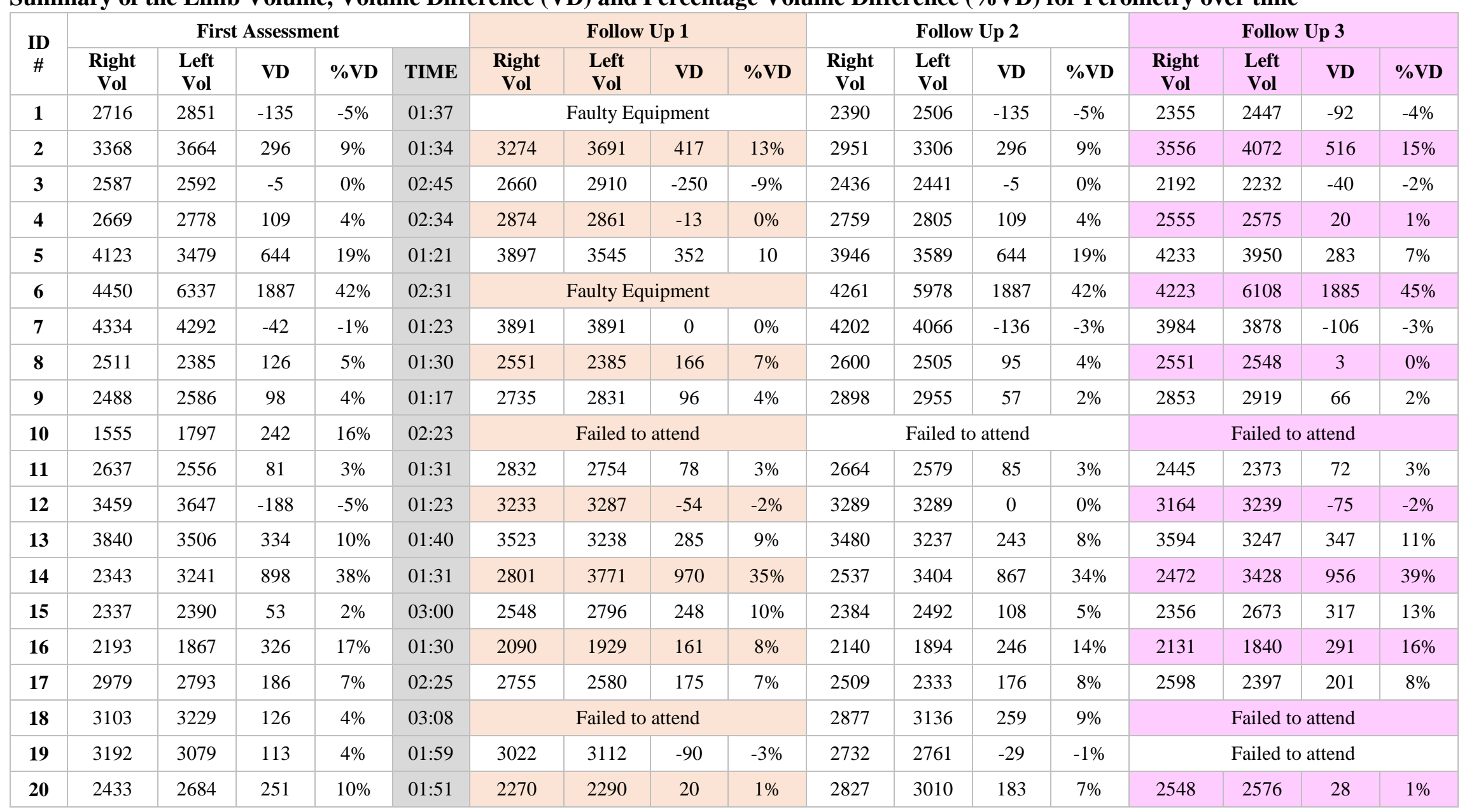


Summary of the Limb Volume, Volume Difference (VD) and Percentage Volume Difference (\%VD) for 3DCI over time

\begin{tabular}{|c|c|c|c|c|c|c|c|c|c|c|c|c|c|c|c|c|c|}
\hline \multirow{2}{*}{$\begin{array}{c}\text { ID } \\
\#\end{array}$} & \multicolumn{5}{|c|}{ First Assessment } & \multicolumn{4}{|c|}{ Follow Up 1} & \multicolumn{4}{|c|}{ Follow Up 2} & \multicolumn{4}{|c|}{ Follow Up 3} \\
\hline & $\begin{array}{c}\text { Right } \\
\text { Vol }\end{array}$ & Left Vol & VD & $\% \mathrm{VD}$ & TIME & $\begin{array}{c}\text { Right } \\
\text { Vol }\end{array}$ & Left Vol & VD & $\% \mathrm{VD}$ & $\begin{array}{c}\text { Right } \\
\text { Vol }\end{array}$ & Left Vol & VD & $\% \mathrm{VD}$ & $\begin{array}{c}\text { Right } \\
\text { Vol }\end{array}$ & Left Vol & VD & $\% \mathrm{VD}$ \\
\hline 1 & 2316 & 2352 & 36 & $2 \%$ & $1: 57$ & 1883.1 & 2149.9 & 266.7 & $14 \%$ & 2326.1 & 2347.2 & 21.1 & $1 \%$ & 2279.7 & 2401.6 & 121.9 & $5 \%$ \\
\hline 2 & 2104.7 & 2281.5 & 176.8 & $8 \%$ & $1: 43$ & 2094.5 & 2548.1 & 453.6 & $22 \%$ & 2228.7 & 2592.6 & 363.9 & $16 \%$ & 2095.9 & 2632.4 & 536.5 & $26 \%$ \\
\hline 3 & 2409.2 & 2158.8 & 250.5 & $12 \%$ & $1: 55$ & 2318.3 & 2323.8 & -5.5 & $0 \%$ & 2318.9 & 2331.9 & -13 & $-1 \%$ & 2159.7 & 2236 & -76.3 & $-3 \%$ \\
\hline 4 & 2588.4 & 2713.3 & 124.9 & $5 \%$ & $2: 03$ & 2538.3 & 2519 & -19.4 & $-1 \%$ & 2333.7 & 2535.7 & 202 & $9 \%$ & 2362.8 & 2420.4 & 57.6 & $2 \%$ \\
\hline 5 & 3625 & 2954.6 & 670.4 & $23 \%$ & $1: 41$ & 3461.5 & 2845.3 & 616.2 & $22 \%$ & 3538.4 & 3078.3 & 460.1 & $15 \%$ & 3591.3 & 3183.1 & 408.2 & $13 \%$ \\
\hline 6 & \multicolumn{4}{|c|}{ Arm too big for analysis } & $1: 58$ & \multicolumn{4}{|c|}{ Arm too big for analysis } & \multicolumn{4}{|c|}{ Arm too big for analysis } & \multicolumn{4}{|c|}{ Arm too big for analysis } \\
\hline 7 & 3710.6 & 3939.6 & 229 & $6 \%$ & $1: 42$ & 3525.9 & 3861.3 & 335.3 & $10 \%$ & 3630.5 & 3893 & 262.5 & $7 \%$ & 3631.2 & 3787.9 & 156.8 & $4 \%$ \\
\hline 8 & 2404.8 & 2256.1 & 148.7 & $7 \%$ & $1: 50$ & 2290 & 2262.5 & 27.1 & $1 \%$ & 2343.6 & 2327.5 & 16.1 & $1 \%$ & 2264.3 & 2299.8 & -35.5 & $-2 \%$ \\
\hline 9 & 2465.6 & 2308.8 & -156.8 & $-6 \%$ & $1: 27$ & 2335.9 & 2314.2 & -21.6 & $-1 \%$ & 2532.4 & 2525.1 & -7.4 & $0 \%$ & 2537.1 & 2572.5 & 35.4 & $1 \%$ \\
\hline 10 & 2442 & 3158 & 716 & $29 \%$ & $1: 33$ & \multicolumn{4}{|c|}{ Failed to attend } & \multicolumn{4}{|c|}{ Failed to attend } & \multicolumn{4}{|c|}{ Failed to attend } \\
\hline 11 & 2705.5 & 2600.6 & 104.9 & $4 \%$ & $1: 41$ & 2872.8 & 2650.6 & 222.2 & $8 \%$ & 2502.2 & 2624 & -121.8 & $-5 \%$ & 2780.5 & 2458.3 & 322.2 & $13 \%$ \\
\hline 12 & 2876.5 & 3025.2 & -148.6 & $-5 \%$ & $1: 43$ & 2477.2 & 2702.6 & -225.4 & $-8 \%$ & 2754.6 & 2666.5 & 88.1 & $3 \%$ & 2820.1 & 2936.5 & -116.5 & $-4 \%$ \\
\hline 13 & 3006.7 & 2809.7 & 197 & $7 \%$ & $1: 30$ & 3008.6 & 2616.7 & 391.9 & $15 \%$ & 3062.3 & 2715.9 & 346.5 & $13 \%$ & 2971.3 & 2582.2 & 389 & $15 \%$ \\
\hline 14 & 2734 & 3581.8 & 847.8 & $31 \%$ & $2: 29$ & 2835.4 & 3750.2 & 914.8 & $32 \%$ & 2775.4 & 3753 & 977.6 & $35 \%$ & 2704.1 & 3523.9 & 819.8 & $30 \%$ \\
\hline 15 & 1878 & 2142.4 & 264.4 & $14 \%$ & $1: 55$ & 1859 & 2253.4 & 394.4 & $21 \%$ & 2010.5 & 2140.9 & 130.4 & $6 \%$ & 1596.3 & 2272.8 & 676.5 & $42 \%$ \\
\hline 16 & 2225.4 & 2015.4 & 210 & $10 \%$ & $1: 34$ & 2191 & 2004.4 & 186.3 & $9 \%$ & 2111.2 & 2025.3 & 85.9 & $4 \%$ & 2144.9 & 2023.1 & 121.7 & $6 \%$ \\
\hline 17 & 2750.4 & 2470.3 & 280.1 & $11 \%$ & $1: 43$ & 3203.3 & 2914 & 289.3 & $10 \%$ & 2826.6 & 2676.7 & 149.9 & $6 \%$ & 2837.4 & 2817.8 & 19.5 & $1 \%$ \\
\hline 18 & 2793.6 & 2962.3 & 168.7 & $6 \%$ & $2: 38$ & \multicolumn{4}{|c|}{ Failed to attend } & 2891.1 & 2997 & 105.8 & $4 \%$ & \multicolumn{4}{|c|}{ Failed to attend } \\
\hline 19 & 2275.8 & 2404.7 & -128.9 & $-5 \%$ & $1: 49$ & 2406.4 & 2577.2 & -170.8 & $-7 \%$ & 2394.9 & 2450.1 & -55.1 & $-2 \%$ & \multicolumn{4}{|c|}{ Failed to attend } \\
\hline 20 & \multicolumn{4}{|c|}{ Images Corrupted } & $01: 55$ & \multicolumn{4}{|c|}{ Images Corrupted } & \multicolumn{4}{|c|}{ Images Corrupted } & \multicolumn{4}{|c|}{ Images Corrupted } \\
\hline
\end{tabular}


Calculated Absolute Volume Difference (AVD) and Percentage Absolute Volume Difference (\%AVD) of the affected upper limb for CTM, Perometry and 3DCI over time

\begin{tabular}{|c|c|c|c|c|c|c|c|c|c|c|c|c|c|c|c|c|c|c|}
\hline \multirow{2}{*}{$\begin{array}{c}\text { ID } \\
\#\end{array}$} & \multicolumn{2}{|c|}{ CTM FU1 } & \multicolumn{2}{|c|}{ CTM FU2 } & \multicolumn{2}{|c|}{ CTM FU3 } & \multicolumn{2}{|c|}{ PERO FU1 } & \multicolumn{2}{|c|}{ PERO FU2 } & \multicolumn{2}{|c|}{ PEROFU3 } & \multicolumn{2}{|c|}{ 3DCI FU1 } & \multicolumn{2}{|c|}{ 3DCI FU2 } & \multicolumn{2}{|c|}{ 3DCI FU3 } \\
\hline & AVD & $\%$ AVD & AVD & $\%$ AVD & AVD & $\%$ AVD & AVD & $\%$ AVD & AVD & $\%$ AVD & AVD & $\%$ AVD & AVD & \%AVD & AVD & $\%$ AVD & AVD & $\% A V D$ \\
\hline 1 & -67 & -3.1 & 193 & 9.0 & 135 & 6.3 & \multicolumn{2}{|c|}{ Fault } & -345 & -12.1 & -404 & -14.2 & -202 & -8.6 & -5 & -0.2 & 50 & 2.1 \\
\hline 2 & 487 & 16.9 & 287 & 10.0 & 438 & 15.2 & 27 & 0.7 & -358 & -9.8 & 408 & 267 & 267 & 11.7 & 311 & 13.6 & 351 & 15.4 \\
\hline 3 & -11 & -0.5 & 4 & 0.2 & 33 & 1.5 & 73 & 2.8 & -151 & -5.8 & -395 & -91 & -91 & -3.8 & -90 & -3.7 & -250 & -10.4 \\
\hline 4 & -199 & -7.8 & -59 & -2.3 & -165 & -6.4 & 83 & 3.0 & 27 & 1.0 & -203 & -194 & -194 & -7.2 & -178 & -6.5 & -293 & -10.8 \\
\hline 5 & 66 & 3.1 & 258 & 12.4 & 336 & 16.1 & -226 & -5.5 & -177 & -4.3 & 110 & -163 & -163 & -4.5 & -87 & -2.4 & -34 & -0.9 \\
\hline 6 & -631 & -9.4 & -423 & -6.3 & -267 & -4.0 & \multicolumn{2}{|c|}{ Fault } & -359 & -5.7 & -229 & -3.6 & \multicolumn{6}{|c|}{ Arm too big for analysis } \\
\hline 7 & -291 & -6.5 & -687 & -15.2 & -392 & -8.7 & -401 & -9.3 & -226 & -5.3 & -414 & -78 & -78 & -2.0 & -47 & -1.2 & -152 & $-3.9 \%$ \\
\hline 8 & 32 & 1.6 & -37 & -1.9 & -61 & -3.2 & 40 & 1.6 & 89 & 3.5 & 40 & -115 & -115 & -4.8 & -61 & -2.5 & -141 & $-5.8 \%$ \\
\hline 9 & 49 & 2.0 & -73 & -3.0 & 114 & 4.8 & 245 & 9.5 & 369 & 14.3 & 333 & 5 & 5 & 0.2 & 216 & 9.4 & 264 & $11.4 \%$ \\
\hline 10 & \multicolumn{6}{|c|}{ Failed to attend } & \multicolumn{6}{|c|}{ Failed to attend } & \multicolumn{6}{|c|}{ Failed to attend } \\
\hline 11 & -20 & -1.2 & -85 & -5.3 & -53 & -3.3 & 195 & 7.4 & 27 & 1.0 & -192 & 167 & 167 & 6.2 & -203 & -7.5 & 75 & $2.8 \%$ \\
\hline 12 & 50 & 2.4 & -1033 & -49.3 & -38 & -1.8 & -226 & -6.5 & -170 & -4.9 & -295 & -399 & -399 & -13.9 & -122 & -4.2 & -56 & $-2.0 \%$ \\
\hline 13 & -58 & -3.4 & -69 & -4.1 & -72 & -4.3 & -317 & -8.3 & -360 & -9.4 & -246 & 2 & 2 & 0.1 & 56 & 1.8 & -35 & $-1.2 \%$ \\
\hline 14 & 103 & 2.5 & -108 & -2.6 & -265 & -6.5 & 530 & 16.4 & 163 & 5.0 & 187 & 168 & 168 & 4.7 & 171 & 4.8 & -58 & $-1.6 \%$ \\
\hline 15 & 3 & 0.1 & 23 & 1.0 & 39 & 1.7 & 406 & 17.0 & 102 & 4.3 & 283 & 111 & 111 & 5.2 & -2 & -0.1 & 130 & $6.1 \%$ \\
\hline 16 & 40 & 3.8 & -1 & 0.0 & -10 & -0.9 & -103 & -4.7 & -53 & -2.4 & -62 & -35 & -35 & -1.6 & -114 & -5.1 & -80 & $-3.6 \%$ \\
\hline 17 & 25 & 1.3 & -46 & -2.3 & -29 & -1.5 & -224 & -7.5 & -470 & -15.8 & -381 & 453 & 453 & 16.5 & 76 & 2.8 & 87 & $3.2 \%$ \\
\hline 18 & \multicolumn{2}{|c|}{ Failed to attend } & -137 & -3.6 & \multicolumn{2}{|c|}{ Failed to attend } & \multicolumn{2}{|c|}{ Failed to attend } & -93 & -2.9 & \multicolumn{2}{|c|}{ Failed to attend } & \multicolumn{2}{|c|}{ Failed to attend } & 35 & 1.2 & \multicolumn{2}{|c|}{ Failed to attend } \\
\hline 19 & -7 & -0.4 & -98 & -6.1 & \multicolumn{2}{|c|}{ Failed to attend } & -170 & -5.3 & -460 & -14.4 & \multicolumn{2}{|c|}{ Failed to attend } & 131 & 5.7 & 119 & 5.2 & \multicolumn{2}{|c|}{ Failed to attend } \\
\hline 20 & -37 & -1.2 & -72 & -2.4 & -74 & -2.5 & -394 & -14.7 & 326 & 12.1 & -108 & -4.0 & \multicolumn{6}{|c|}{ Images Corrupted } \\
\hline
\end{tabular}


APPENDIX 12: The STARD 2015 List: An Updated List of Essential Items for Reporting Diagnostic Accuracy Studies (Bossuyt et al., 2015)

\begin{tabular}{|c|c|c|}
\hline $\begin{array}{l}\text { Section \& } \\
\text { Topic }\end{array}$ & No. & Item \\
\hline \multicolumn{3}{|c|}{ TITLE OR ABSTRACT } \\
\hline & 1 & $\begin{array}{l}\text { Identification as a study of diagnostic accuracy using at least one measure of accuracy } \\
\text { (such as sensitivity, specificity, predictive values, or AUC) }\end{array}$ \\
\hline \multicolumn{3}{|l|}{ ABSTRACT } \\
\hline & 2 & $\begin{array}{l}\text { Structured summary of study design, methods, results, and conclusions (for specific } \\
\text { guidance, see STARD for Abstracts) }\end{array}$ \\
\hline \multicolumn{3}{|c|}{ INTRODUCTION } \\
\hline & 3 & $\begin{array}{l}\text { Scientific and clinical background, including the intended use and clinical role of the } \\
\text { index test }\end{array}$ \\
\hline & 4 & Study objectives and hypotheses \\
\hline \multicolumn{3}{|l|}{ METHODS } \\
\hline $\begin{array}{l}\text { Study } \\
\text { design }\end{array}$ & 5 & $\begin{array}{l}\text { Whether data collection was planned before the index test and reference standard were } \\
\text { performed (prospective study) or after (retrospective study) }\end{array}$ \\
\hline \multirow{4}{*}{ Participants } & 6 & Eligibility criteria \\
\hline & 7 & $\begin{array}{l}\text { On what basis potentially eligible participants were identified (such as symptoms, results } \\
\text { from previous tests, inclusion in registry) }\end{array}$ \\
\hline & 8 & $\begin{array}{l}\text { Where and when potentially eligible participants were identified (setting, location and } \\
\text { dates) }\end{array}$ \\
\hline & 9 & Whether participants formed a consecutive, random or convenience series \\
\hline \multirow{7}{*}{$\begin{array}{l}\text { Test } \\
\text { methods }\end{array}$} & 10a & Index test, in sufficient detail to allow replication \\
\hline & $10 \mathrm{~b}$ & Reference standard, in sufficient detail to allow replication \\
\hline & 11 & Rationale for choosing the reference standard (if alternatives exist) \\
\hline & $12 \mathbf{a}$ & $\begin{array}{l}\text { Definition of and rationale for test positivity cut-offs or result categories of the index test, } \\
\text { distinguishing pre-specified from exploratory }\end{array}$ \\
\hline & $12 b$ & $\begin{array}{l}\text { Definition of and rationale for test positivity cut-offs or result categories of the reference } \\
\text { standard, distinguishing pre-specified from exploratory }\end{array}$ \\
\hline & 13a & $\begin{array}{l}\text { Whether clinical information and reference standard results were available to the } \\
\text { performers/readers of the index test }\end{array}$ \\
\hline & 13b & $\begin{array}{l}\text { Whether clinical information and index test results were available to the assessors of the } \\
\text { reference standard }\end{array}$ \\
\hline \multirow{5}{*}{ Analysis } & 14 & Methods for estimating or comparing measures of diagnostic accuracy \\
\hline & 15 & How indeterminate index test or reference standard results were handled \\
\hline & 16 & How missing data on the index test and reference standard were handled \\
\hline & 17 & $\begin{array}{l}\text { Any analyses of variability in diagnostic accuracy, distinguishing pre-specified from } \\
\text { exploratory }\end{array}$ \\
\hline & 18 & Intended sample size and how it was determined \\
\hline \multicolumn{3}{|l|}{ RESULTS } \\
\hline \multirow{5}{*}{ Participants } & 19 & Flow of participants, using a diagram \\
\hline & 20 & Baseline demographic and clinical characteristics of participants \\
\hline & 21a & Distribution of severity of disease in those with the target condition \\
\hline & 21b & Distribution of alternative diagnoses in those without the target condition \\
\hline & 22 & Time interval and any clinical interventions between index test and reference standard \\
\hline \multirow{3}{*}{ Test results } & 23 & $\begin{array}{l}\text { Cross tabulation of the index test results (or their distribution) by the results of the } \\
\text { reference standard }\end{array}$ \\
\hline & 24 & Estimates of diagnostic accuracy and their precision (such as $95 \%$ confidence intervals) \\
\hline & 25 & Any adverse events from performing the index test or the reference standard \\
\hline \multicolumn{3}{|c|}{ DISCUSSION } \\
\hline & 26 & $\begin{array}{l}\text { Study limitations, including sources of potential bias, statistical uncertainty, and } \\
\text { generalisability }\end{array}$ \\
\hline & 27 & Implications for practice, including the intended use and clinical role of the index test \\
\hline
\end{tabular}


OTHER INFORMATION

28 Registration number and name of registry

29 Where the full study protocol can be accessed

30 Sources of funding and other support; role of funders 


\section{APPENDIX 13: Phase 1: Tests of Normality and Bland-Altman Plots 3DCI}

Tests of normality of the 3DCI data for total volume difference (TVD) had an initial skewness more than three times the standard error, with kurtosis only slightly higher than the standard error. The Shapiro-Wilk test result for 3DCI had a p-value $<0.05$, indicating that the distribution deviates from normal (Laerd Statistics, 2018b). However, the Kolmogorov-Smirnov test had a p-value $>0.05$, thus normality could be assumed. The Q-Q Plot shows the near normal distribution of kurtosis. Square root and logarithms of the data were perforemd, but these overcorrected the data too much. Thus the original data for 3DCI TVD was used for the construction of the Bland-Altman Plots.

Descriptives of 3DCI Total Volume Difference Data

\begin{tabular}{|c|c|c|c|c|}
\hline & & & Statistic & Std. Error \\
\hline \multirow{13}{*}{$\begin{array}{l}\text { Standardized Residual for 3DCI } \\
\text { VD }\end{array}$} & Mean & & .0000 & .12309 \\
\hline & \multirow{2}{*}{$\begin{array}{l}\text { 95\% Confidence Interval for } \\
\text { Mean }\end{array}$} & Lower Bound & -.2458 & \\
\hline & & Upper Bound & .2458 & \\
\hline & 5\% Trimmed Mean & & -.0601 & \\
\hline & Median & & -.1853 & \\
\hline & Variance & & 1.000 & \\
\hline & Std. Deviation & & 1.00000 & \\
\hline & Minimum & & -1.60 & \\
\hline & Maximum & & 2.79 & \\
\hline & Range & & 4.39 & \\
\hline & Interquartile Range & & 1.21 & \\
\hline & Skewness & & .944 & .295 \\
\hline & Kurtosis & & .600 & .582 \\
\hline
\end{tabular}

Tests of Normality of 3DCI Total Volume Difference Data

Kolmogorov-Smirnov ${ }^{\mathrm{a}}$

Shapiro-Wilk

\begin{tabular}{|c|c|c|c|c|c|c|}
\hline & \multicolumn{3}{|c|}{ Kolmogorov-Smirnov ${ }^{\mathrm{a}}$} & \multicolumn{3}{|c|}{ Shapiro-Wilk } \\
\hline & Statistic & df & Sig. & Statistic & df & Sig. \\
\hline Standardized Residual for 3DCI VD & .105 & 66 & .068 & .933 & 66 & .002 \\
\hline
\end{tabular}

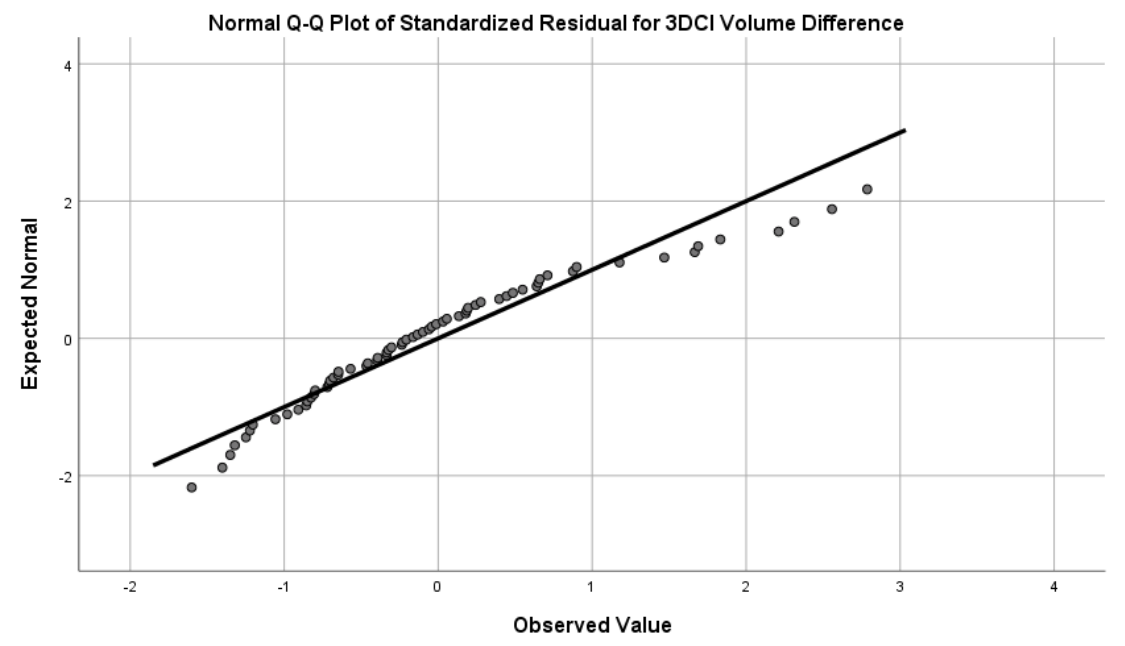




\section{CTM}

Tests of normality of the CTM data for TVD had an initial skewness of nearly 10 times the standard error, and the kurtosis was nearly nine times greater than the standard error. Both the Shapiro-Wilk and Kolmogorov-Smirnov test results had a p-value $<0.05$, indicating that the distribution deviated from normal. The Q-Q plot for CTM supports this outcome as the data deviates significantly from the diagonal line.

Descriptives of CTM Total Volume Difference Data

\begin{tabular}{ll|r|r}
\hline \multirow{3}{*}{$\begin{array}{l}\text { Standardized Residual for } \\
\text { CTVD }\end{array}$} & Statistic & \multicolumn{1}{c}{ Std. Error } \\
\cline { 2 - 4 } & Mean & .0000 & .11625 \\
\hline & Mean & -.2317 & \\
\hline $5 \%$ Trimmed Mean & Lower Bound & .2317 & \\
\cline { 2 - 4 } & Upper Bound & -.1341 & \\
\hline & Median & -.3489 & 1.000 \\
\hline & Variance & 1.00000 & \\
\hline Std. Deviation & -.94 & \\
\hline Minimum & 3.65 & \\
\hline & Maximum & 4.59 & \\
\hline Range & .65 & \\
\hline & Interquartile Range & 2.259 & .279 \\
\hline
\end{tabular}

Tests of Normality of CTM Total Volume Difference Data

\begin{tabular}{lc|c|c|c|c|c|r} 
& \multicolumn{3}{c}{ Kolmogorov-Smirnov } & \multicolumn{4}{c}{ Shapiro-Wilk } \\
& Statistic & df & Sig. & Statistic & df & Sig. \\
\hline Standardized Residual for CTVD & .236 & & 74 & .000 & .707 & 74 & .000 \\
\hline
\end{tabular}

a. Lilliefors Significance Correction

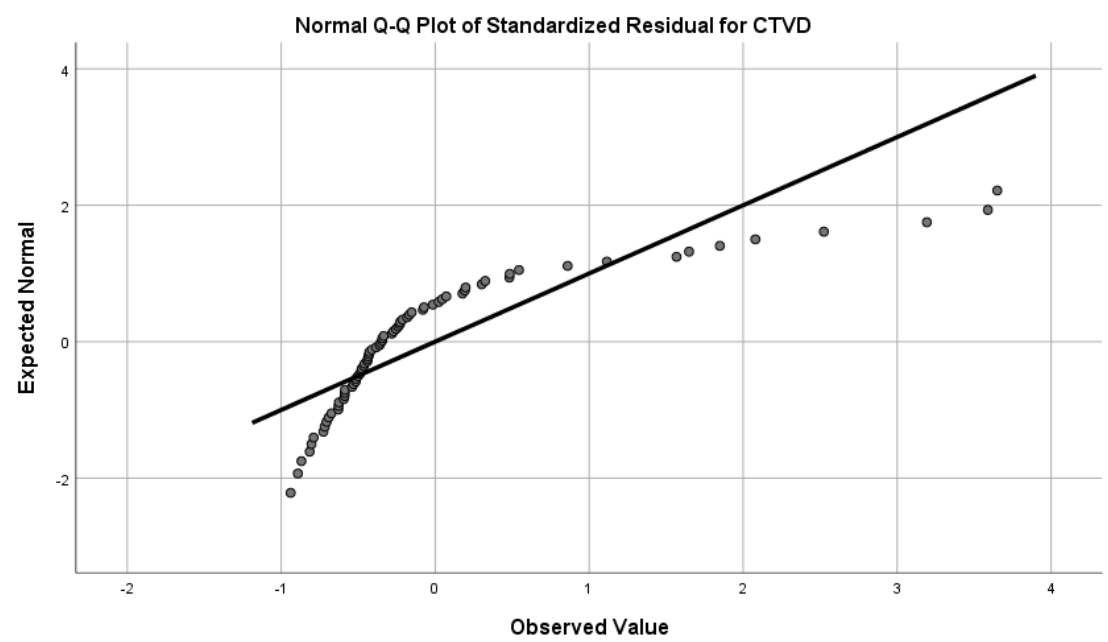

The square root of the data was performed, but had marginal improvement on the distribution of the data. Thus, transforming the data for normalisation was performed using the logarithm of the CTM TVD data. Both the skewness and kurtosis were less than the standard error, and both the Kolmogorov-Smirnov and Shapiro-Wilk tests of 
normality were non-significant ( $\mathrm{p}>0.05$ ), and was confirmed by the improved Q-Q Plot of data following the normative line more closely. Thus, the logarithm of the CTM TVD was used for the Bland Altman plots.

Descriptives of the Logarithm of CTM Total Volume Difference Data

\begin{tabular}{|c|c|c|c|c|}
\hline & & & Statistic & Std. Error \\
\hline \multirow{13}{*}{$\begin{array}{l}\text { Standardized Residual for } \\
\text { LNCTVD }\end{array}$} & Mean & & .0000 & .12599 \\
\hline & \multirow{2}{*}{$\begin{array}{l}95 \% \text { Confidence Interval for } \\
\text { Mean }\end{array}$} & Lower Bound & -.2518 & \\
\hline & & Upper Bound & .2518 & \\
\hline & \multicolumn{2}{|l|}{ 5\% Trimmed Mean } & .0124 & \\
\hline & \multicolumn{2}{|l|}{ Median } & -.0058 & \\
\hline & \multicolumn{2}{|l|}{ Variance } & 1.000 & \\
\hline & \multicolumn{2}{|l|}{ Std. Deviation } & 1.00000 & \\
\hline & \multicolumn{2}{|l|}{ Minimum } & -2.22 & \\
\hline & \multicolumn{2}{|l|}{ Maximum } & 1.96 & \\
\hline & \multicolumn{2}{|l|}{ Range } & 4.18 & \\
\hline & \multicolumn{2}{|l|}{ Interquartile Range } & 1.23 & \\
\hline & \multicolumn{2}{|l|}{ Skewness } & -.048 & .302 \\
\hline & \multicolumn{2}{|l|}{ Kurtosis } & -.189 & .595 \\
\hline
\end{tabular}

Tests of Normality of the Logarithm of CTM Total Volume Difference Data

\begin{tabular}{|c|c|c|c|c|c|c|}
\hline & \multicolumn{3}{|c|}{ Kolmogorov-Smirnov ${ }^{\mathrm{a}}$} & \multicolumn{3}{|c|}{ Shapiro-Wilk } \\
\hline & Statistic & df & Sig. & Statistic & df & Sig. \\
\hline $\begin{array}{l}\text { Standardized Residual for } \\
\text { LNCTVD }\end{array}$ & .048 & 63 & $.200^{*}$ & .983 & 63 & .513 \\
\hline
\end{tabular}

*. This is a lower bound of the true significance.

a. Lilliefors Significance Correction

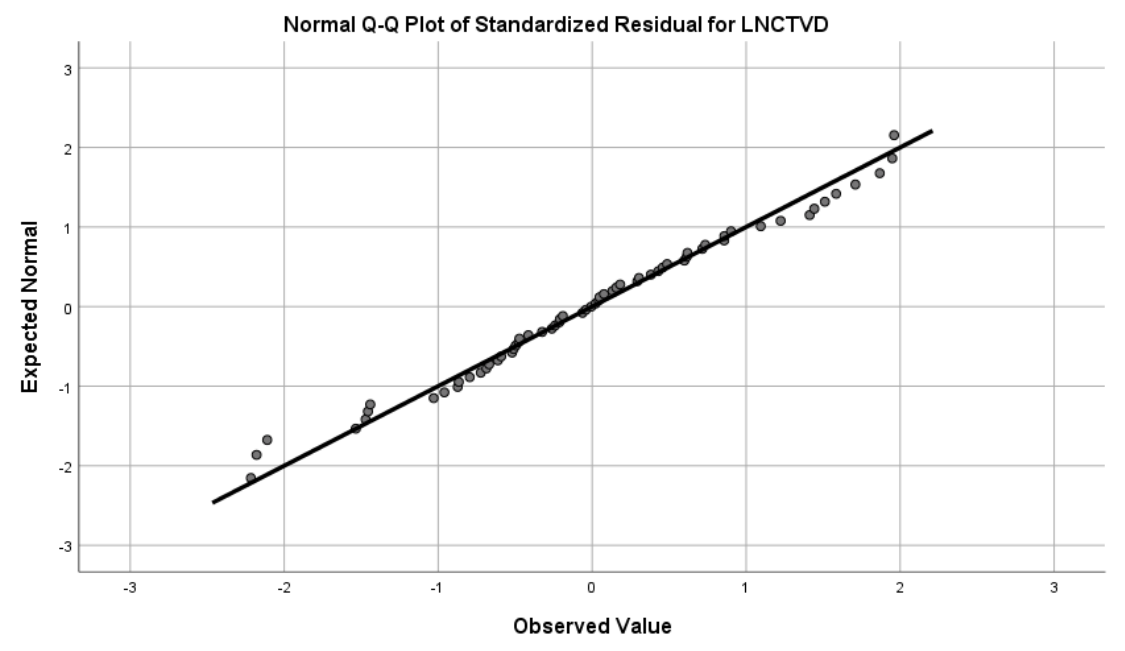




\section{Perometry}

Tests of normality of the perometry data for TVD had an initial skewness of nine times the standard error, and the kurtosis was 13 times greater than the standard error. Both the Shapiro-Wilk and Kolmogorov-Smirnov test results had a p-value $<0.05$, indicating that the distribution deviated from normal. The Q-Q plot for perometry confirmes this outcome as the data deviates significantly from the diagonal line.

\section{Descriptives of the Logarithm of Perometry Total Volume Difference Data}

\begin{tabular}{|c|c|c|c|c|}
\hline \multirow{4}{*}{$\begin{array}{l}\text { Standardized Residual for } \\
\text { PTVD }\end{array}$} & \multirow{2}{*}{\multicolumn{2}{|c|}{ Mean }} & \multirow{3}{*}{$\begin{array}{r}\text { Statistic } \\
.0000 \\
-.2350\end{array}$} & \multirow{2}{*}{$\begin{array}{r}\text { Std. Error } \\
.11785\end{array}$} \\
\hline & & & & \\
\hline & 95\% Confidence Interval for & Lower Bound & & \\
\hline & Mean & Upper Bound & .2350 & \\
\hline & 5\% Trimmed Mean & & -.1382 & \\
\hline & Median & & -.2917 & \\
\hline & Variance & & 1.000 & \\
\hline & Std. Deviation & & 1.00000 & \\
\hline & Minimum & & -1.16 & \\
\hline & Maximum & & 3.84 & \\
\hline & Range & & 5.00 & \\
\hline & Interquartile Range & & .69 & \\
\hline & Skewness & & 2.578 & .283 \\
\hline & Kurtosis & & 7.424 & .559 \\
\hline
\end{tabular}

\begin{tabular}{|c|c|c|c|c|c|c|}
\hline \multicolumn{7}{|c|}{ Tests of Normality of the Logarithm of Perometry Total Volume Difference Data } \\
\hline & \multicolumn{3}{|c|}{ Kolmogorov-Smirnov ${ }^{\mathrm{a}}$} & \multicolumn{3}{|c|}{ Shapiro-Wilk } \\
\hline & Statistic & df & Sig. & Statistic & df & Sig. \\
\hline $\begin{array}{l}\text { Standardized Residual for } \\
\text { PTVD }\end{array}$ & .248 & 72 & .000 & .705 & 72 & .000 \\
\hline
\end{tabular}

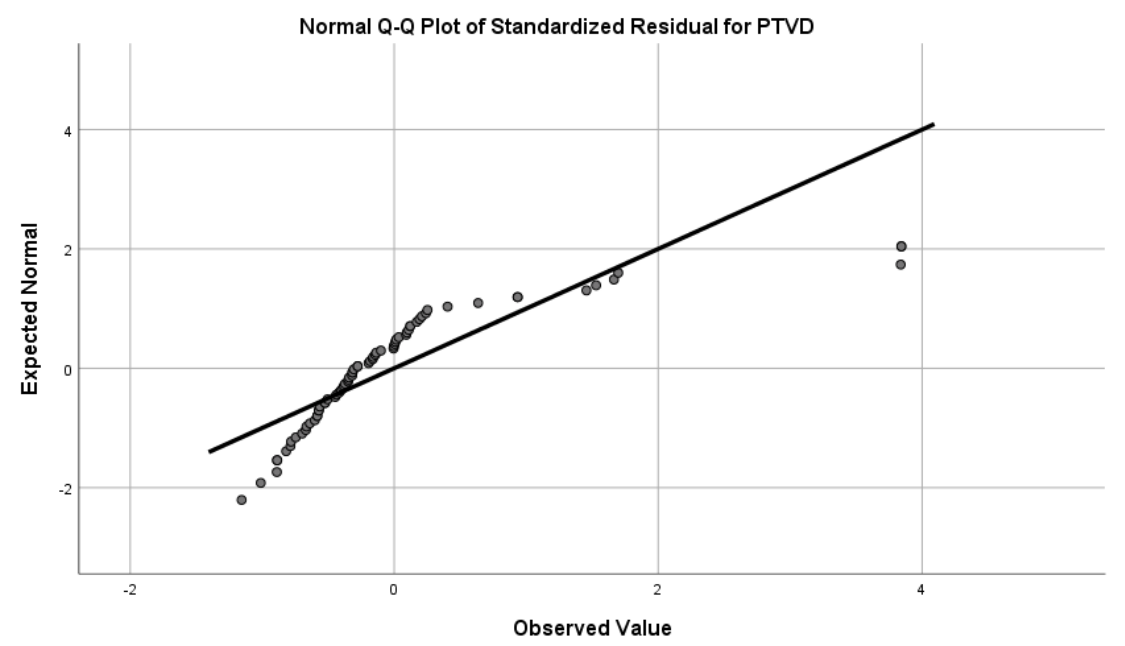

The square root of the data was performed, but had marginal improvement on the distribution of the data. Thus, transforming the data for normalisation was performed using the logarithm of the perometry TVD data. Both the skewness improved significantly and was only twice the standard error, but the kurtosis was still three times 
the standard error. However, The Kolmogorov-Smirnov test was now non-significant with a $p$-value of 0.20 ; however the Shapiro-Wilk tests was still significant at $\mathrm{p}=0.04$. The Q-Q Plot was improved but had one outlier that caused the significant kurtosis value.

Descriptives of the Logarithm of Perometry Total Volume Difference Data

\begin{tabular}{|c|c|c|c|c|}
\hline & & & Statistic & Std. Error \\
\hline \multirow{13}{*}{$\begin{array}{l}\text { Standardized Residual for } \\
\text { LNPTVD }\end{array}$} & Mean & & .0000 & .13608 \\
\hline & \multirow{2}{*}{$\begin{array}{l}\text { 95\% Confidence Interval for } \\
\text { Mean }\end{array}$} & Lower Bound & -.2729 & \\
\hline & & Upper Bound & .2729 & \\
\hline & \multicolumn{2}{|l|}{ 5\% Trimmed Mean } & .0323 & \\
\hline & \multicolumn{2}{|l|}{ Median } & .1125 & \\
\hline & \multicolumn{2}{|l|}{ Variance } & 1.000 & \\
\hline & \multicolumn{2}{|l|}{ Std. Deviation } & 1.00000 & \\
\hline & \multicolumn{2}{|l|}{ Minimum } & -3.51 & \\
\hline & \multicolumn{2}{|l|}{ Maximum } & 1.92 & \\
\hline & \multicolumn{2}{|l|}{ Range } & 5.43 & \\
\hline & \multicolumn{2}{|l|}{ Interquartile Range } & 1.05 & \\
\hline & \multicolumn{2}{|l|}{ Skewness } & -.652 & .325 \\
\hline & \multicolumn{2}{|l|}{ Kurtosis } & 2.031 & .639 \\
\hline
\end{tabular}

\begin{tabular}{|c|c|c|c|c|c|c|}
\hline \multicolumn{7}{|c|}{ Tests of Normality of the Logarithm of Perometry Total Volume Difference Data } \\
\hline & \multicolumn{3}{|c|}{ Kolmogorov-Smirnova } & \multicolumn{3}{|c|}{ Shapiro-Wilk } \\
\hline & Statistic & df & Sig. & Statistic & df & Sig. \\
\hline $\begin{array}{l}\text { Standardized Residual for } \\
\text { LNPTVD }\end{array}$ & .103 & 54 & $.200^{*}$ & .954 & 54 & .039 \\
\hline
\end{tabular}

*. This is a lower bound of the true significance.

a. Lilliefors Significance Correction

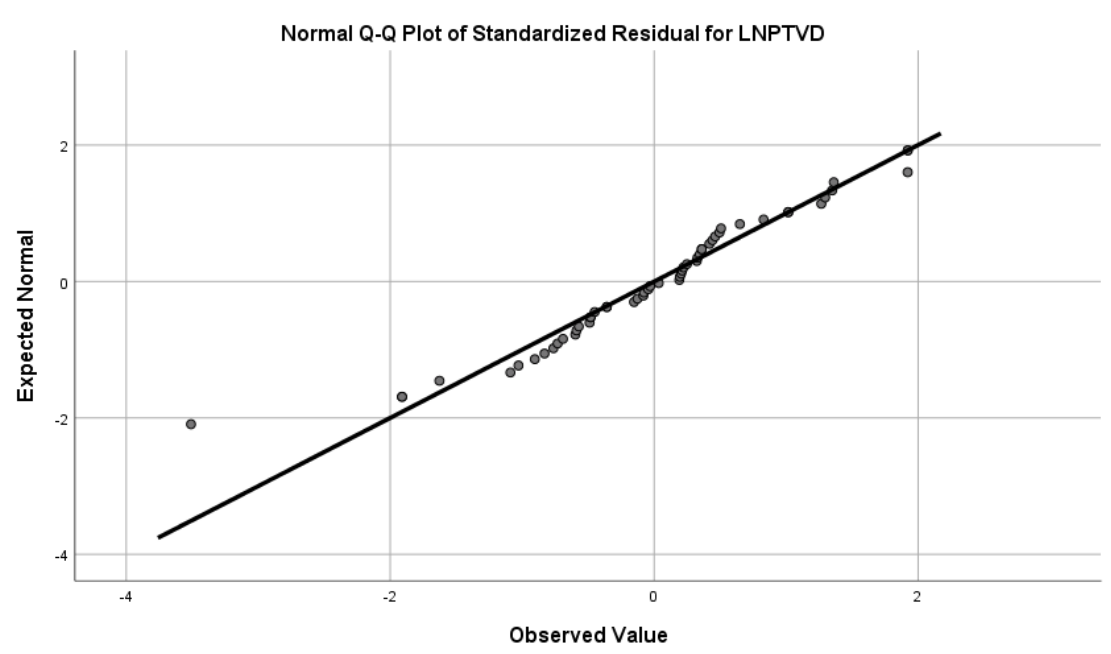

Thus, the perometry TVD data entry for number 68 was removed, which resulted in significantly improved values for the skewness and kurtosis (both less than the standard error), and both the Kolmogorov-Smirnov and Shapiro-Wilk tests of normality were non-significant ( $\mathrm{p}>0.05)$, confirmed by the improved Q-Q Plot of data following the 
normative line more closely. Thus, the logarithm of the adjusted perometry TVD was used for the Bland Altman plots.

Descriptives of the Logarithm of Adjusted Perometry Total Volume Difference Data

\begin{tabular}{|c|c|c|c|c|}
\hline \multirow{14}{*}{$\begin{array}{l}\text { Standardized Residual for } \\
\text { LNCTVD }\end{array}$} & & & Statistic & Std. Error \\
\hline & Mean & & .0000 & .12599 \\
\hline & \multirow{2}{*}{$\begin{array}{l}\text { 95\% Confidence Interval for } \\
\text { Mean }\end{array}$} & Lower Bound & -.2518 & \\
\hline & & Upper Bound & .2518 & \\
\hline & \multicolumn{2}{|l|}{ 5\% Trimmed Mean } & .0124 & \\
\hline & \multicolumn{2}{|l|}{ Median } & -.0058 & \\
\hline & \multicolumn{2}{|l|}{ Variance } & 1.000 & \\
\hline & \multicolumn{2}{|l|}{ Std. Deviation } & 1.00000 & \\
\hline & \multicolumn{2}{|l|}{ Minimum } & -2.22 & \\
\hline & \multicolumn{2}{|l|}{ Maximum } & 1.96 & \\
\hline & \multicolumn{2}{|l|}{ Range } & 4.18 & \\
\hline & \multicolumn{2}{|l|}{ Interquartile Range } & 1.23 & \\
\hline & \multicolumn{2}{|l|}{ Skewness } & -.048 & .302 \\
\hline & \multicolumn{2}{|l|}{ Kurtosis } & -.189 & .595 \\
\hline
\end{tabular}

Tests of Normality of the Logarithm of Adjusted Perometry Total Volume Difference Data

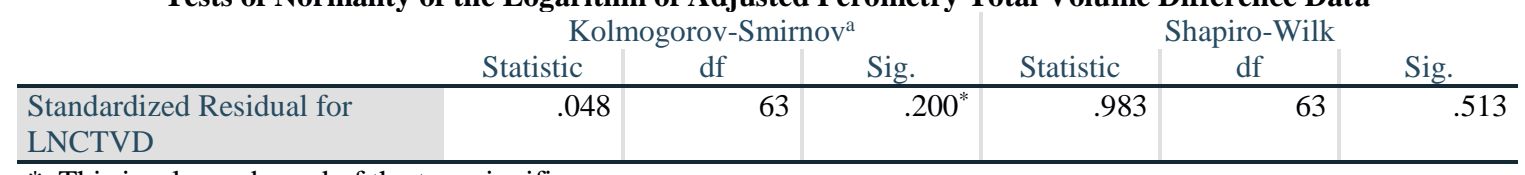

*. This is a lower bound of the true significance.

a. Lilliefors Significance Correction

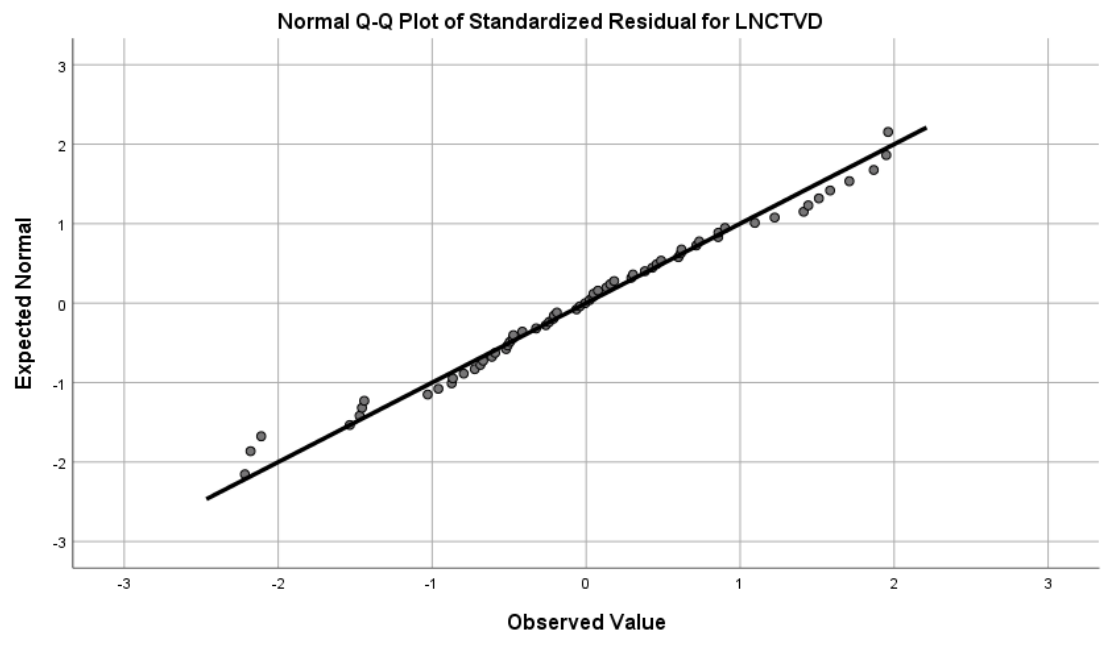


Bland-Altman Plot of 3DCI vs CTM with Coefficients Table

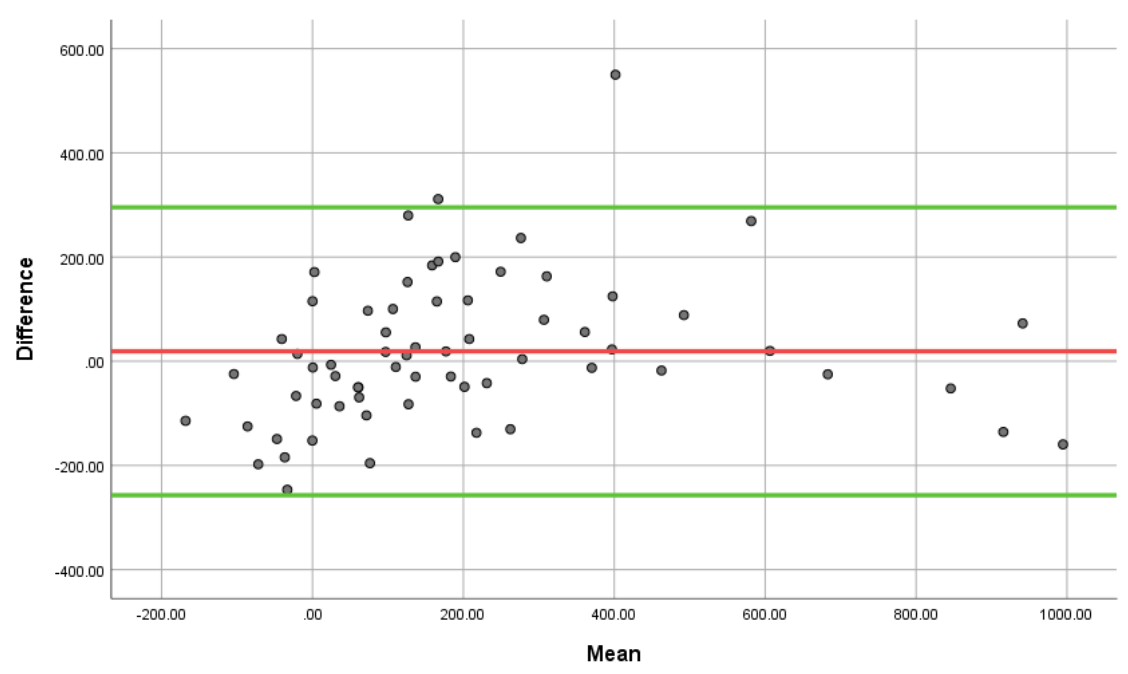

Coefficients $^{a}$

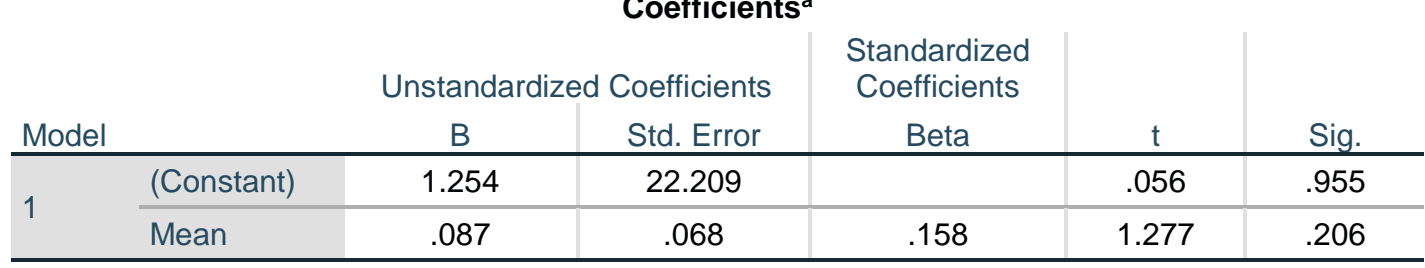

a. Dependent Variable: Difference 3DCI \& CTM

Bland-Altman Plot of 3DCI vs Perometry with Coefficients Table

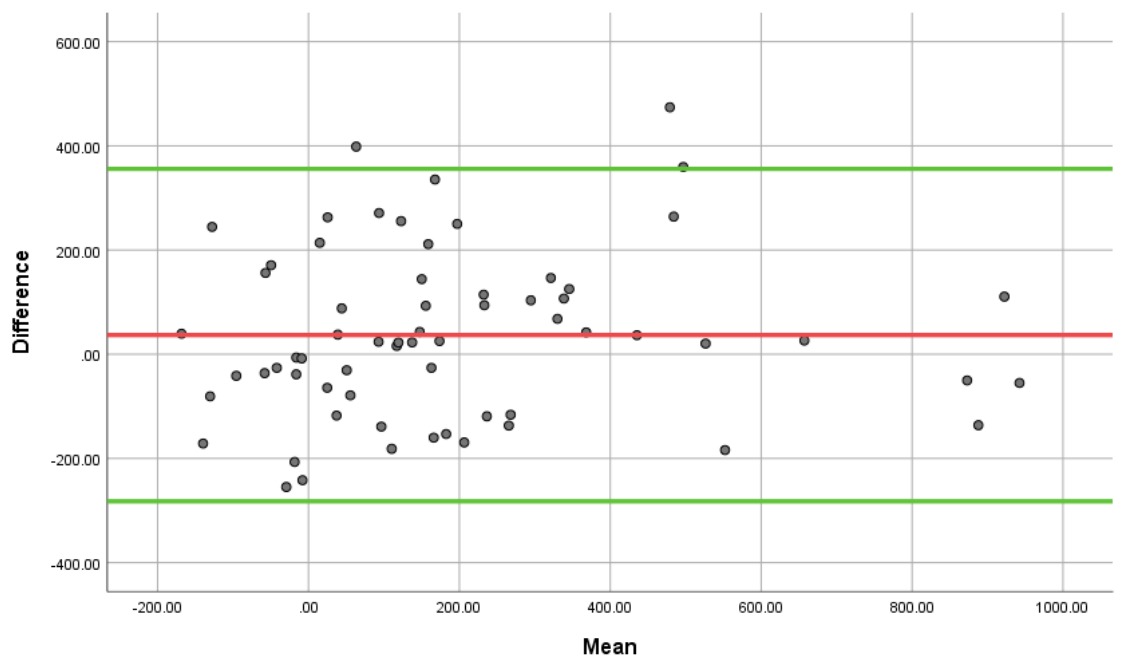

Coefficients $^{a}$

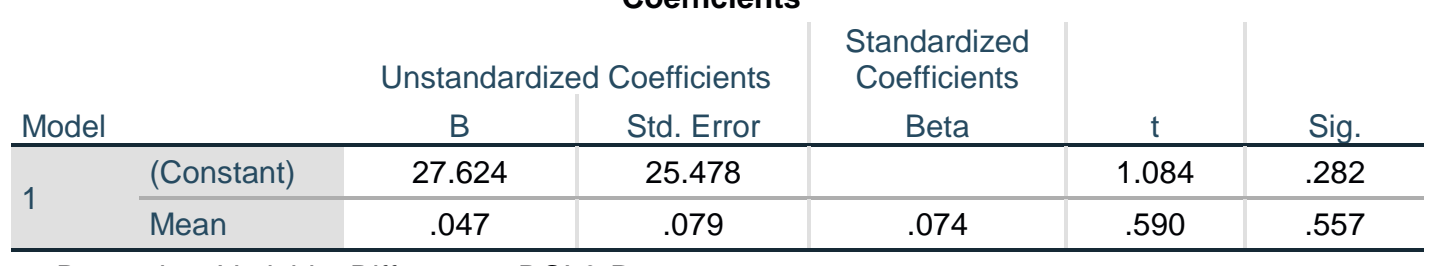

a. Dependent Variable: Difference 3DCI \& Perometer 
Bland-Altman Plot of CTM vs Perometry with Coefficients Table

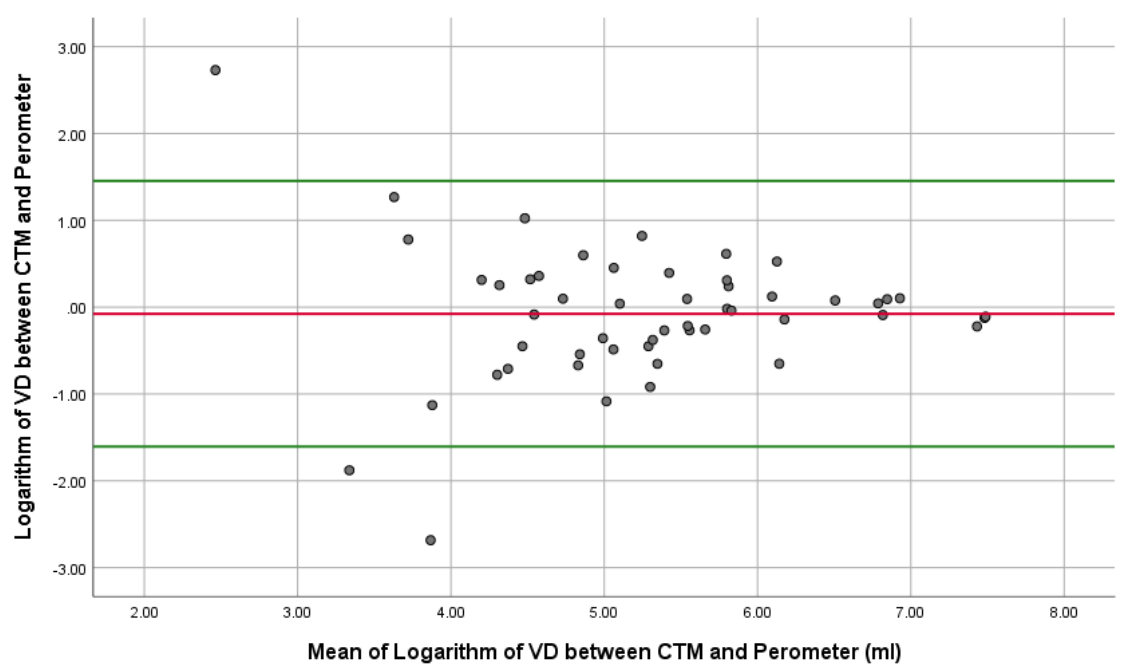

\begin{tabular}{|c|c|c|c|c|c|c|}
\hline \multirow[b]{3}{*}{ Model } & \multicolumn{6}{|c|}{ Coefficients $^{a}$} \\
\hline & & \multicolumn{2}{|c|}{ Unstandardized Coefficients } & \multirow{2}{*}{$\begin{array}{c}\text { Standardized } \\
\text { Coefficients } \\
\text { Beta } \\
\end{array}$} & \multirow[b]{2}{*}{$\mathrm{t}$} & \multirow[b]{2}{*}{ Sig. } \\
\hline & & B & Std. Error & & & \\
\hline \multirow[t]{2}{*}{1} & (Constant) & .094 & .552 & & .170 & .866 \\
\hline & Mean & -.032 & 102 & -.045 & -.316 & .754 \\
\hline
\end{tabular}

a. Dependent Variable: Difference CTM \& Perometer logarithms of VD 


\section{APPENDIX 14: Oedema Maps derived from 3DCI}

Key: cyan $=$ no difference between arms; yellow to red = larger than unaffected side; blue $=$ smaller than unaffected side

\section{$\underline{\text { Participant } 1}$}
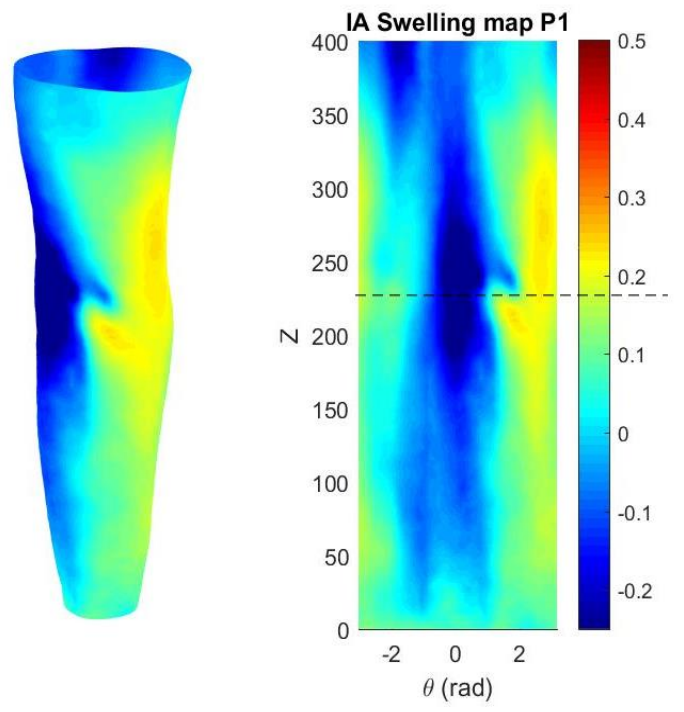

Initial Assessment

$\mathrm{CTM}=4 \%$

Perometer $=-4.7 \%$

$3 \mathrm{DCI}=1.6 \%$

Redundancy $=-1 \%$
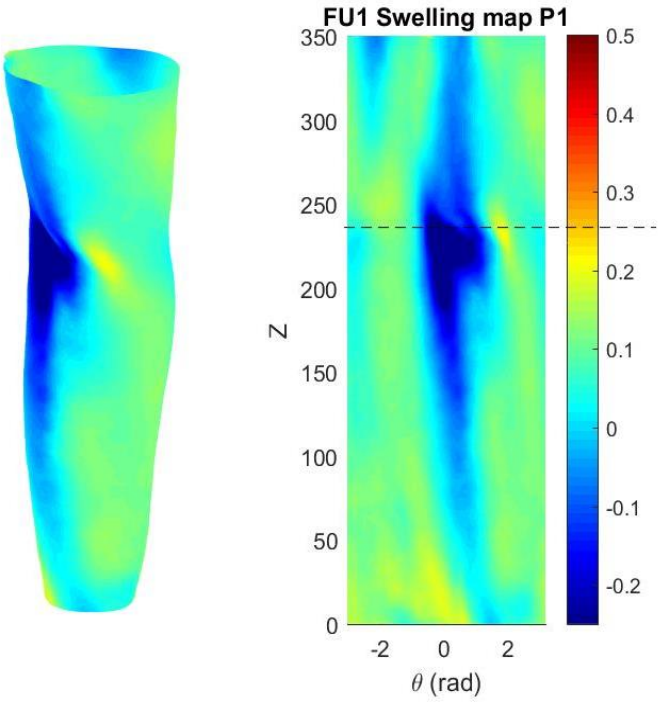

Follow up 1

$\mathrm{CTM}=-1 \%$

Perometer $=$ FAULT

$3 \mathrm{DCI}=14.2 \%$

Redundancy $=$ calculation error
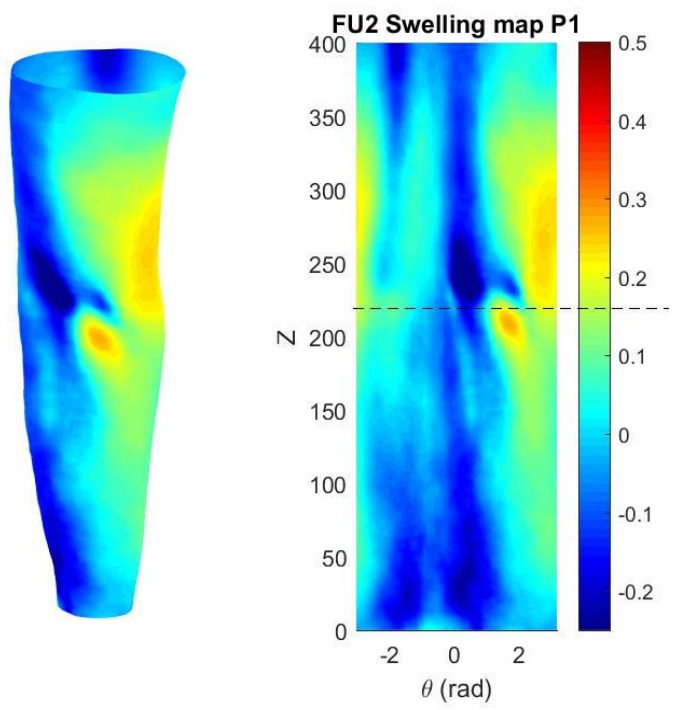

Follow up 2

$\mathrm{CTM}=1 \%$

Perometer $=-4.7 \%$

$3 \mathrm{DCI}=0.9 \%$

Redundancy $=$ calculation error

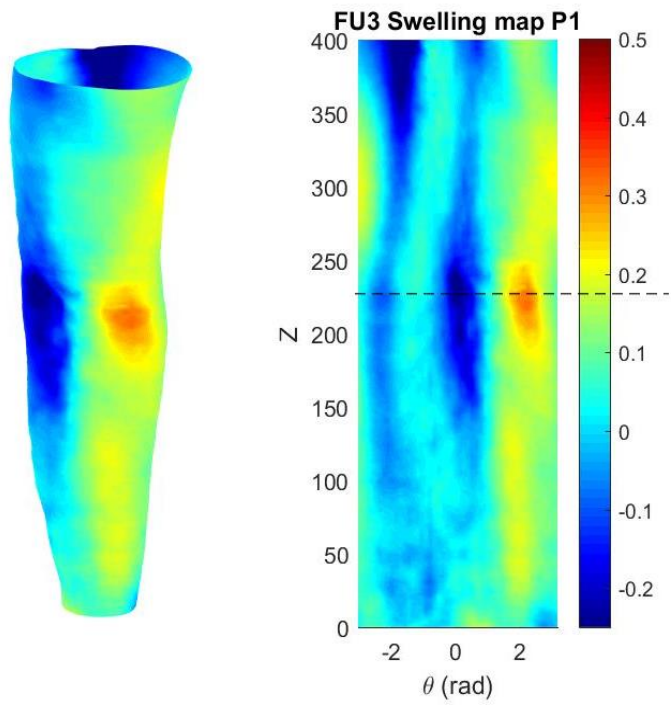

Follow up 3

$\mathrm{CTM}=1 \%$

Perometer $=-3.8 \%$

$3 \mathrm{DCI}=5.3 \%$

Redundancy $=13 \%$ 


\section{$\underline{\text { Participant } 2}$}
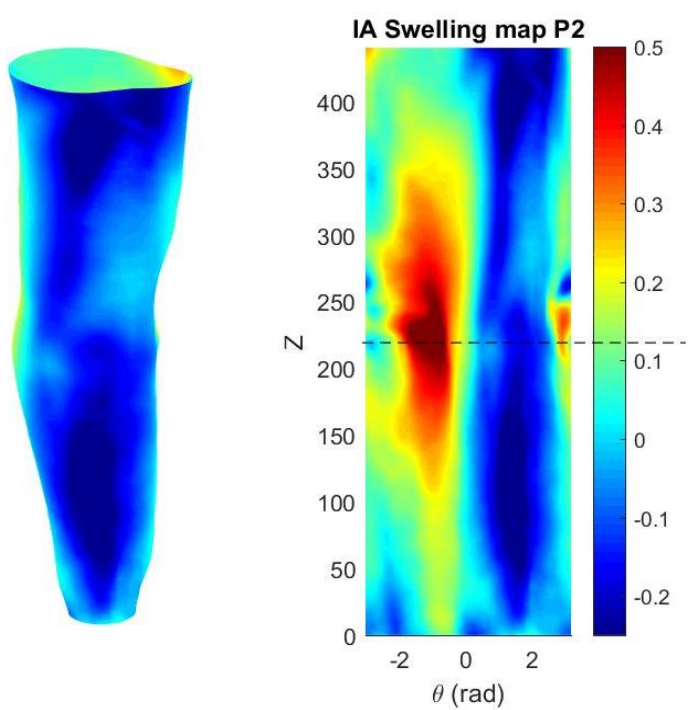

Initial Assessment

$\mathrm{CTM}=9 \%$

Perometer $=8.8 \%$

$3 \mathrm{DCI}=8.4 \%$

Redundancy $=0 \%$
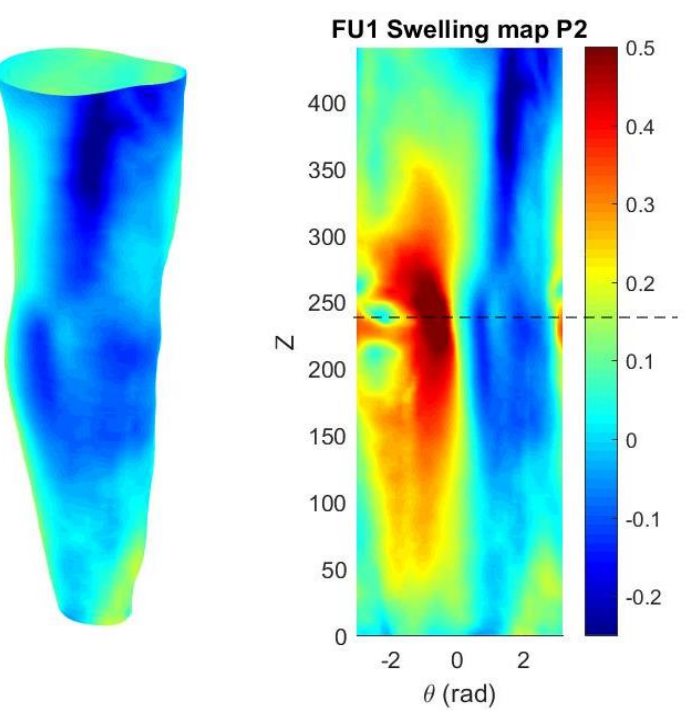

Follow up 1

$\mathrm{CTM}=16 \%$

Perometer $=12.7 \%$

$3 \mathrm{DCI}=21.7 \%$

Redundancy $=26 \%$
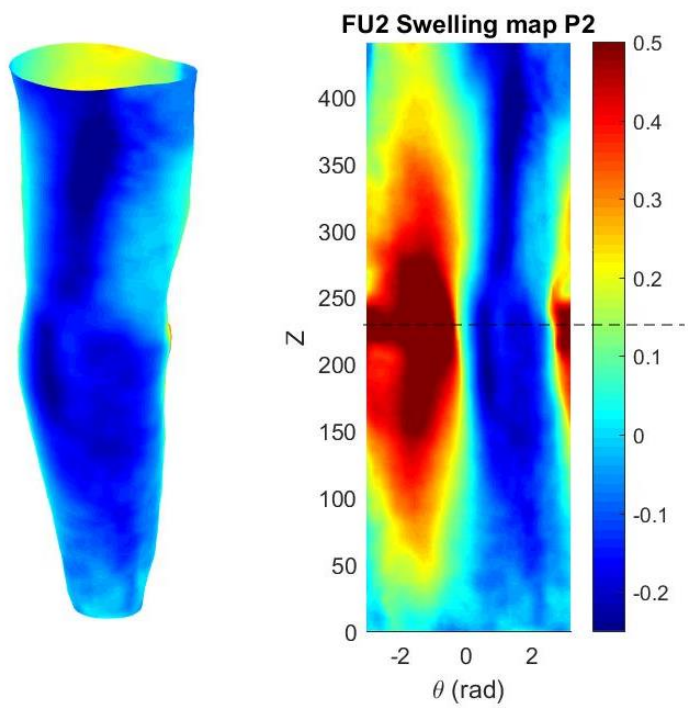

Follow up 2

$\mathrm{CTM}=14 \%$

Perometer $=8.8 \%$

$3 \mathrm{DCI}=16.3 \%$

Redundancy $=5 \%$

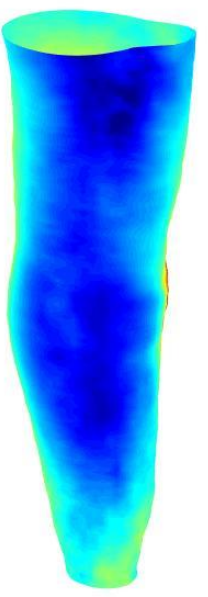

Follow up 3

$\mathrm{CTM}=16 \%$

Perometer $=14.5 \%$

$3 \mathrm{DCI}=25.6 \%$

Redundancy $=22 \%$ 


\section{$\underline{\text { Participant } 3}$}

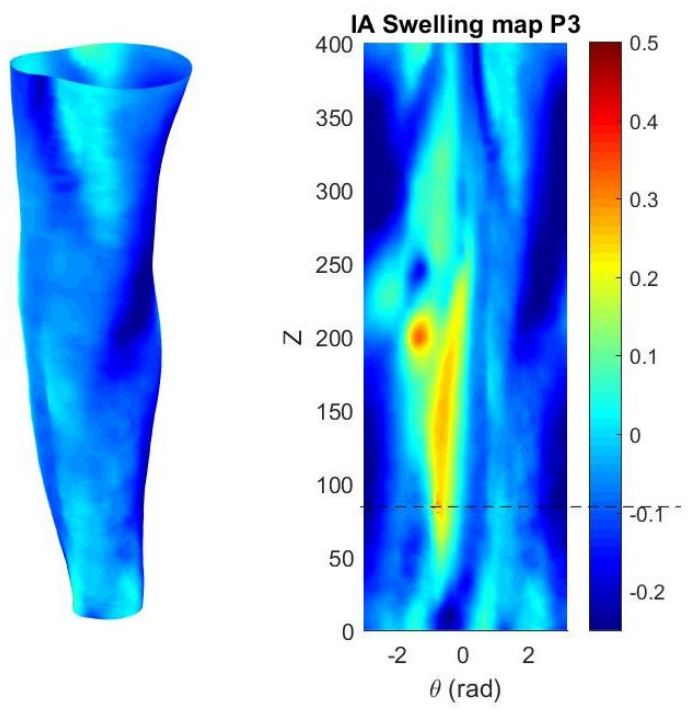

\section{Initial Assessment}

$\mathrm{CTM}=3 \%$

Perometer $=-0.2 \%$

$3 \mathrm{DCI}=11.6 \%$

Redundancy $=14 \%$
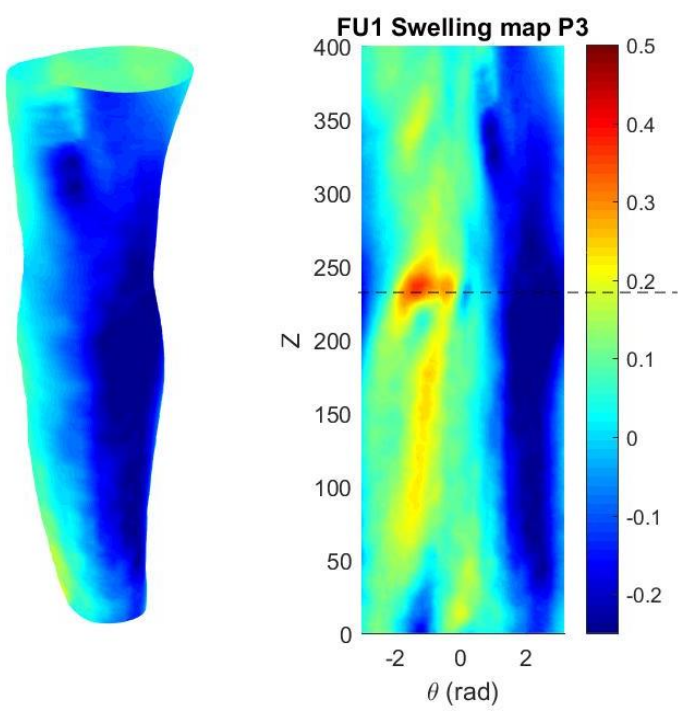

Follow up 1

$\mathrm{CTM}=0 \%$

Perometer $=-8.6 \%$

3 DCI $=-0.2 \%$

Redundancy $=-7 \%$
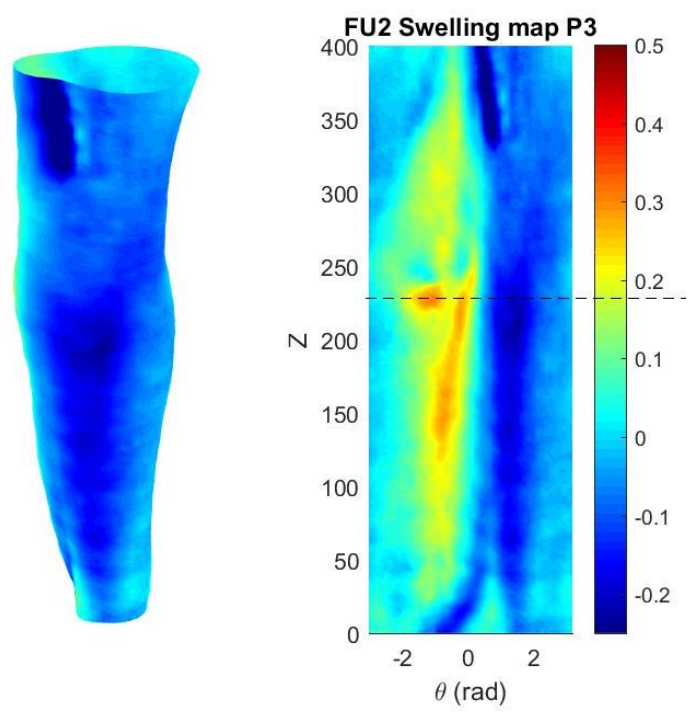

Follow up 2
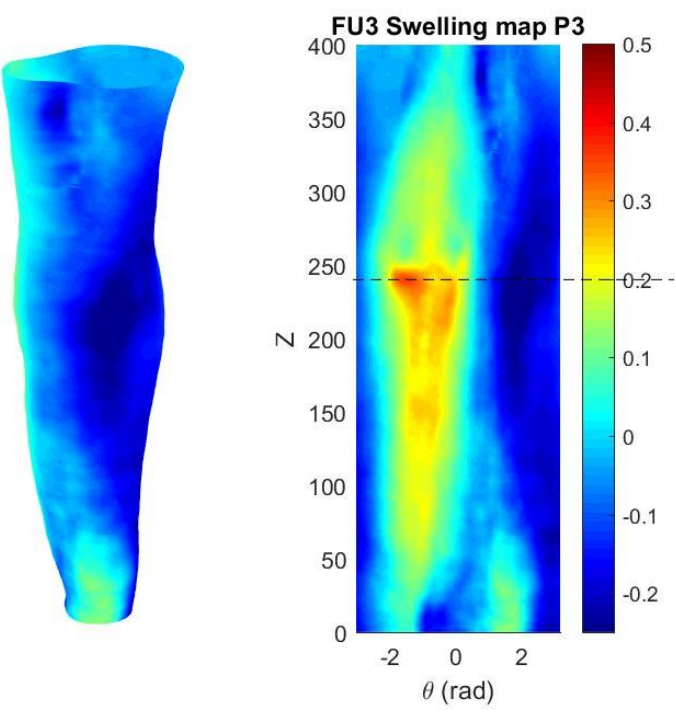

Follow up 3

$\mathrm{CTM}=-1 \%$

$\mathrm{CTM}=4 \%$

Perometer $=-0.2 \%$

Perometer $=-1.8 \%$

$3 \mathrm{DCI}=-0.6 \%$

Redundancy $=14 \%$

$3 \mathrm{DCI}=-3.4 \%$

Redundancy $=-3 \%$ 


\section{$\underline{\text { Participant } 4}$}
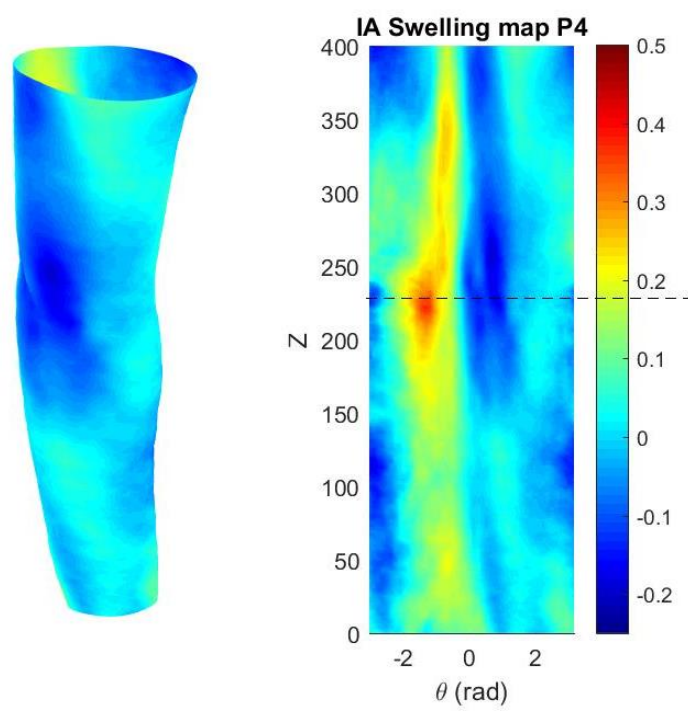

Initial Assessment

$\mathrm{CTM}=3 \%$

Perometer $=4.1 \%$

$3 \mathrm{DCI}=4.8 \%$

Redundancy $=4 \%$
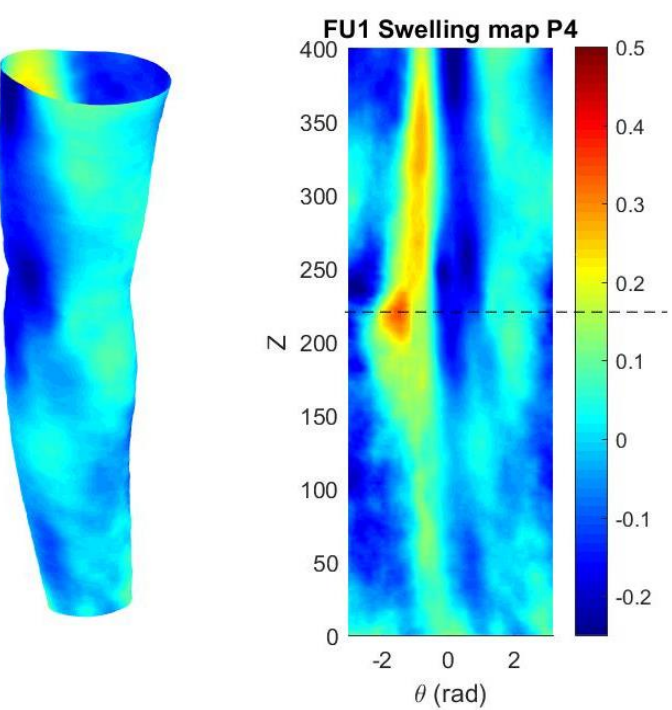

Follow up 1

$\mathrm{CTM}=-3 \%$

Perometer $=-0.5 \%$

3 DCI $=-0.8 \%$

Redundancy $=19 \%$
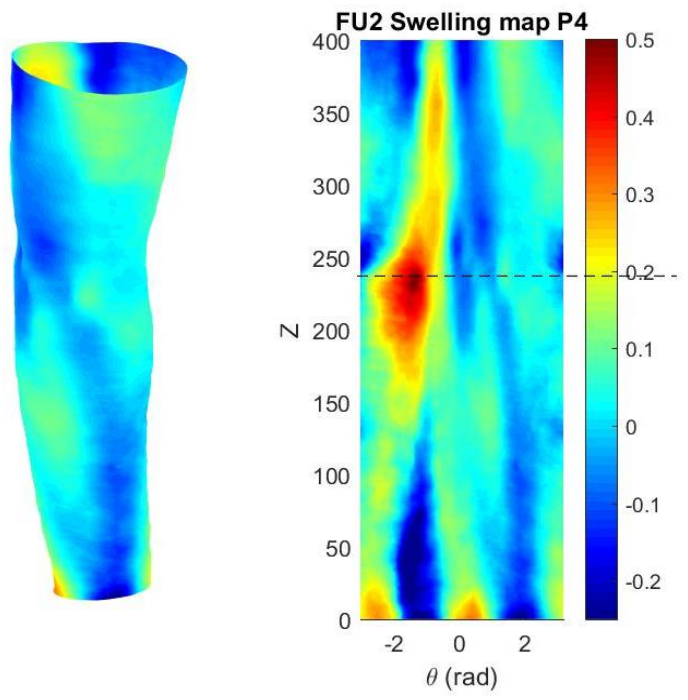

Follow up 2

$\mathrm{CTM}=2 \%$

Perometer $=4.1 \%$
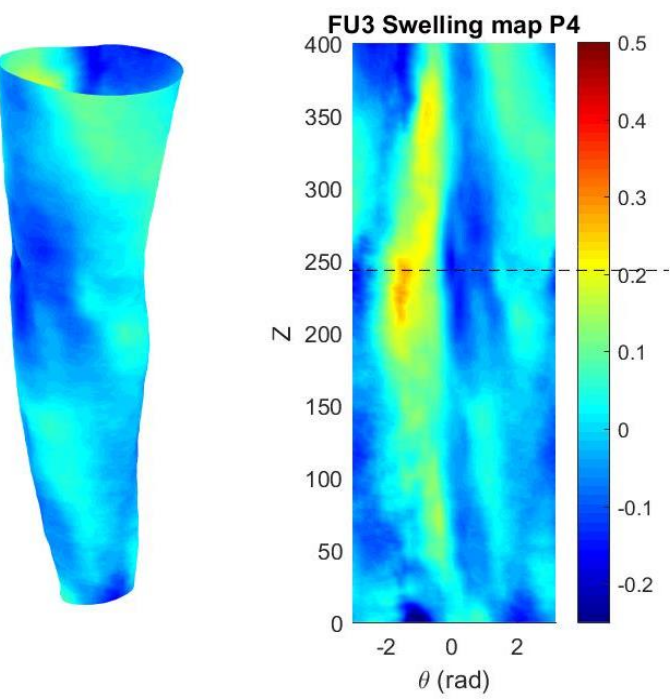

Follow up 3

$\mathrm{CTM}=-2 \%$

Perometer $=0.8 \%$

$3 \mathrm{DCI}=2.4 \%$

Redundancy $=5 \%$

Redundancy $=3 \%$ 


\section{$\underline{\text { Participant } 5}$}

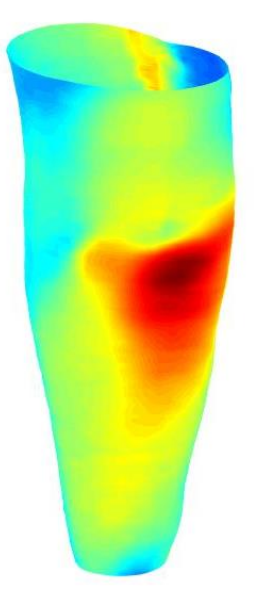

Initial Assessment

$\mathrm{CTM}=21 \%$

Perometer $=18.5 \%$

$3 \mathrm{DCI}=22.7 \%$

Redundancy $=2 \%$

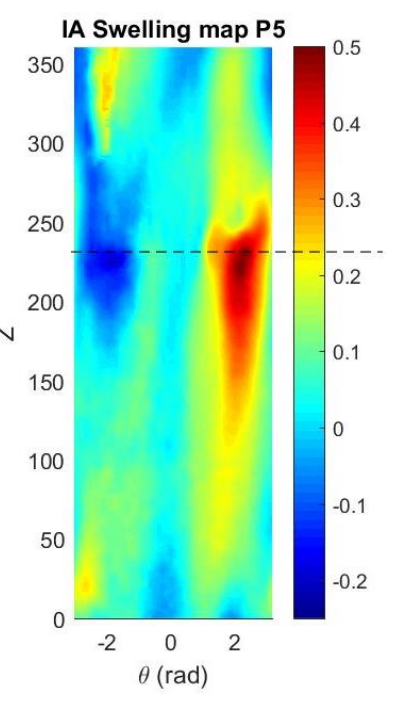




\section{$\underline{\text { Participant } 7}$}

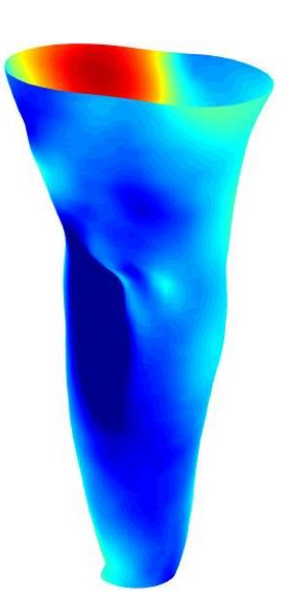

Initial Assessment

$\mathrm{CTM}=5 \%$

Perometer $=-1 \%$

$3 \mathrm{DCI}=6.2 \%$

Redundancy $=6 \%$
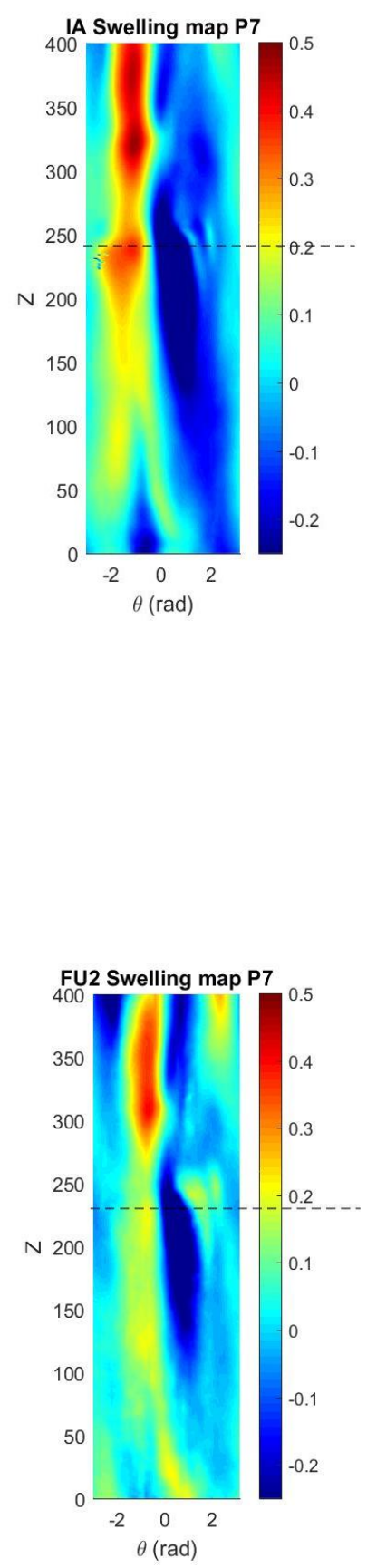

Follow up 2

$\mathrm{CTM}=-1 \%$

Perometer $=-3.2 \%$

$3 \mathrm{DCI}=7.2 \%$

Redundancy $=-42 \%$
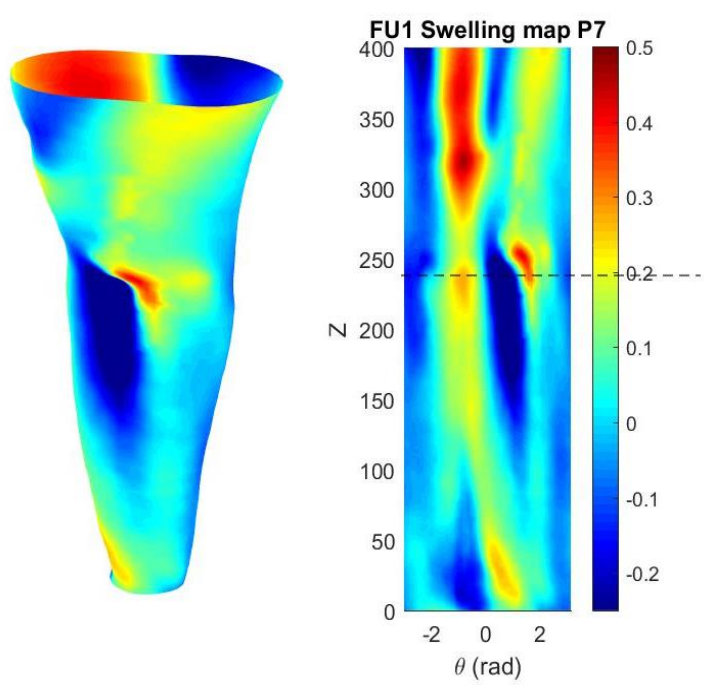

Follow up 1

$\mathrm{CTM}=4 \%$

Perometer $=0 \%$

$3 \mathrm{DCI}=9.5 \%$

Redundancy $=8 \%$
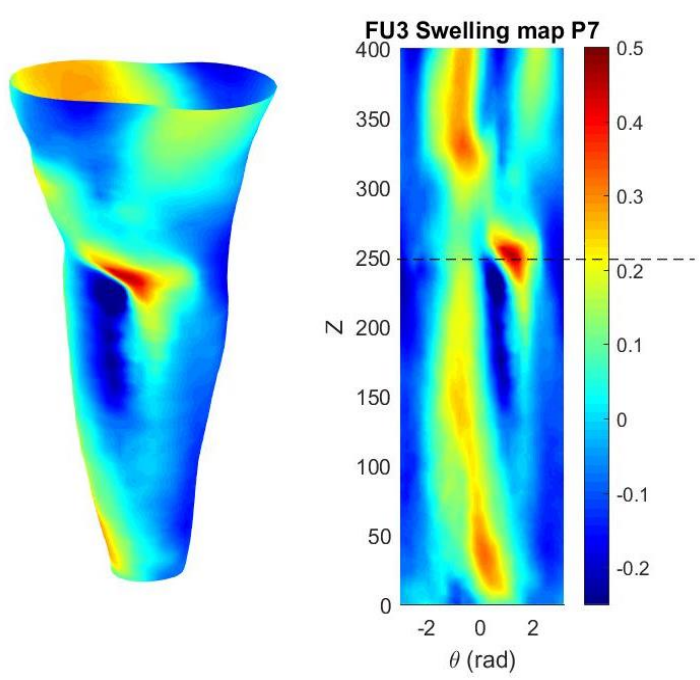

Follow up 3

$\mathrm{CTM}=2 \%$

Perometer $=-2.7 \%$

$3 \mathrm{DCI}=4.3 \%$

Redundancy $=-5 \%$ 


\section{$\underline{\text { Participant } 8}$}

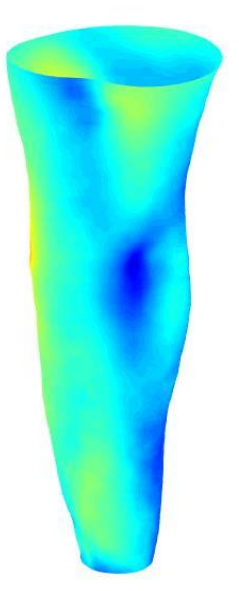

Initial Assessment

$\mathrm{CTM}=10 \%$

Perometer $=5.3 \%$

$3 \mathrm{DCI}=6.6 \%$

Redundancy $=14 \%$
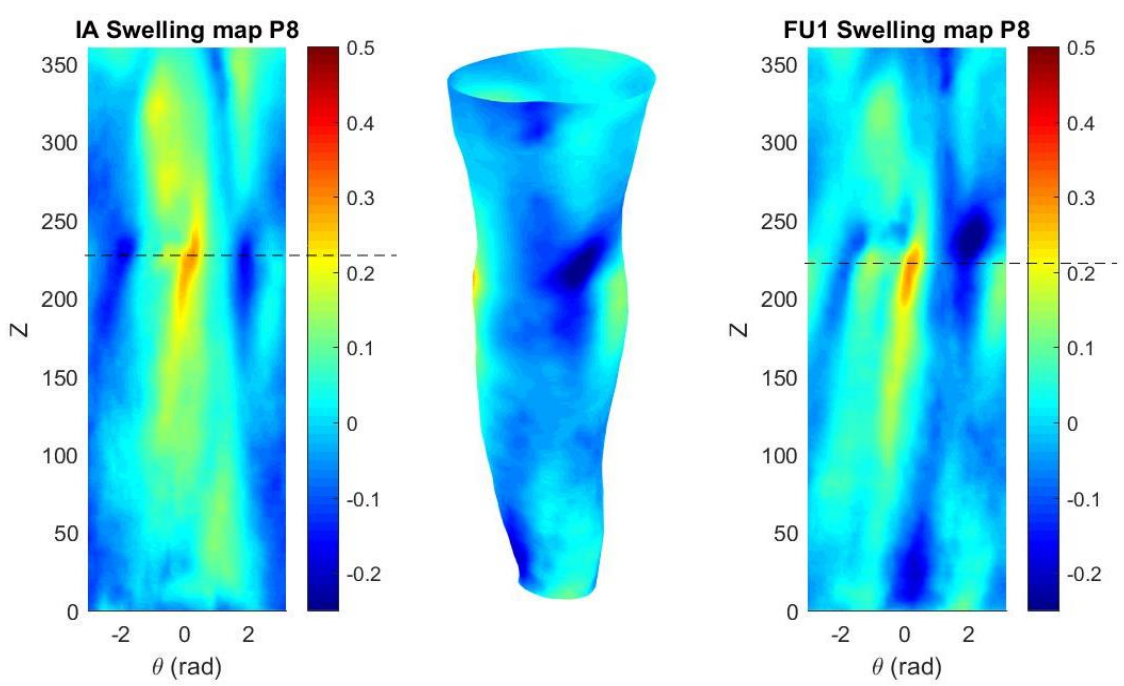

Follow up 1

$\mathrm{CTM}=4 \%$

Perometer $=7 \%$

$3 \mathrm{DCI}=1.2 \%$

Redundancy $=-3 \%$

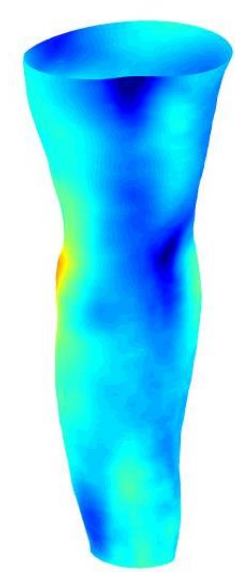

Follow up 2

$\mathrm{CTM}=2 \%$

Perometer $=3.8 \%$

$3 \mathrm{DCI}=0.7 \%$

Redundancy $=-9 \%$
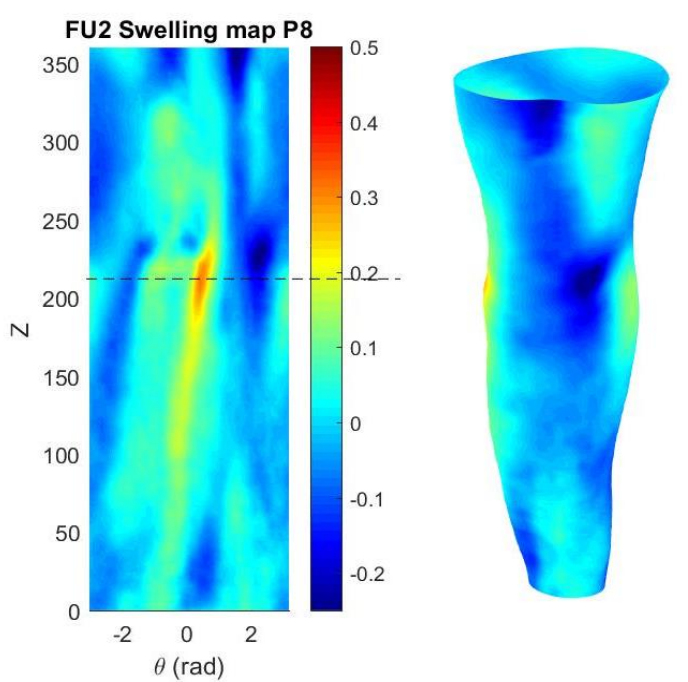

Follow up 3

$\mathrm{CTM}=2 \%$

Perometer $=0.1 \%$

$3 \mathrm{DCI}=-1.5 \%$

Redundancy $=-5 \%$ 


\section{$\underline{\text { Participant } 9}$}
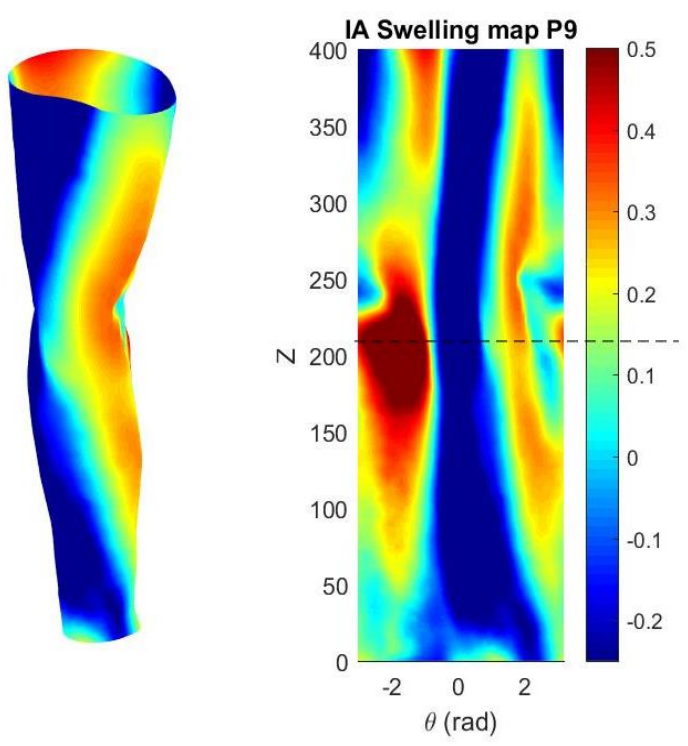

\section{Initial Assessment}

$\mathrm{CTM}=4 \%$

Perometer $=3.9 \%$

$3 \mathrm{DCI}=-6.4 \%$

Redundancy $=-1 \%$
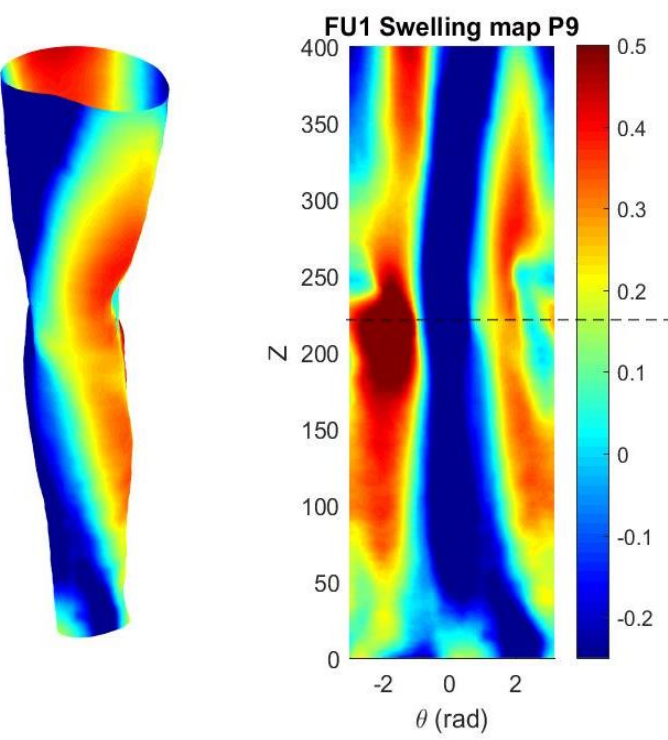

Follow up 1

$\mathrm{CTM}=8 \%$

Perometer $=3.5 \%$

$3 \mathrm{DCI}=-0.9 \%$

Redundancy $=$ calculation error
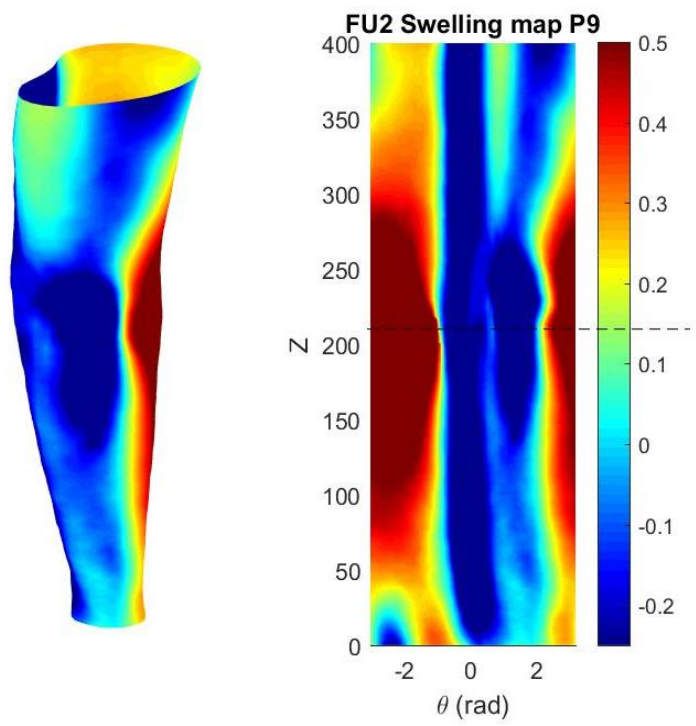

Follow up 2

$\mathrm{CTM}=4 \%$

Perometer $=2 \%$

$3 \mathrm{DCI}=-0.3 \%$

Redundancy $=6 \%$
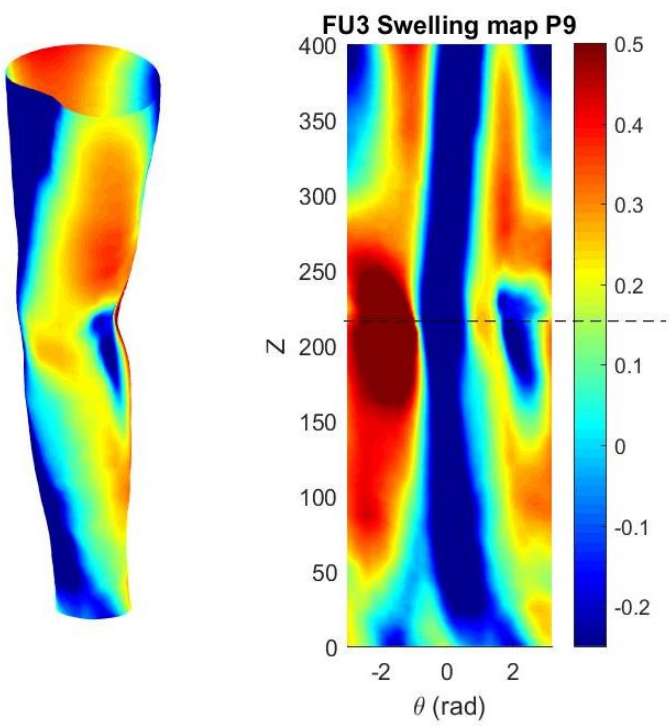

Follow up 3

$\mathrm{CTM}=4 \%$

Perometer $=2.3 \%$

$3 \mathrm{DCI}=1.4 \%$

Redundancy $=-4 \%$ 


\section{Participant 10}

Participant lost to follow up; Initial Assessment image distorted due to mismatched arm position.

\section{Participant 11}
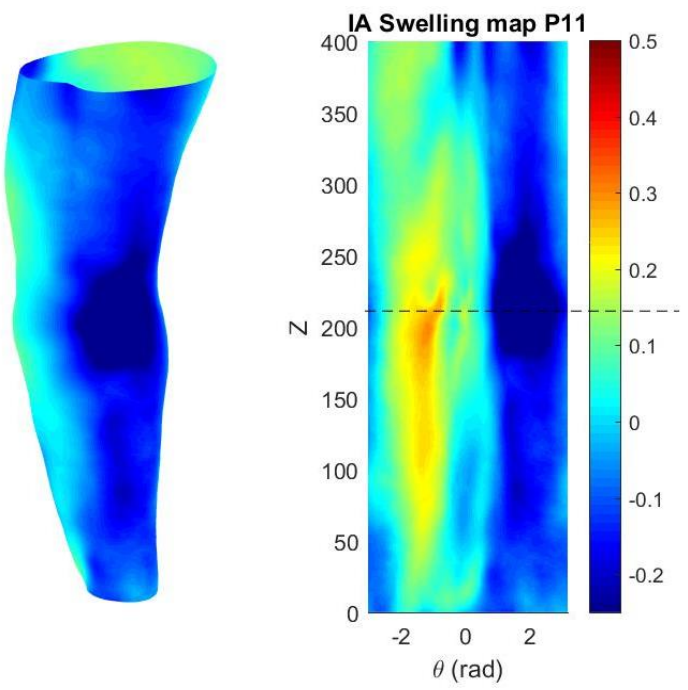

Initial Assessment

$\mathrm{CTM}=5 \%$

Perometer $=3.2 \%$

$3 \mathrm{DCI}=4.0 \%$

Redundancy $=$ calculation error
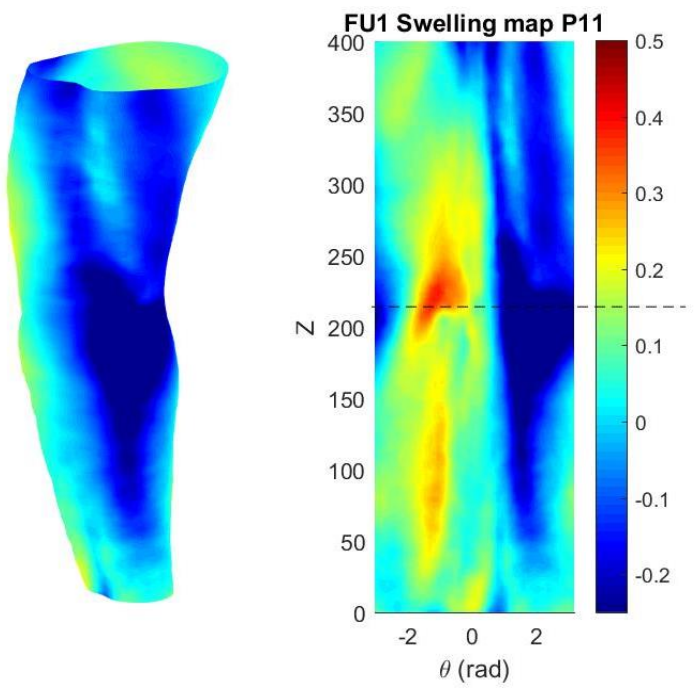

Follow up 1

$\mathrm{CTM}=5 \%$

Perometer $=2.8 \%$

$3 \mathrm{DCI}=8.4 \%$

Redundancy $=-5 \%$
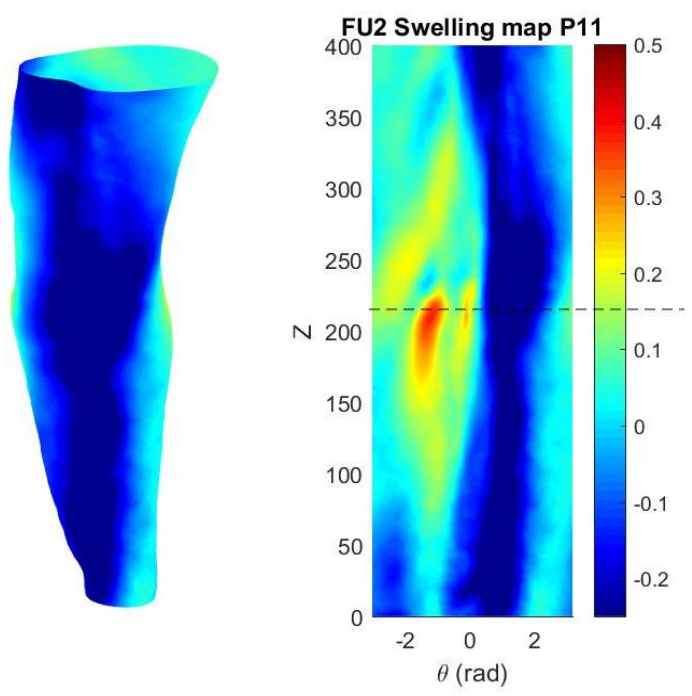

Follow up 2

$\mathrm{CTM}=2 \%$

Perometer $=3.3 \%$
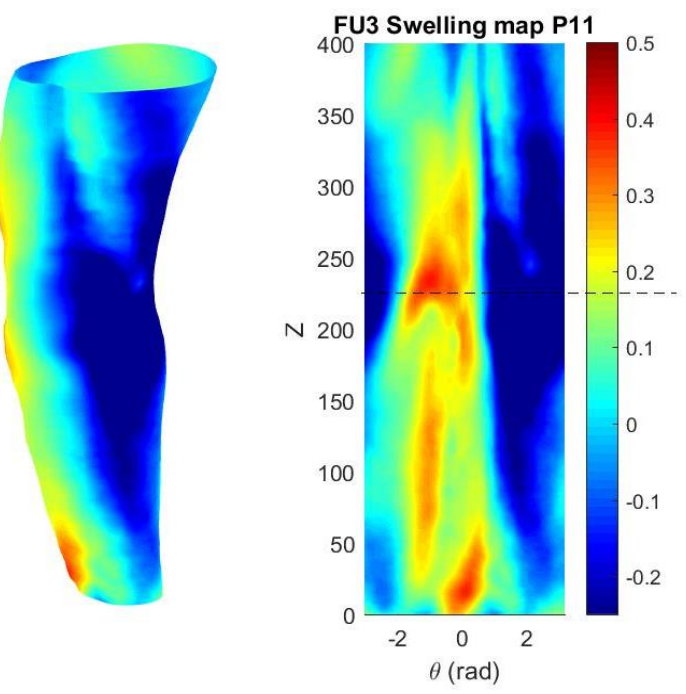

Follow up 3

$\mathrm{CTM}=1 \%$

Perometer $=3 \%$

$3 \mathrm{DCI}=-4.6 \%$

Redundancy $=-41 \%$

$3 \mathrm{DCI}=13.1 \%$

Redundancy $=2 \%$ 


\section{Participant 12}

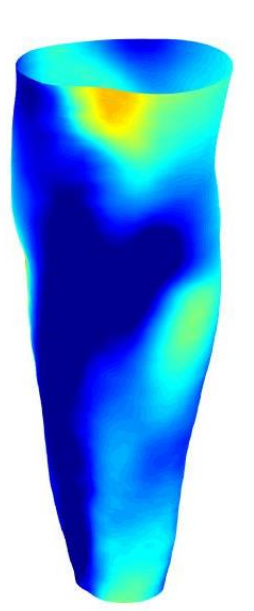

Initial Assessment

$\mathrm{CTM}=-1 \%$

Perometer $=-5.2 \%$

$3 \mathrm{DCI}=-4.9 \%$

Redundancy $=-6 \%$

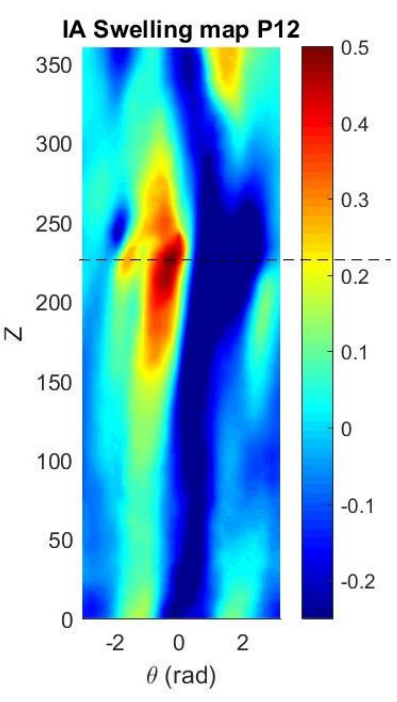

t




\section{$\underline{\text { Participant } 13}$}

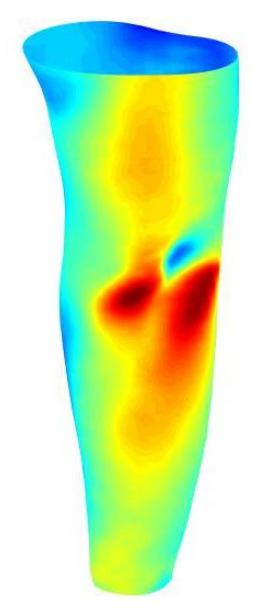

Initial Assessment

$\mathrm{CTM}=13 \%$

Perometer $=9.5 \%$

$3 \mathrm{DCI}=7 \%$

Redundancy $=-1 \%$

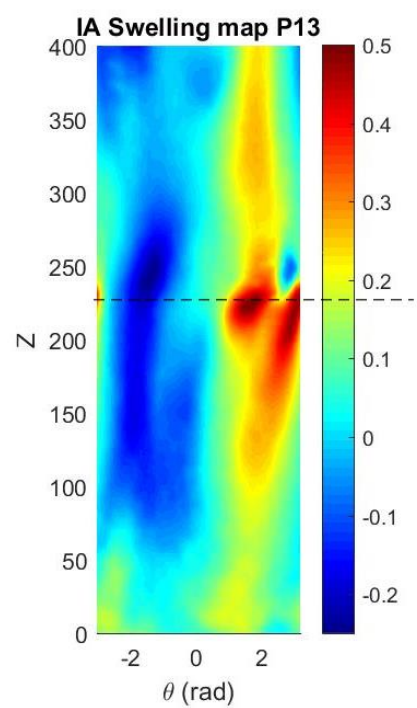

t




\section{Participant 14}

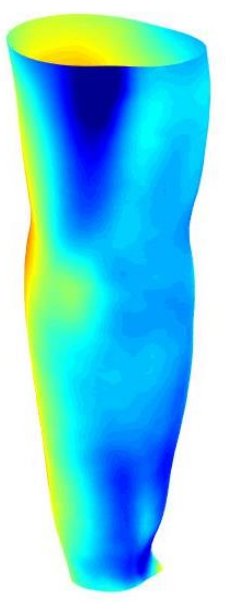

Initial Assessment

$\mathrm{CTM}=32 \%$

Perometer $=38.3 \%$

$3 \mathrm{DCI}=31 \%$

Redundancy $=-15 \%$

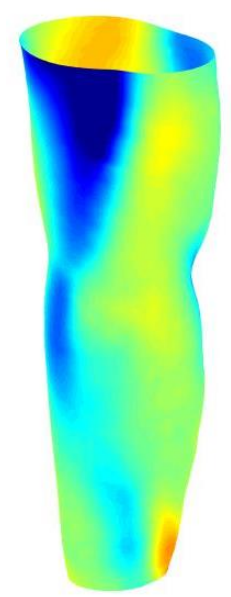

Follow up 2

$\mathrm{CTM}=30 \%$

Perometer $=34.2 \%$

$3 \mathrm{DCI}=35.2 \%$

Redundancy $=14 \%$
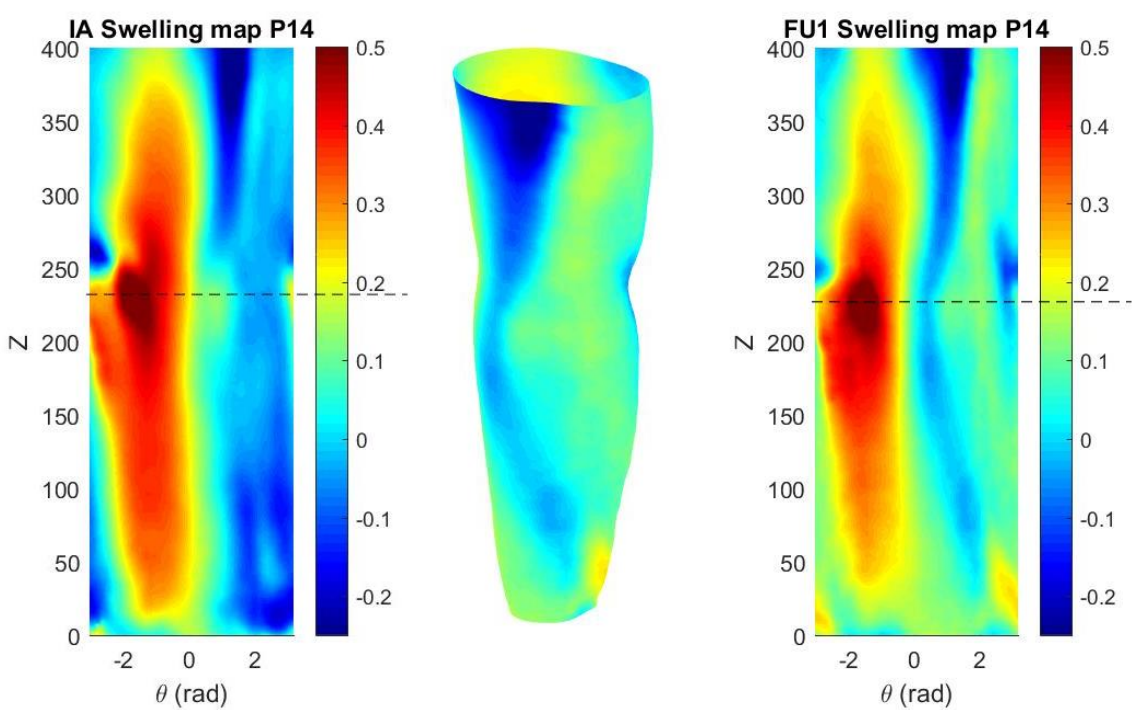

Follow up 1

$\mathrm{CTM}=35 \%$

Perometer $=34.6 \%$

$3 \mathrm{DCI}=32.3 \%$

Redundancy $=10 \%$
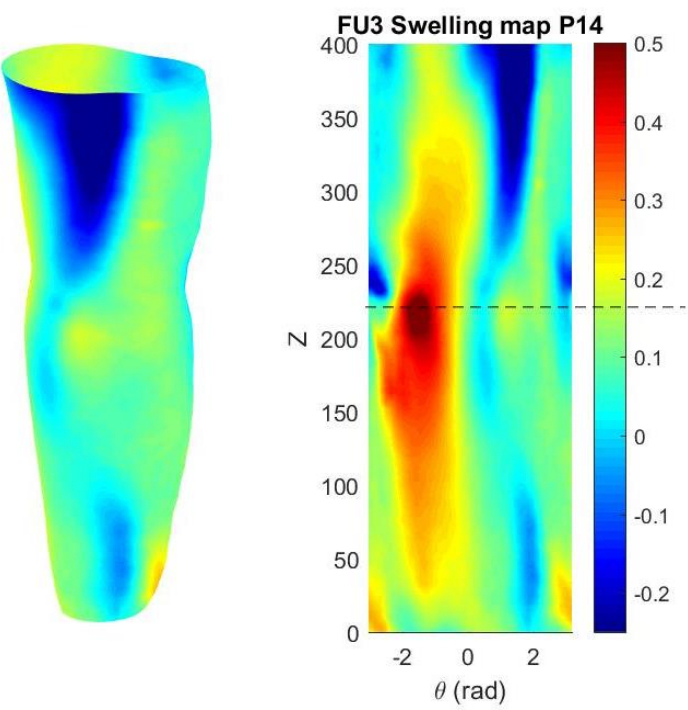

Follow up 3

$\mathrm{CTM}=30 \%$

Perometer $=38.7 \%$

$3 \mathrm{DCI}=30.3 \%$

Redundancy $=14 \%$ 


\section{Participant 15}
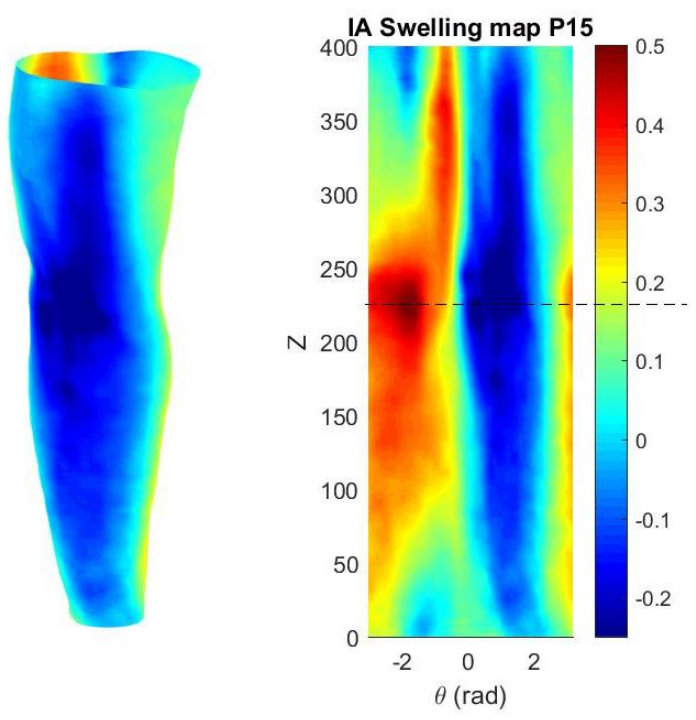

Initial Assessment

$\mathrm{CTM}=7 \%$

Perometer $=2.3 \%$

$3 \mathrm{DCI}=14.1 \%$

Redundancy $=12 \%$
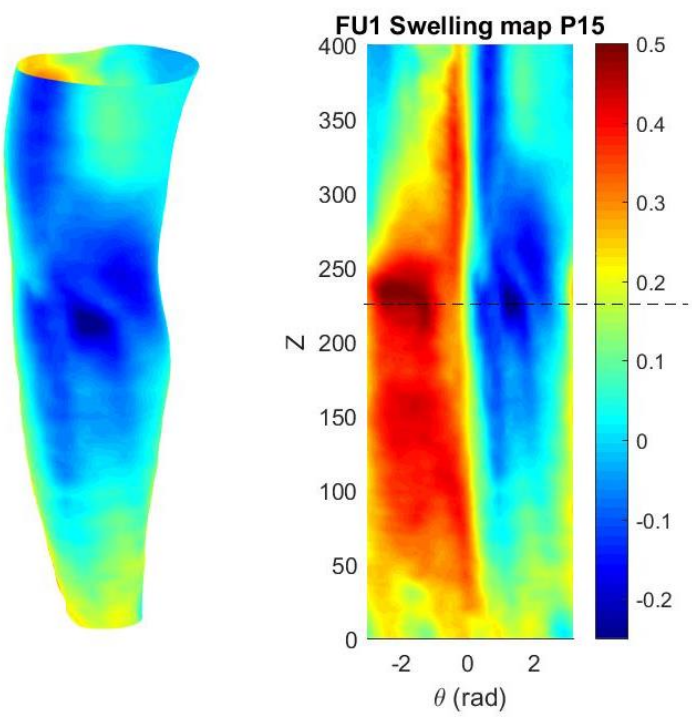

\section{Follow up 1}

$\mathrm{CTM}=8 \%$

Perometer $=9.7 \%$

$3 \mathrm{DCI}=21.2 \%$

Redundancy $=0 \%$
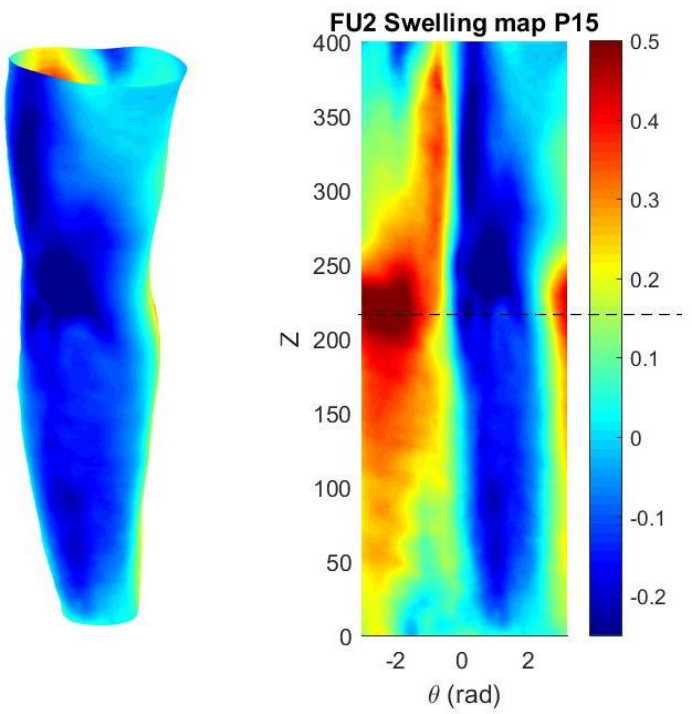

Follow up 2
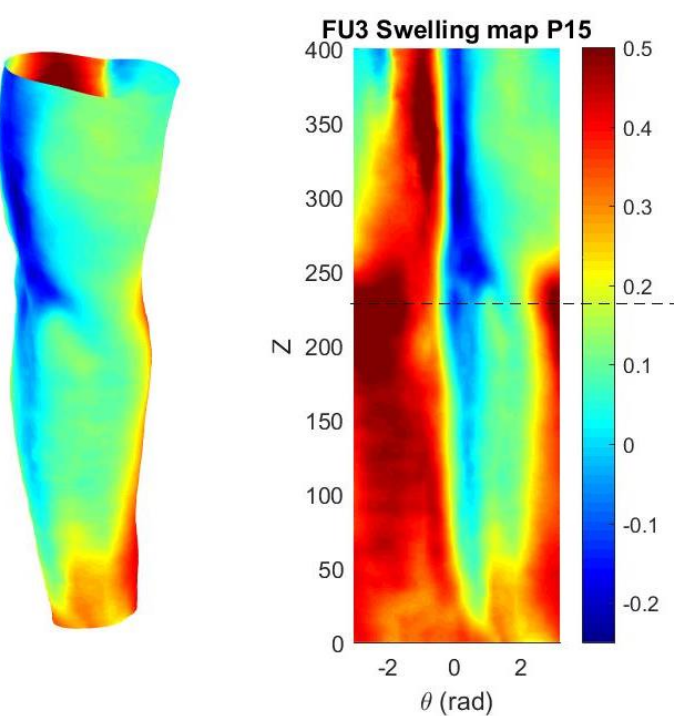

Follow up 3

$\mathrm{CTM}=6 \%$

Perometer $=4.5 \%$

$\mathrm{CTM}=6 \%$

Perometer $=13.5 \%$

$3 \mathrm{DCI}=6.5 \%$

$3 \mathrm{DCI}=42.4 \%$

Redundancy $=17 \%$

Redundancy $=23 \%$ 


\section{Participant 16}
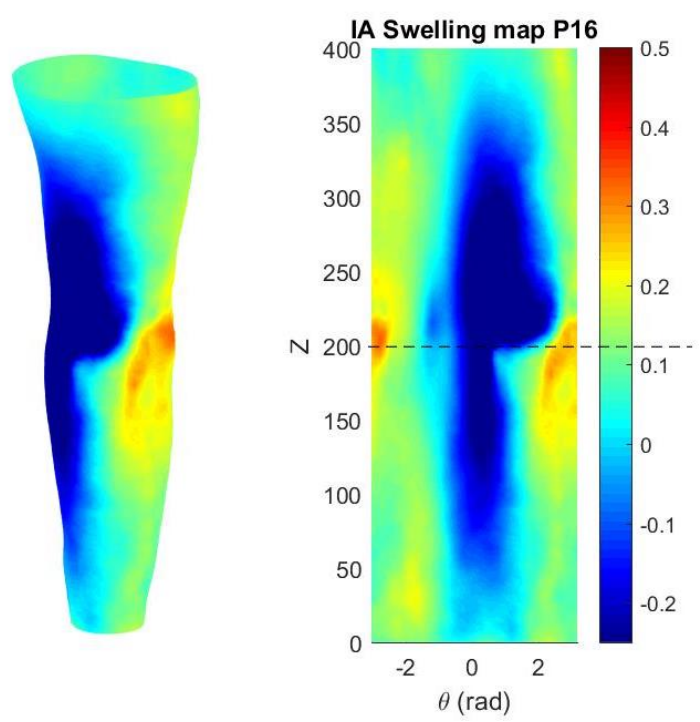

Initial Assessment

$\mathrm{CTM}=14 \%$

Perometer $=17.5 \%$

$3 \mathrm{DCI}=10.4 \%$

Redundancy $=-8 \%$
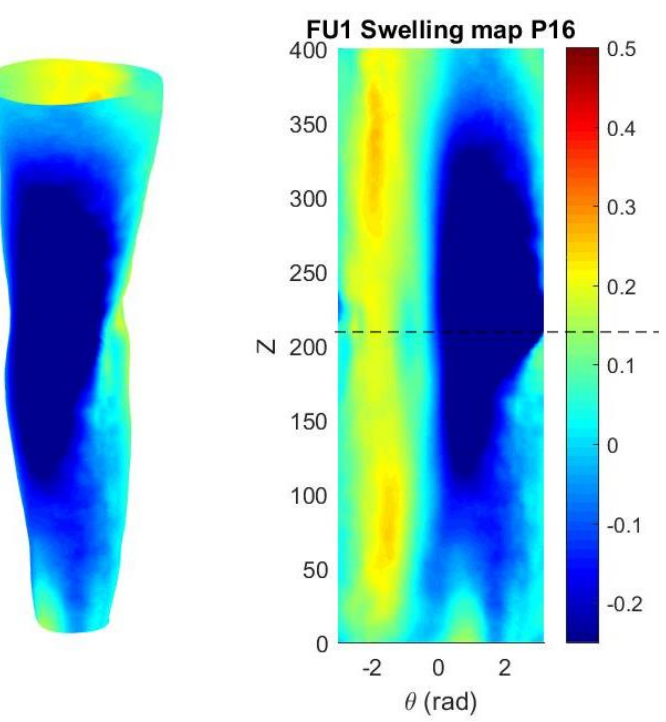

\section{Follow up 1}

$\mathrm{CTM}=9 \%$

Perometer $=8.3 \%$

$3 \mathrm{DCI}=9.3 \%$

Redundancy $=1 \%$
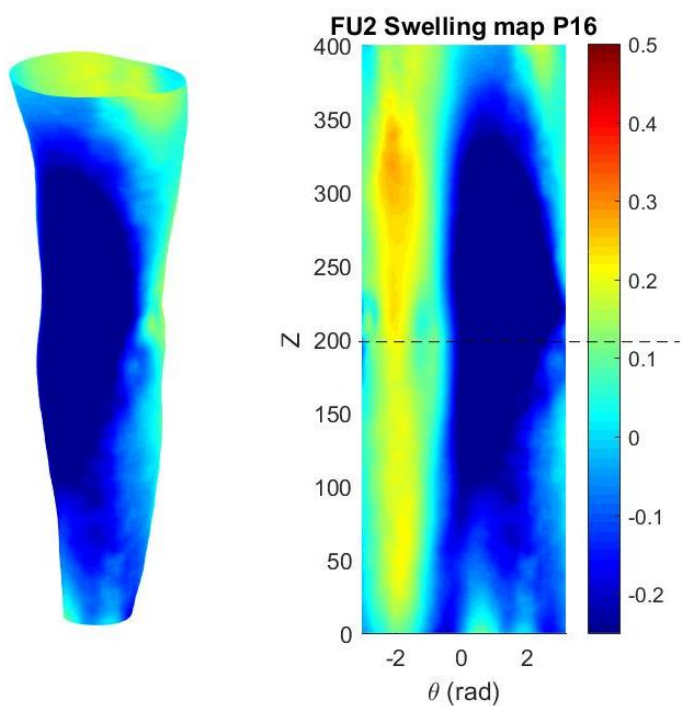

Follow up 2

$\mathrm{CTM}=9 \%$

Perometer $=13 \%$

$3 \mathrm{DCI}=4.2 \%$

Redundancy $=-8 \%$
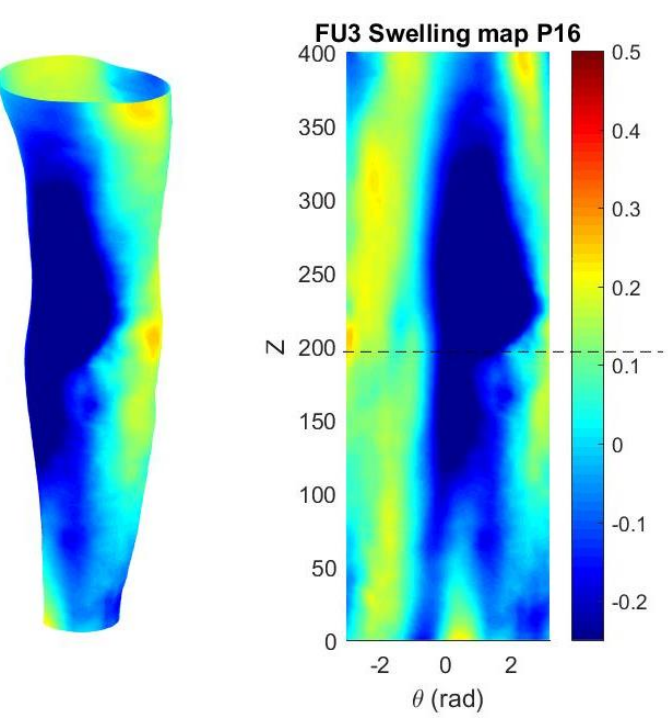

Follow up 3

$\mathrm{CTM}=9 \%$

Perometer $=15.8 \%$

$3 \mathrm{DCI}=6 \%$

Redundancy $=2 \%$ 


\section{Participant 17}
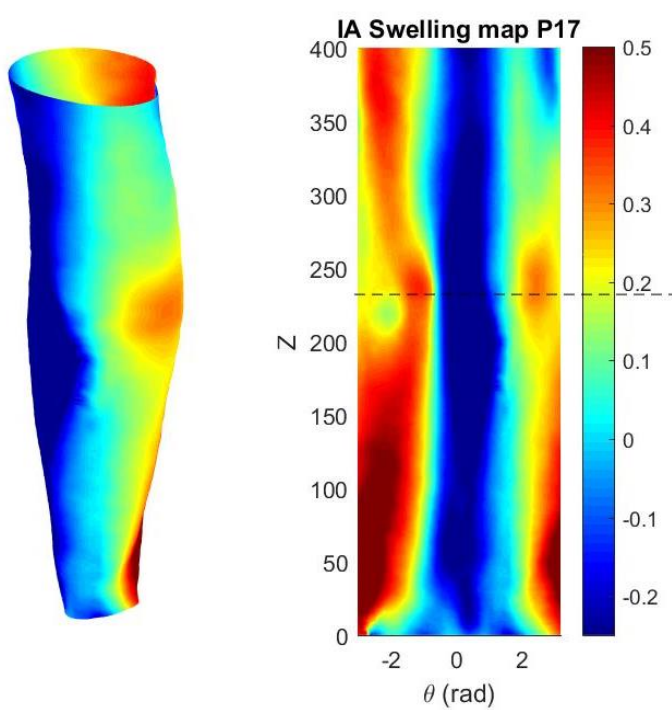

Initial Assessment

$\mathrm{CTM}=10 \%$

Perometer $=6.7 \%$

$3 \mathrm{DCI}=11.3 \%$

Redundancy $=0 \%$
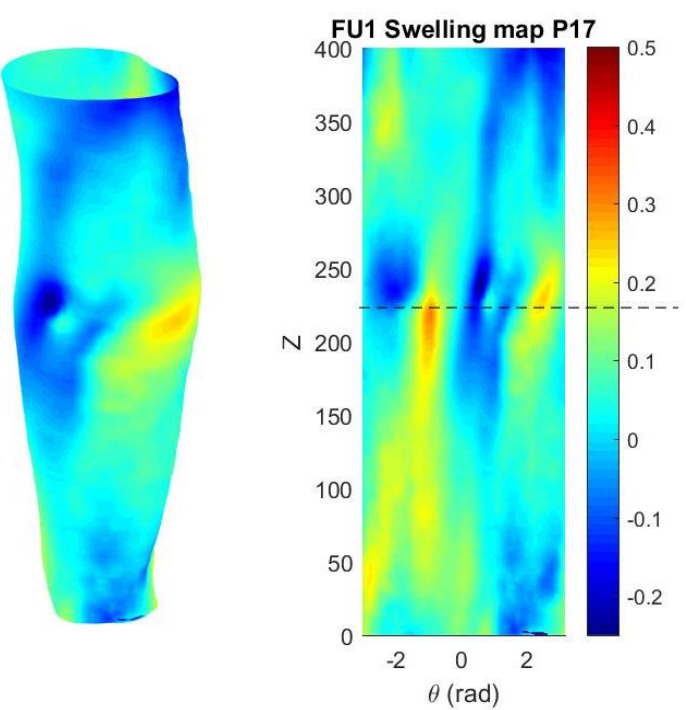

Follow up 1

$\mathrm{CTM}=3 \%$

Perometer $=6.8 \%$

$3 \mathrm{DCI}=9.9 \%$

Redundancy $=-1 \%$
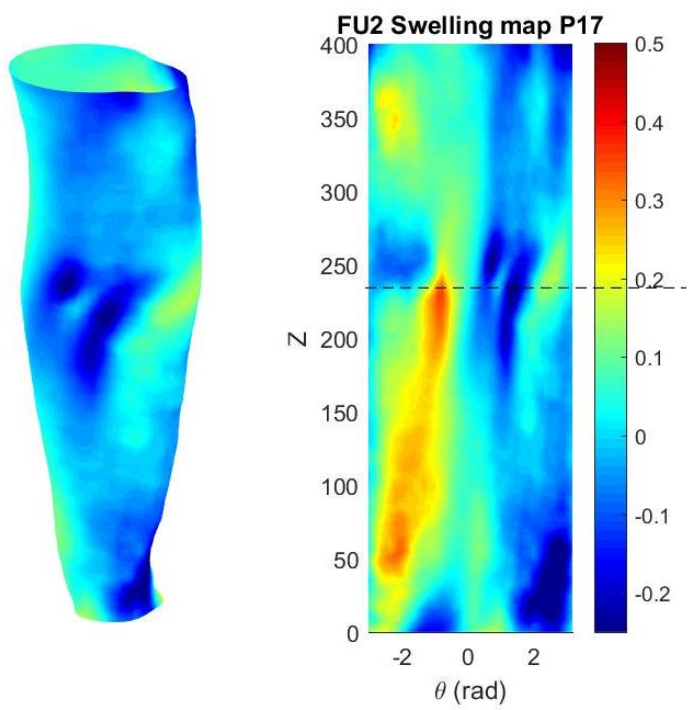

Follow up 2

$\mathrm{CTM}=4 \%$

Perometer $=7.5 \%$

$3 \mathrm{DCI}=5.6 \%$

Redundancy $=-3 \%$
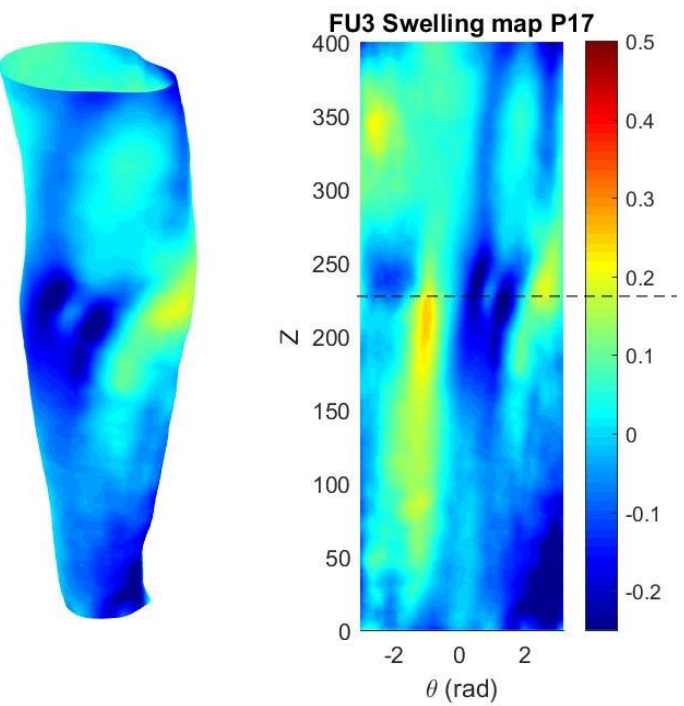

Follow up 3

$\mathrm{CTM}=5 \%$

Perometer $=8.4 \%$

$3 \mathrm{DCI}=0.7 \%$

Redundancy $=11 \%$ 


\section{Participant 18}

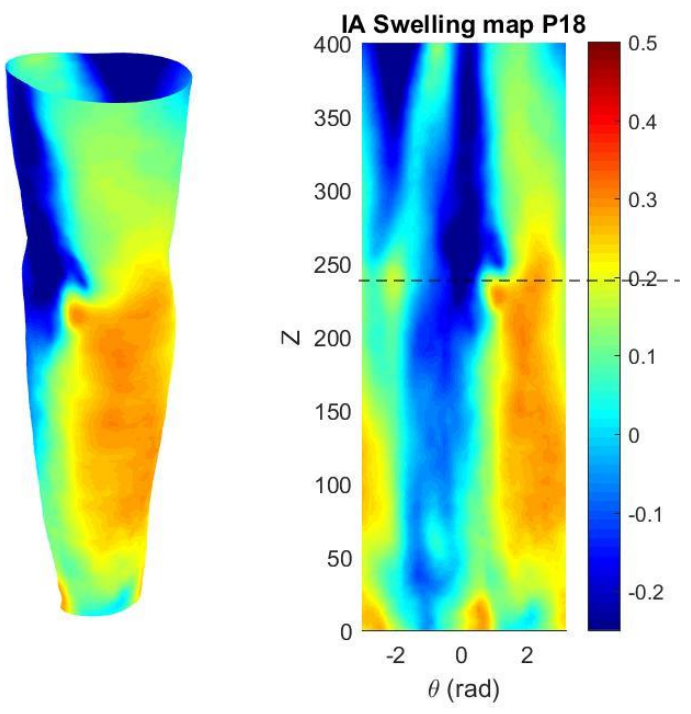

\section{Initial Assessment}

\section{Follow up 1}

$\mathrm{CTM}=6 \%$

Particiapnt unable to attend

Perometer $=4.1 \%$

$3 \mathrm{DCI}=6 \%$

Redundancy $=16 \%$
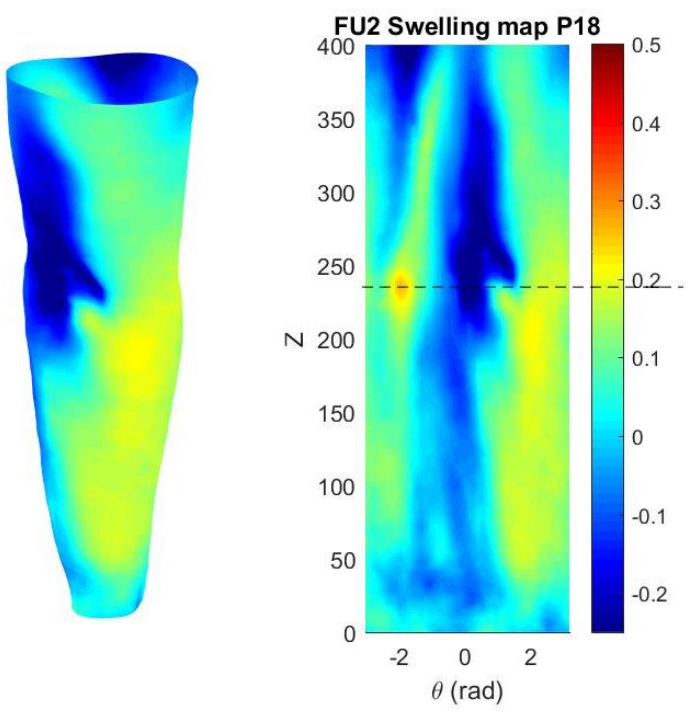

Follow up 2

$\mathrm{CTM}=3 \%$

Perometer $=9 \%$

$3 \mathrm{DCI}=3.7 \%$

Redundancy $=-1 \%$

\section{Follow up 3}

Did not attend 


\section{Participant 19}
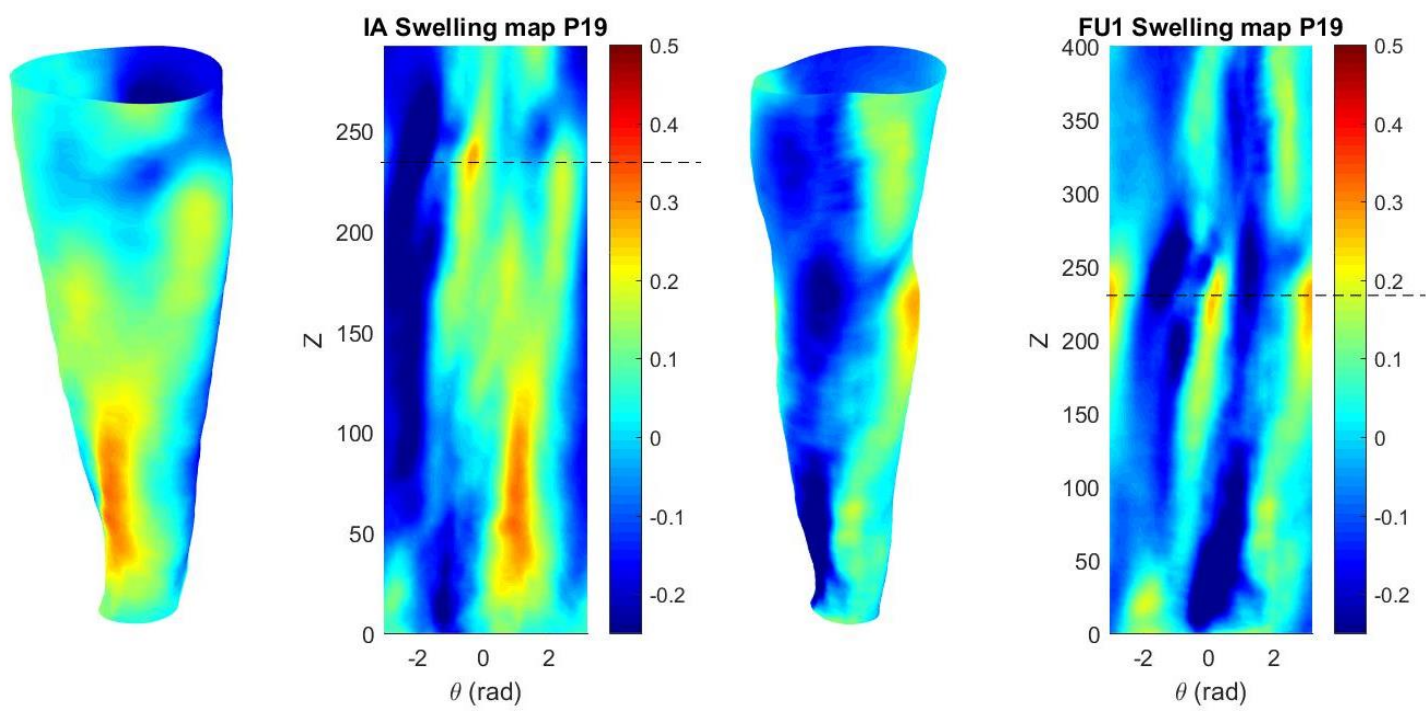

\section{Initial Assessment}

$\mathrm{CTM}=-2 \%$

Perometer $=3.7 \%$

$3 \mathrm{DCI}=-5.4 \%$

Redundancy $=-4 \%$
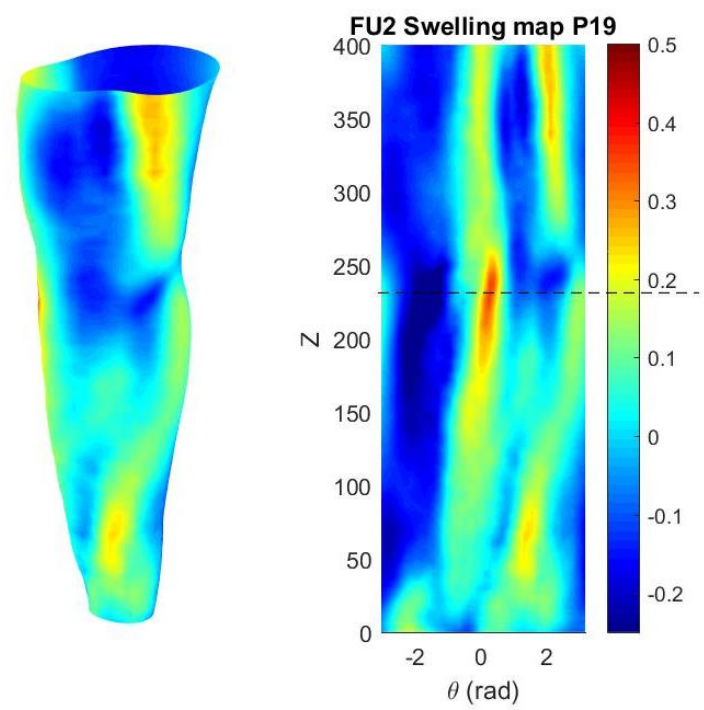

Follow up 2

$\mathrm{CTM}=-1 \%$

Perometer $=-1.1 \%$

$3 \mathrm{DCI}=-2.3 \%$

Redundancy $=0 \%$

\section{Follow up 3}

Did not attend

Follow up 1

$\mathrm{CTM}=-1 \%$

Perometer $=-2.9 \%$

$3 \mathrm{DCI}=-6.6 \%$

Redundancy $=-10 \%$

$\underline{\text { Participant } 20}$ - Data corrupted for all four sessions 


\section{APPENDIX 15: Phase 2: Tests of Normality}

\section{Shape Redundancy}

Tests of normality of the Shape Redundancy (R-value) data for percentage difference had an initial skewness more than four times the standard error, with kurtosis sevent times greater than the standard error. The Shapiro-Wilk test result for R-value had a pvalue $<0.05$, indicating that the distribution deviates from normal (Laerd Statistics, 2018b). The Q-Q Plot supports this outcome as the data deviates significantly from the diagonal line.

\begin{tabular}{|c|c|c|c|c|}
\hline \multicolumn{5}{|c|}{ Descriptives for Shape Redundancy Percentage Differences } \\
\hline & & & Statistic & Std. Error \\
\hline \multirow{13}{*}{$\begin{array}{l}\text { Standardized Residual for } \\
\text { RedundancyP }\end{array}$} & Mean & & .0000 & .12910 \\
\hline & \multirow{2}{*}{$\begin{array}{l}95 \% \text { Confidence Interval for } \\
\text { Mean }\end{array}$} & Lower Bound & -.2583 & \\
\hline & & Upper Bound & .2583 & \\
\hline & 5\% Trimmed Mean & & .0627 & \\
\hline & Median & & -.0249 & \\
\hline & Variance & & 1.000 & \\
\hline & Std. Deviation & & 1.00000 & \\
\hline & Minimum & & -3.68 & \\
\hline & Maximum & & 1.97 & \\
\hline & Range & & 5.64 & \\
\hline & Interquartile Range & & 1.14 & \\
\hline & Skewness & & -1.288 & .309 \\
\hline & Kurtosis & & 4.399 & .608 \\
\hline
\end{tabular}

\begin{tabular}{|c|c|c|c|c|c|c|}
\hline \multicolumn{7}{|c|}{ Tests of Normality for Shape Redundancy Percentage Differences } \\
\hline & \multicolumn{3}{|c|}{ Kolmogorov-Smirnov ${ }^{\mathrm{a}}$} & \multicolumn{3}{|c|}{ Shapiro-Wilk } \\
\hline & Statistic & df & Sig. & Statistic & df & Sig. \\
\hline $\begin{array}{l}\text { Standardized Residual for } \\
\text { Shape Redundancy }\end{array}$ & .122 & 60 & .026 & .888 & 60 & .000 \\
\hline
\end{tabular}

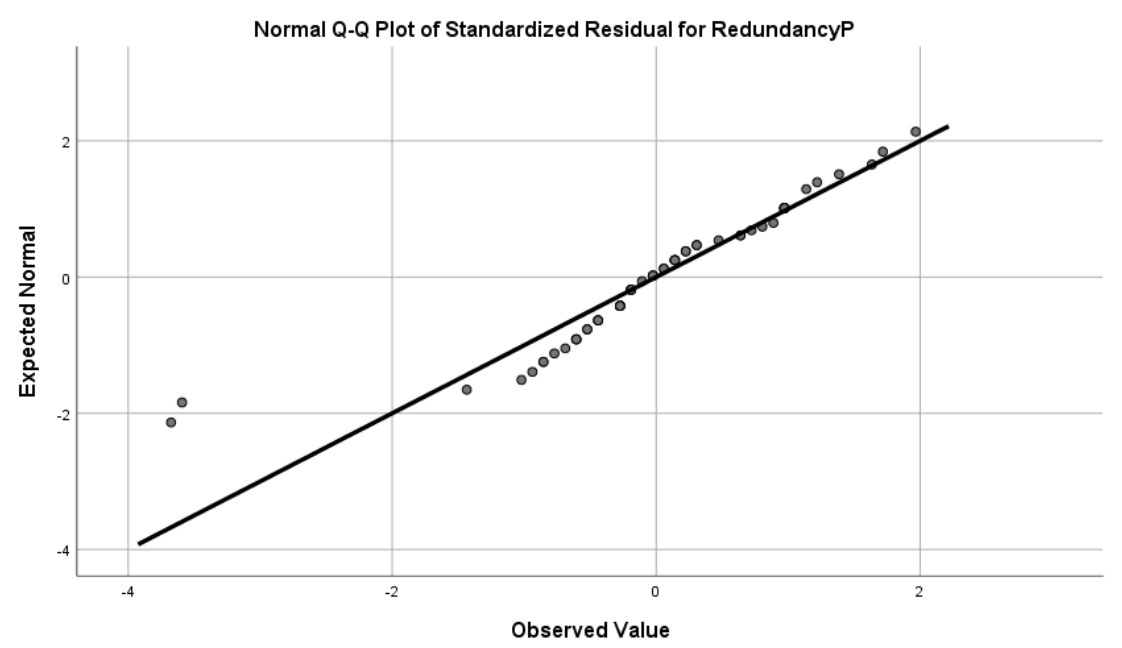

Transforming the data for normalisation was performed using the square root of the Rvalue data. Both the skewness and kurtosis were significantly less than one times the 
standard error, and both the Kolmogorov-Smirnov and Shapiro-Wilk tests of normality were non-significant ( $p>0.05$ ), as confirmed by the improved Q-Q Plot of data following the normative line more closely. The logarithm of the data was also calculated, but this overcorrected the data. Thus, the square root of the Shape Redundancy percentage differences was used for the Bland Altman plots.

\begin{tabular}{|c|c|c|c|c|}
\hline \multicolumn{5}{|c|}{ Descriptives } \\
\hline & & & Statistic & Std. Error \\
\hline \multirow{13}{*}{$\begin{array}{l}\text { Standardized Residual for } \\
\text { SqRtRvalue }\end{array}$} & Mean & & .0000 & .16440 \\
\hline & \multirow{2}{*}{$\begin{array}{l}\text { 95\% Confidence Interval for } \\
\text { Mean }\end{array}$} & Lower Bound & -.3334 & \\
\hline & & Upper Bound & .3334 & \\
\hline & 5\% Trimmed Mean & & .0069 & \\
\hline & Median & & -.0830 & \\
\hline & Variance & & 1.000 & \\
\hline & Std. Deviation & & 1.00000 & \\
\hline & Minimum & & -1.75 & \\
\hline & Maximum & & 1.72 & \\
\hline & Range & & 3.48 & \\
\hline & Interquartile Range & & 1.48 & \\
\hline & Skewness & & -.319 & .388 \\
\hline & Kurtosis & & -.777 & .759 \\
\hline
\end{tabular}

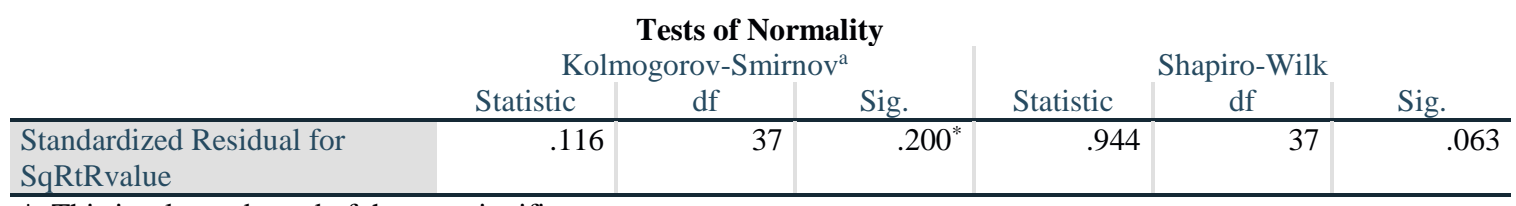

*. This is a lower bound of the true significance.

a. Lilliefors Significance Correction

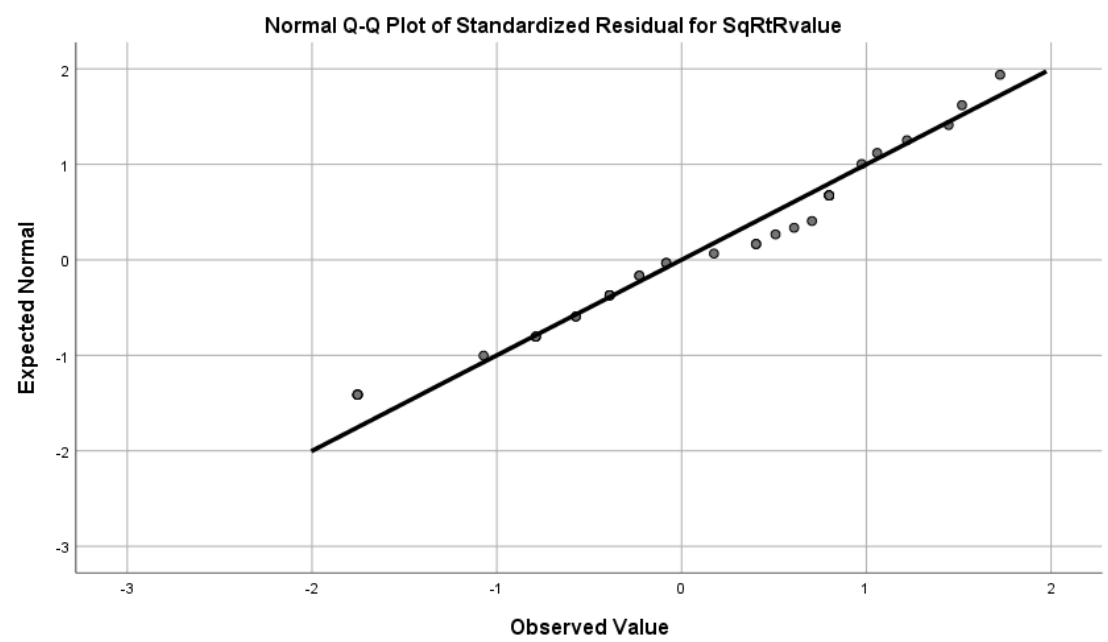

